\title{
Panorama des administrations publiques 2019
}

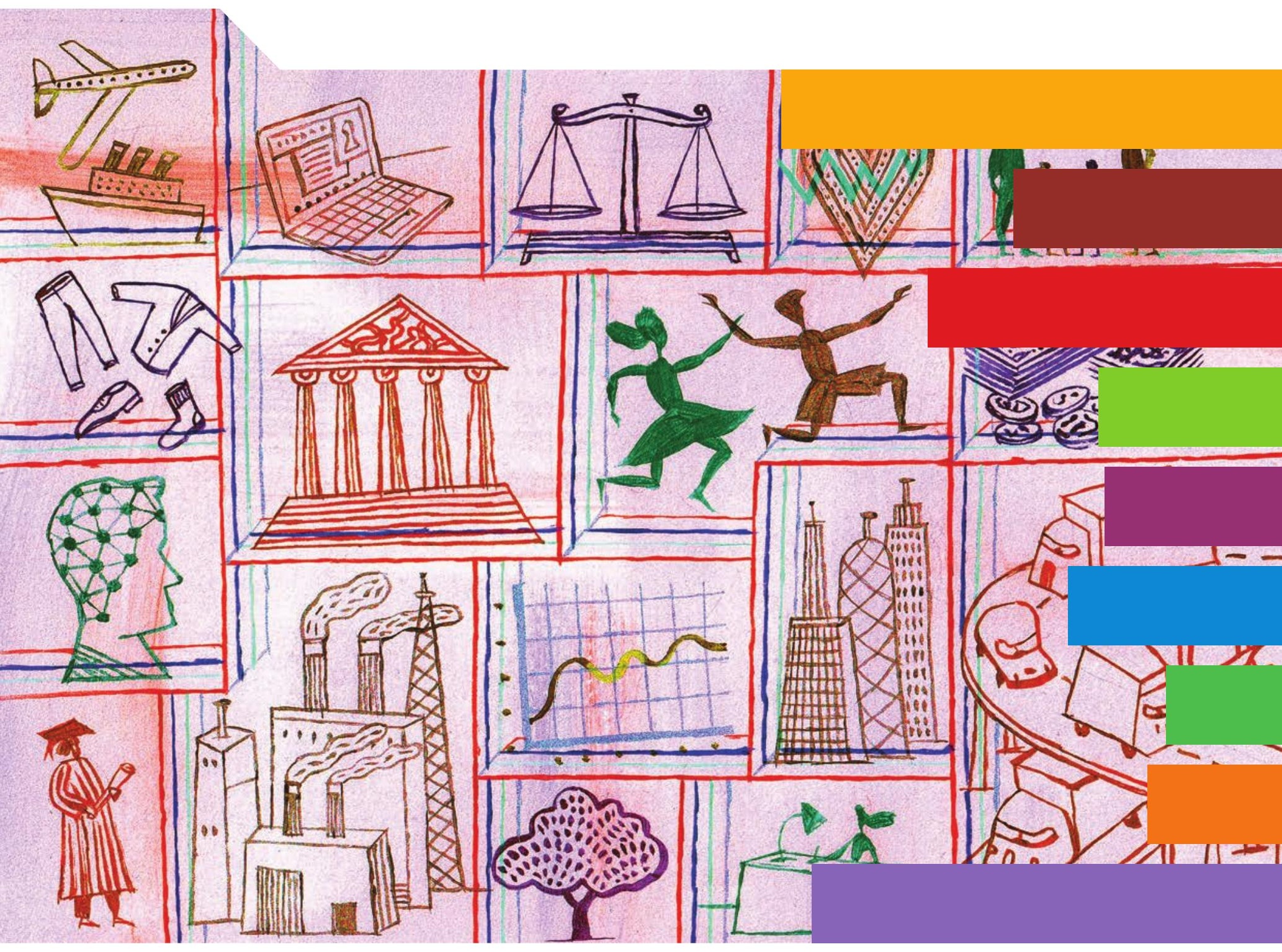

Q) 



\section{Panorama \\ des administrations \\ publiques \\ 2019}

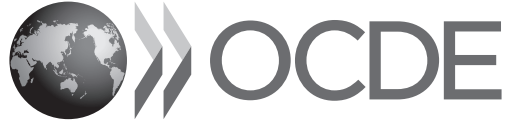


Cet ouvrage est publié sous la responsabilité du Secrétaire général de l'OCDE. Les opinions et les arguments exprimés ici ne reflètent pas nécessairement les vues officielles des pays membres de l'OCDE.

Ce document, ainsi que les données et cartes qu'il peut comprendre, sont sans préjudice du statut de tout territoire, de la souveraineté s'exerçant sur ce dernier, du tracé des frontières et limites internationales, et du nom de tout territoire, ville ou région.

Les données statistiques concernant Israël sont fournies par et sous la responsabilité des autorités israéliennes compétentes. L'utilisation de ces données par l'OCDE est sans préjudice du statut des hauteurs du Golan, de Jérusalem-Est et des colonies de peuplement israéliennes en Cisjordanie aux termes du droit international.

Merci de citer cet ouvrage comme suit:

OCDE (2019), Panorama des administrations publiques 2019, Éditions OCDE, Paris, https://doi.org/10.1787/8be847c0-fr.

ISBN 978-92-64-76224-4 (imprimé)

ISBN 978-92-64-91052-2 (pdf)

ISBN 978-92-64-46277-9 (HTML)

ISBN 978-92-64-90312-8 (epub)

Panorama des administrations publiques

ISSN 2221-4402 (imprimé)

ISSN 2221-4410 (en ligne)

Crédits photo : Illustration de la couverture @ Jeffrey Fisher.

Les corrigenda des publications sont disponibles sur: www.oecd.org/about/publishing/corrigenda.htm.

(c) OCDE 2019

L'utilisation de ce contenu, qu'il soit numérique ou imprimé, est régie par les conditions d'utilisation suivantes : http://www.oecd.org/fr/ conditionsdutilisation. 


\section{Avant-propos}

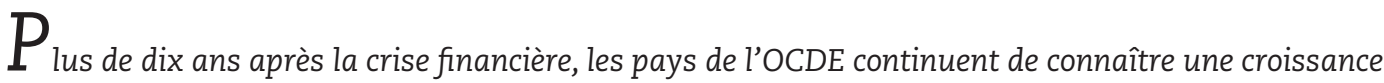
économique relativement atone. Ils subissent aussi le poids d'un endettement public important, et doivent relever les défis liés au vieillissement démographique et à un paysage technologique en pleine mutation. Dans le même temps, les effets de la crise continuent de peser sur la confiance des citoyens à l'égard des institutions publiques et sur la façon dont ils perçoivent la réactivité, l'intégrité et l'efficience du secteur public, ce qui limite l'aptitude des gouvernants à mettre en œuvre les réformes nécessaires. Dans ce contexte, il devient urgent d'adopter, pour l'action publique et la prestation des services publics, une démarche centrée sur l'humain. Une telle démarche implique que les pouvoirs publics tiennent mieux compte, dans la conception, la mise en œuvre et l'évaluation des politiques et services publics, des besoins et des attentes exprimés par les citoyens.

Le Panorama des administrations publiques 2019 constitue la sixième édition de cette publication phare du Comité de la gouvernance publique de l'OCDE. Il est axé sur les progrès accomplis par les pays de l'OCDE s'agissant de mettre l'humain au centre de la gestion publique, de la gouvernance publique et des services publics. Les données montrent que les pays font des progrès sur le plan de leur prestation de services, ce qu'illustre l'évolution favorable des niveaux de satisfaction des citoyens à l'égard des principaux services publics; le recours à des pratiques de gouvernance publique centrées sur l'humain progresse.

La série des Panoramas des administrations publiques a pour objet principal de fournir des données fiables et comparables à l'échelle internationale sur les activités des administrations publiques des pays de l'OCDE, des pays candidats à l'adhésion à l'OCDE et d'autres grandes économies. Ces données peuvent être mises à profit pour comparer les performances des secteurs publics, suivre les évolutions intervenues à l'échelle nationale et internationale et recueillir des éléments probants à l'appui de l'action publique.

Comme pour chaque édition, les indicateurs couvrent l'intégralité du processus de production des administrations publiques : des intrants aux réalisations, en passant par les processus et les produits. L'une des caractéristiques qui fait l'originalité de cette publication est que les données relatives à la gestion et à la gouvernance publiques sont recueillies au moyen d'enquêtes menées par l'OCDE auprès de responsables publics et validées par des experts de l'OCDE. On trouvera dans cette édition des chapitres sur le rôle et les responsabilités de certaines institutions, les pratiques et procédures budgétaires, la gestion des ressources humaines, la gouvernance de la réglementation, les marchés publics et l'ouverture et la transition numérique de l'administration. Les données portant sur les intrants sont axées sur les finances publiques et l'emploi public. Sur le plan des réalisations, on trouvera des indicateurs relatifs aux principaux résultats de l'action publique - tels que la confiance à l'égard des pouvoirs publics, le sentiment d'efficacité politique ou la réduction des inégalités de revenu. Toujours sur le plan des réalisations, on trouvera aussi des indicateurs portant sur l'accessibilité, la réactivité et la qualité des services publics de l'éducation, de la santé et de la justice, ainsi que sur le degré de satisfaction des citoyens à leur égard. 
Les indicateurs présentés dans le Panorama des administrations publiques sont uniques. Ils ont été élaborés pour suivre la mise en œuvre par les pays des principes et recommandations de l'OCDE concernant diverses pratiques de gouvernance publique. Ils constituent donc des éléments de référence reconnus dans de nombreux domaines de la gouvernance publique, et permettent d'éclairer et d'étayer les discussions menées à l'échelle du monde entier sur l'action à mener. 


\section{Remerciements}

$\mathrm{P}$ anorama des administrations publiques 2019 a été prépare par la Direction de la Gouvernance Publique de l'OCDE sous la direction de Marcos Bonturi, directeur. Ces travaux ont été conduits par Zsuzsanna Lonti, sous la direction d'Irène Hors et de Monica Brezzi. La publication a été rédigée par Barbara Baredes, Santiago González, Alessandro Lupi, and Minjoo Son. L'assistance de Clara Delcourt a été très précieuse. D'importantes contributions ont été rédigées et transmises par Daniel Gerson, Donal Mulligan and Pinar Guven (chapitre 3: Emploi publique); Stéphane Jacobzone, Claire Salama, Diego Millan (chapitre 4: Les institutions); Andrew Blazey, Delphine Moretti, Juliana Jansen, Anne Keller, Scherie Nicol, Ana-Maria Ruiz (chapitre 5: Les pratiques et procédures budgétaires); Daniel Gerson and Donal Mulligan (chapitre 6: Gestion des ressources humaines); Christiane ArndtBascle, Benjamin Gerloff, Rebecca Schultz (chapitre 7: La gouvernance de la réglementation); Janos Bertok, Paulo Magina, Matthieu Cahen, Antoine Comps, Fleur D'Souza, Tessa Cullen (chapitre 8: Marchés publics); Barbara Ubaldi, Reginald Dadzie, Jacob Arturo Riviera Perez, Joao Vasconcelos, Zina Akrout, Cecilia Emilsson (chapitre 9: Gouvernement numérique et données publiques ouvertes). Nous remercions Janos Bertok, Edwin Lau, Andrew Blazey, Eva Beuselinck, Stéphane Jacobzone et Barbara Ubaldi pour leurs précieux commentaires au chapitre 1. Nous remercions aussi Andrea Uhrhammer pour le support éditorial, ainsi que Julie Harris et Joelle Palmieri de nous avoir aidés à préparer le document en vue de sa publication. La traduction en français est à mettre au crédit de Maia Kirilovski, Frederic Berri and Maria Eiguren.

Cette publication est le fruit de contributions d'un large éventail de sources et d'experts. Elle a bénéficié de travaux de Comité de la gouvernance publique de l'OCDE et du Groupe de direction chargé du Panorama des administrations publiques (voir annexe G) ; du Comité de statistique et de politique statistique (CSSP) ; du Groupe de travail sur l'emploi et la gestion publics ; du groupe de travail du Groupe de travail des hauts responsables du budget ; du Groupe de travail des hauts responsables de l'administration numérique (E-Leaders) ; du Groupe de travail des experts des marchés publics ; du Groupe d'experts sur l'administration ouverte et novatrice; et du Groupe d'experts sur les données ouvertes. De plus, des commentaires précieux ont été fournis par Gaetan Lafortune, Chris James et Maxime Ladaique (Direction de l'emploi, du travail et des affaires sociales de l'OCDE), Corinne Heckman, Daniel Sanchez Serra et Camila Demoraes (Direction de l'éducation et des compétences de l'OCDE), Carlota Balestra (Direction de statistiques et des données) et Oliver Petzold (Centre de politique et d'administration fiscale) 



\section{Table des matières}

Pour combattre la désillusion à l'égard des pouvoirs publics, il faut des politiques et des services publics centrés sur l'humain $\ldots \ldots \ldots \ldots \ldots \ldots \ldots \ldots \ldots \ldots \ldots \ldots \ldots$

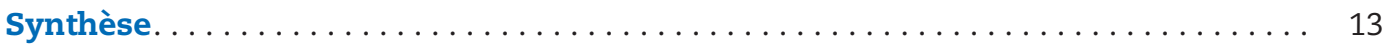

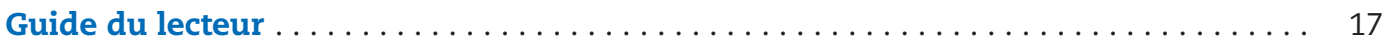

Introduction $\ldots \ldots \ldots \ldots \ldots \ldots \ldots \ldots \ldots \ldots \ldots \ldots \ldots \ldots \ldots \ldots \ldots \ldots \ldots \ldots \ldots \ldots \ldots, 23$

Chapitre 1. Vers des services publics centrés sur l'individu. .............. 29

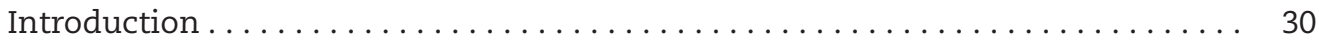

1. Qu'entend-on par « services publics centrés sur l'individu » ?......... 31

2. Parvenir à mettre en place des services publics centrés sur l'individu et évaluer ces services. . . . . . . . . . . . . . . . . . . . . . 32

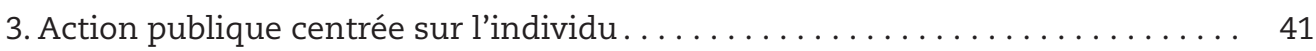

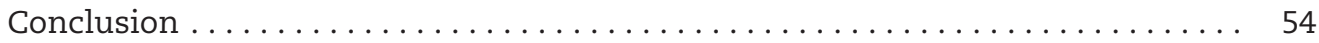

Chapitre 2. Finances publiques et économie .................... 59

Solde budgétaire des administrations publiques ................. 60

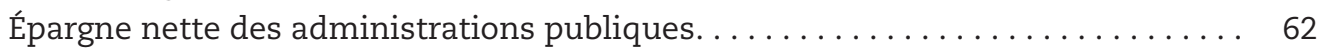

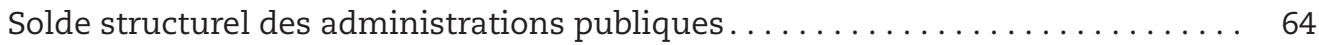

Dette brute des administrations publiques..................... 66

Patrimoine financier net des administrations publiques $\ldots \ldots \ldots \ldots \ldots \ldots 6$

Solde budgétaire et endettement par niveau d'administration . . . . . . . . 70

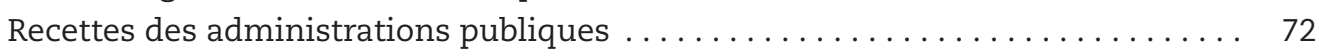

Composition des recettes des administrations publiques $\ldots \ldots \ldots \ldots \ldots \ldots \ldots \quad 74$

Dépenses des administrations publiques ........................ 76

Ventilation par fonction des dépenses des administrations publiques

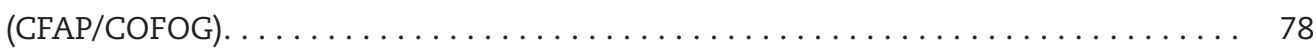

Ventilation par opération économique des dépenses des administrations

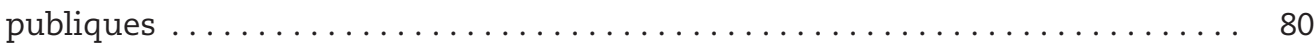

Ventilation par niveau d'administration des recettes et des dépenses

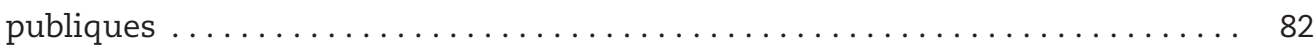

Dépenses d'investissement des administrations publiques............ 84

Coûts de production et externalisation dans les administrations publiques..... 86

Éclairage : ventilation des dépenses des administrations publiques

par fonction de protection sociale et de santé (CFAP) $\ldots \ldots \ldots \ldots \ldots \ldots \ldots$

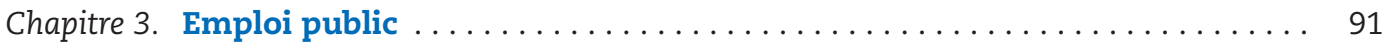

L'emploi dans les administrations publiques .................... 92

L'emploi au sein des différents niveaux d'administration . . . . . . . . . . . 94 
Égalité femmes-hommes dans les administrations publiques. . . . . . . . . 96

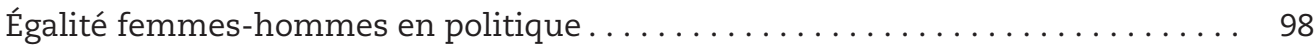

Égalité femmes-hommes dans l'appareil judiciaire. . . . . . . . . . . . . 100

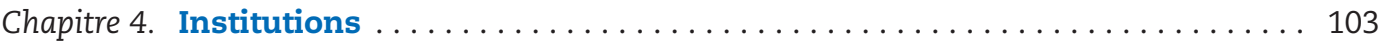

Rôles et responsabilités des centres de gouvernement ............... 104

Rôle du centre de gouvernement dans le gouvernement ouvert . . . . . . . . . . 106

Institutionnalisation des systèmes d'évaluation des politiques publiques . . . . . 108

Capitalisation des évaluations de politiques publiques $\ldots \ldots \ldots \ldots \ldots \ldots \ldots$

Chapitre 5. Pratiques et procédures budgétaires . . . . . . . . . . . . . . . . . 113

Budgétisation des investissements et infrastructures . . . . . . . . . . . . . . 114

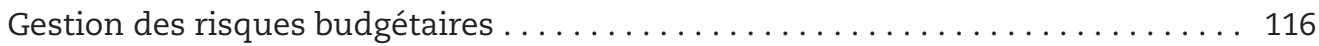

Budgétisation au service de l'égalité femmes-hommes . . . . . . . . . 118

Transparence budgétaire . . . . . . . . . . . . . . . . . . . . 120

Budgétisation au service d'un développement durable et respectueux

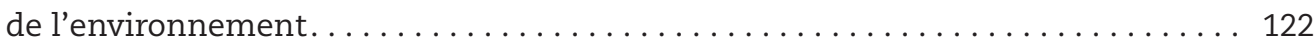

Chapitre 6. Gestion des ressources humaines ...................... 125

Conditions d'emploi des fonctionnaires et autres agents publics . . . . . . . . . 126

Apprentissage et perfectionnement dans la fonction publique............ 128

Gestion de la performance des cadres de haut niveau de la fonction publique . . 130

Chapitre 7. Gouvernance réglementaire ........................ 133

Participation des parties prenantes à l'élaboration de la réglementation . . . . . . 134

Analyse d'impact de la réglementation ........................ 136

Évaluation a posteriori de la réglementation $\ldots \ldots \ldots \ldots \ldots \ldots \ldots \ldots \ldots \ldots$

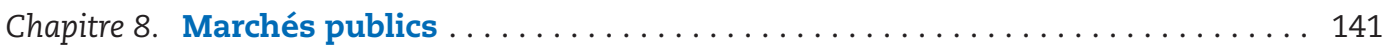

Poids des marchés publics . . . . . . . . . . . . . . . . . . . . . . . . . . . . . 142

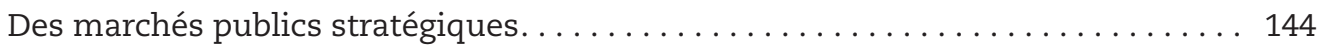

Transformation numérique des marchés publics................... 146

Capacités de passation des marchés publics et performance . . . . . . . . 148

Gestion des risques liés aux marchés publics. . . . . . . . . . . . . . 150

Chapitre 9. Gouvernement numérique et données publiques ouvertes ......... 153

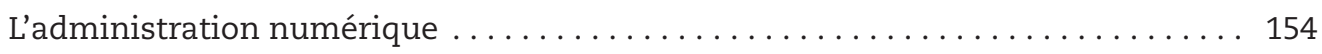

Données publiques ouvertes : clés de la maturité et de la viabilité......... 156

Disponibilité des données : cadres d'action, association des parties prenantes

et diffusion des données . . . . . . . . . . . . . . . . . . . . . . . . . . . 158

Accessibilité des données : des formats ouverts, gratuits et accessibles . . . . . 160

Associer les usagers : encourager l'apprentissage et la réutilisation

des données ouvertes . . . . . . . . . . . . . . . . . . . . . . 162

Chapitre 10. Principaux résultats des pouvoirs publics .................. 165

Confiance à l'égard des pouvoirs publics . . . . . . . . . . . . . . 166

Réactivité perçue des administrations publiques (efficacité politique externe) . . 168

Redistribution des revenus et du patrimoine . . . . . . . . . . . . . . . 170

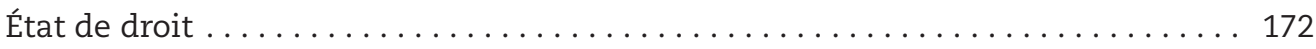




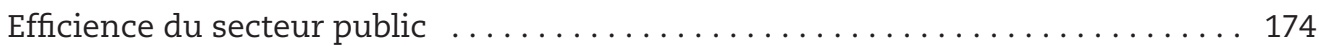

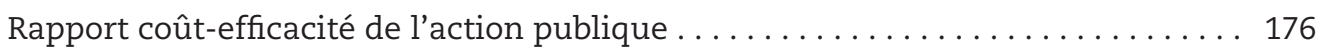

Chapitre 11. Servir les citoyens . . . . . . . . . . . . . . . . . . . . . 179

Tableaux de bord des services rendus aux citoyens. . . . . . . . . . . . . . . 180

Satisfaction des citoyens à l'égard des institutions et des services publics . . . . . 184

Accessibilité financière et géographique des soins de santé . . . . . . . . 186

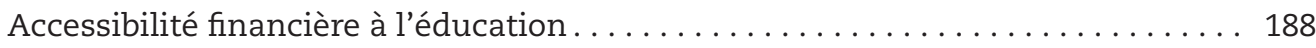

Accès aux services juridiques et judiciaires . . . . . . . . . . . . . . . . 190

Réactivité des systèmes de santé face aux besoins des patients . . . . . . . . . . . 192

Réactivité des systèmes éducatifs face aux besoins des élèves . . . . . . . . . . . . . . 194

Rapidité des services de justice civile. . . . . . . . . . . . . . . . . . . . . 196

Qualité des soins de santé . . . . . . . . . . . . . . . . . . . . . . . . . . 198

Résultats scolaires et équité dans l'éducation . . . . . . . . . . . . . . . . . 200

Efficacité et équité des systèmes judiciaires . . . . . . . . . . . . . . . . . . . . . . 202

Annexe A. Systèmes et sources d'information sur les comptes publics au sein des statistiques sur les comptes nationaux . . . . . . . . . . 205

Annexe B. Méthodologie utilisée pour les agrégats relatifs aux recettes . . . . . . . 207

Annexe C. Classification des fonctions des administrations publiques

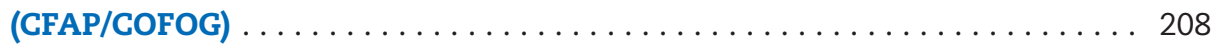

Annexe D. Classification et définition des professions . . . . . . . . . . . 210

Annexe E. Méthodologie employée pour les indices composites sur la budgétisation sensible au genre et sur les politique et gouvernance réglementaires et pour l'indice Ourdata (des données ouvertes,

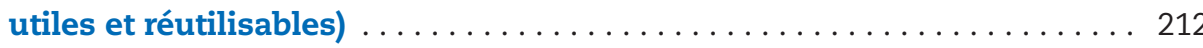

Annexe F. Graphiques supplémentaires disponibles en ligne.............. 214

Annexe G. Membres du groupe de direction $\ldots \ldots \ldots \ldots \ldots \ldots \ldots \ldots \ldots \ldots$

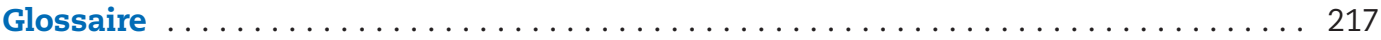




\section{Suivez les publications de l'OCDE sur :}

)

y http://twitter.com/OECD_Pubs

f http://www.facebook.com/OECDPublications

in. http://www.linkedin.com/groups/OECD-Publications-4645871

Youl $h$ ttp://www.youtube.com/oecdilibrary

OECD

http://www.oecd.org/oecddirect/

Ce livre contient des...

En bas des tableaux ou graphiques de cet ouvrage, vous trouverez des StatLinks. Pour télécharger le fichier Excel ${ }^{\circledR}$ correspondant, il vous suffit de retranscrire dans votre navigateur Internet le lien commençant par : $h t t p: / / d x . d o i . o r g$, ou de cliquer sur le lien depuis la version PDF de l'ouvrage. 


\section{Pour combattre la désillusion à l'égard des pouvoirs publics, il faut des politiques et des services publics centrés sur l'humain}

$\mathrm{S}_{\mathrm{i}, \mathrm{s}}$ nous, la plupart des pays de l'OCDE continuent de subir le poids d'un endettement public important : en moyenne, $110 \%$ du PIB en 2017. Un endettement aussi élevé pèse sur l'aptitude des pouvoirs publics à stimuler la croissance économique, mais aussi à relever des défis aussi pressants que le creusement des inégalités ou le sentiment d'insécurité suscité par les mutations technologiques en cours, en lesquelles beaucoup de citoyens voient une menace pour leur emploi. La redistribution par l'impôt et les transferts a chuté. Il y a aussi moins de fonds disponibles pour investir, alors même que la nécessité de moderniser de toute urgence des infrastructures vieillissantes apparaît de plus en plus clairement.

Ces évolutions, parmi d'autres, ont alimenté un mécontentement croissant à l'égard des autorités et des institutions démocratiques. Dans de nombreux pays, les citoyens se demandent si les gouvernants servent l'intérêt général, ou les intérêts d'une élite. De fait, en 2016, seuls $37 \%$ des citoyens des pays de l'OCDE considéraient exercer une influence sur l'action des pouvoirs publics. Ce mécontentement se reflète souvent dans le discours politique et dans les résultats électoraux. Globalement, ces évolutions montrent qu'il est nécessaire de redoubler d'efforts pour renforcer la confiance à l'égard des autorités et des institutions publiques.

Les administrations publiques disposent d'une panoplie d'outils pour renouer le lien avec les citoyens et renforcer l'efficacité de l'action publique. Elles peuvent, notamment, adopter des démarches centrées sur l'humain en matière d'action publique et de prestation des services publics. De telles démarches permettent aux pouvoirs publics de consulter les citoyens sur leurs besoins, en les incitant à participer directement à la conception et à la mise en œuvre des politiques. Elles leur permettent aussi d'évaluer les incidences de leur action sur la société.

Le Panorama des administrations publiques 2019 présente des indicateurs et des analyses qui montrent les progrès accomplis par les pays de l'OCDE s'agissant d'adopter une démarche centrée sur l'humain dans leurs pratiques de gestion publique et de gouvernance publique. Il présente des résultats comparatifs pour les 36 pays de l'OCDE, dans de nombreux domaines de la gouvernance publique.

Les résultats montrent que les pays commencent à proposer des services publics centrés sur l'humain. Dans la majorité des pays, l'accessibilité, la réactivité et la qualité des services publics s'améliorent dans des domaines tels que l'éducation, la santé ou la justice. Ainsi, en 2018, en moyenne de la zone OCDE, $70 \%$ des citoyens étaient satisfaits des soins de santé disponibles, 66 \% étaient satisfaits du système éducatif et $56 \%$ avaient confiance dans le système judiciaire et les tribunaux. 
La gestion et la gouvernance publiques sont elles aussi en train d'évoluer dans ce sens. Dans le cadre de leurs efforts visant à améliorer la transparence, la réactivité, la redevabilité et l'efficience du secteur public, les pays de l'OCDE ouvrent de plus en plus l'accès aux données détenues par les administrations publiques, sous des formats ouverts, libres et accessibles. De plus, un nombre croissant de pays mettant l'accent sur l'impact des décisions budgétaires sur des catégories de population et des domaines d'action spécifiques. Ainsi, le nombre de pays de l'OCDE à pratiquer la budgétisation tenant compte de l'égalité des sexes est passé de 12 en 2016 à 17 en 2018. Par ailleurs, la consultation des parties prenantes sur les projets de textes législatifs et réglementaires représente désormais une pratique très répandue, même s'il faut encore améliorer son calendrier et les dispositifs de retour d'information. Enfin, investir dans la fonction publique est aussi en train de devenir une priorité : en 2019, 24 pays de l'OCDE indiquaient accorder un haut degré de priorité à la formation et au coaching du personnel de direction. En revanche, seuls 17 pays considéraient comme une priorité la formation aux TIC et au numérique.

Le Panorama des administrations publiques 2019 démontre que les indicateurs de gouvernance peuvent être un outil crucial pour nous aider à comprendre les dernières évolutions du domaine de la gouvernance publique et à favoriser et multiplier les bonnes pratiques. Cette publication constitue donc une ressource incontournable pour les responsables publics, les professionnels, les chercheurs et les citoyens.

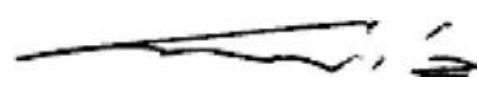

Angel Gurría 


\section{Synthèse}

L indicateurs relatifs aux performances des administrations publiques et aux politiques que les pouvoirs publics sont en train de mettre en œuvre pour rétablir le lien avec le citoyen, améliorer l'égalité et favoriser une croissance plus inclusive. Le chapitre thématique de cette publication est axé sur les performances des services publics " centrés sur l'humain " en termes d'accessibilité, de réactivité et de qualité. On trouvera dans ce Panorama des indicateurs sur les réalisations obtenues dans les domaines de l'éducation, de la santé et de la justice, complétés par des éléments de mesure concernant la façon dont les citoyens perçoivent ces services publics.

Cette publication examine également, au moyen d'indicateurs comparables à l'échelle internationale, les pratiques et réformes de la gouvernance publique sous l'angle de leur centrage sur l'humain, par exemple en matière de budgétisation, de gouvernance de la réglementation, de marchés publics et de données publiques ouvertes.

\section{Principales conclusions}

\section{La persistance d'un endettement public élevé pèse sur l'aptitude des pouvoirs publics à réagir face aux chocs économiques.}

- Le déficit budgétaire moyen se réduit progressivement depuis 2009. Il s'établissait à 2,2 \% du PIB en 2017, ce qui reste moins favorable que son niveau d'avant la crise (1,7 \% du PIB).

- En 2017, la dette brute des administrations publiques s'établissait, en moyenne, à $110 \%$ du PIB dans la zone OCDE, d'où une marge de manœuvre réduite pour les pays.

- Les dépenses des administrations publiques en matière de protection sociale et de santé représentaient, au total, plus de $21 \%$ du PIB des pays de l'OCDE en 2017. Le poids des dépenses liées à ces deux catégories a augmenté par rapport à 2007, principalement sous l'effet du vieillissement démographique : ce poids s'est alourdi de 1,5 point de pourcentage pour les dépenses de protection sociale, et de 1,1 point de pourcentage pour les dépenses de santé.

- L'investissement public représentait, en moyenne, 3,1 \% du PIB en 2017, ce qui reste inférieur de 0,5 point de pourcentage à son niveau de 2007. Il est nécessaire de combler le déficit d'investissement; accroître l'investissement public peut contribuer à la croissance économique et apporter le capital nécessaire à la lutte contre les changements climatiques et à la mise en œuvre des Objectifs de développement durable (ODD).

\section{Si le niveau de l'emploi public s'est globalement maintenu au fil du temps, tous les agents publics ne sont pas traités de façon égale}

- L'emploi dans les administrations publiques représente environ $18 \%$ de l'emploi total à l'échelle de la zone OCDE, soit un niveau inchangé par rapport à 2007. 
- Il existe des disparités persistantes entre les sexes au niveau de l'emploi public. Ainsi, les hommes sont surreprésentés au sein des juridictions de haut niveau (où $67 \%$ des juges sont de sexe masculin) et dans la sphère politique. En 2019, en moyenne de la zone OCDE, les femmes occupent $30 \%$ des sièges de la chambre basse du parlement (ou du parlement monocaméral) et détiennent le tiers des portefeuilles ministériels dans le gouvernement national.

- Au sein de l'administration centrale, les agents publics dotés du statut de fonctionnaire représentent, en moyenne, 68 \% des effectifs. Par rapport aux autres agents publics, ils bénéficient d'une plus grande sécurité de l'emploi et de meilleures possibilités d'évolution de carrière, et ils font l'objet d'une procédure de recrutement plus rigoureuse.

Les pays sont de plus en plus nombreux à chercher à adopter des pratiques budgétaires mettant l'accent sur l'impact des décisions budgétaires sur des catégories de population et des domaines d'action jugés prioritaires

- En 2018, la moitié des pays de l'OCDE interrogés avaient mis en place une budgétisation tenant compte de l'égalité des sexes et un quart environ l'avaient intégrée à leur réglementation.

- En 2018, un quart des pays de l'OCDE interrogés rendaient public l'impact environnemental et climatique des mesures budgétaires ; un nombre similaire de pays fournissaient des éléments d'information sur les effets du budget en termes de bien-être sociétal ; seuls $25 \%$ des pays prenaient en compte les Objectifs de développement durable dans leur système de budgétisation axée sur la performance.

La consultation des parties prenantes sur les projets de textes législatifs et réglementaires est très répandue au sein des pays de l'OCDE. Toutefois, cette consultation intervient généralement tard dans le processus ; de plus, les parties prenantes bénéficient rarement d'un retour d'information sur l'impact de leurs commentaires.

- Dans tous les pays de l'OCDE interrogés, l'association des parties prenantes est obligatoire pour l'élaboration d'au moins certains textes.

- En 2016, dans 28 pays de l'OCDE, le centre de gouvernement consultait directement les parties prenantes sur les politiques publiques.

- Par rapport à 2014, les pays ont légèrement amélioré leurs pratiques d'association des parties prenantes, avec des progrès plus nets pour les textes législatifs : de 2014 à 2017, sur une échelle de 1 à 4 , le score est passé de 2 à 2,2 pour les textes législatifs et de 2 à 2,1 pour les textes réglementaires.

\section{Les pouvoirs publics s'appuient de plus en plus sur les marchés publics pour} poursuivre des objectifs de protection de l'environnement

- Les marchés publics représentaient, en moyenne, 12 \% du PIB des pays de l'OCDE en 2017.

- Tous les pays de l'OCDE avaient mis en place des stratégies en matière de marchés publics verts en 2018 ; en outre, ils sont de plus en plus nombreux à s'appuyer sur les marchés publics pour favoriser la croissance inclusive (29 pays), l'innovation (26 pays) et la conduite responsable des entreprises (22 pays). 
Les pays de l'OCDE continuent de progresser s'agissant de permettre à chacun d'accéder, sous des formats ouverts, libres et accessibles, aux données détenues par les administrations publiques.

- Sur 33 pays de l'OCDE, 30 imposent la gratuité des données publiques ; 29 imposent que ces données soient disponibles sous licence ouverte ; 31 imposent qu'elles soient fournies dans un format lisible par machine ; et 21 traitent comme une priorité le renforcement des aptitudes et capacités de l'administration en matière de réutilisation des données.

- L'indice OURdata (des données ouvertes, utiles et réutilisables), qui a pour objet de comparer les politiques d'ouverture des données publiques et leur mise en œuvre, a progressé entre 2014 et 2017. Cette progression reflète des améliorations pour toutes les dimensions prises en compte par cet indice : la disponibilité des données, leur accessibilité et le soutien public à leur réutilisation. Certains pays antérieurement à la traîne commencent à rattraper les pays de tête que sont la Corée, la France et le Japon.

- En 2016, dans 21 pays de l'OCDE, le centre de gouvernement intervenait dans la conception des stratégies et initiatives liées au gouvernement ouvert et, dans 20 pays, il intervenait également dans leur mise en œuvre.

Si la confiance à l'égard des pouvoirs publics a retrouvé ses niveaux d'avant la crise, le sentiment d'efficacité politique des citoyens reste faible

- Après s'être dégradée à partir de 2007, la confiance des citoyens à l'égard des pouvoirs publics a retrouvé un niveau similaire à celui d'avant la crise, puisqu'elle s'établit désormais à $45 \%$ à l'échelle de la zone OCDE. La confiance à l'égard des pouvoirs publics a progressé dans 16 pays comme l'Allemagne, la Corée, le Japon, la Pologne et la Suisse.

- En 2016, en moyenne, seuls 37 \% des habitants de la zone OCDE avait en le sentiment d'avoir une influence sur l'action des pouvoirs publics (sentiment d'efficacité politique), et cette proportion n'atteignait, au mieux, que $20 \%$ en Italie et en Slovénie.

Dans l'ensemble, le degré de satisfaction des citoyens à l'égard des services de santé et d'éducation et leur niveau de confiance envers le secteur judiciaire ont légèrement augmenté dans les pays de l'OCDE, mais des inégalités persistent selon les catégories de population.

- En 2018, sur l'ensemble de la zone OCDE, 70 \% des citoyens étaient satisfaits des services de santé disponibles ; 66 \% étaient satisfaits du système éducatif et des établissements d'enseignement ; et $56 \%$ avaient confiance dans le système judiciaire et les tribunaux.

- L’accessibilité, la réactivité et la qualité des services publics (en matière d'éducation, de santé et de justice) se sont améliorées dans la plupart des pays. Par exemple, la proportion de jeunes sans emploi et sortis du système éducatif (NEET) est passée de 6,9 \% en 2012 à $5,2 \%$ en 2018.

- Des inégalités persistent entre les catégories de population. Ainsi, en 2017, le pourcentage de besoins non satisfaits en matière d'examens médicaux était supérieur de 3,2 points de pourcentage pour les individus dotés d'un revenu faible que pour les individus dotés d'un revenu élevé. 



\title{
Guide du lecteur
}

\begin{abstract}
A
fin d'interpréter correctement les données figurant dans cette édition 2019 du Panorama des administrations publiques, les lecteurs doivent être au fait des considérations méthodologiques ci-après, qui valent pour un certain nombre d'indicateurs.

À partir du chapitre 2, tous les indicateurs sont présentés sur deux pages. La première page comporte un texte qui explique la pertinence du thème abordé et met en évidence certaines des grandes différences observées entre les pays de l'OCDE. Ce texte est suivi d'une rubrique « Méthodologie et définitions » qui précise les sources des données et fournit d'importantes indications nécessaires à leur interprétation. En fin de première page, on trouve une rubrique "Pour en savoir plus " qui dresse une liste de travaux de référence permettant de replacer dans leur contexte les données fournies. La seconde page présente ces données. Les graphiques présentent les chiffres actuels et, dans la mesure du possible, leur évolution au fil du temps. On trouvera au dernier chapitre un glossaire des principaux termes employés dans la publication.
\end{abstract}

\section{Année civile/exercice budgétaire dans les statistiques de l'OCDE relatives aux comptes nationaux}

Sauf indication contraire, les données tirées des statistiques de l'OCDE relatives aux comptes nationaux portent sur l'année civile.

Les données relatives à l'Australie et à la Nouvelle-Zélande portent sur des exercices budgétaires, qui courent du $1^{\mathrm{er}}$ juillet de l'année indiquée au 30 juin pour l'Australie et du $1^{\mathrm{er}}$ avril de l'année indiquée au 31 mars pour la Nouvelle-Zélande. Pour le Japon, les données relatives aux sous-secteurs des administrations publiques et aux dépenses selon la CFAP/COFOG (Classification des fonctions des administrations publiques) portent sur des exercices budgétaires.

Les données fondées sur le Système de comptabilité nationale (SCN) ont été tirées le 24 juin 2019 des bases de données suivantes : Statistiques de l'OCDE sur les comptes nationaux et Statistiques de finances publiques d'Eurostat.

\section{Portée géographique}

Le Panorama des administrations publiques 2019 présente des chiffres pour les 36 pays de l'OCDE, en fonction des données disponibles. Les données statistiques concernant Israël sont fournies par et sous la responsabilité des autorités israéliennes compétentes. L'utilisation de ces données par l'OCDE est sans préjudice du statut des hauteurs du Golan, de Jérusalem Est et des colonies de peuplement israéliennes en Cisjordanie aux termes du droit international. 
Certains pays non membres tels que la Colombie, le Costa Rica et la Fédération de Russie ${ }^{1}$ (pays candidats à l'adhésion à l'OCDE) ainsi que d'autres grandes économies mondiales (l'Afrique du Sud, le Brésil, la République populaire de Chine, l'Inde et l'Indonésie) ont également fourni des données pour certains indicateurs. Les données relatives aux pays non membres sont présentées séparément, à la fin des tableaux et des graphiques.

\section{Abréviations de noms de pays}

\begin{tabular}{|c|c|c|c|}
\hline \multicolumn{4}{|l|}{ Pays de I'OCDE } \\
\hline Allemagne & DEU & Pays-Bas & NLD \\
\hline Australie & AUS & Pologne & POL \\
\hline Autriche & AUT & Portugal & PRT \\
\hline Belgique & BEL & République slovaque & SVK \\
\hline Canada & CAN & République tchèque & CZE \\
\hline Chili & $\mathrm{CHL}$ & Royaume-Uni & GBR \\
\hline Corée & KOR & Slovénie & SVN \\
\hline Danemark & DNK & Suède & SWE \\
\hline Espagne & ESP & Suisse & CHE \\
\hline Estonie & EST & Turquie & TUR \\
\hline États-Unis & USA & & \\
\hline Finlande & FIN & Pays candidats à l'adhésion à l'OCDE* & \\
\hline France & FRA & Colombie & $\mathrm{COL}$ \\
\hline Grèce & GRC & Costa Rica & $\mathrm{CRI}$ \\
\hline Hongrie & HUN & Fédération de Russie (ci-après, « Russie ») & RUS \\
\hline Irlande & IRL & & \\
\hline Islande & ISL & Autres grandes économies & \\
\hline Israël & ISR & Afrique du Sud (participant auprès du Comité de la gouvernance publique de l'OCDE) & ZAF \\
\hline Italie & ITA & Brésil (participant auprès du Comité de la gouvernance publique de l'OCDE) & BRA \\
\hline Japon & JPN & République populaire de Chine (ci-après, " Chine ») & $\mathrm{CHN}$ \\
\hline Lettonie & LVA & Inde & IND \\
\hline Lituanie & LTU & Indonésie & IDN \\
\hline Luxembourg & LUX & & \\
\hline Mexique & MEX & & \\
\hline Norvège & NOR & & \\
\hline Nouvelle-Zélande & NZL & & \\
\hline
\end{tabular}

* Note : s'agissant de la Fédération de Russie, voir la note de bas de page ci-dessus.

\section{Moyennes et totaux OCDE}

\section{Moyennes}

Dans les graphiques, la moyenne OCDE est présentée, soit comme une moyenne arithmétique non pondérée, soit comme une moyenne pondérée des pays de l'OCDE pour lesquels des données sont disponibles. Elle exclut les données relatives aux pays non membres. Les notes précisent pour quels pays de l'OCDE on ne dispose pas de données.

Quand un graphique présente des données portant sur une ou plusieurs années, la moyenne OCDE englobe tous les pays de l'OCDE pour lesquels on dispose de données. Par exemple, une moyenne OCDE pour 2017 englobe tous les pays actuellement membres de l'OCDE au sujet desquels on dispose de données pour l'année en question, même si, à l'époque, ils n'étaient pas membres de l'Organisation.

${ }^{1}$ S'agissant de la Fédération de Russie, le 12 mars 2014, le Conseil de l'OCDE a « reporté les activités liées au processus d'adhésion de la Fédération de Russie pour le moment ". Pour en savoir plus, voir www. oecd.org/fr/presse/declaration-de-l-ocde-sur-le-statut-du-processus-d-adhesion-avec-la-russie-et-la-cooperationavec-l-ukraine.htm. 
S'agissant des données tirées des statistiques de l'OCDE relatives aux comptes nationaux, les moyennes OCDE sont, sauf indication contraire, des moyennes pondérées. De plus, les moyennes OCDE sont calculées jusqu'en 2017, car tous les pays de l'OCDE n'ont pas de données disponibles pour 2018 (surtout parmi les membres non européens de l'OCDE).

\section{Totaux}

Les totaux OCDE figurent généralement dans les tableaux et représentent la somme des données présentées dans la colonne correspondante au sujet des pays de l'OCDE pour lesquels des données sont disponibles. Les totaux excluent les données relatives aux pays non membres. Les notes précisent pour quels pays de l'OCDE on ne dispose pas de données.

\section{Suppléments en ligne}

Pour plusieurs indicateurs, on trouvera en ligne des tableaux et graphiques supplémentaires présentant des données nationales, ou encore des annexes proposant un complément d'information sur la méthodologie employée pour l'indicateur. Lorsque de tels suppléments sont disponibles, ils sont signalés à la rubrique « Méthodologie et définitions » de l'indicateur. Le Panorama des administrations publiques 2019 offre également l'accès au service StatLinks, qui permet aux lecteurs de télécharger les fichiers Excel correspondant aux données présentées. Les liens StatLinks sont placés dans le coin inférieur droit des tableaux et des graphiques et il est possible de les saisir dans un navigateur web ou, pour les versions électroniques des publications, de cliquer directement dessus.

Les supports complémentaires suivants sont également disponibles en ligne, à l'adresse www.oecd.org/fr/gov/panorama-des-administrations-publiques.htm :

- des fiches par pays qui présentent un certain nombre de données essentielles pour chaque pays, avec une comparaison avec la moyenne OCDE ;

- la base de données statistiques du Panorama des administrations publiques, qui présente des données régulièrement actualisées pour une sélection d'indicateurs quantitatifs (via OECD.Stat) ainsi que des données qualitatives en lien avec les enquêtes de la Direction de la gouvernance publique de l'OCDE, par le truchement d'une plateforme web spécifique ;

- des notes relatives au contexte de chaque pays qui fournissent des renseignements sur certaines grandes caractéristiques de la structure politique et administrative de chaque pays membre.

\section{Indicateurs par habitant}

Certains indicateurs (les indicateurs relatifs aux dépenses, aux recettes et à la dette publique, par exemple) sont présentés par habitant (c'est-à-dire par personne). Les estimations de la population de référence sont fondées sur la notion de résidence du Système de comptabilité nationale. Elles incluent les personnes qui sont résidentes dans un pays pendant un an ou plus, quelle que soit leur nationalité, et comprennent aussi les membres du personnel diplomatique et militaire étranger ainsi que leurs familles, de même que les étudiants effectuant des études à l'étranger et les patients recevant un traitement à l'étranger, même s'ils y restent pendant plus d'un an. La règle d'un an signifie que les résidents habituels vivant à l'étranger pendant moins d'un an sont inclus dans la population, tandis que les visiteurs étrangers (les touristes, par exemple) présents dans le pays pendant moins d'un an sont exclus. Point important à noter dans ce contexte : des individus peuvent être comptabilisés comme employés dans un pays (contribuant au produit 
intérieur brut - PIB - de ce pays par le biais de la production) mais comme résidents d'un autre pays (leurs traitements et salaires étant pris en compte dans le revenu national brut de leur pays de résidence).

\section{Parités de pouvoir d'achat}

Les parités de pouvoir d'achat (PPA) sont les taux de change des monnaies qui égalisent le pouvoir d'achat de différents pays en éliminant les différences de niveaux des prix entre les pays. Lorsqu'on les convertit au moyen des PPA, les dépenses des différents pays sont, de fait, exprimées aux mêmes niveaux de prix. Cela signifie qu'un groupe équivalent de biens et de services aura le même coût dans les deux pays, ce qui permet d'effectuer des comparaisons internationales prenant uniquement en compte les différences au niveau du volume des biens et des services achetés.

Les PPA pour les séries courantes et chronologiques sont produites et actualisées par l'OCDE au moyen d'une procédure spécifique. Les PPA pour une année donnée (année «T ") sont publiées en cinq étapes :

1. À T+2 mois : premières estimations relatives aux PPA, uniquement pour le PIB.

2. À T+6 mois : deuxièmes estimations relatives aux PPA, à partir d'une extrapolation détaillée, pour le PIB, de la consommation individuelle effective des ménages et de la consommation privée des ménages.

3. À T+12 mois : troisièmes estimations relatives aux PPA, intégrant l'ensemble des données relatives aux prix et aux dépenses pour l'année $\mathrm{T}$.

4. À T+24 mois : quatrièmes estimations relatives aux PPA, intégrant l'ensemble des données actualisées relatives aux dépenses.

5. À T+36 mois : estimations finales relatives aux PPA pour l'année T.

Les données antérieures relatives aux PPA jusqu'en 2012 peuvent être révisées au mois de décembre de chaque année afin de tenir compte des modifications apportées aux déflateurs au sein des comptes nationaux. En décembre 2016, les données antérieures relatives aux PPA jusqu'en 2012 ont été exceptionnellement révisées pour tous les pays européens.

On trouvera un complément d'information à l'adresse www.oecd.org/fr/sdd/prix-ppa/.

\section{Indicateurs composites}

On trouvera dans cette publication plusieurs indices composites descriptifs portant sur des domaines étroitement définis liés à la budgétisation sensible au genre à la gouvernance de la réglementation et à l'ouverture des données publiques. Ces indices composites constituent un moyen commode de rassembler des informations éparses d'ordre qualitatif. Les indices composites présentés dans cette publication ont été créés conformément aux étapes définies dans le Handbook on Constructing Composite Indicators (Nardo et al. 2008).

On trouvera à l'Annexe E des précisions sur la méthodologie employée pour construire les indices composites. Si les indices composites ont été élaborés en coopération avec les pays de l'OCDE et selon des fondements théoriques ou de bonnes pratiques, les variables composant les indices et leurs pondérations relatives reposent sur des jugements autorisés et, de ce fait, peuvent évoluer au fil du temps. 


\section{Signes et abréviations}

\begin{tabular}{|c|c|}
\hline Abréviation/acronyme & Signification \\
\hline . & Valeurs manquantes \\
\hline$x$ & Sans objet (sauf indication contraire) \\
\hline $\mathrm{ABC}$ & Autorité budgétaire centrale \\
\hline CFAP (ou COFOG) & Classification des fonctions des administrations publiques \\
\hline CIDO & Charte internationale sur les données ouvertes \\
\hline DPO & Données publiques ouvertes \\
\hline DTS & Droits de tirage spéciaux \\
\hline FMI & Fonds monétaire international \\
\hline GRH & Gestion des ressources humaines \\
\hline GSRH & Gestion stratégique des ressources humaines \\
\hline ID & Directeur de l'information \\
\hline ISO & Organisation internationale de normalisation \\
\hline MARL & Mécanismes alternatifs de règlement des litiges \\
\hline MSFP & Manuel de statistiques de finances publiques \\
\hline OCSC & Office of the Civil Service Commission \\
\hline ODD & Objectifs de développement durable \\
\hline OIT & Organisation internationale du Travail \\
\hline OPB & Offices parlementaires du budget \\
\hline p.p. & Points de pourcentage \\
\hline PEM & Perspectives de l'économie mondiale \\
\hline PIB & Produit intérieur brut \\
\hline PISA & Programme international pour le suivi des acquis des élèves \\
\hline PME & Petites et moyennes entreprises \\
\hline PPA & Parités de pouvoir d'achat \\
\hline PPP & Partenariat public-privé \\
\hline RAM & Rémunération au mérite \\
\hline $\mathrm{R}-\mathrm{D}$ & Recherche-développement \\
\hline $\mathrm{RH}$ & Ressources humaines \\
\hline $\mathrm{RP}$ & Représentation proportionnelle \\
\hline SCN & Système de comptabilité nationale \\
\hline SFP & Statistiques de finances publiques \\
\hline TIC & Technologies de l'information et de la communication \\
\hline TVA & Taxe sur la valeur ajoutée \\
\hline WJP & World Justice Project \\
\hline
\end{tabular}





\section{Introduction}

L fiables et comparables à l'échelle internationale sur les activités des administrations publiques et sur leurs résultats, dans les pays de l'OCDE et dans le reste du monde. Les pays peuvent utiliser ces données pour comparer les performances de leurs administrations, suivre l'évolution de ces performances à l'échelle interne et internationale et apporter des preuves de l'impact de leurs politiques publiques. Les indicateurs du Panorama des administrations publiques sont en train de devenir une référence dans de nombreux domaines de la gouvernance publique et ils dépassent désormais la seule zone OCDE pour couvrir également des pays d'Amérique latine et d'Asie du Sud-Est. En plus des indicateurs de base qui constituent la marque de fabrique de la publication, cette sixième édition propose une sélection de nouveaux indicateurs et des sources de données supplémentaires, ce qui permet de dresser un panorama plus complet des travaux et des résultats des administrations publiques de l'ensemble de la zone OCDE. Dans cette édition, les deux cinquièmes des indicateurs environ se fondent sur des données primaires directement recueillies auprès de services officiels par le truchement d'enquêtes de l'OCDE. Les autres proviennent de sources secondaires et se fondent, soit sur des données administratives (indicateurs sur les finances publiques, par exemple), soit sur des enquêtes menées auprès des ménages (indicateurs sur la confiance ou la satisfaction à l'égard des services publics, par exemple), soit, dans une moindre mesure, sur des avis d'experts recueillis par d'autres organisations (l'indice de l'état de droit du World Justice Project, par exemple).

\section{Les nouveautés du Panorama des administrations publiques 2019}

L'édition 2019 du Panorama des administrations publiques propose à la fois des chapitres communs à chaque édition et de nouvelles rubriques.

Les chapitres communs présentent les dernières données disponibles dans les domaines suivants : les finances et l'économie publiques (chapitre 2) ; l'emploi public (chapitre 3) ; les pratiques et procédures budgétaires (chapitre 5) ; la gestion des ressources humaines (chapitre 6) ; la gouvernance de la réglementation (chapitre 7) ; les marchés publics (chapitre 8) ; et les principaux résultats de l'action publique (chapitre 10). On retrouvera aussi dans cette édition le chapitre « Au service des citoyens » (chapitre 11).

\section{Nouveaux indicateurs}

Dans de nombreux chapitres communs de cette édition 2019, on trouvera de nouveaux indicateurs :

- Le chapitre 5, consacré aux pratiques et procédures budgétaires, évoque des questions d'actualité en lien avec le processus budgétaire, dans des domaines où de nouvelles tendances ou des pratiques communes sont en train d'apparaître ou de s'affermir à l'échelle de la zone OCDE. Il englobe ainsi des indicateurs sur la budgétisation des 
investissements et infrastructures, les risques budgétaires, la budgétisation sensible au genre, la transparence budgétaire et la budgétisation au service d'un développement durable.

- Le chapitre 6 propose de nouveaux indicateurs relatifs à la gestion des ressources humaines qui portent sur les conditions d'emploi des fonctionnaires et des autres agents publics des pays de l'OCDE, la gestion des performances des cadres de haut niveau de la fonction publique et les approches officielles en matière d'apprentissage et de perfectionnement au sein de la fonction publique.

- Le chapitre 7 , consacré à la gouvernance de la réglementation, propose la dernière mise à jour des indicateurs iREG (indicateurs relatifs à la politique et à la gouvernance réglementaires) pour ce qui est de l'association des parties prenantes, de l'analyse d'impact de la réglementation et de l'évaluation a posteriori. Cette actualisation des données permet de présenter des séries chronologiques et de mettre en évidence les évolutions intervenues au sein de la zone OCDE et de l'Union européenne.

- En plus des indicateurs de base relatifs aux marchés publics présentés dans le chapitre 8 , on trouvera de nouvelles données sur les capacités et les performances en matière de passation des marchés, y compris sur le rôle de la centralisation ainsi que sur la gestion des risques dans le cadre des marchés publics.

- Pour mettre en relief la montée en puissance de la logique produits/réalisations, le chapitre 10, qui est consacré aux principaux résultats de l'action publique, propose un nouvel indicateur relatif au pourcentage de citoyens ayant le sentiment d'avoir une influence sur l'action des pouvoirs publics.

\section{Nouvelles rubriques}

Cette édition du Panorama des administrations publiques comporte aussi de nouvelles rubriques :

- Elle propose une série d'indicateurs inédits sur certains aspects de la gouvernance publique. Si le chapitre consacré aux institutions (chapitre 4) inclut, pour la deuxième fois, des indicateurs sur le rôle et les fonctions du centre de gouvernement, il aborde aussi pour la première fois le thème du rôle du centre de gouvernement dans les politiques d'ouverture de l'administration. On y trouvera aussi, pour la première fois, des données probantes sur l'évaluation des politiques publiques et notamment sur les dispositifs institutionnels mis en place en la matière et sur les moyens employés pour encourager la mise à profit des conclusions de ces évaluations.

- Le chapitre 9, qui est consacré aux données ouvertes et à l'administration numérique, propose une actualisation de l'indice OURdata (des données ouvertes, utiles et réutilisables), qui permet d'évaluer l'ouverture des données publiques. On y trouvera une présentation de chacun des sous-indices qui composent l'indice principal. Pour la première fois, on y trouvera aussi des données issues d'une nouvelle enquête sur l'administration numérique, avec des données sur la gouvernance des stratégies en matière d'administration numérique, le rôle de coordination assuré par différents acteurs et les leviers d'action publique pouvant être actionnés.

\section{Définition des administrations publiques}

Les données relatives aux finances publiques sont fondées sur la définition du secteur des " administrations publiques " qui figure dans le Système de comptabilité nationale (SCN). Les administrations publiques recouvrent donc les ministères/départements, organismes 
publics et services ainsi que certaines institutions sans but lucratif au niveau central, au niveau des États fédérés et au niveau local, ainsi que les administrations de sécurité sociale. Les données sur les recettes et les dépenses sont présentées pour l'administration centrale et pour les niveaux d'administration infranationaux (États fédérés et administrations locales) et, s'il y a lieu, pour les administrations de sécurité sociale. Les données sur l'emploi se réfèrent également aux administrations publiques, bien que les données sur l'emploi par sexe se réfèrent au secteur public, qui englobe les administrations publiques et l'emploi dans les entreprises et sociétés résidentes à capitaux publics. Enfin, les données relatives aux pratiques et procédures de gestion publique portent uniquement sur les pratiques et procédures de l'administration centrale.

\section{Cadre et structure de la publication}

Le Panorama des administrations publiques couvre les 36 pays de l'OCDE. Quand elles sont disponibles, des données sont également fournies au sujet des pays candidats à l'adhésion à l'OCDE (la Colombie, le Costa Rica et la Russie) ainsi que sur d'autres grandes économies telles que l'Afrique du Sud, le Brésil, la Chine, l'Inde et l'Indonésie. Ces pays jouent un rôle important et grandissant dans l'économie mondiale et dans les structures politiques internationales.

Cette sixième édition du Panorama des administrations publiques comporte des éléments de contexte ainsi que des indicateurs axés sur les intrants, les processus, les produits et les réalisations. On trouvera au graphique 0.1 le cadre conceptuel du Panorama des administrations publiques.

\section{Contexte}

Des éléments de contexte (en ligne) fournissent des renseignements sur certaines grandes caractéristiques de la structure politique et administrative de chaque pays membre de l'OCDE. Prendre en compte ces éléments de contexte permet de comprendre les grandes différences et similitudes qui existent entre les institutions des divers pays et, donc, de trouver de meilleurs éléments de comparaison. En complément, les fiches par pays (en ligne) offrent, pays par pays, des précisions sur la façon dont les données fournies dans le Panorama des administrations publiques s'appliquent au contexte spécifique des réformes du secteur public dans les pays de l'OCDE et dans certains pays candidats à l'adhésion.

\section{Intrants}

Les intrants font référence aux ressources utilisées par les administrations publiques dans le cadre de leur fonction de production ainsi qu'à la façon dont ces ressources, qui correspondent au travail et au capital, sont combinées. Les chapitres qui décrivent ces intrants sont les suivants : "Finances publiques et économie " (chapitre 2) et «Emploi public » (chapitre 3). Ils proposent des indicateurs relatifs aux dépenses des administrations publiques, à leurs coûts de production, à l'emploi public et à la composition de la fonction publique. Les différences pouvant exister au niveau de ces divers indicateurs peuvent aider à comprendre les capacités différentes des administrations publiques à produire des biens publics et à les fournir aux citoyens.

\section{Processus}

Les processus font référence aux pratiques et aux procédures de gestion publique suivies par les pouvoirs publics pour mettre en œuvre leurs politiques. Ils touchent à la question des moyens employés par les administrations publiques pour remplir leurs fonctions et pour atteindre leurs objectifs. Par conséquent, ils sont souvent essentiels pour assurer le 
respect de l'état de droit, la responsabilité et l'équité ainsi que la transparence de l'action des pouvoirs publics. Les réformes du secteur public sont souvent axées sur ces processus, qui sont donc au cœur de l'attention que la population porte à l'action publique. On trouvera dans cette édition des renseignements sur les institutions, les pratiques et procédures budgétaires, la gestion des ressources humaines, l'intégrité du secteur public, la gouvernance de la réglementation, les marchés publics, les données publiques ouvertes et la gouvernance des stratégies en matière d'administration numérique (chapitres 4 à 9).

\section{Graphique 0.1. Cadre conceptuel du Panorama des administrations publiques 2019}

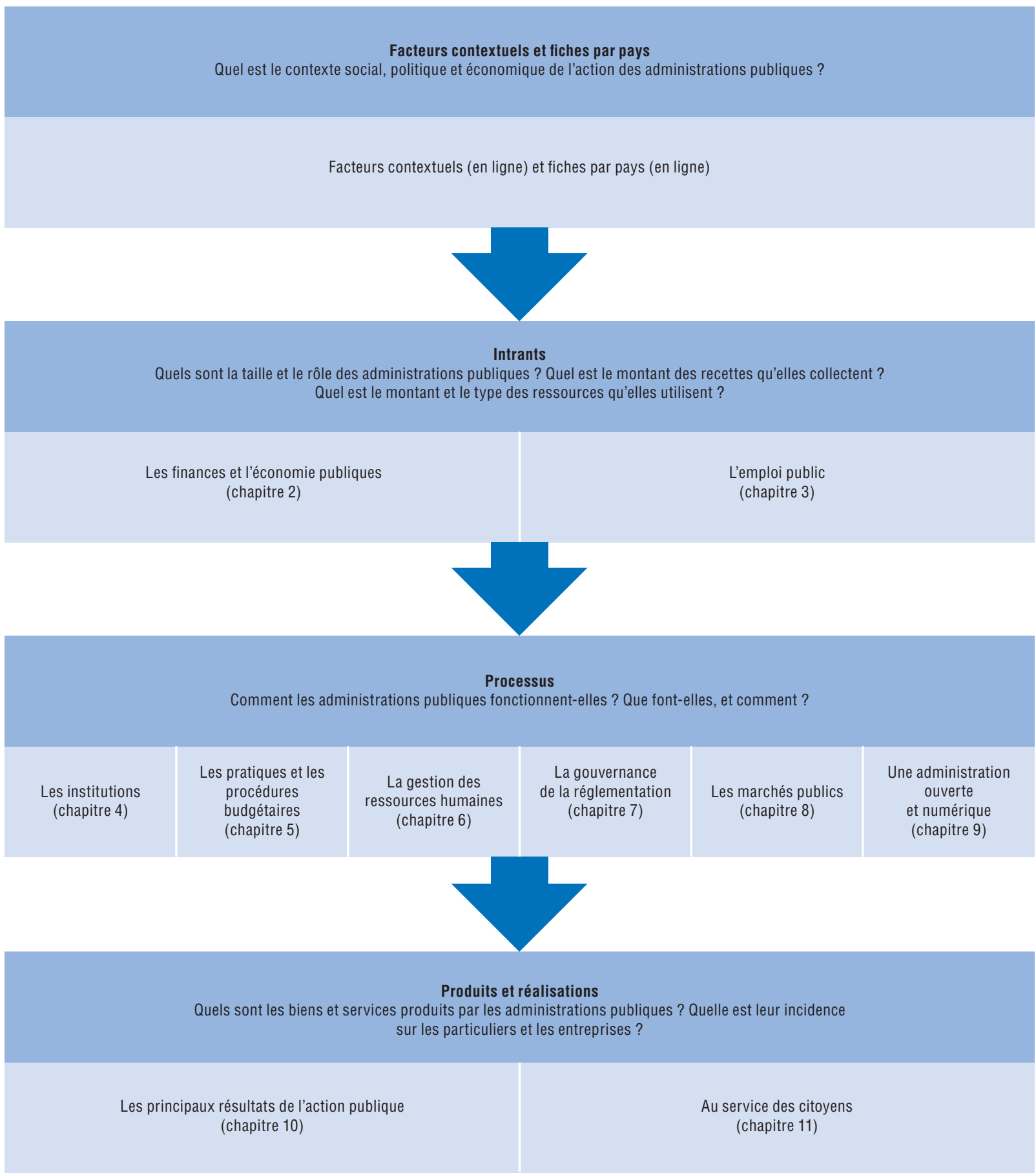




\section{Produits et réalisations}

La frontière entre produits (" outputs ») et réalisations (" outcomes ») peut être floue; les produits correspondent à la quantité de biens et de services produits par les administrations publiques, tandis que les réalisations correspondent aux effets des politiques et des pratiques sur les particuliers et les entreprises. Le succès d'une politique donnée devrait se mesurer à l'aune de ses produits dans un premier temps mais, au bout du compte, en fonction de ses réalisations. De manière générale, les réalisations correspondent aux effets des programmes et services publics sur les citoyens, en termes d'amélioration du bien-être, de l'état de santé, du niveau éducatif et de l'apprentissage, etc. Si ces réalisations peuvent indéniablement être influencées par la qualité des programmes et des services assurés, elles peuvent aussi l'être par d'autres facteurs tels que la situation socio-économique de la population et les facteurs comportementaux individuels.

Dans l'édition 2019 du Panorama des administrations publiques, les éléments de mesure relatifs aux produits et aux réalisations sont fournis dans deux chapitres distincts :

- Le chapitre 10, consacré aux principaux résultats de l'action publique, est axé sur des aspects touchant aux administrations publiques dans leur ensemble, tels que la confiance des citoyens à l'égard des autorités nationales, l'état de droit, la redistribution des revenus et les grands éléments de mesure de l'efficience du secteur public (en fonction des produits) et de son rapport coût-efficacité (en fonction des réalisations).

- Le chapitre 11, intitulé "Au service des citoyens ", suit une démarche sectorielle pour mesurer les produits et les réalisations des activités du secteur public. À partir d'un cadre intégré élaboré en collaboration avec d'autres Directions de l'OCDE et avec des pays de l'OCDE, ce chapitre fournit des éléments permettant de mesurer l'accessibilité, la réactivité et la qualité des services assurés aux citoyens. L'édition de cette année est axée sur trois secteurs : la santé, l'éducation et le système judiciaire.

\section{Activités futures}

Pour produire le Panorama des administrations publiques, l'OCDE travaille en étroite collaboration avec d'autres organisations, parmi lesquelles l'Organisation internationale du Travail (OIT), le World Justice Project, la Commission européenne pour l'efficacité de la justice (CEPEJ), Gallup et la Commission européenne, afin de dresser un panorama complet de la nature et des modalités de l'action publique tout en évitant les doublons au niveau de la collecte des données. Cette coopération va être encore renforcée afin d'assurer la comparabilité des données pour les pays couverts par la publication.

Pour les éditions futures de la publication, il est prévu :

- d'actualiser et d'élargir le recueil de données sur les finances publiques et les dépenses publiques par fonction, notamment pour les pays de l'OCDE non membres de l'Union européenne ;

- de collaborer avec les offices statistiques nationaux en vue de recueillir des données sur les effectifs de la fonction publique par niveau d'administration ainsi que sur les grandes caractéristiques des agents publics (par exemple, sexe, âge) par le truchement du Système de comptabilité nationale ;

- d'élaborer de nouveaux indicateurs composites visant à mesurer les "réalisations intermédiaires ", notamment dans les domaines de la transparence budgétaire, de l'administration numérique, des marchés publics et de la gestion des risques ; 
- de fournir de nouveaux indicateurs relatifs aux réalisations dans des domaines étroitement liés aux grands principes de la gouvernance publique ou dans des secteurs assortis d'un impact important en termes de bien-être des citoyens (par exemple, diversité, y compris dans la sphère professionnelle) ;

- d'approfondir la collaboration déjà engagée entre le Secrétariat et les Directions de l'OCDE quant à l'utilisation pouvant être faite des nouvelles méthodes de collecte et d'analyse des données telles que l'exploration de texte ou les données massives.

\section{Éditions régionales du Panorama des administrations publiques}

La première édition du Panorama des administrations publiques axée sur l'Asie du Sud-Est a été publiée en septembre 2019. Cette édition régionale présente des données pour les dix pays membres de l'ASEAN (Association des nations de l'Asie du Sud-Est) ${ }^{1}$, en les comparant à la moyenne de la zone OCDE ainsi qu'au Japon, à la Corée, à l'Australie et à la NouvelleZélande. On trouvera dans cette édition des données sur les finances et économie publiques, l'emploi public, les pratiques et procédures budgétaires, la gestion des ressources humaines et l'ouverture et la transformation numérique de l'administration ; on y trouvera aussi un chapitre sur le thème "Au service des citoyens".

Par ailleurs, la troisième édition du Panorama des administrations publiques axée sur l'Amérique latine et les Caraibes est en cours de préparation et devrait être publiée en mars 2020. Dans cette publication, l'OCDE proposera les dernières données disponibles sur les administrations publiques de la zone Amérique latine-Caraibes et les comparera aux données relatives aux pays de l'OCDE.

En parallèle, l'édition d'un Panorama des administrations publiques axé sur les Balkans occidentaux ${ }^{2}$ est elle aussi en cours de préparation et devrait être publiée en mai 2020.

\section{L'ensemble des données et des indicateurs relatifs à la gouvernance publique est disponible en ligne}

Toutes les données recueillies par la Direction de la gouvernance publique de l'OCDE en vue, entre autres, de la production du Panorama des administrations publiques (à partir de son édition 2015) peuvent être consultées en ligne à l'adresse https://www.oecd.org/fr/gov/ panorama-des-administrations-publiques.htm.

Les lecteurs qui souhaiteraient utiliser les données présentées dans cette publication dans le cadre de travaux d'analyse ou de recherche sont invités à consulter la documentation complète relative aux définitions, aux sources et aux méthodes qui est fournie dans la publication elle-même et en ligne.

La base de données statistiques du Panorama des administrations publiques présente des indicateurs tant qualitatifs que quantitatifs sur les intrants, les processus, les produits et les réalisations du secteur public et elle est régulièrement actualisée, à mesure que de nouvelles données deviennent disponibles.

\section{Notes}

1. Les dix membres de l'ASEAN sont le Brunei Darussalam, le Cambodge, l'Indonésie, le Laos, la Malaisie, le Myanmar, les Philippines, Singapour, la Thaïlande et le Vietnam.

2. Les six pays couverts par cette publication sont l'Albanie, la Bosnie-Herzégovine, le Kosovo, le Monténégro, la Macédoine du Nord et la Serbie. 
Chapitre 1

\section{Vers des services publics centrés sur l'individu}




\section{Introduction}

Outre le gonflement de la dette et l'incertitude économique, la crise économique de 2008 a suscité du mécontentement au sein des populations qui se demandent si les pouvoirs publics œuvrent véritablement dans l'intérêt général ou seulement pour une minorité de citoyens. Cette désaffection est en train d'éroder les fondations sur lesquelles reposent les systèmes démocratiques des pays membres et non membres de l'OCDE et il est urgent d'agir pour renforcer la légitimité des institutions publiques. Bien qu'on relève enfin, dans de nombreux pays de l'OCDE, des signes d'amélioration de la confiance de la population à l'égard de ses administrations, laquelle s'était détériorée depuis la crise, dans d'autres pays, le niveau de cette confiance reste irrémédiablement plus bas qu'en 2007. Dans le présent chapitre, on défend l'idée que les pouvoirs publics, en adoptant une approche de l'élaboration des politiques et de la prestation de services centrée sur l'individu, peuvent rétablir la confiance dans l'administration publique, rendre leur action plus efficace et mieux relever les défis mondiaux et locaux qui se posent aux pays de l'OCDE.

Dans beaucoup de pays de l'OCDE, le vieillissement de la population modifie à la fois la structure du marché du travail et la demande de services publics. L'allongement de l'espérance de vie s'est traduit par un allongement de la durée des retraites : en 1970, un homme passait en moyenne 11 ans de sa vie à la retraite et une femme 15, mais en 2016, cette durée moyenne était passée à respectivement 18 et 22 ans (OCDE 2017 [1]]). Le taux de fécondité reculant dans la plupart des pays, les dépenses relatives aux pensions de retraite devraient augmenter et la masse des cotisations diminuer. Le plus souvent, des réformes ont été adoptées pour modérer les pressions qui s'exercent sur les finances publiques, en repoussant par exemple l'âge de départ à la retraite et en limitant les retraites anticipées. Cependant, les dépenses devraient aussi s'alourdir dans d'autres secteurs, comme celui de la santé où l'on prévoit qu'elles atteindront 10,2 \% du produit intérieur brut (PIB) d'ici 2030 (Lorenzoni et al. 2016 $[2]]$ ), contre 7,8 \% en 2017.

Une grande partie de la population est vulnérable aux chocs d'ordre financier (par exemple liés à une maladie de longue durée ou à une perte d'emploi). Depuis deux décennies, les écarts de revenu se creusent dans la plupart des pays de l'OCDE et on a enregistré une moindre redistribution des revenus par le biais de la fiscalité et des transferts (Causa, Browne et Vindics 2019 [3] ). Dans les pays de l'OCDE, les $10 \%$ de ménages les plus riches détiennent $52 \%$ du patrimoine net total, alors que les $10 \%$ d'habitants aux revenus les plus élevés concentrent $24 \%$ du revenu total (Balestra et Tonkin $2018_{[4]}$ ). Cela implique que la richesse (comme la détention de capital économique, prenant par exemple la forme d'un bien immobilier) est aujourd'hui deux fois plus forte que les inégalités de revenu ce qui indique que les citoyens n'ont pas tous la même capacité de réaction face à un choc financier.

Par ailleurs, les technologies numériques font évoluer la société et les démocraties, de même que les modalités de participation et d'influence de la population sur la vie civique et politique (Welby $2019_{[5]}$ ). Associées à la disponibilité croissante des données, 
au développement de leur utilisation et aux services fournis par le secteur privé entendus comme des points de repère, ces technologies sont en train de transformer les modes de production et de consommation des biens et des services dans le monde entier. Les attentes de la population quant à la façon dont les administrations publiques devraient travailler et fournir des services s'en trouvent, par voie de conséquence, modifiées. De plus en plus souvent, les citoyens veulent interagir avec leurs administrations par des moyens plus efficients, notamment grâce à des plateformes numériques et comptent bénéficier d'une qualité de service uniforme quelle que soit la voie empruntée pour accéder au service. Les technologies de l'information et de la communication (TIC) ont contribué, lorsqu'elles ont été convenablement appliquées, à une simplification des procédures administratives, à la suppression des opérations sur support papier et à la mise en place de points d'accès uniques aux différentes composantes de l'administration publique. Toutefois, ces nouvelles attentes font peser sur les administrations une pression en faveur d'une prestation de services mieux intégrée et plus proactive dans un contexte opérationnel marqué par des contraintes budgétaires.

On s'intéresse avant tout dans ce chapitre, qui s'appuie sur les données probantes présentées dans le reste de la publication afin de mettre en évidence les réalisations obtenues et les mécanismes de gouvernance en vigueur dans les pays de l'OCDE, à une approche de l'élaboration des politiques et de la prestation de services qui soit centrée sur l'individu. En outre, on y aborde des aspects qu'il faudrait encore s'efforcer d'améliorer et on y donne des exemples de bonnes pratiques. Des données plus détaillées sur les différents domaines de l'action publique figurent dans les autres chapitres de la publication.

\section{Qu'entend-on par " services publics centrés sur l'individu "?}

Des politiques et services publics « centrés sur l'individu » se caractérisent par la prise en compte des besoins et des points de vue exprimés par la population lors de leur élaboration, de leur mise en œuvre ou prestation et de leur évaluation. Pour centrer leur action sur l'individu, les pouvoirs publics peuvent directement associer les citoyens à la prise de décisions, recueillir et analyser des données susceptibles de servir à évaluer la performance des politiques et services publics eu égard aux besoins et aux attentes de la population, mais aussi anticiper ces besoins. Pour simplifier, une approche centrée sur l'individu est une approche suivant laquelle les pouvoirs publics consultent les citoyens sur leurs besoins et les encouragent à participer directement à l'élaboration des politiques ainsi qu'à la conception et à la prestation des services.

Pour que l'action publique soit centrée sur l'individu, il faut également que la fonction publique soit représentative et inclusive de la société en tant que cible des politiques et services publics. Un personnel très diversifié est composé d'individus de milieux diversifiés qui apportent du talent, des compétences et des points de vue distincts assortis d'une expérience particulière. Lorsque les diverses composantes de la population sont représentées dans le secteur public, la prestation des services correspondants peut être mieux adaptée à leurs besoins. De plus, si ils sont gérés de manière constructive, les différents points de vue peuvent aussi renforcer l'innovation (OCDE 2017[6]). En tant que telle, la notion d'action publique soit centrée sur l'individu inclut, tout en allant au-delà, des concepts tels que " centrée sur l'utilisateur " et " axée sur l'utilisateur ", qui se concentrent sur l'engagement des individus (usagers ou citoyens) pour développer des services et des politiques. L'action publique soit centrée sur l'individu prend également en compte les capacités et caractéristiques de la main-d'œuvre comme levier essentiel dans la fourniture 
de services inclusifs. Concevoir et mettre en place des politiques et services publics centrés sur l'individu suppose de surmonter la résistance au changement et les cloisonnements dans l'administration publique, en bâtissant et diffusant un savoir collectif, en simplifiant la circulation de l'information et en intégrant le traitement des données afin de toucher les citoyens où qu'ils se trouvent. Selon cette approche, les relations entre l'administration, les citoyens et les agents du secteur privé concernés sont régies par les principes d'accessibilité, de transparence, d'intégrité, de réactivité, de responsabilité, d'égalité et d'implication des parties prenantes. Les pouvoirs publics font délibérément des efforts pour associer les citoyens à l'élaboration des politiques, c'est-à-dire leur offrir la possibilité de collaborer avec les premiers à toutes les étapes du cycle d'élaboration des politiques ainsi qu'à la conception et à la prestation des services, et pour mettre à leur disposition les ressources et moyens nécessaires à cette collaboration (par exemple informations, données et outils numériques) (OCDE $2017_{[7]}$ ). En conséquence, l'ouverture de l'administration occupe une place centrale dans un modèle de prestation de services centrés sur l'individu.

Pour être inclusive, l'action publique devrait également cibler des groupes de population tels que les jeunes, les personnes âgées, les pauvres et les personnes qui disposent d'un accès limité à l'information et aux technologies et ont le sentiment d'être oubliées. La partie suivante présente pour les pays de l'OCDE des données probantes sur la performance liée à certains services publics et étudie la corrélation entre les indicateurs objectifs de performance des services et le degré de satisfaction des citoyens. La partie « Action publique centrée sur l'individu " présente des exemples de bonnes pratiques et de mesures adoptées par les administrations pour parvenir à concevoir et mettre en place des services publics centrés sur l'individu.

\section{Parvenir à mettre en place des services publics centrés sur l'individu et évaluer ces services}

La présente partie analyse les services publics dans les pays de l'OCDE sous les angles de l'accessibilité, de la réactivité, de la qualité et de la satisfaction des citoyens. Elle reconnaît également que l'augmentation de la satisfaction pourrait améliorer le niveau de confiance, un mécanisme de transmission scientifiquement appelé " hypothèse de micro-performance " (Van de Walle et Bouckaert $2003_{[8]}$; Yang et Holzer $2006_{[9]}$ ). Cette partie a pour socle le cadre d'analyse "Au service des citoyens » du « Panorama des administrations publiques ", prisme très utile pour comparer les performances des pays relatives aux différents services publics. Outre des indicateurs sur les principaux éléments de ce cadre, cette partie fait état du degré de satisfaction à l'égard des services, mesure de performance cruciale portant sur les retombées des services publics.

\subsection{Cadre d'analyse "Au service des citoyens"}

Chacun, tout au long de sa vie, entretient des relations avec les institutions publiques. Ces relations s'inscrivent généralement dans le cadre de la prestation de services tels que la délivrance d'une pièce d'identité ou la mise à disposition de biens publics par les administrations, la fourniture d'un enseignement ou de soins par les établissements scolaires et les hôpitaux publics, ou la possibilité d'adresser une demande en ligne de prestation sociale ou de déposer une réclamation au sujet d'une situation précise. Le cadre décrit cidessous est applicable à tous les types de service et appliqué, à ce jour, dans les secteurs de la santé, de l'éducation et de la justice car ce sont là des secteurs pour lesquels davantage de données sont disponibles et où il existe un consensus entre les différentes politiques 
concernées sur les indicateurs permettant de mesurer chacun d'eux. Toutefois, le cadre pourrait être étendu à d'autres secteurs au fil du temps.

Le terme « services publics » englobe un large éventail de biens et de services fournis à la population au titre de différents aspects de la vie en société et de la vie privée. Le cadre « Au service des citoyens » (tableau 1.1) a été créé par l'OCDE afin d'offrir une structure intégrée mettant en lumière les principaux aspects de la performance d'un service public qu'il est nécessaire d'évaluer selon le critère d'une approche centrée sur l'individu, indépendamment de la nature de ce service. Il permet également d'établir entre les pays une comparaison approximative des performances de différents services publics considérés sous des aspects identiques, bien que les indicateurs correspondant à ces aspects varient en fonction du type de service et ne sont pas directement comparables d'un service à un autre.

Les services publics centrés sur l'individu sont des services qui sont inclusifs (c'est-àdire accessibles à toutes les composantes de la population, y compris les plus vulnérables), adaptés aux besoins et aux attentes des citoyens (autrement dit qui satisfont les personnes ayant davantage besoin de l'aide des pouvoirs publics mais aussi celles qui en ont moins besoin et préfèrent se procurer les services en question par leurs propres moyens) et de grande qualité. Si chaque service a ses spécificités, les aspects pris en compte dans le cadre (tableau 1.1) nous permettent toutefois de comparer et d'évaluer les progrès accomplis par les pays de l'OCDE dans l'établissement d'une approche centrée sur l'individu dans les services publics.

Tableau 1.1. Cadre d'analyse de l'OCDE « Au service des citoyens "

\begin{tabular}{lll}
\hline Accessibilité & Réactivité & Qualité \\
\hline Accessibilité financière & Courtoisie et traitement & Efficacité de la prestation des services et des réalisations \\
Proximité géographique & $\begin{array}{l}\text { Correspondance entre les services } \\
\text { et les besoins spécifiques }\end{array}$ & $\begin{array}{l}\text { Cohérence de la prestation des services et des réalisations } \\
\text { Accès à l'information }\end{array}$ \\
\hline
\end{tabular}

Ces aspects revêtent tous la même importance car le fait d'accorder une trop grande place à l'un d'eux risque de nuire à la performance des deux autres. Ainsi, l'obtention d'un niveau de qualité élevé - du fait, par exemple, de très bons résultats scolaires - pourrait avoir des effets néfastes en termes d'accessibilité - comme par exemple la restriction du bénéfice d'un enseignement aux seuls élèves ou étudiants qui résident dans certains secteurs ou qui en ont les moyens financiers - et d'exclusion des élèves ou étudiants ayant besoin d'une aide supplémentaire - par exemple fourniture d'un enseignement unique, indépendamment de l'intérêt des élèves ou étudiants pour la matière et la compréhension qu'ils en ont.

Le cadre "Au service des citoyens" peut être utilisé comme un prisme théorique permettant de comparer des indicateurs qui, sans cet instrument, ne pourraient l'être (tels le taux de scolarisation et la couverture des soins de santé) ; pour être comparables, les indicateurs en question doivent toutefois porter sur le même aspect (l'accessibilité par exemple). Il n'en reste pas moins que le cadre pourrait encore être complété au moyen d'autres indicateurs ou bien d'auto-évaluations jugés essentiels pour l'amélioration du bien-être.

Par exemple, il existe à ce jour peu de données sur le vécu des patients qui sont comparables au niveau international. L'OCDE a récemment lancé l'enquête PaRIS (PatientReported Indicators Survey ou "Enquête pour l'établissement d'indicateurs fondés sur les déclarations des patients ") afin de recueillir des données sur les résultats de santé et le 
vécu des patients (il s'agit de savoir, par exemple, si les médecins fournissent suffisamment d'explications sur les traitements envisagés). Ces indicateurs peuvent fournir de précieux éléments de preuve sur l'efficacité réelle des traitements mis en œuvre par les médecins (comme les prothèses de genou) en termes d'amélioration de la qualité de vie des patients, en soulageant leur douleur par exemple. Ces éléments de preuve jouent un rôle crucial dans la prise de décisions sur le contenu du panier des traitements et actes médicaux qui doivent être pris en charge par le système public d'assurance maladie.

Les données nécessaires à l'élaboration des indicateurs correspondant à chaque aspect pourraient entre autres provenir des fichiers administratifs disponibles (par exemple dans les ministères d'exécution ou chez les prestataires de services), des enquêtes auprès des ménages réalisées par les offices statistiques nationaux et de sources internationales.

\subsection{Satisfaction à l'égard des services publics}

La satisfaction à l'égard des services publics est considérée comme une retombée fondamentale de l'action publique au titre de laquelle sont pris en compte des éléments essentiels pour la vie des citoyens (OCDE $2017_{[8]}$; OCDE $2017_{[9]}$ ). Il est généralement admis que la satisfaction est influencée par les attentes de la population ainsi que par le vécu des citoyens relatif aux services publics et aux informations sur ces services provenant d'autres sources (médias, internet, connaissances, etc.) (Jakobsen et al. 2015 [10- ; James 2009 [1]]). Une meilleure compréhension des facteurs déterminants de la satisfaction à l'égard des services publics permet aux pouvoirs publics de repérer les domaines dans lesquels des changements s'imposent pour répondre aux besoins de la population et tenir compte de ses préférences. Le degré de satisfaction à l'égard des services publics indique dans quelle mesure ces derniers sont conformes aux souhaits des citoyens et répondent à leurs attentes et leurs besoins. De ce fait, il peut uniquement être exprimé par les individus eux-mêmes, ce qui n'est pas le cas des indicateurs objectifs de performance des services (comme la durée moyenne de règlement d'une affaire dans les tribunaux de première instance qui est issue de données administratives), lesquels ne rendent pas compte de la perception qu'ont les citoyens des services dont ils bénéficient (par exemple savoir si les services publics leur apportent ce qu'ils estiment être le plus important).

Étant donné que la satisfaction à l'égard d'un service est liée en partie à l'accessibilité, à la réactivité et à la qualité de ce service telles qu'elles sont perçues par les citoyens, la mesurer peut apporter des éclairages propices à l'amélioration des services. Écouter les avis exprimés par les divers groupes de population peut contribuer à une réduction des dépenses. Par exemple, des solutions moins coûteuses et plus rapides peuvent être proposées aux personnes qui n'ont pas besoin d'une aide substantielle pour bénéficier d'un service donné, ces personnes affichant alors un degré de satisfaction plus élevé. On peut ainsi faire baisser la pression qui s'exerce sur les modes de prestation de services existants et réaffecter les économies dégagées au soutien d'autres composantes de la population qui requièrent davantage d'attention de la part des pouvoirs publics. En l'absence de solution universelle, l'innovation centrée sur l'individu est indispensable pour que les services publics répondent aux besoins des citoyens.

Lorsqu'ils adoptent une approche centrée sur l'individu, les pouvoirs publics ne peuvent prendre de décisions sur l'affectation des ressources et sur les changements de conception et de prestation des services sans consulter les citoyens ou analyser l'impact de ces décisions sur leur bien-être. Les indicateurs de satisfaction aident les décideurs à comprendre dans quelle mesure les politiques et services publics répondent aux besoins de la population. 
Selon une telle approche, le degré de satisfaction des citoyens devrait être pris en compte parallèlement à l'efficience et au rapport coût-efficacité qui sont des indicateurs servant les pouvoirs publics à évaluer la performance de la prestation de leurs services.

L'enquête Gallup World Poll a révélé qu'en 2018, en moyenne, les citoyens des pays de l'OCDE se disaient satisfaits de leur système de santé à $70 \%$ (proportion identique au chiffre de 2007), et satisfaits de leur système éducatif à $66 \%$ (soit une hausse de 3 points par rapport à 2007). En 2018, 55 \% des citoyens en moyenne se disaient confiants en leur système judiciaire, soit +4 points par rapport à 2007. Bien que de nombreux facteurs puissent influencer les réponses à un tel sondage - tels que l'expérience récente avec des fonctionnaires, la fatigue des répondants et les styles de réponse - et que les échantillons soient petits (1 000 cas par pays), Gallup World Poll permet, au fil du temps et dans les pays de l'OCDE, de comparer la perception des citoyens.

On note cependant des écarts prononcés entre les pays de l'OCDE. Le Danemark, la Norvège, les Pays-Bas et la Suisse bénéficient des niveaux de satisfaction les plus élevés à l'égard des services fournis dans les trois systèmes (santé, éducation et justice). En outre, la Belgique se classe deuxième quant au degré de satisfaction de sa population à l'égard des services de santé $(89 \%)$ et la Finlande troisième pour ce qui est du degré de satisfaction à l'égard des services d'éducation (84\%). Entre 2007 et 2018, le degré de satisfaction des citoyens à l'égard des services de santé a progressé en Estonie (+21 points) et en Lituanie (+19 points), tandis que Israël, les Pays-Bas et la Suisse figurent parmi les pays où le degré de satisfaction des citoyens à l'égard des services d'éducation a augmenté le plus fortement (+16 points dans le premier cas et +12 points dans les deuxième et troisième). À l'inverse, la Turquie a connu la baisse la plus marquée dans ce secteur (-17 points).

\subsection{Performance des services et degré de satisfaction des citoyens}

Les tableaux de bord du cadre d'analyse « Au service des citoyens » ont été présentés dans l'édition 2017 du « Panorama des administrations publiques ». Comme mentionné supra, ils montrent les performances relatives des pays en matière d'accessibilité, de réactivité et de qualité des services d'éducation, de santé et de justice, ainsi que de satisfaction des citoyens à l'égard de ces services. Les indicateurs pris en compte dans les tableaux de bord en question ont été choisis par des experts de l'OCDE en raison de leur aptitude à mesurer les concepts ainsi que la disponibilité des données. Naturellement, les tableaux de bord " Au service des citoyens » donnent seulement un aperçu de l'accessibilité, de la réactivité et de la qualité des services. Ils illustrent néanmoins la satisfaction et la performance à l'égard des services publics dans les pays de l'OCDE. Tandis que plus loin dans la publication, les performances des trois secteurs (éducation, santé et justice) sont comparées cote à cote pour chaque dimension, l'analyse ci-dessous fait état de l'accès, la réactivité et la qualité de chaque service ${ }^{1}$.

En ce qui concerne les soins de santé, les indicateurs sélectionnés pour les tableaux de bord "Au service des citoyens " sont liés à la qualité des politiques de santé dans leur ensemble, y compris la prévention. En ce sens, ils diffèrent de ceux utilisés dans le rapport phare de l'OCDE sur la santé (Panorama de la santé) qui aborde la qualité des soins fournis aux patients (par exemple, hospitalisations évitables, traumatismes obstétricaux). Cette distinction est pertinente car les indicateurs affichés dans les tableaux de bord «Au service des citoyens " reflètent également les comportements de la population en matière d'autosoins (par exemple, en respectant les échéances recommandées pour les checkup médicaux). 
Chaque tableau de bord se concentre sur un service (éducation, santé et justice) et compare toutes les dimensions du cadre. Pour chaque indicateur, les pays sont classés en trois quantiles en fonction de leurs performances: 1) tiers supérieur (en vert); 2) le tiers intermédiaire (orange); et 3) tiers inférieur (rouge). De plus, chaque pays est classé parmi les pays pour lesquels des données sont disponibles, afin de fournir des informations supplémentaires sur les performances (le pays avec la meilleure performance est classé numéro 1). Si plusieurs pays ont la même valeur pour un indicateur, ils se voient attribuer le même rang.

Comme le fait apparaître le tableau 1.2, selon les indicateurs sélectionnés, aucun pays ne surclasse les autres selon l'ensemble des trois critères d'accessibilité, de réactivité et de qualité des politiques de santé. La majorité des pays de l'OCDE affichent de meilleures performances que les autres pays en ce qui concerne un ou deux critères et, pour un critère donné, il est possible qu'ils se placent en haut du classement pour un indicateur et plus bas dans ce classement pour un autre indicateur. De plus, les taux de mortalité liés aux trois causes figurant dans le tableau de bord (crise cardiaque, accident vasculaire cérébral et cancer du sein] dépendent de nombreux autres facteurs, notamment comportementaux (le tabagisme par exemple), que les systèmes de santé ne peuvent complètement maîtriser.

La plupart des pays qui affichent un niveau de satisfaction élevé ont mis en place une couverture santé universelle et enregistrent une faible proportion de besoins non satisfaits en matière de soins (indépendamment du poids des dépenses de santé restant à la charge 
Tableau 1.2. Tableau de bord relatif à l'accessibilité, à la réactivité et à la qualité des politiques et services de santé

\begin{tabular}{|c|c|c|c|c|c|c|c|c|c|c|}
\hline \multirow[b]{3}{*}{ Indicateurs } & \multicolumn{10}{|c|}{ Soins de santé } \\
\hline & \multicolumn{3}{|c|}{ Accessibilité } & \multicolumn{3}{|c|}{ Réactivité } & \multicolumn{3}{|c|}{ Qualité } & \multirow[b]{2}{*}{$\begin{array}{c}\text { Classement } \\
\text { en } \\
\text { termes de } \\
\text { satisfaction } \\
\text { des } \\
\text { citoyens }\end{array}$} \\
\hline & $\begin{array}{c}\text { Couverture } \\
\text { des soins } \\
\text { de santé }\end{array}$ & $\begin{array}{l}\text { Besoins } \\
\text { non } \\
\text { satisfaits } \\
\text { en matière } \\
\text { de soins }\end{array}$ & $\begin{array}{c}\text { Poids du } \\
\text { reste à charge } \\
\text { dans la } \\
\text { consommation } \\
\text { des ménages }\end{array}$ & $\begin{array}{l}\text { Pas } \\
\text { toujours de } \\
\text { rappel du } \\
\text { médecin le } \\
\text { jour même }\end{array}$ & $\begin{array}{l}\text { Délai pour } \\
\text { obtenir un } \\
\text { rendez-vous } \\
\text { avec le } \\
\text { généraliste }\end{array}$ & $\begin{array}{l}\text { Délai pour } \\
\text { obtenir un } \\
\text { rendez-vous } \\
\text { avec un } \\
\text { spécialiste }\end{array}$ & $\begin{array}{c}\text { Taux de } \\
\text { mortalité - } \\
\text { infarctus aigu } \\
\text { du myocarde } \\
\text { (crise } \\
\text { cardiaque) }\end{array}$ & $\begin{array}{c}\text { Taux de } \\
\text { mortalité - } \\
\text { accident } \\
\text { vasculaire } \\
\text { cérébral (AVC) }\end{array}$ & $\begin{array}{c}\text { Taux de } \\
\text { mortalité - } \\
\text { cancer } \\
\text { du sein } \\
\text { chez les } \\
\text { femmes }\end{array}$ & \\
\hline Pays-Bas & 2 & 2 & 8 & 3 & 2 & 1 & 7 & 16 & 26 & 1 \\
\hline Belgique & 4 & 9 & 25 & S.0 & S.0 & S.0 & 9 & 12 & 24 & 2 \\
\hline Norvège & 1 & 7 & 22 & 4 & 1 & 8 & 20 & 9 & 6 & 2 \\
\hline Danemark & 1 & 7 & 17 & S.o & S.0 & S.0 & 5 & 18 & 27 & 3 \\
\hline Suisse & 1 & 4 & 35 & 1 & 4 & 1 & 4 & 1 & 15 & 3 \\
\hline Australie & 1 & S.0 & 16 & 4 & 3 & 5 & 14 & 10 & 9 & 4 \\
\hline Autriche & 2 & 1 & 30 & S.0 & S.0 & S.0 & 24 & 6 & 18 & 5 \\
\hline Nouvelle-Zélande & 1 & S.0 & 4 & 6 & 8 & 4 & 30 & 23 & 16 & 6 \\
\hline Allemagne & 1 & 3 & 11 & 2 & 11 & 3 & 23 & 13 & 28 & 7 \\
\hline Luxembourg & S.0 & 3 & 2 & S.0 & S.0 & S.0 & 10 & 5 & 22 & 8 \\
\hline Finlande & 1 & 17 & 24 & S.0 & S.0 & S.0 & 25 & 22 & 9 & 9 \\
\hline Suède & 1 & 8 & 27 & 7 & 9 & 7 & 21 & 14 & 7 & 9 \\
\hline Royaume-Uni & 1 & 15 & 7 & 8 & 7 & 6 & 15 & 15 & 22 & 10 \\
\hline Slovénie & 1 & 15 & 1 & S.0 & S.0 & S.0 & 27 & 27 & 28 & 11 \\
\hline États-Unis & 10 & S.0 & 14 & 8 & 5 & 2 & 16 & 11 & 9 & 12 \\
\hline Canada & 1 & S.0 & 13 & 9 & 10 & 9 & 17 & 3 & 12 & 13 \\
\hline République tchèque & 1 & 3 & 5 & S.0 & S.0 & S.0 & 19 & 26 & 14 & 13 \\
\hline Japon & 1 & S.0 & 10 & S.0 & S.0 & S.0 & 1 & 13 & 3 & 14 \\
\hline Corée & 1 & S.0 & 34 & S.0 & S.0 & S.0 & 6 & 20 & 1 & 15 \\
\hline France & 2 & 6 & 3 & 5 & 6 & 3 & 2 & 2 & 21 & 16 \\
\hline Israël & 1 & S.0 & 18 & S.0 & S.0 & S.0 & 3 & 4 & 23 & 17 \\
\hline Espagne & 2 & 2 & 26 & S.0 & S.0 & S.0 & 6 & 8 & 5 & 18 \\
\hline Irlande & 1 & 13 & 12 & S.0 & S.0 & S.0 & 29 & 17 & 29 & 19 \\
\hline Portugal & 1 & 10 & 29 & S.0 & S.0 & S.0 & 13 & 28 & 10 & 20 \\
\hline République slovaque & 5 & 11 & 6 & S.0 & S.0 & S.0 & 26 & 33 & 25 & 20 \\
\hline Estonie & 6 & 20 & 19 & S.0 & S.0 & S.0 & 8 & 19 & 11 & 21 \\
\hline Turquie & 3 & 12 & S.0 & S.0 & S.0 & S.0 & 32 & 32 & 2 & 21 \\
\hline Mexique & 11 & S.0 & 23 & S.0 & S.0 & S. 0 & 33 & 21 & 4 & 22 \\
\hline Lituanie & 8 & 8 & 20 & S.0 & S.0 & S. 0 & 11 & 34 & 13 & 23 \\
\hline Pologne & 9 & 16 & 9 & S.0 & S.0 & S. 0 & 12 & 25 & 20 & 24 \\
\hline Italie & 1 & 9 & 21 & S.0 & S.0 & S.0 & 8 & 24 & 17 & 25 \\
\hline Hongrie & 7 & 5 & 28 & S.0 & S.0 & S.0 & 28 & 31 & 30 & 26 \\
\hline Grèce & 1 & 19 & 32 & S.0 & S. 0 & S.0 & 22 & 29 & 19 & 27 \\
\hline Chili & 7 & S.0 & 31 & S.0 & S.0 & S.0 & 31 & 30 & 8 & 28 \\
\hline Lettonie & 1 & 18 & 33 & S. 0 & S.0 & S.0 & 27 & 35 & 26 & 28 \\
\hline Islande & 1 & 14 & 15 & S. 0 & S. 0 & S.0 & 18 & 7 & 28 & S.0 \\
\hline \multirow[t]{2}{*}{ Année } & 2017 & 2018 & 2017 & 2017 & 2017 & 2017 & 2017 & 2017 & 2017 & 2018 \\
\hline & $\begin{array}{l}\text { Tiers supé } \\
\text { Tiers interr } \\
\text { Tiers inféri }\end{array}$ & diaire & & & & & & & & \\
\hline
\end{tabular}

Note : Les pays sont listés par ordre croissant en fonction de leur rang dans la satisfaction. En raison de la disponibilité limitée des données, les codes couleur ne sont pas affichés. Le nombre dans la cellule indique la position de chaque pays parmi ceux disponibles. Pour la couverture des soins de santé, le regroupement a été produit de la manière suivante : groupe du tiers supérieur (entre $95 \%$ et $100 \%$ pour la couverture des soins de santé), groupe du tiers intermédiaire (entre $90 \%$ et $95 \%$ ), groupe du tiers inférieur (moins de $90 \%$ ). Les données sur les besoins en soins non satisfaits proviennent de l'enquête EU-SILC qui demande un examen de la situation, à tout moment au cours des 12 mois précédant l'entretien, et en quoi les répondants ont estimé qu'ils avaient besoin d'un examen médical et ne l'ont pas reçu. Les données ne présentent que le nombre de répondants qui n'ont pas pu l'obtenir à cause de la distance, des temps d'attente ou des coûts. Les paiements directs ne sont pas inclus dans le coût total des soins fournis par le patient, pas plus qu'ils ne sont inclus dans le forfait. Les résultats incluent également pour certains pays des estimations des paiements informels aux prestataires de soins de santé. La question "Après avoir été conseillé ou décidé de consulter un spécialiste, combien de jours, de semaines ou de mois avez-vous dû attendre pour obtenir un rendez-vous ? ". Le temps d'attente pour les médecins et les spécialistes ne représente qu'une partie de ces répondants qui doivent prendre rendez-vous. Le niveau de satisfaction vis-à-vis des soins de santé est basé sur le principe " satisfait » lorsqu'on demande : " Dans la ville où vous habitez, êtes-vous satisfait ou insatisfait de la disponibilité et de la qualité des soins de santé ? ".

Sources : OCDE (2019), Statistiques de l'OCDE sur la santé (base de données); Eurostat (2019), Fonds du Commonwealth (2017) Fonds du Commonwealth International Enquête sur les politiques de santé des personnes âgées, Gallup World Poll 2018 (base de données). 
des patients dans la consommation des ménages). Ils obtiennent en outre des performances relativement meilleures sous certains aspects de la réactivité et de la qualité des services. Par exemple, bien qu'on n'y observe pas les taux de mortalité globale les plus bas par crise cardiaque, l'Australie et la Norvège font partie des pays qui bénéficient des plus faibles de taux de mortalité dans les 30 jours suivant une admission à l'hôpital pour cette pathologie (voir l'indicateur de qualité des soins de santé au chapitre 11 consacré au cadre d'analyse "Au service des citoyens").

Les données sur la couverture des soins de santé datent de 2017, à l'exception de l'Espagne et du Japon, qui datent de 2014. Les données sur les besoins en soins non satisfaits pour l'Allemagne, la France, l'Irlande, la Lituanie, le Luxembourg, la Norvège, le Royaume-Uni et la Suisse concernent 2017 ; concernant l'Islande, les données concernent 2016 et non 2018. Les données relatives à la part des dépenses de santé remboursables au Chili datent de 2014. Les données sur l'infarctus aigu du myocarde, les maladies vasculo-cérébrales et la mortalité par cancer du sein pour les pays suivants Allemagne, Australie, Belgique, Chili, Corée, Espagne, Estonie, États-Unis, Finlande, Grèce, Israël, Luxembourg, Mexique, Norvège, Pays-Bas, Pologne, Portugal, Royaume-Uni, Suède, Suisse et Turquie et font référence à 2016 au lieu de 2017. Les données sur le Canada, le Danemark, la France, l'Irlande, l'Italie, la Lettonie et la Slovénie sont de 2015 et non 2017. Pour les données relatives à Israël, voir http://doi.org/10.1787/888932315602. En ce qui concerné l'éducation, la plupart des pays dont les citoyens sont très satisfaits du système éducatif, parmi lesquels la Norvège et la Suisse, par exemple, affichent des performances élevées pour au moins un des indicateurs de qualité, de réactivité et d'accessibilité (voir tableau 1.3). Ces pays ont en commun des dépenses privées relativement faibles en matière d'éducation (imputables pour l'essentiel aux ménages) de l'enseignement primaire à l'enseignement supérieur et, à l'exception du Luxembourg, des résultats relativement élevés à l'enquête du Programme international pour le suivi des acquis des élèves (PISA). Ces pays répondent également aux besoins des élèves et étudiants et des établissements scolaires, dans une certaine mesure, en faisant appel à des méthodes d'enseignement flexibles ou en proposant une aide à l'apprentissage, et en fournissant des outils pédagogiques adaptés. 


\section{Tableau 1.3. Tableau de bord relatif à l'accessibilité, à la réactivité et à la qualité} des services d'éducation

\begin{tabular}{|c|c|c|c|c|c|c|c|c|c|c|}
\hline & \multicolumn{10}{|c|}{ Services d'éducation } \\
\hline & \multicolumn{3}{|c|}{ Accessibilité } & \multicolumn{3}{|c|}{ Réactivité } & \multicolumn{3}{|c|}{ Qualité } & \multirow[b]{2}{*}{ Satisfaction } \\
\hline & $\begin{array}{l}\text { Dépenses } \\
\text { privées } \\
\text { consacrées } \\
\text { à l'éducation } \\
\text { (de l'ensei- } \\
\text { gnement } \\
\text { primaire au } \\
\text { supérieur) }\end{array}$ & $\begin{array}{c}\text { Taux de } \\
\text { scolarisation } \\
\text { des enfants } \\
\text { de } 4 \text { ans }\end{array}$ & $\begin{array}{c}\text { Taux } \\
\text { d'accès à } \\
\text { l'ensei- } \\
\text { gnement } \\
\text { supérieur }\end{array}$ & $\begin{array}{l}\text { Indice } \\
\text { d'insuffi- } \\
\text { sance des } \\
\text { outils péda- } \\
\text { gogiques }\end{array}$ & $\begin{array}{l}\text { Disponibilité } \\
\text { d'une aide à } \\
\text { l'apprentis- } \\
\text { sage dans } \\
\text { les établis- } \\
\text { sements } \\
\text { scolaires }\end{array}$ & $\begin{array}{l}\text { Recours } \\
\text { à des } \\
\text { méthodes } \\
\text { d'ensei- } \\
\text { gnement } \\
\text { flexibles }\end{array}$ & $\begin{array}{l}\text { Score } \\
\text { moyen en } \\
\text { sciences à } \\
\text { l'enquête } \\
\text { PISA }\end{array}$ & $\begin{array}{l}\text { Score } \\
\text { moyen en } \\
\text { mathéma- } \\
\text { tiques à } \\
\text { l'enquête } \\
\text { PISA }\end{array}$ & $\begin{array}{c}\text { Score moyen } \\
\text { en } \\
\text { compréhension } \\
\text { de l'écrit à } \\
\text { l'enquête PISA }\end{array}$ & \\
\hline Norvège & 2 & 8 & 12 & 18 & 30 & 11 & 18 & 14 & 7 & 1 \\
\hline Suisse & S.0 & 32 & 3 & 4 & 23 & 20 & 12 & 3 & 22 & 2 \\
\hline Danemark & S.0 & 3 & 4 & 11 & 1 & 7 & 15 & 7 & 15 & 3 \\
\hline Finlande & 1 & 28 & 21 & 23 & 15 & 14 & 3 & 8 & 2 & 3 \\
\hline Irlande & 10 & 1 & S.0 & 28 & 28 & 19 & 13 & 13 & 3 & 4 \\
\hline Pays-Bas & 22 & 9 & 14 & 12 & 22 & 17 & 11 & 6 & 12 & 4 \\
\hline Slovénie & 12 & 22 & 11 & 8 & 24 & S.0 & 7 & 9 & 11 & 5 \\
\hline Luxembourg & 3 & 14 & 28 & 15 & 2 & 31 & 26 & 26 & 28 & 6 \\
\hline Belgique & 7 & 2 & 9 & 25 & 26 & 27 & 14 & 10 & 17 & 7 \\
\hline Pologne & 13 & 27 & 7 & 5 & 13 & 22 & 16 & 12 & 10 & 8 \\
\hline Autriche & 6 & 18 & 13 & 10 & 36 & 34 & 20 & 15 & 25 & 9 \\
\hline Canada & 26 & S.0 & S.0 & 1 & 7 & 2 & 4 & 5 & 1 & 9 \\
\hline République tchèque & 17 & 23 & 17 & 16 & 18 & 30 & 23 & 21 & 24 & 9 \\
\hline France & 16 & 1 & S.0 & 14 & 16 & 28 & 21 & 19 & 16 & 9 \\
\hline Nouvelle-Zélande & 28 & 10 & 1 & 17 & 8 & 3 & 6 & 16 & 8 & 9 \\
\hline Royaume-Uni & 31 & 1 & 10 & 19 & 3 & 10 & 9 & 20 & 19 & 9 \\
\hline Australie & 33 & 24 & S.0 & 3 & 6 & 4 & 8 & 18 & 13 & 10 \\
\hline Allemagne & 18 & 13 & 20 & 22 & 25 & 33 & 10 & 11 & 9 & 11 \\
\hline Mexique & 25 & 21 & 23 & 33 & 34 & 6 & 36 & 36 & 36 & 11 \\
\hline Portugal & 20 & 19 & 19 & 24 & 12 & 1 & 17 & 22 & 18 & 12 \\
\hline Israël & 23 & 4 & 15 & 32 & 17 & 21 & 31 & 31 & 29 & 13 \\
\hline Espagne & 24 & 5 & 6 & 27 & 32 & 15 & 24 & 25 & 21 & 13 \\
\hline États-Unis & 32 & 31 & 25 & 6 & 4 & 5 & 19 & 32 & 20 & 14 \\
\hline Suède & 4 & 16 & 18 & 9 & 5 & 12 & 22 & 17 & 14 & 15 \\
\hline Estonie & 9 & 20 & S.0 & 20 & 21 & 23 & 2 & 4 & 4 & 16 \\
\hline Japon & 29 & 12 & 5 & 36 & 9 & 35 & 1 & 1 & 6 & 17 \\
\hline Italie & 15 & 15 & 24 & 35 & 35 & 29 & 27 & 23 & 26 & 18 \\
\hline République slovaque & 19 & 29 & 22 & 21 & 20 & 32 & 32 & 30 & 34 & 18 \\
\hline Chili & 34 & 26 & 2 & 7 & 33 & 8 & 34 & 34 & 33 & 19 \\
\hline Grèce & 8 & 30 & 26 & 30 & 27 & 25 & 33 & 33 & 32 & 20 \\
\hline Lettonie & 11 & 17 & S.0 & 13 & 14 & 9 & 25 & 27 & 23 & 20 \\
\hline Corée & 30 & 7 & S.0 & 31 & 29 & 24 & 5 & 2 & 5 & 21 \\
\hline Hongrie & 21 & 11 & 27 & 34 & 19 & 26 & 28 & 29 & 31 & 22 \\
\hline Lituanie & 14 & 25 & 8 & 29 & 10 & 16 & 29 & 28 & 30 & 23 \\
\hline Turquie & 27 & 33 & S.0 & 26 & 31 & 18 & 35 & 35 & 35 & 24 \\
\hline Islande & 5 & 6 & 16 & 2 & 11 & 13 & 30 & 24 & 27 & S.0 \\
\hline Année & 2016 & 2017 & 2017 & 2015 & 2015 & 2015 & 2015 & 2015 & 2015 & 2018 \\
\hline & Tiers supér & & & & & & & & & \\
\hline & Tiers intern & édiaire & & & & & & & & \\
\hline & Tiers inféri & & & & & & & & & \\
\hline
\end{tabular}

Note : Les pays sont listés par ordre croissant en fonction de leur rang dans la satisfaction. Le nombre dans la cellule indique la position de chaque pays parmi tous les pays pour lesquels des données sont disponibles. L'indice de pénurie de matériel pédagogique a été calculé sur la base des réponses fournies par les directeurs d'école sur la mesure dans laquelle la capacité de leur école à dispenser un enseignement était entravée (" pas du tout ", " très peu ", " dans une certaine mesure » ou " beaucoup ") en raison d'une pénurie ou d'une insuffisance d'infrastructures matérielles, telles que les bâtiments scolaires, les systèmes de chauffage et de climatisation et les locaux d'enseignement ; et du matériel pédagogique, tel que des manuels scolaires, du matériel de laboratoire, du matériel didactique et des ordinateurs. La moyenne de l'indice est égale à zéro et l'écart-type est de 1 dans les pays de l'OCDE. La disponibilité de l'aide à l'étude indique si, quand le personnel de l'école aide les élèves à faire leurs devoirs, cela est indiqué par les directeurs d'école. L'indicateur sur l'utilisation de méthodes d'enseignement adaptatives couvre le nombre d'élèves déclarant que leurs enseignants apportent une aide individuelle lorsqu'un élève a des difficultés à comprendre un sujet ou une tâche dans " plusieurs leçons " et " lors de chaque leçon ou presque chaque ". Le degré de satisfaction à l'égard de l'éducation est basé sur la proportion de répondants qui ont déclaré être " satisfaits » à la question : "Dans la ville ou la région où vous habitez, êtes-vous satisfait ou insatisfait du système éducatif et des écoles ? ”.

L'enseignement primaire au Canada comprend le préprimaire et le préprimaire en Irlande comprend l'éducation de la petite enfance. En Australie, aux États-Unis, en Nouvelle-Zélande et au Royaume-Uni, la part importante des dépenses privées consacrées à l'éducation est associée à une part importante des étudiants bénéficiant de prêts et de bourses.

Les données pour le Danemark et la Suisse ne sont pas disponibles. Les données relatives aux dépenses privées d'éducation en Grèce concernent 2015 et le Chili 2017. Pour les données relatives à Israël, voir http://doi.org/10.1787/888932315602.

Source : OCDE (2019), Statistiques de l'OCDE sur l'éducation (base de données), OCDE (2015) PISA (base de données), Gallup World Poll 2018 (base de données). 
Le moindre degré de satisfaction constaté dans des pays qui obtiennent de bons résultats à l'enquête PISA pourrait s'expliquer par les tensions financières qu'induisent les frais de scolarité ou les apprentissages périscolaires pour les ménages, et par le fait que les élèves sont contraints d'obtenir de bons résultats. Ainsi que le révèle le cadre d'analyse "Au service des citoyens ", l'ensemble des services d'éducation réactifs doit être accessible à tous les citoyens pour qu'ils soient satisfaits.

Enfin, concernant le système judiciaire, le tableau 1.4 montre que la plupart des pays dans lesquels la confiance dans le système judiciaire est forte affichent des performances relativement meilleures pour les indicateurs sélectionnés. Le niveau de confiance coïncide en particulier avec les indicateurs de qualité (bonne mise en application de la justice civile, absence d'influence indue des autorités et non-recours à la violence en cas de griefs).

Tableau 1.4. Tableau de bord relatif à l'accessibilité, à la réactivité et à la qualité du système judiciaire

\begin{tabular}{|c|c|c|c|c|c|c|c|c|c|}
\hline \multirow[b]{3}{*}{ Indicateurs } & \multicolumn{9}{|c|}{ Judiciary system } \\
\hline & \multicolumn{2}{|c|}{ Accessibilité } & \multicolumn{3}{|c|}{ Réactivité } & \multicolumn{3}{|c|}{ Qualité } & \multirow[b]{2}{*}{ Confiance } \\
\hline & $\begin{array}{l}\text { La justice } \\
\text { civile est } \\
\text { accessible } \\
\text { et } \\
\text { abordable }\end{array}$ & $\begin{array}{l}\text { Les mécanismes } \\
\text { alternatifs de } \\
\text { règlement des } \\
\text { litiges sont } \\
\text { accessibles, } \\
\text { impartiaux et } \\
\text { efficaces }\end{array}$ & $\begin{array}{l}\text { Temps disponible } \\
\text { lors d'une } \\
\text { affaire civile, } \\
\text { commerciale, } \\
\text { administrative ou } \\
\text { autre en première } \\
\text { instance }\end{array}$ & $\begin{array}{c}\text { Temps } \\
\text { disponible } \\
\text { Iors d'affaires } \\
\text { contentieuses } \\
\text { civiles et } \\
\text { commerciales }\end{array}$ & $\begin{array}{c}\text { Temps } \\
\text { disponible } \\
\text { Iors d'affaires } \\
\text { administratives } \\
\text { (cours } \\
\text { suprêmes) }\end{array}$ & $\begin{array}{c}\text { Bonne } \\
\text { mise en } \\
\text { application } \\
\text { de la justice } \\
\text { civile }\end{array}$ & $\begin{array}{c}\text { Absence } \\
\text { d'influence } \\
\text { indue des } \\
\text { autorités sur la } \\
\text { justice civile }\end{array}$ & $\begin{array}{l}\text { Non-recours } \\
\text { à la violence } \\
\text { en cas de } \\
\text { griefs }\end{array}$ & \\
\hline Norvège & 9 & 1 & 12 & 10 & S.0 & 2 & 1 & 4 & 1 \\
\hline Danemark & 4 & 4 & 1 & 12 & S.0 & 4 & 2 & 2 & 2 \\
\hline Suisse & S.0 & S.0 & 9 & 3 & 8 & S.0 & S.0 & S.0 & 3 \\
\hline Finlande & 17 & 19 & 9 & 16 & 11 & 6 & 8 & 5 & 4 \\
\hline Luxembourg & S.0 & S.0 & S.0 & 2 & S.0 & S.0 & S.0 & S.0 & 4 \\
\hline Canada & 23 & 16 & S.0 & S.0 & S.0 & 13 & 7 & 7 & 5 \\
\hline Allemagne & 2 & 5 & S.o & 13 & 15 & 5 & 3 & 12 & 5 \\
\hline Pays-Bas & 1 & 6 & 6 & 4 & 7 & 3 & 6 & 18 & 5 \\
\hline Autriche & 10 & 20 & 4 & 6 & 16 & 8 & 9 & 9 & 6 \\
\hline Royaume-Uni & 26 & 17 & S.0 & S.0 & 17 & 14 & 13 & 15 & 7 \\
\hline Irlande & S.0 & S.0 & S.0 & S.0 & S.0 & S.0 & S.0 & S.0 & 8 \\
\hline Suède & 3 & 26 & 10 & 11 & 3 & 1 & 5 & 1 & 9 \\
\hline Nouvelle-Zélande & 8 & 12 & S.0 & S.0 & S.0 & 15 & 12 & 11 & 10 \\
\hline Japon & 11 & 2 & S.0 & S.o & S.0 & 12 & 16 & 3 & 11 \\
\hline Australie & 20 & 9 & S.o & S.o & S.0 & 10 & 4 & 13 & 12 \\
\hline France & 15 & 7 & 15 & 21 & 14 & 11 & 15 & 22 & 13 \\
\hline Belgique & 6 & 15 & S.0 & S.0 & 19 & 9 & 11 & 17 & 14 \\
\hline Lituanie & S.0 & S.0 & 3 & 1 & 1 & S.0 & S.0 & S.0 & 14 \\
\hline États-Unis & 27 & 18 & S.0 & S.0 & S.0 & 17 & 19 & 16 & 15 \\
\hline République tchèque & 18 & 11 & 11 & 8 & 18 & 18 & 17 & 6 & 16 \\
\hline Grèce & 16 & 23 & S.0 & 24 & 22 & 25 & 23 & 26 & 16 \\
\hline Israël & S.0 & S.0 & 16 & 20 & 2 & S.0 & S.0 & S.0 & 17 \\
\hline Estonie & 7 & 8 & 2 & 7 & 3 & 16 & 10 & 10 & 18 \\
\hline Portugal & 14 & 10 & S.0 & 19 & 20 & 24 & 14 & 24 & 19 \\
\hline Hongrie & 24 & 27 & 4 & 9 & 4 & 26 & 27 & 8 & 20 \\
\hline Pologne & 21 & 13 & 7 & 14 & 5 & 20 & 25 & 19 & 20 \\
\hline Turquie & 25 & 24 & S.0 & 22 & 6 & 22 & 28 & 23 & 20 \\
\hline République slovaque & S.0 & S.0 & 8 & 5 & 9 & S.0 & S.0 & S.0 & 21 \\
\hline Espagne & 5 & 14 & 14 & 18 & 13 & 23 & 22 & 21 & 22 \\
\hline Mexique & 28 & 28 & S.0 & S.0 & S. 0 & 27 & 26 & 28 & 23 \\
\hline Italie & 22 & 25 & 17 & 23 & 21 & 28 & 20 & 25 & 24 \\
\hline Slovénie & 12 & 22 & 5 & 17 & 12 & 21 & 24 & 14 & 24 \\
\hline Lettonie & S.0 & S.0 & 13 & 15 & 10 & S.0 & S.0 & S.0 & 25 \\
\hline Chili & 13 & 21 & S.0 & S.0 & S.0 & 19 & 21 & 27 & 26 \\
\hline Corée & 19 & 3 & S.0 & S.0 & S.o & 7 & 18 & 20 & n.d. \\
\hline Islande & S.0 & S.0 & S.o & S.o & S.0 & S.0 & S.0 & S.0 & S.o \\
\hline Année & 2019 & 2019 & 2016 & 2016 & 2016 & 2019 & 2019 & 2019 & 2018 \\
\hline & $\begin{array}{l}\text { Tiers supé } \\
\text { Tiers intern } \\
\text { Tiers inféri }\end{array}$ & diaire & & & & & & & \\
\hline
\end{tabular}




\section{Tableau 1.4. Tableau de bord relatif à l'accessibilité, à la réactivité et à la qualité du système judiciaire (suite)}

Note: Les pays sont listés par ordre croissant en fonction de leur rang dans la satisfaction. Le nombre dans la cellule indique la position de chaque pays parmi tous les pays pour lesquels des données sont disponibles. L'état de droit, établi par World Justice Projects, repose sur une enquête auprès de 1000 répondants (représentatifs) dans les trois plus grandes villes de chaque pays et sur une enquête auprès d'experts en droit civil (praticiens et universitaires). Chaque dimension de l'index présente un score allant de 0 à 1 ; un score plus élevé signifie une meilleure performance sur la dimension. L'accès et le caractère abordable de la justice civile permettent de mesurer entre autres la connaissance des droits et des mécanismes de résolution des litiges, des coûts des services juridiques, de l'existence d'une discrimination à l'égard des minorités. L'accessibilité, l'impartialité et l'efficacité des mécanismes alternatifs de résolution des litiges mesurent entre autres les coûts de tels mécanismes, le temps requis pour parvenir à un règlement, l'exécution des décisions. On évalue le degré d'absence d'influence indue en se demandant dans quelle mesure un plaignant est susceptible de gagner un procès contre l'État, dans quelle mesure l'administration est susceptible de respecter cette décision et de chercher à influencer le tribunal. L'application effective de la justice civile mesure l'exécution des décisions de justice et le respect des délais. L'enquête sur l'efficacité et la rapidité du système de jugement pénal rend compte du temps nécessaire pour traduire un suspect en justice, de la durée de sa détention provisoire, ainsi que de la connaissance de si les auteurs de crimes violents sont arrêtés et traduits en justice. Le recours à la violence comprend l'intimidation ou l'attaque de l'auteur d'un délit, par exemple. Le contrôle effectif de la criminalité inclut notamment la perception par les citoyens d'être en sécurité lorsqu'ils marchent la nuit et d'être victimes d'un crime au cours de la ou des trois dernières années (selon la question). Le temps de décision indique le temps estimé nécessaire pour résoudre une affaire, ce qui implique le temps nécessaire à un tribunal de première instance pour parvenir à une décision. Il est calculé en divisant le nombre d'affaires en cours lors d'une année donnée par le nombre d'affaires résolues au cours de la même période, multiplié par 365. Les affaires civiles et commerciales litigieuses font référence à des différends entre parties, tels que des divorces contentieux. Les affaires non contentieuses concernent des procédures non contestées, par exemple les ordres de paiement non contestés. Les affaires commerciales sont traitées par des tribunaux spécialisés dans certains pays et par des tribunaux civils dans d'autres. Les affaires administratives concernent des litiges entre citoyens et autorités locales, régionales ou nationales. Il existe des tribunaux spécialisés dans ce type de différends dans certains pays et des tribunaux civils dans d'autres. Les pays diffèrent dans la manière dont ils administrent la justice et répartissent les responsabilités entre les tribunaux ; par conséquent, les comparaisons entre pays doivent être considérées avec prudence. Il existe des différences dans les types de tribunaux et entre les cas inclus dans cet exercice, ainsi que différentes méthodes de collecte et de catégorisation des données. La confiance dans le pouvoir judiciaire repose sur les réponses à la question « Dans ce pays, avez-vous confiance en chacun des éléments suivants ou non ? Qu'en est-il du système judiciaire et des tribunaux ? ". Les données sont exprimées en proportion des répondants ayant répondu " oui ". Les données sur la confiance pour la Corée ne sont pas affichées (s.o). Sur les données pour Israël, voir http://doi.org/10.1787/888932315602.

Source: World Justice Project (2019), état de droit Index 2019, base de données de la CEPEJ (2017).

Ces trois tableaux de bord fournissent un indice selon lequel la satisfaction des citoyens à l'égard des services publics est liée aux performances conjuguées en matière d'accessibilité, de réactivité et de qualité. La satisfaction à l'égard des soins de santé et la confiance dans le pouvoir judiciaire font état de liens plus clairs avec les mesures de performance que la satisfaction à l'égard du système éducatif. Par exemple, le pourcentage de personnes déclarant avoir confiance dans le système judiciaire est corrélé à l'application effective de la justice civile $(r=0,67)$ et la satisfaction à l'égard des soins de santé est négativement corrélée aux besoins de soins non satisfaits des personnes à faible revenu $(r=-0,57)$. La disponibilité limitée des données et le nombre total d'observations ne permettent pas de réaliser une analyse plus poussée tenant compte de l'ampleur des effets relatifs et des liens entre les différents aspects. Cela étant, cet aperçu indique - comme le fait que, dans certains pays, les citoyens sont relativement plus satisfaits d'un ou deux services publics que des autres - qu'il est possible de tirer des enseignements (par exemple décentralisation, ouverture de l'administration...) pour apprendre de la gouvernance d'un secteur au profit des autres secteurs.

\section{Action publique centrée sur l'individu}

Pour que l'action publique puisse être centrée sur l'individu, il convient de mettre en place des processus inclusifs, des données probantes et des structures permettant de veiller à ce que les politiques et leur mise en œuvre reflètent et intègrent les points de vue des personnes qu'elle touche. Il est crucial de centrer davantage l'action publique sur le citoyen ; dans l'ensemble de la zone OCDE, les habitants jugent les services et avantages produits par le secteur public difficiles d'accès et sont nombreux à trouver qu'ils ne reçoivent pas tous les avantages qu'ils devraient, au vu des impôts qu'ils paient (OCDE 2019 ${ }_{\text {[12] }}$ ). L'ouverture 
de l'administration et la transition numérique devraient permettre, à terme, de recueillir et d'analyser davantage de données sur la satisfaction des usagers à l'égard de certains services publics (soins médecins, enseignants, etc.) et de s'appuyer sur des outils tels que les enquêtes et les données massives (big data) pour mieux comprendre les besoins des usagers et les intégrer à la conception et à la prestation des politiques publiques.

On constate que trois grands piliers favorisent une action publique centrée sur le citoyen. Premièrement, une démarche centrée sur l'individu exige de la transparence, de l'ouverture et une véritable association des citoyens. Deuxièmement, les services publics doivent être conçus et organisés à partir des besoins de la population, ce qui implique de disposer de données sur les préférences des usagers et sur leur utilisation des services publics et de s'en inspirer pour la conception et la prestation de ces services. Il faut aussi analyser et évaluer tous les types d'activités publiques, afin de favoriser l'amélioration continue, mais aussi la transparence sur les succès et les échecs rencontrés (OCDE à paraître $\left.{ }_{[13]}\right)$. Troisièmement, une action publique centrée sur l'individu passe par une fonction publique dotée des compétences et des aptitudes requises pour répondre aux besoins des usagers et pour les anticiper.

\subsection{Une gouvernance ouverte, transparente, participative et redevable}

L'ouverture, le dialogue, la transparence et la redevabilité sont des notions liées ; cette partie présente des exemples issus de divers pays de l'OCDE qui illustrent les voies que les pays peuvent suivre en la matière, pour un large éventail de domaines de la gouvernance publique. Aujourd'hui, la gamme des dispositifs et des outils que les pays peuvent employer pour nouer avec les citoyens un dialogue permanent et constructif est plus large que jamais, notamment grâce aux technologies de l'information et des communications, aux plateformes numériques et aux données publiques ouvertes. Les pays de l'OCDE ont largement adopté les technologies numériques, aussi bien comme moyen d'assurer des services aux citoyens que comme source de gains d'efficience, grâce à la simplification et à l'automatisation des processus. Les TIC représentent néanmoins un outil puissant afin d'aider les pouvoirs publics à comprendre et anticiper les besoins ; à évaluer, redéfinir et moderniser les services publics ; et à placer les besoins des usagers au cœur de la conception des services publics. De plus, les technologies émergentes telles que l'intelligence artificielle (IA) et les chaînes de blocs (blockchain) représentent un potentiel considérable pour rendre les services publics plus « intelligents »-c'est-à-dire plus agiles, plus efficients, plus conviviaux et, par conséquent, plus dignes de confiance (Ubaldi et al. $2019_{[14]}$ ).

Réussir la transformation numérique des services publics implique de se mettre à la place de l'usager lors de la conception des solutions numériques, afin de réduire les obstacles à l'accès aux services. Toutefois, assurer avec efficience des services en ligne signifie décloisonner l'administration publique et renforcer la collaboration entre ses composantes (par exemple en mutualisant l'information et en procédant à une planification conjointe, avec des budgets communs). Les portails en ligne nationaux peuvent également contribuer à la transition vers un " gouvernement numérique ", en favorisant par exemple l'intégration des services (voir encadré 1.1). Ils combinent des données, des systèmes et des processus pour offrir aux citoyens un point d'accès unique aux services officiels. Selon les dernières données disponibles (2014), 19 pays de l'OCDE sur 21, qui ont participé à l'enquête sur l'administration numérique, déclaraient disposer d'un portail en ligne permettant d'assurer des services aux administrés. 


\section{Encadré 1.1. Principales caractéristiques d'une démarche cohérente en matière d'administration numérique}

La Recommandation du Conseil sur les stratégies numériques gouvernementales formulait, autour de trois piliers, 12 principes visant à permettre de bien concevoir, élaborer et mettre en œuvre les stratégies relatives aux outils numériques, de façon à accompagner la transformation vers l'administration numérique. Depuis l'adoption de cette Recommandation en 2014, l'OCDE a œuvré aux côtés des pays et la réflexion s'est restructurée autour de six dimensions considérées comme dotées d'une forte influence sur le degré de maturité de l'administration numérique d'un pays :

1. La conception numérique : volonté de l'administration d'aborder le numérique en ayant analysé l'ensemble des activités stratégiques nécessaires à une transformation réussie et durable en modifiant la culture de la prestation des services publics.

2. Un secteur public axé sur les données : reconnaissance de l'importance des données en tant qu'outil fondamental permettant aux composantes du secteur public de collaborer dans le but d'anticiper les besoins, d'organiser la prestation des services publics, de comprendre les changements et d'y réagir.

3. L'ouverture par défaut : volonté des pouvoirs publics de collaborer par-delà les frontières administratives ainsi qu'avec des acteurs extérieurs à la sphère publique ; il s'agit d'un marqueur important d'une culture qui intégrera les principes de transparence et de redevabilité qui sous-tendent les modes de travail numériques.

4. Un fonctionnement axé sur l'usager : démarche de prestation des services publics appuyée par une culture d'ouverture et mue par une ambition visant à pratiquer une conception numérique des services publics afin d'intégrer et de se laisser guider par les besoins des usagers plutôt que par les hypothèses des pouvoirs publics.

5. Une administration plateforme : construction d'un écosystème visant à apporter aux agents publics le soutien et les moyens nécessaires pour mener des politiques publiques et assurer des services publics en tirant parti des possibilités de collaborer entre autres avec les particuliers, les entreprises et la société civile.

6. La proactivité : aptitude des administrations à anticiper et à répondre rapidement aux besoins des administrés grâce la mise en œuvre des cinq dimensions susmentionnées. Une administration transformée permet de traiter les problèmes de bout en bout, plutôt que de procéder au coup par coup à la numérisation de telle ou telle composante.

Un dispositif d'identification numérique légalement reconnu (la signature numérique par exemple) permet aux usagers d'accéder à de multiples services en ligne de l'administration à partir du portail national destiné aux citoyens. L'identification numérique permet une prestation de services plus sophistiquée, répondant mieux aux besoins et aux attentes des administrés. Selon les dernières données disponibles de 2014, 20 pays de l'OCDE sur 21 ont mis en place des dispositifs d'identification numérique. L'expérience des pays montre que pour faire adopter plus largement les dispositifs numériques, il est essentiel de couvrir un éventail de services aussi large que possible, de les rendre très simples à utiliser et d'assurer l'interopérabilité des systèmes.

Il est également essentiel d'intégrer les nouvelles technologies à une administration numérique pour parvenir au gouvernement ouvert, notion définie dans la Recommandation $\mathrm{du}$ Conseil sur le gouvernement ouvert comme « une culture de gouvernance qui promeut les principes de transparence, d'intégrité, de redevabilité et de participation des parties 
prenantes, au service de la démocratie et de la croissance inclusive ". Le gouvernement ouvert (on parle aussi d'" administration ouverte ») est une démarche axée sur le citoyen qui vise à créer de la valeur publique grâce à des dispositifs collaboratifs en matière de conception et de mise en œuvre de l'action publique. Les dernières données disponibles montrent que les 36 pays de l'OCDE ont lancé des initiatives d'ouverture de l'administration. Ces initiatives sont intégrées, soit à une stratégie de gouvernement ouvert, soit à d'autres plans ou stratégies (voir graphique 1.1). Par ailleurs, en 2016, dans 21 pays de l'OCDE, le centre de gouvernement participait à la conception des stratégies et des initiatives d'ouverture de l'administration; dans 20 pays, il participait également à leur mise en œuvre. De telles initiatives ont été lancées dans plusieurs domaines d'action et sont souvent transversales. Toutefois, l'existence de ces dispositifs ne signifie pas nécessairement que les pays tirent pleinement parti des possibilités offertes par le gouvernement ouvert. Ainsi, pour que les administrés continuent de participer à de telles initiatives, il est crucial de veiller à ce qu'elles améliorent concrètement leur vécu. Il faut donc suivre l'élaboration de ces initiatives, évaluer leur impact et s'appuyer sur l'information ainsi recueillie pour améliorer l'action menée.

\section{Graphique 1.1. Existence d'une stratégie en matière de gouvernement ouvert, 2015}

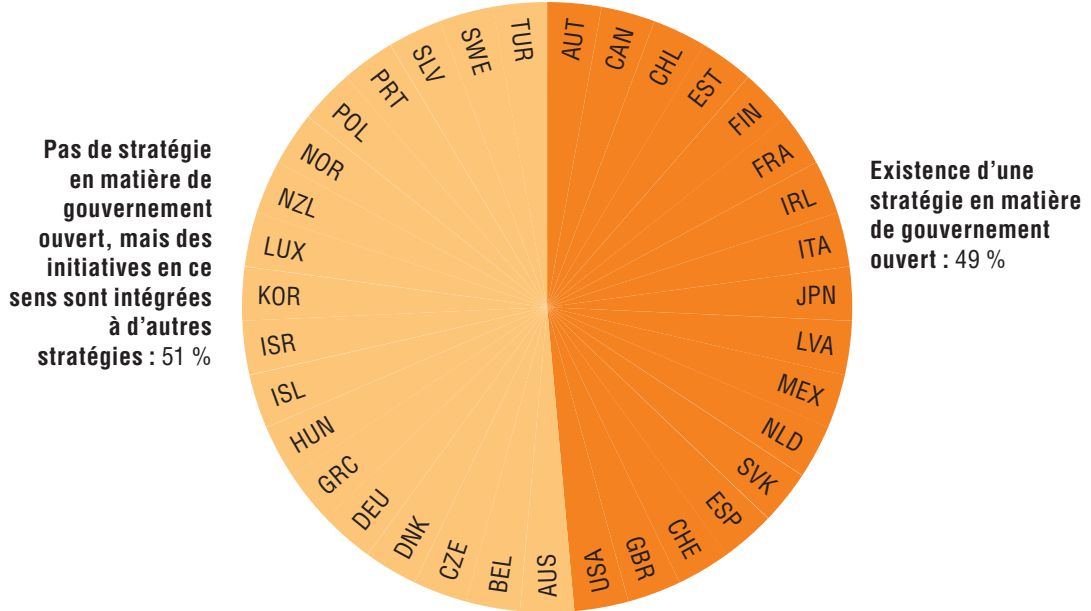

Note : Pour les données concernant Israël, voir http://doi.org/10.1787/888932315602.

Source : OCDE (2015 $[15]$ ), « Enquête sur la coordination de l'overture de l'administration et la participation des citoyens au cycle de l'action publique ».

Le succès des initiatives lancées en matière de gouvernement ouvert dépend, en grande partie, de l'aptitude à associer véritablement les parties prenantes concernées : particuliers, entreprises, consommateurs et employés. En dialoguant avec les parties prenantes et en tenant compte de leur vécu, de leurs connaissances, de leurs points de vue et de leurs idées, les administrations publiques obtiennent des renseignements précieux sur lesquels baser leurs décisions stratégiques. L'information recueillie auprès des parties prenantes peut permettre d'éviter certains effets non escomptés et certains problèmes concrets lors de la mise en œuvre de politiques ou de textes (OCDE 2018 ${ }_{[16]}$ ).

L'association des parties prenantes illustre en quoi les politiques d'ouverture de l'administration peuvent favoriser l'émergence de services publics centrés sur l'individu. En 2016, 28 pays de l'OCDE indiquaient que leur centre de gouvernement consultait 
directement les parties prenantes sur les politiques publiques. De plus, les pays de l'OCDE sont globalement attachés à l'association des parties prenantes dans le cadre de l'élaboration des textes aussi bien législatifs que réglementaires. C'est ce que révèlent les indicateurs de l'OCDE relatifs à la politique et à la gouvernance réglementaires (iREG), qui portent sur quatre aspects : méthodologie ; adoption systématique ; transparence ; et contrôle et suivi de la qualité. Toutefois, il existe une marge de progression s'agissant d'ouvrir davantage les consultations au grand public et de les rendre plus utiles au sein du processus de l'action publique.

Sur tous les territoires étudiés (c'est-à-dire les pays de l'OCDE, les pays candidats à l'adhésion et l'Union européenne), l'association des parties prenantes est obligatoire pour l'élaboration d'au moins certains textes. En général, les exigences formelles et les pratiques de consultation sont moins contraignantes pour les textes réglementaires (voir graphique 1.2). Les parties prenantes sont souvent associées à un stade tardif de l'élaboration d'un texte, une fois que la marche à suivre pour résoudre un problème a été déterminée. Cela prive les parties prenantes de la possibilité d'intervenir à un stade où elles pourraient soumettre des options alternatives à l'appréciation des responsables publics.

\section{Graphique 1.2. Participation des parties prenantes à l'élaboration des textes réglementaires, 2017}

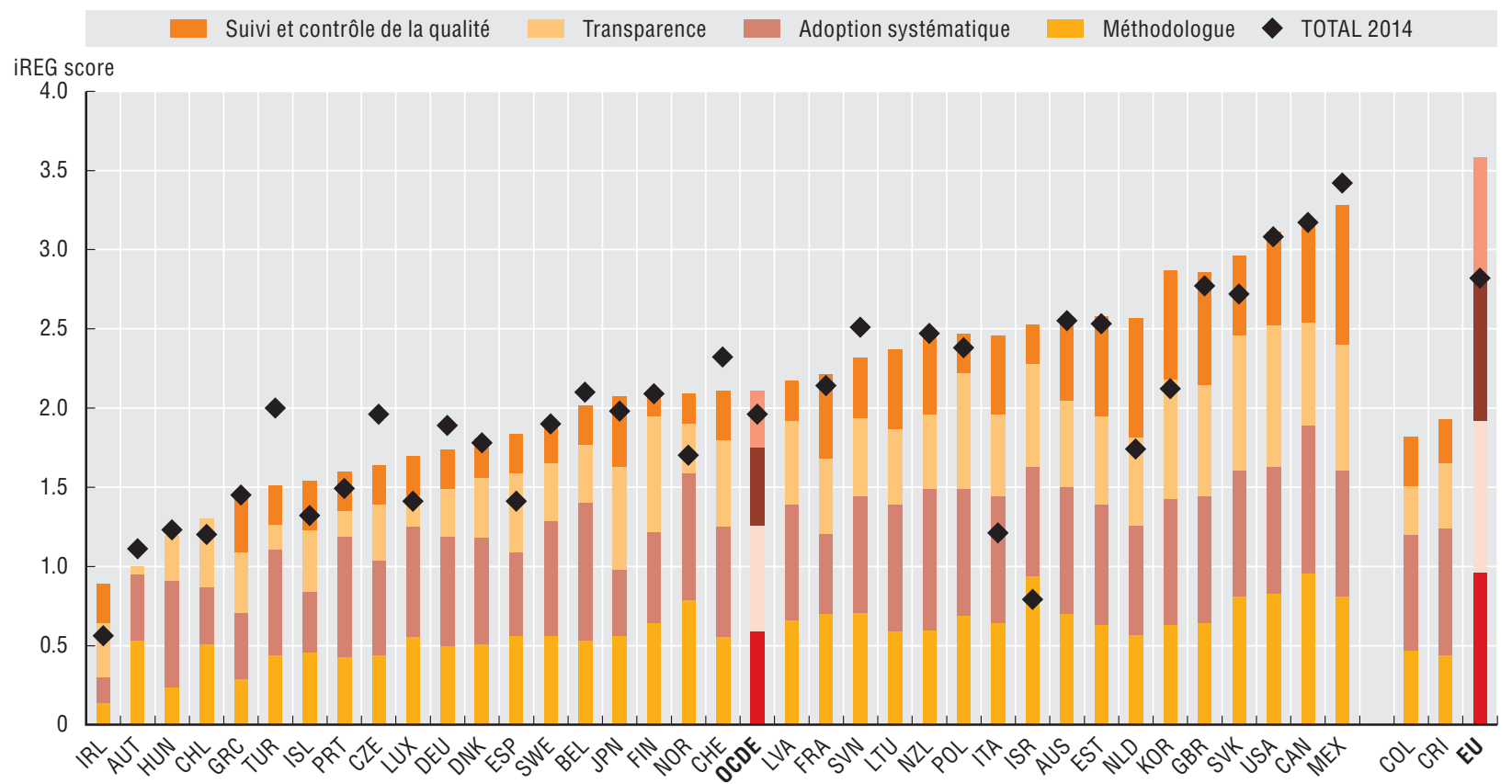

Note : Pour les données concernant Israël, voir http://doi.org/10.1787/888932315602. Les données pour les pays de l'OCDE sont basées sur les 34 pays de l'OCDE qui étaient membres en 2014 et de l'Union européenne. Les données sur les nouveaux pays membres de l'OCDE et les pays en voie d'adhésion (Colombie, Costa Rica, Lettonie et Lituanie) ne sont disponibles que pour 2017. Plus le nombre de pratiques réglementaires préconisées dans la recommandation de 2012 qu'un pays a mise en œuvre est grand, plus son score iREG est élevé. Le score iREG varie de 0 à 4, 0 étant le plus bas et 4 le plus élevé. L'indicateur ne couvre que les pratiques de l'exécutif.

Source : OCDE Indicateurs relatifs à la politique et à la gouvernance réglementaires (iREG) 2015 [17] et 2018 [18], http://oe.cd/ireg.

L'association des parties prenantes à l'élaboration des textes réglementaires a modérément progressé. Ces progrès tiennent, pour l'essentiel, à une meilleure transparence du système - notamment à l'accessibilité publique des informations relatives aux consultations prévues, des commentaires formulés par les parties prenantes et des réponses fournies suite 
à ces commentaires. Certaines améliorations ont aussi été apportées à la méthodologie employée dans le but d'associer les parties prenantes, notamment aux premiers stades de l'élaboration des textes (voir graphique 1.2).

Les pays ont tout intérêt à progresser davantage sur les terrains de la transparence et du contrôle de leurs systèmes d'association des parties prenantes. Par exemple, de nombreux pays publient en ligne les commentaires formulés dans le cadre des consultations et les transmettent aux décideurs, mais la plupart des pays n'ont pas pour pratique systématique d'informer la population à l'avance qu'une consultation va être réalisée, ni de revenir vers elle sur la façon dont les commentaires ont été pris en compte. Cela peut dissuader les parties prenantes de participer à d'autres consultations, voire peser sur l'engagement civique et sur la propension à respecter les textes - ce qui est à l'opposé de la raison d'être de l'association des parties prenantes (Lind et Arndt 2016). Sur le plan du contrôle, la plupart des pays ne procèdent pas à des évaluations régulières des performances de leurs dispositifs d'association des parties prenantes.

Enfin, l'évaluation de l'action publique peut renforcer la transparence et la redevabilité du secteur public, surtout quand les résultats des évaluations sont ouvertement débattus, mis en lien avec les décisions adoptées et accessibles pour les professionnels comme pour le grand public. La plupart des pays de l'OCDE se sont dotés de certains dispositifs pour encourager la mise à profit des conclusions des évaluations de politiques publiques (intégration des conclusions au cycle budgétaire, examen des conclusions en conseil des ministres, dispositif invitant la direction à réagir).

\subsection{Mieux assurer les services publics grâce à une administration fondant davantage son action sur les données}

Pour centrer les services publics sur l'individu, les administrations publiques ont besoin de données sur les besoins, préférences, préoccupations et attentes des administrés, ainsi que sur le rôle que ceux-ci entendent jouer pour influer sur les politiques et services publics et pour contribuer à leur élaboration. Qu'elles soient locales ou nationales, les administrations accumulent un volume considérable de données dans le cadre de la prestation des services publics, de la mise en œuvre des textes législatifs et réglementaires et de la réalisation d'opérations financières. Ces données ont des attributs et des valeurs qui pourraient être mis à profit pour mieux adapter les services publics aux besoins de la population. De plus en plus, les pouvoirs publics voient dans les données un actif stratégique susceptible d'améliorer l'action publique ainsi que la conception et la prestation des services publics (OCDE 2014 [20]).

La mise à profit des données au sein du secteur public permet de créer de la valeur publique par le truchement de trois types d'activités :

- Anticipation et planification : utilisation des données dans le cadre de la conception des politiques, de la planification des interventions, de l'anticipation des évolutions possibles et de la prévision des besoins.

- Prestation des services : utilisation des données pour fournir des éclairages et améliorer la mise en œuvre de l'action publique, la réactivité des pouvoirs publics et la prestation des services publics.

- Évaluation et suivi : utilisation des données pour mesurer l'impact, contrôler les décisions et suivre les performances. L'évaluation et le suivi s'appuient sur une combinaison de données d'enquête et administratives dans le but de contribuer ex ante à la conception des services publics et ex post à l'évaluation de la performance. Les compétences en 
matière d'évaluation des politiques au sein du gouvernement sont le plus souvent attribuées au centre du gouvernement et aux ministères des Finances afin d'assurer une cohérence, des incitations et des normes de qualité appropriées (OCDE à paraitre ${ }_{[23]}$ ).

\section{Encadré 1.2. Exemples de recours aux données massives par des pays de l'OCDE}

Les autorités irlandaises utilisent principalement les données massives dans le cadre d'activités de cartographie. Le service cartographique irlandais (Ordnance Survey Ireland) utilise un service dénommé Geohive qui offre un accès facile aux données géospatiales d'accès public. Combinées aux données issues d'autres sources, ces données cartographiques aident à analyser les évolutions du marché immobilier et les risques d'inondation. L'Irlande a aussi créé Pobal, un site internet couplé à un service d'aide qui fournit des renseignements sur le niveau de pauvreté des zones, sur les services disponibles en matière de petite enfance et sur les autres services publics dont les citoyens peuvent bénéficier. Il s'agit d'une ressource à l'usage des responsables publics, mais aussi les citoyens et les associations.

En Corée, un projet relatif à l'analyse des données massives dans le secteur public favorise depuis 2014 une démarche scientifique et fondée sur des données probantes pour la gestion de l'administration centrale, des administrations locales et des institutions publiques. La Corée a mis au point un modèle normalisé d'analyse des données massives au sein du secteur public, de façon à ce que les données créés dans une composante du secteur public puissent être comparées aux données créés dans les autres composantes de ce secteur. Les modèles produits grâce à ce dispositif fournissent des éclairages pour l'action à mener dans des domaines tels que le tourisme, les transports, la vidéosurveillance et le logement social, le tout avec 16 modèles normalisés ayant été fournis à 175 organisations depuis 2017. Une telle normalisation limite les divergences dans les analyses faites par différentes institutions, notamment entre l'administration centrale et les administrations locales, ce qui permet de mener, à partir de tel ou tel ensemble de données, une action fondée sur une analyse plus exacte et plus complète.

À l'heure actuelle, l'Union européenne finance plusieurs projets transfrontaliers portant sur l'utilisation des données massives, y compris le projet MIDAS (Meaningful Integration of Data Analytics and Services). Il s'agit d'une plateforme qui vise à cartographier, acquérir, gérer, modéliser, traiter et exploiter les données existantes et hétérogènes sur la santé, ainsi que d'autres données publiques et des données ouvertes extérieures afin de permettre la création d'une information concrète et fondée sur des données probantes. On escompte que cette plateforme permettra aux responsables publics de prendre de meilleures décisions à long terme et qu'elle améliorera les soins dispensés sur l'ensemble du continent, que ce soit à l'échelon régional, national ou européen.

Collaboration mondiale : les données publiques présentent souvent un intérêt mondial et peuvent être mises à profit pour prévenir et résoudre des problèmes d'envergure mondiale. On peut citer l'exemple du Groupe sur l'observation de la Terre (GEO), initiative de collaboration intergouvernementale visant à intégrer et partager les données issues de l'observation de la Terre. Son Système mondial des systèmes d'observation de la Terre (GEOSS), infrastructure publique mondiale qui crée en temps réel des données environnementales exhaustives, a pour objet de fournir des renseignements et des analyses à l'intention d'un large éventail d'utilisateurs et de décideurs du monde entier.

Les données massives (big data), c'est-à-dire les données à fort volume, à forte vélocité et à forte variété (Kim et al. 2014 [21] ), font partie des types de données qui peuvent aboutir à la création de valeur dans le cadre des services publics. On demande de plus en plus aux 
pouvoirs publics d'intégrer ces technologies à leur mode de fonctionnement. Les données massives aident les administrations publiques à produire des indicateurs reflétant des données récentes, à repérer beaucoup plus vite les points d'inflexion et à prendre des décisions plus judicieuses et plus rapides face aux situations d'urgence (par exemple, catastrophes naturelles, pandémies ou crises économiques ; voir encadré 1.2).

Centrer la prestation des services publics sur l'individu implique aussi de permettre aux usagers d'accéder et de réutiliser tous types de données publiques. L'ouverture des données publiques permet de conférer plus de pouvoir aux particuliers, aux entreprises, aux organisations de la société civile, aux chercheurs et aux journalistes en leur offrant plus de possibilités d'accéder et d'utiliser les données. Par conséquent, de nombreux pays de l'OCDE utilisent les données publiques ouvertes (DPO) pour alimenter un écosystème permettant de faire apparaître des services et des solutions novateurs grâce à des initiatives lancées par des entreprises privées ou par des citoyens (qui créent des applications utilisant des données publiques, accèdent à des données ouvertes de façon automatisée par le truchement d'applications ou encore programment des interfaces permettant de mieux suivre la passation des marchés publics, par exemple).

L'indice OURdata de l'OCDE (données publiques ouvertes, utiles et réutilisables) permet de comparer l'action menée par les pays pour concevoir et mettre en œuvre des politiques et initiatives d'ouverture des données publiques. Il prend en compte la disponibilité des données, leur accessibilité et le soutien public à leur réutilisation en vue de créer de la valeur économique et sociale. Il représente un prolongement du cadre d'analyse de l'OCDE en matière de données publiques ouvertes, qui est lui-même lié aux principes de la Charte internationale sur les données ouvertes (Lafortune et Ubaldi $2018_{[22]}$ ). L'indice OURdata aide les pays à évaluer leurs forces et à repérer les domaines dans lesquels une action peut s'avérer nécessaire.

Les politiques d'ouverture des données publiques ont contribué à une montée en puissance des interactions avec les usagers ou les professionnels des données en vue de créer de la valeur publique. Dans l'ensemble des pays de l'OCDE, le score global de l'indice a progressé par rapport à 2017. À l'heure actuelle, 90 \% des pays de l'OCDE pour lesquels on dispose de données imposent la gratuité des données publiques ; 87 \% imposent que les données soient disponibles sous licence ouverte ; et $93 \%$ imposent que les données soient fournies dans un format lisible par machine (voir graphique 1.3).

De manière générale, les pouvoirs publics ont aussi intensifié leurs efforts sur le plan du soutien à la réutilisation des DPO. Désormais, 64 \% des pays de l'OCDE traitent comme une priorité la collaboration et les manifestations de co-création avec les usagers, ainsi que le renforcement des capacités et des aptitudes au sein de l'administration. Les pays sont plus nombreux qu'en 2017 à étudier les incidences possibles de l'ouverture des données publiques, en menant des travaux de recherche ou en recueillant des exemples de réutilisations des données (voir graphique 1.3). Il serait néanmoins encore possible de progresser sur le plan de l'intégration des données ouvertes et de l'exploitation de leurs avantages. Ainsi, les administrations publiques pourraient aller plus loin dans la définition de normes en termes de format de données et de procédures de publication, afin de davantage améliorer la qualité et l'accessibilité des données. De plus, la mise en place, sur les portails de données publiques ouvertes, de canaux ou d'autres dispositifs permettant les retours d'information peut inciter les usagers des données ouvertes à apporter leur contribution (OCDE 2018 $\left.{ }_{[25]}\right)$. 


\section{Graphique 1.3. Indice OURdata (données ouvertes, utiles et réutilisables), 2017 et 2019}

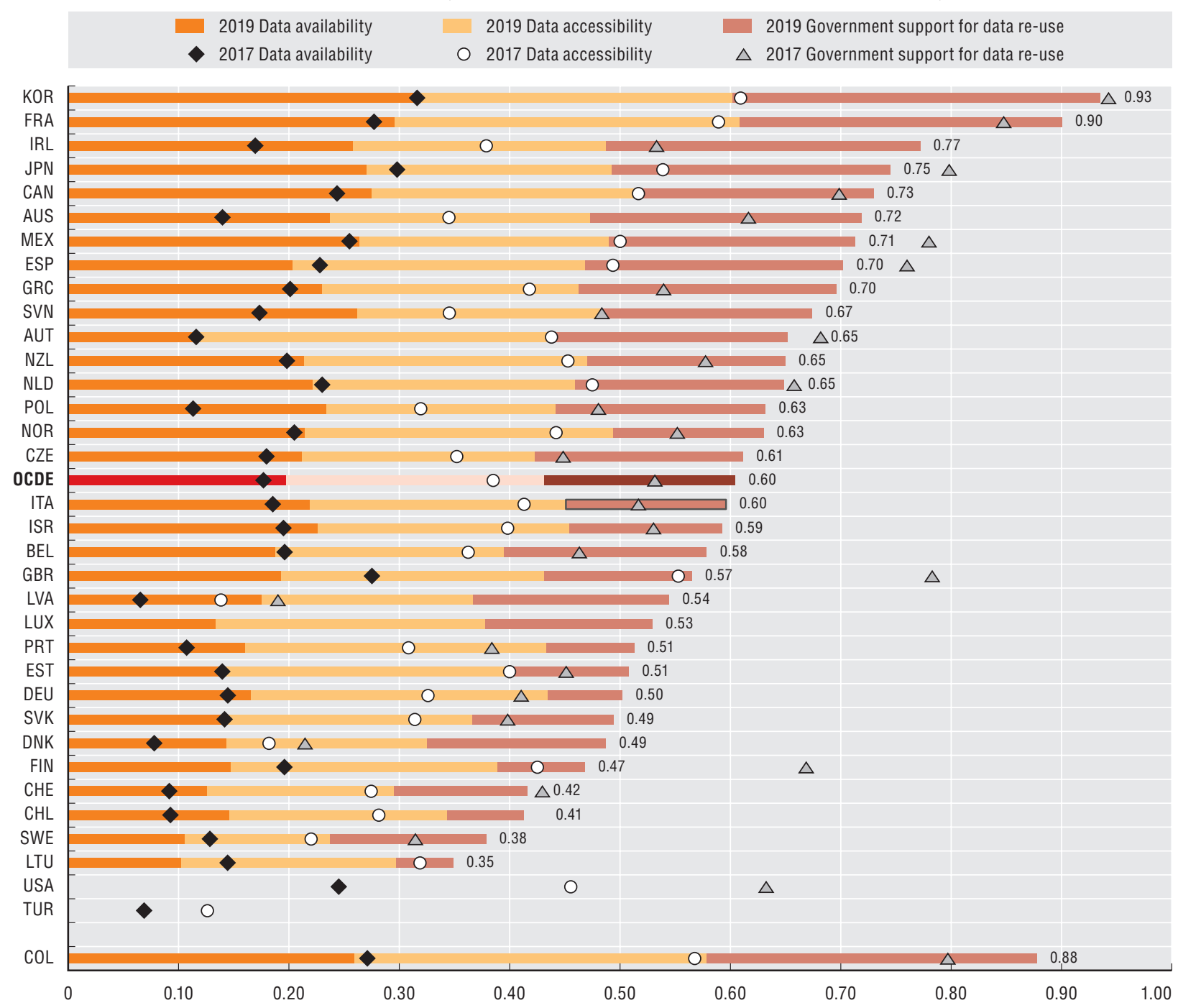

Sources : OCDE $\left(2016_{[23]}\right)$, « Enquête sur les données publiques ouvertes »; OCDE $\left(2018_{[24]}\right)$, « Enquête sur les données publiques ouvertes ». StatLink नils https://doi.org/10.1787/888934050997

\subsection{Une fonction publique qui reflète la société, pour une meilleure prestation des services publics}

La diversité et l'inclusion au sein de la fonction publique représentent des aspects importants si l'on veut centrer les services publics sur l'individu. Les administrations publiques reconnaissent de plus en plus l'importance de disposer d'une main-d'œuvre publique reflétant la société et représentant toutes les caractéristiques socio-économiques et autres caractéristiques personnelles, afin que les besoins, aspirations et expériences de nombreux citoyens soient pris en compte dans la prise de décision et que les lacunes dans la prestation des services puissent être mieux comprises. À son tour, l'inclusion implique la mise en pratique de la diversité et les soutien, valorisation et respect de toutes les expériences et points de vue du milieu du travail et leur mise en œuvre de manière bénéfique. 
En fonction du domaine ou du secteur considéré, une fonction publique plus représentative peut contribuer à exploiter des connaissances, des réseaux et des points de vue antérieurement négligés afin de mieux élaborer et mettre en œuvre les politiques publiques (OCDE 2011 [26] ; OCDE 2014 [27]). À son tour, une fonction publique diversifiée est également vue comme cruciale pour bâtir un secteur public plus efficient et empathique, comme le montre le nombre croissant de stratégies d'inclusion et de diversification menées dans les pays de l'OCDE (Marina 2015 ${ }_{\text {[28] }}$; Edlin et Delamore 2018 ${ }_{[29]}$ ). Or, l'efficience et l'empathie sont ce qui caractérise un secteur public centré sur l'individu. Les différentes catégories de population qui s'attendent légitimement à être représentées au sein du secteur public se sont étoffées au fil des ans et recouvrent désormais les minorités, sexuelles et religieuses, les pauvres, les personnes âgées, les personnes en situation de handicap ainsi que d'autres groupes minoritaires tels que les personnes appartenant à des peuples autochtones (White et Rice $2015_{[30]}$ ). Toutefois, si l'on dispose de données sur le genre au sein de la fonction publique, on n'a que peu d'éléments s'agissant des membres de minorités, sexuelles ou religieuses, ce qui s'explique, en grande partie, par le caractère sensible de telles données.

Dans la zone OCDE, en moyenne, les femmes représentaient $60 \%$ de l'emploi public en 2017. Plusieurs raisons peuvent expliquer cette surreprésentation des femmes au sein du secteur public. Globalement, le secteur public continue d'offrir des conditions d'emploi, plus stables et plus compatibles avec la vie de famille que le secteur privé. De plus, de nombreux métiers du secteur public sont désormais principalement féminins (enseignantes, infirmières). En revanche, même si la sous-représentation des femmes aux postes de décision recule, elles n'occupent en moyenne qu'environ un tiers des postes. On constate un niveau similaire de représentation des femmes dans les rangs des parlementaires, des ministres et des juges de tribunaux du plus haut niveau (voir les parties A, B et C du graphique 1.4). Les pays de l'OCDE ont mis en œuvre plusieurs stratégies pour parvenir à une meilleure égalité entre les femmes et les hommes aux plus hauts rangs de l'administration. Ils ont, par exemple, fait de l'égalité entre les sexes un objectif majeur de leurs stratégies de diversité et fixé des objectifs en termes d'embauche ou de promotion (OCDE 2014 ${ }_{[27]}$ ).

Il est possible de recourir à d'autres types de pratiques pour lutter contre les nombreuses inégalités qui se sont ancrées au cœur même des politiques publiques et de l'affectation des ressources publiques. Ces pratiques ont tendance à mettre l'accent sur l'impact de différentes politiques sur des catégories de population et des domaines d'action jugés importants. Par exemple, la « budgétisation sensible au genre » consiste en l'application systématique d'outils et de processus d'analyse, dans le cadre habituel de la procédure budgétaire, pour examiner l'impact des décisions budgétaires sur les femmes et les hommes, pour mettre en relief les sources d'inégalités entre les sexes et pour éclairer, mener en priorité et financer des politiques tenant compte de l'égalité des sexes. Selon la Recommandation du Conseil de l'OCDE sur l'égalité femmes-hommes dans la vie publique (2015) et sa boîte à outils, la budgétisation sensible au genre est un volet crucial de toute stratégie systémique des pouvoirs publics visant à promouvoir l'égalité des sexes. 


\section{Graphique 1.4. Égalité femmes-hommes au parlement, dans les ministères et dans les tribunaux de haut niveau}

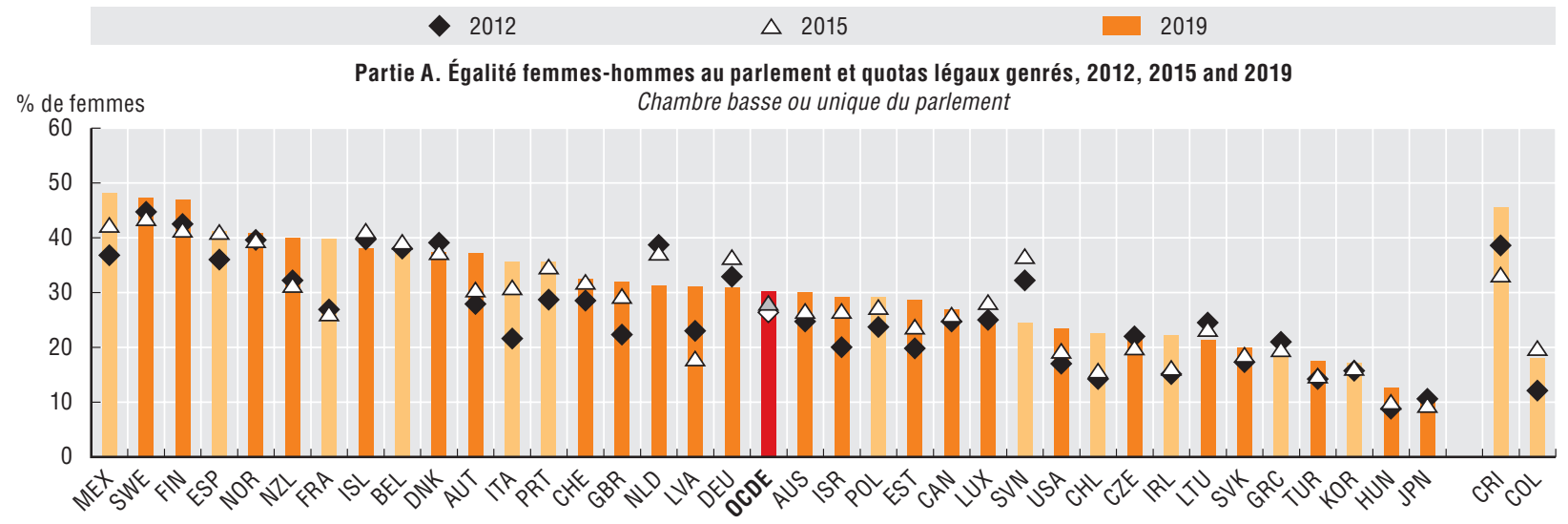

Note : Pour la Finlande, les données 2019 ont été fournies par les autorités nationales. Concernant les données sur Israël, voir http://doi. org/10.1787/888932315602.

Sources : Union interparlementaire (UIP), PARLINE (base de données) ; base de données mondiales sur les quotas de femmes de l'Institut international pour la démocratie et l'assistance électorale (IDEA).

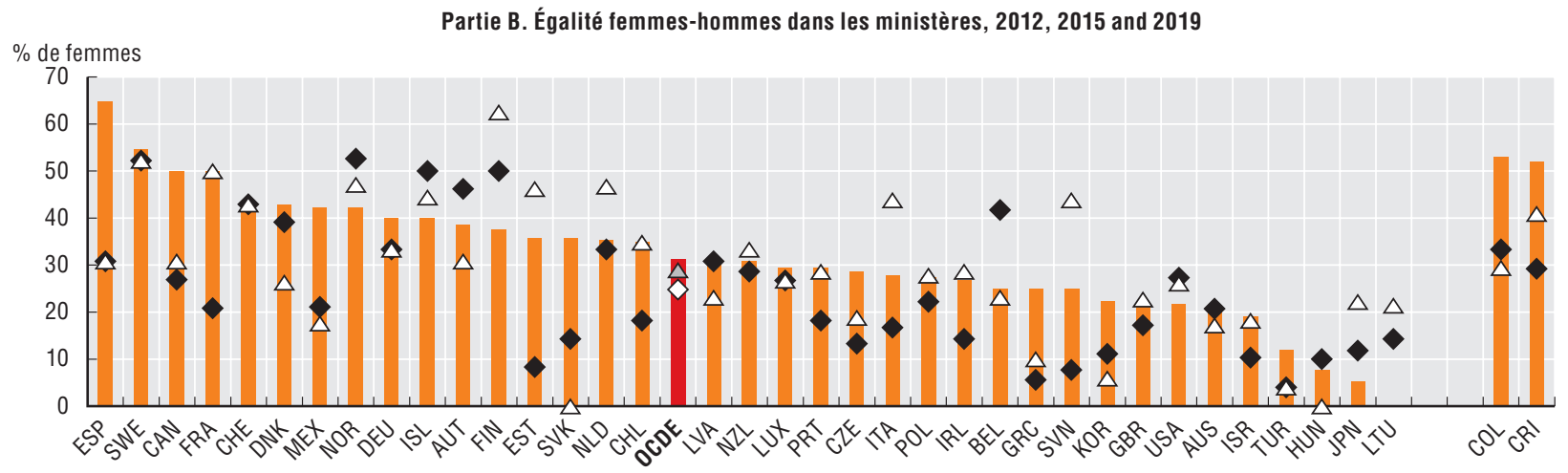

Note : Concernant les données sur Israël, voir http://doi.org/10.1787/888932315602.

Source : Union interparlementaire (UIP), « Femmes en politique » (2012, 2015 et 2019).

Partie C. Égalité femmes-hommes chez les juges de carrière, par niveau de juridiction, 2016

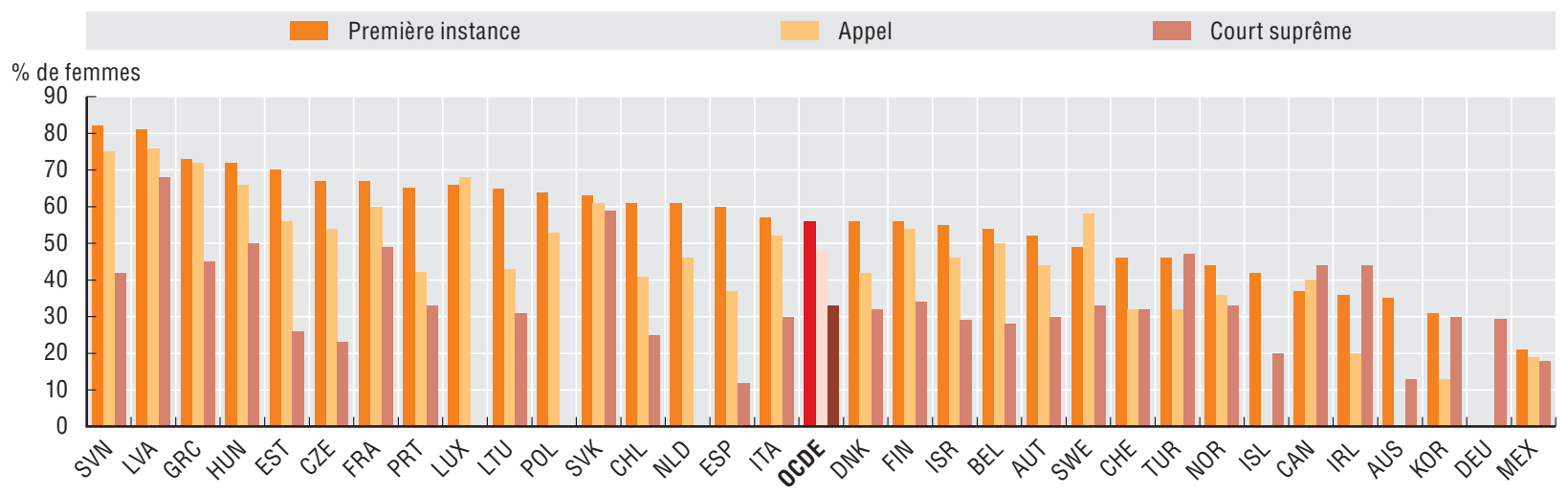

Note : Pour les données concernant Israël, voir http://doi.org/10.1787/888932315602. Les données pour l'Allemagne et le Portugal ont été fournies par les autorités nationales.

Sources : Données de la Commission européenne pour l'efficacité de la justice (CEPEJ) du Conseil de l'Europe, 2016 ; OCDE (2017[31]), " Enquête sur les pratiques sensibles au genre dans le pouvoir judiciaire ». 
Le nombre de pays de l'OCDE à avoir instauré une budgétisation sensible au genre est passé de 12 en 2016 à 17 en 2018 (Allemagne, Autriche, Belgique, Canada, Chili, Corée, Espagne, Finlande, Irlande, Islande, Israël, Italie, Japon, Mexique, Portugal et Suède). Il peut être difficile de mettre en place une démarche efficace et pérenne en la matière, pour différentes raisons : les gouvernements successifs n'accordent pas tous la même importance à l'égalité des sexes ; les ressources budgétaires sont limitées ; ou, plus généralement, il est difficile d'instaurer de nouvelles pratiques ou procédures en matière de gestion des finances publiques. Afin d'offrir à la budgétisation sensible au genre un statut reconnu et pérenne parmi les instruments de l'action publique et afin de la préserver, autant que possible, des fluctuations liées au contexte économique et politique, un peu plus de la moitié des pays qui la pratiquent à l'heure actuelle ont intégré cette budgétisation à leur législation. La mise en œuvre de la budgétisation sensible au genre varie selon les pays, mais elle englobe généralement l'adoption d'outils tels que l'analyse des situations de départ en matière d'égalité des sexes, l'analyse d'impact ex ante et ex post des politiques visant à améliorer cette égalité et l'évaluation des besoins en matière d'égalité des sexes $\left(\right.$ OCDE $2019_{\text {[32] }}$ ). Dans la plupart des pays, la budgétisation sensible au genre n'en est qu'à ses débuts.

La première version ici proposée par l'OCDE d'un indice composite sur la budgétisation sensible au genre vise à permettre aux responsables publics et au grand public de suivre les progrès réalisés en la matière au fil du temps. Cet indice est axé sur le cadre de gouvernance, les outils de mise en œuvre et le degré d'opportunité du contexte en place (voir graphique 1.5). Les pays dotés d'un score élevé, tels que l'Espagne et le Mexique, ont créé un cadre complet de budgétisation sensible au genre : les principaux aspects liés à la gouvernance sont en place, un large éventail d'outils sont appliqués au cours du cycle budgétaire et la budgétisation sensible au genre bénéficie d'appuis institutionnels balayant un large spectre. Toutefois, un tel indice ne permet pas de mesurer le niveau de succès du dispositif considéré. Pour évaluer ce succès, il est préférable d'examiner dans quelle mesure l'affectation des ressources publiques contribue à la réalisation des objectifs fondamentaux en matière d'égalité femmes-hommes. De plus, un peu plus du cinquième des pays de l'OCDE ont aussi mis en œuvre d'autres pratiques visant à faciliter l'accès des femmes aux débouchés économiques offerts par les clients publics, par exemple en aidant les femmes chefs d'entreprise à accéder aux marchés publics (voir le chapitre 8, consacré à la passation stratégique des marchés publics).

Pour assurer des services publics centrés sur l'humain, il est indispensable de pouvoir s'appuyer sur une fonction publique professionnelle, c'est-à-dire apte à relever les défis de l'action publique et à assurer les services publics avec efficacité. Les agents publics devraient donc être qualifiés, impartiaux, guidés par des valeurs et respectueux de l'éthique. Pour relever des défis transversaux aussi complexes que la poursuite des Objectifs de développement durable (ODD) et pour renforcer les capacités au service d'une décentralisation efficace, des compétences stratégiques sont nécessaires. Les agents publics vont devoir encourager la collaboration, gérer les risques, anticiper et faire preuve de résilience. Quel que soit le type de fonction publique en place (c'est-àdire que les postes soient essentiellement occupés par des fonctionnaires de carrière ou non), il est nécessaire de la doter des valeurs et des compétences requises pour relever des défis de gouvernance complexes, de mettre en relief l'attractivité de l'emploi public par rapport au reste du marché du travail et d'assurer la qualité et l'intégrité des mécanismes de recrutement. 


\section{Graphique 1.5. Premier passage à un indice composite relatif à la budgétisation sensible au genre, 2018}

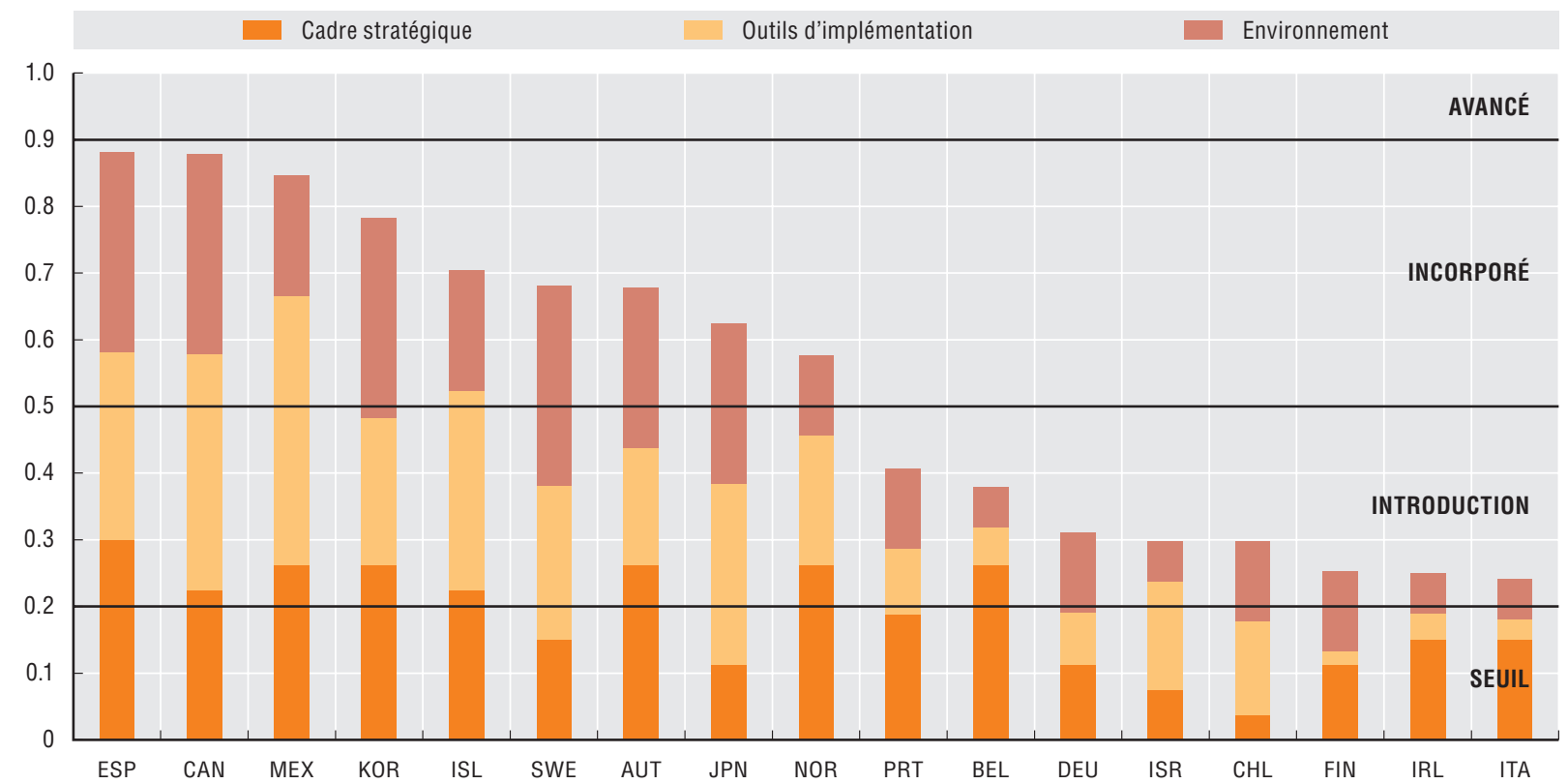

Note : données fournies uniquement pour les pays de l'OCDE ayant instauré une budgétisation sensible au genre. Concernant les données sur Israël, voir http://doi.org/10.1787/888932315602.

Source : OCDE (2019 $\left.{ }_{[32]}\right)$, Concevoir et mettre en œuvre la budgétisation sensible au genre: une voie pour agir, Éditions OCDE, Paris, https://www. oecd.org/gov/budgeting/designing-and-implementing-gender-budgeting-a-path-to-action.pdf.

Pour faire face à des problématiques complexes, surtout à l'ère numérique, les agents publics doivent posséder les bonnes compétences, les bonnes connaissances et les bonnes attitudes. Pour que la fonction publique puisse favoriser ces aptitudes dans le service public, elle doit d'abord les comprendre, ajuster ses descriptions de poste en conséquence et offrir des possibilités de formation ou de recyclage. Des investissements stratégiques et ciblés dans l'apprentissage et le perfectionnement sont essentiels pour que la fonction publique réussisse à suivre le rythme, face à l'évolution rapide des exigences des citoyens et des outils technologiques. De plus, il peut être important d'offrir des possibilités de formation si l'on souhaite attirer et motiver des collaborateurs de qualité. Dans sa Recommandation de 2019 sur le leadership et les aptitudes de la fonction publique, le Conseil de l'OCDE recommande aux adhérents de créer une culture et un environnement propices à l'apprentissage au sein de la fonction publique, bien au-delà des cours de formation traditionnels.

Les données présentées aux parties A et B du graphique 1.6 semblent montrer que l'importance de l'apprentissage et du perfectionnement est largement reconnue par les exécutifs des pays de l'OCDE, comme l'illustre par exemple le nombre de pays désormais dotés de stratégies de formation applicables à l'ensemble de leur fonction publique. La formation des dirigeants est prioritaire dans les deux-tiers des pays de l'OCDE démontre le rôle vital attribué à ce groupe en tant que catalyseur de réformes stratégiques dans l'ensemble de la fonction publique. On constate, en revanche, qu'un peu moins de la moitié des pays de l'OCDE qualifient la formation aux technologies de l'information ou au numérique de priorité au sein de l'administration centrale. Il s'agit là d'un score relativement faible, au vu de l'importance de telles compétences. Les programmes de mobilité peuvent eux aussi être utiles pour l'apprentissage et le perfectionnement ; pourtant, seule la moitié environ des pays de l'OCDE y ont recours. 
Être centré sur l'individu, c'est aussi être à l'écoute du retour d'information fourni par les agents publics et en tirer les conséquences. Les enquêtes menées auprès des agents publics permettent aux entités publiques de mesurer et de comparer l'engagement des agents publics dans leur travail, ainsi que leur façon de percevoir leur travail et leur environnement de travail. De telles enquêtes peuvent être une source d'éclairages précieux pour les décisions à prendre concernant l'amélioration du bien-être, le leadership et l'inclusion au travail. Des enquêtes menées dans le secteur privé montrent que l'engagement des employés est corrélé à la productivité au travail. Les pays de l'OCDE sont une très grande majorité (plus de 85 \%) à mener des enquêtes auprès des agents publics. Toutefois, la teneur de ces enquêtes varie, de même que l'usage qui en est fait. Dans la plupart des pays, ces enquêtes ont pour ambition de mettre à profit pour mesurer les engagement, motivation, satisfaction et implication des agents publics, ainsi que leur bien-être. Elles sont moins employées, en revanche, pour évaluer les aspects liés à la diversité et à l'inclusion.

\section{Graphique 1.6. Initiatives en matière d'apprentissage et de perfectionnement et priorités de formation dans l'administration publique, 2019}

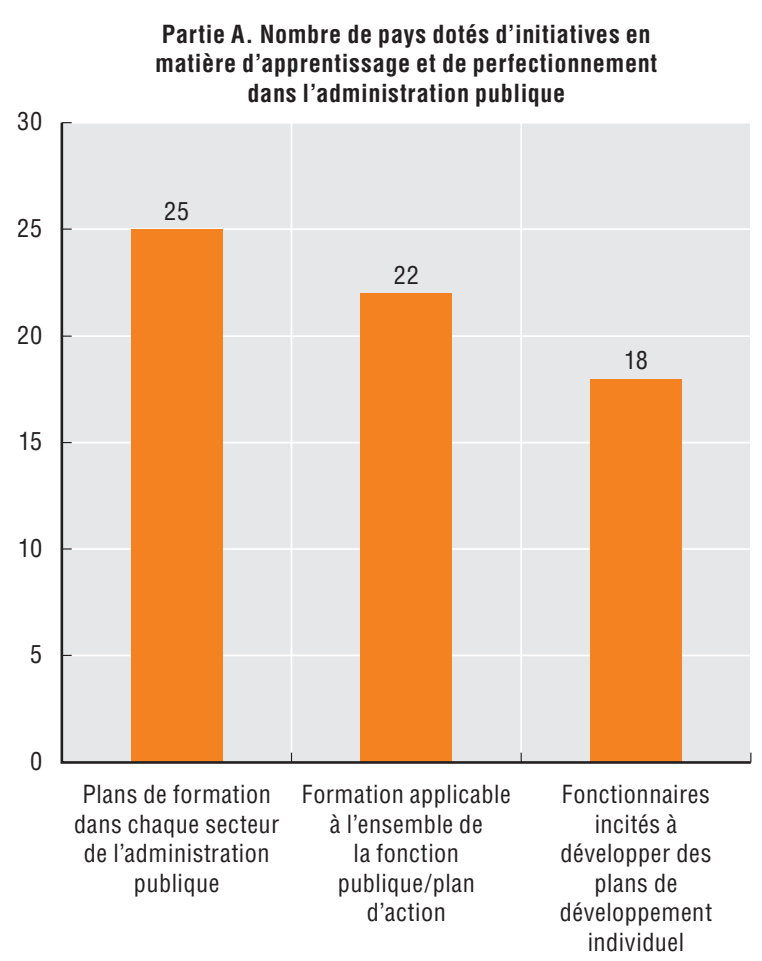

Note : données pour le total des répondants de 36 pays de l'OCDE.
Partie B. Nombre de pays priorisant la formation au sein de l'administration centrale

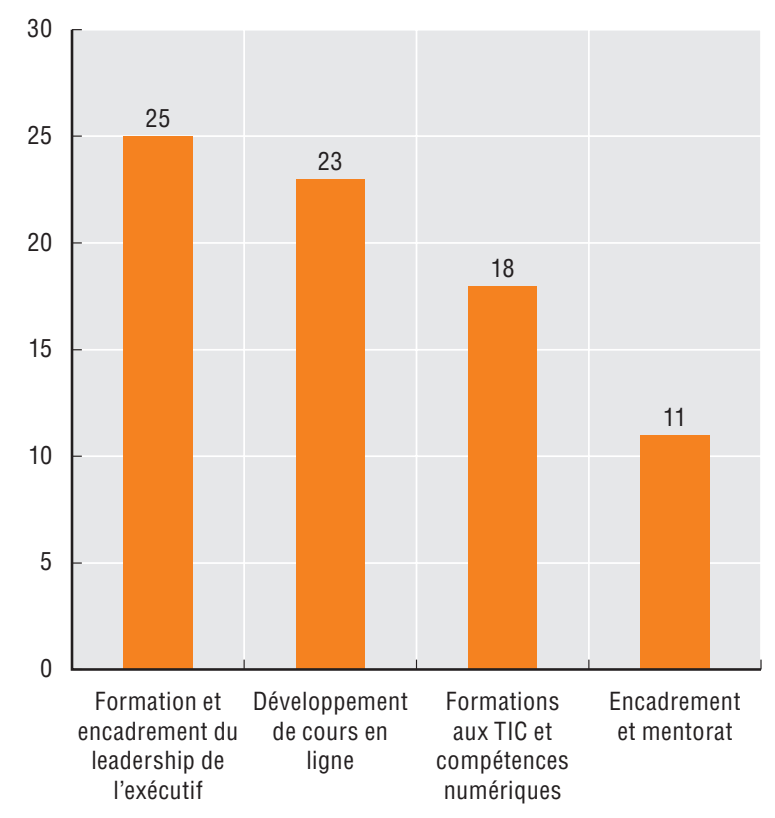

Source : OCDE (2019[33] $)$, Enquête de l'OCDE sur la gestion stratégique des ressources humaines ».

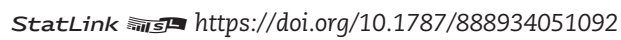

\section{Conclusion}

Dans un contexte de ralentissement de la croissance économique, de vieillissement démographique et de désenchantement à l'égard des administrations publiques, adopter une approche de la gouvernance publique et des services publics centrée sur l'individu peut favoriser l'utilisation efficiente de ressources publiques limitées, renforcer la légitimité des institutions publiques et rétablir la confiance à l'égard des compétences et des valeurs de la 
fonction publique. Des services publics centrés sur l'individu prennent l'individu comme point de départ et tiennent compte de ses besoins, aspirations et comportements. Dans le même temps, il convient de constituer un socle de données probantes, à partir de la consultation de données existantes ou de la création de nouvelles données, afin d'éclairer l'action publique, d'assurer une prestation de services sur mesures et de suivre l'évolution des politiques au fil du temps - autant d'actions qui devraient améliorer les niveaux de satisfaction. Pour assurer des services publics centrés sur l'individu, il faut s'appuyer sur une administration mettant l'individu au cœur de son projet et toujours guidée vers la recherche de solutions, dans une démarche de curiosité et d'empathie, et il faut chercher en permanence à comprendre le rapport des administrés au monde. Une administration publique centrée sur l'individu se fie à la collaboration avec les acteurs concernés pour mieux comprendre les difficultés considérables que certains rencontrent dans leur vie quotidienne et pour les associer à la formulation de solutions.

Pour ancrer solidement la notion de services publics centrés sur l'individu, il faut aussi se doter d'une fonction publique qui soit à l'image de la société dans son ensemble. Les femmes, les minorités et les personnes en situation de handicap doivent y être mieux représentées, notamment aux plus hauts rangs de la sphère administrative et politique. Les administrations publiques pourraient aussi s'employer à renforcer les capacités de la fonction publique en termes de stratégie et d'innovation. Elles pourraient notamment répertorier et acquérir les compétences requises pour adopter les nouvelles technologies, innover et faire face au changement. Les administrations publiques pourraient également élaborer leurs politiques en s'appuyant sur les nouveaux outils numériques, sur un dialogue constructif et sur une participation citoyenne, selon des modalités favorisant la transparence et la redevabilité.

Ce chapitre présente des données probantes et évoque plusieurs pratiques mises en place par les pays de l'OCDE pour mettre l'individu au centre des services publics. Il montre aussi qu'il s'agit d'une démarche nouvelle et complexe, dans le cadre de laquelle les pays sont en train d'apprendre les uns au contact des autres. Il existe encore une marge de progression vers plus de transparence, plus de dialogue véritable et moins de frontières et silos, lesquels entravent l'innovation. Ce chapitre convient aussi qu'il est important d'axer son action sur les réalisations qui comptent aux yeux des citoyens et d'évaluer en quoi le travail accompli par les administrations publiques contribue à ces réalisations. S'employer à mieux comprendre les articulations entre les pratiques de l'administration et leurs effets sur les perceptions et les expériences des administrés, comme en témoigne la satisfaction à l'égard des services et la confiance des institutions, pourrait contribuer à resserrer les liens entre administrations et administrés.

Enfin, créer des services publics centrés sur l'individu implique d'examiner de façon holistique les données disponibles (y compris les données de masse) et de répertorier les lacunes à combler. Il va certainement falloir recueillir des données probantes plus nombreuses et de meilleure qualité. Néanmoins, il est possible de tirer des éclairages précieux d'une analyse combinée des différentes dimensions du cadre d'analyse " Au service des citoyens " de l'OCDE, ainsi que des niveaux de satisfaction à l'égard des services publics. Cette analyse permet de mettre en relief les principaux aspects et les modèles de prestation susceptibles d'améliorer le niveau de satisfaction et de mieux refléter une démarche centrée sur l'individu en matière de prestation des services publics. 


\section{Note}

1. Certains indicateurs affichés dans les tableaux de bord de ce chapitre diffèrent de ceux du chapitre 11. Ce chapitre a pour but de rendre compte de la relation de satisfaction entre chaque service et les dimensions de l'accès, de la réactivité et de la qualité. Le chapitre 11, en revanche, cherche à montrer comment les pays se comportent en termes de dimensions du cadre plutôt que dans chaque service. Pour cette raison, ce chapitre présente les indicateurs de réactivité et de qualité de l'éducation de l'édition 2017 du Panorama des administrations publiques (c'est-à-dire l'indice de pénurie de matériel pédagogique, la disponibilité de l'aide aux études dans les écoles, l'utilisation de méthodes adaptatives d'enseignement, le score moyen PISA en sciences, le score moyen PISA en mathématiques et le score moyen PISA en lecture), bien qu'aucune donnée nouvelle n'ait été publiée à leur sujet (les résultats du cycle PISA 2018 seront publiés en décembre 2019). Au chapitre 11, les indicateurs de réactivité ne sont pas affichés en raison du manque de couverture par pays, ce qui limiterait toute comparaison potentielle.

\section{Références}

Balestra, C. et Tonkin, R. (2018), "Inequalities in household wealth across OECD countries: Evidence from the OECD Wealth Distribution Database", OECD Statistics Working Papers, $n^{\circ}$ 2018/01, Éditions OCDE, Paris, https://doi.org/10.1787/7e1bf673-en.

Causa, O., Browne, J. et Vindics, A. (2019), "Income redistribution across OECD countries: Main findings and policy implications", Documents d'orientation du Département des affaires économiques de l'OCDE, n²3, Éditions OCDE, Paris, https://doi.org/10.1787/3b63e61c-en.

Edlin, M. et Delamore, S. (2018), "Ready to serve the public? The role of empathy in public service education programs", Journal of Public Affairs Education, vol. 24, n³, pp. 300-320.

Jakobsen, M. et Jensen, R. (2015), “Common Method Bias in Public Management Studies”, International Public Management Journal, vol. 18, n 1, pp. 3-30, http://dx.doi.org/10.1080/10967494.2014.997906. [10]

James, O. (2009), "Evaluating the expectations disconfirmation and expectations anchoring approaches to citizen satisfaction with local public services", Journal of Public Administration Research and Theory, vol. 19, n 1, pp. 107-123.

Kim, G. H. et al. (2014), "Big Data Applications in the Government Sector: A Comparative Analysis among Leading Countries", Communications of the ACM, vol. 57, pp. 78-85, doi:10.1145/2500873.

Lafortune, G. et Ubaldi, B. (2018), “OECD 2017 OURdata Index: Methodology and results”, Documents de travail de l'OCDE sur la gouvernance publique, $\mathrm{n}^{\circ}$ 30, Éditions OCDE, Paris, https://doi.org/ 10.1787/2807d3c8-en.

Lind, E. et Arndt, C. (2016), "Perceived Fairness and Regulatory Policy: A Behavioural Science Perspective on Government-Citizen Interactions", OECD Regulatory Policy Working Papers, $n^{\circ} 6$, Éditions OCDE, Paris, http://dx.doi.org/10.1787/1629d397-en.

Lorenzoni, L. et al. (2019), "Health Spending Projections to 2030: New results based on a revised OECD methodology", Documents de travail de l'OCDE sur la santé, n 110, Éditions OCDE, Paris, https://doi. org/10.1787/5667f23d-en.

OCDE (2019), Designing and Implementing Gender Budgeting: A path to action, Éditions OCDE, Paris, www. oecd.org/gou/budgeting/designing-and-implementing-gender-budgeting-a-path-to-action.pdf.

OCDE (2019), OECD Survey on Strategic Human Resource Management.

OCDE (2019), Risks that Matter: Main Findings from the 2018 OECD Risks that Matter Survey, Éditions OCDE, Paris, https://www.oecd.org/social/ministerial/Risks-That-Matter-Early-Results-2017.pdf.

OCDE (2018), OECD Regulatory Policy Outlook 2018, Éditions OCDE, Paris, https://dx.doi.org/10.1787/ 9789264303072-en.

OCDE (2018), Open Government Data Report: Enhancing Policy Maturity for Sustainable Impact, OECD Digital Government Studies, Éditions OCDE, Paris, https://dx.doi.org/10.1787/9789264305847-en.

OCDE (2018), Open Government Data Survey.

OCDE (2017), Core skills for Public Sector Innovation, Éditions OCDE, Paris, https://oecd-opsi.org/wp-content/ uploads/2018/07/OECD_OPSI-core_skills_for_public_sector_innovation-201704.pdf.

OCDE (2017), Fostering Innovation in the Public Sector, Éditions OCDE, Paris, https://doi.org/10.1787/ 9789264270879-en. 
OCDE (2017), Panorama des administration 2017, Éditions OCDE, Paris, https://dx.doi.org/10.1787/gou glance-2017-en.

OCDE (2017), How's Life? 2017: Measuring Well-being, Éditions OCDE, Paris, https://dx.doi.org/10.1787/ how_life-2017-en.

OCDE (2017), Indicators of Regulatory Policy and Governance Survey.

OCDE (2017), Panorama des pensions 2017 : les indicateurs de l'OCDE et du G20, Éditions OCDE, Paris, https://doi.org/10.1787/pension_glance-2017-fr.

OCDE (2017), Survey on Gender-sensitive Practices in the Judiciary.

OCDE (2016), Open Government Data Survey.

OCDE (2015), Survey on Open Government Co-ordination and Citizen Participation in the Policy Cycle.

OCDE (2014), Indicators of Regulatory Policy and Governance Survey.

OCDE (2014), Recommendation of the Council on Digital Government Strategies, Éditions OCDE, https://legalinstruments.oecd.org/en/instruments/OECD-LEGAL-0406.

OCDE (2014), Women, Government and Policy Making in OECD Countries: Fostering Diversity for Inclusive Growth, Éditions OCDE, Paris, https://dx.doi.org/10.1787/9789264210745-en.

OCDE (2011), Public Servants as Partners for Growth: Toward a Stronger, Leaner and More Equitable Workforce, Éditions OCDE, Paris, https://dx.doi.org/10.1787/9789264166707-en.

OCDE (à paraître), Data-driven Public Sector Report, Éditions OCDE.

Rice, M. (dir.) (2015), Managing Diversity in Communities, Workplace and Societies, Routledge.

Rice, M. (dir.) (2015), The Multiple Dimensions of Diversity and Culture, Routledge.

Ubaldi, B. et al. (2019), "State of the art in the use of emerging technologies in the public sector", Documents de travail de l'OCDE sur la gouvernance publique, $\mathrm{n}^{\circ}$ 31, Éditions OCDE, Paris, https://doi.org/ 10.1787/932780bc-en.

Welby, B. (2019), “The impact of digital government on citizen well-being”, Documents de travail de l'OCDE sur la gouvernance publique, Éditions OCDE, Paris. 


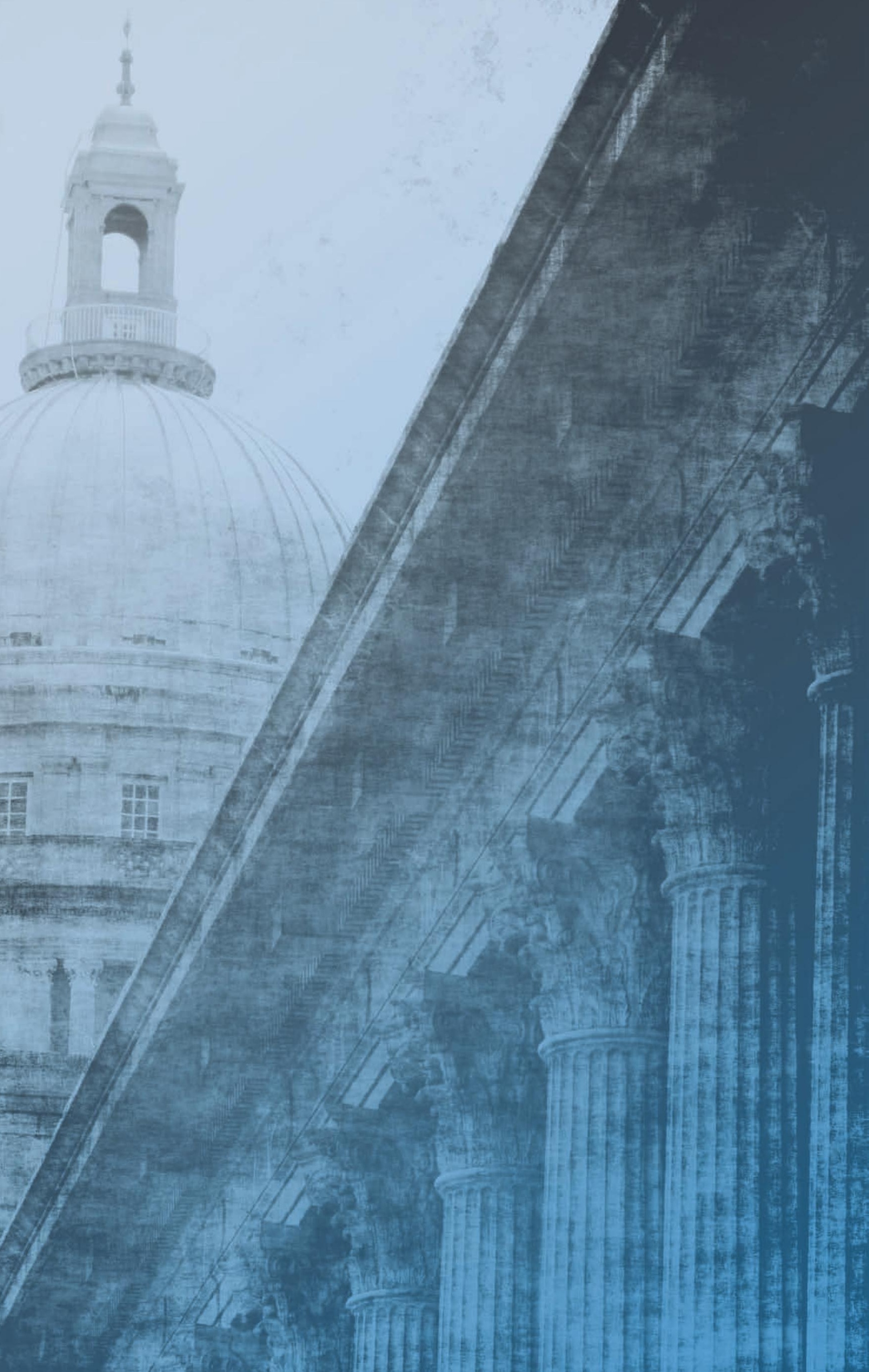




\section{FINANCES PUBLIQUES ET ÉCONOMIE}

Solde budgétaire des administrations publiques

Épargne nette des administrations publiques

Solde structurel des administrations publiques

Dette brute des administrations publiques

Patrimoine financier net des administrations publiques

Solde budgétaire et endettement par niveau d'administration

Recettes des administrations publiques

Composition des recettes des administrations publiques

Dépenses des administrations publiques

Ventilation par fonction des dépenses des administrations publiques (CFAP/COFOG)

Ventilation par opération économique des dépenses des administrations publiques

Ventilation par niveau d'administration des recettes et des dépenses publiques

Dépenses d'investissement des administrations publiques

Coûts de production et externalisation dans les administrations publiques

Éclairage : ventilation des dépenses des administrations publiques par fonction de protection sociale et de santé (CFAP) 
Le solde budgétaire, différence entre les recettes et les charges des administrations publiques, indique pour une année donnée dans quelle mesure les secondes sont financées par les premières. Il y a déficit lorsque les administrations dépensent plus qu'elles n'encaissent; dans le cas contraire, il y a excédent. Le solde primaire, c'est-à-dire le solde budgétaire total, déduction faite des paiements d'intérêts nets sur la dette publique, est un indicateur essentiel de la viabilité à court terme.

En 2017, le déficit moyen des administrations publiques dans les pays de l'OCDE représentait 2,2 \% du produit intérieur brut (PIB). Il avait culminé en 2009 (à 8,7 \% du PIB) à cause de la crise économique de 2007-2008. Par la suite, les déficits sont restés relativement élevés, mais ont diminué lentement et progressivement jusqu'au niveau actuel. En 2017, plus d'untiers des pays de l'OCDE ont dégagé un excédent, la Norvège affichant le plus important $(4,9 \%$ du PIB) devant la Corée (2,8 \% du PIB). À l'opposé, les déficits les plus élevés ont été enregistrés aux États-Unis (4,1\%), en Espagne (3,1\%), au Portugal et au Japon (3\% pour ces deux pays). Dans la majorité des pays où les données sont disponibles pour 2018, le solde budgétaire des administrations publiques s'est amélioré par rapport à 2017 - la plus grande variation étant constatée au Portugal $(+2,5$ points), pays qui reste toutefois en déficit, et en Norvège (+2,3 points). S'agissant du Portugal, cela peut s'expliquer par l'augmentation des recettes courantes, en particulier des recettes fiscales et des cotisations sociales, expliquée en grande partie par l'évolution de l'activité économique et de l'emploi, conjuguée au fait qu'en 2017, le solde budgétaire a été impacté négativement par un événement exceptionnel lié à la recapitalisation d'une institution financière publique. En ce qui concerne la Norvège, malgré des cours du pétrole bas en termes relatifs, l'amélioration découle des mesures visant à protéger le pays de la volatilité des marchés pétroliers, notamment l'adoption d'une règle budgétaire plus prudente ciblant un déficit structurel hors activités pétrolières équivalent à $3 \%$ du montant du fonds souverain au lieu de 4 \% (OCDE 2018).

Une succession de déficits fait augmenter la dette, d'où un alourdissement de la charge des intérêts et donc un gonflement des déficits. Le solde primaire indique dans quelle mesure un État peut honorer ses obligations financières sans devoir s'endetter davantage. En 2017, près des trois-quarts des pays de l'OCDE ont affiché un excédent primaire, le plus fort ayant été dégagé par la Grèce (3,6 \%) à la suite d'un assainissement budgétaire de grande ampleur qui a renforcé la crédibilité et réduit l'incertitude, ce qui contribue au retour de la croissance économique. Au cours de la même période, c'est au Japon que le déficit primaire a été le plus marqué $(2,7 \%)$ du fait d'une croissance inférieure aux attentes, d'une série de budgets rectificatifs et de retards dans le relèvement prévu de la taxe sur la consommation. Le Japon a besoin d'un vaste plan d'assainissement budgétaire, comprenant des coupes dans certaines dépenses et des hausses d'impôt ciblées, ainsi que d'un meilleur cadre budgétaire permettant d'assurer l'application de ce plan (OCDE 2019).

\section{Méthodologie et définitions}

Les données relatives au solde budgétaire des administrations publiques sont tirées de la base de données des Statistiques de l'OCDE sur les comptes nationaux, qui sont établies conformément au Système de comptabilité nationale (SCN). Ce dernier constitue un ensemble de concepts, de définitions, de nomenclatures et de règles approuvés au plan international en matière de comptabilité nationale. Le cadre du SCN 2008 est désormais en vigueur dans tous les pays de l'OCDE (voir l'Annexe A pour plus de précisions sur les systèmes de déclaration et les sources). Selon la terminologie du SCN, les administrations publiques se composent de l'administration centrale, des administrations des États fédérés, des administrations locales et des administrations de sécurité sociale.

Le solde budgétaire des administrations publiques, également présenté comme leur capacité $(+)$ ou leur besoin (-) de financement, est égal à la différence entre le montant total de leurs dépenses et celui de leurs recettes. Les recettes comprennent les impôts, les cotisations sociales nettes, les dons et les autres rentrées. Les dépenses comprennent la consommation intermédiaire, la rémunération des personnels, les subventions, les revenus de la propriété (dont les paiements d'intérêts), les prestations sociales, les autres dépenses courantes (essentiellement les transferts courants) et les dépenses en capital (transferts en capital et investissements).

Le solde primaire correspond au solde budgétaire, déduction faite des paiements d'intérêts nets au titre des engagements des administrations publiques (c'est-à-dire les intérêts versés moins les intérêts perçus).

Le produit intérieur brut (PIB) est la mesure habituelle de la valeur des biens et services produits par un pays au cours d'une période donnée.

\section{Pour en savoir plus}

OCDE (2019), OECD Economic Surveys: Japan 2019, Éditions OCDE, Paris, https://doi.org/10.1787/fd63f374-en.

OCDE (2018), OECD Economic Surveys: Norway 2018, Éditions OCDE, Paris, http://doi.org/10.1787/eco_surveys-nor-2018-en.

\section{Notes relatives aux graphiques}

Informations sur les données concernant Israël : http://doi.org/ 10.1787/888932315602.

2.1. Les données sur le Chili et la Turquie ne sont pas prises en compte dans la moyenne de la zone OCDE car il manque des séries chronologiques ou les principaux agrégats non financiers des administrations publiques. Les données sur le Brésil, la Chine et l'Indonésie portent sur 2016 au lieu de 2017. Les données sur la Russie portent sur 2015 au lieu de 2017.

2.2. Les données sur le Chili ne sont pas disponibles. Celles sur la Turquie ne sont pas prises en compte dans la moyenne de la zone OCDE car il manque des séries chronologiques. Les données sur le Brésil et l'Indonésie portent sur 2016 au lieu de 2017. 
2.1. Solde budgétaire des administrations publiques en pourcentage du PIB, 2007, 2017 et 2018

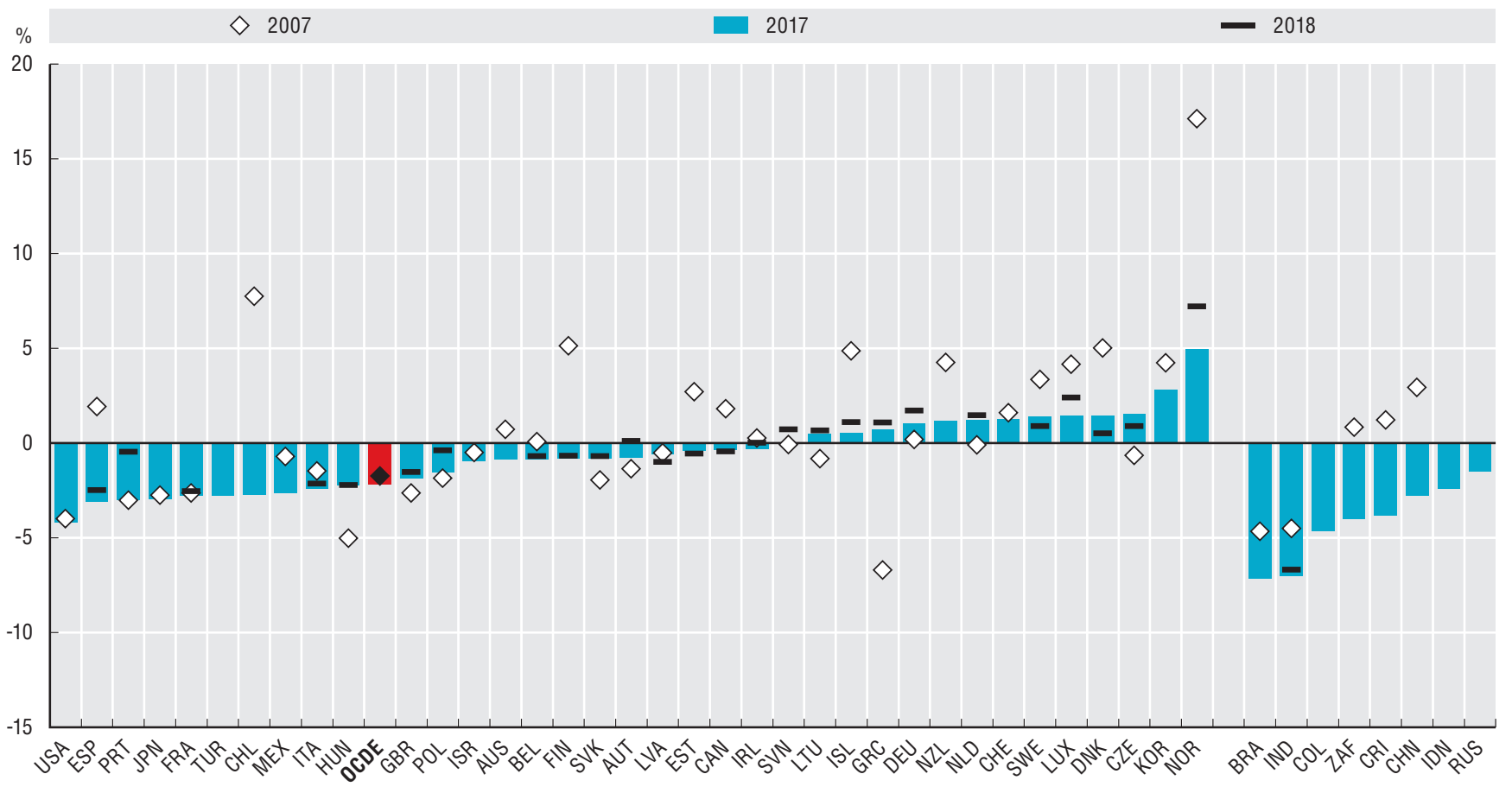

Sources : Statistiques de l'OCDE sur les comptes nationaux (base de données). Les données sur l'Inde sont tirées des Perspectives de l'économie mondiale du FMI (avril 2019).

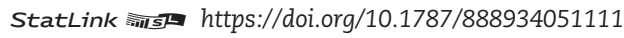

2.2. Solde primaire des administrations publiques et charges nettes d'intérêts en pourcentage du PIB, 2017 et 2018

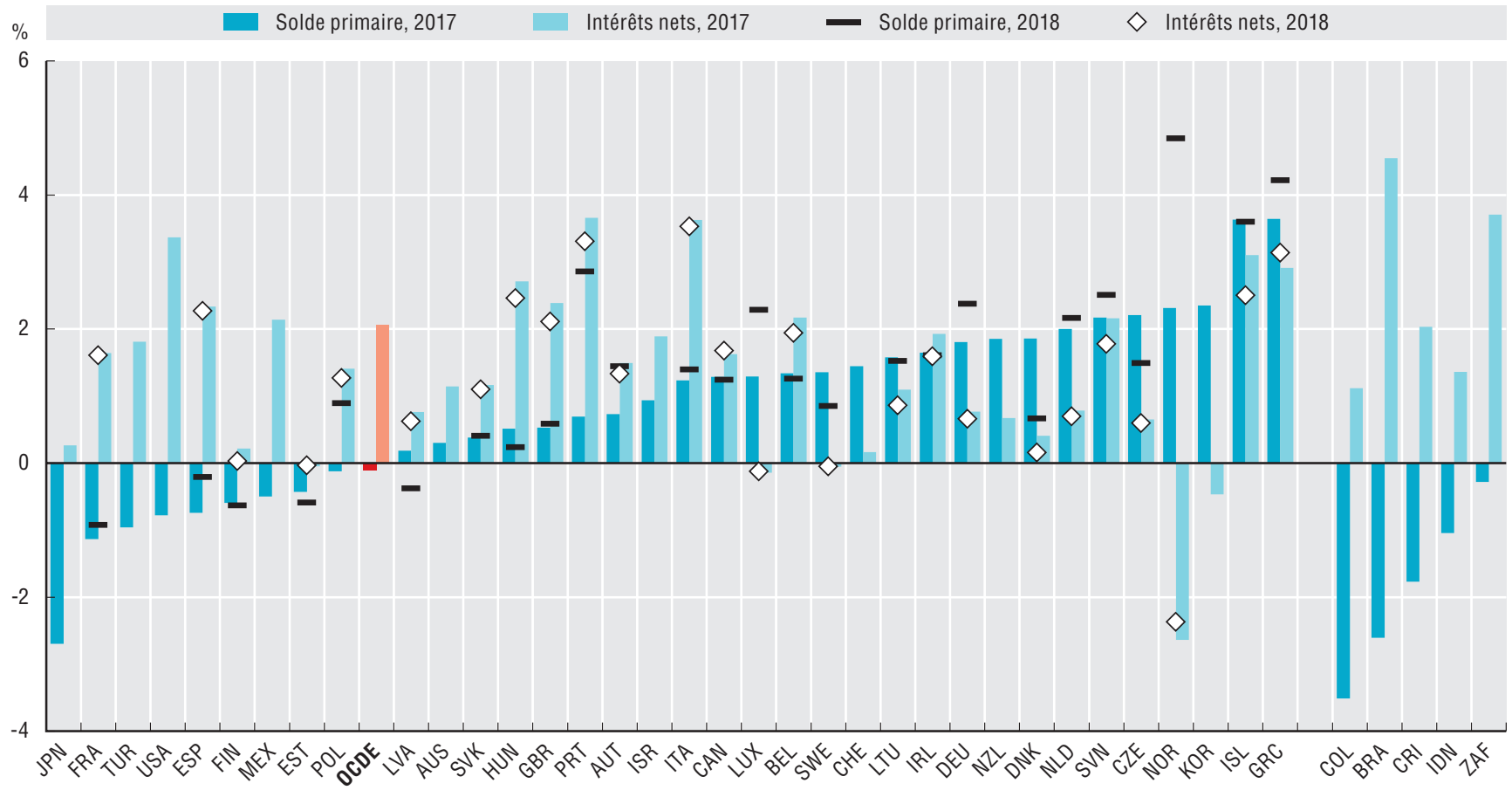

Source : Statistiques de l'OCDE sur les comptes nationaux (base de données). 
L'épargne nette correspond à la différence entre les recettes et les dépenses courantes ou au solde budgétaire hors dépenses en capital. Elle ne prend pas en compte les dépenses d'investissement ni les transferts en capital, par exemple à destination des entreprises publiques ou des institutions financières. L'épargne nette est en général associée à la " règle d'or " des finances publiques qui préconise qu'au cours d'un cycle économique les recettes courantes couvrent les dépenses courantes. Cela implique aussi qu'on devrait s'endetter uniquement pour investir en faveur de la croissance et ainsi donner une orientation à long terme à la politique budgétaire.

En 2017, l'épargne nette moyenne des administrations publiques des pays de l'OCDE représentait $-1,8 \%$ du produit intérieur brut (PIB), mais elle était positive dans un peu plus de $50 \%$ des pays. C'est aux États-Unis que l'épargne nette négative était la plus creusée (-4,9\% du PIB), en partie $\mathrm{du}$ fait d'un changement de tendance intervenu en 2016, lorsque le gouvernement fédéral a augmenté ses dépenses après plusieurs années d'assainissement. On prévoit une poursuite de cette tendance car la politique budgétaire a été sensiblement assouplie au début de 2018, avec la conjonction d'une réforme fiscale et d'un relèvement des plafonds de dépenses par le Congrès en 2018 et 2019, laquelle a entraîné de nouvelles dépenses (OCDE 2018a). À l'inverse, la Norvège a enregistré le taux d'épargne nette positif le plus élevé $(6,9 \%)$ en 2017 ; en effet, conformément au cadre budgétaire, les prélèvements sur le fonds souverain norvégien (constitué par exemple des recettes provenant de la production pétrolière en mer) couvrent le déficit hors activités pétrolières dans la limite d'un plafond fixé par la règle budgétaire, tout en préservant les intérêts des générations futures. Il est donc improbable que l'épargne nette devienne négative tant que ce dispositif sera en vigueur.

En 2007, seuls 8 pays de l'OCDE affichaient une épargne nette négative contre 17 en 2017. Or, dans tous les pays où le taux d'épargne nette était positif en 2007, ce taux avait baissé 10 ans plus tard, sauf en Autriche (+0,1 point), en République tchèque ( $+0,4$ point), aux Pays-Bas ( $+0,8$ point) et en Allemagne (+1,2 point), qui ont déclaré un niveau plus élevé d'épargne. Ces pays ont été à l'avant-garde de la promotion et de l'application de politiques d'austérité.

La différence entre la capacité ou le besoin de financement (c'est-à-dire le solde budgétaire) et l'épargne nette réside dans le montant des dépenses en capital qui peuvent être soit des investissements, soit des transferts en capital. En moyenne, dans les pays de l'OCDE, le solde (capacité ou besoin de financement) dépassait de 0,38 point le taux d'épargne nette en 2017. C'est en Islande et en Turquie que l'écart négatif était le plus marqué, s'élevant respectivement à $-2,88$ points et $-2,86$ points. En Turquie, la situation est imputable à l'augmentation sensible des investissements publics depuis 2016 et à une très forte hausse des garanties d'emprunts (OCDE 2018b). En Islande, la restructuration du système bancaire s'est terminée en 2016, mais la décision concernant l'utilisation de certains reliquats de fonds spéculatifs implantés dans le pays avant 2008 a été laissée en suspens jusqu'au premier trimestre 2017, période à laquelle la plupart de ces fonds ont finalement été libérés (Baldursson). En outre, l'Islande a intensifié ses investissements en infrastructures pour répondre aux besoins consécutifs aux restrictions budgétaires et aux tensions générées par le secteur du tourisme.

\section{Méthodologie et définitions}

Les données sont tirées de la base de données des Statistiques de l'OCDE sur les comptes nationaux, qui sont établies conformément au Système de comptabilité nationale (SCN) ; ce dernier constitue un ensemble de concepts, de définitions, de nomenclatures et de règles approuvés au plan international en matière de comptabilité nationale. Le cadre du SCN 2008 est désormais en vigueur dans tous les pays de l'OCDE (voir l'Annexe A pour plus de précisions). Selon la terminologie du SCN, les administrations publiques se composent de l'administration centrale, des administrations d'États fédérés, des administrations locales et des administrations de sécurité sociale.

L'épargne nette des administrations publiques correspond à la différence entre les recettes courantes et les dépenses courantes, amortissement compris. Pour le calcul de l'épargne brute, les coûts d'amortissement ne sont pas déduits des dépenses courantes. L'épargne brute majorée des transferts nets en capital (c'est-à-dire les transferts en capital reçus moins les transferts en capital versés) et minorée des investissements bruts (c'est-à-dire la formation brute de capital et les acquisitions moins les cessions d'actifs non financiers non produits) constitue le solde budgétaire des prêts ou emprunts nets. La capacité ou le besoin de financement reflète la situation budgétaire après comptabilisation des dépenses en capital : une capacité de financement, c'est-à-dire un excédent, signifie que les administrations fournissent des ressources financières à d'autres secteurs, alors qu'un besoin de financement, ou déficit, signifie que les administrations ont besoin de ressources financières provenant d'autres secteurs pour financer une partie de leurs dépenses. Par rapport à la capacité ou au besoin de financement, l'épargne nette présente l'avantage d'éviter les possibles distorsions ponctuelles dues à des transferts en capital exceptionnels et éventuellement massifs. Elle permet, en outre, d'éviter de trop restreindre l'investissement public durant les périodes d'austérité, sans augmenter le déficit.

\section{Pour en savoir plus}

Baldursson, F., Portes, R. et Thorlaksson, E. (2017), Iceland's Capital Controls and the Resolution of its Problematic Bank Legacy, http://doi.org/10.13140/RG.2.2.36548.73604.

OCDE (2018a), OECD Economic Surveys: United States 2018, Éditions OCDE, Paris, http://doi.org/10.1787/eco_surveys-usa-2018-en.

OCDE (2018b), OECD Economic Surveys: Turkey 2018, Éditions OCDE, Paris. https://doi.org/10.1787/eco_surveys-tur-2018-en.

\section{Notes relatives aux graphiques}

Les données sur le Chili ne sont pas disponibles. Concernant les données sur Israël, voir http://doi.org/10.1787/888932315602. Les données sur la Turquie ne sont pas prises en compte dans la moyenne de la zone OCDE car il manque des séries chronologiques. Les données sur le Brésil et l'Indonésie portent sur 2016. Les données sur la Russie portent sur 2015 au lieu de 2017.

2.5. (Transferts nets en capital en pourcentage du PIB) est disponible en ligne à l'Annexe $\mathrm{F}$. 
2.3. Épargne nette des administrations publiques en pourcentage du PIB, 2007, 2017 et 2018

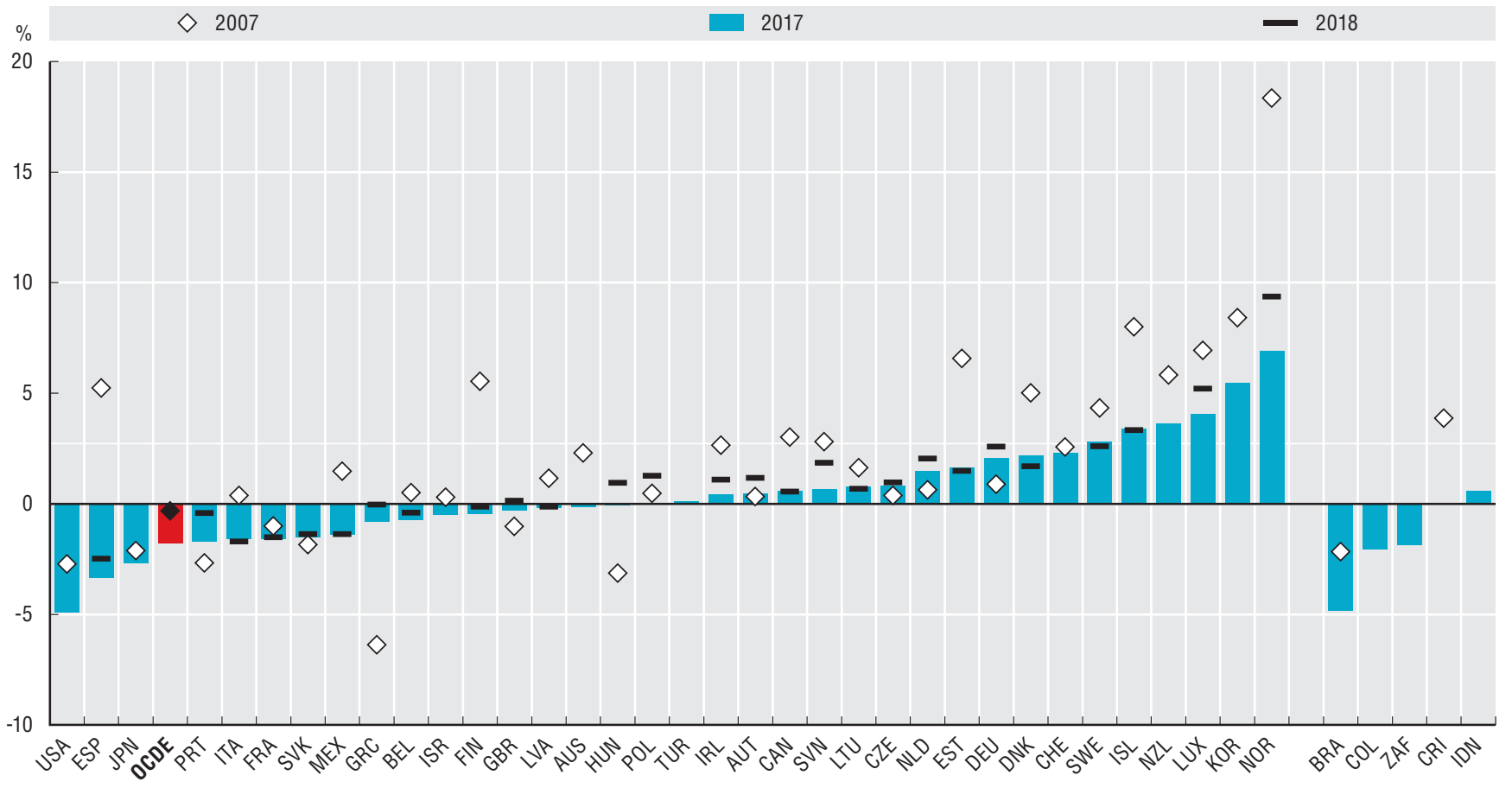

Source : Statistiques de l'OCDE sur les comptes nationaux (base de données).

StatLink 光ist https://doi.org/10.1787/888934051149

\subsection{Comparaison entre l'épargne nette et la capacité ou le besoin de financement des administrations publiques} en pourcentage du PIB, 2017 et 2018

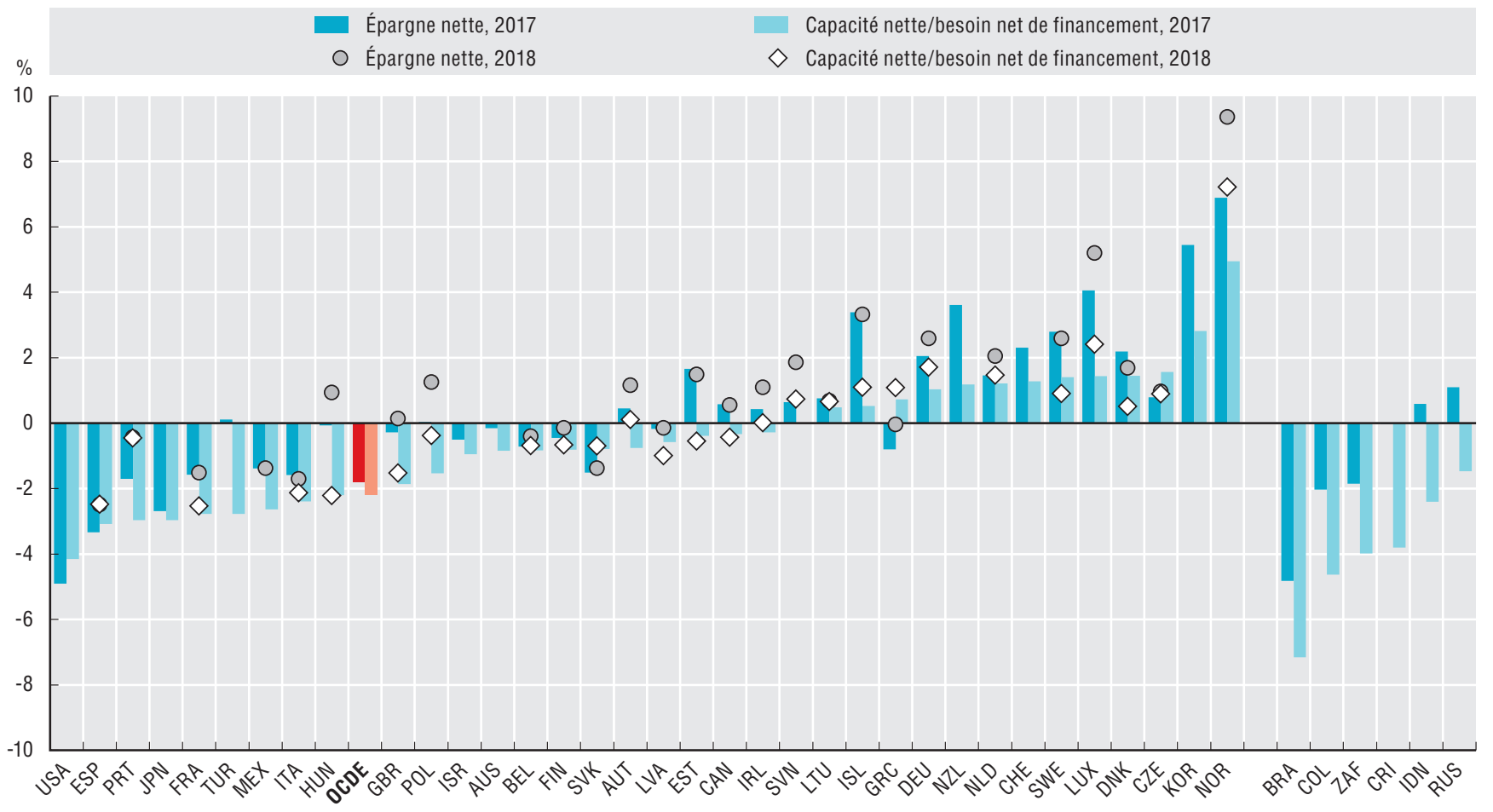

Source : Statistiques de l'OCDE sur les comptes nationaux (base de données). 
Les cycles économiques et les événements ponctuels peuvent influer notablement sur le solde budgétaire des administrations publiques. Les recettes de ces dernières (notamment les recettes fiscales) tendent à diminuer pendant la phase descendante des cycles économiques car il y a ralentissement des activités imposées. Au même moment, les dépenses publiques sont susceptibles d'augmenter parce que davantage de personnes perdent leur emploi et ont droit à des prestations d'assistance sociale ou à des allocations de chômage. Le gouvernement peut également décider d'engager des dépenses supplémentaires (à des fins d'investissement, par exemple) pour compenser les effets du tassement de l'activité privée. Le seul solde budgétaire des administrations publiques ne reflète donc pas pleinement la situation sous-jacente des finances publiques. Le solde structurel, qui tient compte des effets imputables au cycle économique et aux événements ponctuels, fait mieux ressortir les tendances de fond qui permettent d'évaluer la viabilité à long terme des finances publiques. Son estimation nécessite celle des composantes cyclique et structurelle du solde budgétaire et de la production, appelé aussi produit intérieur brut (PIB) potentiel - situation dans laquelle l'économie fonctionne à pleine capacité sans incidence sur l'inflation. L'écart de production mesure la différence entre PIB réel et PIB potentiel.

En 2017, le solde budgétaire structurel des pays de l'OCDE correspondait à un déficit moyen de 2,3\% du PIB potentiel. Aux États-Unis, en particulier, et malgré des perspectives économiques favorables, le déficit corrigé des variations cycliques était, à 4,7 \%, le plus élevé. Ce chiffre s'explique, entre autres choses, par les effets de baisses d'impôts pas encore compensées par l'accélération attendue de la croissance et par une augmentation des dépenses de santé. En outre, la mondialisation et l'automatisation ont déplacé des travailleurs, en particulier dans les bastions industriels, et beaucoup ont eu des difficultés à trouver un nouvel emploi. Dans certaines régions, cela a eu pour conséquence un chômage élevé, un recul du taux d'activité et une montée de la pauvreté, le sousemploi accentuant la pression exercée sur les programmes d'aide au revenu (OCDE 2018a). En revanche, la Grèce a affiché l'excédent structurel le plus élevé pour la même année (6,4%). Elle a considérablement assaini ses finances publiques en améliorant la discipline fiscale et la maîtrise des dépenses, d'où un dépassement des objectifs de 2015 à 2017. Ces bons résultats ont sans doute suscité un regain de confiance qui a atténué les effets de contraction de l'activité produits par l'assainissement budgétaire. Du fait de sa performance budgétaire et des nouveaux progrès attendus, la Grèce n'est plus soumise depuis 2017 à la procédure prévue par l'Union européenne (UE) en cas de déficit excessif (OCDE 2018b).

En 2018, comparativement à 2017, près des deux-tiers des pays de l'OCDE ont connu une dégradation du solde budgétaire structurel de leurs administrations publiques, avec un déficit moyen de $2,8 \%$. La plus forte hausse du déficit a été observée en Israël ( $+2,1$ points), en raison de réductions d'impôts, d'une augmentation des subventions et des difficultés du gouvernement à obtenir un consensus politique sur l'adoption d'une réforme budgétaire. À l'opposé, c'est au Luxembourg et en Allemagne qu'on observe un solde structurel amélioré
(+1 point). Dans les deux cas, cela s'explique par une croissance économique solide et une politique budgétaire prudente. Dans le cas de l'Allemagne toutefois on prévoit un ralentissement de l'activité car l'économie se heurte à une insuffisance des capacités, y compris sur le plan de l'emploi (OCDE 2018c).

Le solde primaire structurel est aussi corrigé de l'incidence des charges d'intérêts nettes (c'est-à-dire les intérêts payés moins les intérêts perçus) sur les engagements des administrations publiques. On observe une dégradation générale de cet indicateur similaire à celle du solde structurel. Alors que le solde primaire structurel dans les pays de l'OCDE s'établissait en moyenne à -0,5\% de leur PIB potentiel en 2017, ce déficit devrait se creuser encore de -0,4 point de 2018 à 2020. Cette prévision négative est alimentée par une plus grande incertitude causée par des facteurs comme la guerre commerciale que se livrent les États-Unis et la Chine et ses conséquences sur les autres économies, ou les effets potentiels du Brexit, ces facteurs étant tous susceptibles d'affaiblir la croissance mondiale.

\section{Méthodologie et définitions}

Ces données sont tirées de la base de données des Perspectives économiques de l'OCDE, $\mathrm{n}^{\circ} 105$. Le solde budgétaire structurel, ou solde sous-jacent, correspond au solde budgétaire présenté dans le cadre du Système de comptabilité nationale (SCN), corrigé de deux facteurs : le stade du cycle économique (mesuré par l'écart de production) et les opérations budgétaires ponctuelles. Le PIB potentiel n'est pas directement observable et les estimations sont sujettes à d'importantes marges d'erreur. Parmi les facteurs ponctuels, on peut citer les opérations budgétaires exceptionnelles et non périodiques ainsi que les écarts par rapport à la tendance en matière de transferts en capital nets. On trouvera davantage de précisions dans les "Sources et méthodes " des Perspectives économiques de l'OCDE (www.oecd.org/fr/ eco/perspectives/sources-et-methodes.htm).

\section{Pour en savoir plus}

OCDE (2018), OECD Economic Surveys: United States 2018, Éditions OCDE, Paris, http://doi.org/10.1787/eco_surveys-usa-2018-en.

OCDE (2018), OECD Economic Surveys: Greece 2018, Éditions OCDE, Paris, http://doi.org/10.1787/eco_surveys-grc-2018-en.

OCDE (2018c), OECD Economic Surveys: Germany 2018, Éditions OCDE, Paris, http://doi.org/10.1787/eco_surveys-deu-2018-en.

\section{Notes relatives aux graphiques}

Les données sur le Chili, le Mexique, la République slovaque et la Turquie ne sont pas disponibles. Concernant les données sur Israël, voir http://doi.org/10.1787/888932315602. 
2.6. Solde structurel des administrations publiques en pourcentage du PIB potentiel, 2007, 2017 et 2018

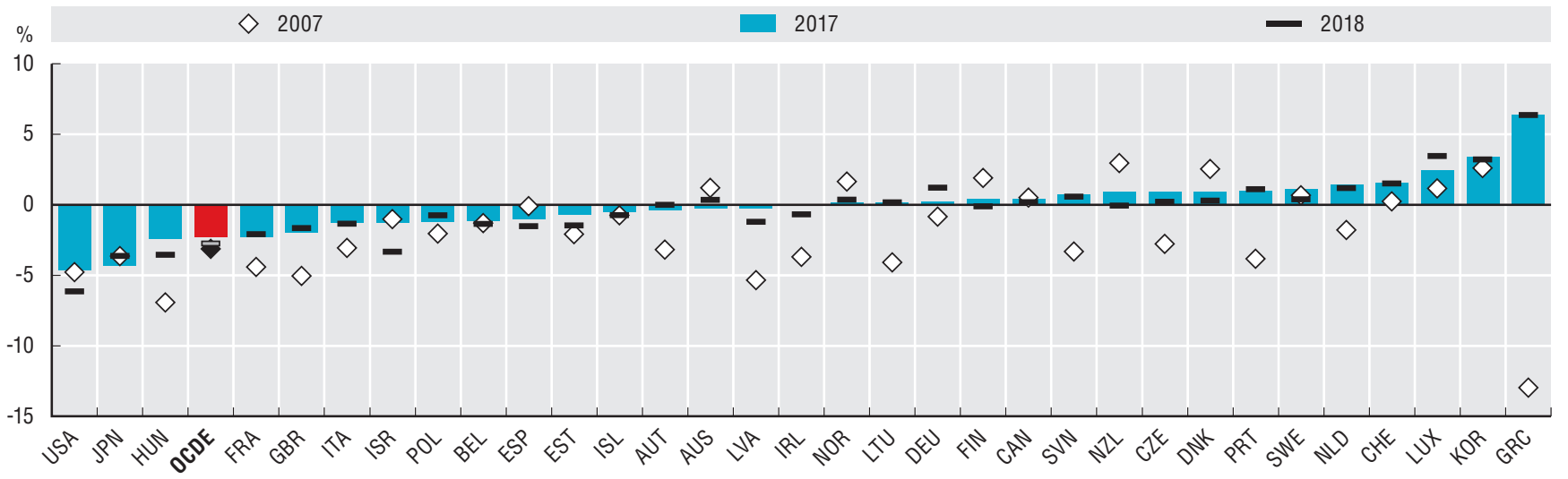

Source : Perspectives économiques de l’OCDE, n 105, mai 2019.

2.7. Solde primaire structurel des administrations publiques en pourcentage du PIB potentiel, 2007,2017 et 2018

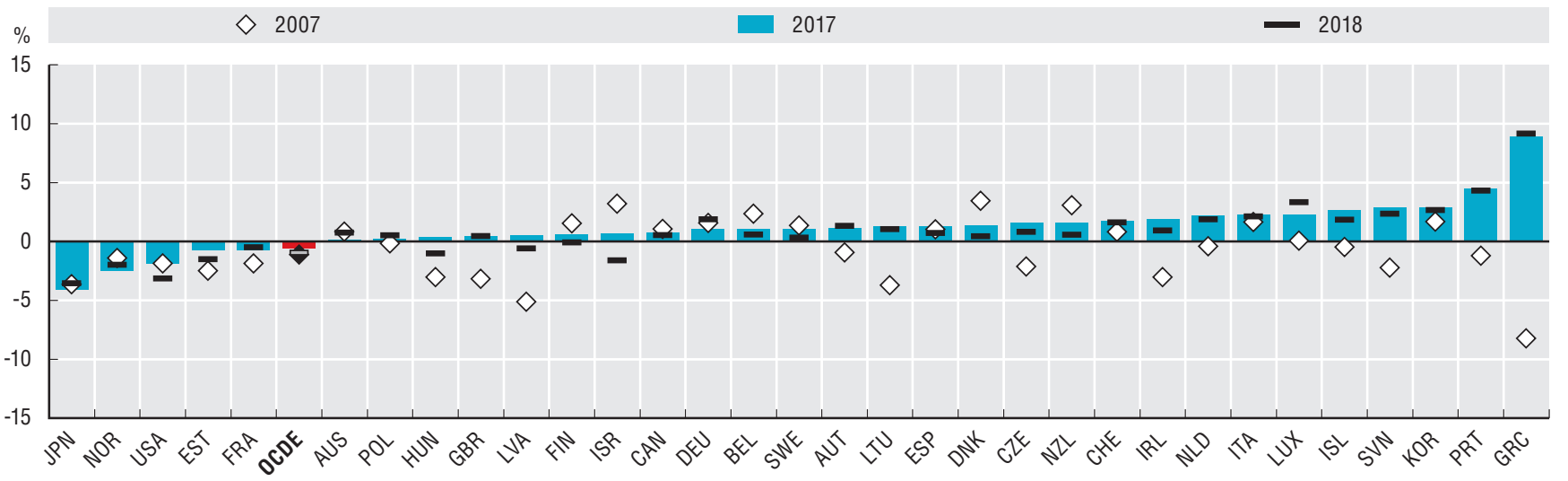

Source : Perspectives économiques de l’OCDE, $\mathrm{n}^{\circ}$ 105, mai 2019.

StatLink 解 https://doi.org/10.1787/888934051206

2.8. Solde primaire structurel prévu des administrations publiques en pourcentage du PIB potentiel, 2019 et 2020 et variation entre 2018 et 2020

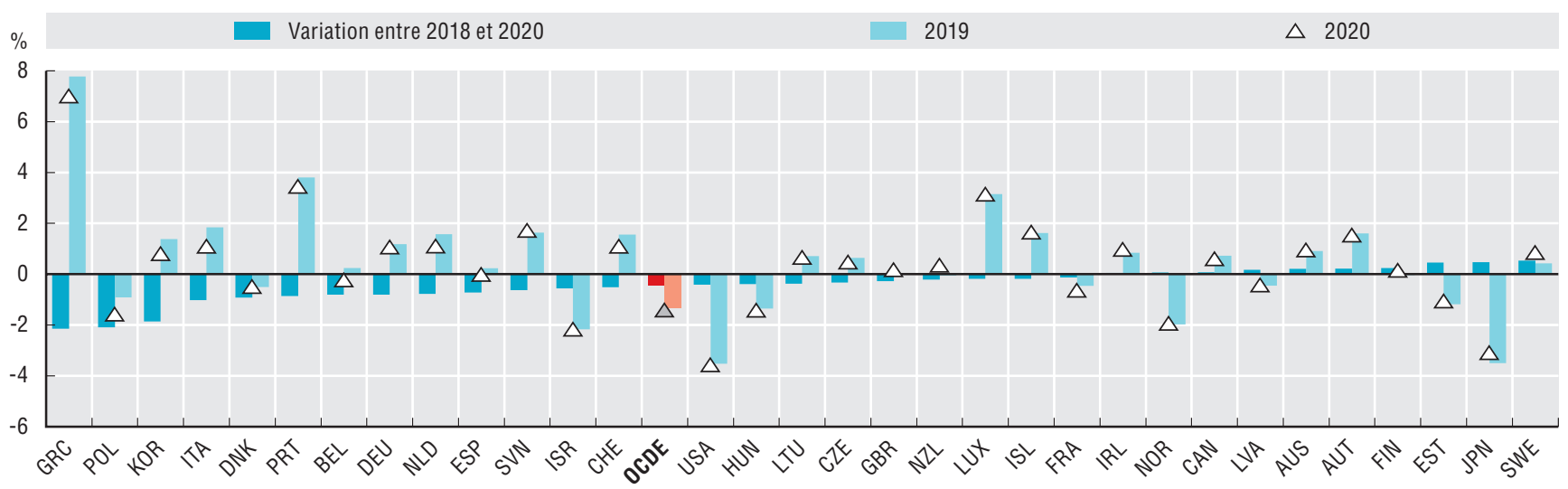

Source : Perspectives économiques de l’OCDE, n 105, mai 2019. 
Le niveau de la dette brute des administrations a d'importantes répercussions sur la stabilité des finances publiques et sur l'économie dans son ensemble. L'endettement public peut servir à financer des dépenses courantes ou à investir dans le capital physique, mais un coût est supporté sous la forme de charges d'intérêts et il convient de s'appuyer sur l'appréciation objective des lacunes en matière de capacités économiques, des besoins de développement des infrastructures et des priorités sectorielles ou sociales, ainsi que sur une analyse prudente des coûts et des avantages.

En 2017, la dette brute des administrations publiques des pays de l'OCDE représentait en moyenne $110 \%$ du produit intérieur brut (PIB). Ce pourcentage a augmenté de 37,3 points entre 2007 et 2017. Une telle hausse s'explique par le ralentissement de l'économie, l'adoption de politiques budgétaires anticycliques à caractère expansionniste et les opérations exceptionnelles de sauvetage d'institutions financières menées dans de nombreux pays jusqu'au début des années 2010. À l'opposé de cette tendance, l'Estonie et le Chili affichaient en 2017 les niveaux d'endettement les plus bas avec, respectivement, $13 \%$ et $29,6 \%$ de leur PIB.

De 2007 à 2017, les plus fortes progressions de la dette brute ont eu lieu en Grèce (+75,9 points) et en Espagne (+72,8 points). En Grèce, la dette se stabilise car l'État a dégagé ces dernières années des excédents primaires significatifs. Toutefois, la réduire nécessitera de nouvelles réformes propres à stimuler la croissance du PIB, le maintien d'excédents primaires substantiels mais réalistes et une restructuration supplémentaire, consistant par exemple à bloquer les taux d'intérêt à leur bas niveau actuel (OCDE 2018a). En Espagne, la dette publique recule, comme le montre sa baisse de 1,3 point entre 2017 et 2018 ; les progrès récents sont dus à une situation économique favorable. Néanmoins, dans un contexte de poursuite de la reprise, l'administration devra s'en tenir à ses objectifs budgétaires à moyen terme pour garantir une diminution durable de la dette publique (OCDE 2018b).

La dette brute par habitant a depuis 2007 augmenté à un rythme annuel de $5 \%$ dans les pays de l'OCDE pour atteindre en moyenne 53641 USD. Pourtant, la tendance s'est lentement inversée ces dernières années, comme le montre la baisse moyenne du ratio de la dette au PIB de 2,2 points enregistrée en 2017 par rapport à 2016. Dans les pays de l'OCDE, la dette publique des administrations se compose surtout de titres de créance (83\%), suivis des emprunts (8,6\%).

\section{Méthodologie et définitions}

Ces données sont tirées de la base de données des Statistiques de l'OCDE sur les comptes nationaux et de la base de données d'Eurostat sur les Statistiques relatives aux finances publiques, qui sont établies conformément au Système de comptabilité nationale (SCN). Le cadre du SCN 2008 est désormais en vigueur dans tous les pays de l'OCDE (voir l'Annexe A).

La dette est définie comme une sous-catégorie spécifique d'éléments de passif caractérisée par la nature des instruments financiers qu'elle englobe ou non. Généralement, elle est considérée comme l'ensemble des éléments de passif en vertu desquels un débiteur est tenu de verser à un créancier intérêts ou capital à une ou plusieurs dates futures. Tous les instruments de dette constituent des éléments de passif, mais certains de ces éléments, tels que les participations et les produits dérivés financiers, ne sont pas des instruments de dette. On obtient donc le montant de la dette en additionnant les éléments de passif suivants, pour autant qu'ils figurent au bilan financier des administrations publiques : numéraire et dépôts, titres de créance, emprunts, autres engagements (c'est-à-dire assurances, pensions, garanties standard, autres comptes à payer et, dans certains cas, droits de tirage spéciaux). Dans le SCN, la plupart des instruments de dette sont évalués au prix du marché lorsque c'est pertinent (mais il arrive que certains pays n'appliquent pas ce principe d'évaluation, notamment aux titres de créance).

Le traitement des engagements des administrations publiques au titre des régimes de retraite de leurs agents diffère selon les pays, ce qui rend toute comparaison internationale difficile. Certains pays de l'OCDE, tels que l'Australie, le Canada, les États-Unis, l'Islande et la Suède, comptabilisent ces engagements, qu'ils soient provisionnés ou non, dans la dette publique. Pour ces pays, on calcule un ratio d'endettement public corrigé en déduisant les engagements non provisionnés au titre de pensions. On trouvera des précisions en suivant le StatLinks. La dette publique est ici comptabilisée en brut, c'est-à-dire non ajustée de la valeur des actifs publics. La définition de la dette employée dans le SCN diffère de celle du Traité de Maastricht, qui sert à évaluer les situations budgétaires dans l'Union européenne. Pour plus d'informations sur le calcul de la dette publique par habitant, voir Recettes des administrations publiques (page 72).

\section{Pour en savoir plus}

OCDE (2018a), OECD Economic Surveys: Greece 2018, Éditions OCDE, Paris, http://doi.org/10.1787/eco_surveys-grc-2018-en.

OCDE (2018b), OECD Economic Surveys: Spain 2018, Éditions OCDE, Paris, https://doi.org/10.1787/eco_surveys-esp-2018-en.

\section{Notes relatives aux graphiques}

Les données sur l'Australie, le Canada, les États-Unis, l'Islande et la Suède sont présentées après ajustement (c'est-à-dire exclusion faite des engagements non provisionnés au titre de pensions). Concernant les données sur Israël, voir http://doi.org/10.1787/888932315602. Les données sur le Chili, le Mexique et la Turquie ne sont pas prises en compte dans la moyenne de la zone OCDE. Les données sur la Colombie et la Russie portent sur 2016 et celles sur le Brésil portent sur 2015 au lieu de 2017.

2.9 et 2.10. Les données de 2017 sur l'Islande et celles de 2007 sur la Corée sont fondées sur des estimations de l'OCDE.

2.11. Les données sur l'Islande ne sont pas disponibles.

2.12. (Taux annuel moyen de progression en termes réels de la dette publique par habitant, 2007-2017 et 2017-2018) est consultable en ligne (voir l'Annexe F). 
2.9. Dette brute des administrations publiques en pourcentage du PIB, 2007, 2017 et 2018

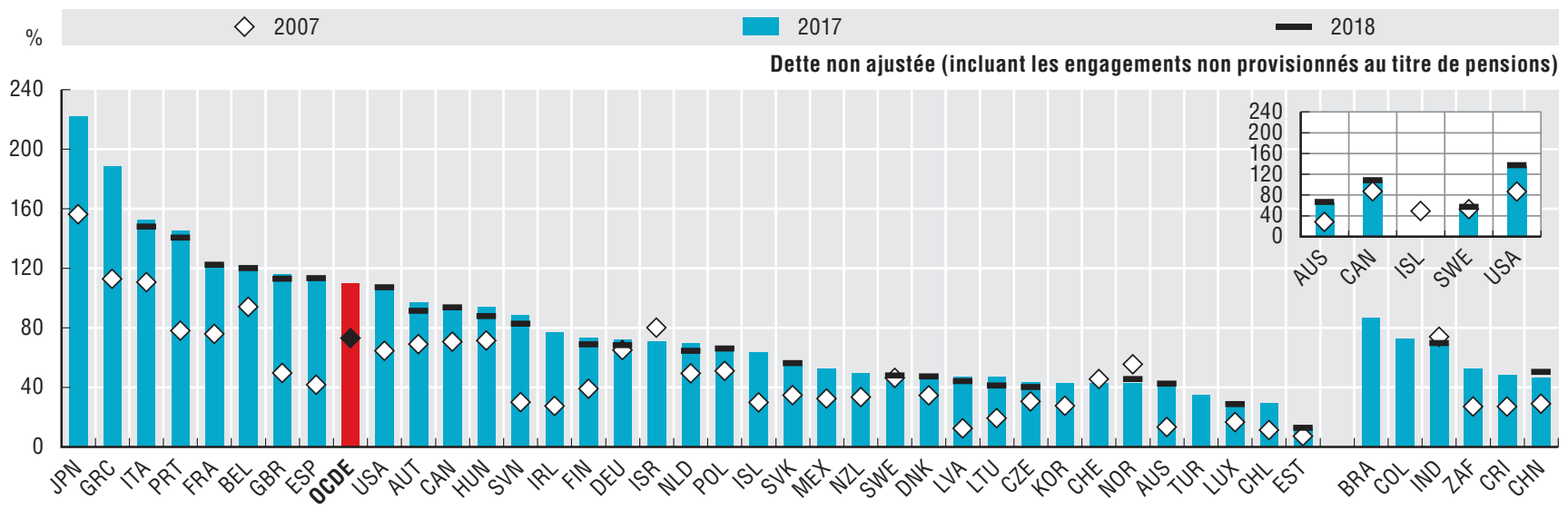

Sources : Statistiques de l'OCDE sur les comptes nationaux (base de données); statistiques d'Eurostat relatives aux finances publiques (base de données). Les données sur les autres grandes économies (sauf le Brésil) et sur le Costa Rica sont tirées des Perspectives de l'économie mondiale du FMI (avril 2019).

StatLink ants https://doi.org/10.1787/888934051244

\subsection{Dette brute des administrations publiques par habitant, 2007, 2017 et 2018}

$\diamond 2007 \quad 2017 \quad 2018$

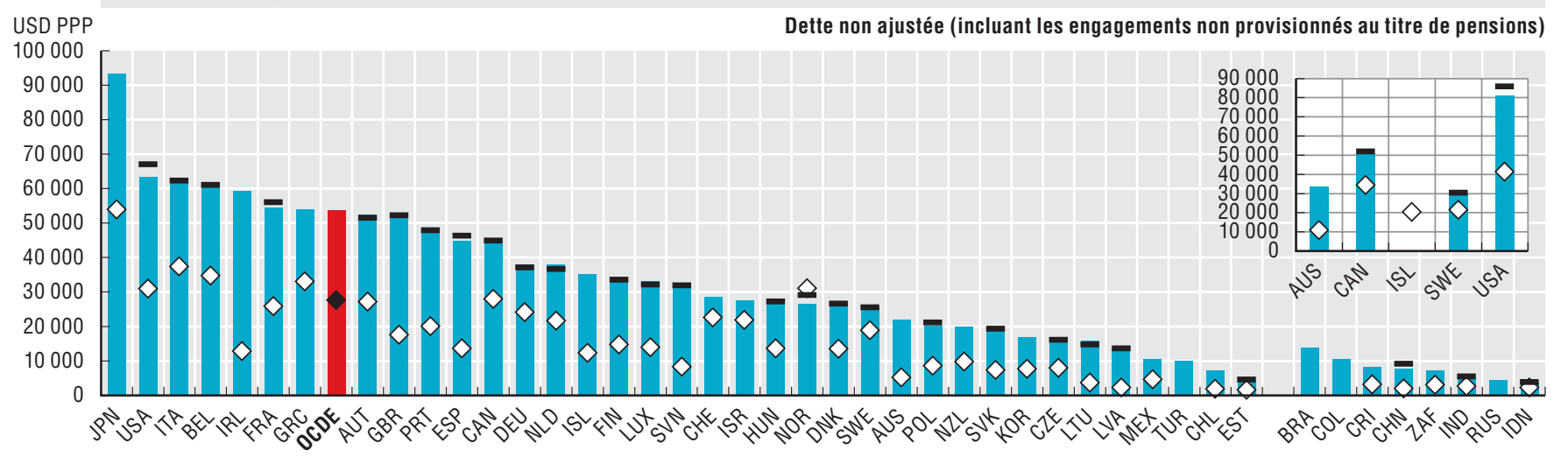

Sources: Statistiques de l'OCDE sur les comptes nationaux (base de données), statistiques d'Eurostat relatives aux finances publiques (base de données). Les données sur les autres grandes économies (sauf le Brésil) et sur le Costa Rica sont tirées des Perspectives de l'économie mondiale du FMI (avril 2019).

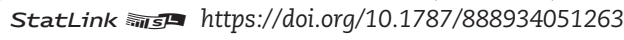

2.11. Composition de la dette brute des administrations publiques par catégorie d'instruments financiers, 2017

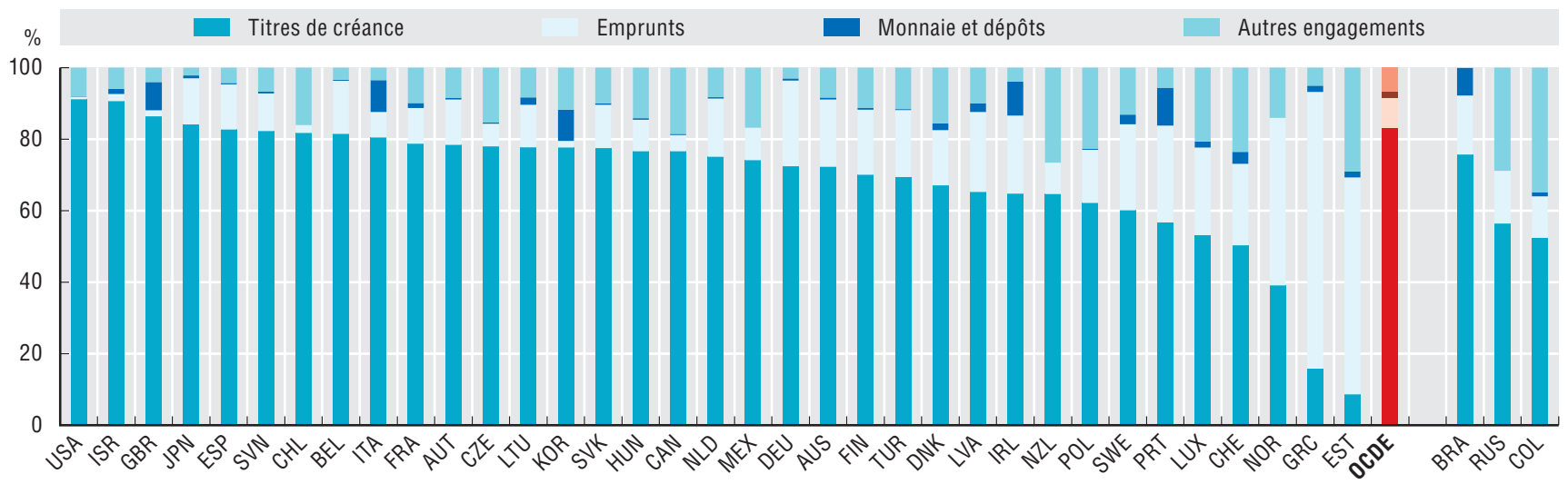

Sources : Statistiques de l'OCDE sur les comptes nationaux (base de données), statistiques d’Eurostat relatives aux finances publiques (base de données). StatLink ails https://doi.org/10.1787/888934051282 
Le patrimoine financier net des administrations publiques, c'est-à-dire la différence entre leurs avoirs et leurs engagements, illustre la capacité des administrations à honorer leurs obligations financières et peut donner une image plus exacte de la situation budgétaire d'un pays. Les avoirs représentent une source supplémentaire de financement et de revenu à la disposition des administrations publiques ; les engagements reflètent les dettes accumulées au fil du temps. Une hausse durable du patrimoine financier net est un signe de bonne santé financière. En revanche, le patrimoine financier peut être entamé par la dette publique, ce qui est alors le signe d'une dégradation de la situation budgétaire susceptible d'ébranler la confiance et d'accroître les risques.

En 2017, le patrimoine financier net des administrations publiques des pays de l'OCDE ressortait à $-70 \%$ du produit intérieur brut (PIB), ce qui signifie que les administrations avaient en moyenne beaucoup plus d'engagements que d'avoirs. Le passif financier net dépassait le PIB dans quatre pays de l'OCDE : la Grèce (-149\% du PIB), l'Italie (-125\%), le Japon (-124\%) et le Portugal (-108\%). Ces pays ont récemment accumulé une dette substantielle, en particulier pendant les années postérieures à la crise économique de 2007-2008. En 2017 également, 8 des 35 pays de l'OCDE ont fait état d'un patrimoine financier net positif, la Norvège affichant le patrimoine le plus étoffé $(+308 \%$ du PIB), devant la Finlande (+59\%). En Norvège, ce taux s'explique par les 1000 milliards USD du fonds souverain national, qui est géré par la Banque centrale pour le compte du ministère des Finances et est composé d'actions, d'obligations et de placements immobiliers. Ce fonds a été créé en 1990 à des fins de gestion des ressources pétrolières dans l'intérêt des générations actuelles et futures. En Finlande, la taille du patrimoine financier net résulte de la bonne performance des régimes de retraite.

Une décennie après la crise financière internationale, le patrimoine financier net des administrations publiques des pays de l'OCDE est, à quelques exceptions près, loin d'avoir retrouvé ses niveaux antérieurs. Toutefois, on constate entre 2017 et 2018 une amélioration moyenne de 0,4 points dans la plupart des pays disposant de données. Font exception les États-Unis (1,3 point), le Chili (1 point) et la France (0,2 point), dont la récente dégradation du patrimoine financier net est surtout imputable à de nouvelles émissions de dette publique.

En moyenne, le patrimoine financier net par habitant des pays de l'OCDE représentait -34 591 USD à PPA en 2017, soit plus du double de la moyenne de la zone OCDE en 2007 (-15 523 USD à PPA). En 2017, le patrimoine financier net par habitant le plus élevé était celui de la Norvège (+191 667 USD à PPA) et le plus faible celui du Japon (-51 921 USD à PPA).

\section{Méthodologie et définitions}

Ces données sont tirées de la base de données des Statistiques de l'OCDE sur les comptes nationaux et de la base de données d'Eurostat sur les Statistiques relatives aux finances publiques, qui sont établies conformément au Système de comptabilité nationale (SCN) ; ce dernier constitue un ensemble de concepts, de définitions, de nomenclatures et de règles approuvés au plan international en matière de comptabilité nationale.
Le cadre du SCN 2008 est désormais en vigueur dans tous les pays de l'OCDE (voir l'Annexe A pour plus de précisions sur les systèmes de déclaration et les sources).

Le patrimoine financier net du secteur des administrations publiques correspond à la différence entre la valeur totale de ses actifs financiers et celle de l'encours de ses engagements. Le SCN définit ces actifs financiers et les engagements correspondants comme étant les suivants, pour autant qu'ils figurent au bilan financier du secteur institutionnel : or monétaire et droits de tirage spéciaux (DTS) ; numéraire et dépôts ; titres de créance ; emprunts ; actions et parts de fonds de placement ; assurances, pensions et garanties standard ; produits financiers dérivés et options sur titres des salariés ; comptes à recevoir et à payer. Dans le SCN, l'actif et le passif financiers sont évalués au prix du marché, lorsque c'est pertinent (mais il arrive que certains pays n'appliquent pas ce principe d'évaluation, notamment aux titres de créance). Les données sont fondées sur l'actif et le passif financiers consolidés sauf pour le Brésil, le Chili, le Mexique, la Nouvelle-Zélande et la Russie.

Cet indicateur peut servir de mesure représentative de la dette publique nette ; en effet, comme pour la définition de la dette brute, il est possible de limiter la définition de la dette nette à la différence entre la dette brute et les actifs financiers correspondant à des instruments de dette (conformément à la définition qui figure dans le document intitulé "Statistiques de la dette du secteur public: Guide pour les statisticiens et les utilisateurs ").

Le cadre institutionnel de comptabilisation des engagements non provisionnés des administrations publiques à l'égard de leurs agents peut avoir une incidence sur le patrimoine financier net des administrations dans divers pays, ce qui rend toute comparaison internationale difficile. C'est le cas pour certains pays membres de l'OCDE tels que l'Australie, le Canada, les États-Unis, l'Islande et la Suède. Par conséquent, comme pour la dette publique brute, un patrimoine financier net ajusté est calculé pour ces pays. Pour plus d'informations sur le calcul du patrimoine financier net par habitant, voir Recettes des administrations publiques, page 72 .

\section{Pour en savoir plus}

OCDE (2019), " Panorama des comptes nationaux », Statistiques de l'OCDE sur les comptes nationaux (base de données), OCDE, Paris, https://doi.org/10.1787/data-00369-fr.

\section{Notes relatives aux graphiques}

Les données sur l'Australie, le Canada, les États-Unis, l'Islande et la Suède sont présentées après ajustement (c'est-à-dire exclusion faite des engagements non provisionnés au titre de pensions). Les données sur la Corée et l'Islande ne sont pas disponibles. Concernant les données sur Israël, voir http://doi.org/10.1787/888932315602. Les données sur le Chili, le Mexique et la Turquie ne sont pas prises en compte dans la moyenne de la zone OCDE. Les données sur la Colombie et la Russie portent sur 2016 au lieu de 2017 et celles concernant le Brésil portent sur 2015 au lieu de 2017 
2.13. Patrimoine financier net des administrations publiques en pourcentage du PIB, 2007, 2017 et 2018

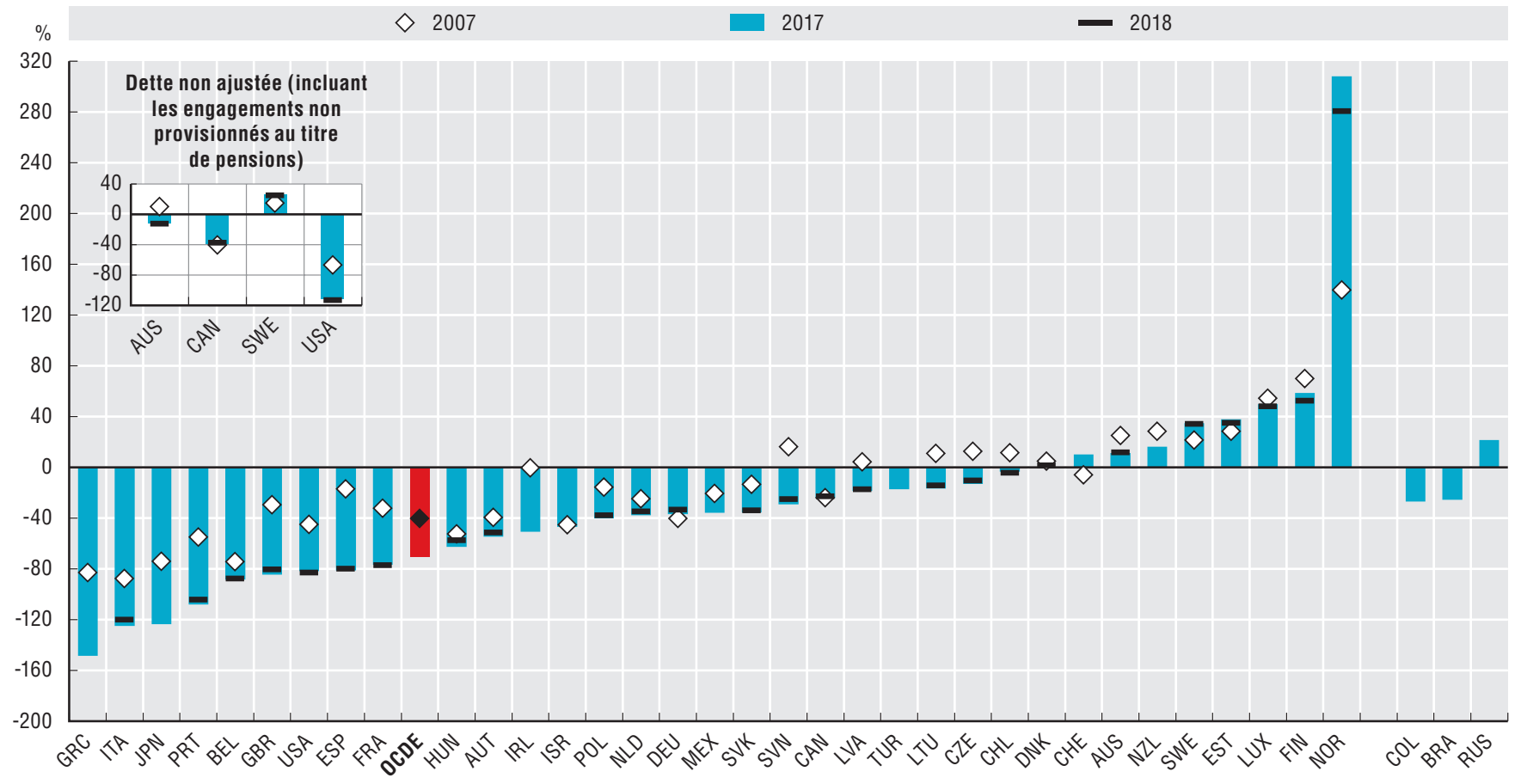

Sources : Statistiques de l'OCDE sur les comptes nationaux (base de données) ; statistiques d'Eurostat relatives aux finances publiques (base de données).

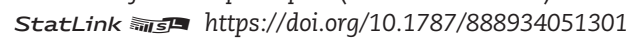

2.14. Patrimoine financier net des administrations publiques par habitant, 2007, 2017 et 2018

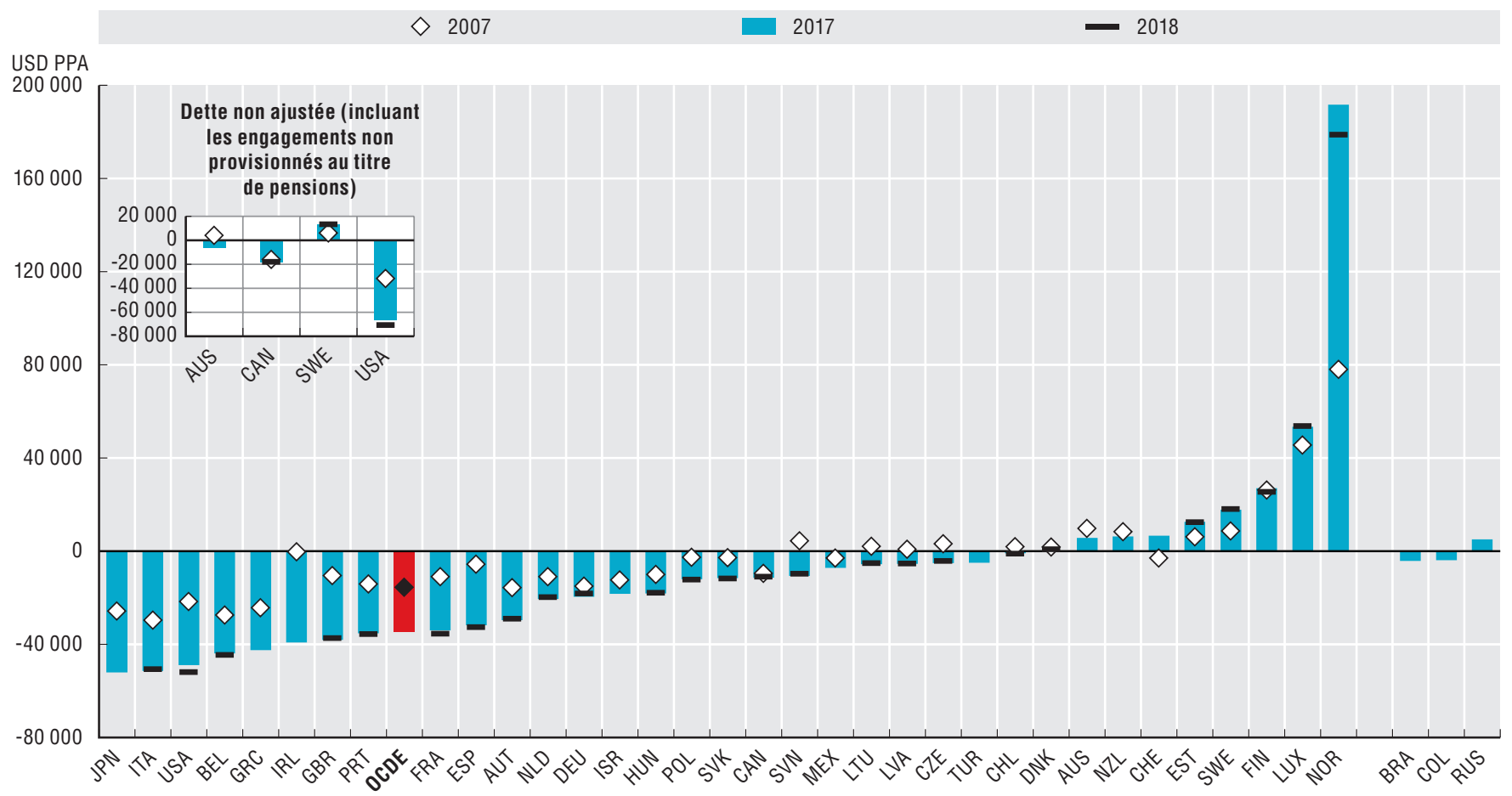

Sources : Statistiques de l'OCDE sur les comptes nationaux (base de données); statistiques d'Eurostat relatives aux finances publiques (base de données). 
Les différents systèmes administratifs accordent à leurs administrations infranationales un degré d'autonomie plus ou moins important en matière de collecte et d'utilisation de ressources, de sorte que les résultats budgétaires de ces différents niveaux d'administration peuvent varier considérablement. Néanmoins, afin de ne pas créer de mauvaises incitations, les administrations infranationales sont souvent soumises à des règles budgétaires strictes, en particulier en ce qui concerne l'endettement destiné à financer des déficits.

En 2017, les administrations centrales des pays de l'OCDE accusaient un déficit budgétaire moyen de 1,9\% du produit intérieur brut (PIB), alors que celui des administrations infranationales ressortait à 0,5\%. La même année, le solde budgétaire des administrations centrales s'échelonnait de $-4,4 \%$ du PIB au Portugal à $+5,4 \%$ en Norvège, 9 pays seulement dégageant un excédent. Parmi les pays fédéraux, les États-Unis affichaient le déficit le plus fort $(1,5 \%)$ au niveau des États fédérés, devant le Canada (0,6\%) et l'Australie (0,4\%).

Au niveau des administrations locales, en 2017, la République tchèque avait l'excédent le plus important $(0,8 \%)$ et l'Islande le déficit le plus lourd (1,3\%). En République tchèque, les économies locales se développent régulièrement; pour rendre cette croissance tout à fait bénéfique et inclusive, on a adopté une règle d'endettement des administrations locales et mis en place des mécanismes de péréquation destinés à remédier à leurs différences de capacité (OCDE 2018). En Islande, les administrations locales sont responsables des infrastructures, de l'aménagement du territoire et de l'environnement à leur niveau ; leur principale source de recettes est l'impôt sur le revenu. Les dépenses liées au tourisme et le besoin urgent de modernisation des infrastructures suscitent des difficultés pour les communes, car leurs ressources ne sont pas suffisantes pour faire face à ces pressions grandissantes, d'où un décalage entre les recettes et les dépenses (OCDE 2017). En 2018, on a constaté un changement important concernant les soldes budgétaires entre le gouvernement central et les fonds de sécurité sociale en Lituanie, imputable à l'annulation par le gouvernement central de la dette du fonds national d'assurance sociale.

Les administrations infranationales peuvent emprunter afin de financer des déficits à leur niveau. En moyenne, la dette des administrations centrales des pays de l'OCDE représentait en 2017 95,7 \% du PIB et celle des administrations infranationales $20,3 \%$. Dans les pays fédéraux, la dette brute des États fédérés n'était pas négligeable, le niveau le plus élevé étant constaté au Canada (47\% du PIB), devant l'Espagne (27\% du PIB). Au Canada, l'endettement global en proportion du PIB baisse, mais on observe une évolution contrastée entre 2017 et 2018 : la dette de l'État fédéral a diminué (de 2,3 points) alors que celle des provinces et territoires a augmenté (de 1,2 point). Du fait d'un durcissement des règles d'endettement des administrations locales, la dette de ces dernières représentait en moyenne $7,2 \%$ du PIB dans les pays de l'OCDE, soit sensiblement moins qu'au niveau central ou des États fédérés.

\section{Méthodologie et définitions}

Les données sont tirées de la base de données des Statistiques de l'OCDE sur les comptes nationaux, qui sont établies conformément au Système de comptabilité nationale
(SCN) ; ce dernier constitue un ensemble de concepts, de définitions, de nomenclatures et de règles approuvés au plan international en matière de comptabilité nationale. Le cadre du SCN 2008 est désormais en vigueur dans tous les pays de l'OCDE (voir l'Annexe A pour plus de précisions). Selon la terminologie du SCN, les administrations publiques se composent de l'administration centrale, des administrations des États fédérés, des administrations locales et des administrations de sécurité sociale. Les administrations des États fédérés n'existent que dans les neuf pays membres de l'OCDE à structure fédérale : l'Allemagne, l'Australie, l'Autriche, la Belgique, le Canada, l'Espagne (pays considéré comme quasi fédéral), les États-Unis, le Mexique et la Suisse. Le solde budgétaire des administrations publiques, également présenté comme la capacité (+) ou le besoin (-) de financement de ces dernières, est égal à la différence entre le montant total de leurs dépenses et celui de leurs recettes. Pour plus de renseignements sur la dette, voir Recettes des administrations publiques, page 72 .

\section{Pour en savoir plus}

OCDE (2018), OECD Economic Surveys: Czech Republic 2018, Éditions OCDE, Paris, https://doi.org/10.1787/eco_surveys-cze2018-en.

OCDE (2017), OECD Economic Surveys: Iceland 2017, Éditions OCDE, Paris, http://doi.org/10.1787/eco_surveys-isl-2017-en.

\section{Notes relatives aux graphiques}

Les administrations locales sont comprises dans les administrations des États fédérés en ce qui concerne l'Australie et les États-Unis. Il n'existe pas de régime public d'assurance sociale en Australie. Aux États-Unis, en Irlande, en Norvège et au Royaume-Uni, les administrations de sécurité sociale font partie de l'administration centrale. Les données sur le Chili et la Turquie ne sont pas prises en compte dans la moyenne de l'OCDE du fait de l'absence de séries chronologiques. Concernant les donn.es sur Isra.l, voir http:// doi.org/10.1787/888932315602. Pour le Japon, les données sur les sous-secteurs des administrations publiques portent sur l'exercice budgétaire.

2.15. Les données sur l'Indonésie portent sur 2016 au lieu de 2017.

2.16. Les données sur l'Islande ne sont pas disponibles.

2.16 et 2.17. Les données sur le Chili, la Corée et le Mexique ne sont pas disponibles. Les données sur l'Australie, le Canada, les États-Unis et la Suède sont présentées après ajustement (c'est-à-dire exclusion faite des engagements non provisionnés au titre de pensions) Les données sur les États-Unis, la Nouvelle-Zélande et la Suisse sont présentées sur une base non consolidée. Les données sur la Colombie portent sur 2016 au lieu de 2017.

2.17. Les données sont consolidées au sein des sous-secteurs des administrations publiques. Toutefois, les flux entre les niveaux d'administration sont intégrés. Les données sur l'Islande ne sont pas prises en compte dans la moyenne de la zone OCDE du fait de l'absence de séries chronologiques. 
2.15. Solde budgétaire aux différents niveaux d'administration, en \% du PIB, 2017 et 2018

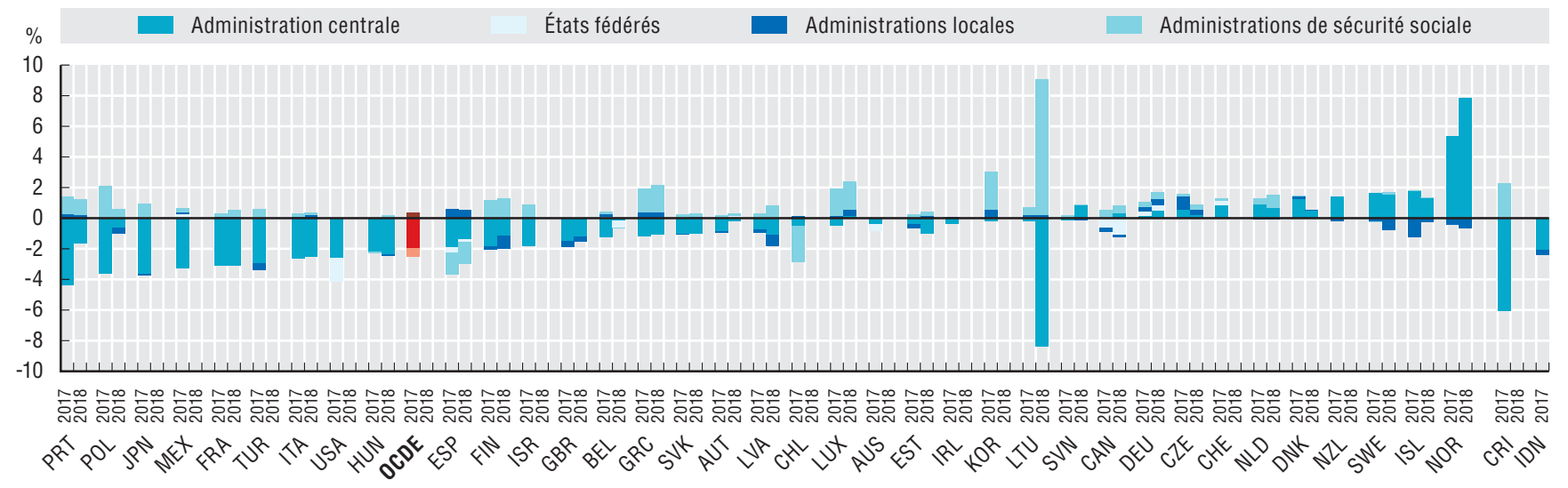

Source : Statistiques de l’OCDE sur les comptes nationaux (base de données).

StatLink 光is https://doi.org/10.1787/888934051339

\subsection{Dette brute aux différents niveaux d'administration, en \% du PIB, 2017 et 2018}

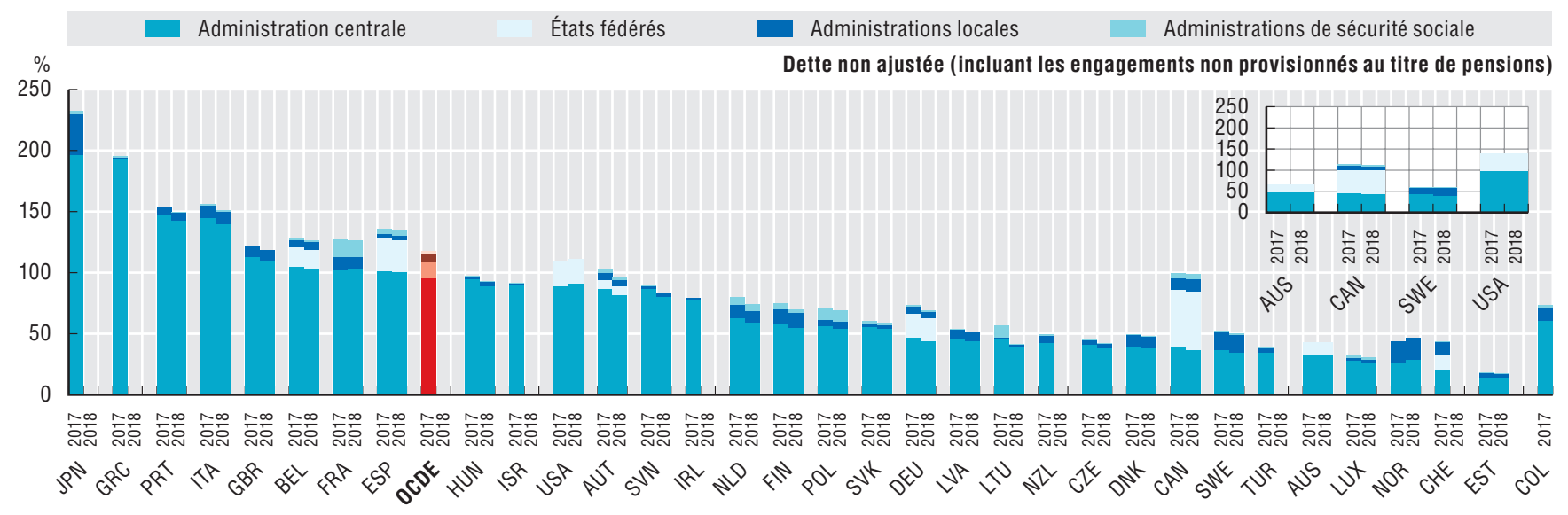

Sources : Statistiques de l'OCDE sur les comptes nationaux (base de données); statistiques d'Eurostat relatives aux finances publiques (base de données).

StatLink (ints https://doi.org/10.1787/888934051358

2.17. Répartition de la dette publique brute par niveau d'administration, 2007 et 2017

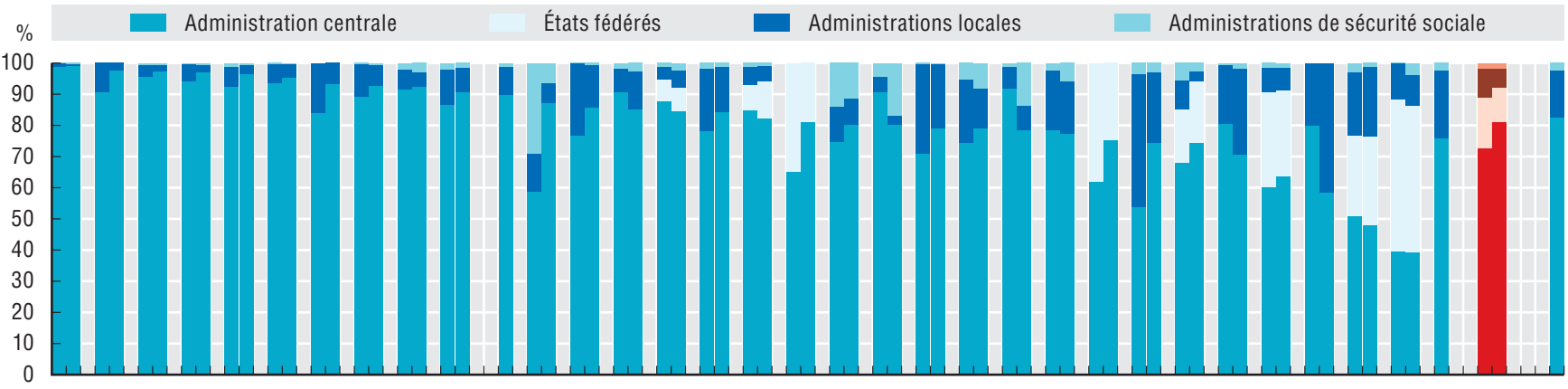

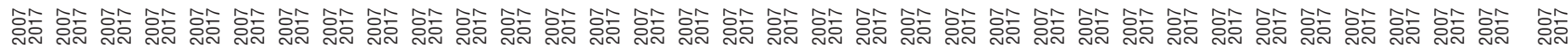

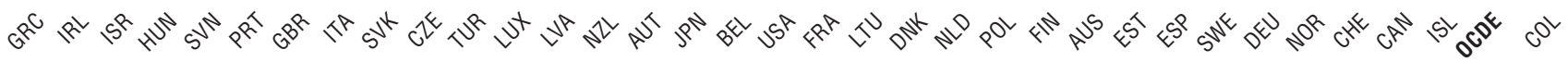

Sources : Statistiques de l'OCDE sur les comptes nationaux (base de données), statistiques d'Eurostat sur les finances publiques (base de données). 
Les recettes publiques servent à financer les biens et les services fournis à la population (comme dans les domaines de la santé et de la défense), tout en permettant à l'État d'assurer son rôle en matière de redistribution (par le versement de subventions et de prestations sociales). Les principales sources de recettes sont les impôts et les cotisations sociales. Les choix de politique publique des gouvernements, par exemple en matière de santé et de retraite, ainsi que les fluctuations du cycle économique déterminent le montant nécessaire de recettes pour servir les citoyens et honorer les obligations financières (telles que la dette). Bien qu'il soit possible de couvrir pendant un certain temps les déséquilibres par l'endettement, il faut à long terme équilibrer les comptes pour assurer la viabilité des finances publiques.

En 2017, les recettes publiques des pays de l'OCDE s'élevaient en moyenne à $38,2 \%$ du produit intérieur brut (PIB), en hausse de 1 point depuis 2007. Elles représentent plus de la moitié du PIB en Norvège (54,9\%), en France (53,7 \%), en Finlande (53,4 \%), au Danemark $(52,6 \%)$ et en Belgique (51,3\%). À l'opposé, c'est au Mexique (23,7\%) et en Irlande (26\%) que les recettes publiques en proportion du PIB sont les plus faibles. L'Irlande a enregistré un recul de ses recettes publiques de 10,2 points de PIB depuis 2007. Ce recul s'explique par une croissance du PIB plus rapide que la progression des recettes publiques (en termes nominaux), du fait en partie de taux d'imposition des sociétés et des personnes physiques relativement faibles. À l'inverse, la Grèce a augmenté ses recettes publiques de 7,7 points au cours de la même période ; toutefois, il faut interpréter ce résultat avec précaution car il est imputable à une baisse du PIB plus rapide que celle des recettes. De 2007 à 2017, les recettes des administrations publiques en pourcentage du PIB sont restées assez stables au Canada (-0,5 point), en Hongrie (-0,1 point), aux États-Unis (+0,4 point) ainsi qu'en Australie et en Autriche (+0,5 point dans les deux cas).

Une autre manière d'évaluer comparativement le volume des recettes des administrations publiques consiste à ramener leur montant à la population. En 2017, les recettes publiques moyennes par habitant dans les pays de l'OCDE s'établissaient à 17535 USD, en hausse de 4302 USD à PPA par rapport à 2007. Cet indicateur a augmenté dans tous les pays entre 2007 et 2017 et la tendance s'est poursuivie en 2018. Les recettes publiques par habitant sont le plus élevées au Luxembourg (47 749 USD à PPA de 2017), ce qui s'explique en partie par la forte proportion de travailleurs transfrontaliers, non comptabilisés comme résidents, qui payent en conséquence l'impôt sur le revenu au Luxembourg sans être considérés comme faisant partie de sa population. La Norvège arrive en deuxième position, avec 34134 USD à PPA en 2017, grâce aux recettes substantielles tirées de la vente de pétrole. À l'opposé, les recettes publiques par habitant sont le plus faibles au Mexique (4 658 USD à PPA), en Turquie (8 840 USD à PPA) et en Lettonie (10 565 USD à PPA) parce que ces pays ont des taux d'imposition relativement bas ou des assiettes fiscales relativement étroites.

Entre 2007 et 2017, le taux annuel moyen de croissance des recettes réelles des administrations publiques par habitant a été de $0,89 \%$ dans les pays de l'OCDE. La République slovaque a connu la plus forte hausse $(+3,7 \%)$, suivie de la Corée $(+2,9 \%)$ et de la Pologne (+2,9\%). C'est aussi en Pologne que ce taux de croissance a été en 2017-2018 le plus élevé (+9,1 points). Elle a réussi à améliorer la discipline fiscale, en particulier pour la taxe sur la valeur ajoutée (TVA), en mettant en place un mécanisme de paiement fractionné en vertu duquel les clients règlent directement la TVA aux fournisseurs via un compte dédié. Ainsi, l'OCDE estime qu'en Pologne, les pertes dues à la fraude fiscale pourraient avoir diminué de quelque $25 \%$ en 2017 (OCDE 2018). En revanche, entre 2007 et 2017, c'est en Grèce et en Espagne que les recettes annuelles moyennes ont le plus baissé, respectivement de 0,9 et 0,8 point ; cela s'explique très probablement par le rétrécissement de la base imposable, qui est imputable aux graves effets de la crise financière de 2007-2008 et par l'extrême lenteur du redressement à l'origine d'un niveau de chômage durablement élevé. Néanmoins, dans ces deux cas, le taux de croissance a sensiblement progressé en 2017-2018, les mesures d'assainissement commençant à porter leurs fruits.

\section{Méthodologie et définitions}

Les données relatives aux recettes publiques sont tirées de la base de données des Statistiques de l'OCDE sur les comptes nationaux, qui sont établies conformément au Système de comptabilité nationale (SCN). Ce dernier constitue un ensemble de concepts, de définitions, de nomenclatures et de règles approuvés au plan international en matière de comptabilité nationale. Le cadre du SCN 2008 est désormais en vigueur dans tous les pays de l'OCDE (voir l'Annexe A pour plus de précisions sur les systèmes de déclaration et les sources). Selon la terminologie du SCN, les administrations publiques se composent de l'administration centrale, des administrations des États fédérés, des administrations locales et des administrations de sécurité sociale. Les recettes comprennent les impôts, les cotisations sociales nettes, les dons et les autres rentrées. Le produit intérieur brut (PIB) est la mesure habituelle de la valeur des biens et services produits par un pays au cours d'une période donnée. On a calculé les recettes publiques par habitant en convertissant les recettes totales en USD à la parité de pouvoir d'achat (PPA) OCDE/Eurostat pour le PIB et en divisant le résultat obtenu par la population du pays. La PPA correspond au nombre d'unités monétaires du pays $B$ nécessaire à l'acquisition d'une même quantité de biens et de services dans le pays A.

\section{Pour en savoir plus}

Lequiller, F. et Blades, D. (2014), Understanding National Accounts: Second Edition, Éditions OCDE, Paris, http://doi. org/10.1787/9789264214637-en.

\section{Notes relatives aux graphiques}

Les données sur le Chili ne sont pas disponibles. Concernant les donn.es sur Isra.l, voir http://doi.org/10.1787/888932315602.

Les données sur la Turquie ne sont pas prises en compte dans la moyenne de la zone OCDE car il manque des séries chronologiques.

Les données sur le Brésil et l'Indonésie portent sur 2016 au lieu de 2017 et celles concernant la Russie portent sur 2015 au lieu de 2017 
2.18. Recettes des administrations publiques en pourcentage du PIB, 2007, 2017 et 2018

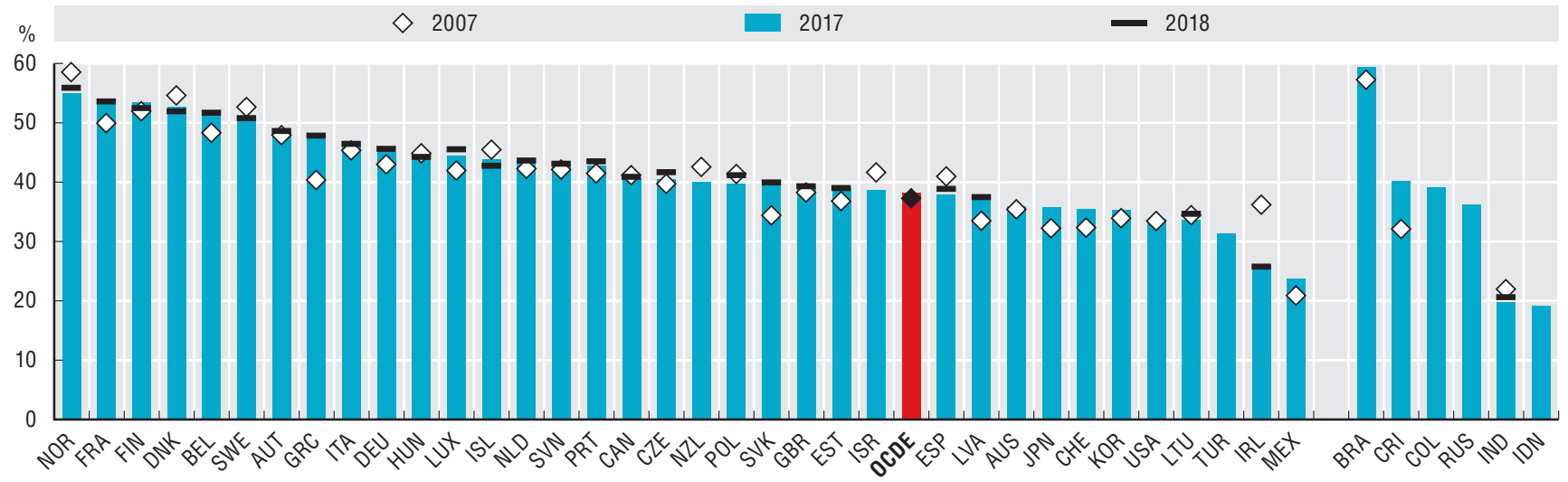

Sources : Statistiques de l'OCDE sur les comptes nationaux (base de données). Les données sur l'Inde sont tirées des Perspectives de l'économie mondiale du FMI (avril 2019).

2.19. Recettes des administrations publiques par habitant, 2007, 2017 et 2018

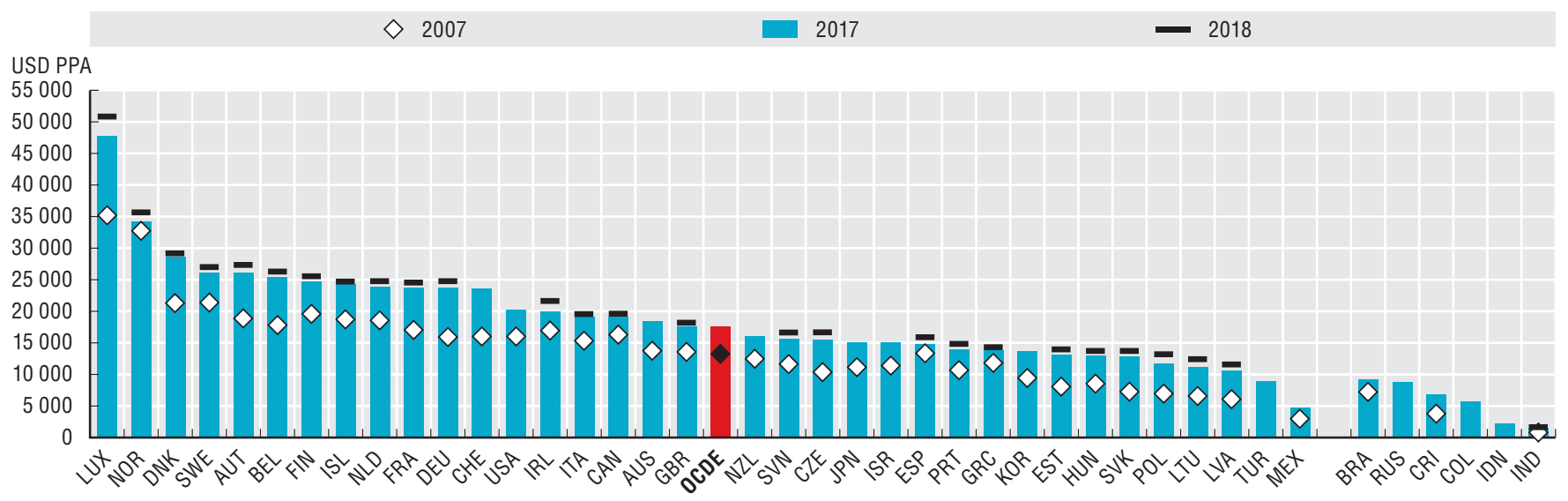

Sources: Statistiques de l'OCDE sur les comptes nationaux (base de données). Les données sur l'Inde sont tirées des Perspectives de l'économie mondiale du FMI (avril 2019).

StatLink त्गाड़ https://doi.org/10.1787/888934051415

2.20. Taux annuel moyen de croissance des recettes publiques par habitant en termes réels, 2007-2017 et 2017-2018

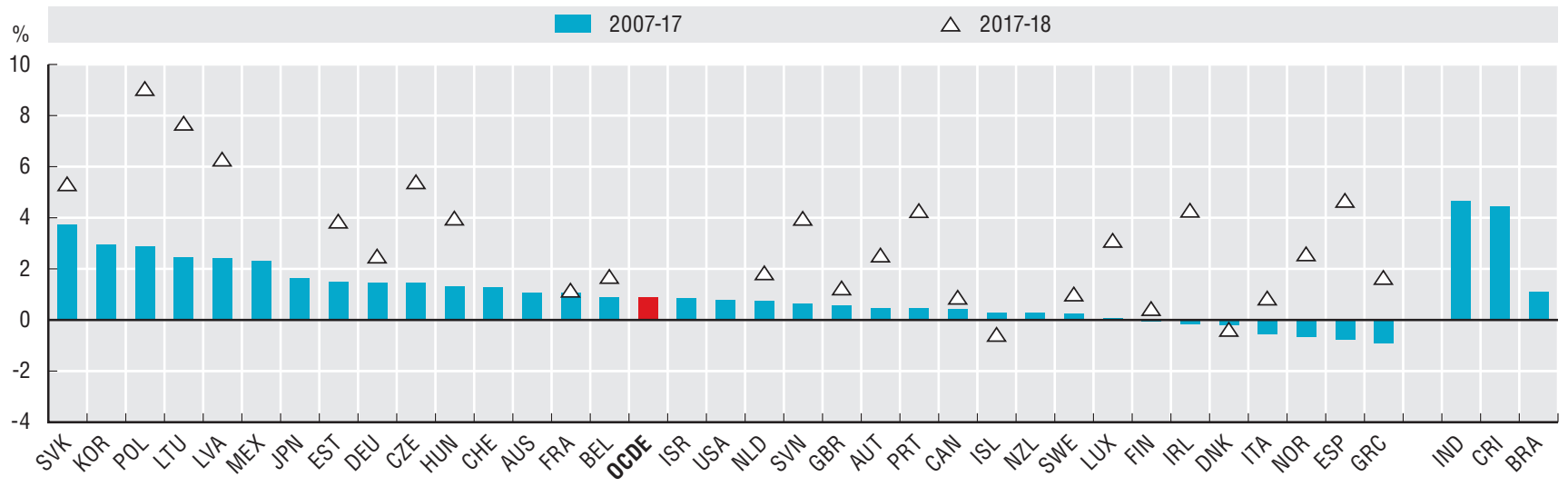

Sources: Statistiques de l'OCDE sur les comptes nationaux (base de données). Les données sur l'Inde sont tirées des Perspectives de l'économie mondiale du FMI (avril 2019). 
Les principales sources de recettes des administrations publiques sont les impôts (comme ceux sur le revenu ou sur le patrimoine) et les cotisations sociales versées directement par ou pour le compte des salariés. Une moindre proportion des recettes publiques provient des ventes de biens et de services réalisées par les administrations (par exemple les redevances acquittées par les usagers en contrepartie de la prestation de services), des dons et d'autres sources (par exemple les revenus du patrimoine).

En 2017, $60 \%$ en moyenne des recettes perçues par les administrations des pays de l'OCDE provenaient des impôts, $25 \%$ des cotisations sociales nettes, $8 \%$ des ventes de biens et de services et $7 \%$ des dons et autres sources. La composition de ces recettes est très différente selon les pays. Les impôts représentent $88 \%$ des recettes publiques du Danemark qui précède sur ce plan l'Australie, la Nouvelle-Zélande et la Suède (plus de $79 \%$ ), alors qu'ils représentent seulement $46 \%$ de celles de la République slovaque. Plus que dans n'importe quelle autre économie de l'OCDE, les recettes publiques reposent en République slovaque sur les cotisations sociales nettes, qui représentent 37,5\% des recettes en question. C'est au Mexique et en Norvège que la part des recettes publiques provenant des dons et autres sources est la plus importante (respectivement $34 \%$ et $23 \%$ ), car l'exploitation de ressources naturelles (comme le pétrole et le gaz) y est une source importante de rentrées budgétaires. La contribution des ventes de biens et de services est la plus substantielle en Finlande (13\%), en Suisse (13\%) et aux États-Unis (12\%) ; cela indique que la population contribue proportionnellement davantage au financement des services publics dans ces pays, essentiellement par le biais de redevances ou de droits d'usage.

Dans les pays de l'OCDE, la part des dons et autres sources dans les recettes publiques s'est réduite (respectivement -0,6 et $-0,4$ point). Par rapport aux autres membres de l'OCDE, c'est en Lituanie que la composition des recettes des administrations publiques a le plus évolué entre 2007 et 2017 : la contribution relative des cotisations sociales nettes a augmenté de 10 points et celle des ventes de biens et de services de 1,7 point, alors que la part des impôts a reculé de 9,4 points et celle des dons et autres sources de 2,3 points. Ces variations s'expliquent, au moins en partie, par le taux élevé des cotisations de sécurité sociale qui sont principalement à la charge des employeurs, par le vieillissement rapide de la population, par l'émigration et par la rigidité du marché du travail qui favorise l'emploi informel et restreint la base d'imposition. Pour résoudre partiellement ce problème dans le cadre de son Nouveau modèle social, la Lituanie a adopté une vaste réforme, mise en œuvre par étapes en 2017 et 2018, des relations entre employeurs et salariés, des relations entre les chômeurs et l'administration et des régimes de retraite, qui s'étend également à certains aspects du système fiscal (OCDE 2018)

Les impôts sur le revenu et sur les bénéfices sont la principale source de recettes fiscales dans les pays de l'OCDE (34\% de ces recettes en 2016), précédant de peu les impôts sur les biens et les services (33\%). Dans certains pays, toutefois, la composition des impôts s'écarte de la moyenne de l'OCDE. En 2016, la part des impôts sur le revenu et sur les bénéfices dans le prélèvement fiscal total était la plus faible en Hongrie, en Lituanie et en Slovénie (environ $19 \%$ ) ; elle était la plus élevée au Danemark (63\%), en Australie (57\%) et en NouvelleZélande (56 \%). La contribution des impôts sur les biens et les services (par exemple la TVA) était la plus importante au Chili (55\%), en Turquie (44\%) et en Estonie (43\%), mais la plus faible aux États-Unis (17\%), au Japon (20\%) et en Suisse (21\%). En 2016, les impôts sur le patrimoine constituaient la principale source de rentrées publiques en Islande (34\%), en hausse de 28 points par rapport à 2007. Néanmoins, cette hausse résulte d'une opération ponctuelle par laquelle le pays a prélevé une " contribution de stabilité "sur les actifs patrimoniaux d'entités qui opéraient auparavant en tant que banques commerciales ou caisses d'épargne, afin d'assouplir le contrôle des mouvements de capitaux imposé pendant la crise.

\section{Méthodologie et définitions}

Les données relatives aux recettes publiques sont tirées de la base de données des Statistiques de l'OCDE sur les comptes nationaux, qui sont établies conformément au Système de comptabilité nationale (SCN) ; ce dernier constitue un ensemble de concepts, de définitions, de nomenclatures et de règles approuvés au plan international en matière de comptabilité nationale. Le cadre du SCN 2008 est désormais en vigueur dans tous les pays de l'OCDE (voir l'Annexe A pour plus de précisions sur les systèmes de déclaration et les sources). Les recettes publiques comprennent les impôts (sur la consommation, le revenu, le patrimoine et le capital, par exemple), les cotisations sociales nettes (au titre de la retraite, de l'assurance maladie et de la sécurité sociale, déduction faite le cas échéant des charges liées aux régimes d'assurance sociale), le produit des ventes de biens et de services (par exemple la production marchande des établissements publics et les droits d'entrée) et les dons et autres sources (dons courants et en capital, revenus du patrimoine et subventions, etc.). Ces agrégats ont été construits à partir de sous-comptes budgétaires (voir l'Annexe B). Les données présentées au graphique 2.23 proviennent des Statistiques des recettes publiques de l'OCDE. Les recettes fiscales ne sont pas définies de la même façon par l'OCDE et dans le SCN, en particulier dans le cas des cotisations obligatoires de sécurité sociale. Selon le SCN, les impôts sont des paiements obligatoires, sans contrepartie, en numéraire ou en nature, effectués par des unités institutionnelles aux administrations publiques. Les cotisations sociales nettes correspondent aux cotisations effectives ou imputées qui sont versées aux régimes d'assurance sociale afin de garantir le droit à des prestations sociales. Elles peuvent être obligatoires ou facultatives et les régimes peuvent être provisionnés ou non. Les Statistiques des recettes publiques de l'OCDE traitent les cotisations de sécurité sociale obligatoires comme des impôts, alors que le SCN les considère comme des cotisations sociales nettes, parce que l'obtention de prestations sociales dépend dans la plupart des pays du versement de cotisations appropriées même si le montant des prestations n'a pas nécessairement de rapport avec celui des cotisations.

\section{Pour en savoir plus}

OCDE (2018), OECD Economic Surveys: Lithuania 2018, Éditions OCDE, Paris, https://doi.org/10.1787/eco_surveys-ltu-2018-en.

\section{Notes relatives aux graphiques}

Concernant les données sur Israël, voir http://doi.org/10.1787/888932315602.

2.21 et 2.22. Les données sur le Chili ne sont pas disponibles. Celles sur la Turquie ne sont pas prises en compte dans la moyenne de la zone OCDE car il manque des séries chronologiques. L'Australie ne perçoit pas de recettes sous forme de cotisations sociales car il n'existe pas de régime public d'assurance sociale dans ce pays. Les données sur la Russie portent sur 2015 au lieu de 2017.

2.23. Pour les pays membres à la fois de l'OCDE et de l'Union européenne, l'imposition totale inclut les droits de douane perçus pour le compte de l'Union européenne. La dernière année pour laquelle des données sont disponibles sur tous les pays de l'OCDE est 2016. La moyenne de la zone OCDE n'est pas pondérée.

2.24 à 2.26. La ventilation des recettes par niveau d'administration est consultable en ligne à l'Annexe F. 


\section{FINANCES PUBLIQUES ET ÉCONOMIE}

Composition des recettes des administrations publiques

2.21. Composition des recettes des administrations publiques, 2017 et 2018

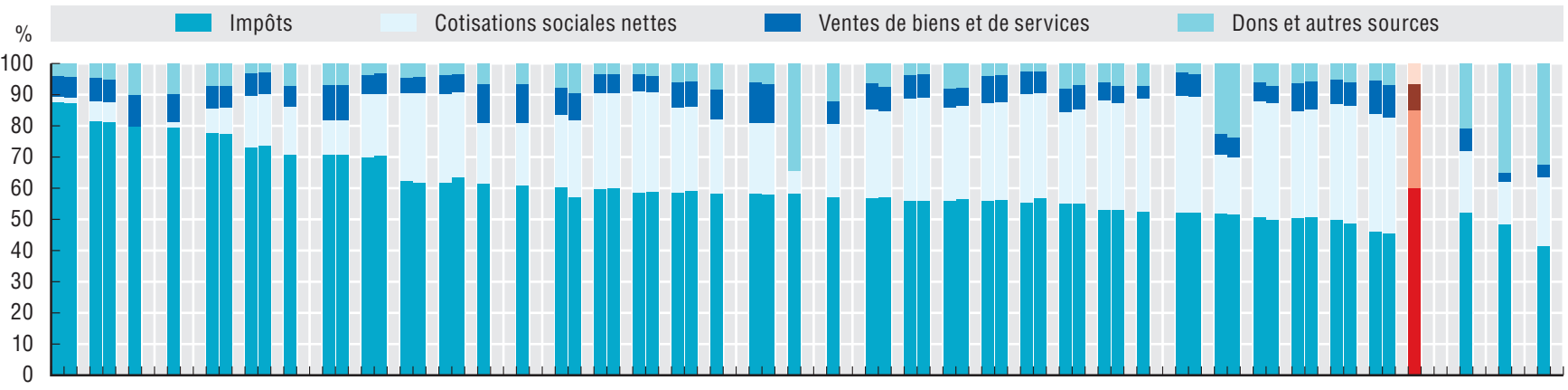

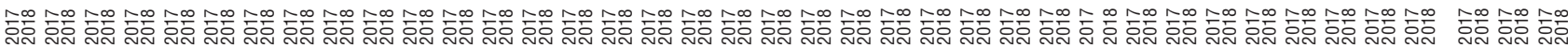

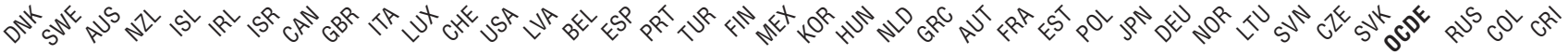

Source: Statistiques de l'OCDE sur les comptes nationaux (base de données).

StatLink 年15 https://doi.org/10.1787/888934051453

\subsection{2. Évolution de la composition des recettes des administrations publiques, 2007 à 2017}

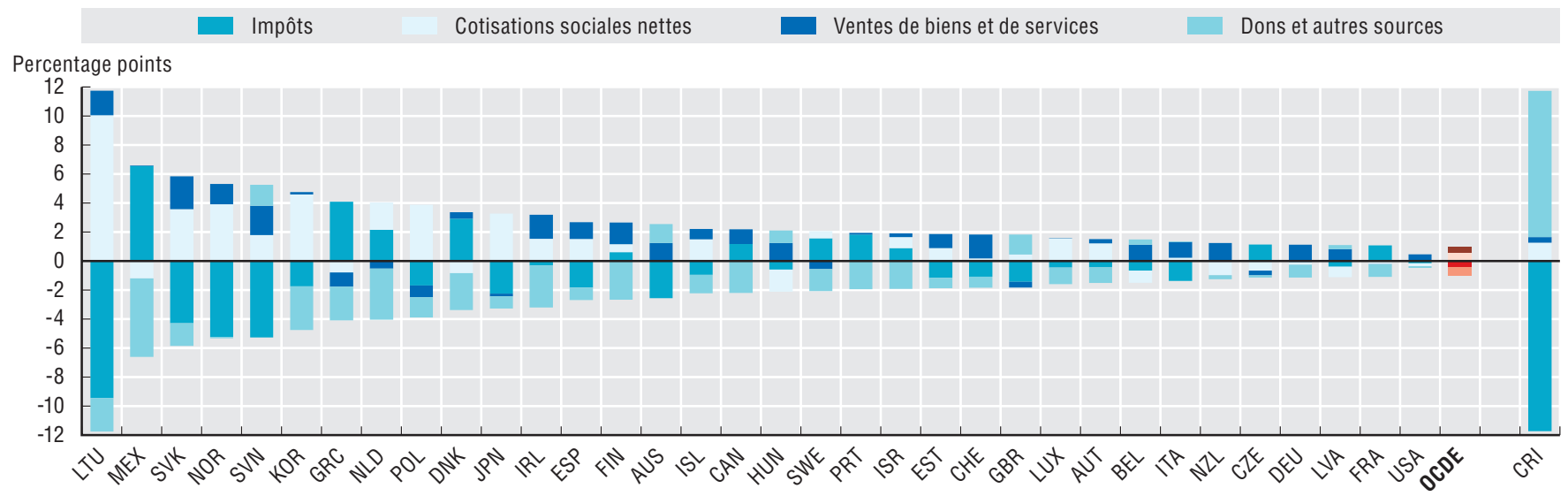

Source: Statistiques de l'OCDE sur les comptes nationaux (base de données).

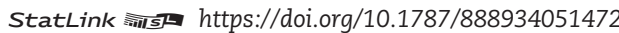

2.23. Ventilation des recettes fiscales en pourcentage de leur montant total, 2007 et 2016

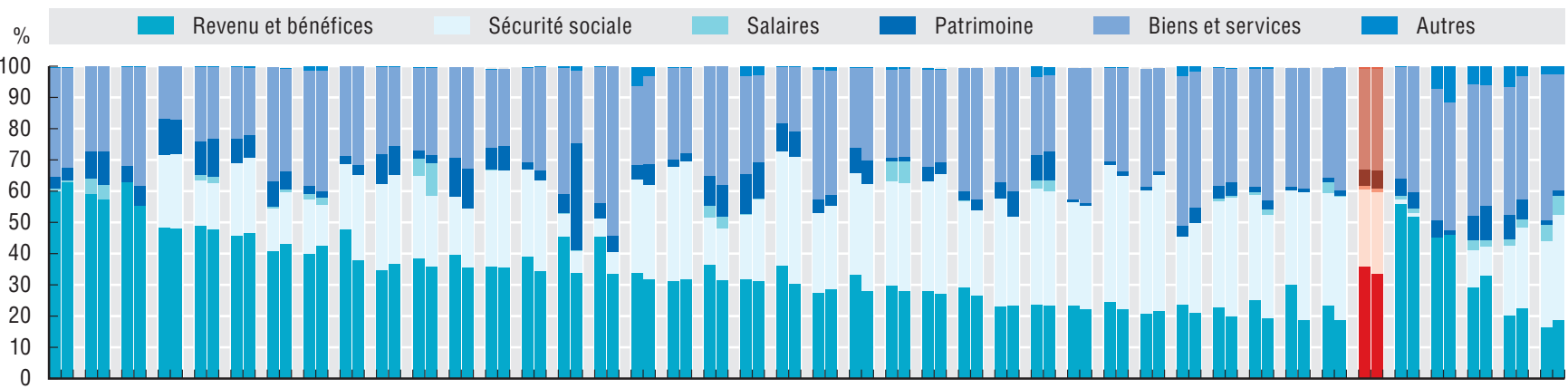

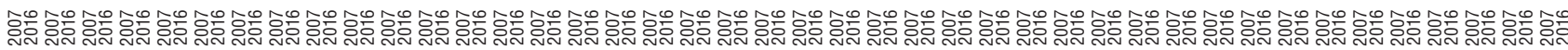

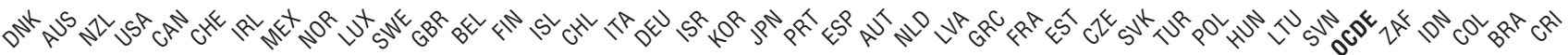

Source: Statistiques de l'OCDE sur les comptes nationaux (base de données). 
L'État est chargé de fournir à la population des biens et des services, dont certains sont exclusivement de son ressort (comme l'administration de la justice), et de redistribuer les revenus (par exemple sous la forme de prestations sociales et de subventions). Les dépenses des administrations publiques, financées essentiellement par les impôts et les cotisations sociales, sont en général moins flexibles que les recettes, car elles sont moins sensibles au cycle économique et résultent de décisions passées et présentes en matière d'action publique qui garantissent certains droits.

En 2017, les dépenses des administrations publiques ont représenté en moyenne des pays de l'OCDE 40,4\% du produit intérieur brut (PIB), chiffre en hausse de 1,4 point par rapport à 2007. La France est le pays qui a dépensé le plus, tant en 2017 (55,5 \% du PIB) qu'en 2007 (52,6 \%). Entre 2007 et 2018, parmi les pays disposant de données, la plus forte progression des dépenses publiques s'est produite en Norvège $(+7,3$ points) du fait de la mise en œuvre prolongée d'une politique budgétaire anticyclique à caractère expansionniste. Toutefois, comme le montre la réduction déjà intervenue entre 2017 et 2018 (-1,3 point), la Norvège évolue désormais vers la neutralité des comptes publics et s'attache davantage à l'efficience de ses dépenses.

En 2017, l'Irlande et le Mexique ont enregistré les dépenses publiques les plus faibles, à 25,3 \% du PIB chacun. Entre 2007 et 2017, c'est en Irlande que le ratio dépenses/PIB a baissé le plus fortement (-9,6 points), essentiellement en raison de la croissance très rapide du PIB nominal. En 2015, quelques entreprises multinationales y ont délocalisé leurs actifs de propriété intellectuelle, ce qui a entraîné une hausse massive du stock de capital puis une augmentation des exportations dans le cadre de la production manufacturière sous-traitée (OCDE 2018b). Le Mexique a pour sa part accru ses dépenses publiques entre 2007 et 2017 (+4,7 points), notamment celles à caractère social (pour la santé et en faveur des personnes âgées, par exemple), mais il lui est encore possible d'améliorer leur efficience en évitant les doubles emplois et les déperditions dans les programmes d'aide sociale (OCDE 2019a).

Dans les pays de l'OCDE, le montant moyen des dépenses publiques par habitant était en 2017 de 18441 USD à PPA contre 13852 USD à PPA en 2007. C'est au Luxembourg que les dépenses publiques par habitant étaient le plus élevées en 2017 (46 208 USD à PPA), ayant progressé de 16454 USD à PPA entre 2007 et 2018. Le chiffre relativement élevé enregistré au Luxembourg s'explique en partie par la forte proportion de travailleurs transfrontaliers, non comptabilisés comme résidents - le dénominateur du ratio (la population) est donc moindre que s'ils l'étaient - il résulte aussi des mesures anticycliques prises pour lutter contre la crise. Au Luxembourg, les dépenses publiques devraient continuer d'augmenter au cours des prochaines années à cause de la hausse substantielle des coûts liés au vieillissement de la population (OCDE 2019b).

Entre 2007 et 2017, le taux annuel moyen de croissance des dépenses publiques réelles par habitant s'est établi à $1 \%$ en termes réels dans les pays de l'OCDE. Pendant cette période, la Grèce et l'Italie ont été les seuls pays où les dépenses publiques par habitant ont diminué de respectivement $2,6 \%$ et $0,4 \%$. Pourtant, elles ont progressé dans ces deux pays en 2017-2018 (respectivement de $0,9 \%$ et $0,4 \%$ ). La plus forte croissance annuelle moyenne des dépenses publiques par habitant entre
2007 et 2017 a été relevée en Corée (+3,5\%). Alors que les dépenses publiques totales y restent faibles, cette croissance est due au déploiement d'un effort durable sur le plan social au cours de la décennie écoulée, illustrant une volonté d'améliorer le bien-être dans ce pays.

\section{Méthodologie et définitions}

Les données relatives aux dépenses publiques sont tirées de la base de données des Statistiques de l'OCDE sur les comptes nationaux, qui sont établies conformément au Système de comptabilité nationale (SCN) ; ce dernier constitue un ensemble de concepts, de définitions, de nomenclatures et de règles approuvés au plan international en matière de comptabilité nationale. Le cadre du SCN 2008 est désormais en vigueur dans tous les pays de l'OCDE (voir l'Annexe A pour plus de précisions). Selon la terminologie du SCN, les administrations publiques se composent de l'administration centrale, des administrations des États fédérés, des administrations locales et des administrations de sécurité sociale. Les dépenses comprennent la consommation intermédiaire, la rémunération des personnels, les subventions, les revenus de la propriété versés (dont les paiements d'intérêts), les prestations sociales, les autres dépenses courantes (essentiellement les transferts courants) et les dépenses en capital (transferts en capital et investissements). Le produit intérieur brut (PIB) est la mesure habituelle de la valeur des biens et services produits par un pays au cours d'une période donnée. On a calculé les dépenses publiques par habitant en convertissant les dépenses publiques totales en USD aux parités de pouvoir d'achat (PPA) OCDE/Eurostat pour le PIB et en divisant le résultat obtenu par la population du pays. La PPA correspond au nombre d'unités monétaires du pays B nécessaire à l'acquisition d'une même quantité de biens et de services dans le pays A.

\section{Pour en savoir plus}

OCDE (2019a), OECD Economic Surveys: Mexico 2019, Éditions OCDE.

OCDE (2019b), Études économiques de l>OCDE : Luxembourg 2019, Éditions OCDE, Paris, https://doi.org/10.1787/2a4a718c-fr.

OCDE (2018), OECD Economic Surveys: Ireland 2018, Éditions OCDE, Paris. http://doi.org/10.1787/eco_surveys-irl-2018-en.

\section{Notes relatives aux graphiques}

Concernantles données sur Israël, voir http://doi.org/10.1787/888932315602.

2.27 et 2.28. Les données sur le Chili ne sont pas disponibles. Celles sur la Turquie ne sont pas prises en compte dans la moyenne de la zone OCDE car il manque des séries chronologiques. Les données sur le Brésil et l'Indonésie portent sur 2016 au lieu de 2017. Celles sur la Russie portent sur 2015 au lieu de 2017.

2.29. Les données sur le Chili et la Turquie ne sont pas disponibles. Celles sur le Brésil portent sur 2007-2016 au lieu de 2007-2017. 


\subsection{Dépenses des administrations publiques en pourcentage du PIB, 2007, 2017 et 2018}

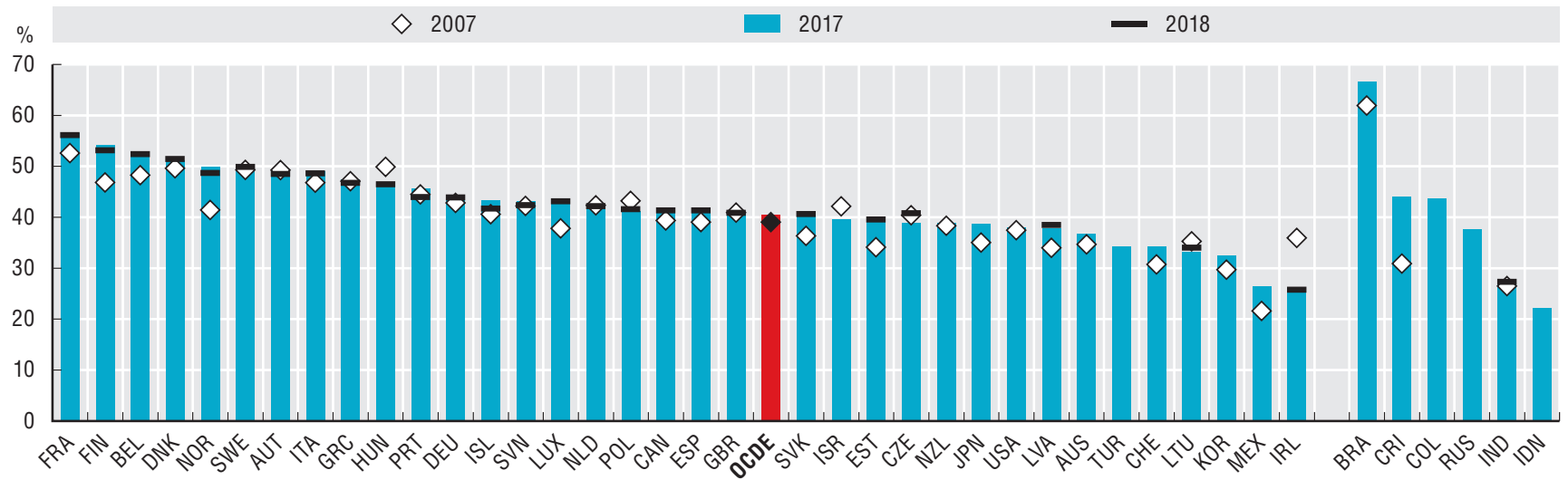

Sources: Statistiques de l'OCDE sur les comptes nationaux (base de données). Les données sur l'Inde sont tirées des Perspectives de l'économie mondiale du FMI (avril 2019).

\subsection{Dépenses des administrations publiques par habitant, 2007, 2017 et 2018}

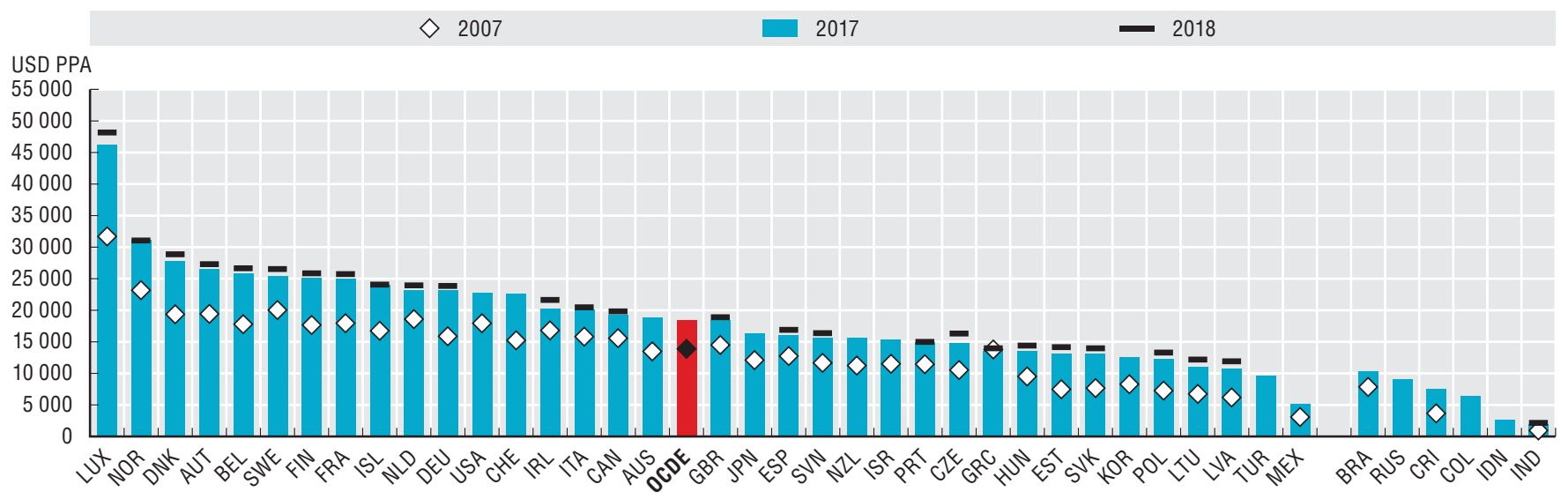

Sources : Statistiques de l'OCDE sur les comptes nationaux (base de données). Les données sur l'Inde sont tirées des Perspectives de l'économie mondiale du FMI (avril 2019).

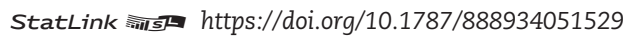

2.29. Taux annuel moyen de croissance des dépenses publiques par habitant en termes réels, 2007-2017 et 2017-2018

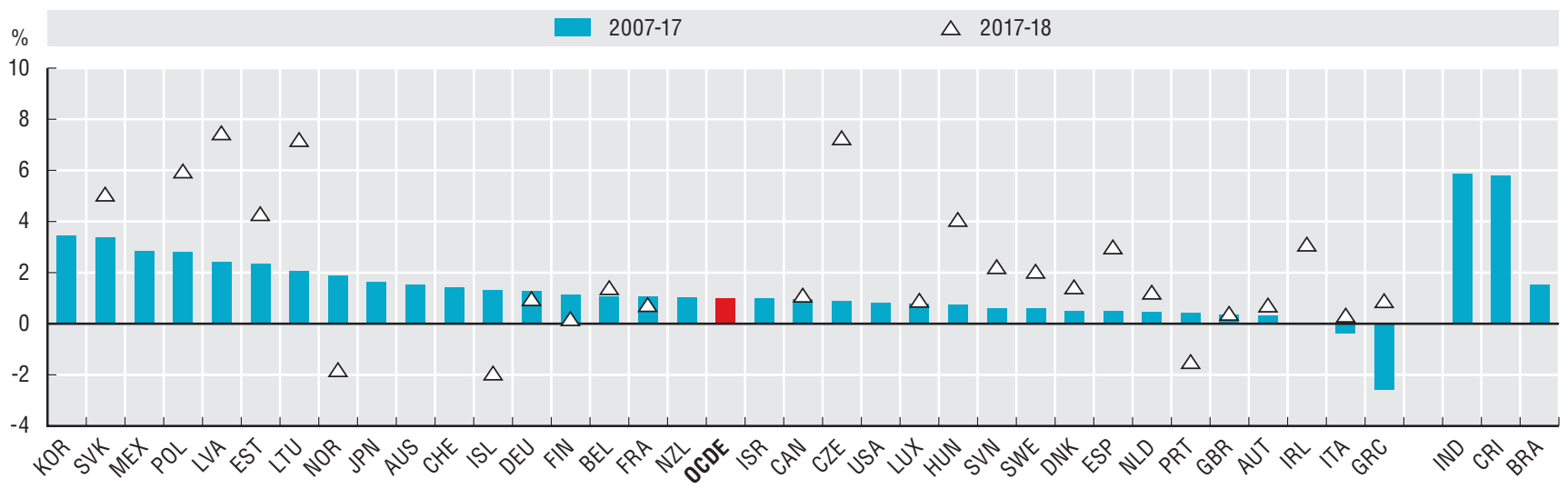

Sources: Statistiques de l'OCDE sur les comptes nationaux (base de données). Les données sur l'Inde sont tirées des Perspectives de l'économie mondiale du FMI (avril 2019). 
Les dépenses des administrations publiques ont des finalités très diverses, telles que la prestation de services de santé, d'éducation et de justice à l'intention de la population ainsi que le maintien de l'ordre et de la sûreté publics. Leur ventilation par fonction permet de mettre en exergue les priorités des pouvoirs publics et les défis auxquels ils sont confrontés, mais aussi de suivre l'évolution dans le temps de ces priorités et défis. Sa modification peut résulter de choix stratégiques ou de tendances socioéconomiques, comme les changements démographiques (par exemple le vieillissement de la population), du cycle économique et de l'application d'accords internationaux.

En 2017, dans les pays de l'OCDE, l'essentiel des ressources publiques était affecté à la protection sociale -13,3\% du produit intérieur brut (PIB) -, qui recouvre, par exemple, les pensions de vieillesse, d'invalidité et de maladie, les aides au logement et les allocations de chômage. Les pays qui dépensent le plus, en proportion de leur PIB, pour la protection sociale sont la Finlande $(24,9 \%)$, la France $(24,3 \%)$ et le Danemark $(22,4 \%)$, alors que ceux qui dépensent le moins sont le Chili, (5,2\%), la Corée $(5,6 \%)$ et les États-Unis (7,6\%).

Le second poste de dépenses le plus important est la santé (7,8 \% du PIB dans les pays de l'OCDE en 2017) ; il englobe entre autres les produits, les appareils et les équipements médicaux, ainsi que les services hospitaliers et ambulatoires. Les États-Unis (9,3\%), la Norvège (8,5 \%) et le Danemark $(8,4 \%)$ consacrent la plus grande partie de leurs dépenses de santé en proportion du PIB. Bien que seulement un tiers environ de la population des États-Unis soit couvert par le système public d'assurance maladie, les coûts et les frais administratifs par habitant dépassent les chiffres constatés dans les autres pays. Les pays qui dépensent le moins en matière de santé sont la Suisse (2,2 \%), la Lettonie (3,5\%) et le Chili (4,0\%). En Suisse, le système de santé repose surtout sur des régimes d'assurance obligatoires privés.

En 2017, les services publics généraux (par exemple la gestion de la dette publique, le fonctionnement des organes exécutifs et législatifs centraux, les transferts entre niveaux d'administration) représentaient 5,4 \% du PIB dans les pays de l'OCDE. Les pays affectant le plus de ressources à cette fonction étaient la Grèce (8,3\%), l'Italie $(8,2 \%)$ et l'Islande $(8,1 \%)$, où les opérations sur la dette forment, dans les trois cas, la composante la plus importante de cette sous-catégorie. En moyenne, les services publics généraux absorbaient un peu plus de ressources que l'éducation (5,1\%).

Le changement climatique est une préoccupation croissante dans les pays de l'OCDE, comme le prouve leur adhésion à des accords internationaux tels que l'Accord de Paris sur le climat. Néanmoins, peu de ressources sont consacrées à la lutte contre le changement climatique. La protection de l'environnement comprend notamment la gestion des déchets, l'atténuation de la pollution et la préservation de la biodiversité et des paysages. En 2017, c'était la fonction la moins bien dotée (0,5\% du PIB au total). Aux Pays-Bas (1,4\%), en Grèce (1,3\%) et au Japon $(1,2 \%)$, ces dépenses étaient plus de deux fois supérieures à la moyenne de la zone OCDE mais au Chili, aux États-Unis et en Finlande elles restaient négligeables en proportion du PIB.

Les dépenses en faveur de la protection sociale ont progressé de 1,5 point de PIB depuis 2007, principalement en raison du vieillissement des populations et de la hausse des allocations de chômage. En Finlande et en Norvège, leur augmentation (respectivement $+5,7$ et $+4,6$ points) a été plus de trois fois supérieure à la moyenne de la zone OCDE. À l'inverse, la Hongrie et l'Irlande ont réduit ces dépenses de respectivement 3,2 et 3,6 points. L'Irlande a revu à la baisse les allocations de chômage et les allocations familiales en réaction à la crise économique et financière de 2007-2008. La Hongrie a raccourci la durée d'indemnisation du chômage et encore durci l'accès aux pensions d'invalidité. En moyenne, les dépenses de santé ont aussi pris plus de place : en 2017, 1,1 point de PIB supplémentaire y était consacré. C'est en Norvège et aux ÉtatsUnis que la progression des dépenses de santé a été la plus forte (+1,6 point), devant le Japon (+1,5 point). À l'opposé, c'est en Irlande et au Portugal que ces dépenses ont le plus baissé $(-1,1$ point $)$.

\section{Méthodologie et définitions}

Les données relatives aux dépenses publiques sont tirées de la base de données des Statistiques de l'OCDE sur les comptes nationaux et de la base de données d'Eurostat sur les Statistiques relatives aux finances publiques, qui sont établies conformément au Système de comptabilité nationale (SCN) ; ce dernier constitue un ensemble de concepts, de définitions, de nomenclatures et de règles approuvés au plan international en matière de comptabilité nationale. Le cadre du SCN 2008 est désormais en vigueur dans tous les pays de l'OCDE (voir l'Annexe A). Les données sur les dépenses publiques par fonction sont ventilées selon la Classification des fonctions des administrations publiques (CFAP), en vertu de laquelle les dépenses sont réparties suivant 10 fonctions (niveau 1) : les services généraux des administrations publiques ; la défense ; l'ordre et la sécurité publics ; les affaires économiques ; la protection de l'environnement ; le logement et les équipements collectifs ; la santé ; les loisirs, la culture et le culte ; l'éducation; la protection sociale. On trouvera à l'Annexe $C$ des précisions sur les catégories de dépenses. D'autres informations sur la ventilation des dépenses des administrations sont disponibles en ligne (Annexe $F$ (2.32 à 2.40)) par fonction de niveau 1 de la CFAP (différents niveaux d'administration) et par certaines fonctions prioritaires de niveau 2 de la CFAP (hors indicateur sur les dépenses en matière de protection sociale et de santé).

\section{Notes relatives aux graphiques}

Les données sur le Canada, le Mexique, la Nouvelle-Zélande et la Turquie ne sont pas disponibles. Concernant les données sur Israël, voir http://doi.org/10.1787/888932315602. Les données sur la Corée portent sur 2016.

2.30. Les données sur le Chili et l'Islande ne sont pas prises en compte dans la moyenne de la zone OCDE car il manque des séries chronologiques. Les données sur le Chili portent sur 2016 et non 2017.

2.31. Les données sur le Chili et l'Islande ne sont pas disponibles. 
2.30. Dépenses des administrations publiques par fonction, en pourcentage du PIB, 2017

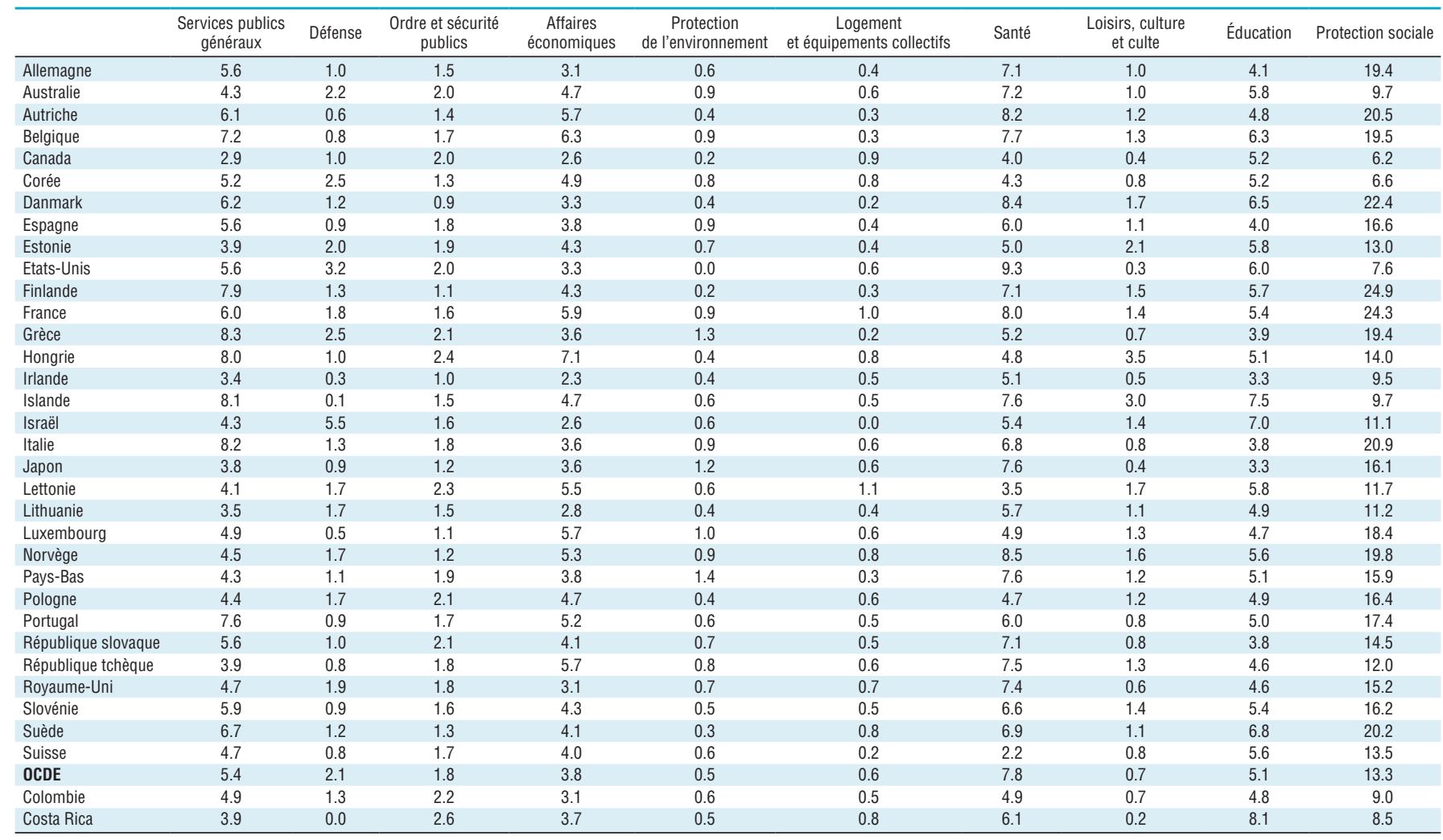

Sources : Statistiques de l'OCDE sur les comptes nationaux (base de données) ; statistiques d'Eurostat relatives aux finances publiques (base de données). Pour l'Australie, les données sont tirées des statistiques sur les finances publiques fournies par le Bureau australien des statistiques.

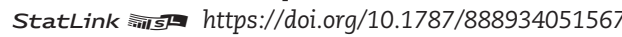

2.31. Évolution des dépenses des administrations publiques par fonction, en pourcentage du PIB, de 2007 à 2017

\begin{tabular}{|c|c|c|c|c|c|c|c|c|c|c|}
\hline & $\begin{array}{l}\text { Services publics } \\
\text { généraux }\end{array}$ & Défense & $\begin{array}{c}\text { Ordre et sécurité } \\
\text { publics }\end{array}$ & $\begin{array}{c}\text { Affaires } \\
\text { économiques }\end{array}$ & $\begin{array}{c}\text { Protection } \\
\text { de l'environnement }\end{array}$ & $\begin{array}{c}\text { Logement } \\
\text { et équipements collectifs }\end{array}$ & Santé & $\begin{array}{l}\text { Loisirs, culture } \\
\text { et culte }\end{array}$ & Éducation & Protection sociale \\
\hline Allemagne & -0.5 & 0.1 & 0.1 & -0.1 & 0.1 & -0.4 & 0.8 & 0.2 & 0.2 & 0.6 \\
\hline Australie & 0.8 & 0.9 & 0.3 & 0.8 & 0.2 & -0.2 & 1.0 & 0.2 & 1.1 & 0.5 \\
\hline Autriche & -1.5 & -0.2 & 0.1 & -0.2 & -0.1 & -0.1 & 0.8 & -0.2 & 0.2 & 1.1 \\
\hline Belgique & -1.5 & -0.2 & 0.0 & 0.8 & 0.2 & -0.1 & 1.0 & 0.0 & 0.8 & 2.8 \\
\hline Corée & 0.0 & 0.1 & 0.1 & -1.1 & -0.1 & -0.3 & 1.1 & 0.1 & 0.6 & 2.1 \\
\hline Danmark & -0.5 & -0.2 & 0.0 & 0.3 & -0.1 & -0.1 & 0.7 & 0.0 & 0.5 & 0.9 \\
\hline Espagne & 0.6 & -0.1 & 0.0 & -1.4 & -0.1 & -0.5 & 0.3 & -0.5 & 0.0 & 3.7 \\
\hline Estonie & 0.6 & 0.8 & -0.2 & 0.0 & -0.1 & -0.3 & 0.7 & 0.1 & -0.1 & 3.8 \\
\hline Etats-Unis & -0.5 & -0.8 & -0.1 & -0.3 & 0.0 & 0.0 & 1.6 & 0.0 & -0.2 & 0.9 \\
\hline Finlande & 1.2 & -0.1 & 0.0 & -0.1 & -0.1 & 0.0 & 0.5 & 0.4 & -0.1 & 5.7 \\
\hline France & -1.3 & 0.1 & 0.2 & 1.6 & 0.1 & -0.1 & 0.6 & 0.0 & 0.1 & 2.7 \\
\hline Grèce & -3.3 & -0.3 & 0.6 & -0.6 & 0.5 & 0.0 & -0.8 & 0.1 & 0.3 & 3.7 \\
\hline Hongrie & -1.5 & -0.3 & 0.4 & 0.6 & -0.2 & -0.2 & -0.1 & 2.0 & -0.5 & -3.2 \\
\hline Irlande & 0.0 & -0.1 & -0.5 & -1.3 & -0.6 & -1.2 & -1.1 & -0.1 & -1.1 & -3.6 \\
\hline Israël & -3.3 & -1.5 & 0.1 & 0.1 & 0.0 & -0.3 & 0.5 & 0.1 & 0.7 & 0.6 \\
\hline Italie & -0.4 & 0.1 & 0.0 & -0.6 & 0.1 & -0.1 & 0.1 & 0.0 & -0.7 & 3.3 \\
\hline Japon & -0.5 & 0.1 & 0.0 & 0.2 & 0.0 & -0.1 & 1.5 & 0.0 & -0.1 & 2.8 \\
\hline Lettonie & 0.2 & 0.2 & -0.1 & 0.6 & -0.3 & -0.1 & -0.5 & 0.0 & 0.2 & 3.7 \\
\hline Lithuanie & -0.6 & -0.2 & -0.2 & -1.4 & -0.5 & 0.1 & 0.4 & 0.0 & -0.4 & 0.5 \\
\hline Luxembourg & 0.3 & 0.2 & 0.2 & 1.1 & -0.1 & 0.0 & 0.6 & 0.0 & 0.2 & 2.7 \\
\hline Norvège & -1.3 & 0.1 & 0.3 & 1.6 & 0.3 & 0.2 & 1.6 & 0.4 & 0.7 & 4.6 \\
\hline Pays-Bas & -1.3 & -0.1 & 0.0 & -0.5 & -0.1 & -0.1 & 0.8 & -0.2 & 0.1 & 1.5 \\
\hline Pologne & -1.0 & -0.2 & -0.1 & -0.3 & -0.2 & -0.5 & 0.2 & 0.0 & -0.8 & 0.8 \\
\hline Portugal & 0.6 & -0.3 & -0.1 & 1.1 & 0.0 & -0.3 & -1.1 & -0.2 & -1.2 & 2.7 \\
\hline République slovaque & 1.1 & 0.2 & 0.3 & -0.3 & 0.0 & -0.1 & 1.0 & 0.0 & 0.3 & 1.3 \\
\hline République tchèque & -0.4 & -0.3 & -0.2 & -0.7 & -0.2 & -0.4 & 0.7 & -0.1 & -0.1 & 0.1 \\
\hline Royaume-Uni & 0.5 & -0.2 & -0.5 & 0.2 & -0.2 & -0.4 & 1.0 & -0.3 & -1.0 & 0.9 \\
\hline Slovénie & 0.4 & -0.5 & -0.1 & 0.2 & -0.3 & -0.1 & 0.7 & 0.2 & -0.5 & 0.9 \\
\hline Suède & -0.9 & -0.4 & 0.0 & 0.2 & 0.0 & 0.1 & 0.5 & 0.1 & 0.5 & 0.0 \\
\hline Suisse & 0.1 & 0.0 & 0.2 & 0.3 & 0.0 & 0.1 & 0.5 & 0.0 & 0.7 & 1.5 \\
\hline OCDE & -0.5 & -0.3 & 0.0 & -0.1 & 0.0 & -0.2 & 1.1 & 0.0 & -0.1 & 1.5 \\
\hline
\end{tabular}

Sources : Statistiques de l'OCDE sur les comptes nationaux (base de données) ; statistiques d'Eurostat relatives aux finances publiques (base de données). Pour l'Australie, les données sont tirées des statistiques sur les finances publiques fournies par le Bureau australien des statistiques. 
Les administrations publiques utilisent leurs ressources de différentes manières : en effectuant des transferts (subventions et prestations sociales, par exemple), en achetant des biens et des services (comme des vaccins pour les hôpitaux), en rémunérant leurs agents, en réalisant des investissements, etc. Ces dépenses leur permettent d'assurer leurs principales missions, à savoir la fourniture de biens et de services à la population et, en fonction du contrat social, la redistribution des revenus. La ventilation des dépenses publiques par opération économique apporte une indication sur la composition de ces dépenses et sur la marge de manœuvre dont disposent les administrations pour modifier l'affectation des fonds publics.

En 2017, les administrations publiques des pays de l'OCDE ont consacré $40,9 \%$ de leurs dépenses au versement de prestations sociales (pensions et autres), soit une hausse de 4,2 points par rapport à 2007. La rémunération des agents publics était la seconde opération économique par ordre d'importance (22,8 \% des dépenses publiques), en baisse de 1,3 point par rapport à 2007 .

Le Japon a dépensé le plus en prestations sociales (54,9\%) et le Mexique le moins (9,7\%). Ces différences sont imputables en grande partie à la diversité des situations démographiques : les pensions versées aux personnes âgées constituent la charge la plus importante au Japon, alors qu'au Mexique, la société reste relativement jeune et le système de protection sociale relativement peu développé. C'est en Hongrie que la part des prestations sociales dans les dépenses publiques a baissé le plus fortement (-6,3 points depuis 2007), cette évolution étant due à une réduction des allocations de chômage et à un durcissement des conditions d'obtention des pensions d'invalidité.

Le Japon, l'Allemagne et les Pays-Bas, soit les trois pays ayant dépensé le plus en prestations sociales, sont ceux où la part de la rémunération des agents publics dans les dépenses est le plus faible (respectivement $14 \%, 17,1 \%$ et $19,6 \%$ ), du fait que l'emploi public y est relativement limité (pour plus d'informations, voir la double page sur l'emploi dans le secteur public). En revanche, l'Islande et le Mexique ont consacré la plus forte proportion de leurs dépenses à la rémunération des agents publics (respectivement $32,4 \%$ et $32 \%$ ), mais pas en raison de la taille de l'emploi public. En Islande, les prestations sociales sont plutôt faibles, car seules les pensions de base sont financées par l'impôt, l'essentiel du système étant géré par des caisses privées, d'où le poids relativement plus élevé des dépenses destinées à la rémunération des agents publics. En outre, en Islande, comme dans les autres pays nordiques, l'emploi dans le secteur public est relativement développé. Au Mexique, le secteur public est assez réduit, ce qui met en évidence le montant élevé des rémunérations, en particulier celle des hauts fonctionnaires. C'est en République tchèque et en République slovaque que la part des dépenses affectées à la rémunération des agents publics a le plus augmenté depuis 2007 (+2,9 points). En République slovaque, malgré des hausses modérées, ces rémunérations restent les plus faibles des pays de l'OCDE. Cette catégorie de dépenses a diminué le plus sensiblement au Portugal (-5,5 points), en raison de la réduction des rémunérations et des effectifs dans le secteur public intervenue de 2010 à 2015 en réaction à la crise économique de 2007-2008.

En 2017, les administrations publiques des pays de l'OCDE ont consacré 9,5\% de leurs dépenses à l'investissement, soit une baisse de 1,5 point par rapport à 2007. Toutefois, il y a eu des variations au cours de cette période car, en général, l'investissement a nettement augmenté pendant la mise en œuvre des mesures contrecycliques adoptées après la crise. En 2017, parmi les pays de l'OCDE, c'est la Corée qui a dépensé le plus dans ce domaine (18,8\%), malgré une diminution de 4,8 points par rapport à 2007 .

\section{Méthodologie et définitions}

Les données relatives aux dépenses publiques sont tirées de la base de données des Statistiques de l'OCDE sur les comptes nationaux, qui sont établies conformément au Système de comptabilité nationale (SCN) ; ce dernier constitue un ensemble de concepts, de définitions, de nomenclatures et de règles approuvés au plan international en matière de comptabilité nationale. Le cadre du SCN 2008 est désormais en vigueur dans tous les pays de l'OCDE (voir l'Annexe A pour plus de précisions sur les systèmes de déclaration et les sources). Les dépenses comprennent les opérations économiques suivantes : la consommation intermédiaire (c'est-à-dire les biens et les services consommés au cours du processus de production dans le territoire économique et pendant la période de comptabilisation) ; la rémunération des personnels ; les subventions ; les revenus de la propriété versés (essentiellement constitués de paiements d'intérêts) ; les prestations sociales (autres que les transferts en nature) et les transferts sociaux en nature aux ménages assurés par l'intermédiaire de producteurs marchands; les autres dépenses courantes (essentiellement les transferts courants, mais aussi d'autres dépenses mineures telles que les autres impôts sur la production, les impôts sur le revenu et le patrimoine, etc., ainsi que l'ajustement pour variation des droits à pension) ; les dépenses en capital (transferts en capital et investissements). Toutes ces opérations à l'échelle de l'ensemble des administrations publiques sont comptabilisées sur une base consolidée (on procède à une compensation des opérations entre les différents niveaux d'administration).

\section{Pour en savoir plus}

OCDE (2019), OECD Economic Surveys: Hungary 2019, Éditions OCDE, Paris, https://doi.org/10.1787/eco_surveys-hun-2019-en.

OCDE (2018), OECD Economic Surveys: Korea 2018, Éditions OCDE, Paris http://doi.org/10.1787/eco_surveys-kor-2018-en.

\section{Notes relatives aux graphiques}

Les données sur le Chili ne sont pas disponibles. Concernant les donn. es sur Isra.l, voir http://doi.org/10.1787/888932315602. Celles sur la Turquie ne sont pas prises en compte dans la moyenne de la zone OCDE car il manque des séries chronologiques.

Les données sur le Brésil et l’Indonésie portent sur 2016 et celles sur la Russie portent sur 2015 au lieu de 2017.

2.42. (Ventilation des dépenses de l'administration centrale, par opération économique, 2017 et 2018), est consultable en ligne (voir l'Annexe F). 
2.41. Ventilation des dépenses des administrations publiques par opération économique, 2017 et 2018 et évolution de 2007 à 2017

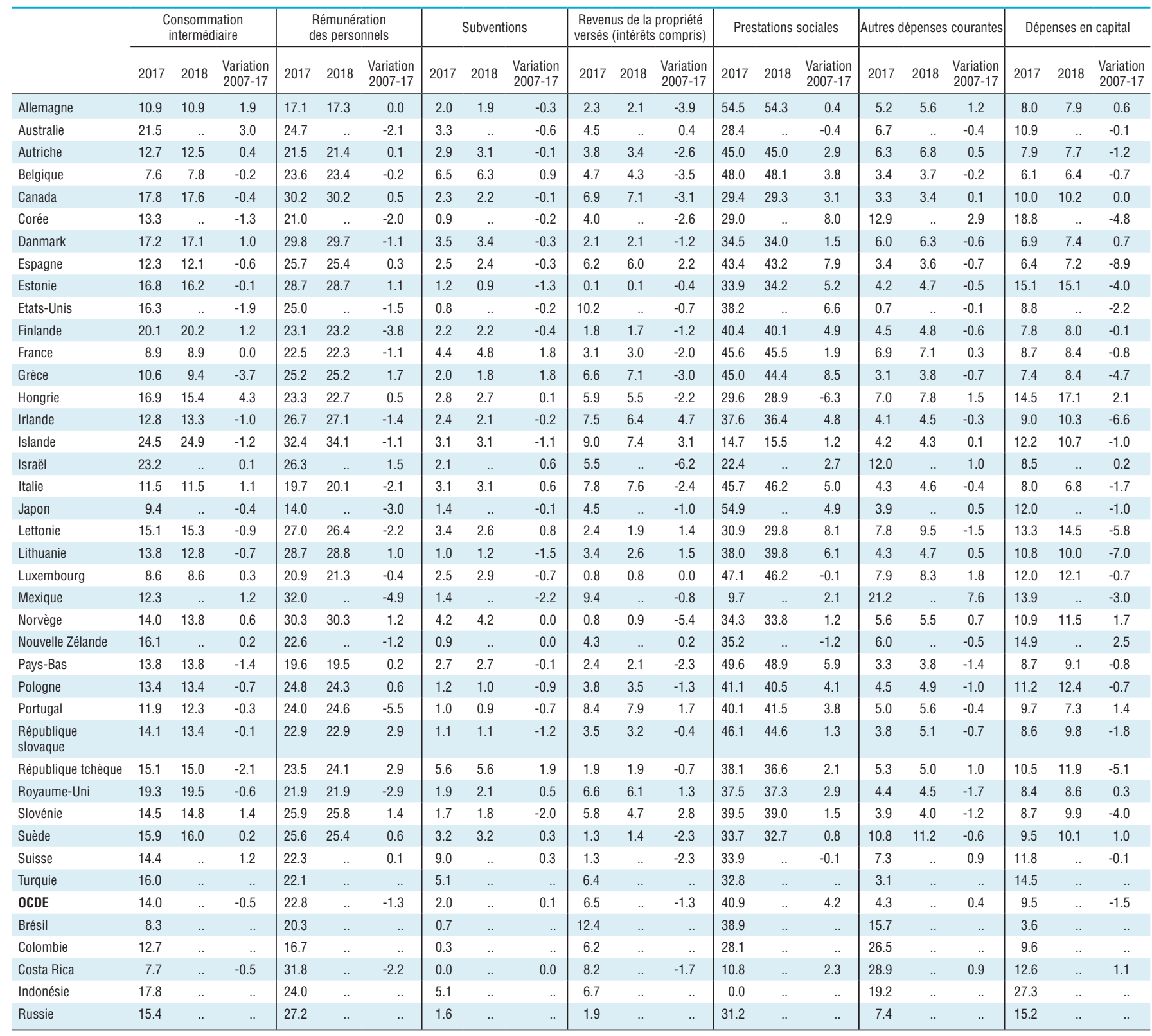

Sources: Statistiques de l'OCDE sur les comptes nationaux (base de données). Pour l'Australie, les données sont tirées à la fois des statistiques sur les comptes nationaux et des statistiques sur les finances publiques fournies par le Bureau australien des statistiques. 
Les administrations publiques sont composées de différents niveaux (administrations centrales, des États fédérés et locales), dont la capacité à percevoir des recettes varie. Celle des administrations infranationales dépend du degré de fédéralisme budgétaire. Dans les pays où les administrations infranationales ont des possibilités limitées en matière de prélèvement d'impôts, les transferts de l'administration centrale constituent leur principale source de recettes.

Dans une récente étude, l'OCDE a constaté que la décentralisation des dépenses et des recettes publiques tendait à dynamiser la croissance économique lorsque cette décentralisation était aussi poussée pour les dépenses que pour les recettes et lorsque l'intégration de l'économie aux marchés internationaux était relativement forte. Même si la décentralisation favorise l'efficience, ses effets sur l'équité diffèrent selon les pays (Kim et Dougherty 2018).

Il existe des différences entre les pays de l'OCDE en termes de décentralisation des recettes. En moyenne, c'est à l'administration centrale que revient la plus forte proportion de recettes $(52,9 \%)$. L'administration centrale encaisse plus de $80 \%$ des recettes publiques en Irlande (95\%), au Royaume-Uni (91,6\%), en Nouvelle-Zélande $(89,2 \%)$, en Norvège $(83,8 \%)$ et en Estonie (81,3\%). En Allemagne, pays fédéral, cette proportion tombe à $28 \%$ et ce sont les cotisations sociales qui constituent la plus grande part des recettes. En France, la sécurité sociale représente $47,2 \%$ des recettes publiques (proportion la plus forte des pays de l'OCDE) et l'administration centrale 36,9 \%. Même si en moyenne les collectivités locales encaissent la plus faible part des recettes publiques dans les pays de l'OCDE (10,5\%), elles en représentent environ un tiers en Corée (33,9\%) et en Suède (33,3\%).

On s'attend à ce que les administrations infranationales aient une plus grande autonomie budgétaire dans les pays fédéraux. Or, le niveau de recettes varie selon les niveaux. Au Canada, les recettes des provinces sont supérieures à celles de l'administration centrale, en proportion du total des recettes publiques (44\% contre 35,6\%). Bien que le gouvernement fédéral canadien lève tous les impôts, les provinces ont la possibilité de créer les leurs. En Australie et aux États-Unis, les États fédérés perçoivent à peu près $40 \%$ des recettes. En Autriche, en revanche, ils n'en encaissent que 3,9\% et l'échelon local 6,3 \%.

Sur le plan des dépenses, il y a aussi de grandes différences entre les niveaux d'administration. L'administration centrale est en moyenne à l'origine de $41 \%$ des dépenses publiques, dans les pays de l'OCDE. Comme suite à l'augmentation des recettes, c'est en Irlande, en Nouvelle-Zélande et au Royaume-Uni que l'administration centrale dépense le plus (respectivement 91,5\%, $88,3 \%$ et $76,7 \%$ ). La sécurité sociale pèse le plus lourd dans les dépenses publiques au Japon (49,7\%), en France $(45,1 \%)$ et en Allemagne $(43,4 \%)$. Au Danemark, bien que l'administration centrale recouvre la part la plus importante des recettes, les collectivités locales réalisent $64 \%$ des dépenses. La situation est similaire en Suède (50,5 \% de dépenses locales) et en Finlande $(39,6 \%)$. Au Canada et en Suisse, ce sont respectivement les échelons de la province et du canton qui dépensent le plus (47,1\% dans le premier cas et $37 \%$ dans le second).

\section{Méthodologie et définitions}

Les données relatives aux recettes et aux dépenses publiques sont tirées de la base de données des Statistiques de l'OCDE sur les comptes nationaux qui sont établies conformément au Système de comptabilité nationale (SCN) ; ce dernier constitue un ensemble de standards en matière de comptabilité nationale. Le cadre du SCN 2008 est désormais en vigueur dans tous les pays de l'OCDE (voir l'Annexe A pour plus de précisions). Selon la terminologie du SCN, les administrations publiques se composent des administrations centrale, des États fédérés, locales et de sécurité sociale. Les administrations des États fédérés n'existent que dans les 9 pays membres de l'OCDE dotés d'une structure fédérale : l'Allemagne, l'Australie, l'Autriche, la Belgique, le Canada, l'Espagne (pays considéré comme quasi fédéral), les États-Unis, le Mexique et la Suisse. Les données ne tiennent pas compte des transferts entre niveaux d'administration, sauf pour l'Australie, la Corée, le Costa Rica, l'Indonésie et la Turquie, l'objectif étant d'appréhender la contribution de chaque sous-secteur aux recettes et dépenses totales des administrations publiques, qui sont consolidées à ce niveau.

Les recettes publiques comprennent les impôts (sur la consommation, le revenu et le patrimoine, par exemple), les cotisations sociales nettes (au titre de la retraite, de l'assurance maladie et de la sécurité sociale), le produit des ventes de biens et services (par exemple la production marchande des établissements publics) et les dons et autres sources (dons courants et en capital, revenus du patrimoine et subventions, etc.). Ces agrégats ont été construits à partir de sous-comptes budgétaires (voir l'Annexe B). Les dépenses comprennent la consommation intermédiaire, la rémunération des agents, les subventions, les revenus de la propriété versés (essentiellement constitués de paiements d'intérêts), les prestations sociales, les autres dépenses courantes (principalement les transferts courants) et les dépenses en capital (transferts en capital et investissements).

\section{Pour en savoir plus}

Kim, J. et Dougherty, S. (dir.) (2018), Fiscal Decentralisation and Inclusive Growth, OECD Fiscal Federalism Studies, Éditions OCDE, Paris/KIPF, Séoul, https://doi.org/10.1787/ 9789264302488-en.

\section{Notes relatives aux graphiques}

En Australie et aux États-Unis, les administrations locales sont englobées dans celles des États fédérés.

L'Australie n'a pas de régime public d'assurance sociale. Aux États-Unis, en Irlande, en Nouvelle-Zélande, en Norvège et au Royaume-Uni, les administrations de sécurité sociale font partie de l'administration centrale.

Les données sur le Chili ne sont pas disponibles. Concernant les données sur Israël, voir http://doi.org/10.1787/888932315602. S'agissant du Japon, les données sur les sous-secteurs des administrations publiques portent sur l'exercice budgétaire. Celles sur la Turquie ne sont pas prises en compte dans la moyenne de l'OCDE car il manque des séries chronologiques.

Les données sur l'Indonésie portent sur 2016 au lieu de 2017.

2.45 et 2.46. (Modifications de la collecte des recettes et des dépenses par niveau de gouvernement de 2007 à 2017) sont consultables en ligne à l'Annexe $\mathrm{F}$. 
2.43. Ventilation des recettes des administrations publiques entre les différents niveaux d'administration, 2017 et 2018

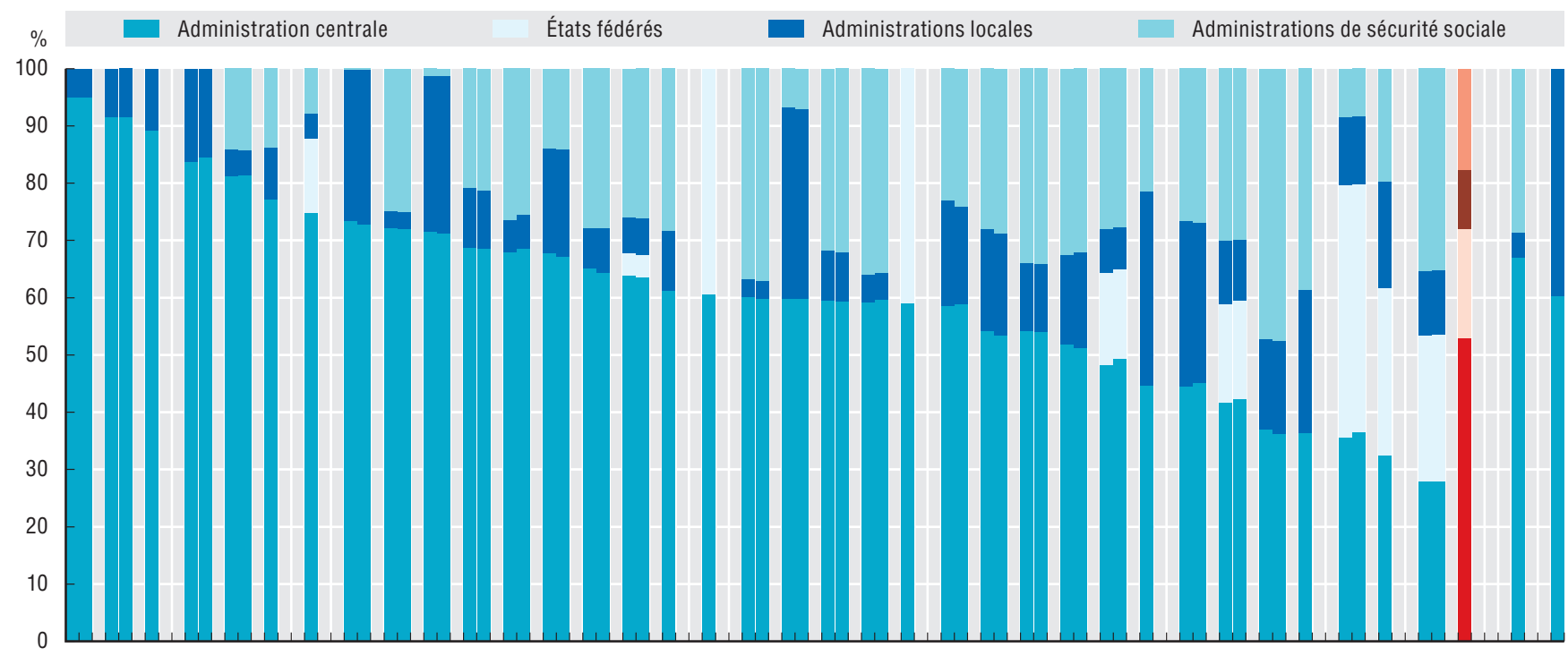

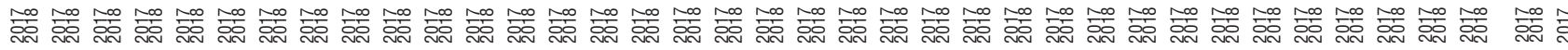

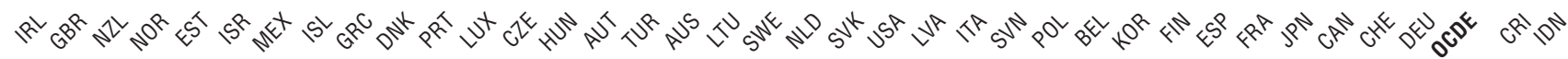

Source : Statistiques de l'OCDE sur les comptes nationaux (base de données).

StatLink 年ISt https://doi.org/10.1787/888934051624

2.44. Ventilation des dépenses des administrations publiques entre les différents niveaux d'administration, 2017 et 2018

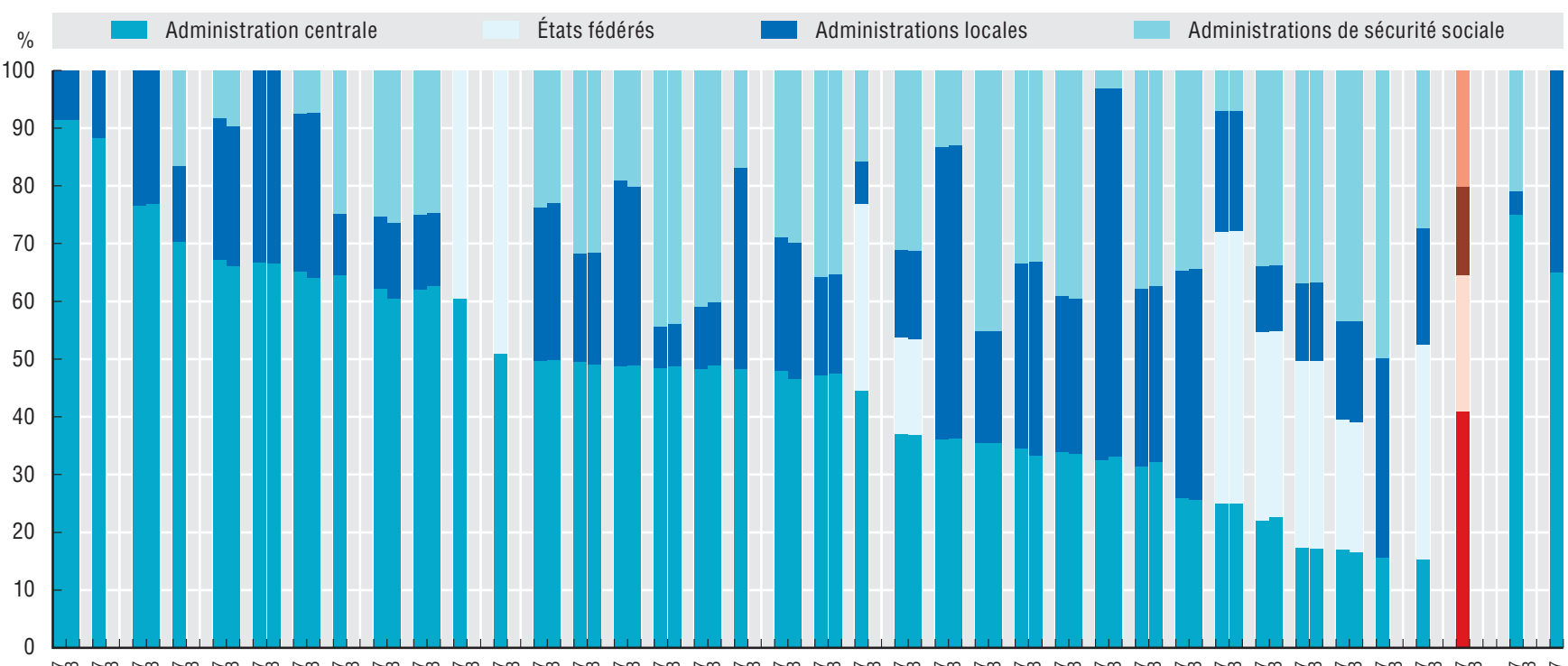

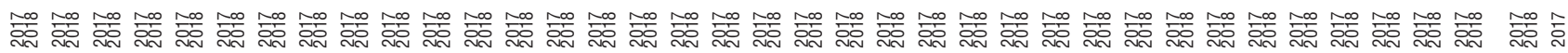

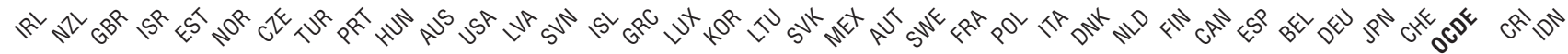

Source : Statistiques de l'OCDE sur les comptes nationaux (base de données). 
L'investissement public peut améliorer la productivité et promouvoir la croissance économique, tout en favorisant le bien-être de la société. Il porte en général sur les infrastructures de transport, les systèmes de défense, la construction (d'écoles et d'hôpitaux, par exemple) et sur d'autres actifs corporels ou incorporels.

En 2017, les pouvoirs publics des pays de l'OCDE ont investi en moyenne $3,1 \%$ du produit intérieur brut (PIB), soit une baisse de 0,5 point par rapport à 2007. C'est en Estonie et en Norvège que la part des dépenses publiques consacrée à l'investissement, en proportion du PIB, a été le plus importante (5,3\%). En Estonie, l'investissement public stratégique est une priorité nationale comme le montrent notamment le recensement au niveau central des actifs publics qu'il est indispensable de développer, la concentration d'efforts sur le renforcement de la qualité des infrastructures publiques et la prise en compte d'objectifs verts dans les décisions d'investissement. À l'opposé, Israël est le pays qui a le moins investi $(1,4 \%)$. Afin de combler l'écart en matière d'infrastructures - stock relativement faible d'infrastructures publiques - par rapport aux autres économies développées, Israël a rendu publique une stratégie nationale à l'horizon 2030 prévoyant la réalisation de projets dans le secteur des transports, principalement, mais aussi dans ceux de l'énergie, de la désalinisation de l'eau et du traitement des déchets. Malgré la diversité des modalités envisagées pour la mise en œuvre de cette stratégie, l'investissement public devrait donc progresser.

Entre 2007 et 2017, l'investissement public a le plus augmenté en Norvège (+1,6 point), du fait d'un effort substantiel en faveur des transports qui s'est intensifié ces dernières années ; si les projets dans ce secteur sont bien choisis et réalisés, ils pourront aider le pays à se détacher progressivement des activités en rapport avec le pétrole (OCDE 2018). À l'opposé, les investissements publics ont le plus fortement diminué en Irlande (-2,9 points), en Espagne (-2,7 points) et en Grèce (-2,3 points) car ces pays, sérieusement touchés par la crise de 2007-2008, ont accompli de considérables efforts d'assainissement budgétaire, notamment en réduisant leurs investissements.

Dans les pays de l'OCDE, les investissements ont représenté $7,7 \%$ des dépenses publiques en 2017, chiffre en baisse par rapport aux 9,3\% de 2007. En 2017, ce sont la Corée (15,6\%), l'Estonie $(13,5 \%)$ et la Lettonie $(11,7 \%)$ qui ont dépensé le plus en investissements. À l'opposé, Israël, le Portugal et la Belgique leur ont consacré le montant le plus faible en proportion des dépenses publiques (respectivement $3,6 \%, 4,1 \%$ et 4,4\%). Au Portugal et en Belgique, c'est le résultat de mesures d'assainissement, alors qu'en Israël, comme on l'a indiqué plus haut, ce constat s'explique par la faiblesse structurelle de l'investissement. La ventilation des investissements par fonction varie beaucoup : en moyenne, plus d'un tiers (34,4\%) est destiné aux affaires économiques, dont les transports, puis viennent la défense $(15,4 \%)$ et l'éducation (14,1\%). Les investissements dans la protection de l'environnement ne représentent que $4 \%$ du total ; ils sont le plus importants aux Pays-Bas (12,2 \%) et au Japon (11,3\%), deux pays très exposés aux risques environnementaux et naturels.

Les dépenses d'investissement par niveau d'administration varient beaucoup et diffèrent selon que le pays est doté ou non d'une structure fédérale. Tandis qu'en 2018, l'essentiel des investissements publics était imputable aux administrations infranationales en Belgique (89,8 \%) et au Canada (87,8 \%), l'administration centrale était à l'origine de plus des troisquarts de ces investissements en Grèce et en Hongrie, contre un peu moins d'un quart pour les administrations locales.

\section{Méthodologie et définitions}

Les données sont tirées de la base de données des Statistiques de l'OCDE sur les comptes nationaux, qui sont établies conformément au Système de comptabilité nationale (SCN) ; ce dernier constitue un ensemble de concepts, de définitions, de nomenclatures et de règles approuvés au plan international en matière de comptabilité nationale. Le cadre du SCN 2008 est désormais en vigueur dans tous les pays de l'OCDE (voir l'Annexe A pour plus de précisions). L'investissement des administrations publiques correspond à la formation brute de capital et aux acquisitions, moins les cessions d'actifs non financiers non produits. La formation brute de capital fixe (appelée aussi investissement fixe) est la principale composante de l'investissement ; dans les administrations publiques, il s'agit surtout des infrastructures de transport, mais aussi des immeubles de bureaux, des logements, des établissements scolaires, des hôpitaux, etc. Dans le cadre du SCN 2008, les dépenses de recherche-développement sont aussi prises en compte dans l'investissement fixe. La somme des investissements et des transferts en capital constitue les dépenses en capital des administrations publiques. Celles-ci se composent de l'administration centrale, des administrations des États fédérés, des administrations locales et des administrations de sécurité sociale. Les administrations des États fédérés n'existent que dans les neuf pays membres de l'OCDE dotés d'une structure fédérale : l'Allemagne, l'Australie, l'Autriche, la Belgique, le Canada, l'Espagne (pays considéré comme quasi fédéral), les États-Unis, le Mexique et la Suisse.

\section{Pour en savoir plus}

OCDE (2018), OECD Economic Surveys: Norway 2018, Éditions OCDE, Paris, http://doi.org/10.1787/eco_surveys-nor-2018-en.

\section{Notes relatives aux graphiques}

2.47 et 2.48. Les données sur le Chili et la Turquie ne sont pas prises en compte dans la moyenne de la zone OCDE car il manque des séries chronologiques. Concernant les données sur Israël, voir http://doi.org/10.1787/888932315602. Les données sur le Brésil, la Chine et l'Indonésie portent sur 2016 au lieu de 2017. Les données sur la Russie portent sur 2015 au lieu de 2017

2.49. Les données sur le Chili ne sont pas disponibles. Celles sur l'Indonésie portent sur 2016 au lieu de 2017. Les données sur la Turquie ne sont pas prises en compte dans la moyenne de l'OCDE car il manque des séries chronologiques. En Australie et aux États-Unis, les administrations locales sont englobées dans les administrations des États fédérés. L'Australie n'a pas de régime public d'assurance sociale. Aux États-Unis, en Irlande, en Norvège, en Nouvelle-Zélande et au Royaume-Uni, les administrations de sécurité sociale font partie de l'administration centrale.

2.50. (Investissement public en pourcentage de l'investissement total) et 2.51. (Composition de l'investissement des administrations publiques, par fonction) sont consultables en ligne (voir l'Annexe F). 
2.47. Investissement des administrations publiques en pourcentage du PIB, 2007, 2017 et 2018

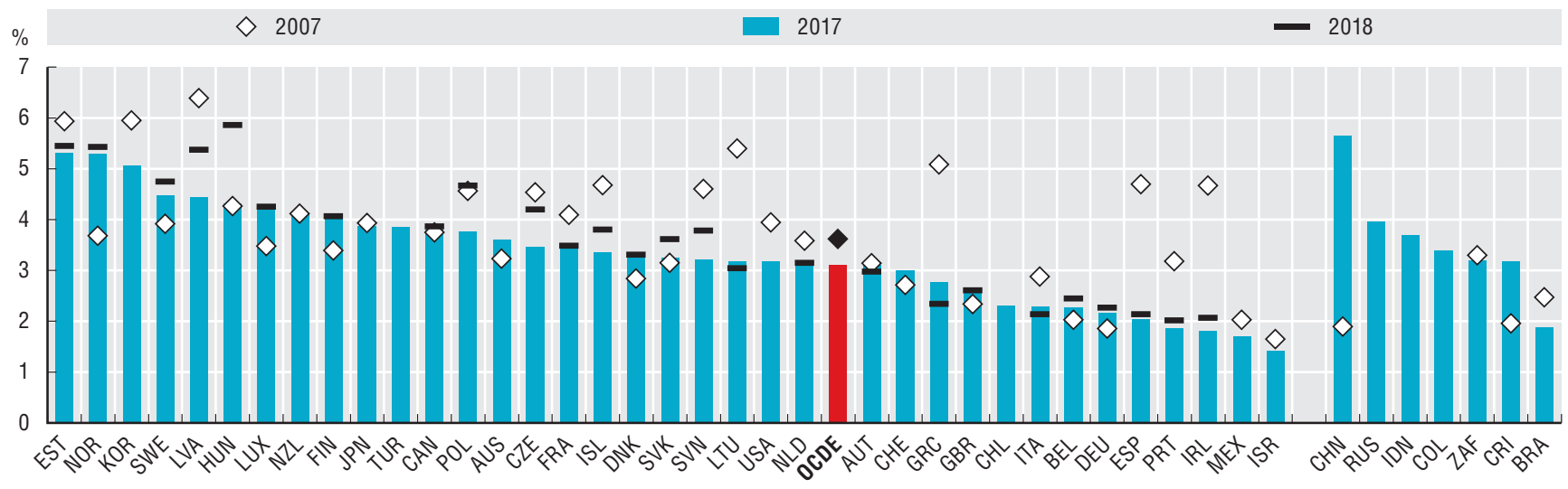

Source : Statistiques de l'OCDE sur les comptes nationaux (base de données).

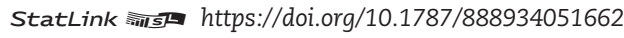

2.48. Investissement des administrations publiques en proportion de leurs dépenses totales, 2007,2017 et 2018

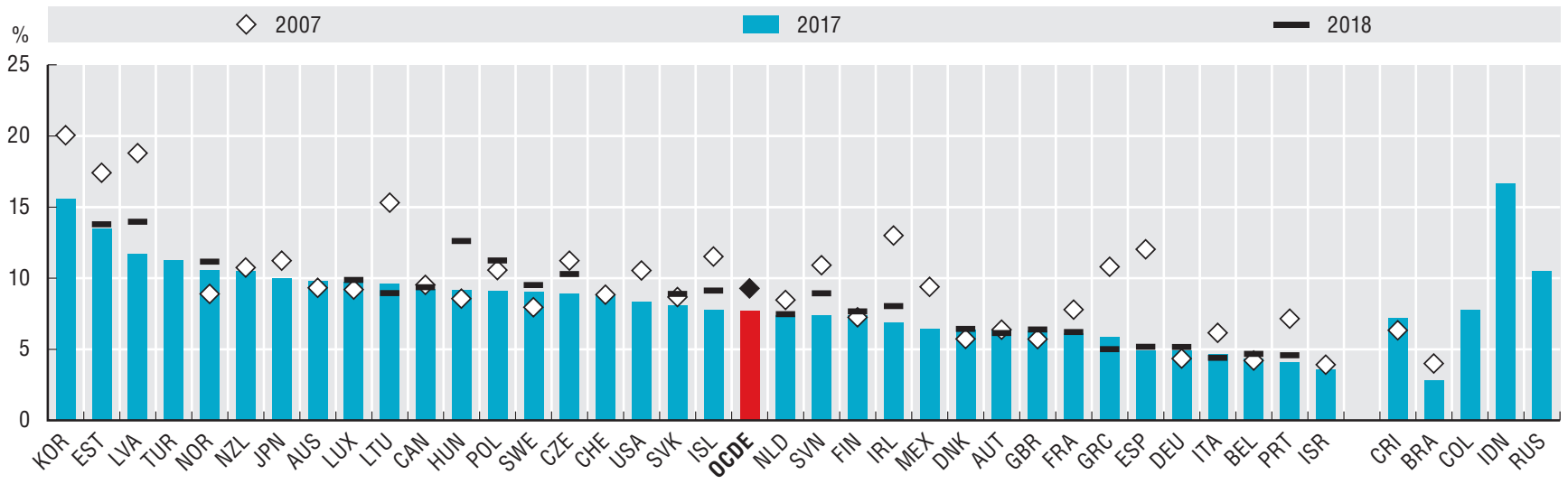

Source : Statistiques de l'OCDE sur les comptes nationaux (base de données).

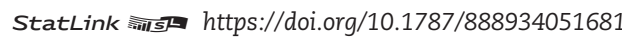

2.49. Ventilation des dépenses d'investissement par niveau d'administration, 2017 et 2018

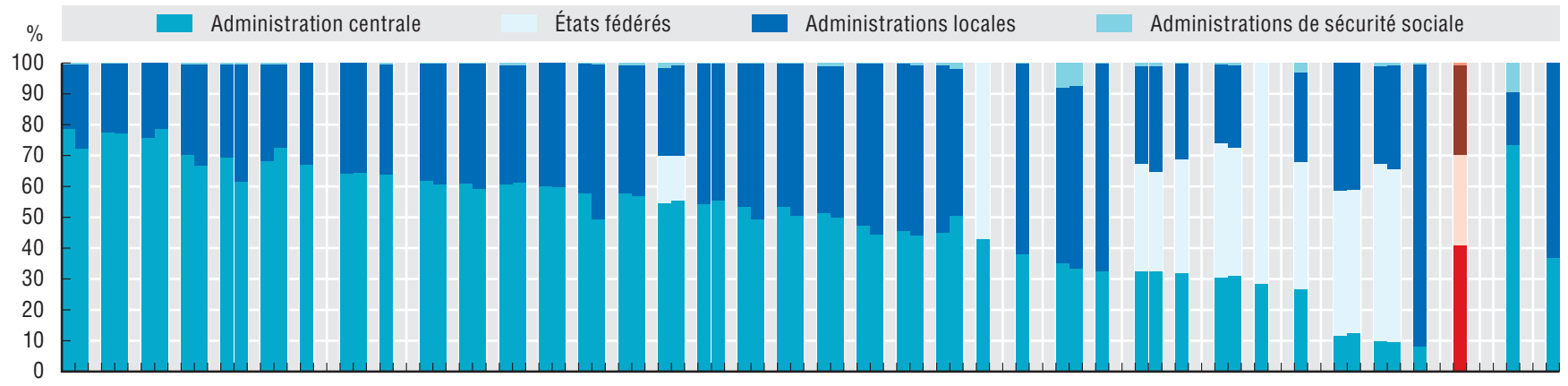

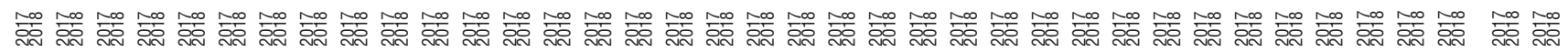

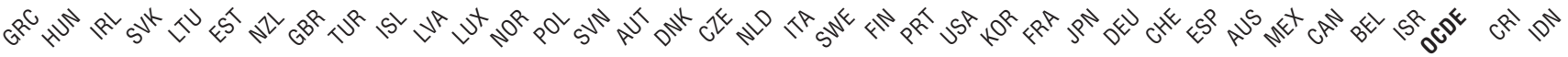
Source : Statistiques de l’OCDE sur les comptes nationaux (base de données). 
Pour produire les biens et les services fournis aux citoyens, les administrations publiques utilisent leurs propres agents, leur capital et effectuent des achats auprès d'entités à but non lucratif ou privées. Il y a deux manières d'externaliser : soit en faisant l'acquisition de biens et de services qui serviront d'intrants (il s'agit alors de consommation intermédiaire), soit en sous-traitant à des prestataires privés ou sans but lucratif la fourniture de biens et de services.

Dans les pays de l'OCDE, les coûts de production des administrations publiques représentaient 20,6\% du produit intérieur brut (PIB) en 2017. La rémunération des agents publics en est la principale composante $(9,2 \%)$, précédant le coût des biens et des services utilisés et financés par les administrations publiques (8,7\%). Ces proportions sont restées relativement stables par rapport à 2007. Les ces pays nordiques comme la Suède (30 \%), en Finlande (29,7 \%) et au Danemark $(28,1 \%)$ connaissent les coûts de production les plus élevés, ce qui s'explique par la prestation généralisée de services à financement public et par des coûts relativement élevés. En revanche, parmi les pays de l'OCDE, le Mexique est celui dont les coûts de production sont le plus bas (11,8\%) car, entre autres facteurs, les services publics y sont moins développés et de moindre qualité, les catégories les plus aisées de la population se tournant vers des prestataires de services privés.

Alors que dans les pays de l'OCDE les coûts de production des administrations publiques ont en moyenne augmenté de 0,4 point de PIB entre 2007 et 2017, la rémunération des agents publics a reculé de 0,2 point. Les plus fortes baisses ont été observées en Irlande (-3,1 points) et au Portugal (-2,2 points). Depuis 2008, l'Irlande et le Portugal ont mis en œuvre des réformes consistant par exemple à appliquer une baisse générale des rémunérations, à réduire ou supprimer des allocations, à diminuer la rémunération ou les primes liées aux résultats et à geler les salaires. L'Irlande a également mis en œuvre une réforme de la rémunération visant spécifiquement les hauts fonctionnaires. Pendant la même période, les coûts de main-d'œuvre ont augmenté en Norvège (+3,1 points), où l'emploi dans le secteur public a progressé entre 2007 et 2017.

La composition des coûts de production des administrations publiques varie selon les pays de l'OCDE. En 2017, la rémunération des agents publics représentait en moyenne $44,8 \%$ de ces coûts, les biens et les services utilisés et financés par les administrations $42,3 \%$ et les autres coûts de production $12,9 \%$. Toutefois, au Mexique, par exemple, la rémunération des agents publics représentait $71,5 \%$ des coûts de production, les biens et les services utilisés et financés par les administrations publiques 27.5 \% et les autres coûts de production $1 \%$. La répartition était aussi sensiblement différente au Japon : les biens et les services utilisés et financés par les administrations publiques représentaient 58,7 \% de leurs coûts de production, les coûts de rémunération $25,5 \%$ et les autres coûts de production $15,7 \%$.

En 2017, les biens et les services utilisés par les administrations publiques représentaient en moyenne 5,7\% du PIB dans les pays de l'OCDE et ceux financés en vue d'être fournis aux citoyens par le secteur privé $3 \%$. Aux Pays-Bas, les services à financement public ont absorbé 10,2 \% du PIB (proportion la plus élevée des pays de l'OCDE), surtout parce que le système de santé y est géré par l'État mais complété par des dispositifs d'assurance privée. Le Japon est le deuxième pays de l'OCDE quant au niveau de financement de biens et de services fournis par le secteur privé (8,8 \% du PIB), alors que ce pays consacre seulement $3,7 \%$ de son PIB à la consommation intermédiaire. Cette faible proportion est le signe d'un modèle où la prestation de services est largement confiée au secteur privé, tandis que l'État conserve un rôle essentiel en réglementant et en finançant partiellement la fourniture de biens et de services.

\section{Méthodologie et définitions}

Le concept et la méthode de calcul des coûts de production reposent sur la classification des dépenses des administrations publiques propre au Système de comptabilité nationale (SCN). Le cadre du SCN 2008 est désormais en vigueur dans tous les pays de l'OCDE (voir l'Annexe A pour plus de précisions).

Les coûts de production des administrations publiques comprennent : 1) la rémunération des agents publics, c'est-à-dire la rémunération de ces derniers en numéraire et en nature, à laquelle s'ajoutent les cotisations obligatoires (et imputées) des employeurs au régime d'assurance sociale et les versements facultatifs pour le compte des agents ; 2) les biens et services utilisés par les administrations publiques, qui sont la première composante des dépenses d'externalisation - selon la terminologie du SCN, ces coûts de production englobent la consommation intermédiaire (acquisition des éléments intermédiaires nécessaires à la production des administrations) ; 3) les biens et les services financés par les administrations publiques, seconde composante des dépenses d'externalisation ; 4) les autres coûts de production, c'est-à-dire les éléments restants : la consommation de capital fixe (amortissement) et les autres impôts sur la production, moins les autres subventions à la production. Les données tiennent compte de l'emploi public et de la consommation intermédiaire destinés à la production des administrations publiques à leur usage. Les coûts de production présentés ici ne sont pas égaux à la valeur de la production dans le SCN.

\section{Pour en savoir plus}

OCDE (2012), Competitive Neutrality: Maintaining a Level Playing Field between Public and Private Business, Éditions OCDE, Paris, https://doi.org/10.1787/9789264178953-en.

\section{Notes relatives aux graphiques}

L'Afrique du Sud, les États-Unis, l'Islande, le Mexique et l'Indonésie ne font pas figurer distinctement dans leurs comptes nationaux les biens et les services financés par les administrations publiques. Les données sur le Chili ne sont pas disponibles. Concernant les donn. es sur Isra.l, voir http://doi.org/10.1787/888932315602. Celles sur la Turquie ne sont pas prises en compte dans la moyenne de la zone OCDE car il manque des séries chronologiques.

Les données sur le Brésil et l'Indonésie portent sur 2016 au lieu de 2017 et celles sur la Russie sur 2015 au lieu de 2017.

2.55. (Composition des dépenses d'externalisation des administrations publiques, 2017 et 2018) est consultable en ligne (voir l'Annexe F) 
2.52. Coûts de production en pourcentage du PIB, 2007, 2017 et 2018

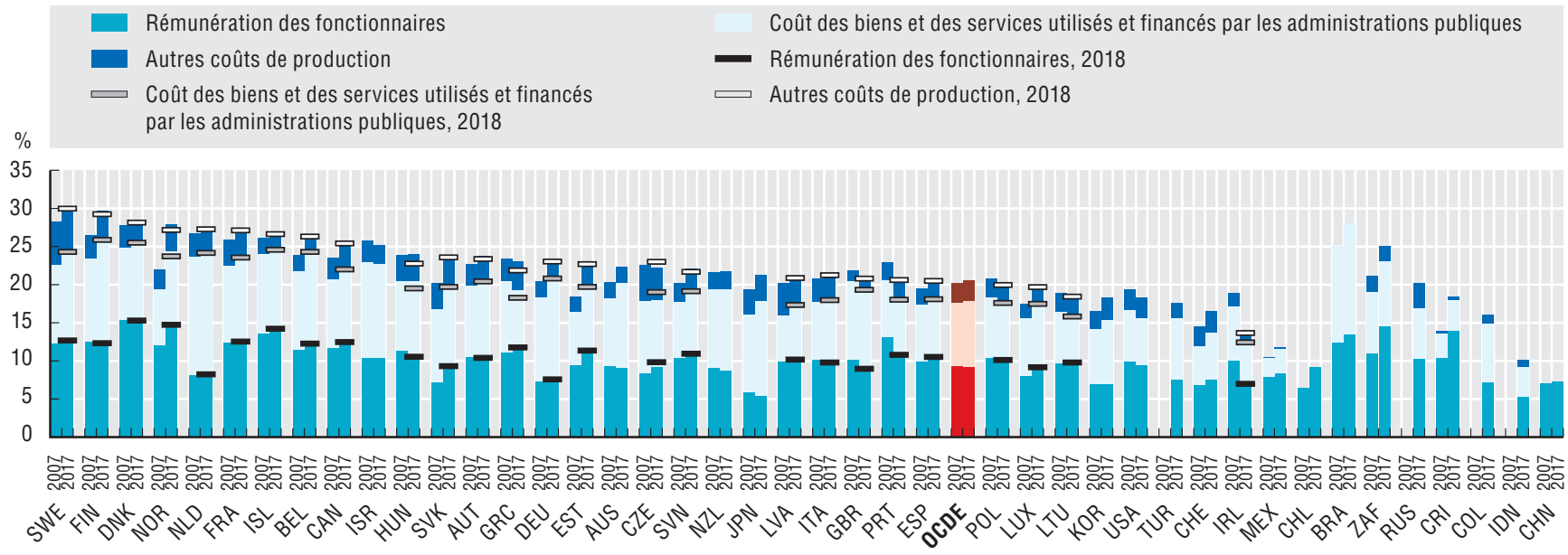

Sources: Statistiques de l'OCDE sur les comptes nationaux (base de données). Pour l'Australie, les données sont tirées à la fois des statistiques sur les comptes nationaux et des statistiques sur les finances publiques fournies par le Bureau australien des statistiques.

StatLink anist https://doi.org/10.1787/888934051719

\subsection{Ventilation des coûts de production, 2017 et 2018}

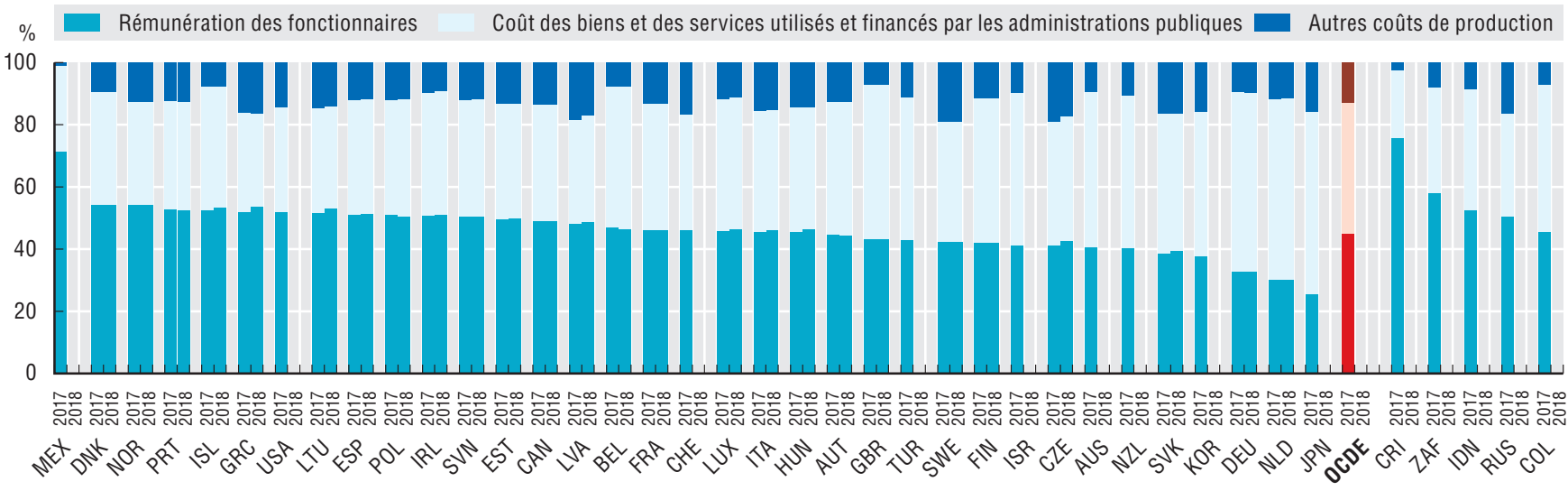

Sources: Statistiques de l'OCDE sur les comptes nationaux (base de données). Pour l'Australie, les données sont tirées à la fois des statistiques sur les comptes nationaux et des statistiques sur les finances publiques fournies par le Bureau australien des statistiques.

StatLink ailst https://doi.org/10.1787/888934051738

\subsection{Dépenses d'externalisation des administrations publiques en pourcentage du PIB, 2017 et 2018}

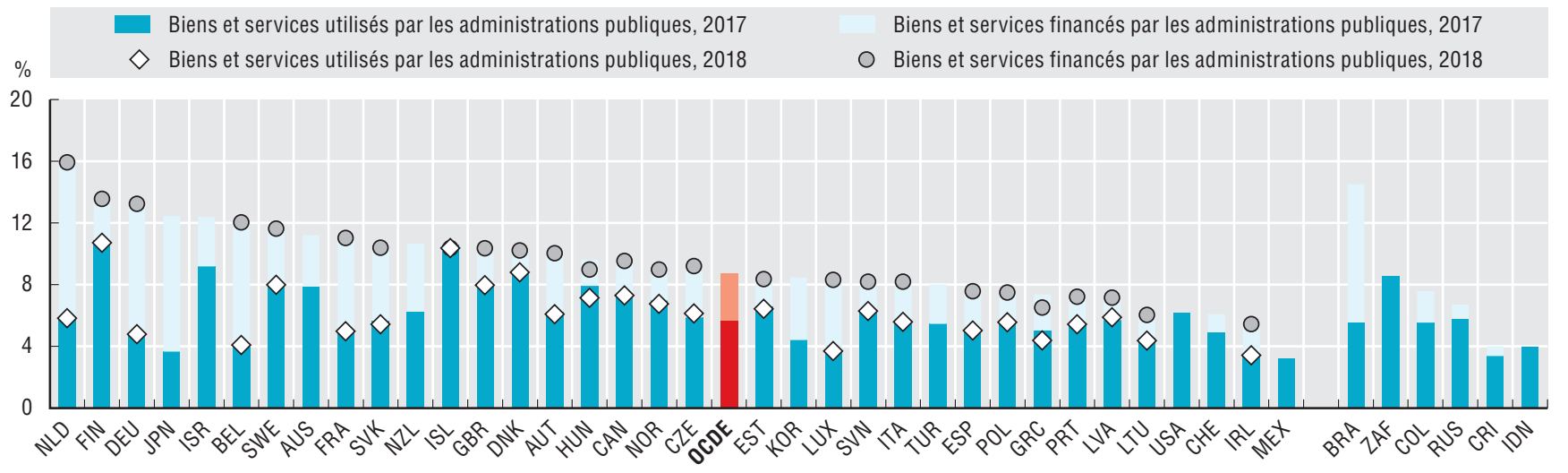

Sources: Statistiques de l'OCDE sur les comptes nationaux (base de données). Pour l'Australie, les données sont tirées à la fois des statistiques sur les comptes nationaux et des statistiques sur les finances publiques fournies par le Bureau australien des statistiques.

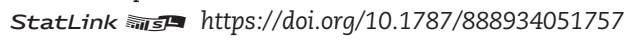


Entre autres missions, les pouvoirs publics sont chargés d'assurer l'accès aux soins et de gérer les prestations sociales destinées à certaines catégories de population, telles que les pensions, les allocations de chômage et les allocations familiales. Ces services ayant un coût élevé, la santé et la protection sociale figurent parmi les principaux postes de dépenses des administrations publiques. Les évolutions démographiques et technologiques ont accentué leur importance relative au cours des cinquante dernières années.

Ainsi, le nombre de personnes ayant atteint l'âge de la retraite a augmenté par rapport à la population en âge de travailler, de même que la durée de la retraite. Selon des études de l'OCDE, on comptait en 2015 dans ses pays membres 28 personnes âgées de 65 ans ou plus pour 100 personnes âgées de 20 à 64 ans contre 18 en 1970. Les projections montrent que le ratio sera en 2060 de 57 personnes de 65 ans ou plus pour 100 personnes de 20 à 64 ans (OCDE 2019). En outre, alors qu'en 1970 un homme passait en moyenne 11 ans à la retraite et une femme 15 ans, ces chiffres étaient respectivement de 18 et 22 ans en 2016 (OCDE 2017).

En 2017, selon les dernières données disponibles, plus de la moitié des fonds consacrés à la protection sociale a été affectée aux pensions de retraite, ce qui équivaut en moyenne à $10 \%$ du produit intérieur brut (PIB) des pays de l'OCDE. En Finlande, en Grèce, en France et en Italie, ces pensions représentent plus de $13 \%$ du PIB. En revanche, l'Islande, l'Irlande et Israël sont les moins dépensiers dans ce domaine, avec des taux respectifs de 3,0 \%, 3,4 \% et 5,0 \% du PIB. Les pensions jouent un rôle social essentiel puisqu'elles constituent la principale source de revenu des personnes âgées dans la majorité des pays de l'OCDE. Bien que le taux de remplacement (c'est-à-dire la capacité d'un régime de retraite à remplacer la rémunération d'une activité) varie entre les pays de l'OCDE et dépende à terme de l'indexation ou non des retraites sur la même valeur de référence que les salaires, l'objectif de la plupart des pays membres est de protéger les travailleurs à faible revenu de la pauvreté pendant la vieillesse en leur assurant des taux de remplacement supérieurs (OCDE 2017).

En absorbant $15 \%$ des dépenses de protection sociale, les indemnités de maladie et les pensions d'invalidité, équivalentes en moyenne à 2,8 \% du PIB, viennent en deuxième position. La Norvège, le Danemark et les Pays-Bas dépensent le plus sur ce plan, soit respectivement 5,7 \%, 4,4 \% et 4,1 \% du PIB. Le Japon ne dépense que $0,9 \%$ de son PIB pour les pensions d'invalidité et les indemnités de maladie en raison de multiples facteurs, notamment les conditions d'ouverture du droit à une pension d'invalidité, la proportion relativement faible de la population en âge de travailler faisant état d'un handicap et le petit nombre de demandes d'admission au bénéfice de ces dispositifs, d'acceptations de ces demandes et de recours connexes (Rajnes 2010).

L'Autriche, l'Estonie, le Japon, le Luxembourg et la Pologne sont les seuls pays qui dépensent plus en aides à la famille et à l'enfance qu'en indemnités de maladie et pensions d'invalidité. Sachant que ces pays affichent des taux de fécondité inférieurs à la moyenne de la zone OCDE, améliorer ces aides constitue une priorité stratégique (OCDE 2019).

En termes de santé, la plus grande part est consacrée aux services hospitaliers (3,1\% du PIB en moyenne en 2017), suivis des services de soins ambulatoires (1\%). Par analogie avec les indemnités de maladie et les pensions d'invalidité, les dépenses en services hospitaliers (hôpitaux généraux et spécialisés, centres médicaux et maternités, ainsi que les services de soins infirmiers et pour personnes en convalescence) en Norvège, au Danemark et aux Pays-Bas sont supérieures à la moyenne de l'OCDE.

\section{Méthodologie et définitions}

Les données relatives aux dépenses publiques sont tirées de la base de données des Statistiques de l'OCDE sur les comptes nationaux et de la base de données d'Eurostat sur les Statistiques relatives aux finances publiques, qui sont établies conformément au Système de comptabilité nationale (SCN) ; ce dernier constitue un ensemble de concepts, de définitions, de nomenclatures et de règles approuvés au plan international en matière de comptabilité nationale. Le cadre du SCN 2008 est désormais en vigueur dans tous les pays de l'OCDE (voir l'Annexe A pour plus de précisions). Les données sur les dépenses publiques par fonction sont ventilées selon la Classification des fonctions des administrations publiques (CFAP) en 10 fonctions de niveau 1 (voir l'Annexe C pour davantage d'informations). La fonction correspondant aux dépenses de santé est subdivisée en 6 fonctions de niveau 2 : produits, appareils et matériels médicaux ; services ambulatoires ; services hospitaliers ; services de santé publique ; recherche-développement dans le domaine de la santé ; santé n.c.a. La fonction correspondant aux dépenses de protection sociale est subdivisée en 9 fonctions de niveau 2 : maladie et invalidité ; vieillesse (c'est-à-dire pensions de retraite) ; survivants ; famille et enfants ; chômage ; logement ; exclusion sociale n.c.a. ; recherche-développement dans le domaine de la protection sociale ; protection sociale n.c.a. Dans la base de données de l'OCDE sur la répartition des revenus, la pauvreté des personnes âgées correspond aux personnes de plus de 65 ans dont le revenu est inférieur à la moitié du revenu équivalent médian du ménage national.

\section{Pour en savoir plus}

OCDE (2019), Panorama de la société 2019 : les indicateurs sociaux de l'OCDE, Éditions OCDE, Paris, https://doi.org/10.1787/soc glance-2019-en.

OCDE (2018), Panorama des pensions 2017 : les indicateurs de l'OCDE et du G20, Éditions OCDE, Paris, http://doi.org/10.1787/ pension_glance-2017-en.

Rajnes, D. (2010), Permanent Disability Social Insurance Programs in Japan, Social Security Bulletin, vol. 70, $n^{\circ} 1$, pp. 61-84.

\section{Notes relatives aux graphiques}

Les données sur les pays non européens membres de l'OCDE (sauf Israël et le Japon) et la Turquie ne sont pas disponibles. Concernant les données sur Israël, voir http://doi.org/10.1787/888932315602. « Europe OCDE " correspond aux pays européens de l'OCDE ; les données sur l'Islande ne sont pas prises en compte dans la moyenne de la zone OCDE car il manque des séries chronologiques.

2.58 à 2.61. (Structure des dépenses publiques par fonction de gouvernement de protection sociale et de santé en 2017 et évolution depuis 2019) sont disponibles en ligne à l'Annexe F. 
2.56. Ventilation par fonction de protection sociale des dépenses des administrations publiques, en pourcentage du PIB, 2017

\begin{tabular}{|c|c|c|c|c|c|c|c|c|c|}
\hline & Maladie et invalidité & Vieillesse & Survivants & Famille et enfants & Chômage & Logement & $\begin{array}{c}\text { Exclusion sociale } \\
\text { non classée ailleurs } \\
\text { (n.c.a.) }\end{array}$ & $\begin{array}{l}\text { Recherche-développement } \\
\text { dans le domaine de la } \\
\text { protection sociale }\end{array}$ & $\begin{array}{c}\text { Protection sociale } \\
\text { n.c.a. }\end{array}$ \\
\hline Allemagne & 3.22 & 9.35 & 1.85 & 1.63 & 1.64 & 0.33 & 0.62 & 0.00 & 0.72 \\
\hline Autriche & 1.82 & 12.51 & 1.38 & 2.16 & 1.30 & 0.11 & 1.04 & 0.01 & 0.20 \\
\hline Belgique & 3.29 & 9.19 & 1.72 & 2.19 & 1.65 & 0.21 & 1.13 & 0.01 & 0.16 \\
\hline Danmark & 4.44 & 8.24 & 0.01 & 4.39 & 2.26 & 0.67 & 1.88 & 0.02 & 0.47 \\
\hline Espagne & 2.37 & 9.11 & 2.22 & 0.70 & 1.60 & 0.02 & 0.33 & 0.00 & 0.20 \\
\hline Estonie & 2.13 & 6.79 & 0.06 & 2.53 & 1.15 & 0.02 & 0.18 & 0.04 & 0.14 \\
\hline Finlande & 3.14 & 13.80 & 0.67 & 3.05 & 2.26 & 0.56 & 1.05 & 0.02 & 0.31 \\
\hline France & 2.94 & 13.36 & 1.51 & 2.38 & 1.94 & 0.96 & 1.06 & 0.00 & 0.18 \\
\hline Grèce & 1.54 & 13.77 & 2.11 & 0.65 & 0.48 & 0.02 & 0.78 & 0.02 & 0.05 \\
\hline Hongrie & 2.58 & 6.96 & 0.97 & 2.08 & 0.30 & 0.15 & 0.78 & 0.00 & 0.19 \\
\hline Irlande & 1.90 & 3.42 & 0.56 & 1.34 & 1.09 & 0.82 & 0.30 & 0.00 & 0.05 \\
\hline Islande & 3.11 & 3.04 & 0.01 & 2.02 & 0.49 & 0.42 & 0.32 & 0.00 & 0.33 \\
\hline Israël & 2.63 & 4.97 & 0.58 & 1.42 & 0.27 & 0.16 & 0.54 & 0.00 & 0.48 \\
\hline Italie & 1.82 & 13.35 & 2.62 & 1.56 & 1.11 & 0.04 & 0.36 & 0.00 & 0.01 \\
\hline Japon & 0.91 & 10.88 & 1.50 & 1.85 & 0.27 & 0.00 & 0.29 & 0.00 & 0.43 \\
\hline Lettonie & 2.15 & 6.90 & 0.17 & 1.19 & 0.49 & 0.11 & 0.38 & 0.00 & 0.27 \\
\hline Lithuanie & 3.08 & 5.75 & 0.31 & 1.06 & 0.57 & 0.05 & 0.25 & 0.00 & 0.15 \\
\hline Luxembourg & 2.84 & 9.89 & 0.00 & 3.74 & 1.06 & 0.08 & 0.68 & 0.00 & 0.16 \\
\hline Norvège & 6.71 & 7.31 & 0.19 & 3.52 & 0.49 & 0.11 & 0.95 & 0.04 & 0.44 \\
\hline Pays-Bas & 4.11 & 6.55 & 0.07 & 1.35 & 1.62 & 0.47 & 1.69 & 0.01 & 0.00 \\
\hline Pologne & 2.37 & 8.99 & 1.66 & 2.67 & 0.37 & 0.04 & 0.21 & 0.00 & 0.12 \\
\hline Portugal & 1.29 & 11.73 & 1.73 & 1.07 & 0.80 & 0.03 & 0.23 & 0.00 & 0.56 \\
\hline République slovaque & 2.91 & 7.77 & 0.81 & 1.21 & 0.20 & 0.00 & 0.25 & 0.00 & 1.36 \\
\hline République tchèque & 2.10 & 7.34 & 0.54 & 1.01 & 0.17 & 0.22 & 0.38 & 0.00 & 0.21 \\
\hline Royaume-Uni & 2.45 & 8.35 & 0.05 & 1.28 & 0.10 & 1.10 & 1.58 & 0.00 & 0.28 \\
\hline Slovénie & 2.20 & 9.18 & 1.27 & 1.85 & 0.50 & 0.02 & 0.88 & 0.00 & 0.26 \\
\hline Suède & 4.11 & 10.31 & 0.25 & 2.45 & 1.29 & 0.28 & 1.41 & 0.00 & 0.08 \\
\hline Suisse & 2.93 & 6.81 & 0.31 & 0.48 & 1.19 & 0.02 & 1.53 & 0.00 & 0.27 \\
\hline OCDE-Europe & 2.78 & 9.97 & 1.35 & 1.71 & 1.20 & 0.43 & 0.86 & 0.00 & 0.29 \\
\hline Colombie & 0.01 & 6.40 &.. & 0.83 & .. & 0.29 & 1.16 & .. & 0.26 \\
\hline Costa Rica & 0.62 & 4.72 & 0.69 & 0.24 & 0.00 & 0.00 & 0.01 & 0.00 & 2.20 \\
\hline
\end{tabular}

Sources : Statistiques de l'OCDE sur les comptes nationaux (base de données) ; statistiques d'Eurostat relatives aux finances publiques (base de données).

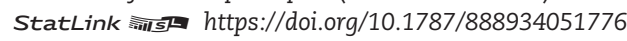

2.57. Ventilation par fonction de santé des dépenses des administrations publiques, en pourcentage du PIB, 2017

\begin{tabular}{|c|c|c|c|c|c|c|}
\hline & $\begin{array}{l}\text { Produits, appareils et matériels } \\
\text { médicaux }\end{array}$ & Services ambulatoires & Services hospitaliers & Services de santé publique & $\begin{array}{l}\text { Recherche-développement } \\
\text { dans le domaine de la santé }\end{array}$ & $\begin{array}{l}\text { Santé non classée } \\
\text { ailleurs (n.c.a.) }\end{array}$ \\
\hline Allemagne & 1.60 & 2.13 & 2.72 & 0.06 & 0.08 & 0.51 \\
\hline Autriche & 1.12 & 1.47 & 4.64 & 0.18 & 0.46 & 0.31 \\
\hline Belgique & 0.79 & 2.66 & 3.91 & 0.13 & 0.02 & 0.17 \\
\hline Danmark & 0.55 & 1.18 & 5.96 & 0.15 & 0.20 & 0.34 \\
\hline Espagne & 0.97 & 4.60 & .. & 0.09 & 0.25 & 0.03 \\
\hline Estonie & 0.66 & 0.54 & 3.64 & 0.04 & 0.12 & 0.05 \\
\hline Finlande & 0.63 & 3.15 & 3.10 & 0.03 & 0.12 & 0.04 \\
\hline France & 1.43 & 2.91 & 3.39 & 0.11 & 0.09 & 0.10 \\
\hline Grèce & 1.49 & 0.48 & 3.20 & 0.00 & 0.04 & 0.03 \\
\hline Hongrie & 0.90 & 1.36 & 2.15 & 0.13 & 0.03 & 0.24 \\
\hline Irlande & 0.76 & 1.86 & 1.95 & 0.14 & 0.01 & 0.42 \\
\hline Islande & 0.49 & 1.80 & 5.09 & 0.02 & 0.00 & 0.16 \\
\hline Israël & 0.69 & 1.57 & 2.87 & 0.11 & 0.00 & 0.11 \\
\hline Italie & 0.81 & 2.59 & 2.99 & 0.27 & 0.07 & 0.09 \\
\hline Japon & 1.26 & 2.97 & 2.78 & 0.45 & 0.01 & 0.16 \\
\hline Lettonie & 0.55 & 0.93 & 1.88 & 0.05 & 0.00 & 0.09 \\
\hline Lithuanie & 0.74 & 1.46 & 2.11 & 0.06 & 0.00 & 1.29 \\
\hline Luxembourg & 3.44 & 1.06 & 0.07 & 0.05 & 0.19 & 0.07 \\
\hline Norvège & 0.54 & 2.00 & 5.03 & 0.25 & 0.41 & 0.31 \\
\hline Pays-Bas & 0.75 & 2.49 & 3.50 & 0.22 & 0.34 & 0.27 \\
\hline Pologne & 0.06 & 1.46 & 2.89 & 0.07 & 0.08 & 0.12 \\
\hline Portugal & 0.35 & 2.08 & 3.30 & 0.04 & 0.11 & 0.12 \\
\hline République slovaque & 1.48 & 2.22 & 3.02 & 0.04 & 0.00 & 0.35 \\
\hline République tchèque & 0.90 & 1.57 & 3.40 & 1.34 & 0.05 & 0.21 \\
\hline Royaume-Uni & 0.52 & 1.00 & 5.37 & 0.22 & 0.16 & 0.16 \\
\hline Slovénie & 0.95 & 2.21 & 2.73 & 0.34 & 0.08 & 0.27 \\
\hline Suède & 0.75 & 3.03 & 2.55 & 0.22 & 0.18 & 0.19 \\
\hline Suisse & 0.00 & 0.19 & 1.70 & 0.13 & 0.11 & 0.05 \\
\hline OCDE-Europe & 0.97 & 2.23 & 3.14 & 0.16 & 0.13 & 0.22 \\
\hline Colombie & 4.58 & .. & .. & 0.21 & 0.03 & 0.07 \\
\hline Costa Rica & 0.27 & 2.32 & 3.03 & 0.13 & 0.12 & 0.24 \\
\hline
\end{tabular}

Sources : Statistiques de l'OCDE sur les comptes nationaux (base de données) ; statistiques d'Eurostat relatives aux finances publiques (base de données). 


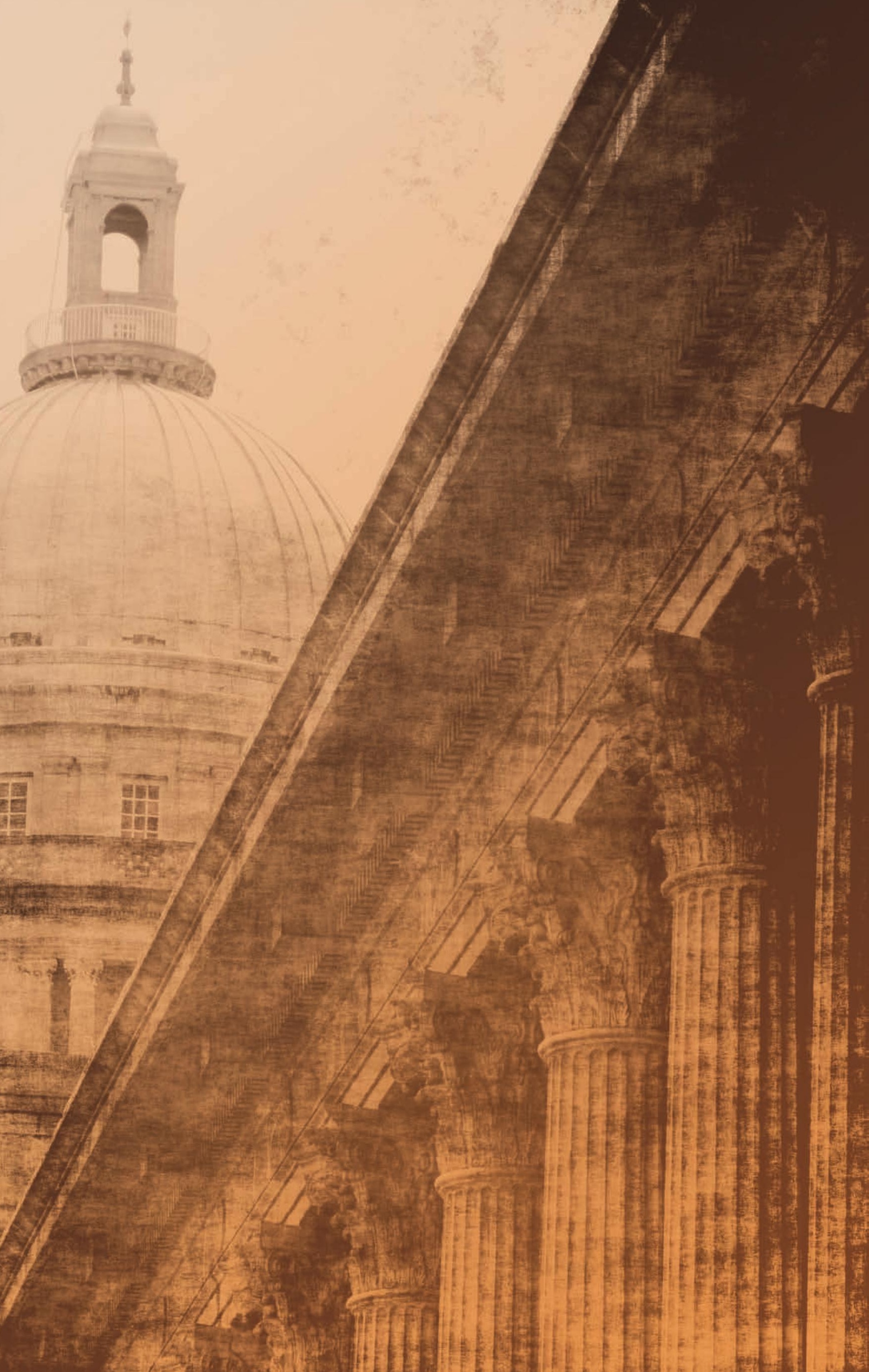


Les administrations publiques des pays de l'OCDE effectuent de nombreuses tâches qui sont fondamentales pour le bon fonctionnement des sociétés. La diversité de ces tâches traduit en partie l'existence de cultures administratives distinctes selon les pays et l'expression d'attentes différentes par leurs populations. Les fortes disparités constatées relativement au poids de l'emploi public dans l'emploi total mettent en évidence la variété des fonctions exercées par les administrations ainsi que le large éventail de mécanismes de prestation à leur disposition pour la fourniture de services. Ces mécanismes peuvent être assurés par des agents publics ou être fournis grâce à divers partenariats avec le secteur privé ou non lucratif. Dans certains pays, la grande majorité des professionnels de santé, des enseignants et des membres des services d'urgence, par exemple, sont directement employés par l'administration. Ailleurs, ceux-ci et d'autres types de professionnels sont employés par des entités du secteur privé ou des organisations à but non lucratif.

Le volume de l'emploi dans les administrations publiques varie fortement d'un pays à l'autre de l'OCDE. Le taux d'emploi dans les administrations publiques est le plus élevé dans les pays nordiques où il atteint dans certains cas (Norvège) plus de $30 \%$ de l'emploi total. À l'inverse, au Japon et en Corée, respectivement moins de $6 \%$ et moins de $8 \%$ des actifs occupés, travaillent dans l'administration publique. La moyenne de la zone OCDE s'établit à $18 \%$.

En général, le taux d'emploi dans les administrations publiques est resté relativement stable au fil du temps. Certains pays de l'OCDE ont fait part de réductions considérables de l'emploi dans les administrations publiques, réductions qui s'expliquent par la crise financière de 2007-2008, mais la part moyenne des effectifs des administrations publiques dans l'emploi total, exprimée en pourcentage à l'échelle des pays de l'OCDE, n'a que légèrement baissé en 2017 (17,7 \%) par rapport à 2007 (17,8 \%). Au Royaume-Uni, en Israël et en Turquie cependant, le taux d'emploi dans les administrations publiques a reculé entre 2007 et 2017 de respectivement 3,1, 2,9 et 2,3 points, tandis qu'en Norvège, il a progressé sur la même période (d'environ 1 point de pourcentage).

En ce qui concerne le taux annuel de croissance des effectifs des administrations publiques, la moyenne de la zone OCDE reste relativement stable : $+0,6 \%$ sur la période $2007-09$, puis $-0,2 \%$ en 2011-12 et $+0,4 \%$ en 2016-17. Beaucoup de pays enregistrent un ralentissement de la croissance de ces effectifs pendant la période 2011-12, du fait des mesures d'austérité puis, pour près de la moitié d'entre eux, une reprise en 2017 au plus tard. Cependant, l'examen individuel des différents pays considérés dessine un tableau plus nuancé. Par exemple, l'Irlande affiche la croissance la plus forte en 2016-17, à savoir $3,5 \%$, tandis que l'Estonie enregistre les réductions d'effectifs les plus importantes, supérieures à $5 \%$, sur la même période.

\section{Méthodologie et définitions}

Ces données sont tirées de la base de données des Statistiques de l'OCDE sur les comptes nationaux, qui sont établies conformément au Système de comptabilité nationale (SCN). Ce dernier constitue un ensemble de concepts, définitions, nomenclatures et règles approuvés au plan international en matière de comptabilité nationale. Par " emploi dans les administrations publiques ", on entend l'emploi à tous les niveaux d'administration (administration centrale, administrations d'États fédérés, administrations locales et administrations de sécurité sociale) ainsi que dans les ministères proprement dits, les organismes et directions publics et les institutions à but non lucratif contrôlées par les pouvoirs publics. Les données correspondent au nombre total de personnes employées directement par ces institutions. L'emploi total englobe toutes les personnes qui exercent une activité productive entrant dans le domaine de la production au sens des comptes nationaux. Les personnes employées comprennent toutes celles qui, au cours d'une période déterminée, ont occupé un emploi rémunéré ou exercé un travail indépendant.

\section{Pour en savoir plus}

OCDE (2019), Recommandation du Conseil sur le leadership et les aptitudes de la fonction publique, OCDE, Paris, https:// legalinstruments.oecd.org/fr/instruments/OECD-LEGAL-0445.

OCDE (2017), "Skills for a High Performing Civil Service", Examens de l'OCDE sur la gouvernance publique, Éditions OCDE, Paris, https://doi.org/10.1787/9789264280724-en.

OCDE (2016), Engaging Public Employees for a High-Performing Civil Service, Examens de l'OCDE sur la gouvernance publique, Éditions OCDE, Paris, https://doi.org/10.1787/9789264267190-en.

\section{Notes relatives aux graphiques}

On ne dispose pas de données pour l'Australie, le Chili, l'Islande et la Nouvelle-Zélande. Concernant les données sur Israël, voir http://doi. org/10.1787/888932315602. Les données relatives à la Corée et à la Suisse ne sont pas prises en compte dans la moyenne de la zone OCDE en raison de l'absence de certaines séries chronologiques. Les données sur la situation en Pologne avant 2010 reposent sur des estimations.

3.1. En ce qui concerne le Costa Rica, le Mexique et la Suisse, les données portent sur 2016 au lieu de 2017. Les données sur les États-Unis se rapportent à 2008 et non 2009.

3.2. Les données relatives à la période 2007-09 correspondent au taux de croissance annuelle moyen. Les données relatives au Costa Rica, au Mexique et à la Suisse portent sur 2015-16 au lieu de 2016-17. Les données sur les États-Unis se rapportent à 2008-09 et non 2007-09. 
3.1. Emploi dans les administrations publiques, en pourcentage de l'emploi total, 2007, 2009 et 2017

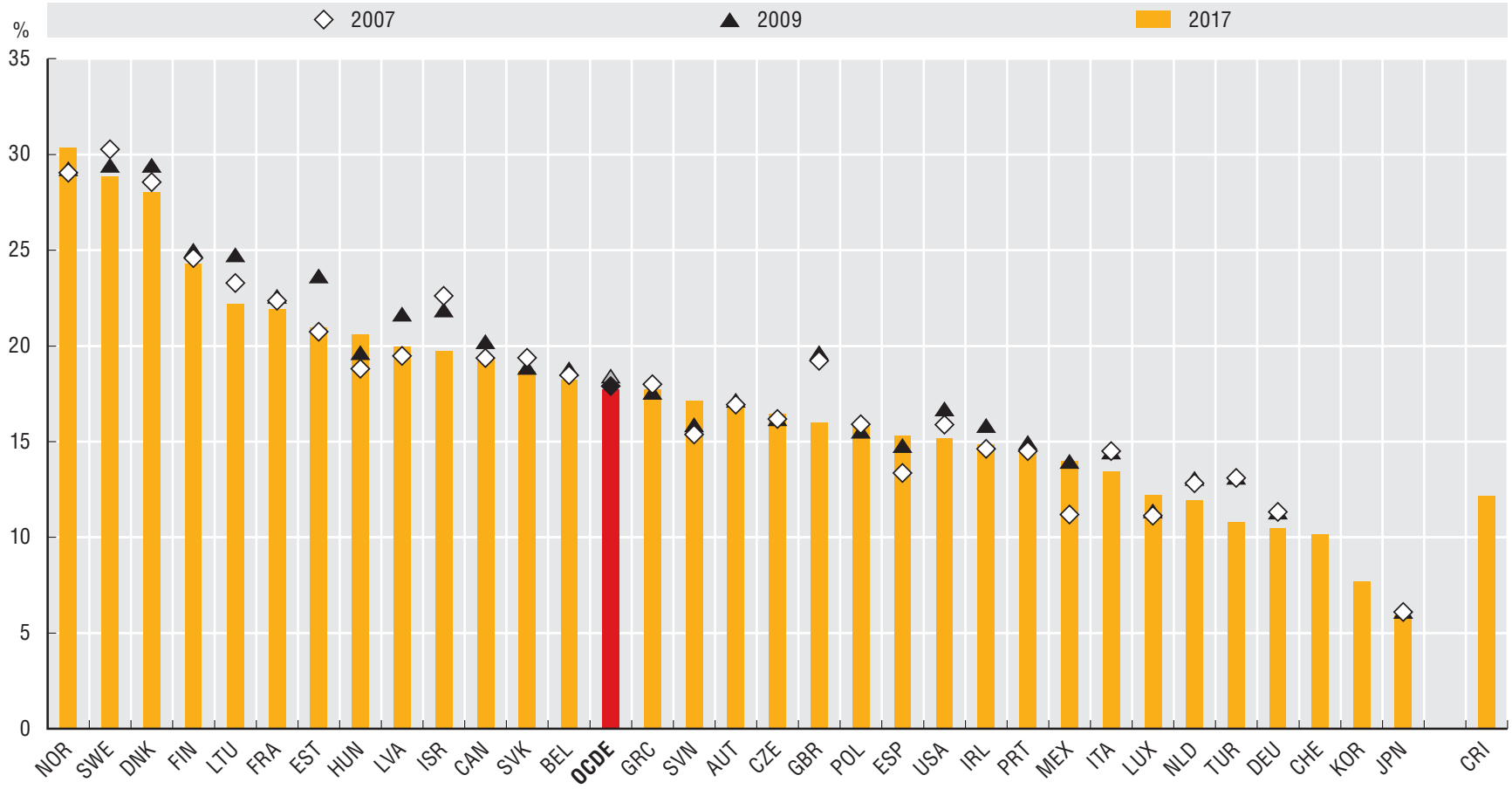

Sources : Statistiques de l'OCDE sur les comptes nationaux (base de données). Les données relatives à la Corée, aux États-Unis, au Japon, au Mexique, à la Suisse et à la Turquie proviennent de l'Organisation internationale du travail (OIT), ILOSTAT (base de données), Emploi public par secteurs et sous-secteurs des comptes nationaux.

3.2. Taux de croissance annuelle de l'emploi public, 2007-09, 2012 et 2017

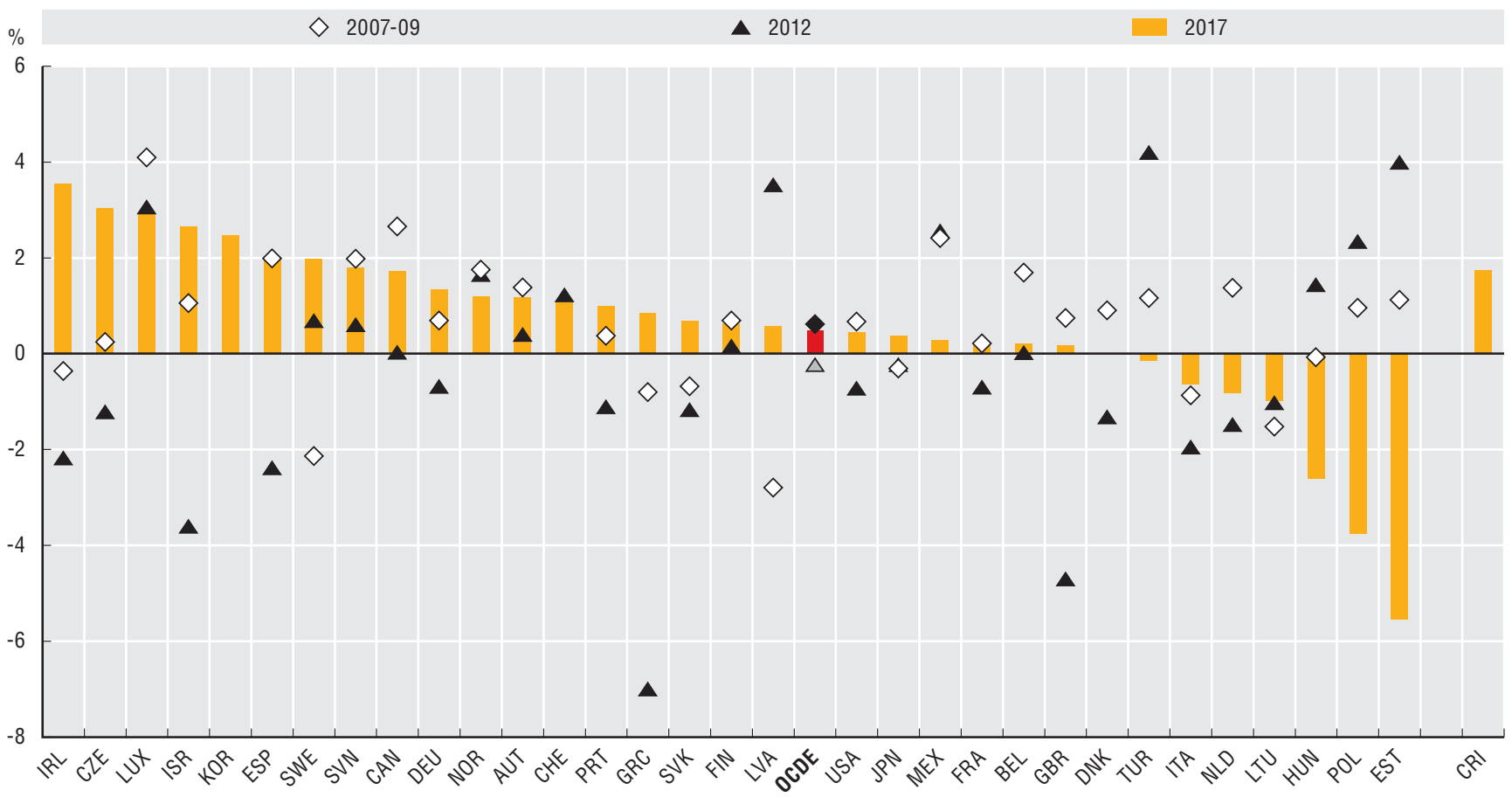

Sources : Statistiques de l'OCDE sur les comptes nationaux (base de données). Les données relatives à la Corée, aux États-Unis, au Japon, au Mexique, à la Suisse et à la Turquie proviennent de l'Organisation internationale du travail (OIT), ILOSTAT (base de données), Emploi public par secteurs et sous-secteurs des comptes nationaux. 
La proportion de personnel employé aux échelons administratifs infranationaux constitue un indicateur du niveau de décentralisation des administrations publiques. La concentration d'une part importante des effectifs dans les administrations infranationales signifie généralement que les administrations régionales et locales assument de nombreuses responsabilités en matière de prestation de services publics. Si la décentralisation peut accroître la réactivité des administrations face aux besoins et aux priorités d'ordre local, elle peut aussi entraîner des disparités dans la prestation des services publics au sein d'un même pays.

En 2017, les administrations publiques comptaient plus d'employés au niveau infranational qu'au niveau central, malgré des différences d'un pays à l'autre. Certains États fédéraux, comme l'Allemagne, la Belgique et la Suisse, sont caractérisés par des effectifs d'agents publics plus élevés dans les administrations infranationales, tandis que dans d'autres pays reposant sur un modèle d'État unitaire, tels l'Irlande, Israël et la Turquie, la plupart des agents publics travaillent à l'échelon central.

Entre 2011 et 2017, le pourcentage d'agents publics employés au niveau central est resté relativement stable, la tendance observée sur la période 2009-2014 se poursuivant. Il semble donc que, dans les pays où les effectifs d'agents publics ont fait l'objet d'ajustements sur cette période (voir Emploi dans les administrations publiques), ces ajustements ont, dans l'ensemble, été opérés de façon équilibrée entre administrations centrale et infranationales et n'ont pas sensiblement évolué depuis la crise financière de 2008. Seule la Hongrie a connu un changement important au cours de cette période : la proportion d'agents publics employés à l'échelon central a bondi de près de 27 points. Cette hausse s'explique par la réorganisation de l'administration publique territoriale ayant eu lieu pendant cette période. En outre, la proportion d'agents publics employés dans l'administration centrale au Royaume-Uni ainsi qu'aux Pays-Bas a augmenté de 8,5 et 5 points respectivement, entre 2011 et 2017. Dans les deux cas, la situation résultait d'une augmentation des effectifs employés à l'échelon central et d'une diminution des effectifs aux échelons infranationaux, ce qui a entraîné une réduction globale des effectifs des administrations publiques au cours de la période.

\section{Méthodologie et définitions}

Ces données ont été rassemblées par l'Organisation internationale du travail (OIT) dans le cadre de sa base de données ILOSTAT. Elles sont fondées sur les définitions du Système de comptabilité nationale (SCN) et recouvrent l'emploi public à l'échelon central et aux échelons infranationaux. L'administration infranationale englobe les administrations d'États fédérés et les administrations locales, y compris les régions, les provinces et les municipalités. Ensemble, le niveau central et les niveaux infranationaux forment les administrations publiques. Les pays ont également fourni des données sur l'emploi dans les administrations de sécurité sociale, qui font partie des administrations publiques et englobent toutes les unités institutionnelles aux échelons central, des États fédérés et local dont l'activité principale consiste à fournir des prestations sociales. Dans la mesure où les administrations de sécurité sociale relèvent de divers niveaux d'administration, l'emploi dans cette catégorie a été, sauf indication contraire, comptabilisé séparément. Cela étant, dans la plupart des pays, à l'exception de l'Allemagne, de la France et du Mexique, les administrations de sécurité sociale emploient peu d'agents et représentent un faible pourcentage des effectifs totaux. Les données correspondent au nombre total d'agents employés directement par chacun de ces différents niveaux d'administration et de ces différentes administrations de sécurité sociale. Les pays suivants sont considérés comme des États fédéraux dans la série de données : l'Allemagne, la Belgique, l'Espagne (considérée comme un pays quasi fédéral), les États-Unis, le Mexique et la Suisse.

\section{Pour en savoir plus}

OCDE (2019), Recommandation du Conseil sur le leadership et les aptitudes de la fonction publique, OCDE, Paris, https:// legalinstruments.oecd.org/fr/instruments/OECD-LEGAL-0445.

OCDE (2017), Skills for a High Performing Civil Service, Examens de l'OCDE sur la gouvernance publique, Éditions OCDE, Paris, https://doi.org/10.1787/9789264280724-en.

OCDE (2016), Engaging Public Employees for a High-Performing Civil Service, Examens de l'OCDE sur la gouvernance publique, Éditions OCDE, Paris, https://doi.org/10.1787/9789264267190-en.

\section{Notes relatives aux graphiques}

On ne dispose pas de données pour l'Australie, l'Autriche, le Canada, le Chili, l'Islande, le Japon, le Luxembourg, la Nouvelle-Zélande et la Slovénie. Les données pour le Costa Rica, l'Espagne, l'Estonie et la Grèce sont basées sur l'Enquête sur la population active. L'emploi au sein des administrations de sécurité sociale n'est pas différencié (c'est-à-dire qu'il est comptabilisé dans le cadre unique de l'emploi au sein de l'administration centrale ou des administrations infranationales) pour les pays suivants : l'Espagne, l'Estonie, les États-Unis, l'Irlande, la Norvège, le Royaume-Uni et la Suisse. Les données concernant la France, la Hongrie, le Mexique et la Suisse se rapportent à 2016. Dans le cas de la Pologne, les autres institutions sans but lucratif (ISBL) ont été ventilées entre l'administration centrale et les niveaux d'administration infranationaux.

3.4. Les données concernant la Lituanie se rapportent à 2010 et non 2011. 
3.3. Répartition de l'emploi public entre les divers niveaux d'administration, 2017

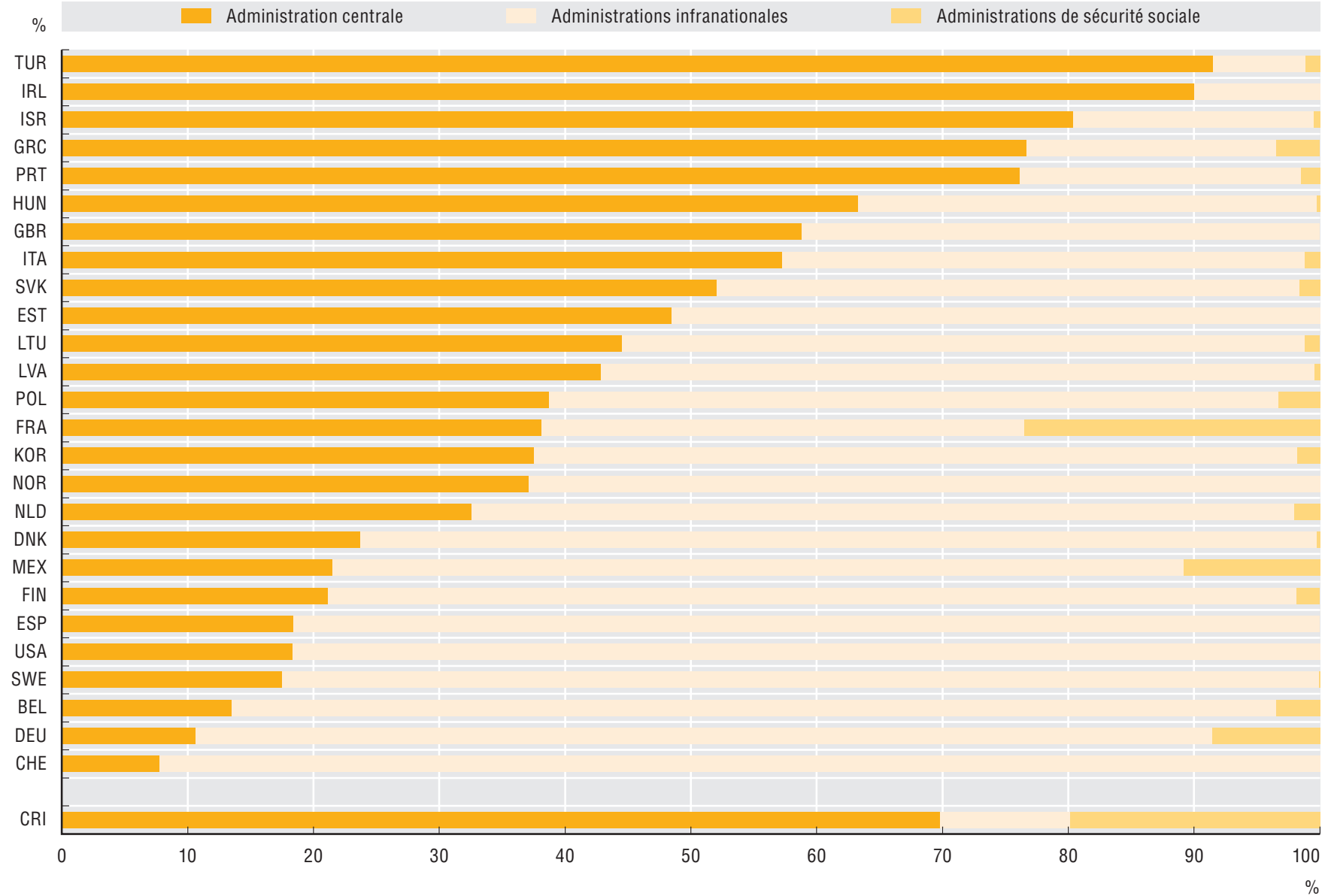

Source : Organisation internationale du travail (OIT), ILOSTAT (base de données), Emploi public par secteurs et sous-secteurs des comptes nationaux.

\subsection{Pourcentage d'agents publics employés au niveau central, 2011 et 2017}

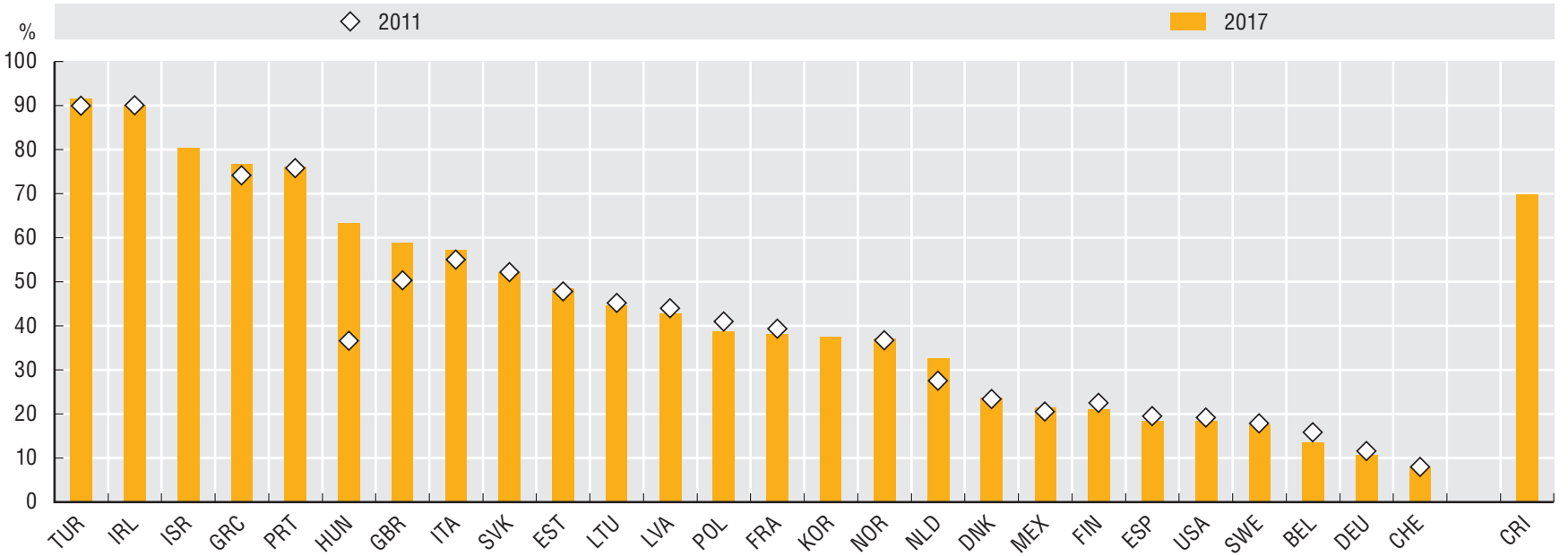

Sources : Organisation internationale du travail (OIT), ILOSTAT (base de données), Emploi public par secteurs et sous-secteurs des comptes nationaux.

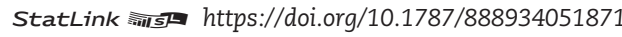


Une égale représentation des femmes et des hommes dans l'emploi public est un indicateur important de progrès sur la voie de la constitution d'un effectif de personnel plus diversifié et plus représentatif de la société. Les administrations publiques sont censées montrer la voie à suivre et fixer des normes en matière de respect de l'égalité femmes-hommes et de promotion de la diversité en leur sein. Par conséquent, encourager une plus grande diversité des effectifs d'agents publics peut servir la performance des institutions et favoriser la mise en œuvre de politiques et la prestation de services qui répondent mieux aux besoins de la population.

Dans la zone OCDE, les femmes sont surreprésentées dans l'effectif total des administrations publiques (59,6\%), malgré d'importantes disparités entre les pays. En 2017, seul un quart des postes que comptent les administrations publiques turques étaient occupés par des femmes, alors que cette proportion se situait entre $69 \%$ et $71,3 \%$ dans les pays nordiques (Danemark, Finlande, Norvège et Suède). Certains pays, comme la Grèce et le Mexique, étaient proches d'un état de parité des sexes au sein de leurs effectifs d'agents publics (respectivement $51,5 \%$ et $48 \%$ ). L'évolution observée à l'échelle de la zone OCDE montre un accroissement de la part des femmes dans l'emploi public de 2011 à 2017. Au Japon, par exemple, ce chiffre a progressé de près de 3 points entre 2011 et 2017.

Dans les pays de l'OCDE, la proportion de femmes est généralement plus élevée dans l'emploi public (60,1\%) que dans l'emploi total (45,9\%). Ce constat se vérifie dans tous les pays en question sauf les Pays-Bas et la Turquie, où les femmes sont en proportion légèrement moins nombreuses à travailler dans les administrations publiques. L'écart entre la part des postes d'agent public occupés par des femmes et la part des femmes dans l'emploi total atteint pas moins de 20 points dans des pays tels que le Danemark, la Finlande, la Norvège et la Suède. Parallèlement, c'est aussi dans ces pays que la proportion de femmes dans l'emploi total est la plus forte. L'une des raisons pour lesquelles les femmes employées dans les administrations publiques sont plus nombreuses que dans l'emploi total, en termes relatifs, réside dans le fait que certains des principaux métiers exercés dans le cadre des administrations publiques, par exemple ceux d'enseignante et d'infirmière, sont traditionnellement considérés comme des «métiers de femme ". Lutter contre de tels stéréotypes de genre et s'assurer que les femmes ont accès à des professions diverses dans les administrations est un objectif crucial poursuivi par l'ensemble des pays de l'OCDE. La transition vers une diversité et une inclusivité plus grandes - et une efficacité renforcée - des effectifs d'agents publics passe notamment par la garantie donnée aux femmes de pouvoir accéder et concourir avec les mêmes chances que les hommes à des postes de direction et de décision (où les hommes sont traditionnellement dominants).

\section{Méthodologie et définitions}

Ces données sur l'emploi dans le secteur public ont été réunies par l'OIT dans le cadre de sa base de données ILOSTAT. Elles sont, sauf indication contraire, extraites de l'Enquête sur la population active. L'emploi public englobe à la fois l'emploi dans les administrations publiques et l'emploi dans les entreprises et sociétés résidentes à capitaux publics. Les données correspondent au nombre total de personnes employées directement par ces institutions, quel que soit le type de contrat de travail qui les lie et la durée du travail. Les personnes employées regroupent toutes celles en âge de travailler qui, au cours d'une brève période déterminée, ont occupé un emploi rémunéré ou exercé un travail indépendant.

\section{Pour en savoir plus}

OCDE (2019), Recommandation du Conseil sur le leadership et les aptitudes de la fonction publique, OCDE, Paris, https:// legalinstruments.oecd.org/fr/instruments/OECD-LEGAL-0445.

OCDE (2017), Skills for a High Performing Civil Service, Examens de l'OCDE sur la gouvernance publique, Éditions OCDE, Paris, https://doi.org/10.1787/9789264280724-en.

OCDE (2014), Women, Government and Policy Making in OECD countries: Fostering Diversity for Inclusive Growth, Éditions OCDE, Paris, https://doi.org/10.1787/9789264210745-en.

\section{Notes relatives aux graphiques}

On ne dispose pas de données pour l'Autriche, l'Irlande, l'Islande, Israël, le Luxembourg, la Nouvelle-Zélande, la République tchèque et la Slovénie. Les données relatives à l'Allemagne, au Danemark et à la Suisse proviennent de fichiers administratifs ou d'enquêtes auprès des entités concernées. Les données pour le Japon portent sur2010 au lieu de 2011. Les données pour le Brésil portent sur 2012 au lieu de 2011. Les données pour la Suisse portent sur 2015 au lieu de 2017 et celles pour l'Indonésie sur 2016 au lieu de 2017. 


\section{5. Égalité femmes-hommes dans l'emploi du secteur public, 2011 et 2017}

$\diamond 2011$

2017

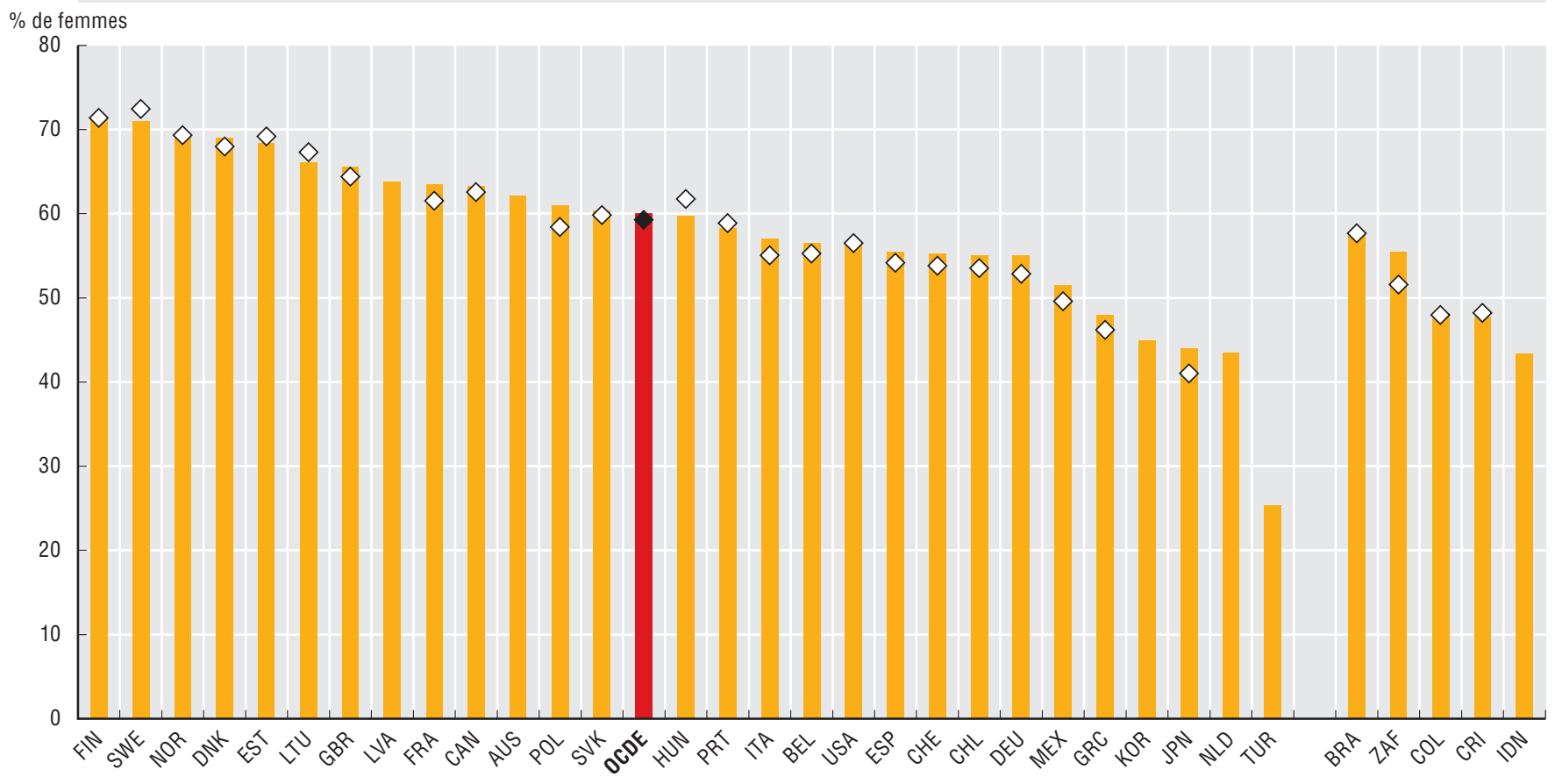

Source : Organisation internationale du travail (OIT), ILOSTAT (base de données), Employment by sex and institutional sector. Les données relatives à la Corée, l'Italie et le Portugal ont été fournies par les autorités du pays.

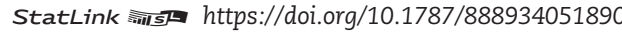

\section{6. Égalité femmes-hommes dans l'emploi total, 2011 et 2017}

$\diamond 2011$

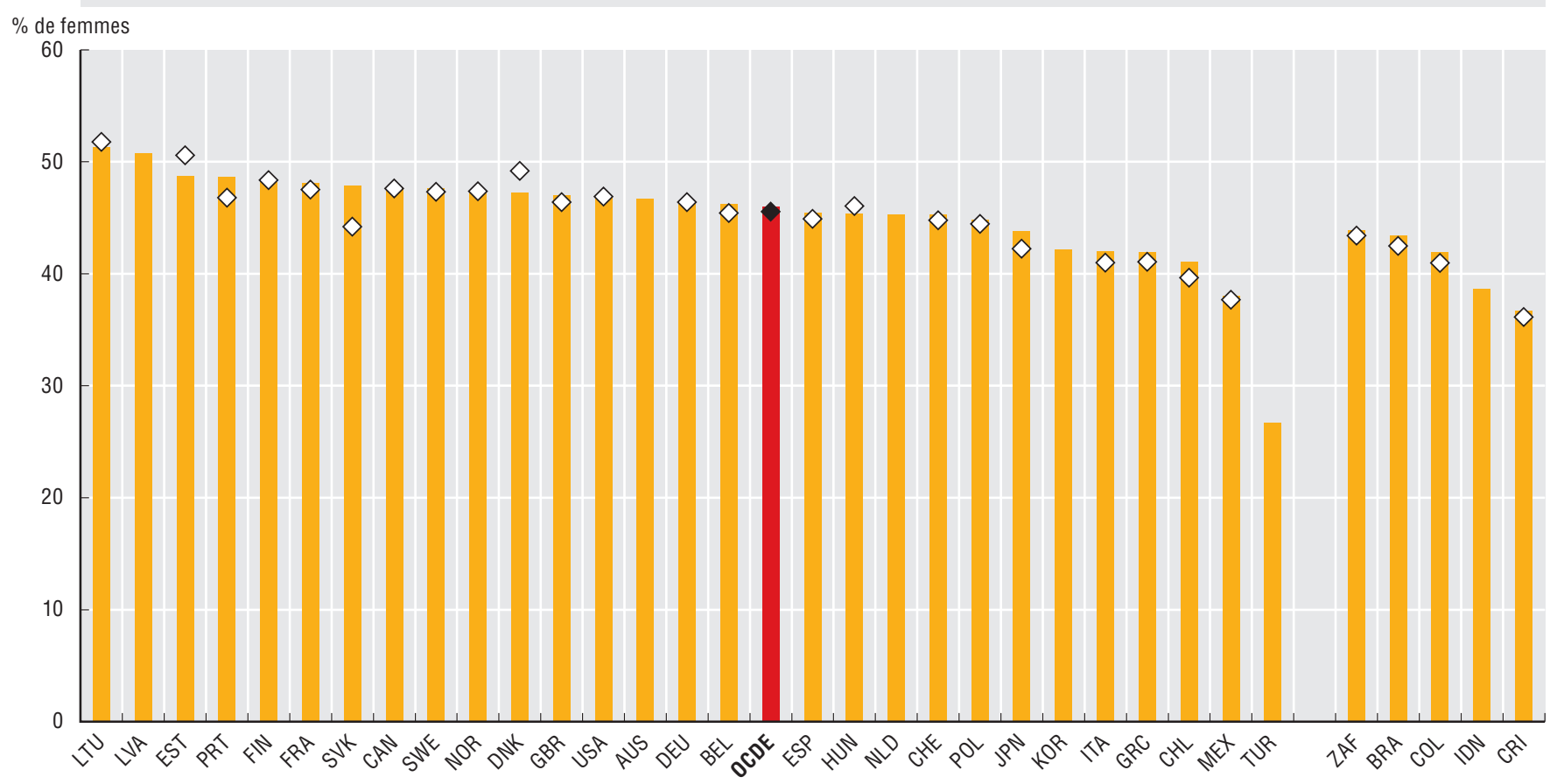

Source : Organisation internationale du travail (OIT), ILOSTAT (base de données), Employment by sex and institutional sector. Les données relatives à la Corée et au Portugal ont été fournies par les autorités du pays. 
Les gouvernements et les parlements sont des acteurs essentiels du déploiement d'efforts institutionnels visant à promouvoir l'égalité femmes-hommes et la diversité dans la vie publique. Les lois et politiques à l'origine du cadre législatif et réglementaire de promotion de l'égalité femmeshommes, ainsi que de prévention des discriminations de genre et de lutte contre ces discriminations, peuvent être mises en chantier, débattues et adoptées au sein du parlement. Ces lois et politiques aident à façonner les comportements sociétaux à l'égard de la place des femmes, de leurs capacités et de leurs responsabilités. Compte tenu du rôle ainsi joué par les parlements, il est important que ces derniers s'emploient eux-mêmes à répondre aux besoins des femmes et des hommes et expriment les valeurs qu'ils s'attachent à porter haut dans le cadre des processus législatifs. Toutefois, l'égalité femmeshommes en politique ne se limite pas à l'égale occupation des sièges et des fonctions : il est également crucial d'obtenir un engagement coordonné à l'échelle de l'administration tout entière et de mettre en place des mécanismes clairement définis et efficaces permettant de traduire les politiques, les services et les budgets publics en retombées concrètes favorables pour les hommes et les femmes provenant de milieux divers.

En 2019, les femmes occupaient, dans les pays de l'OCDE, en moyenne $30,1 \%$ des sièges de la chambre basse du parlement ou du parlement lui-même dans le cas des institutions monocamérales, variant de plus de $45 \%$ en Finlande, au Mexique et en Suède à moins de $15 \%$ en Hongrie et au Japon. On observe dans les pays de l'OCDE une évolution globalement positive de la représentation des femmes à la chambre basse ou unique du parlement, cet indicateur ayant progressé en moyenne de 3,8 points entre 2012 et 2019. Au cours de cette période, la France, l'Italie et le Mexique ont enregistré une augmentation régulière et forte (supérieure à 10 points) du nombre de femmes élues à la chambre basse ou unique du parlement. Le plus souvent, ce résultat peut s'expliquer par les changements intervenus dans le paysage politique et par des initiatives favorisant l'égalité femmes-hommes. En France, par exemple, la décision du parti actuellement au pouvoir de présenter des candidates dans les circonscriptions électorales qu'il était susceptible de remporter a eu un effet positif sur ses résultats aux législatives de 2017, aucun parti n'ayant auparavant obtenu une proportion d'élues aussi forte. Faire en sorte qu'un équilibre perdure en termes de représentation des femmes et des hommes dans le processus décisionnel exige une vigilance permanente. Entre 2015 et 2019, l'Allemagne, les Pays-Bas et la Slovénie ont connu à cet égard d'importants reculs (respectivement $-5,6,-6$ et $-12,3$ points).

En moyenne des pays de l'OCDE, les femmes occupaient un tiers des postes de ministre du gouvernement fédéral ou central en 2019, proportion en augmentation de plus de 6 points de pourcentage depuis 2012. La part des femmes dans un gouvernement peut constituer un signal fort de son engagement en faveur de l'égalité femmes-hommes. De plus en plus de pays de l'OCDE expriment une telle volonté politique : les gouvernements du Canada, de la France et de la Suède respectent la parité des sexes depuis 2015. En Espagne, les femmes détenaient près de $65 \%$ des portefeuilles ministériels en 2019 , soit une hausse notable de près de 34 points par rapport à 2015. Malgré une évolution dans l'ensemble positive, plusieurs pays ont vu leur situation se dégrader. En effet, entre 2015 et 2019, la proportion de femmes au sein des gouvernements finlandais, lithuanien et slovène a reculé de respectivement de $25,21,19,17$ et 16 points de pourcentage.

\section{Méthodologie et définitions}

Ces données concernant l'égalité femmes-hommes au parlement se rapportent au partage des postes à la chambre basse du parlement ou au parlement luimême dans le cas des institutions monocamérales. Elles proviennent de la base de données PARLINE de l'Union interparlementaire (UIP). Les quotas légaux sont inscrits dans le droit électoral du pays, dans sa législation relative aux partis politiques ou dans d'autres textes de loi comparables. Par définition, les quotas fondés sur le droit électoral ou sur la législation relative aux partis politiques obligent toutes les entités politiques à les appliquer de la même façon. Les données relatives aux quotas sont issues de la base de données mondiale sur les quotas de femmes de l'Institut international pour la démocratie et l'assistance électorale (IDEA).

Les données sur l'égalité femmes-hommes aux postes de ministres dans les gouvernements nationaux sont tirées de la base de données sur les femmes en politique tenue par l'UIP. Elles correspondent au pourcentage de ministres de sexe féminin en poste au $1^{\mathrm{er}}$ janvier de chaque année de référence. Elles indiquent la part des femmes dans l'effectif total des ministres, y compris les vice-premiers ministres et les vice-ministres. Les premiers ministres ou chefs de gouvernement ont aussi été pris en compte lorsqu'ils détenaient un portefeuille ministériel. Les vice-présidents et les dirigeants d'une instance officielle ou d'un organisme public n'ont pas été comptabilisés.

\section{Pour en savoir plus}

OCDE (2019), Fast Forward to Gender Equality: Mainstreaming Implementation and Leadership, OCDE, Paris, https://doi. org/10.1787/g2g9faa5-en.

OCDE (2018), L'égalité des sexes au Canada : intégration, gouvernance et budgétisation, Éditions OCDE, Paris.

OCDE (2016), Recommandation de 2015 du Conseil de l'OCDE sur l'égalité hommes-femmes dans la vie publique, Éditions OCDE, Paris.

\section{Notes relatives aux graphiques}

3.7. Les barres de couleur orange pâle représentent les pays où un quota légal de candidates était en vigueur en avril 2019 pour la chambre basse ou unique du parlement. Les données correspondent à la proportion de parlementaires de sexe féminin enregistrée au $1^{\text {er }}$ janvier 2019, au $1^{\text {er }}$ décembre 2015 et au 31 octobre 2012. Les pourcentages représentent le nombre de femmes parlementaires en proportion du total des sièges pourvus.

3.8. Les données correspondent au pourcentage de ministres de sexe féminin en poste au $1^{\mathrm{er}}$ janvier de chaque année de référence.

Concernant données sur Israël, voir http://dx.doi.org/10.1787/888932315602. 
3.7. Égalité femmes-hommes au parlement et quotas légaux genrés, 2012, 2015 et 2019

Chambre basse ou unique du parlement

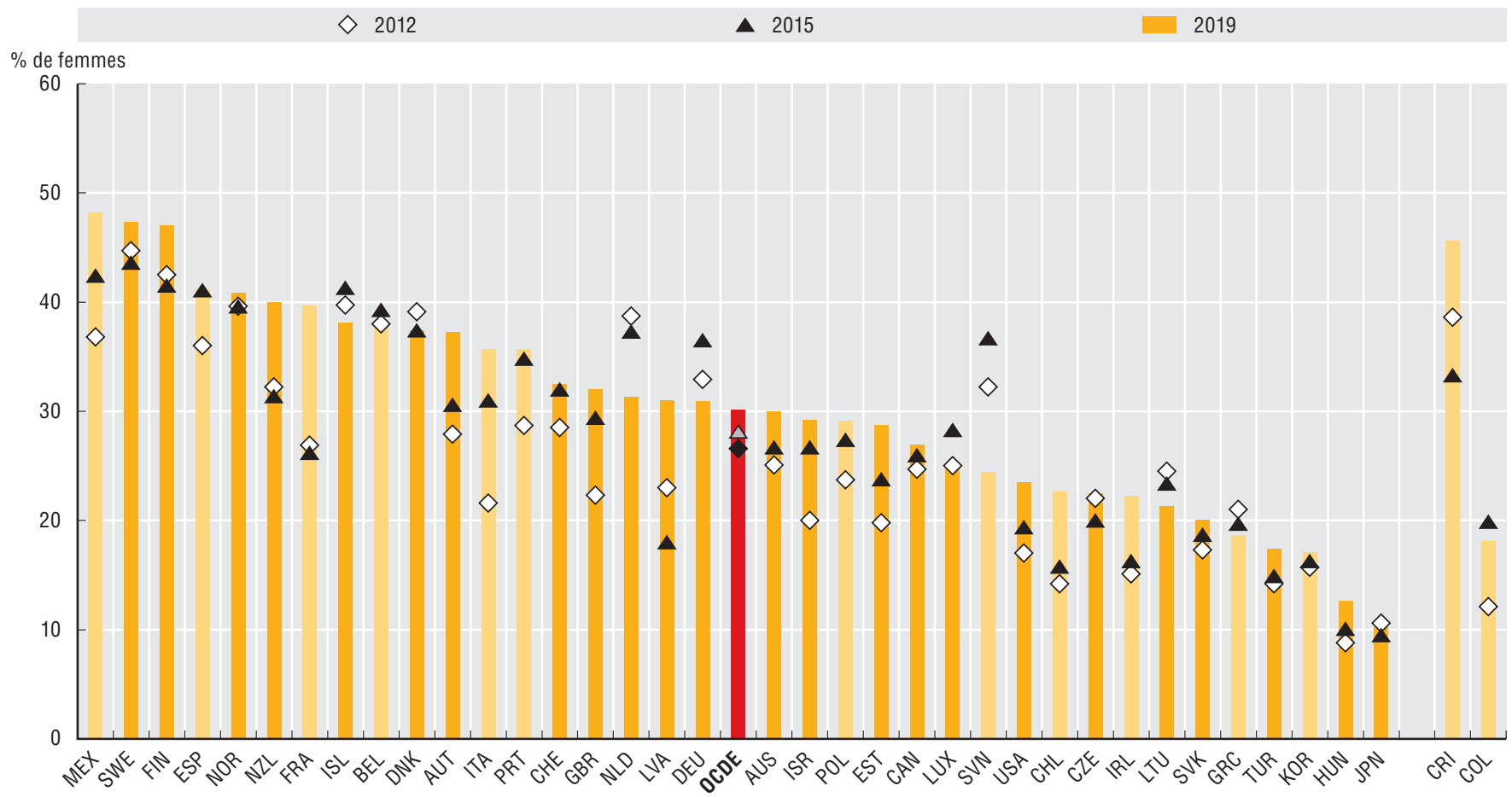

Sources : Union interparlementaire (UIP), PARLINE (base de données) ; base de données mondiales sur les quotas de femmes de l'Institut international pour la démocratie et l'assistance électorale (IDEA). Les données relatives à la Finlande ont été fournies par les autorités du pays.

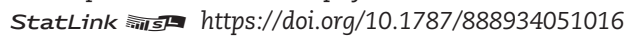

\section{8. Égalité femmes-hommes dans les ministères, 2012, 2015 et 2019}

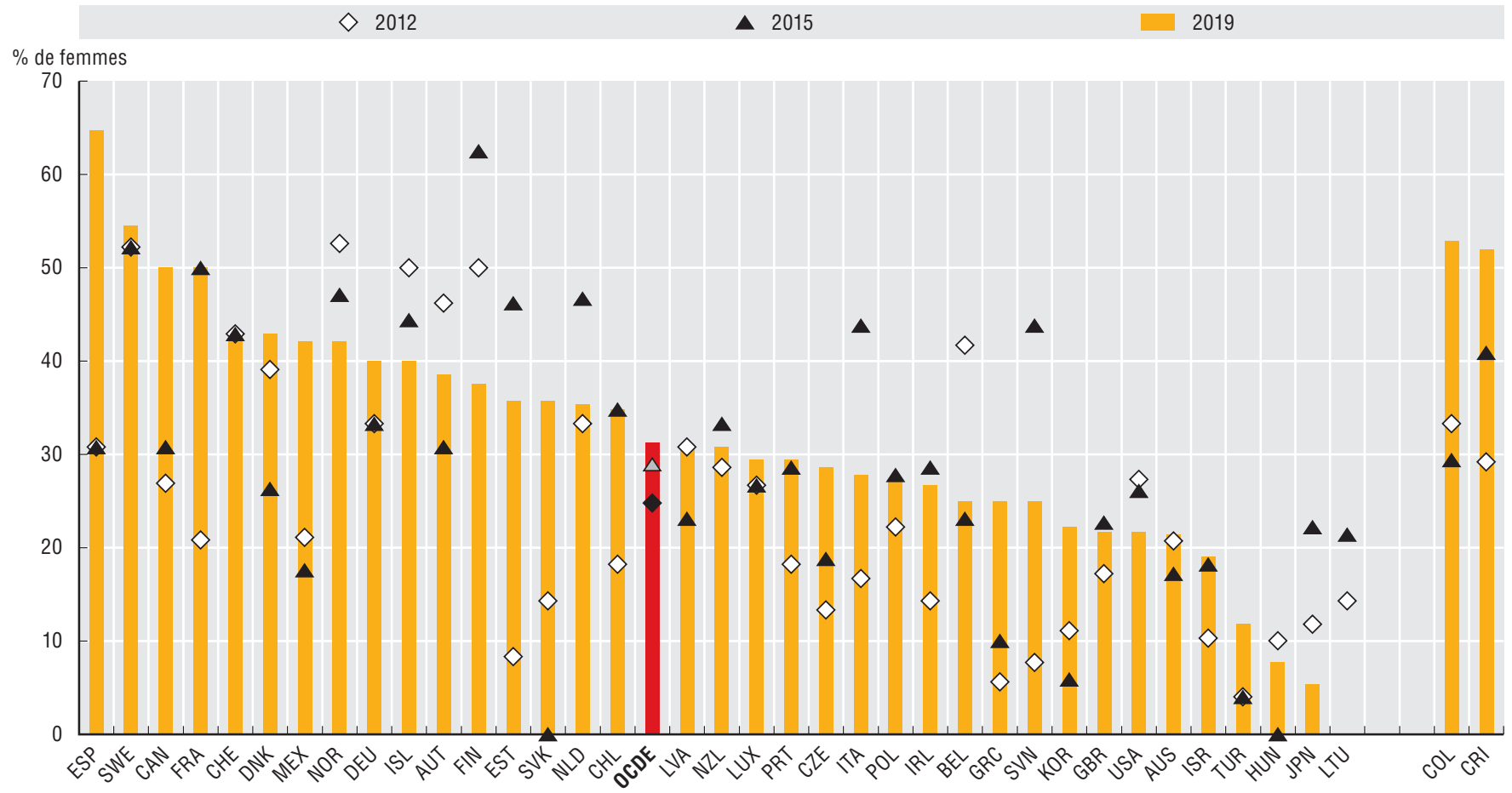

Source : Union interparlementaire (UIP), base de données sur les femmes en politique (2012, 2015 et 2019). 
Les pays de l'OCDE soulignent de plus en plus qu'une égalité garantie entre les sexes au sein du pouvoir judiciaire est une question de gouvernance fondamentale en rapport avec l'équité, la transparence et l'application effective du principe de légalité. Une certaine diversité des effectifs de la justice, alors composés d'hommes et de femmes de différents milieux, peut conduire à une diversification des avis et des points de vue au sein du corps judiciaire. Elle peut aussi renforcer l'intégrité de ses membres, en développant la confiance des citoyens dans les services rendus par la justice. Une plus forte représentation des femmes dans les professions judiciaires, en particulier à un niveau hiérarchique élevé, peut contribuer à éliminer les stéréotypes de genre et à inciter davantage les femmes à faire respecter leurs droits.

D'importants progrès en matière d'égalité femmes-hommes au sein des effectifs de la justice ont été réalisés dans divers pays de l'OCDE. En 2016, les femmes représentaient en moyenne $50 \%$ de l'ensemble des juges de carrière, allant de $20 \%$ au Mexique à 77 \% en Lettonie. La Grèce, l'Irlande, le Luxembourg, la Suisse et la Turquie ont enregistré entre 2010 et 2016 une augmentation d'au moins 10 points de pourcentage dans la proportion de femmes dans leur appareil judiciaire. Parmi les pays de l'OCDE pour lesquels des séries chronologiques sont disponibles, on a observé un accroissement général de la proportion de femmes dans les effectifs de juges de carrière, celle-ci étant passée de $44 \%$ en 2010 à $50 \%$ en 2016. Lorsqu'on compare les ratios femmes-hommes dans la magistrature des différents pays, il est important de prendre en compte les spécificités des systèmes juridiques et des schémas de développement professionnel nationaux. Il existe par exemple des différences entre le système de droit romain et le système de common law : dans le premier système, les femmes peuvent être recrutées directement après leurs études de droit, avant qu'elles ne soient confrontées à d'éventuelles interruptions de carrière, tandis que dans le second système, elles sont soumises à une obligation réglementaire d'acquérir au moins cinq ou sept ans d'expérience dans l'exercice d'une profession juridique, une fois leur diplôme obtenu, pour prétendre à un poste de magistrat. Ainsi, au Canada, les juges fédéraux sont choisis parmi les professionnels du droit en exercice qui sont inscrits depuis au moins 10 ans au barreau d'une province ou d'un territoire ou directement parmi les juges provinciaux ou territoriaux. Par voie de conséquence, cette contrainte est susceptible de retarder le démarrage de la carrière des femmes au sein de l'appareil judiciaire, les obstacles potentiels à la continuité de leur carrière limitant la proportion de femmes nommées à un poste élevé.

Malgré une augmentation récente de leur nombre total dans les effectifs de la justice, les femmes restent sous-représentées dans les juridictions de niveau supérieur. En 2016, dans les pays de l'OCDE, elles occupaient en moyenne $56 \%$ et $48 \%$ des postes au sein respectivement des tribunaux de première instance et d'appel, mais seulement $33 \%$ des postes dans les juridictions de dernier ressort. Ce déséquilibre peut s'expliquer par la persistance de préjugés et de stéréotypes de genre dont continuent d'être victimes les jeunes femmes, en particulier dans la mesure où, souvent, elles ne correspondent pas à l'image que l'on se fait d'un juge. En outre, le manque d'incitations et de possibilités de développement professionnel dont souffrent les femmes tout au long de la phase de recrutement peut également nuire à leur représentation parmi les avocats senior qui forment d'ordinaire le vivier dans lequel sont choisis les hauts magistrats.

\section{Méthodologie et définitions}

Les données sur l'égalité femmes-hommes chez les juges de carrière correspondent à la proportion totale de femmes qui occupaient un poste de magistrat en 2010 et en 2016, tous niveaux de juridiction confondus. Elles proviennent de la Commission européenne pour l'efficacité de la justice (CEPEJ) du Conseil de l'Europe et de l'Enquête sur les pratiques tenant compte de l'égalité femmes-hommes dans l'appareil judiciaire menée en 2017 par l'OCDE.

Les données relatives à l'égalité femmes-hommes chez les juges de carrière par niveau de juridiction correspondent à la proportion de femmes qui occupaient en 2016 un poste de magistrat dans une juridiction de première instance, d'appel ou de juridiction suprême. Elles proviennent de la CEPEJ et des résultats de l'Enquête 2017 de l'OCDE sur les pratiques tenant compte de l'égalité femmes-hommes dans l'appareil judiciaire.

Les tribunaux de première instance sont les juridictions dans lesquelles s'ouvrent les procédures judiciaires ; les tribunaux d'appel réexaminent des décisions rendues par des juridictions inférieures ; les juridictions suprêmes sont situées au sommet de la hiérarchie des nombreuses juridictions et fonctionnent essentiellement comme des tribunaux d'appel, réexaminant les décisions de juridictions inférieures ou de niveau intermédiaire.

Les juges de carrière sont ceux qui sont recrutés, formés et rémunérés pour exercer la fonction de juge à titre d'activité professionnelle principale dans le cadre d'un tribunal de première instance, d'un tribunal d'appel ou d'une juridiction suprême.

\section{Pour en savoir plus}

OCDE (2019), Fast Forward to Gender Equality: Mainstreaming, Implementation and Leadership, Éditions OCDE, Paris, https:// doi.org/10.1787/g2g9faa5-en.

OCDE (2016), Recommandation de 2015 du Conseil de l'OCDE sur l'égalité hommes-femmes dans la vie publique, Éditions OCDE, Paris, https://doi.org/10.1787/9789264252875-fr.

\section{Notes relatives aux graphiques}

La moyenne de la zone OCDE correspond à une moyenne arithmétique simple.

3.9. Les données relatives à la Lettonie et au Luxembourg portent sur 2014 au lieu de 2016. Les données relatives au Danemark, à Israël et au Luxembourg ne sont pas inclues dans la moyenne de l'OCDE en raison de l'absence de séries chronologiques.

Concernant les données concernant sur Israël, voir http://dx.doi.org/ $10.1787 / 888932315602$. 


\section{9. Égalité femmes-hommes chez les juges de carrière, 2010 et 2016}

Tous niveaux de juridiction confondus

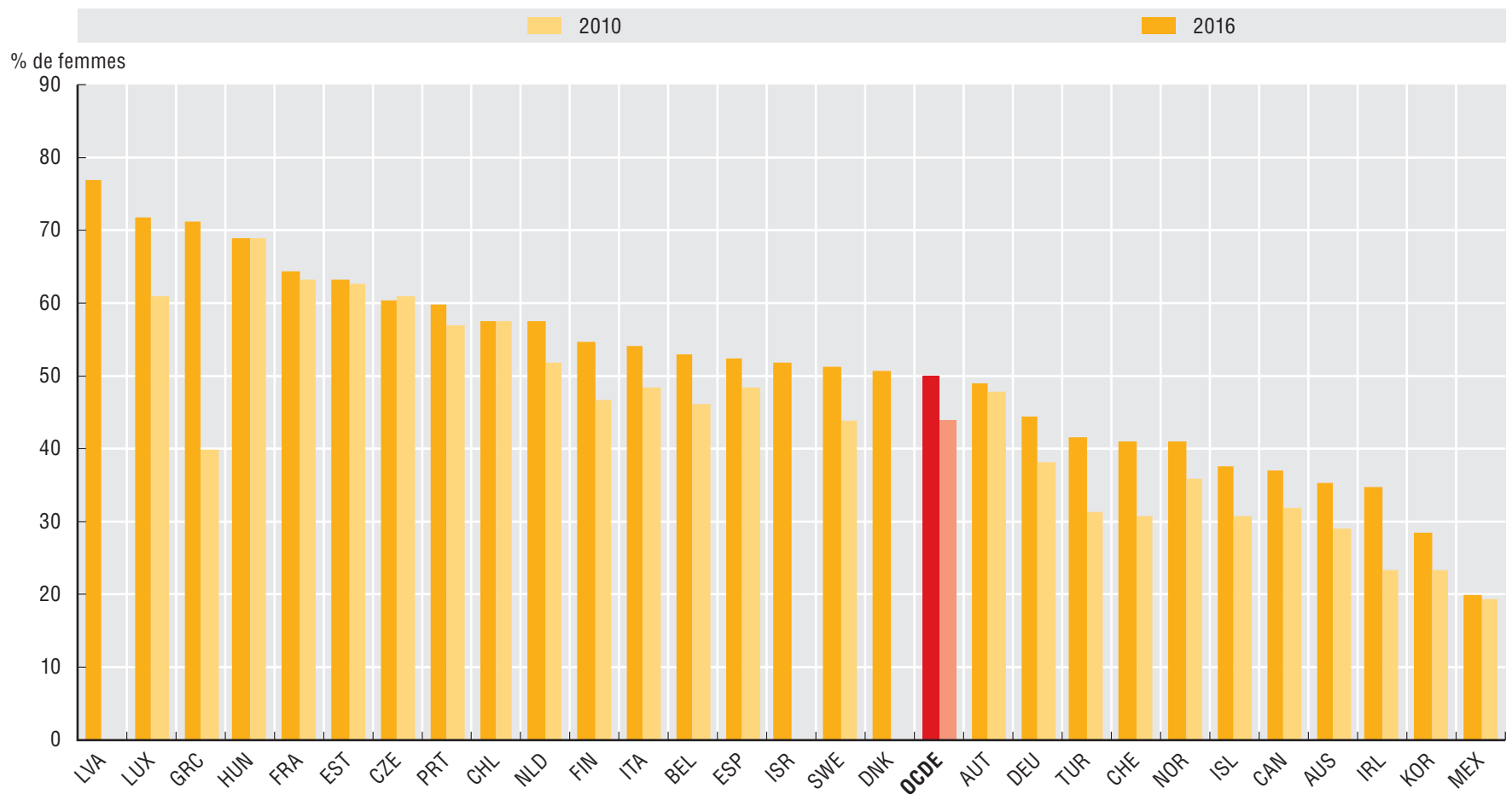

Source : Enquête 2017 de l'OCDE sur les pratiques tenant compte de l'égalité femmes-hommes dans l'appareil judiciaire. Les données relatives à l'Allemagne et le Portugal ont été fournies par les autorités du pays.

StatLink त्नाज़ https://doi.org/10.1787/888934051928

3.10. Égalité femmes-hommes chez les juges de carrière, par niveau de juridiction, 2016

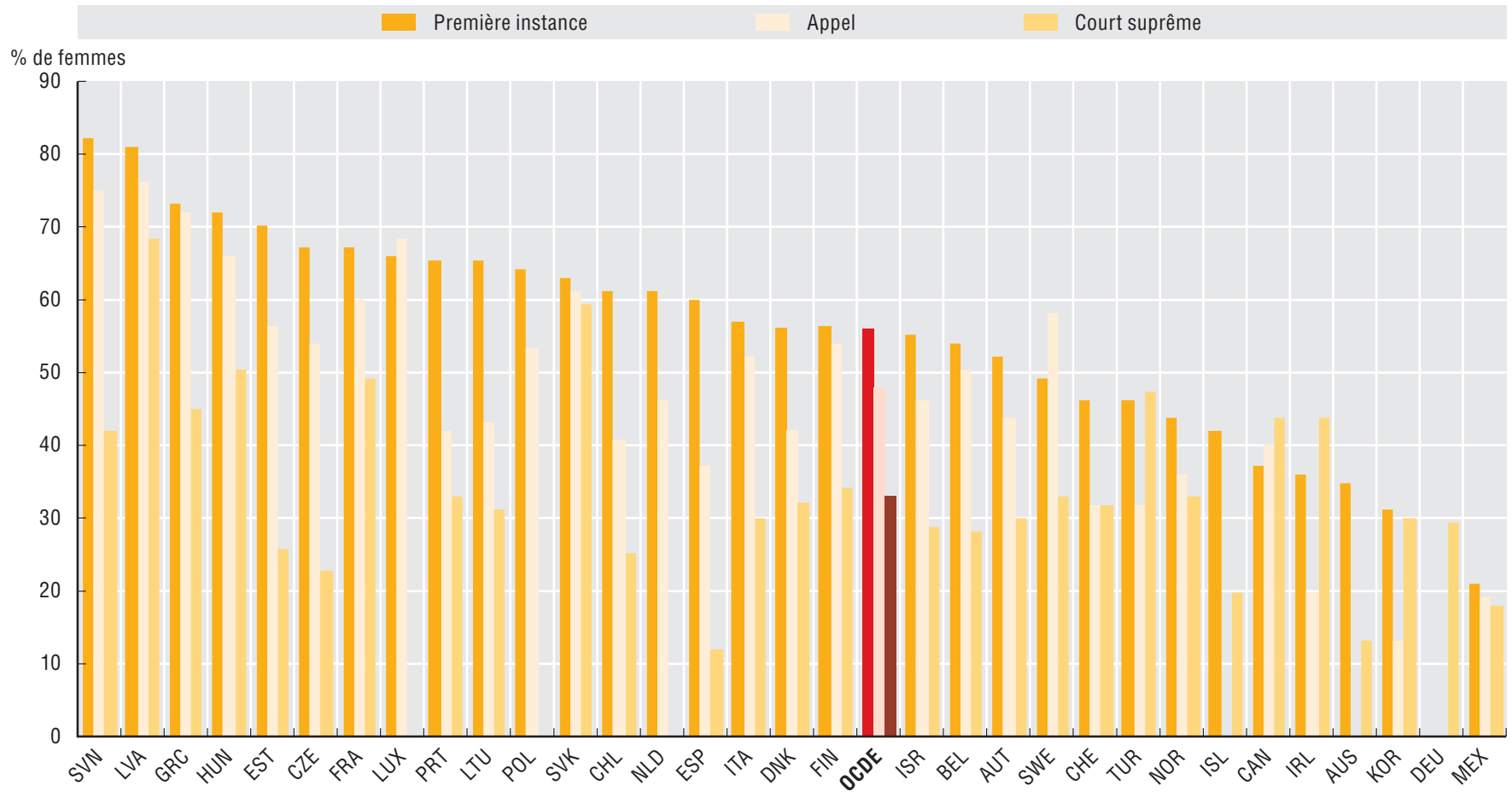

Sources : Commission européenne pour l'efficacité de la justice (CEPEJ) du Conseil de l'Europe (2016) ; enquête 2017 de l'OCDE sur les pratiques tenant compte de l'égalité femmes-hommes dans l'appareil judiciaire. Les données relatives à l'Allemagne et le Portugal ont été fournies par les autorités du pays. 


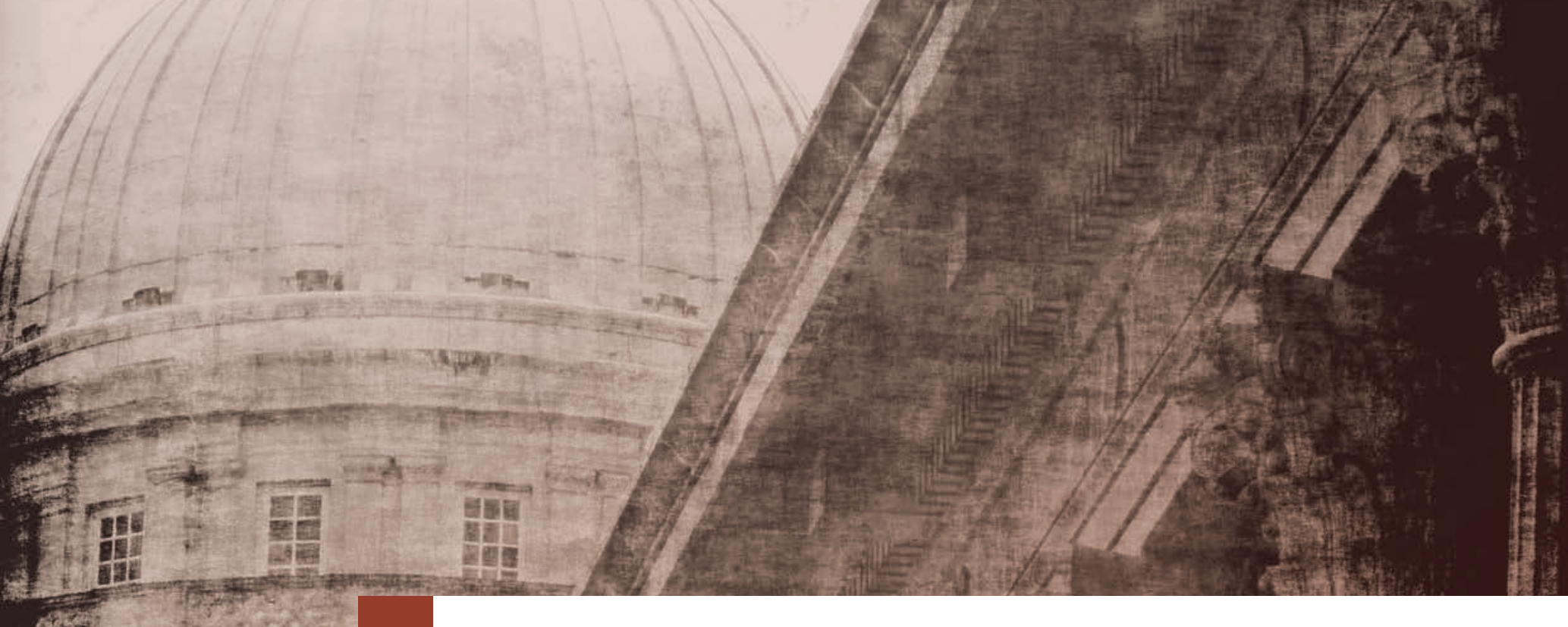

\section{INSTITUTIONS}

Rôles et responsabilités des centres de gouvernement Rôle du centre de gouvernement dans le gouvernement ouvert Institutionnalisation des systèmes d'évaluation des politiques publiques

Capitalisation des évaluations de politiques publiques 
Le centre de gouvernement, qui porte différentes appellations selon les pays - Bureau du Cabinet, Bureau du Président, Conseil privé, Secrétariat général du Gouvernement, etc. -, est la structure chargée d'assurer un appui au Premier ministre et au Conseil des ministres (c'est-à-dire la réunion régulière des ministres). Le centre de gouvernement comprend l'organe placé au service du chef de gouvernement et du Conseil des ministres ainsi que l'unité spécifiquement placée au service du chef de gouvernement (le Secrétariat du Premier ministre, par exemple).

Le périmètre des responsabilités confiées à cette structure varie considérablement selon les pays. En Grèce, le centre de gouvernement assure à titre exclusif onze missions. Il est notamment chargé de la communication avec le grand public et des formulation et analyse des politiques publiques. En Irlande et au Japon, seule la préparation du Conseil des ministres relève de la responsabilité exclusive du centre de gouvernement. Quand on tient compte aussi bien des responsabilités exclusives que des responsabilités partagées, on constate que les pays où le centre de gouvernement exerce l'influence la plus large sont le Mexique, les États-Unis et la Grèce (seize domaines de responsabilité au total). À l'autre extrémité de l'éventail, aux Pays-Bas, le centre de gouvernement est chargé de fonctions de coordination et de communication mais n'intervient pas sur le fond des politiques - il n'est ainsi pas chargé de préparer le programme gouvernemental ni d'analyser les politiques.

Tous les pays de l'OCDE confient la préparation des Conseils des ministres au centre de gouvernement. La préparation de ces réunions est généralement coordonnée grâce à des notes d'information, à des comités ministériels et à des réunions interministérielles ou interservices.

De plus, le centre de gouvernement joue un rôle de pré-examen des questions soumises au Conseil des ministres. Tous les centres de gouvernement veillent au respect des procédures de préparation et de présentation de ces questions et la majorité d'entre eux vérifient également que chaque question a fait l'objet d'une consultation suffisante (des parties prenantes extérieures, par exemple). En Estonie, le ministère de la Justice intervient également quand la question est un projet de loi. Au contraire, en Finlande, au Japon et en Suisse, le centre de gouvernement n'intervient pas pour vérifier que la procédure de consultation a été respectée. Dans 28 pays, le centre de gouvernement est chargé de vérifier que les questions soumises au Conseil des ministres sont conformes au programme gouvernemental. En Finlande, au Japon et en Norvège, cette responsabilité revient au ministère qui porte le projet.

S'agissant du contrôle du chiffrage des propositions soumises au Conseil des ministres, le centre de gouvernement assume seul cette responsabilité dans 11 pays et la partage avec d'autres acteurs dans 9 pays. Le ministère des Finances intervient dans ce contrôle en Australie, en Belgique, au Canada, en Espagne, en Estonie, en Islande, en Norvège, aux Pays-Bas, en République slovaque, en République tchèque, en Slovénie et en Turquie.

Dans l'ensemble de l'OCDE, la tâche consistant à coordonner les politiques à l'échelle de l'ensemble du gouvernement incombe au centre de gouvernement dans tous les pays, que ce soit à titre exclusif ou en collaboration avec une autre entité. 4 pays (l'Australie, l'Estonie, l'Islande et le Mexique) indiquent que leur ministère des Finances participe aussi à cette tâche et 4 autres (l'Irlande, l'Italie, la Norvège et la Suède) précisent que chaque ministère est chargé de veiller à cette coordination. Les mécanismes privilégiés en matière de coordination sont la tenue régulière de conseils des ministres (citée par 28 pays), suivie de l'organisation de réunions ad hoc entre hauts fonctionnaires
(26 pays). L'Australie et les Pays-Bas ont recours à un large éventail d'outils, y compris la gestion de la performance et la production de directives écrites aux ministères. À l'inverse, l'Espagne et la Hongrie n'assurent la coordination que par la tenue régulière de Conseils des ministres.

Le centre de gouvernement exerce des fonctions de planification stratégique dans tous les pays de l'OCDE, à l'exception de la Turquie où cette tâche est assurée par le ministère du Développement. Dans 6 pays (Chili, Estonie, Islande, Lituanie, Mexique et Royaume-Uni), le centre de gouvernement partage cette responsabilité avec le ministère des Finances.

La planification et la gestion de la transition politique (arrivée d'un nouveau gouvernement) relèvent de la seule responsabilité du centre de gouvernement dans 21 pays et sont partagées avec d'autres entités dans 11 autres pays. Dans 5 de ces 11 pays, il appartient à chaque ministère d'informer la nouvelle équipe gouvernementale. Les relations avec le parlement relèvent de la responsabilité du centre de gouvernement dans tous les pays de l'OCDE. Dans 16 pays de l'OCDE, le centre de gouvernement a la responsabilité exclusive de cette tâche. Dans 18 autres pays, il s'agit d'une responsabilité partagée avec les divers ministères, en fonction du thème examiné.

\section{Méthodologie et définitions}

Ces données proviennent des résultats de l'enquête 2017 de l'OCDE sur l'organisation et les fonctions du centre de gouvernement à laquelle 34 pays de l'OCDE et 4 non-membres ont participé. Les personnes sondées sont de hauts responsables qui appuient et conseillent directement le chef de gouvernement et le Conseil des ministres. Leurs réponses portent sur l'année 2016.

Le centre de gouvernement est généralement composé du ministère ou secrétariat général de la présidence, du cabinet du Premier ministre et du secrétariat du Conseil des ministres, même si les fonctions correspondantes peuvent dans certains cas être assurées par des services basés au sein d'autres entités (par exemple, le ministère des Finances ou du Plan, le Bureau du budget).

\section{Pour en savoir plus}

OCDE (2018), Centre Stage II: The Organisation and Functions of the Centre of Government in OECD Countries, https://www.oecd.org/ gov/centre-stage-2.pdf.

OCDE (2014), Centre Stage II: The Organisation and Functions of the Centre of Government in OECD Countries, https://www.oecd.org/ gov/Centre-Stage-Report.pdf.

\section{Notes relatives aux graphiques}

Concernant les données sur Israël, voir http://doi.org/10.1787/888932315602

On ne dispose pas de données pour la Corée et la Pologne. Les données sur l'Italie, là où elles sont disponibles, concernent 2019.

On trouvera à l'Annexe $\mathrm{F}$ (en ligne) des indications sur le rôle du centre de gouvernement et d'autres entités dans le pré-examen des questions soumises au Conseil des ministres (4.3) et sur les mécanismes de coordination des politiques (4.4). 


\section{Rôles et responsabilités des centres de gouvernement}

4.1. Responsabilités du centre de gouvernement, 2016

\begin{tabular}{|c|c|c|c|c|c|c|c|}
\hline Pays & $\begin{array}{l}\text { Préparation du conseil des } \\
\text { ministres }\end{array}$ & Coordination des politiques & $\begin{array}{l}\text { Préparation de la transition } \\
\text { politique }\end{array}$ & Planification stratégique & $\begin{array}{l}\text { Préparation du programme } \\
\text { gouvernemental }\end{array}$ & $\begin{array}{l}\text { Suivi de la politique } \\
\text { gouvernementale }\end{array}$ & $\begin{array}{c}\text { Relations } \\
\text { avec le parlement }\end{array}$ \\
\hline Allemagne & $\bullet$ & $\bullet$ & $\bullet$ & $\bullet$ & $\bullet$ & $\bullet$ & 1 \\
\hline Australie & $\bullet$ & I & I & - & I & I & I \\
\hline Autriche & - & I & - & I & I & - & I \\
\hline Belgique & - & - & I & - & - & - & I \\
\hline Canada & - & - & - & - & - & - & - \\
\hline Chili & $\bullet$ & $\bullet$ & 1 & I & 1 & $\bullet$ & $\bullet$ \\
\hline Danemark & - & - & - & I & - & I & I \\
\hline Espagne & - & - & - & - & • & ! & - \\
\hline Estonie & - & I & - & I & - & - & - \\
\hline Etats-Unis & - & - & $\bullet$ & - & ! & ! & ! \\
\hline Finlande & $\bullet$ & $\bullet$ & $\bullet$ & $\bullet$ & 1 & $\bullet$ & $\bullet$ \\
\hline France & - & $\bullet$ & - & - & $\bullet$ & ! & - \\
\hline Grèce & - & - & $\bullet$ & $\bullet$ & $\bullet$ & $\bullet$ & $\bullet$ \\
\hline Hongrie & - & - & - & - & o & - & - \\
\hline Irlande & - & 1 & I & I & 0 & I & I \\
\hline Islande & $\bullet$ & $\bullet$ & $\bullet$ & 1 & $\bullet$ & 1 & $\bullet$ \\
\hline Israël & - & - & $\bullet$ & - & ! & - & I \\
\hline Italie & - & I & - & - & - & I & I \\
\hline Japon & - & I & I & I & I & 0 & I \\
\hline Lettonie & $\bullet$ & $\bullet$ & $\bullet$ & $\bullet$ & $\bullet$ & $\bullet$ & 1 \\
\hline Lituanie & - & - & - & ! & - & - & ! \\
\hline Luxembourg & - & - & - & - & I & I & - \\
\hline Mexique & - & 1 & ! & ! & I & 1 & I \\
\hline Norvège & $\bullet$ & 1 & $\bullet$ & 1 & 1 & O & 1 \\
\hline Nouvelle Zélande & - & - & 1 & - & - & - & - \\
\hline Pays-Bas & - & - & I & - & o & O & I \\
\hline Portugal & - & $\bullet$ & 0 & - & ! & 1 & - \\
\hline République Slovaque & $\bullet$ & $\bullet$ & 1 & $\bullet$ & $\bullet$ & $\bullet$ & $\bullet$ \\
\hline République Tchèque & $\bullet$ & $\bullet$ & $\bullet$ & 1 & 0 & 1 & 1 \\
\hline Royaume-Uni & - & - & - & I & - & I & - \\
\hline Slovénie & - & - & ! & I & ! & - & - \\
\hline Suède & $\bullet$ & 1 & $\bullet$ & $\bullet$ & $\bullet$ & I & 1 \\
\hline Suisse & $\bullet$ & 1 & 1 & $\bullet$ & $\bullet$ & $\bullet$ & 1 \\
\hline Turquie & - & - & & o & - & 1 & - \\
\hline \multicolumn{8}{|l|}{ Total OCDE } \\
\hline - & 34 & 24 & 21 & 20 & 18 & 16 & 16 \\
\hline 1 & 0 & 10 & 11 & 13 & 12 & 15 & 18 \\
\hline $\mathrm{O}$ & 0 & 0 & 1 & 1 & 4 & 3 & 0 \\
\hline Brésil & $\bullet$ & - & 1 & I & 1 & I & - \\
\hline Costa Rica & - & - & - & - & I & - & - \\
\hline
\end{tabular}

Source : Enquête 2017 de l'OCDE sur l'organisation et les fonctions du centre de gouvernement.

4.2. Mécanismes employés pour coordonner en amont l'examen des points inscrits à l'ordre du jour du Conseil des ministres, 2016

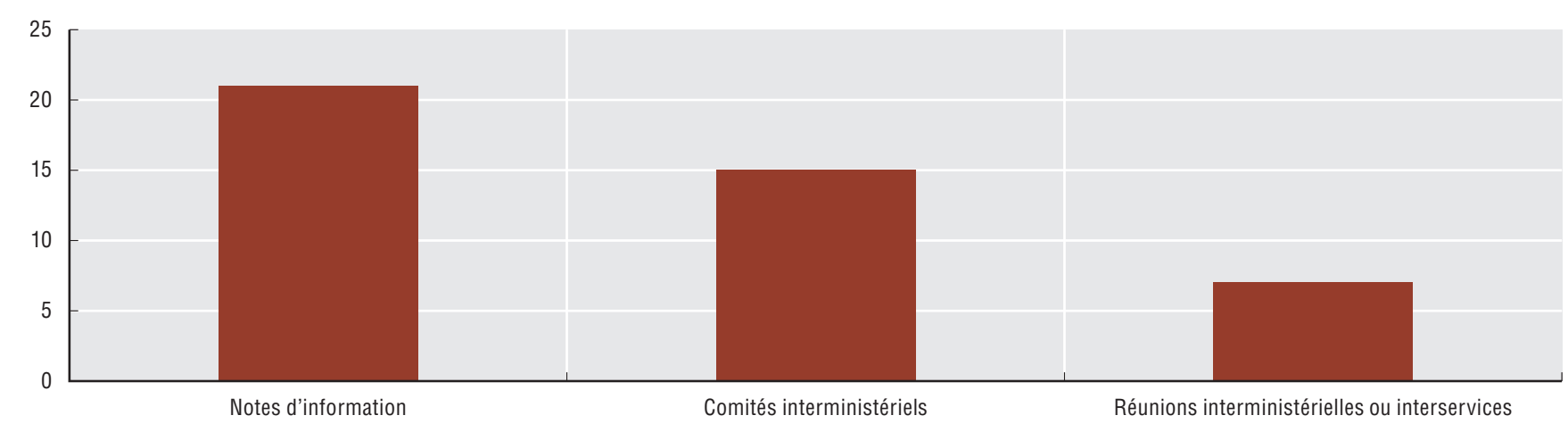

Source : Enquête 2017 de l'OCDE sur l'organisation et les fonctions du centre de gouvernement. 
On définit le gouvernement ouvert (c'est-à-dire l'ouverture de l'administration) comme une culture de gouvernance qui promeut les principes de transparence, d'intégrité, de redevabilité et de participation des parties prenantes, au service de la démocratie et de la croissance inclusive (OCDE 2017). Les stratégies et initiatives d'ouverture de l'administration étant de nature transversale, elles requièrent de robustes dispositifs de coordination institutionnelle. De tels dispositifs permettent aux pays de veiller à ce que les objectifs soient atteints et à ce qu'un large éventail de parties prenantes soient suffisamment associées à l'action publique, y compris les institutions indépendantes, les organisations de la société civile, le monde universitaire et les médias, parmi d'autres. Le centre de gouvernement occupe une position stratégique en termes de coordination, de mise en œuvre, de suivi et d'évaluation des stratégies et initiatives liées au gouvernement ouvert, car celles-ci exigent une démarche commune à l'ensemble de la sphère publique et, sur le plan politique, autorisent une influence, un engagement, un soutien et un leadership forts.

Dans la plupart des pays de l'OCDE, le centre de gouvernement joue un rôle important dans la conception, la mise en œuvre, la diffusion, la coordination, le suivi et l'évaluation des stratégies et initiatives liées au gouvernement ouvert. En Australie, en Estonie, en Israël, en Lettonie, en Lituanie, au Mexique, en République tchèque et au Royaume-Uni, le centre de gouvernement est associé à l'ensemble du cycle de vie de ces stratégies. Au Canada, en France, au Japon et en Slovénie, le centre de gouvernement n'intervient qu'à une étape de ce processus. En Belgique, au Danemark, en Espagne, en Finlande, en Irlande, en Norvège, en Suède et en Suisse, d'autres institutions que le centre de gouvernement prennent en charge le programme national de gouvernement ouvert. Par exemple, en Espagne, ce rôle est joué par le ministère de la Politique térritoriale et de l'Administration publique, et en Finlande, par le minisère des Finances.

Dans 28 pays de l'OCDE, le centre de gouvernement consulte directement les parties prenantes sur les politiques. Parmi les parties prenantes ainsi consultées, les pays interrogés citent principalement les citoyens et les partenaires sociaux (les syndicats, par exemple), puis les universités. L'Australie, l'Estonie, la Grèce, l'Irlande, le Japon, la Lituanie, le Mexique et le Royaume-Uni consultent un large éventail de parties prenantes, y compris les organisations de la société civile, les médias, les institutions étatiques indépendantes et les représentants des échelons administratifs infranationaux. 24 pays associent ces consultations avec le cycle de vie des politiques publiques et la totalité de ces pays procèdent à de telles consultations à l'étape de la rédaction des politiques. Par exemple, la République tchèque a organisé des consultations pour le rapport d'autoévaluation de fin de cycle de son deuxième Plan d'action national au titre du Partenariat pour un gouvernement ouvert (PGO), pour l'élaboration de son troisième Plan d'action national ainsi que pour la rédaction de textes relatifs aux activités de lobbying et des lanceurs d'alerte. En Australie, en Grèce, en Hongrie, en Israël, en Lettonie, au Mexique et en République tchèque, le centre de gouvernement consulte aussi les parties prenantes à l'étape de la définition des priorités stratégiques et lors de la mise en œuvre, du suivi et de l'évaluation des politiques.

Les centres de gouvernement recourent à des plateformes numériques pour recueillir les suggestions ou les réactions des citoyens sur leurs projets de politiques. Certains pays tels que la Lituanie ont créé à cet effet une plateforme unique de gouvernement ouvert. D'autres créent un portail dédié aux retours des citoyens pour chaque projet de politique. Ainsi, au moment de la prise en compte de la réforme du système électoral fédéral en 2016-2017, le Bureau du Conseil privé canadien a créé une plateforme en ligne sur laquelle les citoyens étaient invités à donner leur avis sur les pratiques démocratiques. Après avoir rempli le questionnaire correspondant, les personnes sondées recevaient des renseignements leur permettant de situer leur propre point de vue par rapport aux autres avis exprimés lors de la consultation. Le site s'appuyait sur une interface intuitive pour inciter les citoyens en général et notamment les jeunes, à participer. Cela a permis au centre de gouvernement de faire connaître la réforme et de recueillir des éléments d'information sur l'avis des citoyens.

\section{Méthodologie et définitions}

Ces données proviennent des résultats de l'enquête 2017 de l'OCDE sur l'organisation et les fonctions du centre de gouvernement, à laquelle 34 pays de l'OCDE et 4 nonmembres ont participé. Les répondants sont de hauts responsables qui appuient et conseillent directement le chef de gouvernement et le conseil des ministres. Leurs réponses portaient sur l'année 2016.

Les unités du centre du gouvernement comprennent le ministère ou le secrétariat général de la présidence, le bureau du Premier ministre et le Cabinet des ministres, bien que d'autres parties du gouvernement puissent assumer ces fonctions.

Le document Stratégie en matière de gouvernement ouvert définit un programme en matière de gouvernement ouvert pour l'administration centrale ou pour tous les niveaux de gouvernement infranationaux, ou encore pour toute institution publique ou tout domaine thématique. Il énonce de grandes initiatives en matière de gouvernement ouvert ainsi que des objectifs à court, moyen et long termes et des indicateurs.

Initiatives en matière de gouvernement ouvert regroupe des actions entreprises par les pouvoirs publics ou par une institution publique afin d'atteindre des objectifs précis dans ce domaine, qui peuvent aller de la rédaction de textes de loi à la mise en place d'activités spécifiques telles que des consultations en ligne.

\section{Pour en savoir plus}

OCDE (2017), Recommandation du Conseil sur le gouvernement ouvert, https://legalinstruments.oecd.org/fr/instruments/OECDLEGAL-0438.

OCDE (2017), Gouvernement ouvert : contexte mondial et perspectives, Éditions OCDE, Paris, https://doi.org/10.1787/9789264280984-fr.

\section{Notes relatives aux graphiques}

Concernant les données sur Israël, voir http://doi.org/10.1787/888932315602

On ne dispose pas de données pour la Corée et la Pologne. Les données sur l'Italie, là où elles sont disponibles, concernent 2019.

On trouvera à l'Annexe F (en ligne) des indications sur les étapes du cycle de l'action publique auxquelles le centre de gouvernement consulte les parties prenantes (4.7).

PANORAMA DES ADMINISTRATIONS PUBLIQUES 2019 @ OCDE 2019 


\section{Rôle du centre de gouvernement dans le gouvernement ouvert}

4.5. Rôle du centre de gouvernement dans les stratégies et initiatives en matière de gouvernement ouvert, 2016

\begin{tabular}{|c|c|c|c|c|c|c|}
\hline Pays & Conception & Mise en œuvre & Communication & Coordination & Suivi & Évaluation \\
\hline Allemagne & $\bullet$ & $\bullet$ & 0 & 0 & 0 & 0 \\
\hline Australie & $\bullet$ & $\bullet$ & - & $\bullet$ & $\bullet$ & $\bullet$ \\
\hline Autriche & $\bullet$ & $\bullet$ & 0 & $\bullet$ & 0 & $\bullet$ \\
\hline Belgique & 0 & 0 & 0 & O & 0 & 0 \\
\hline Canada & 0 & 0 & 0 & 0 & $\bullet$ & 0 \\
\hline Chili & $\bullet$ & $\bullet$ & $\bullet$ & $\bullet$ & O & O \\
\hline Danemark & 0 & 0 & 0 & 0 & 0 & 0 \\
\hline Espagne & O & 0 & 0 & O & O & O \\
\hline Estonie & $\bullet$ & $\bullet$ & $\bullet$ & $\bullet$ & $\bullet$ & $\bullet$ \\
\hline Etats-Unis & $\bullet$ & $\bullet$ & $\bullet$ & $\bullet$ & $\bullet$ & O \\
\hline Finlande & 0 & 0 & 0 & 0 & 0 & 0 \\
\hline France & O & O & O & $\bullet$ & O & 0 \\
\hline Grèce & $\bullet$ & 0 & $\bullet$ & $\bullet$ & $\bullet$ & $\bullet$ \\
\hline Hongrie & $\bullet$ & $\bullet$ & O & 0 & O & O \\
\hline Irlande & 0 & 0 & 0 & 0 & 0 & 0 \\
\hline Islande & $\bullet$ & O & $\bullet$ & $\bullet$ & $\bullet$ & 0 \\
\hline Israël & $\bullet$ & $\bullet$ & $\bullet$ & $\bullet$ & $\bullet$ & $\bullet$ \\
\hline Italie & $\bullet$ & $\bullet$ & $\bullet$ & $\bullet$ & $\bullet$ & O \\
\hline Japon & 0 & $\bullet$ & 0 & 0 & 0 & 0 \\
\hline Lettonie & $\bullet$ & $\bullet$ & $\bullet$ & $\bullet$ & $\bullet$ & $\bullet$ \\
\hline Lituanie & $\bullet$ & $\bullet$ & $\bullet$ & $\bullet$ & $\bullet$ & $\bullet$ \\
\hline Luxembourg & $\bullet$ & $\bullet$ & $\bullet$ & O & 0 & 0 \\
\hline Mexique & $\bullet$ & $\bullet$ & $\bullet$ & $\bullet$ & $\bullet$ & $\bullet$ \\
\hline Norvège & O & O & O & O & O & O \\
\hline Nouvelle Zélande & $\bullet$ & $\bullet$ & $\bullet$ & $\bullet$ & $\bullet$ & O \\
\hline Pays-Bas & $\bullet$ & $\bullet$ & $\bullet$ & O & 0 & O \\
\hline Portugal & O & 0 & O & O & 0 & 0 \\
\hline République Slovaque & $\bullet$ & $\bullet$ & $\bullet$ & $\bullet$ & 0 & $\bullet$ \\
\hline République Tchèque & $\bullet$ & $\bullet$ & $\bullet$ & $\bullet$ & $\bullet$ & $\bullet$ \\
\hline Royaume-Uni & $\bullet$ & $\bullet$ & $\bullet$ & $\bullet$ & $\bullet$ & $\bullet$ \\
\hline Slovénie & 0 & 0 & $\bullet$ & 0 & 0 & 0 \\
\hline Suède & 0 & 0 & 0 & 0 & 0 & 0 \\
\hline Suisse & 0 & 0 & 0 & 0 & 0 & 0 \\
\hline Turquie & $\bullet$ & $\bullet$ & $\bullet$ & $\bullet$ & 0 & 0 \\
\hline \multicolumn{7}{|l|}{ Total OCDE } \\
\hline - Oui & 21 & 20 & 19 & 18 & 14 & 11 \\
\hline O Non & 13 & 14 & 15 & 16 & 20 & 23 \\
\hline Brésil & $\bullet$ & 0 & O & O & O & 0 \\
\hline Costa Rica & - & 0 & - & $\bullet$ & - & 0 \\
\hline
\end{tabular}

Source : Enquête 2017 de l'OCDE sur l'organisation et les fonctions du centre de gouvernement.

4.6. Parties prenantes associées aux consultations menées par les centres de gouvernement, 2016

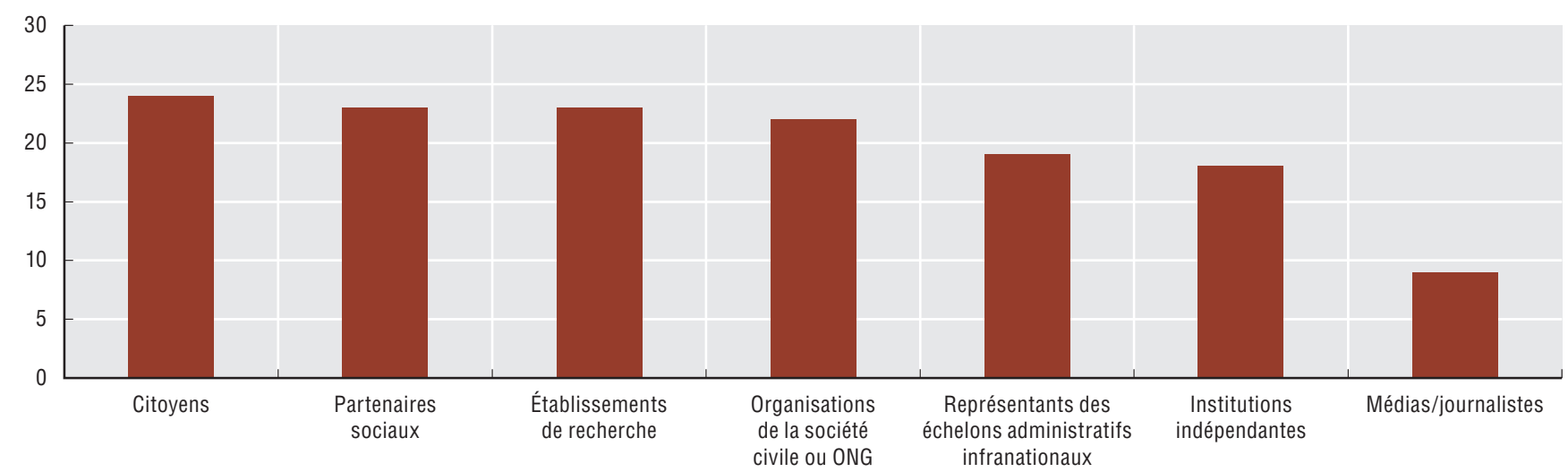

Source : Enquête 2017 de l'OCDE sur l'organisation et les fonctions du centre de gouvernement. 
On peut définir l'évaluation des politiques publiques comme l'évaluation structurée et objective de la conception, de la mise en œuvre ou des résultats d'une initiative d'action publique à venir, en cours ou achevée. L'objectif de l'évaluation peut être d'apprécier l'efficience, l'efficacité, l'impact ou la durabilité d'une politique donnée. L'évaluation permet d'identifier pourquoi et en quoi une politique a réussi ou échoué et de comprendre comment mieux articuler les décisions et leurs retombées. Il s'agit par conséquent d'un aspect crucial de toute action publique éclairée par des données probantes et donc de toute bonne gouvernance.

Il est possible de mesurer l'importance accordée à l'évaluation des politiques publiques en examinant la place qui lui est accordée au sein du cadre juridique des pays. L'Allemagne, le Mexique et la Suisse ont inscrit cette évaluation dans leur Constitution. Les États-Unis en ont fait une part intégrante d'une loi adoptée en 2010 (Government Performance and Results Act Modernization Act GPRA), qui représentait une vaste réforme de modernisation de la gestion publique, lancée dans une optique d'amélioration des performances et des résultats de l'administration.

Les dispositifs institutionnels varient selon les pays car l'évaluation des politiques publiques peut être confiée au pouvoir exécutif, au pouvoir législatif ou à l'institution supérieure de contrôle des finances publiques. La plupart des pays membres et partenaires de l'OCDE ont explicitement confié à une ou plusieurs entités de l'exécutif la responsabilité d'évaluer les politiques publiques à l'échelle de l'ensemble de l'administration, à l'exception du Danemark et de la Suède. 21 pays de l'OCDE ont partagé cette responsabilité entre plusieurs entités du pouvoir exécutif, tandis que 12 autres l'ont confiée à une seule entité de l'exécutif. La France et la République slovaque ont partagé cette responsabilité entre plusieurs entités.

Le centre de gouvernement est le lieu où la politique d'évaluation est la plus institutionnalisée. La Corée, la Grèce, la Lettonie et la Turquie lui ont confié la responsabilité exclusive de l'évaluation des politiques publiques à l'échelle de l'ensemble du gouvernement. Le ministère des Finances a été choisi par 22 pays et, en Irlande, en Norvège, aux Pays-Bas et en Suisse, il ne partage cette mission avec aucune autre entité. L'Autriche, la Belgique et le Japon ont confié cette responsabilité d'évaluation générale des politiques publiques exclusivement à leur ministère de la Réforme du secteur public (ou équivalent), tandis que la République tchèque a opté pour son ministère du Développement régional. Outre les institutions déjà mentionnées, les États-Unis, la France, le Mexique et la Pologne ont également confié des compétences à une ou plusieurs entités autonomes.

Parmi les pays à avoir confié des responsabilités d'évaluation générale des politiques publiques au centre de gouvernement, 19 ont chargé celui-ci de promouvoir le recours aux évaluations. Au Canada et en Nouvelle-Zélande, c'est la seule responsabilité qui a été confiée au centre de gouvernement. 16 pays ont chargé le centre de gouvernement de définir et de mettre à jour la politique d'évaluation. 14 pays ont indiqué que le centre de gouvernement est chargé d'élaborer des lignes directrices et 13 autres qu'il est chargé de créer des incitations en faveur de la conduite d'évaluations. L'Islande n'a confié au centre de gouvernement qu'un rôle de centre de connaissances et de plateforme d'échange entre toutes les composantes de l'administration.

De tous les pays de l'OCDE, le Portugal est celui qui a confié au centre de gouvernement les responsabilités les plus larges en matière d'évaluation des politiques publiques, puisqu'il est responsable de 14 tâches différentes, dont celles de réaliser des évaluations, de former des évaluateurs, de favoriser l'association des parties prenantes et d'assurer le suivi des rapports. Les ministères des Finances ont été chargés de promouvoir l'utilisation et la définition de directives dans 12 pays, tandis que les ministères de la réforme du Secteur public servent de centre de connaissances, offrent une plate-forme d'échange et élaborent des directives dans 7 pays.

\section{Méthodologie et définitions}

Ces données sont issues des résultats de l'enquête sur l'évaluation des politiques, lancée par l'OCDE en mai 2018. Cette enquête avait pour objet d'évaluer les principales caractéristiques et le degré de maturité du système mis en place par les pays pour évaluer leurs politiques publiques, selon trois grands aspects : la nature et le degré d'institutionnalisation de l'évaluation (grandes tendances, pratiques, défis) ; l'action menée pour assurer la qualité des évaluations ; et l'action menée pour promouvoir le recours aux évaluations.

Les personnes sondées appartenaient au centre de gouvernement et à des ministères sectoriels (ministère de la Santé, ministère de la Réforme du secteur public ainsi que, si le pays le souhaitait, un troisième ministère) de pays membres de l'OCDE et de certains pays non membres. En mars 2019, 37 pays membres ou partenaires-clés de l'OCDE avaient répondu à l'enquête principale sur les pratiques d'évaluation au sein du centre de gouvernement.

Les réponses reflètent le point de vue des pays sur leurs pratiques et procédures actuelles. Les données portent uniquement sur les administrations centrales ou fédérales ; elles ne tiennent pas compte des pratiques d'évaluation en place à l'échelon des États fédéraux ou à l'échelon local.

\section{Pour en savoir plus}

OCDE (à paraître), Institutionalisation, Quality and Use of Policy Evaluation: Governance Lessons from Countries' Experience, Éditions OCDE, Paris.

OCDE (2018), Enquête sur l'évaluation des politiques publiques www.oecd.org/gou/policy-monitoring-evaluation.htm.

\section{Notes relatives aux graphiques}

Concernant les données sur Israël, voir http://doi.org/10.1787/888932315602

Les données relatives au Danemark et à la Suède ne sont pas indiquées car ces pays n'ont pas attribué de compétences d'évaluation générale des politiques publiques à une institution au sein de l'ex. On ne dispose pas de données pour le Luxembourg.

4.8. Reflète les réponses fournies à la question « Quelle(s) institution(s) relevant de l'exécutif a(ont) des compétences en matière d'évaluation [générale] des politiques publiques? ".

4.9. Reflète les réponses fournies à la question « Veuillez indiquer toutes les missions et responsabilités de [cette/ces institution(s)] en lien avec l'évaluation [générale] des politiques publiques ".

On trouvera à l'Annexe F (en ligne) des indications sur les cadres juridiques dans lesquels s'inscrit l'évaluation générale des politiques publiques (4.10). 
4.8. Entités de l'exécutif dotées de compétences en matière d'évaluation générale des politiques publiques, 2018

Centre de gouvernement

Ministère de la Planification / du Développement ou équivalent Entité autonome

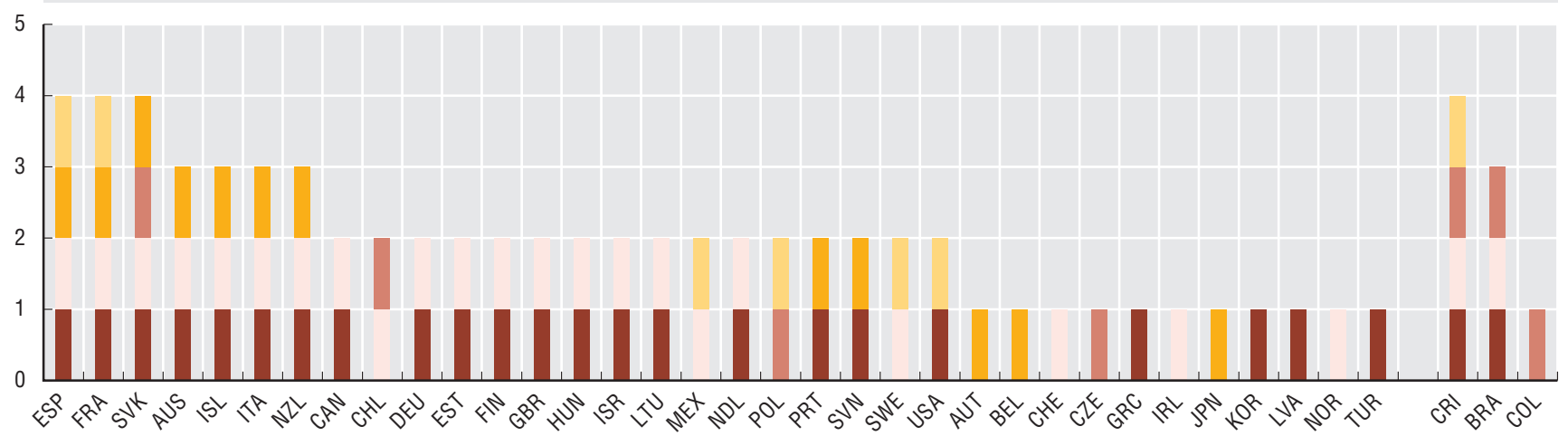

Source : Enquête 2018 de l'OCDE sur l'évaluation des politiques publiques.

4.9. Missions et responsabilités du centre de gouvernement en lien avec l'évaluation générale des politiques publiques, 2018

\begin{tabular}{|c|c|c|c|c|c|c|c|}
\hline Pays & $\begin{array}{l}\text { Définir et mettre } \\
\text { à jour la politique } \\
\text { d'évaluation }\end{array}$ & $\begin{array}{l}\text { Élaborer des lignes } \\
\text { directrices sur } \\
\text { l'évaluation des } \\
\text { politiques publiques }\end{array}$ & $\begin{array}{l}\text { Créer des incitations } \\
\text { en faveur de la conduite } \\
\text { d'évaluations des } \\
\text { politiques publiques }\end{array}$ & $\begin{array}{l}\text { Obliger les institutions } \\
\text { à mener des évaluations } \\
\text { spécifiques }\end{array}$ & $\begin{array}{l}\text { Veiller au niveau } \\
\text { de qualité des } \\
\text { évaluations }\end{array}$ & $\begin{array}{l}\text { Promouvoir } \\
\text { le recours aux } \\
\text { évaluations }\end{array}$ & $\begin{array}{l}\text { Servir de centre de } \\
\text { connaissances et fournir } \\
\text { une plateforme d'échange }\end{array}$ \\
\hline Allemagne & - & 므 & - & 0 & - & อ & on \\
\hline Australie & 0 & 口 & 0 & - & 0 & ๑ே & $\Delta$ \\
\hline Autriche & $\Delta$ & $\boldsymbol{\Delta}$ & 0 & $\Delta$ & 0 & $\Delta$ & $\Delta$ \\
\hline Belgique & 0 & 0 & 0 & 0 & 0 & 0 & 0 \\
\hline Canada & 口 & 口 & 0 & 口 & 0 & 밈 & 口 \\
\hline Chili & $\nabla$ & D* & 0 & 口 & $\bullet$ & a & $\square$ \\
\hline Corée & $\bullet$ & - & 0 & ○ & 0 & $\bullet$ & $\bullet$ \\
\hline Danemark & 0 & 0 & 0 & 0 & 0 & 0 & 0 \\
\hline Espagne & $\boldsymbol{O A}$ & $\Delta$ & a & $\nabla \mathbf{\Delta \nabla}$ & $\Delta$ & $\boldsymbol{\Delta}$ & $\Delta$ \\
\hline Estonie & 0 & e日 & $\bullet$ & 0 & 0 & - & 0 \\
\hline États-Unis & 0 & 0 & 0 & - & • & 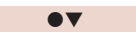 & $\theta \nabla$ \\
\hline Finlande & 0 & - & 0 & 0 & - & - & 0 \\
\hline France & 0 & 0 & 0 & 미 & 0 & 0 & 0 \\
\hline Grèce & $\bullet$ & $\bullet$ & 0 & $\bullet$ & $\bullet$ & $\bullet$ & 0 \\
\hline Hongrie & 0 & 0 & 0 & 0 & च & 0 & $\bullet$ \\
\hline Irlande & $\square$ & 口 & 口 & E & 0 & 口 & - \\
\hline Islande & 0 & 0 & 0 & 0 & 0 & 0 & On \\
\hline Israël & - & - & อே & 미 & 0 & - & - \\
\hline Italie & onA & $\boldsymbol{D} \Delta$ & - & - & 0 & 묘 & - \\
\hline Japon & $\Delta$ & $\Delta$ & 0 & 0 & $\Delta$ & $\Delta$ & $\Delta$ \\
\hline Lettonie & - & - & $\bullet$ & - & - & $\bullet$ & - \\
\hline Lituanie & $\bullet$ & an & $\bullet$ & 0 & 0 & 미 & - \\
\hline Mexique & $\nabla$ & $\bar{\nabla}$ & 0 & $\bar{\nabla}$ & $\boldsymbol{\nabla}$ & $\bar{\nabla}$ & 0 \\
\hline Norvège & $\mathbf{\square}$ & 口 & 0 & 0 & 0 & 口 & 口 \\
\hline Nouvelle-Zélande & $\overline{0}$ & $\overline{0}$ & $\mathbf{\square}$ & 0 & 0 & $\bar{\bullet}$ & $\overline{0}$ \\
\hline Pays-Bas & 口 & - & $\bar{\square}$ & 0 & 0 & -10 & 口 \\
\hline Pologne & $\bullet$ & 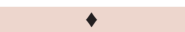 & 0 & 0 & $\bullet$ & 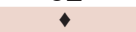 & 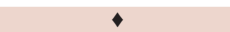 \\
\hline Portugal & $\bullet \boldsymbol{\Delta}$ & $\boldsymbol{O A}$ & $\boldsymbol{0} \Delta$ & $\bullet \Delta$ & $\bullet \Delta$ & $\bullet \Delta$ & $\boldsymbol{B} \Delta$ \\
\hline République slovaque & 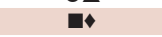 & $\mathbf{D \Delta}$ & a & $\mathbf{n}$ & 口A & - & a \\
\hline République tchèque & $\bullet$ & $\bullet$ & $\bullet$ & $\bullet$ & $\bullet$ & $\bullet$ & 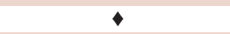 \\
\hline Royaume-Uni &.. & .. &.. &.. &.. &.. &.. \\
\hline Slovénie & $\bullet \Delta$ & $\ddot{\Delta}$ & $\bullet$ & - & ○ & $\bullet \boldsymbol{\Delta}$ & $\boldsymbol{\Delta}$ \\
\hline Suède & 0 & $\overline{0}$ & 0 & 0 & 0 & $\bar{\nabla}$ & $\overline{0}$ \\
\hline Suisse & $\mathbf{\square}$ & 0 & $\mathbf{\square}$ & $\mathrm{O}$ & $\mathbf{\square}$ & 0 & $\mathbf{\square}$ \\
\hline Turquie & 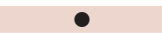 & 0 & 0 & - & 0 & 0 & 0 \\
\hline \multicolumn{8}{|l|}{ Total OCDE } \\
\hline - Centre de gouvernement & 15 & 13 & 14 & 13 & 10 & 19 & 12 \\
\hline - Ministère des Finances ou équivalent & 9 & 12 & 8 & 9 & 4 & 13 & 9 \\
\hline $\begin{array}{l}\text { A Ministère de la Réforme } \\
\text { du secteur public ou équivalent }\end{array}$ & 6 & 7 & 2 & 4 & 4 & 5 & 7 \\
\hline - Ministère de la Planification ou équivalent & 4 & 4 & 1 & 1 & 3 & 3 & 3 \\
\hline$\nabla$ Entité autonome & 1 & 2 & 0 & 2 & 2 & 4 & 1 \\
\hline O Pas d'institution & 7 & 7 & 14 & 13 & 15 & 5 & 9 \\
\hline Brésil & 0 & ○ & - & 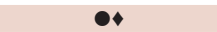 & 0 & 0 & 0 \\
\hline Colombie & 0 & $\bullet$ & 0 & 0 & $\bullet$ & $\bullet$ & 0 \\
\hline Costa Rica & $\theta \bullet \nabla$ & $0 \diamond \nabla$ & 口十 & - & $\bullet \nabla$ & $\theta \bullet \nabla$ & $\bullet$ \\
\hline
\end{tabular}

Source : Enquête 2018 de l'OCDE sur l'évaluation des politiques publiques. 
La mise en place des évaluations de politiques publiques joue un rôle important car elle boucle le cycle de l'action publique. Elle permet, dans le cas des évaluations ex ante, d'estimer l'impact des initiatives envisagées et, dans le cas des évaluations ex post, d'évaluer les résultats et les retombées des politiques mises en œuvre. En offrant la possibilité d'évaluer les retombées et impact des politiques et de les mettre en lien avec les décisions des responsables publics, elle favorise l'apprentissage mutuel et met la redevabilité au premier plan. L'évaluation des politiques publiques est coûteuse en termes de temps, de ressources humaines et de moyens financiers. Les pouvoirs publics doivent donc veiller à ce que les moyens ainsi investis produisent de la valeur publique ; à cette fin, ils doivent faire en sorte qu'au moment de la prise de décision, les conclusions des évaluations soient prises en compte.

La plupart des pays de l'OCDE se sont dotés de dispositifs visant à encourager la mise à profit des conclusions des évaluations de politiques publiques (par exemple, l'intégration des conclusions au cycle budgétaire ou l'examen des conclusions en Conseil des ministres), mais la Belgique, l'Espagne, l'Islande, la République tchèque, la Slovénie et la Turquie font exception à la règle. À l'heure actuelle, le Japon et le Mexique disposent de cinq dispositifs différents pour encourager la mise à profit des conclusions des évaluations de politiques et la Lettonie en compte quatre. 8 pays de l'OCDE en ont deux et 8 autres en ont trois. 9 pays ne comptent qu'un seul dispositif mis en place.

21 pays intègrent explicitement les conclusions des évaluations à leur cycle budgétaire. Ce dispositif est le seul employé pour encourager l'utilisation de ces conclusions en France, en Israël, au Royaume-Uni et en Suède. Au Canada, les conclusions éclairent obligatoirement les décisions du Conseil du Trésor en matière de dépenses et de répartitions budgétaires puisqu'elles sont intégrées au dispositif applicable en matière de redevabilité.

Les conclusions sont présentées au plus haut niveau politique dans 11 pays et ce dispositif est le seul en place pour favoriser leur prise en compte en Hongrie et en Lituanie. En Corée, par exemple, les ministères sectoriels informent le Conseil des ministres des mesures prises en conséquence des critiques formulées lors de l'évaluation annuelle. Les États-Unis ont créé un Conseil interagences sur la politique d'évaluation (Interagency Council on Evaluation Policy). Co-présidé par des représentants du Bureau de la gestion et du budget (OMB) et du ministère du Travail, ce Conseil rassemble une dizaine de membres de haut rang d'organismes publics et se réunit chaque mois pour examiner les conclusions des évaluations.

Dans 10 pays, un dispositif invitant la direction à réagir a été mis en place afin de promouvoir la mise à profit des conclusions des évaluations ; en Nouvelle-Zélande c'est le seul dispositif existant en la matière. 11 pays de l'OCDE (Allemagne, Estonie, États-Unis, Finlande, Irlande, Japon, Lettonie, Mexique, Norvège, Pologne et Royaume-Uni) recourent pour leur part à des plateformes de coordination à l'échelle de l'ensemble du gouvernement. La Norvège a choisi une telle plateforme comme seul outil pour encourager la capitalisation des conclusions des évaluations.

Dans 8 de ces pays, les plateformes en ligne ont pour objet de partager les conclusions des évaluations avec les spécialistes et les administrations locales. Par exemple, en Norvège, la création d'un service web qui rassemble les conclusions de toutes les évaluations réalisées par l'administration centrale vise à accroître l'utilisation des données probantes dans tous les domaines d'action nationaux et pour des évaluations à venir. Dans 7 pays, la plateforme fait en sorte que les données probantes visent à éclairer l'élaboration des politiques publiques. D'autres fonctions, telles que la cartographie de la fonction de courtage des données, sont moins fréquemment exercées, même si l'Estonie et les États-Unis se distinguent en la matière. En Allemagne et au Mexique, les plateformes de coordination ont quatre fonctions différentes et, au Japon et en Pologne, elles n'ont aucune des fonctions susmentionnées.

\section{Méthodologie et définitions}

Ces données sont issues des résultats de l'enquête sur l'évaluation des politiques publiques lancée par l'OCDE en mai 2018. Les personnes sondées appartenaient au centre de gouvernement et à des ministères sectoriels (ministère de la Santé, ministère de la Réforme du secteur public ainsi que, si le pays le souhaitait, un troisième ministère) de pays membres de l'OCDE et de certains pays non membres.

L'évaluation des politiques publiques est définie, dans l'enquête, comme l'évaluation structurée et objective d'une initiative de réforme ou d'une politique publique en cours ou achevée, de sa conception, de sa mise en œuvre et de ses résultats. Le but est de déterminer, entre autres, la pertinence et la réalisation des objectifs, l'efficience, l'efficacité, l'impact et la durabilité. Le terme " évaluation » désigne également le processus de détermination de la valeur ou portée d'une politique publique.

\section{Pour en savoir plus}

OCDE (à paraître), Institutionalisation, Quality and Use of Policy Evaluation: Governance Lessons from Countries' Experience, Éditions OCDE, Paris.

OCDE (2019), Building Capacity for Evidence Informed Policy Making. Lessons from the countries experience, Éditions OCDE, Paris.

OCDE (2018), Survey on Policy Evaluation, Éditions OCDE, Paris, www.oecd.org/gov/policy-monitoring-evaluation.htm

\section{Notes relatives aux graphiques}

Concernant les données sur Israël, voir $h$ ttp://doi.org/10.1787/888932315602. On ne dispose pas de données pour le Luxembourg.

4.11. Reflète les réponses à la question " Dans votre administration, par quels moyens l'utilisation des conclusions des évaluations des politiques publiques est-elle encouragée ?".

4.12. Reflète les réponses à la question "Quelles fonctions remplit cette plateforme de coordination?". 
4.11. Moyens employés pour encourager la mise à profit des conclusions des évaluations de politiques publiques dans les pays de l'OCDE, 2018

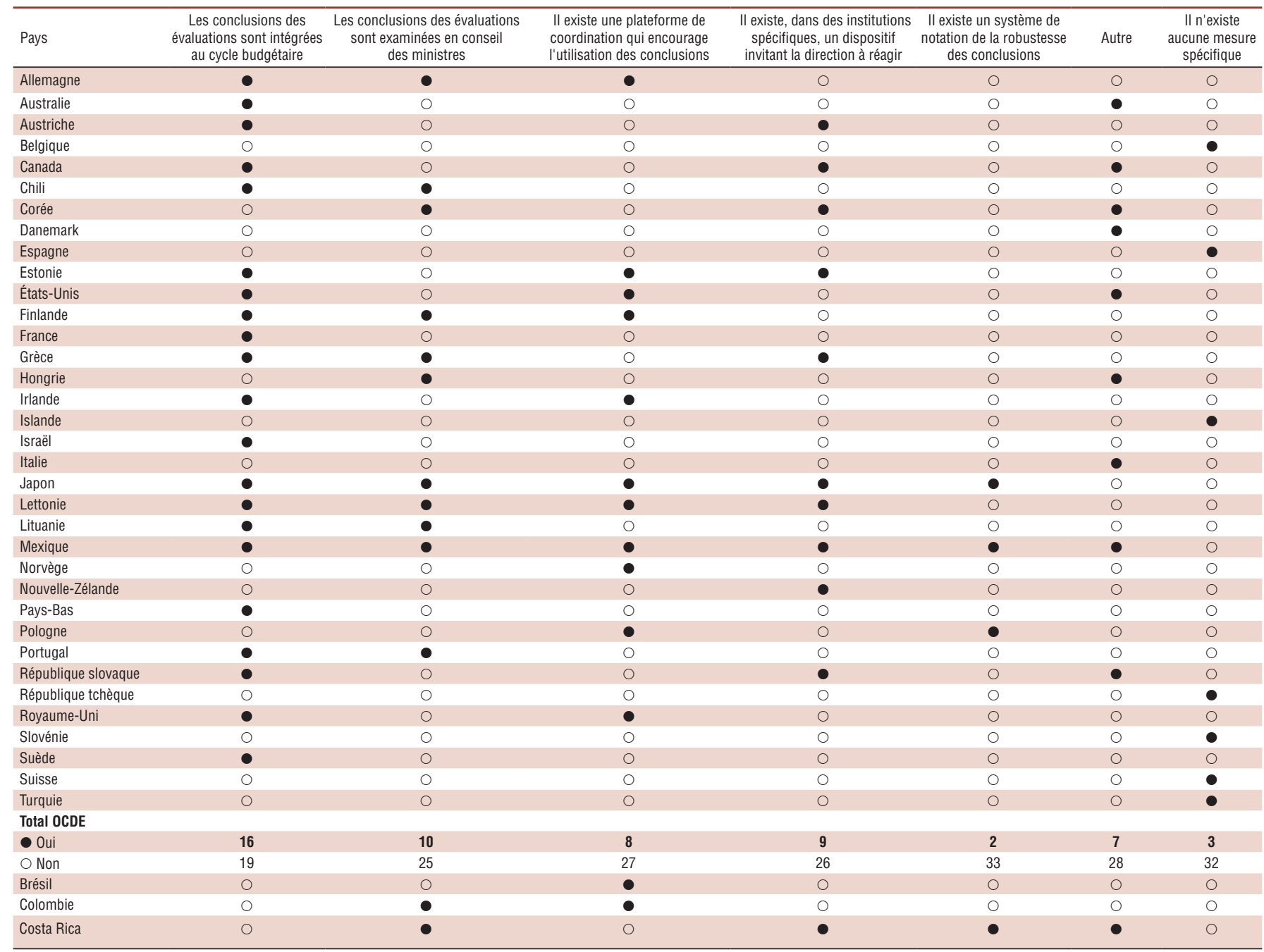

Source : Enquête 2018 de l'OCDE sur l'évaluation des politiques publiques.

4.12. Fonctions exercées par la plateforme de coordination à l'échelle de l'ensemble de l'administration pour encourager la mise à profit des conclusions des évaluations de politiques publiques, 2018
Cartographier la fonction de courtage des données
Veiller au calendrier et au format
Faire en sorte que l'évaluation vise à éclairer l'élaboration des politiques
Faciliter la coopération internationale
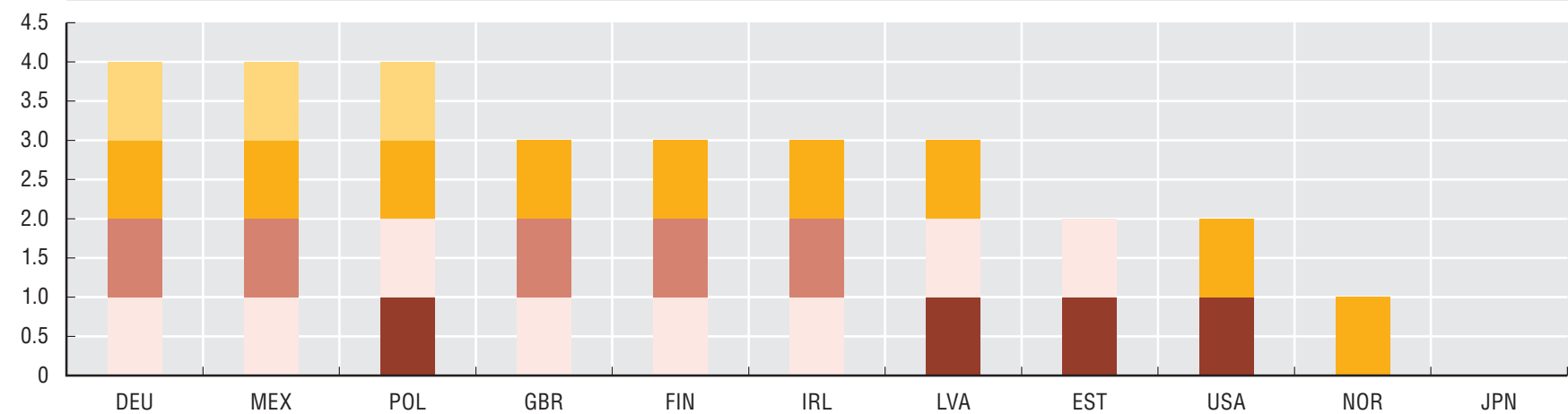

Source : Enquête 2018 de l'OCDE sur l'évaluation des politiques publiques. 


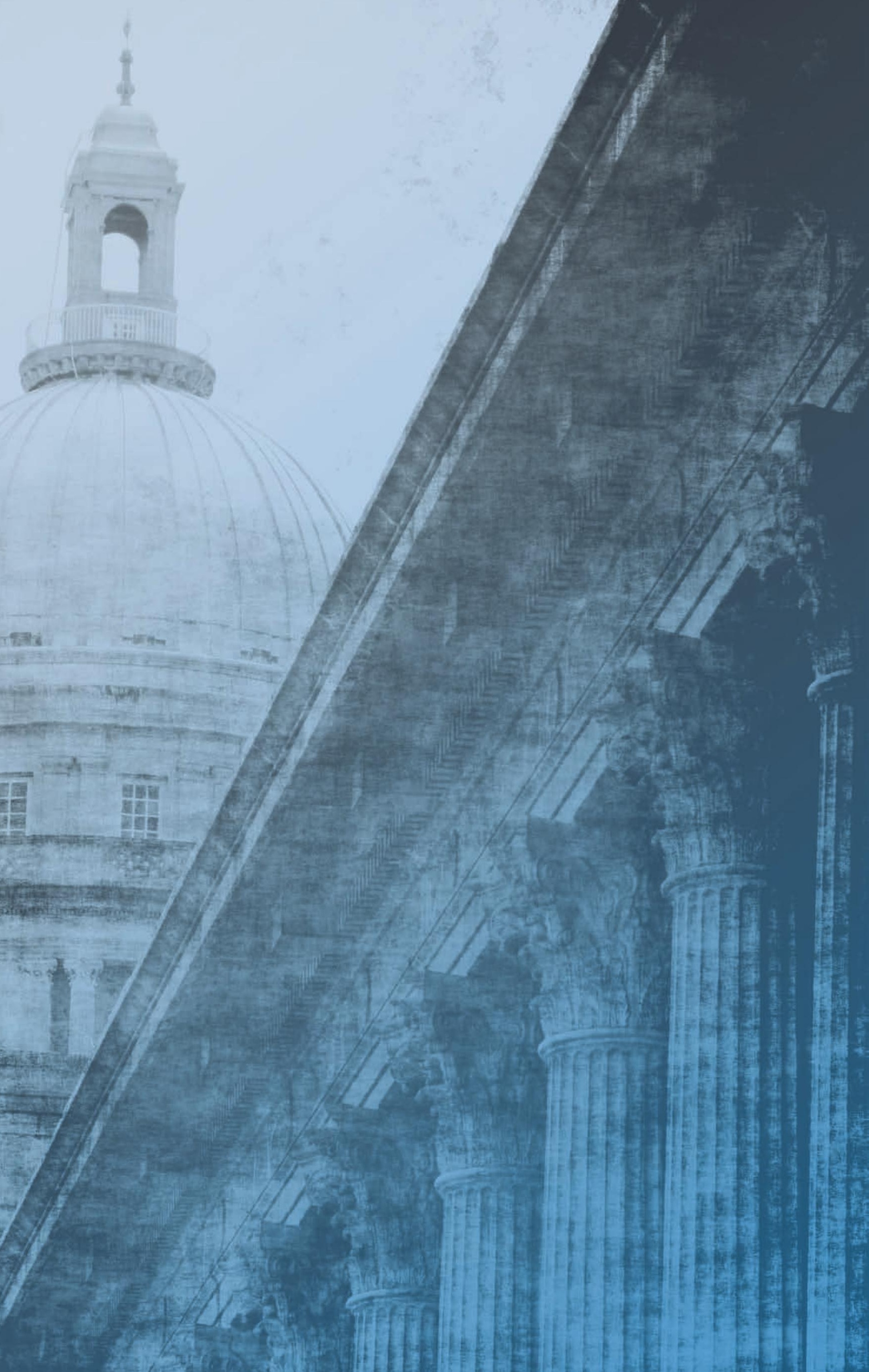


Un cadre solide de budgétisation des investissements est indispensable pour que le budget respecte de manière efficiente et cohérente les priorités stratégiques nationales. Les Principes de gouvernance budgétaire de l'OCDE mettent en évidence: 1) fonder les plans d'investissement sur une évaluation objective préalable des lacunes en matière de capacité économique, des besoins de développement des infrastructures et des priorités sectorielles ou sociales ; 2) procéder à une évaluation prudente des coûts et avantages de ces investissements, de leur faisabilité budgétaire, des degrés de priorité respectifs des différents projets et de l'optimisation des dépenses publiques ; 3) évaluer les décisions d'investissement indépendamment du mécanisme de financement correspondant ; et 4) élaborer et mettre en place un cadre national de soutien à l'investissement public.

Les possibilités d'inclure les dépenses d'investissement dans le processus budgétaire sont variées et vont de l'intégration totale dans les dépenses courantes à l'établissement d'un budget entièrement distinct. Chaque méthode comportant ses avantages et ses inconvénients. L'intégration totale peut présenter l'avantage d'améliorer la planification, de faciliter la coordination et de gagner en souplesse ; l'établissement de budgets séparés peut permettre d'éviter que des postes de dépenses obligatoires n'évincent des postes budgétaires à caractère discrétionnaire comme les investissements (Posner, Ryu and Tkachenko 2009). D'après les dernières données disponibles, $74 \%$ des pays interrogés indiquent que les ministères sectoriels soumettent de manière globale les dépenses d'investissement et les dépenses courantes à l'autorité budgétaire centrale. Dans les autres pays (26\%), les demandes de crédits à des fins d'investissement et leur approbation par l'autorité budgétaire centrale sont totalement distinctes du processus de décision qui décide des dépenses courantes.

De même, on note des progrès en matière de planification stratégique à long terme puisque plus de la moitié des pays de l'OCDE déclarent avoir défini de grandes orientations transversales sur le long terme. Cette pratique est nouvelle dans certains pays comme le Luxembourg et la Norvège. Si les raisons incitant à établir des stratégies à long terme diffèrent selon les pays, elles se rapportent principalement aux priorités stratégiques et à la conjoncture économique du pays. L'engorgement des réseaux de transport, les évolutions démographiques et les déséquilibres en matière de développement régional sont les principaux facteurs qui déterminent l'élaboration de plans d'infrastructures stratégiques dans les pays de l'OCDE ayant participé à l'enquête. Une bonne pratique, actuellement mise en œuvre par certains pays comme l'Irlande et la Norvège, consiste à dresser une liste restreinte de projets prioritaires qui peut servir de base à la " planification d'une réserve de projets » et à la communication.

La construction et l'exploitation des infrastructures s'étendent généralement sur une longue période. Même si c'est au cours des phases de préparation et de construction que sont mobilisées la majorité des ressources, il importe malgré tout d'établir clairement qui est chargé du suivi et de l'évaluation des projets pendant la durée de vie des infrastructures. À cet effet, dans la plupart des pays (69\%), une règle formelle charge le ministère sectoriel ou l'organisme public concerné d'évaluer la performance de chaque projet. Parmi eux, dans $31 \%$ des pays interrogés, cette règle est définie et gérée par l'administration centrale, alors que dans $38 \%$ des pays, même s'il existe un mandat général, il incombe au service d'exécution concerné de définir cette règle.

\section{Méthodologie et définitions}

Ces données proviennent des résultats de l'enquête 2018 de l'OCDE sur la budgétisation des investissements et la gouvernance des infrastructures. L'enquête a été menée début 2018 auprès de 26 pays de l'OCDE. Les participants étaient en majorité de hauts responsables du ministère des Finances central ou fédéral ainsi que d'autres ministères sectoriels compétents.

La gouvernance des infrastructures englobe une série de procédures, outils et normes d'échange, prises de décision et suivi utilisés par les pouvoirs publics et leurs partenaires fournisseurs de services d'infrastructure. Elle renvoie donc aux échanges internes entre institutions publiques ainsi qu'aux échanges entretenus par ces dernières avec les usagers du secteur privé et avec les citoyens.

On peut définir l'optimisation des dépenses publiques comme la combinaison optimale, aux yeux des autorités, en termes de quantité, de qualité, de caractéristiques et de prix (c'est-à-dire de coût), telle qu'elle est envisagée sur la durée entière du projet.

\section{Pour en savoir plus}

OCDE (2019), Budgeting Practices and Procedures in OECD Countries, Éditions OCDE, Paris, https://doi.org/10.1787/9789264307957-en.

OCDE (2017), Getting Infrastructure Right: A Framework for Better Governance, Éditions OCDE, Paris, https://doi.org/10.1787/ 9789264272453-en.

Posner, P. (2009), "Public-private Partnerships: The relevance of budgeting", OECD Journal on Budgeting, Vol. 2009/1, pp. 49-74.

\section{Notes relatives aux graphiques}

Aucune donnée n'est disponible pour la Belgique, le Canada, la Corée, les États-Unis, la Finlande, l'Islande, la Lettonie et la Pologne. Concernant les données sur Israël, voir http://doi.org/10.1787/888932315602. Les données sur la Lituanie n'ont pas été collectées.

5.1. En Allemagne, les dépenses d'investissement et les dépenses courantes sont présentées séparément dans le budget mais sont négociées et décidées avec le budget global.

5.2. La République slovaque a indiqué que le bureau du vice-premier ministre chargé de l'Investissement et de l'information était chargé de l'élaboration du plan national d'investissement.

5.3. Les données sur le Portugal ne sont pas disponibles pour cette graphique. 
5.1. Distinction entre les demandes de crédits concernant des investissements et les demandes de crédits concernant les dépenses courantes, 2018

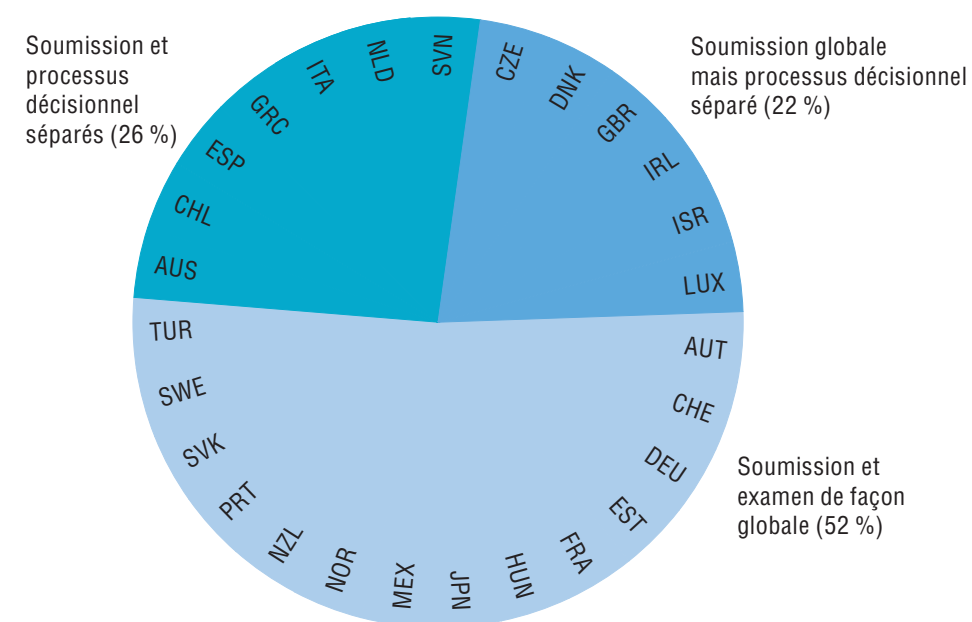

Source : OCDE (2018), Enquête OCDE sur la budgétisation des investissements et la gouvernance des infrastructures.

StatLink ains https://doi.org/10.1787/888934052099

5.2. Existence de plans stratégiques à long terme relatifs aux infrastructures, 2018

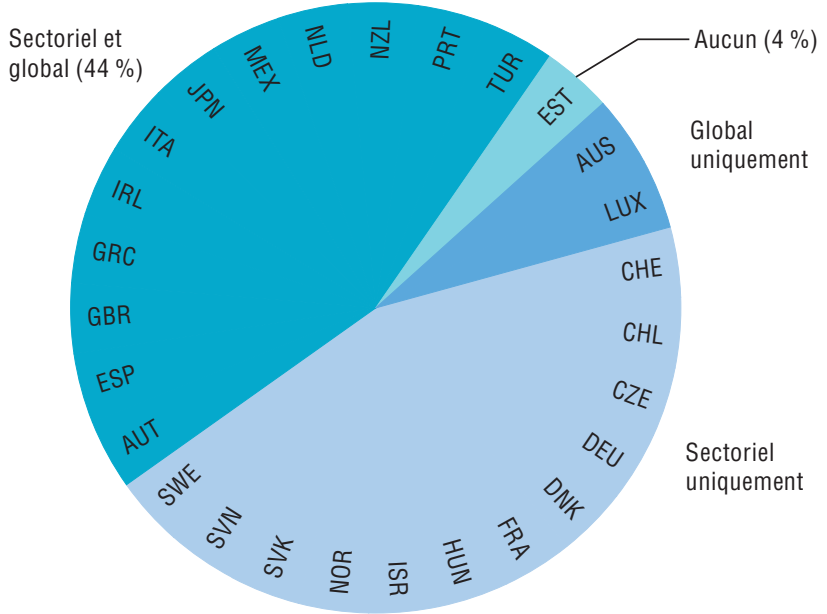

Source: OCDE (2018), Enquête OCDE sur la budgétisation des investissements et la gouvernance des infrastructures.

StatLink ants https://doi.org/10.1787/888934052118

5.3. Règle formelle de mise en œuvre d'une évaluation de performance pour chaque projet, 2018

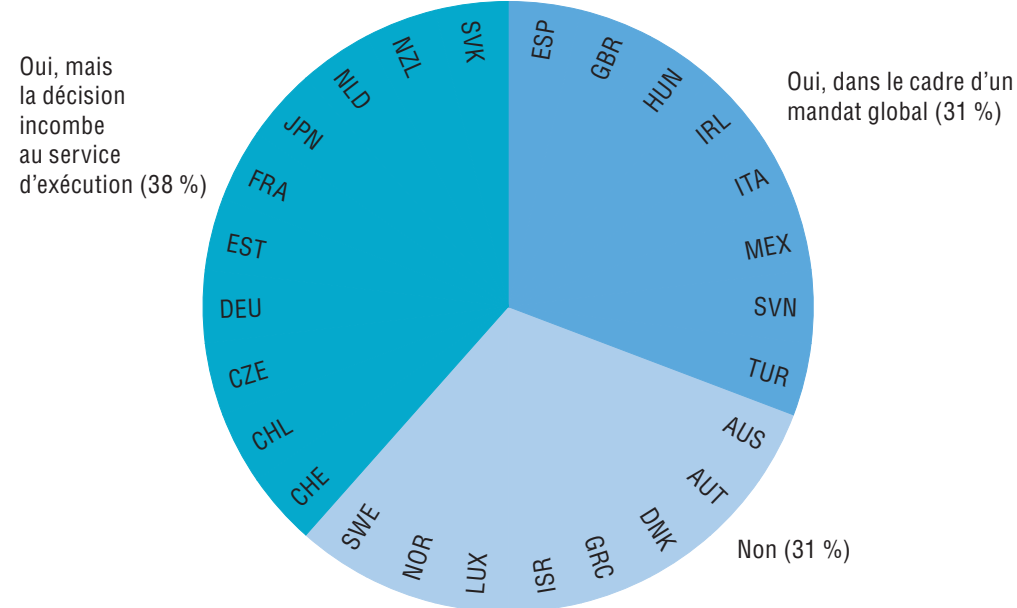

Source : OCDE (2018), Enquête OCDE sur la budgétisation des investissements et la gouvernance des infrastructures. 
Dans les pays de l'OCDE comme dans d'autres pays, c'est avant tout à l'exécutif qu'il incombe de garantir le bon fonctionnement du système économique dans son ensemble. À cet effet, il doit déployer un certain nombre d'outils et assurer différentes missions. Par conséquent, les sources de risques pour les finances publiques sont nombreuses et menacent en permanence de déjouer les prévisions ou de faire échouer les projets du gouvernement.

En dépit du caractère par définition incertain des risques budgétaires, il importe que les décideurs y soient sensibilisés et puissent les cerner afin d'accroître la capacité du gouvernement à agir en conséquence et à rebondir. Ainsi, la Recommandation du Conseil sur la gouvernance budgétaire de 2015 conseille aux gouvernements d'“ identifier, évaluer et gérer avec prudence les risques en matière de viabilité à long terme et autres risques budgétaires".

En 2018, la tendance était claire : les pays de l'OCDE ont, dans leur majorité, pris des mesures pour renforcer la gestion des risques budgétaires. Environ $60 \%$ d'entre eux ont mis en place un cadre ou ont adopté des lignes directrices - sous forme de directive supranationale, de texte législatif ou réglementaire national, ou encore de document d'orientation, dans le but de surveiller les risques budgétaires. La mise en œuvre d'un tel cadre est souvent du ressort du ministère des Finances. Certains pays, comme l'Autriche et le Royaume-Uni, ont toutefois pris le parti de constituer des comités interministériels dédiés, afin d'améliorer la coordination entre les différents acteurs de la surveillance et de la gestion des risques budgétaires. En Belgique, au Luxembourg, en République tchèque et au Royaume-Uni, l'institution budgétaire indépendante identifie et analyse les risques budgétaires dans le cadre de sa fonction de contrôle des prévisions économiques ou budgétaires du gouvernement (OCDE 2019).

La présentation de rapports publics sur les risques budgétaires est une pratique de plus en plus répandue : environ $75 \%$ des pays de l'OCDE publient des informations sur leurs risques budgétaires. Toutefois, les moyens de diffusion utilisés ainsi que l'ampleur et le degré de précision des renseignements publiés varient considérablement. Ces informations figurent souvent dans la documentation budgétaire annuelle, avec les prévisions économiques et budgétaires. Le Canada, par exemple, a introduit dans son plan budgétaire un chapitre sur les "Risques d'amélioration et de détérioration des perspectives économiques et budgétaires ». Seuls la Finlande et le Royaume-Uni ont instauré des rapports spécifiques sur les risques budgétaires. D'autres pays, comme la Suède, publient des informations de qualité sur des risques précis dans différents rapports mais n'en donnent pas une vision consolidée.

Les pays de l'OCDE surveillent les mêmes types de risques budgétaires. Une vaste majorité d'entre eux observent de près les principales variables sur lesquelles reposent les prévisions budgétaires et les prévisions macroéconomiques, telles que la dette publique, la croissance et la démographie, la stabilité du secteur financier, mais aussi des revendications potentielles sur les ressources budgétaires en vertu de garanties accordées par l'État. Au sein de ces catégories générales, chaque pays peut identifier des risques spécifiques liés à son contexte national. Ainsi, les risques liés à la dette publique peuvent englober les risques de financement (risques de liquidité et de refinancement), les risques de marché (risques de taux et de change), les risques de crédit, les risques juridiques et opérationnels et les risques de modèle. Les risques liés à la situation macroéconomique peuvent comprendre l'impact budgétaire d'une baisse inattendue de la production et les risques liés à la démographie peuvent entraîner l'augmentation des dépenses liées au vieillissement, notamment les dépenses publiques de pensions et de santé (ce qui est le cas au Mexique). Les pays sont moins nombreux à considérer les litiges et les garanties liés aux partenariats public-privé (PPP), à la recapitalisation des entreprises publiques, au renflouement des administrations infranationales ou encore aux catastrophes naturelles comme des risques budgétaires. Parmi les autres risques budgétaires évoqués figurent les dépenses fiscales (France) et les dépenses obligatoires.

\section{Méthodologie et définitions}

Les données proviennent des résultats de l'Enquête 2018 de l'OCDE sur les pratiques et les procédures budgétaires. Elles ont été recueillies principalement auprès de hauts responsables du budget des pays de l'OCDE, dont les réponses représentent le propre point de vue des pays sur leurs pratiques actuelles. Sauf indication contraire, les réponses se rapportent à l'administration centrale ou fédérale.

Selon Kopits (2014), les risques budgétaires dénotent l'incertitude associée aux perspectives des finances publiques et peuvent être définis comme la probabilité de différences significatives entre la performance budgétaire réelle et attendue à moyen et long terme.

Bilan : état financier qui rend compte des actifs, des passifs et de la situation nette d'un État à un moment précis.

\section{Pour en savoir plus}

Kopits, G. (2014), "Coping with fiscal risk: Analysis and practice”, Revue de l'OCDE sur la gestion budgétaire, Vol. 14, n 1, https:// doi.org/10.1787/budget-14-5jxrgssdqnit.

Moretti, D. et Youngberry, T. (2018), "Getting added value out of accruals reforms", Revue de l'OCDE sur la gestion budgétaire, Vol. 18, n 1, https://doi.org/10.1787/budget-18-5j81804hpumt.

OCDE (2019), Budgeting and Public Expenditures in OECD Countries 2019, Éditions OCDE, Paris, https://doi.org/10.1787/ 9789264307957-en.

\section{Notes relatives aux graphiques}

Informations sur les données en Israël : http://dx.doi.org/10.1787/ 888932315602. Aucune donnée n'est disponible pour les États-Unis. 
5.4. Existence d'un cadre ou de lignes directrices à l'appui de la gestion des risques budgétaires, 2018

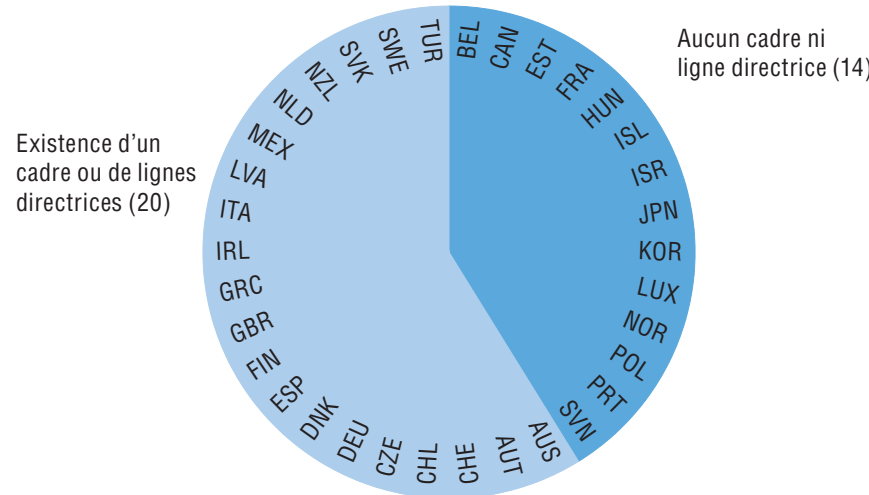

Source: OCDE (2018), Enquête de l'OCDE sur les pratiques et les procédures budgétaires, Éditions OCDE, Paris.

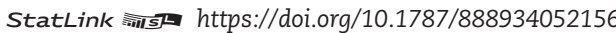

\subsection{Publication des risques financiers} dans un rapport, 2018

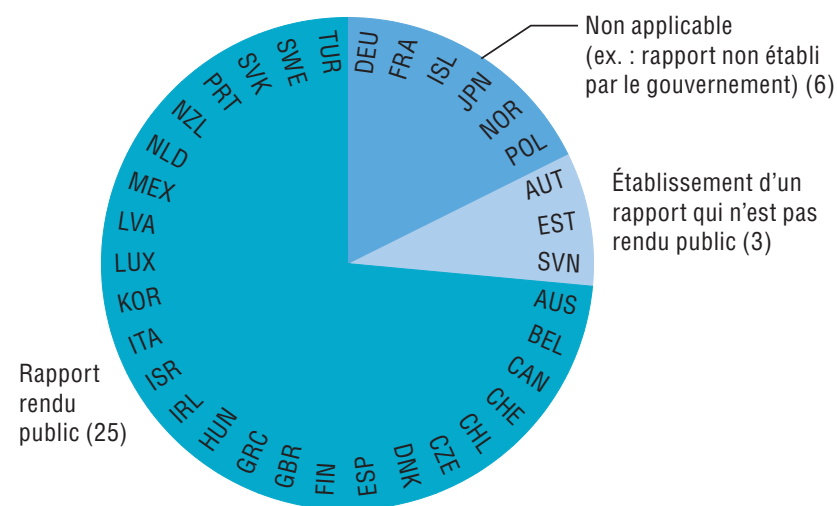

Source : OCDE (2018), Enquête de l'OCDE sur les pratiques et les procédures budgétaires, Éditions OCDE, Paris.

StatLink नiाIst https://doi.org/10.1787/888934052175

5.6. Risques budgétaires surveillés par les pays, 2018

\begin{tabular}{|c|c|c|c|c|c|c|c|c|c|c|c|}
\hline & $\begin{array}{l}\text { Chocs macro- } \\
\text { économiques }\end{array}$ & $\begin{array}{l}\text { Crise du secteur } \\
\text { financier }\end{array}$ & $\begin{array}{l}\text { Evolution } \\
\text { des intérêts } \\
\text { de la dette }\end{array}$ & $\begin{array}{c}\text { Evolutions } \\
\text { démographiques }\end{array}$ & $\begin{array}{l}\text { Catastrophes } \\
\text { naturelles }\end{array}$ & $\begin{array}{l}\text { Garanties } \\
\text { publiques }\end{array}$ & $\begin{array}{l}\text { Litiges et } \\
\text { procédures } \\
\text { judiciaires }\end{array}$ & $\begin{array}{l}\text { Partenariats public-privé } \\
\text { et/ou initiatives financières } \\
\text { envers le secteur privé }\end{array}$ & $\begin{array}{l}\text { Dégradation de } \\
\text { l'environnement }\end{array}$ & $\begin{array}{l}\text { Administrations } \\
\text { locales et/ou } \\
\text { assimilées }\end{array}$ & $\begin{array}{c}\text { Entreprises } \\
\text { publiques }\end{array}$ \\
\hline Allemagne & 0 & 0 & 0 & $\bullet$ & 0 & • & 0 & 0 & $\bullet$ & 0 & $\bullet$ \\
\hline Australie & • & $\bullet$ & - & $\bullet$ & 0 & - & - & 0 & 0 & 0 & 0 \\
\hline Autriche & $\bullet$ & $\bullet$ & - & $\bullet$ & - & - & $\bullet$ & 0 & - & • & $\bullet$ \\
\hline Belgique & $\bullet$ & 0 & - & $\bullet$ & 0 & O & 0 & O & 0 & 0 & 0 \\
\hline Canada & • & 0 & - & • & 0 & • & - & 0 & 0 & 0 & - \\
\hline Chili & 0 & $\bullet$ & 0 & 0 & 0 & - & $\bullet$ & $\bullet$ & 0 & 0 & 0 \\
\hline Corée & $\bullet$ & 0 & - & 0 & 0 & $\bullet$ & 0 & 0 & 0 & 0 & 0 \\
\hline Danemark & • & 0 & - & • & 0 & - & 0 & 0 & 0 & 0 & 0 \\
\hline Espagne & • & $\bullet$ & - & 0 & 0 & - & - & 0 & 0 & - & • \\
\hline Estonie & $\bullet$ & 0 & 0 & 0 & 0 & 0 & 0 & 0 & 0 & 0 & 0 \\
\hline Finlande & $\bullet$ & - & - & • & 0 & - & 0 & - & 0 & - & $\bullet$ \\
\hline France & - & - & - & $\bullet$ & 0 & - & - & - & 0 & 0 & 0 \\
\hline Grèce & 0 & 0 & - & $\bullet$ & 0 & $\bullet$ & 0 & 0 & 0 & - & 0 \\
\hline Hongrie & $\bullet$ & $\bullet$ & - & $\bullet$ & 0 & - & • & - & 0 & $\bullet$ & • \\
\hline Irlande & $\bullet$ & 0 & - & $\bullet$ & 0 & - & 0 & - & 0 & 0 & 0 \\
\hline Islande & $\bullet$ & $\bullet$ & - & 0 & 0 & - & 0 & - & $\bullet$ & 0 & 0 \\
\hline Israël & $\bullet$ & $\bullet$ & - & 0 & 0 & • & 0 & 0 & 0 & 0 & 0 \\
\hline Italie & $\bullet$ & $\bullet$ & - & $\bullet$ & $\bullet$ & - & 0 & 0 & 0 & 0 & 0 \\
\hline Japon & $\bullet$ & 0 & $\bullet$ & $\bullet$ & $\bullet$ & $\bullet$ & $\bullet$ & 0 & 0 & 0 & 0 \\
\hline Lettonie & 0 & 0 & O & $\bullet$ & 0 & $\bullet$ & $\bullet$ & - & 0 & - & $\bullet$ \\
\hline Luxembourg & $\bullet$ & 0 & - & $\bullet$ & 0 & 0 & 0 & 0 & 0 & 0 & 0 \\
\hline Mexique & $\bullet$ & $\bullet$ & $\bullet$ & $\bullet$ & $\bullet$ & $\bullet$ & $\bullet$ & $\bullet$ & $\bullet$ & $\bullet$ & $\bullet$ \\
\hline Norvège & - & 0 & 0 & 0 & 0 & - & 0 & 0 & 0 & 0 & 0 \\
\hline Nouvelle-Zélande & 0 & 0 & 0 & 0 & 0 & $\bullet$ & • & - & $\bullet$ & 0 & $\bullet$ \\
\hline Pays-Bas & - & $\bullet$ & $\bullet$ & $\bullet$ & $\bullet$ & - & - & - & - & - & $\bullet$ \\
\hline Pologne & $\bullet$ & 0 & - & 0 & 0 & $\bullet$ & 0 & $\mathrm{O}$ & 0 & 0 & 0 \\
\hline Portugal & $\bullet$ & $\bullet$ & - & $\bullet$ & 0 & $\bullet$ & 0 & - & 0 & $\bullet$ & $\bullet$ \\
\hline République slovaque & $\bullet$ & $\bullet$ & • & $\bullet$ & 0 & $\bullet$ & - & $\bullet$ & 0 & 0 & 0 \\
\hline République tchèque & $\bullet$ & 0 & $\bullet$ & $\bullet$ & 0 & • & $\bullet$ & - & $\bullet$ & - & - \\
\hline Royaume-Uni & $\bullet$ & $\bullet$ & $\bullet$ & $\bullet$ & 0 & - & $\bullet$ & $\mathrm{O}$ & 0 & - & 0 \\
\hline Slovénie & $\bullet$ & 0 & $\bullet$ & $\bullet$ & 0 & 0 & $\bullet$ & 0 & 0 & 0 & 0 \\
\hline Suède & $\bullet$ & $\bullet$ & $\bullet$ & $\bullet$ & 0 & - & 0 & $\bullet$ & 0 & • & $\bullet$ \\
\hline Suisse & $\bullet$ & $\bullet$ & - & $\bullet$ & $\bullet$ & - & - & 0 & - & 0 & $\bullet$ \\
\hline Turkey & $\bullet$ & - & - & $\bullet$ & - & - & - & - & 0 & - & $\bullet$ \\
\hline \multicolumn{12}{|l|}{ Total OCDE } \\
\hline - Oui & 29 & 18 & 28 & 25 & 7 & 30 & 18 & 15 & 8 & 13 & 15 \\
\hline O No & 5 & 16 & 6 & 9 & 27 & 4 & 16 & 19 & 26 & 21 & 19 \\
\hline
\end{tabular}

Source : OCDE (2018), Enquête de l'OCDE sur les pratiques et les procédures budgétaires. 
Les inégalités entre les sexes font encore partie intégrante de nombreux domaines d'action publique et sont souvent renforcées par les décisions relatives à l'affectation et à l'utilisation des ressources publiques. La budgétisation au service de l'égalité femmes-hommes (ou budgétisation sensible au genre) est une pratique pouvant garantir l'intégration systématique de cette problématique dans les décisions budgétaires. Selon la Recommandation du Conseil de l'OCDE sur l'égalité hommes-femmes dans la vie publique (2015), il s'agit d'un outil essentiel dans toute stratégie systémique des pouvoirs publics visant l'obtention de résultats en faveur de l'égalité des sexes. L'application effective de la budgétisation au service de l'égalité femmes-hommes peut contribuer à réduire les inégalités entre les sexes, en permettant aux administrations publiques de mieux prendre conscience des effets différenciés de leurs mesures budgétaires sur les citoyens et en privilégiant les projets qui concourent à la réduction de ces inégalités.

Les résultats de l'Enquête 2018 de l'OCDE sur les pratiques et les procédures budgétaires montrent que la budgétisation au service de l'égalité femmes-hommes connaît une popularité grandissante auprès des pays de l'OCDE : près de la moitié d'entre eux, soit 17 pays, l'ont désormais introduite à leur processus budgétaire, contre seulement 12 pays en 2016. Parmi ces 17 pays, un peu plus de la moitié est dotée de dispositions légales qui appuient cette pratique (Autriche, Belgique, Canada, Corée, Espagne, Islande, Italie, Mexique, Norvège et Portugal). L'existence d'un tel socle juridique peut permettre de préserver la continuité de la budgétisation au service de l'égalité femmeshommes et ce indépendamment de l'évolution à court terme du contexte économique ou politique.

Les pays connaissent une application de la budgétisation au service de l'égalité femmes-hommes variable, L'indice composite préliminaire de l'OCDE relatif à cette pratique a pour objet de synthétiser des données qualitatives discrètes sur ses principaux aspect et d'aider les décideurs et le public à déterminer dans quelle mesure la pratique a été adoptée et est appliquée. Cet indice composite vise à rendre compte de trois éléments constitutifs de la budgétisation au service de l'égalité femmeshommes : le cadre stratégique, les outils d'implémentation et le degré de faisabilité du contexte. Chacun de ces éléments est évalué indépendamment des autres, ce qui permet aux pays de mesurer leur performance pour chaque aspect. Bien que les outils d'implémentation soient primordiaux aux fins de la budgétisation au service de l'égalité femmes-hommes, un bon cadre stratégique et un contexte favorable peuvent contribuer à rendre cette pratique plus efficace. En terme de pondération de ces trois éléments constitutifs, un coefficient supérieur est donc attribué aux outils de mise en œuvre (40\%) par rapport au cadre stratégique et au degré de faisabilité du contexte (30\% chacun). La valeur de l'indice composite est comprise entre 0 et 1 . Une valeur élevée pour chacun des trois aspects pris en compte indique que le pays a créé un fort cadre stratégique de budgétisation au service de l'égalité femmes-hommes, qu'il dispose d'une vaste panoplie d'outils de mise en œuvre appliqués à toutes les étapes du cycle budgétaire et un environnement puissant pour soutenir la pratique. Les pays ont été classés en plusieurs catégories : la pratique de la budgétisation au service de l'égalité femmes-hommes peut y être quasi systématique (indice égal ou supérieur à 0,9 ), répandue (indice compris entre 0,5 et 0,9 ), en phase d'instauration (indice compris entre 0,2 et 0,5 ) ou minimale si la pratique est peu implantée (indice inférieur à 0,2 ). L'indice montre qu'aucun pays n'a encore recours de façon systématique à la budgétisation au service de l'égalité femmes-hommes. Néanmoins, l'Espagne, le Canada, le Mexique, la Corée, l'Islande, la Suède, l'Autriche, le Japon et la Norvège affichent tous une pratique répandue de cette budgétisation. En Espagne, au Mexique et en Islande, par exemple, l'application de la budgétisation au service de l'égalité femmes-hommes est encadrée par une stratégie nationale d'égalité des sexes. Ces trois pays sont aussi dotés de dispositions légales appuyant cette pratique et disposent d'une vaste panoplie d'outils de mise en œuvre ainsi que, généralement, de données désagrégées par sexe. Une évolution future de cet indice consisterait à passer de l'existence d'un cadre, d'outils et d'un contexte favorable, à un instrument à la fois d'évaluation du degré de bon fonctionnement des systèmes de budgétisation au service de l'égalité femmeshommes et de détermination de la mesure dans laquelle ces systèmes contribuent à la réalisation des objectifs fondamentaux en matière d'égalité des sexes.

\section{Méthodologie et définitions}

Ces données proviennent des résultats de l'Enquête 2018 de l'OCDE sur les pratiques et les procédures budgétaires. Elles ont été recueillies principalement auprès de hauts responsables du budget des pays de l'OCDE, dont les réponses représentent le propre point de vue des pays sur leurs pratiques et procédures actuelles. Ces données portent uniquement sur l'administration centrale ou fédérale et ne tiennent pas compte des niveaux infranationaux d'administration.

L'indice composite regroupe 15 variables pondérées en fonction de l'avis d'experts. Pour plus de détails sur les questions qui ont été posées, la structure de pondération et le calcul de l'indice composite, voir l'Annexe E. L'indice composite est la somme des valeurs normalisées de ses trois éléments constitutifs, qui varient chacune entre 0 et 1. Les variables prises en compte dans l'indice ont été sélectionnées par des experts de l'OCDE en fonction de leur pertinence puis passées en revue par les délégués nationaux à la Réunion d'experts de la budgétisation au service de l'égalité femmes-hommes, organisée sous les auspices du Réseau des hauts responsables du budget.

\section{Pour en savoir plus}

Downes, R. et Nicol, S. (2019), "Designing and implementing gender budgeting - a path to action", https://www.oecd.org/ gov/budgeting/designing-and-implementing-gender-budgeting-apath-to-action.pdf.

OCDE (2019), Budgeting and Public Expenditure in OECD countries 2019, Éditions OCDE, Paris, https://doi.org/10.1787/ 9789264307957-en

OCDE (2015), Recommandation du Conseil de l'OCDE sur l'égalité hommes-femmes dans la vie publique, https:// legalinstruments.oecd.org/en/instruments/OECD-LEGAL-0418.

\section{Notes relatives aux graphiques}

Aucune donnée n'est disponible pour les États-Unis. Informations sur les données en Israël : http://dx.doi.org/10.1787/888932315602.

5.7. La France et la Turquie signalent que l'introduction de la budgétisation au service de l'égalité femmes-hommes est à l'étude.

5.8. N'affiche que les données pour les pays de l'OCDE qui ont introduit la budgétisation sen au service de l'égalité femmes-hommes. Les données pour le Canada reflètent l'introduction de la Loi canadienne sur la budgétisation sensible au genre en décembre 2018. Les informations destinées aux pays continueront à être mises à jour à mesure que de nouvelles informations seront collectées. 
5.7. Existence d'une budgétisation sensible au genre, 2018

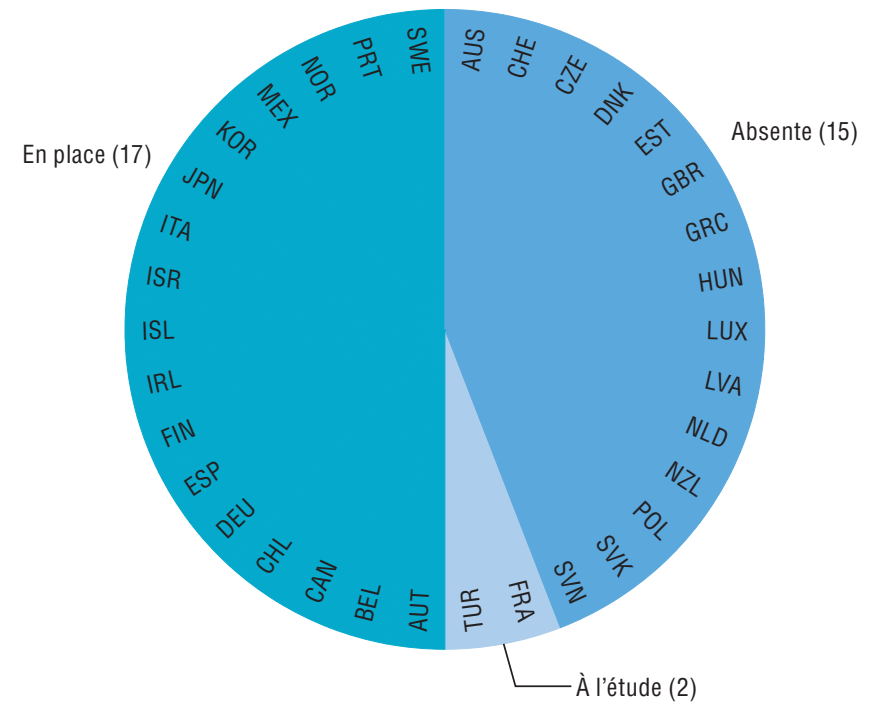

Source : OCDE (2018), Enquête de l’OCDE sur les pratiques et les procédures budgétaires.

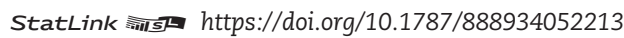

5.8. Premier passage à un indice composite relatif à la budgétisation sensible au genre, 2018

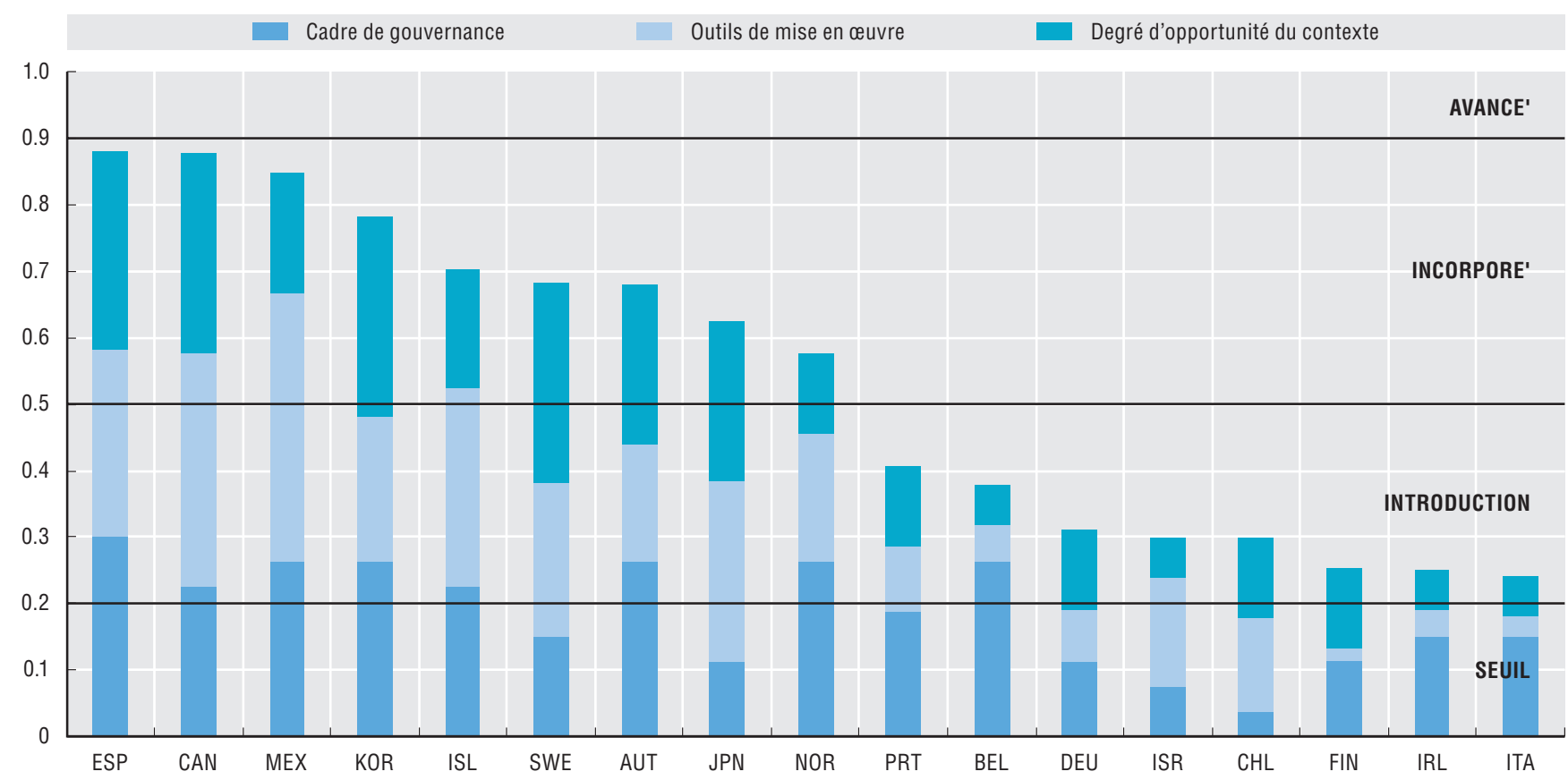

Source : OCDE (2018), Enquête de l'OCDE sur les pratiques et les procédures budgétaires. 
La transparence budgétaire désigne la divulgation complète, en temps opportun et de façon systématique, de l'ensemble des informations budgétaires. Recouvrant plusieurs dimensions, cette notion aborde la clarté, l'exhaustivité, la fiabilité, l'actualité, l'accessibilité et l'exploitabilité des informations publiées sur les finances publiques, ainsi que la participation des citoyens dans le processus budgétaire. Parmi les principales retombées positives de la transparence budgétaire, on peut citer l'amélioration des redevabilité, légitimité, intégrité, inclusivité et qualité des décisions budgétaires, chacune étant propre à développer la confiance entre les citoyens et les pouvoirs publics. Au-delà de la publication d'informationsclés sur le budget, la transparence budgétaire englobe aussi la participation citoyenne dans le processus budgétaire (OCDE 2017, 2019). L'OCDE guide et encourage activement l'adoption de pratiques visant à accroître la transparence budgétaire, comme en témoignent les principes énoncés dans Transparence budgétaire - Les meilleures pratiques de l'OCDE (2001), Recommandation sur la gouvernance budgétaire (2015) et Panoplie OCDE des instruments de la transparence budgétaire (2017).

D'une manière générale, la transparence budgétaire progresse depuis quelques années dans les pays de l'OCDE, une tendance due essentiellement à la publication des principaux rapports budgétaires. Tous les pays de l'OCDE pour lesquels des données sont disponibles publient désormais un projet de budget puis le budget approuvé (34 pays) et une majorité d'entre eux met à disposition l'ensemble des données connexes à ces documents dans un format lisible par machine (par exemple, les données ouvertes) respectivement 24 et (27 pays). On observe une tendance analogue concernant la publication de guides sur ces documents budgétaires destinés aux citoyens («budget du citoyen "). Ces documents synthétiques et faciles à comprendre ont pour objet de présenter simplement et clairement les décisions budgétaires. Si en 2012, 14 pays de l'OCDE publiaient ce type de guide (42\%), ils sont désormais produits dans 23 (68\% des pays étudiés). Les 9 pays ayant commencé dès 2012 à élaborer un " budget du citoyen " sont le Danemark, l'Espagne, la France, la Hongrie, le Portugal, la République tchèque, le Royaume-Uni, la Slovénie et la Turquie. En 2018, ces guides étaient le plus souvent publiés pour le budget approuvé (17 pays) et la proposition de budget de l'exécutif (11 pays) et le sont plus rarement pour les nombreux autres documents budgétaires, même si ces derniers et les jeux de données s'y rapportant, étaient largement accessibles.

Il incombe aux parlements d'approuver la proposition de budget de l'exécutif en veillant à ce que le budget reflète les préférences des citoyens tout en respectant les contraintes budgétaires. Dans tous les pays pour lesquels on dispose de données, la palette des documents budgétaires fournis par les pays de l'OCDE à leur parlement ne cesse de s'élargir depuis 2012, signe des efforts des pays en faveur de la transparence et d'une supervision budgétaire renforcée. En 2018, la totalité des pays étudiés de l'OCDE présentaient leurs hypothèses macroéconomiques à l'organe législatif, $97 \%$ lui soumettaient leurs priorités budgétaires et $82 \%$ proposaient un éclairage à moyen terme sur les recettes et dépenses publiques totales. Une autre tendance en lien avec la supervision budgétaire observée dans les pays de l'OCDE est le rôle grandissant des parlements au cours de la phase ex ante du processus budgétaire. Le nombre de pays déclarant que leur gouvernement soumet un rapport d'orientation budgétaire au parlement est passé de 19 en 2012 à 22 en 2018. Si en 2012, seuls 3 pays de l'OCDE indiquaient qu'un débat d'orientation budgétaire avait lieu au parlement, ils n'étaient pas moins de 15 en 2018. Pour plus de la moitié d'entre eux, ce débat donnait lieu à un rapport adressé au gouvernement (notamment au Canada, en France, en Grèce, en Italie et en Suède).

Au-delà de ces aspects essentiels d'une budgétisation ouverte, transparente et inclusive, on peut envisager d'élargir la notion de transparence budgétaire afin d'englober des mesures complémentaires de gouvernance budgétaire. L'exhaustivité des informations budgétaires mises à disposition dans le cadre des efforts de transparence budgétaire revêt à cet égard une importance toute particulière.

\section{Méthodologie et définitions}

Ces données proviennent des résultats des enquêtes 2012 et 2018 de l'OCDE sur les pratiques et les procédures budgétaires et de l'enquête 2018 du Réseau des responsables budgétaires des parlements sur les pratiques des parlements en matière budgétaire. Concernant les deux premières enquêtes, les données ont été principalement recueillies auprès de hauts responsables du budget du ministère central ou fédéral des Finances. Les données de l'enquête de 2012 se rapportent aux 34 pays alors membres de l'OCDE. Pour l'enquête 2018, on dispose de données pour tous les pays de l'OCDE à l'exception des États-Unis. L'Enquête de l'OCDE sur les pratiques des parlements en matière budgétaire a été menée principalement auprès de responsables budgétaires des parlements des pays de l'OCDE. Les réponses aux enquêtes représentent le propre point de vue des pays sur leurs pratiques et procédures actuelles. Les données ouvertes désignent des données numériques accessibles dont les caractéristiques techniques et juridiques permettent la libre utilisation, réutilisation et redistribution par quiconque, à tout moment, en tout lieu. Un « budget du citoyen » est une synthèse en langage simple des principaux volets du budget annuel ou d'autres documents budgétaires. Il utilise un langage accessible afin d'aider un lecteur non spécialiste à comprendre des informations techniques.

\section{Pour en savoir plus}

OCDE (2019), Budgeting and Public Expenditures in OECD Countries 2019, Éditions OCDE, Paris, https://doi.org/10.1787/ 9789264307957-en.

OECD (2017), OECD Budget Transparency Toolkit: Practical Steps for Supporting Openness, Integrity and Accountability in Public Financial Management, Éditions OCDE, Paris.

OCDE (2015), Recommandation du Conseil sur la Gouvernance budgétaire, Éditions OCDE, Paris, http://www.oecd.org/mena/ governance/Recommandation-du-Conseil-sur-la-gouvernancebudg\%C3\%A9taire.pdf

\section{Notes relatives aux graphiques}

Concernantles données sur Israël, voir http://doi.org/10.1787/888932315602. 5.9 et 5.10. Aucune donnée n'est disponible pour les États-Unis

5.11. Aucune donnée n'est disponible pour le Mexique. 
5.9. Méthodes retenues pour la mise à disposition et la transparence des principales informations budgétaires, 2018

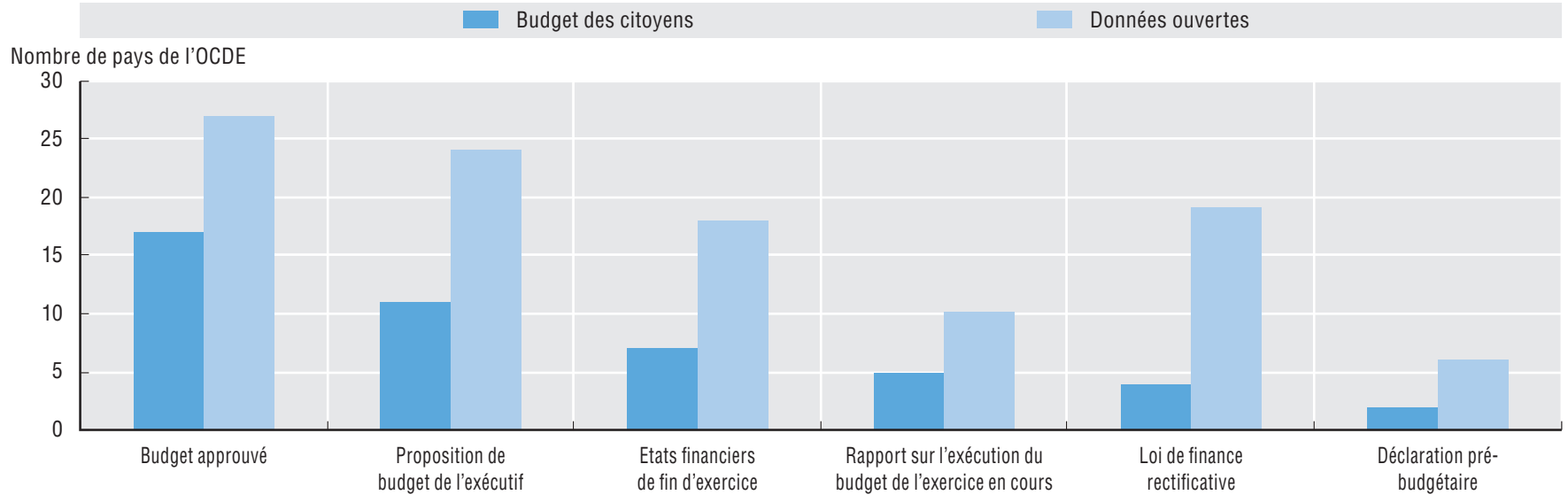

Source : OCDE (2018), Enquête de l'OCDE sur les pratiques et les procédures budgétaires.

\subsection{Documents budgétaires présentés au parlement, 2018}

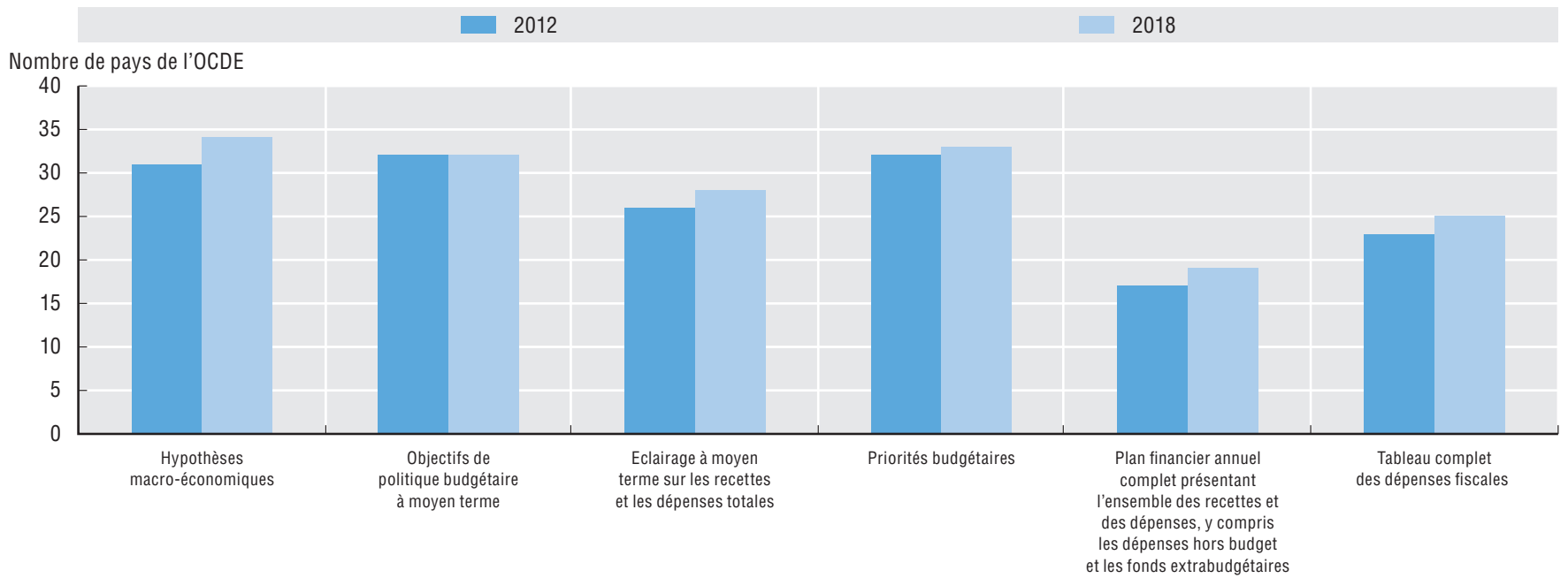

Source : OCDE $(2012,2018)$, Enquête de l'OCDE sur les pratiques et les procédures budgétaires.

5.11. Le parlement tient-il un débat d'orientation budgétaire ?, 2018

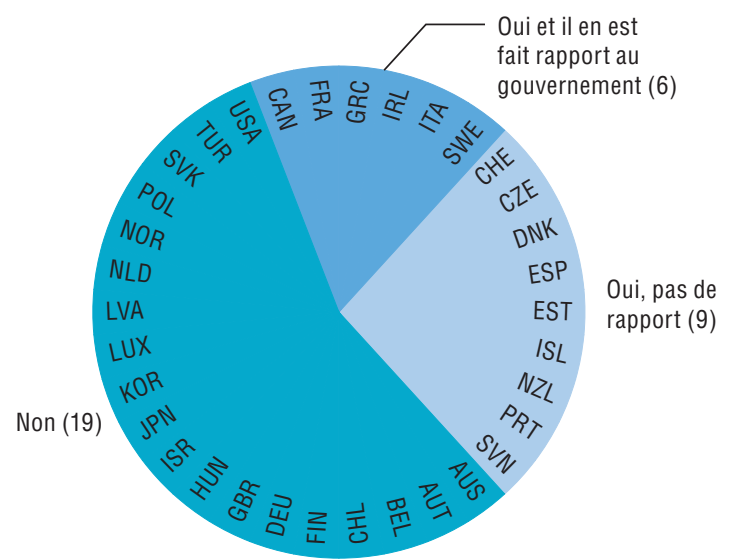

Source : OCDE (2018), Enquête du Réseau des responsables budgétaires des parlements sur les pratiques des parlements en matière budgétaire. 
La procédure et les mécanismes d'affectation des fonds publics sont des leviers d'action essentiels pour la réalisation d'un développement durable. Au cours des dernières décennies, de nombreux pays à travers le monde se sont engagés à atteindre des objectifs nationaux et internationaux de développement durable, dont ceux de l'Accord de Paris sur le climat ainsi que les Objectifs de développement durable (ODD), ce qui suppose que ces pays renforcent les mesures prises en la matière au niveau national. Afin de soutenir les États dans cette démarche, l'OCDE s'intéresse à de nouvelles approches de la planification et de la préparation du budget, en encourageant les autorités publiques à plus de transparence et de dialogue avec les citoyens et en leur recommandant des dispositifs de suivi et d'évaluation susceptibles d'appuyer la réalisation de leurs engagements nationaux et internationaux. Le Projet collaboratif de Paris sur les budgets verts (Paris Collaborative on Green Budgeting) fait partie des initiatives lancées à cet effet par l'OCDE. Son objectif est de travailler en étroite collaboration avec les pays en vue d'aligner les processus relatifs aux recettes et aux dépenses publiques sur les objectifs environnementaux et climatiques.

La budgétisation « verte » vise à donner aux décideurs, au parlement et au public une vision plus claire de l'impact potentiel des choix de budgétisation sur l'environnement. Disposer d'informations exhaustives sur les effets à long terme des politiques pourrait influencer les décisions en matière de collecte de recettes et d'affectation de ressources, car cela permettrait d'y intégrer les intérêts des générations futures et la question de la durabilité des ressources. Or, les données disponibles concernant les effets des choix budgétaires sur l'environnement peuvent s'avérer limitées ou ne pas être utilisées de façon systématique. En 2018, 20 \% des pays étudiés de l'OCDE ont publié des données portant sur l'impact environnemental et climatique des mesures budgétaires. Seules l'Allemagne et l'Italie ont poussé la démarche jusqu'à dévoiler l'impact que produirait sur l'environnement l'exécution du budget telle qu'elle est prévue. De même, les informations concernant les effets du budget sur les indicateurs de bien-être sociétal sont rares - elles sont disponibles dans $26 \%$ des pays interrogés -, tandis que les études d'impact sur l'environnement sont plus courantes lorsqu'il s'agit des investissements en capital. Si la plupart des pays de l'OCDE réalisent des études de ce type au début de l'élaboration des projets d'infrastructure publique, seuls 12 d'entre eux évaluent les émissions potentielles de dioxine de carbone de chaque projet.

La prise en compte de la dimension environnementale dans tous les domaines d'action publique visés par le budget (de l'environnement et du climat aux transports en passant par la santé et les finances) devrait favoriser l'harmonisation des mesures d'incitation et engendrer une approche de l'élaboration des politiques cohérente, éclairée sur le plan environnemental et étayée par les décisions budgétaires. Dans la plupart des pays de l'OCDE, le changement climatique figure dans les programmes budgétaires du ministère qui est chargé au premier chef de cette question (par exemple, le ministère de l'Environnement). Peu de pays, parmi lesquels le Canada, la Finlande et l'Irlande prennent systématiquement en compte les questions environnementales dans l'ensemble de leurs programmes budgétaires. L'existence d'informations sur la performance budgétaire pourrait contribuer à une réflexion plus systématique sur la durabilité, ainsi qu'à un renforcement de la transparence et de la redevabilité, notamment en matière de progrès accomplis en vue de la réalisation des objectifs environnementaux et de tenue des autres engagements internationaux connexes. Seuls $25 \%$ des pays étudiés de l'OCDE ont commencé soit à prendre systématiquement en compte les ODD dans leur système de budgétisation axée sur la performance (6\%) soit à aligner étroitement ce dernier sur les objectifs en question (19 \%). Par exemple, depuis 2016 en Norvège, chacun des ODD est confié à un ministère coordonnateur qui doit rendre compte de l'avancement de la réalisation de l'objectif au niveau de ses diverses cibles dans les documents budgétaires. Au Mexique, la mesure d'harmonisation systématique des programmes budgétaires au regard des ODD, par l'intermédiaire d'un système d'évaluation de la performance, a été adoptée en 2018.

\section{Méthodologie et définitions}

Ces données proviennent de l'édition 2017-18 de l'Enquête de l'OCDE sur les pratiques et les procédures budgétaires, de l'édition 2018 de l'Enquête de l'OCDE sur la budgétisation axée sur la performance, évaluation et contrôle et de l'édition 2018 de l'Enquête de l'OCDE sur la budgétisation des investissements et la gouvernance des infrastructures. Les participants à ces enquêtes étaient en majorité de hauts responsables du ministère des Finances et d'autres ministères sectoriels compétents.

Le Développement durable répond aux besoins actuels sans compromettre la capacité des générations futures à satisfaire leurs propres besoins. Cette notion suppose la préservation des ressources naturelles en vue de leur croissance et de leur développement futur.

Le Projet collaboratif de Paris sur les budgets verts est la première initiative internationale et intersectorielle visant à aider les pouvoirs publics dans leurs efforts d'intégration des engagements de durabilité environnementale et de croissance verte dans les cadres budgétaires et d'action publique nationaux. Le Projet collaboratif de Paris est un moyen de promouvoir à la fois les travaux préparatoires d'ordre analytique et méthodologique, l'apprentissage mutuel et l'échange de bonnes pratiques, d'expérimenter des outils novateurs et de lancer des projets pilotes et enfin de canaliser les connaissances acquises afin d'offrir une aide ciblée aux pouvoirs publics.

Dans la plupart des pays de l'OCDE, le budget est présenté au parlement sous la forme d'un ensemble de programmes budgétaires ou bien par catégorie fonctionnelle correspondant à des domaines d'activité et ce éventuellement en complément ou en remplacement de sa présentation par catégorie administrative et économique. La budgétisation par programme est caractéristique d'un système de budgétisation axée sur la performance.

\section{Pour en savoir plus}

OCDE, Paris Collaborative on Green Budgeting, http://www.oecd.org/ environment/green-budgeting/.

\section{Notes relatives aux graphiques}

Concernantles données sur Israël, voir http://doi.org/10.1787/888932315602. Les données sur les États-Unis ne sont pas disponibles.

5.12. La Grèce n'a pas répondu à cette question. L'Irlande est en train de systématiquement intégrer les considérations climatiques dans ses programmes budgétaires.

5.13. La Belgique, l'Irlande et l'Italie n'ont pas répondu à cette question.

5.15. "Prise en compte des conséquences environnementales et climatiques dans les projets d'infrastructure publique » est disponible en ligne à l'Annexe $\mathrm{F}$. 
5.12. Informations concernant l'impact des décisions budgétaires sur l'environnement et le bien-être général publiées dans le budget, 2018

\begin{tabular}{|c|c|c|}
\hline Pays & Effets sur l'environnement ou sur le changement climatique & Effets sur une sélection d'indicateurs du bien-être sociétal \\
\hline Allemagne & $\odot$ & $\odot$ \\
\hline Australie & 凶 & 凶 \\
\hline Autriche & $\theta$ & 凶 \\
\hline Belgique & 凶 & 凶 \\
\hline Canada & 凶 & 凶 \\
\hline Chili & 凶 & 凶 \\
\hline Corée & 凶 & 凶 \\
\hline Danemark & 凶 & 凶 \\
\hline Espagne & $\bar{\theta}$ & $\bar{\ominus}$ \\
\hline Estonie & 凶 & 凶 \\
\hline Finlande & 凶 & 凶 \\
\hline France & $\ominus$ & $\ominus$ \\
\hline Grèce & 凶 & 凶 \\
\hline Hongrie & $\theta$ & $\theta$ \\
\hline Irlande & 凶 & 凶 \\
\hline Islande & 凶 & 凶 \\
\hline Israël & $凶$ & 凶 \\
\hline Italie & $\odot$ & $\odot$ \\
\hline Japon & 凶 & 凶 \\
\hline Lettonie & 冈 & $\ominus$ \\
\hline Luxembourg & $凶$ & $凶$ \\
\hline Mexique & $\ominus$ & $\ominus$ \\
\hline Norvège & $\theta$ & 凶 \\
\hline Nouvelle-Zélande & 凶 & 凶 \\
\hline Pays-Bas & 凶 & ○ \\
\hline Pologne & 凶 & 凶 \\
\hline Portugal & 凶 & 凶 \\
\hline République slovaque & 凶 & 凶 \\
\hline République tchèque & $\theta$ & $\theta$ \\
\hline Royaume-Uni & 凶 & 凶 \\
\hline Slovénie & 凶 & 凶 \\
\hline Suède & 凶 & 凶 \\
\hline Suisse & 凶 & 凶 \\
\hline Turkey & 凶 & 凶 \\
\hline \multicolumn{3}{|l|}{ Total OCDE } \\
\hline Informations non publiées $囚$ & 25 & 25 \\
\hline Informations publiées pour chaque mesure $\ominus$ & 7 & 6 \\
\hline Informations publiées pour l'ensemble du budget $\odot$ & 2 & 3 \\
\hline
\end{tabular}

Source : OCDE (2018), Enquête de l'OCDE sur les pratiques et les procédures budgétaires, OCDE, Paris.

\subsection{Processus d'intégration du changement climatique dans le budget, 2018}

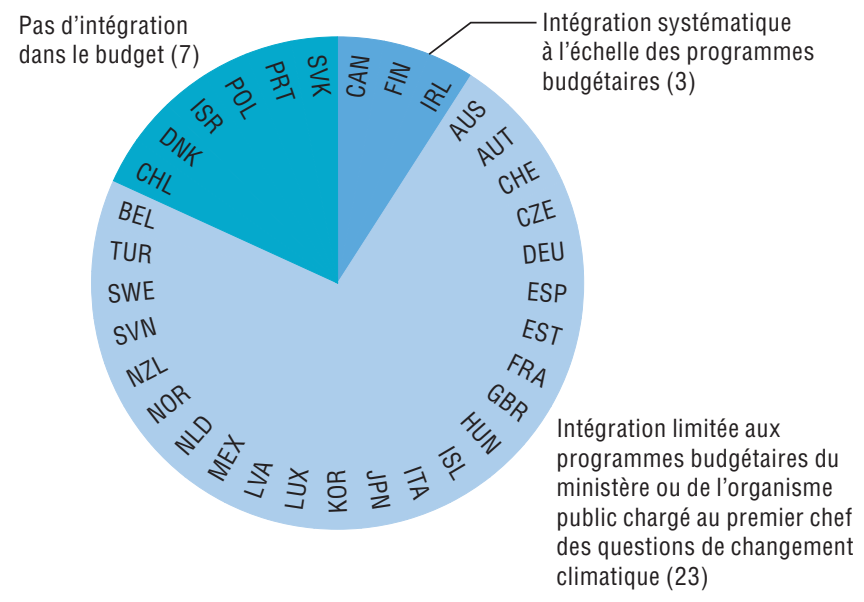

Source : OCDE (2018), Enquête de l'OCDE sur les performances budgétaires. StatLink तiाst https://doi.org/10.1787/888934052308

\subsection{Prise en compte des Objectifs de Développement Durable dans les systèmes} de budgétisation axée sur la performance, 2018

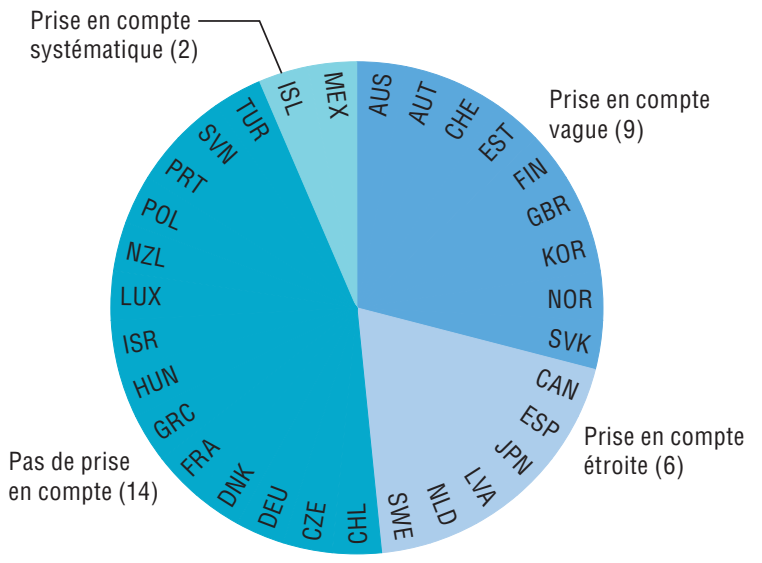

Source : OCDE (2018), Enquête de l'OCDE sur les performances budgétaires.

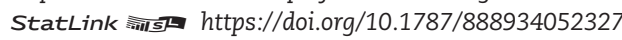




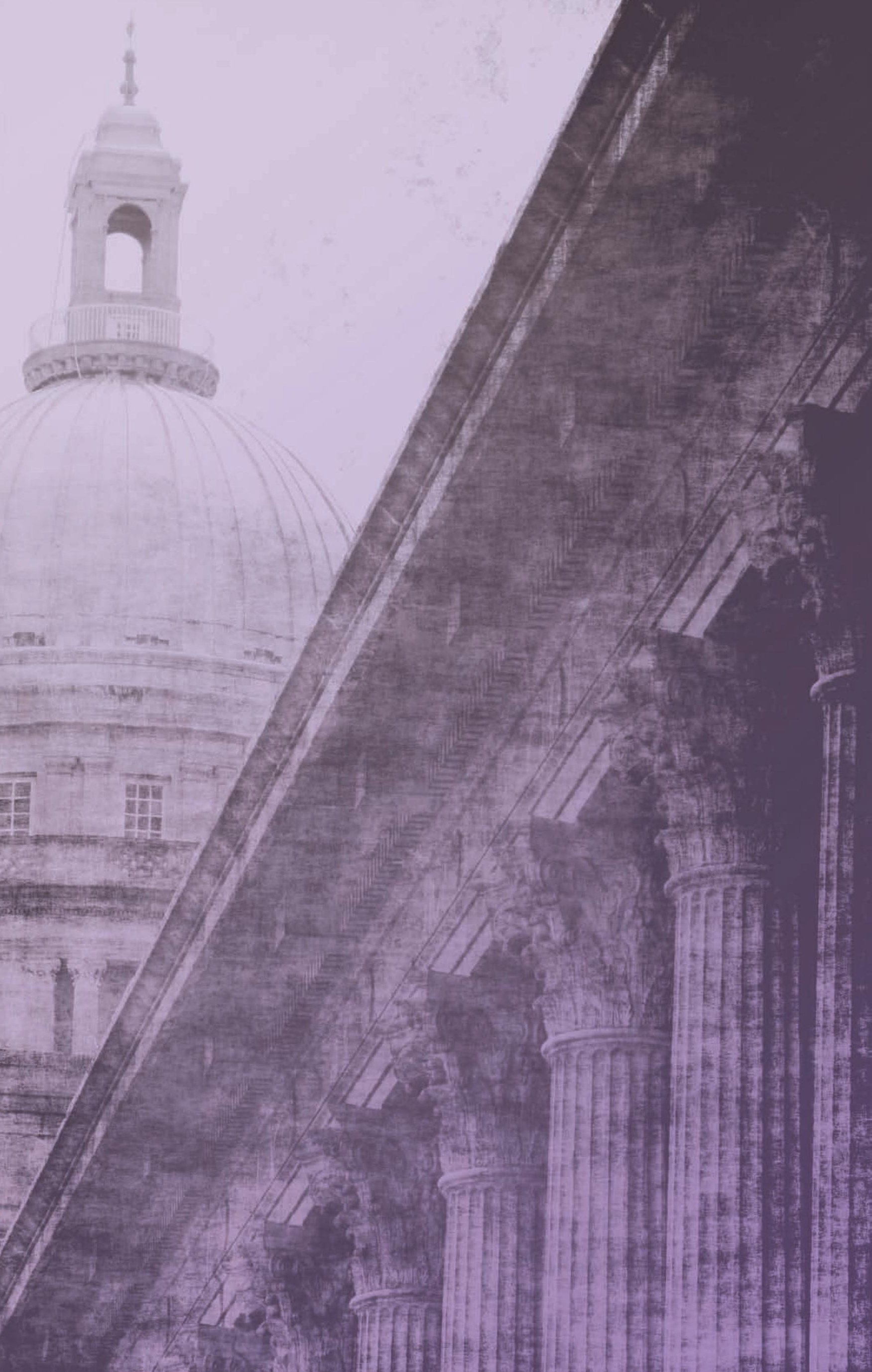




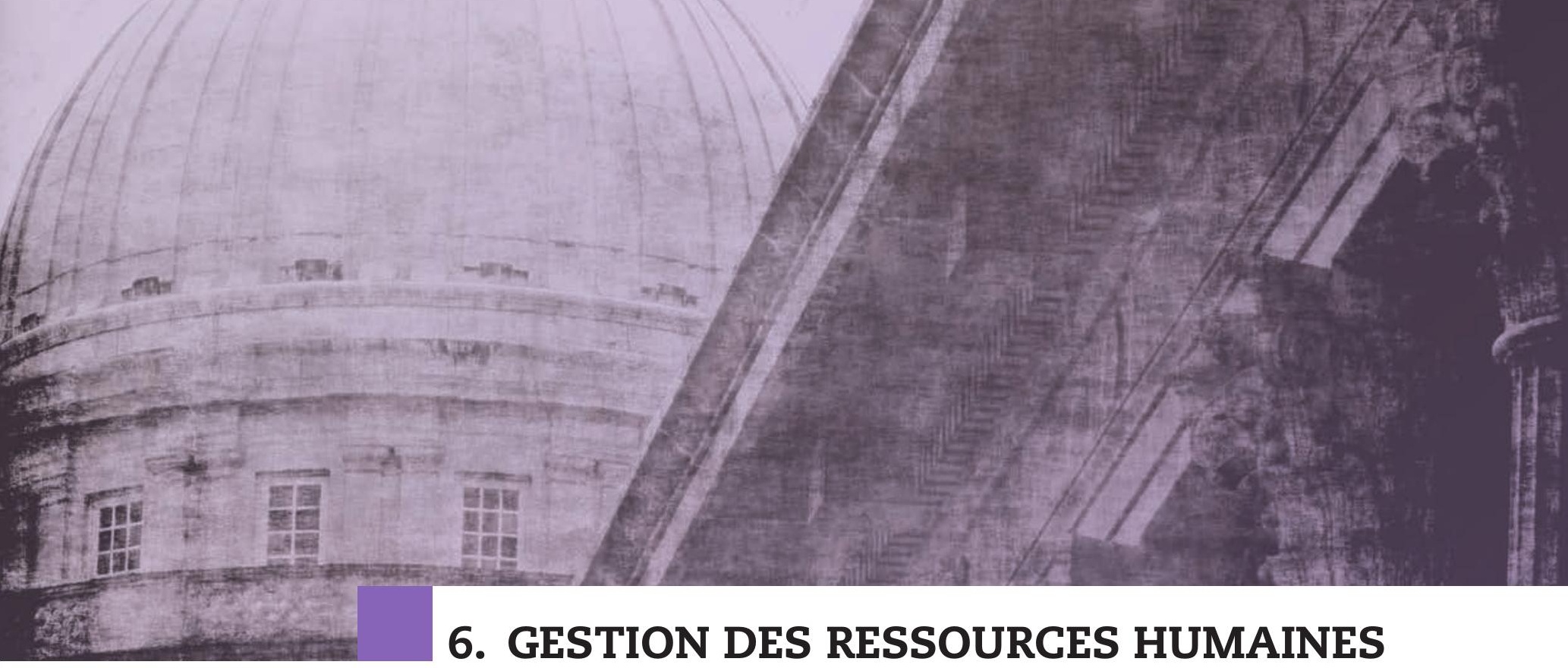

Conditions d'emploi des fonctionnaires et autres agents publics Apprentissage et perfectionnement dans la fonction publique Gestion de la performance des cadres de haut niveau de la fonction publique 
Les administrations font travailler leur personnel selon des conditions diverses, liées aux "modalités contractuelles" qui s'appliquent à leur emploi. L'une des distinctions les plus courantes est celle qui s'opère entre les personnes embauchées avec le statut de fonctionnaire (auquel s'applique généralement un cadre juridique distinct) et les personnes embauchées dans un cadre juridique différent tel que, par exemple, celui du droit du travail classique, avec d'autres conditions d'emploi. Les modalités contractuelles qui s'appliquent peuvent influer entre autres sur la rémunération, la sécurité de l'emploi, l'évaluation des performances et l'accès à la formation. Elles ont également un impact manifeste sur l'aptitude des systèmes d'emploi public à attirer et à garder des employés qualifiés et à les motiver de façon à ce qu'ils donnent le meilleur d'euxmêmes au service des objectifs de l'action publique. Quand elles sont employées à bon escient, ces différentes modalités contractuelles peuvent offrir aux administrations publiques la souplesse nécessaire pour former et diriger des effectifs dotés de la gamme souhaitée de compétences. En revanche, si les administrations publiques n'ont pas de vision claire de la combinaison idéale à trouver entre les diverses modalités contractuelles possibles pour les différents métiers du secteur public, elles s'exposent à un risque de morcellement de leur marché du travail interne.

La plupart des pays de l'OCDE présentent un point commun en la matière : leur administration centrale est essentiellement composée de fonctionnaires. La part des agents d'administration centrale dotés du statut de fonctionnaire s'élevait, en 2018, à $68 \%$ en moyenne des pays de l'OCDE pour lesquels des données étaient disponibles. En Italie, en Pologne, en Slovénie, aux Pays-Bas et en Suisse et tous les agents de l'administration centrale sont des fonctionnaires. À l'autre extrémité de l'éventail, la Suède n'opère pas de distinction entre les fonctionnaires et les " autres employés ». De même, le Danemark, le Mexique et l'Irlande comptent relativement peu de fonctionnaires au sein des effectifs de leur secteur public. La distinction entre ces statuts est importante, car les rôles et les responsabilités des fonctionnaires et des autres agents publics varient considérablement d'un pays à l'autre.

Dans $67 \%$ des pays de l'OCDE, les fonctionnaires bénéficient d'une sécurité de l'emploi supérieure à celle des autres agents publics. Dans $58 \%$ des pays, les fonctionnaires suivent une procédure de recrutement plus rigoureuse, bénéficient de plus grandes possibilités d'évolution de carrière et sont soumis à une grille de rémunération différente. Un peu moins de $50 \%$ des pays de l'OCDE ont mis en place un régime spécifique de gestion des performances pour les fonctionnaires et, dans $39 \%$ des pays de l'OCDE, les fonctionnaires doivent respecter des valeurs et des règles particulières de déontologie. S'agissant de la couverture médicale et des droits à la retraite, la différence semble moins marquée entre les fonctionnaires et les autres agents publics.

Les grands défis que les administrations publiques vont à l'avenir devoir relever les obligeront à réévaluer en permanence la gamme de compétences et d'aptitudes dont elles ont besoin (OCDE 2017). Pour attirer les candidats et garder les agents, il sera crucial de mettre en place des modalités d'emploi claires, transparentes et conformes aux réalités du marché du travail et ce surtout dans les domaines menacés par des pénuries de compétences.

\section{Méthodologie et définitions}

Ces données sont tirées de l'enquête 2016 de l'OCDE sur la gestion stratégique des ressources humaines (SHRM) et ont fait l'objet d'une actualisation en 2019. Les réponses ont été fournies, pour la plupart, par de hauts fonctionnaires des services de gestion des ressources humaines de l'administration centrale et les données font référence aux pratiques de gestion des ressources humaines (RH) de l'administration centrale. Tous les pays de l'OCDE ont répondu à l'enquête ainsi que deux pays candidats à l'adhésion : la Colombie et le Costa Rica. Les définitions de la fonction publique et du périmètre de l'administration centrale varient considérablement d'un pays à l'autre et doivent être prises en compte dans la comparaison. Aux fins de cette enquête, le terme "fonctionnaire » désigne exclusivement les agents publics couverts par un cadre juridique public particulier ou par d'autres dispositions spécifiques, tandis que la catégorie des " autres employés » recouvre les personnes embauchées sous un type différent de cadre juridique, tel que le droit du travail dans le secteur privé qui connaît des règles d'emploi différentes. Les données ne portent pas sur les consultants auxquels le secteur public fait appel dans un autre cadre d'emploi.

\section{Pour en savoir plus}

OCDE (2019), Recommandation du Conseil sur le leadership et les aptitudes de la fonction publique, OECD/LEGAL/0445.

OCDE (2017), "Skills for a High Performing Civil Service", Examens de l'OCDE sur la gouvernance publique, Éditions OCDE, Paris, https://doi.org/10.1787/9789264280724-en.

\section{Notes relatives aux graphiques}

Les données concernant l'Islande, la Turquie et la Colombie se rapportent à 2016. Pour les données relatives à Israël, voir http://doi. org/10.1787/888932315602.

6.1. Les données pour le Danemark, Israël, le Japon, le Mexique et la Nouvelle-Zélande se rapportent à 2019. Les données pour l'Allemagne, la Corée, la France, et la Pologne se rapportent à 2017. Les données pour les États-Unis, l'Italie et la Lettonie et se rapportent à 2016. Les données pour la Slovénie se rapportent à 2015. Les données sur les autres agents du secteur public n'étaient pas disponibles pour la Hongrie, la Norvège et la République slovaque. Pour la Corée, les données relatives aux fonctionnaires ont été compilées par le ministère de la Gestion du personnel et celles concernant les " autres agents publics " ont été compilées par le ministère de l'Emploi et du Travail. Pour le Portugal, les " autres agents publics " incluent les cadres dirigeants à durée déterminée. Les fonctionnaires définis comme des agents de la fonction publique relevant d'un cadre juridique public spécifique ou d'autres dispositions spécifiques se réfèrent au Danemark à « tjenstemøend ». 
6.1. Fonctionnaires et autres agents de l'administration centrale, 2018

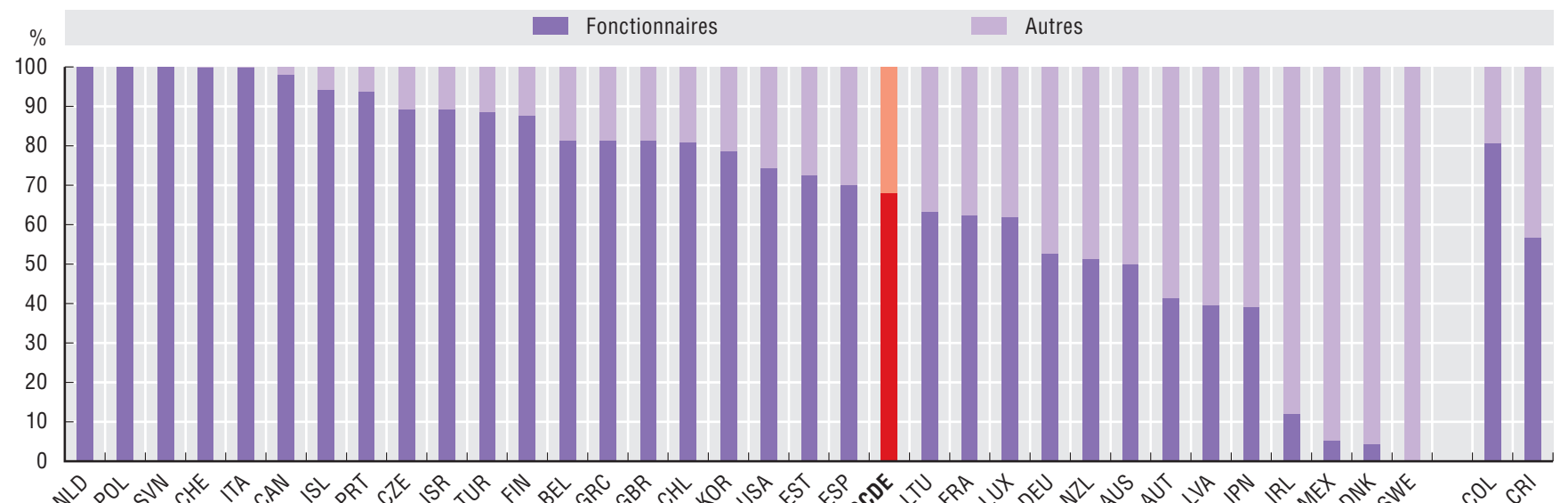

Source : Enquête 2019 de l'OCDE sur la gestion stratégique des ressources humaines (SHRM).

StatLink न्ताIst https://doi.org/10.1787/888934052346

6.2. Différences entre le cadre d'emploi des fonctionnaires et celui des autres agents de l'administration centrale, 2019

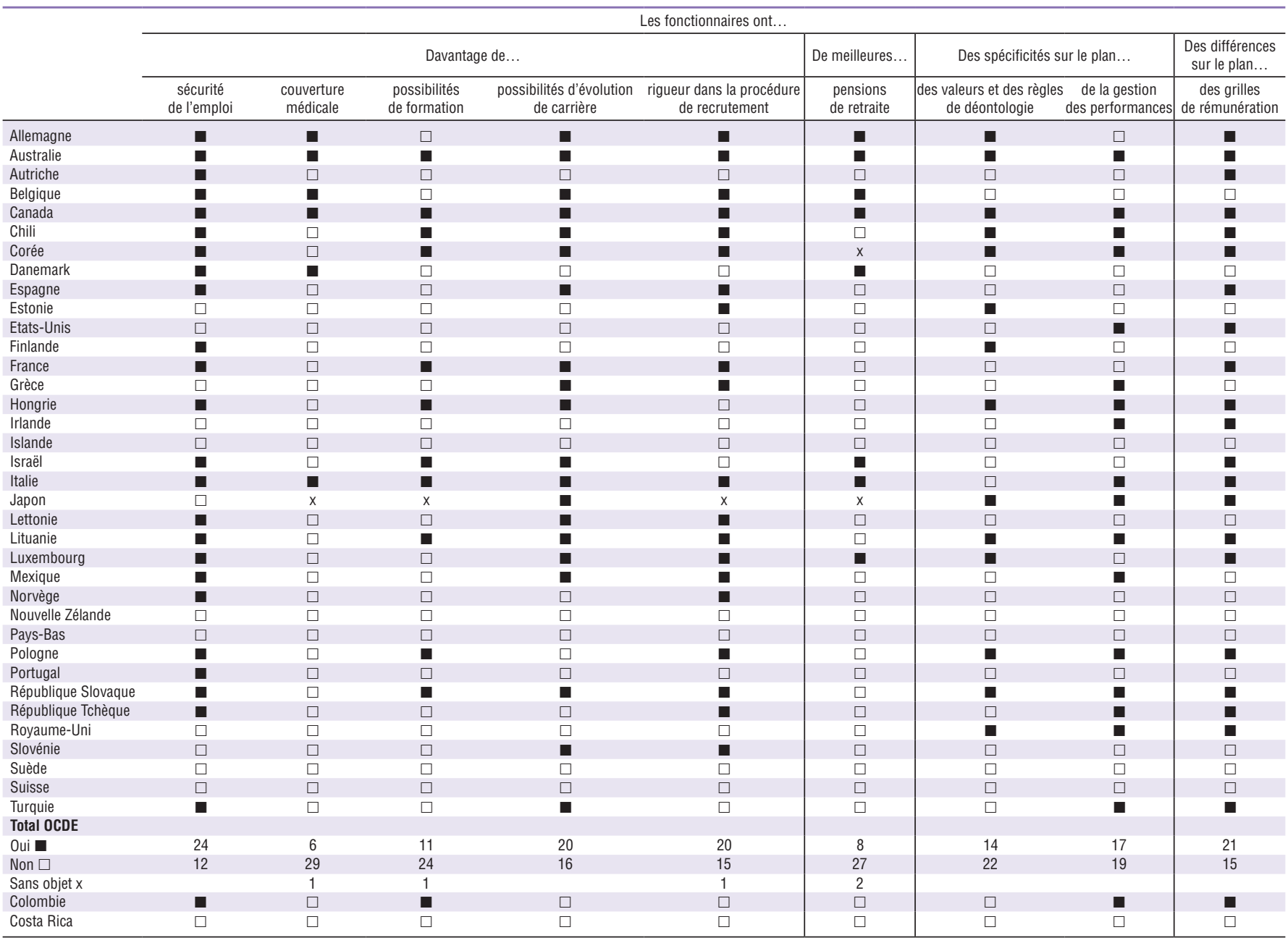

Source : Enquête 2019 de l'OCDE sur la gestion stratégique des ressources humaines (SHRM). 
Traditionnellement, le modèle de recrutement et de perfectionnement suivi par de nombreux pays pour leur fonction publique consiste à recruter des individus en début de carrière, puis à les perfectionner grâce à une formation spécifique. Toutefois, dans de nombreux pays de l'OCDE, les coupes effectuées dans les budgets de formation lors des récentes périodes d'austérité ont réduit l'accès aux cours traditionnels de formation (OCDE 2016). Or, des investissements stratégiques et ciblés dans l'apprentissage et le perfectionnement sont essentiels pour que la fonction publique réussisse à s'adapter à l'évolution rapide des exigences des citoyens et des outils technologiques. De plus, offrir des possibilités de formation peut aider à attirer et à motiver des collaborateurs de qualité. Dans sa Recommandation de 2019 sur le leadership et les aptitudes de la fonction publique, le Conseil de l'OCDE recommande aux pays membres de créer une culture et un environnement propices à l'apprentissage au sein de la fonction publique, bien au-delà des cours traditionnels de formation.

En 2019, environ $61 \%$ des pays de l'OCDE (22 sur 36) ont adopté des stratégies ou des plans d'action en matière de formation à l'échelle de la fonction publique tout entière, alors qu'ils n'étaient qu'un peu moins de $50 \%$ en 2016. En 2016, le Portugal a changé de démarche en matière de renforcement des compétences dans la fonction publique. Ainsi, sa stratégie de formation actuelle, qui s'applique à l'ensemble de la fonction publique, englobe la formation initiale (obligatoire pour la plupart des agents), la formation continue et l'autoformation. De fait, environ $70 \%$ des pays élaborent des plans institutionnels d'apprentissage au sein de leur administration centrale. En revanche, seule la moitié des pays (18) exige des fonctionnaires qu'ils élaborent eux-mêmes un plan individuel d'apprentissage. Les données semblent montrer qu'en matière de formation, l'accent est mis sur la formation et le coaching du personnel de direction, et que les modèles mixtes d'apprentissage sont de plus en plus recherchés. Le coaching et le mentorat des agents n'appartenant pas au personnel de direction constituent une priorité de formation dans 9 pays de l'OCDE, parmi lesquels l'Allemagne et la Corée.

Quand ils sont utilisés de façon stratégique, les programmes de mobilité peuvent s'inscrire dans le cadre d'initiatives de formation et de perfectionnement et constituer un outil de développement précieux pour les employés et les administrations. Cependant, seulement un peu plus de la moitié des pays de l'OCDE se sont dotés de programmes spécifiques visant à encourager la mobilité au sein de la fonction publique. De même, moins du tiers des pays de l'OCDE indiquent que leurs fonctionnaires ont droit chaque année à un temps minimum de formation.

Ces données semblent montrer que l'importance de l'apprentissage et du perfectionnement est largement reconnue par les pays de l'OCDE, comme l'illustre, par exemple, le nombre de pays désormais dotés de stratégies de formation applicables à l'ensemble de leur fonction publique. La priorité accordée à la formation du personnel de direction dans les deux-tiers des pays de l'OCDE démontre le rôle important attribué à ce groupe en tant que catalyseur de réformes stratégiques dans l'ensemble de la fonction publique. Par exemple, l'Allemagne a créé un groupe de coaching destiné au personnel de direction et les États-Unis ont recours à un centre spécialisé dans le perfectionnement de ce personnel, le Center for Leadership Development. Le niveau relativement faible des investissements consacrés aux initiatives de coaching et de mentorat indique qu'il serait possible d'aller plus loin en matière d'apprentissage et de développement en mettant à profit les connaissances présentes au sein des institutions.

\section{Méthodologie et définitions}

Ces données ont été initialement recueillies au sein des résultats de l'enquête 2016 de l'OCDE sur la gestion stratégique des ressources humaines (SHRM) et ont fait l'objet d'une actualisation en 2019. Les réponses ont été fournies, pour la plupart, par de hauts fonctionnaires des services de gestion des ressources humaines de l'administration centrale et les données font référence aux pratiques de gestion des ressources humaines de l'administration centrale. Tous les pays de l'OCDE ont répondu à l'enquête, ainsi que deux pays candidats à l'adhésion : la Colombie et le Costa Rica.

Les définitions de la fonction publique et du périmètre de l'administration centrale varient considérablement selon les pays et doivent être prises en compte dans la comparaison. Aux fins de cette enquête, le terme "fonctionnaire " désigne exclusivement les agents publics couverts par un cadre juridique public particulier ou par d'autres dispositions spécifiques. Pour en savoir plus sur la classification des cadres de haut niveau, voir l'Annexe D.

\section{Pour en savoir plus}

OCDE (2019), Recommandation du Conseil sur le leadership et les aptitudes de la fonction publique, OECD/LEGAL/0445.

OCDE (2017), "Skills for a High Performing Civil Service", Examens de l'OCDE sur la gouvernance publique, Éditions OCDE, Paris, https://doi.org/10.1787/9789264280724-en.

OCDE (2016), "Engaging Public Employees for a High-Performing Civil Service”, Examens de l'OCDE sur la gouvernance publique, Éditions OCDE, Paris, https://doi.org/10.1787/9789264267190-en.

\section{Notes relatives aux graphiques}

Les données relatives à la Colombie, à l'Islande et à la Turquie se réfèrent à l'année 2016. Concernant les données sur Israël, voir http://doi. org/10.1787/888932315602. 
6.3. Initiatives en matière d'apprentissage et de perfectionnement et priorités de formation au sein de l'administration centrale, 2019

\begin{tabular}{|c|c|c|c|c|c|c|c|}
\hline & \multicolumn{3}{|c|}{ Planification des activités d'apprentissage et de formation } & \multicolumn{4}{|c|}{ Priorités en matière de formation } \\
\hline & $\begin{array}{c}\text { Stratégie et/ou plan } \\
\text { d'action en matière de } \\
\text { formation pour l'ensemble } \\
\text { de la fonction publique }\end{array}$ & $\begin{array}{l}\text { Plan institutionnel d'apprentissage } \\
\text { au sein de chaque entité de } \\
\text { l'administration centrale }\end{array}$ & $\begin{array}{l}\text { Obligation pour chaque } \\
\text { fonctionnaire d'élaborer } \\
\text { un plan individuel } \\
\text { d'apprentissage }\end{array}$ & $\begin{array}{l}\text { Création de formations } \\
\text { en ligne }\end{array}$ & $\begin{array}{l}\text { Formation et coaching } \\
\text { du personnel de } \\
\text { direction }\end{array}$ & $\begin{array}{l}\text { Formation aux TIC } \\
\text { ou au numérique }\end{array}$ & $\begin{array}{r}\text { Coaching/ } \\
\text { mentorat }\end{array}$ \\
\hline Allemagne & $\square$ & $\mathbf{\square}$ & $\square$ & $\square$ & $\mathbf{a}$ & $\mathbf{\square}$ & $\mathbf{\square}$ \\
\hline Australie & $\mathbf{\square}$ & $\overline{\mathbf{n}}$ & $\mathbf{\square}$ & $\square$ & $\overline{\mathbf{a}}$ & $\overline{\mathbf{a}}$ & $\bar{\square}$ \\
\hline Autriche & $\bar{\square}$ & $\overline{\mathbf{n}}$ & $\bar{\square}$ & $\mathbf{\square}$ & $\bar{\square}$ & $\overline{\mathbf{n}}$ & $\square$ \\
\hline Belgique & $\overline{\mathbf{a}}$ & $\square$ & $\overline{\mathbf{a}}$ & $\overline{\mathbf{n}}$ & 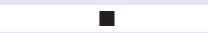 & $\square$ & $\overline{\mathbf{a}}$ \\
\hline Canada & $\bar{\square}$ & $\square$ & $\bar{\square}$ & $\bar{\square}$ & $\overline{\mathbf{n}}$ & $\square$ & $\square$ \\
\hline Chili & $\square$ & च & $\square$ & च & $\mathbf{n}$ & $\square$ & $\square$ \\
\hline Corée & च & च & 口 & च & च & च & च \\
\hline Danemark & $\square$ & $\square$ & 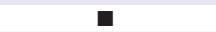 & $\bar{\square}$ & 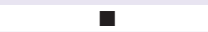 & $\bar{\square}$ & $\square$ \\
\hline Espagne & $\bar{\square}$ & $\bar{\square}$ & $\square$ & $\bar{\square}$ & $\bar{\square}$ & $\bar{\square}$ & $\square$ \\
\hline Estonie & 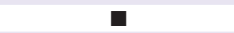 & $\bar{\square}$ & $\square$ & $\bar{\square}$ & $\bar{\square}$ & $\square$ & $\bar{\square}$ \\
\hline Etats-Unis & $\bar{\square}$ & $\overline{\mathbf{n}}$ & $\square$ & $\square$ & $\overline{\mathbf{n}}$ & $\square$ & $\bar{\square}$ \\
\hline Finlande & $\square$ & $\bar{\square}$ & 口 & 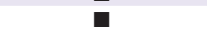 & $\overline{\mathbf{n}}$ & 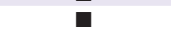 & $\bar{\square}$ \\
\hline France & - & $\bar{\square}$ & $\bar{\square}$ & $\bar{\square}$ & $\overline{\mathbf{n}}$ & $\bar{\square}$ & 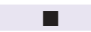 \\
\hline Grèce & $\bar{\square}$ & $\square$ & $\square$ & $\square$ & $\square$ & $\bar{\square}$ & $\square$ \\
\hline Hongrie & [ & [ & [ & $\mathbf{a}$ & $\square$ & [ & $\square$ \\
\hline Irlande & $\overline{\mathbf{a}}$ & $\bar{\square}$ & $\overline{\mathbf{a}}$ & $\bar{\square}$ & 口 & $\bar{\square}$ & 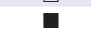 \\
\hline Islande & $\bar{\square}$ & 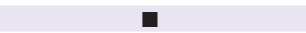 & $\overline{\mathbf{a}}$ & $\square$ & $\bar{\square}$ & $\square$ & $\bar{\square}$ \\
\hline Israël & [ & [ & $\square$ & $\mathbf{a}$ & $\mathbf{0}$ & $\square$ & $\square$ \\
\hline Italie & $\overline{\mathbf{n}}$ & $\bar{\square}$ & $\square$ & $\bar{\square}$ & $\bar{\square}$ & 口 & $\square$ \\
\hline Japon & च & a & $\square$ & $\square$ & $\square$ & $\square$ & a \\
\hline Lettonie & $\square$ & $\bar{\square}$ & $\square$ & 口 & 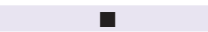 & $\square$ & $\bar{\square}$ \\
\hline Lituanie & 口 & $\overline{\mathbf{D}}$ & $\square$ & $\bar{\square}$ & $\bar{\square}$ & $\square$ & $\square$ \\
\hline Luxembourg & 口 & $\square$ & 口 & $\bar{\square}$ & $\bar{\square}$ & 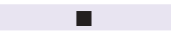 & 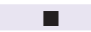 \\
\hline Mexique & 口 & $\square$ & $\square$ & $\bar{\square}$ & $\bar{\square}$ & $\square$ & $\square$ \\
\hline Norvège & $\square$ & $\square$ & च & $\bar{\square}$ & $\square$ & 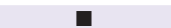 & $\square$ \\
\hline Nouvelle Zélande & $\square$ & $\mathbf{\square}$ & a & $\square$ & $\overline{\mathbf{a}}$ & $\square$ & $\square$ \\
\hline Pays-Bas & $\square$ & $\square$ & $\square$ & च & $\square$ & $\mathbf{\square}$ & $\square$ \\
\hline Pologne & 口 & 口 & 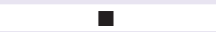 & $\bar{\square}$ & $\square$ & $\bar{\square}$ & $\square$ \\
\hline Portugal & $\bar{\square}$ & $\bar{\square}$ & $\overline{\mathbf{n}}$ & $\bar{\square}$ & 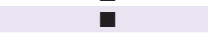 & 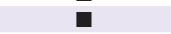 & $\square$ \\
\hline République Slovaque & $\overline{\mathbf{n}}$ & $\overline{\mathbf{a}}$ & $\overline{\mathbf{a}}$ & $\square$ & $\overline{\mathbf{v}}$ & $\bar{\square}$ & 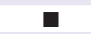 \\
\hline République Tchèque & $\bar{\square}$ & $\bar{\square}$ & $\bar{\square}$ & $\square$ & $\square$ & $\square$ & $\square$ \\
\hline Royaume-Uni & $\overline{\mathbf{n}}$ & $\overline{\mathbf{a}}$ & $\overline{\mathbf{n}}$ & $\square$ & - & $\square$ & $\square$ \\
\hline Slovénie & $\bar{\square}$ & $\overline{\mathbf{a}}$ & $\bar{\square}$ & 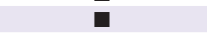 & $\bar{\square}$ & $\square$ & $\square$ \\
\hline Suède & $\square$ & $\overline{\mathbf{n}}$ & $\mathbf{\square}$ & $\overline{\mathbf{n}}$ & $\overline{\mathbf{n}}$ & 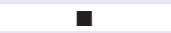 & $\square$ \\
\hline Suisse & $\bar{\square}$ & $\square$ & $\square$ & $\bar{\square}$ & $\bar{\square}$ & $\bar{\square}$ & $\square$ \\
\hline Turquie & $\square$ & च & $\square$ & $\mathbf{\square}$ & $\overline{\mathbf{v}}$ & $\overline{\mathbf{n}}$ & $\square$ \\
\hline \multicolumn{8}{|l|}{ Total OCDE } \\
\hline Oui $\mathbf{\square}$ & 22 & 25 & 18 & 23 & 25 & 18 & 11 \\
\hline Non $\square$ & 14 & 11 & 18 & 13 & 11 & 18 & 25 \\
\hline Colombie & 口 & - & a & $\mathbf{\square}$ & $\square$ & $\square$ & $\square$ \\
\hline Costa Rica & $\bar{\square}$ & $\overline{\mathbf{n}}$ & $\bar{\square}$ & $\bar{\square}$ & 口 & 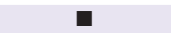 & $\square$ \\
\hline
\end{tabular}

Source : Enquête 2019 de l'OCDE sur la gestion stratégique des ressources humaines (SHRM).

6.4. Existence de programmes spécifiques visant à encourager la mobilité au sein de la fonction publique, 2016

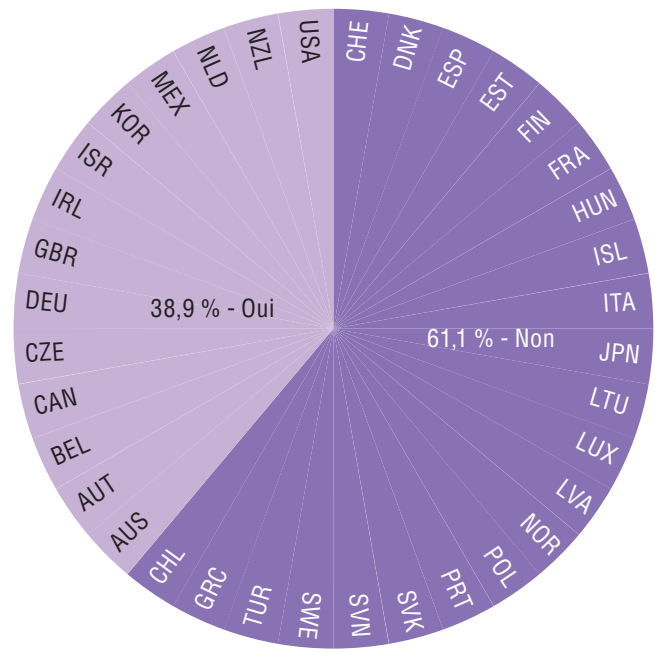

Source: Enquête 2019 de l'OCDE sur la gestion stratégique des ressources humaines (SHRM).

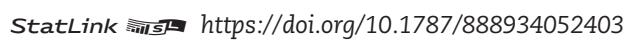

6.5. Droit des fonctionnaires à un certain nombre de jours de formation par an, 2019

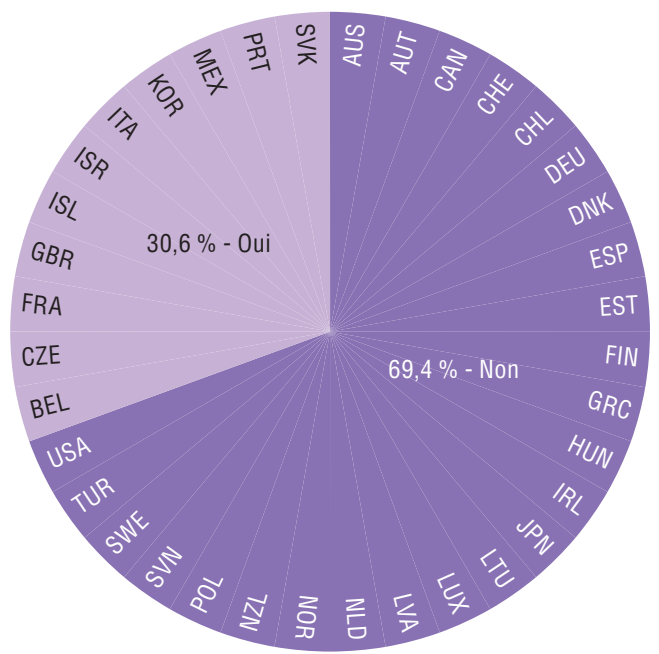

Source : Enquête 2019 de l'OCDE sur la gestion stratégique des ressources humaines (SHRM).

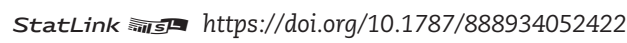


Les hauts fonctionnaires et hauts responsables de la fonction publique travaillent à la frontière entre la sphère politique et la sphère administrative. À cette fin, ils s'appuient souvent sur leurs vastes connaissances institutionnelles et sur leurs nombreux contacts acquis tout au long d'une carrière riche et variée. Ils assument non seulement la responsabilité d'un large éventail de produits et de réalisations, mais sont également chargés de superviser les procédures, les budgets et les moyens humains correspondants. De plus en plus, les fonctions publiques des pays de l'OCDE recourent à des systèmes de gestion de la performance dans le but d'orienter l'action de leurs dirigeants et encadrants et de faire coïncider leurs incitations avec les comportements et réalisations souhaités. Quand ils sont bien conçus et bien utilisés, les systèmes de gestion de la performance peuvent assurer la responsabilisation sur les résultats et renforcer d'importantes compétences en termes de professionnalisme et de leadership. Toutefois, d'anciens travaux de l'OCDE semblent montrer que les systèmes de suivi de la performance et les modalités contractuelles, tous deux caractérisés par un aspect technocratique et uniforme, ont produit des résultats mitigés, de même que les réformes visant à instaurer une rémunération sur le résultat (OCDE 2015).

Dans les deux-tiers des pays de l'OCDE où il existe un régime de gestion du rendement pour les cadres supérieurs, les fonctionnaires du plus haut rang font souvent l'objet d'accords spécifiques en matière de performances, qui sont conclus avec leur ministre (15 pays) ou avec le directeur de la fonction publique (7 pays). Des pays tels que la Norvège et les PaysBas recourent aux deux types d'accords. Dans un peu plus de la moitié des pays de l'OCDE, les cadres de haut niveau peuvent bénéficier d'une prime s'ils remplissent certains critères de performance. Si seulement $17 \%$ des pays associent les perspectives de promotion à la réalisation de bonnes performances, 19 pays de l'OCDE autorisent le licenciement en cas de résultats de performance faibles.

Dans un peu plus des trois-quarts des pays de l'OCDE, les cadres de haut niveau sont engagés pour une durée déterminée, ce qui peut refléter le souhait, pour ces pays, de favoriser la mobilité et de se garder la possibilité de disposer de nouvelles compétences et expériences. Un peu plus de $60 \%$ des pays de l'OCDE lient la reconduction de l'engagement des cadres de haut niveau à l'évaluation de leurs performances. Toutefois, environ $70 \%$ des pays où ce lien est établi, les cadres de haut niveau ne sont pas tenus de respecter un seuil minimum de performance.

Les données recueillies semblent montrer que les administrations centrales mettent davantage l'accent sur les mécanismes permettant de réagir en cas de mauvaises performances plutôt que sur l'instauration d'une gestion plus stratégique des performances. Cette option vise à repérer, fidéliser et promouvoir ceux qui réalisent de bonnes performances, comme par exemple l'importance accordée à la gestion des talents par la Commission australienne de la fonction publique (APSC). Plus de $60 \%$ des pays de l'OCDE n'indiquent pas avoir mis en place de politiques visant à repérer les cadres à hauts potentiels à un stade précoce de leur carrière.

\section{Méthodologie et définitions}

Ces données ont initialement été collectées lors de l'enquête 2016 de l'OCDE sur la gestion stratégique des ressources humaines (SHRM) et ont fait l'objet d'une actualisation en 2019. Les réponses ont été fournies, pour la plupart, par de hauts fonctionnaires des services de gestion des ressources humaines de l'administration centrale et les données concernent les pratiques de gestion des ressources humaines de l'administration centrale. Tous les pays de l'OCDE ont répondu à l'enquête, ainsi que deux pays candidats à l'adhésion : la Colombie et le Costa Rica.

Les définitions de la fonction publique et du périmètre de l'administration centrale varient considérablement selon les pays et doivent être prises en compte dans la comparaison. Aux fins de cette enquête, le terme "fonctionnaire " désigne exclusivement les agents publics couverts par un cadre juridique public particulier ou par d'autres dispositions spécifiques. Les cadres de haut niveau sont des cadres de niveau D1 et D2 et sont parfois qualifiés de " hauts fonctionnaires " ou de " cadres senior ". Le qualificatif " senior " indique uniquement leur rang et non leur âge ou leur ancienneté au sein de la fonction publique ou à leur poste actuel. Ainsi, les cadres de haut niveau peuvent par exemple être plus jeunes et avoir moins d'années d'expérience que des cadres moyens. Les catégories D1 et D2 pour lesquelles des données sont présentées ici sont inspirées de la Classification internationale type des professions (CITP-08) établie par l'Organisation internationale du Travail (OIT). On trouvera une définition détaillée de chacun des niveaux à l'Annexe D. Un accord de performance est un accord passé entre un agent et son supérieur hiérarchique qui fixe les objectifs à l'aune desquels les performances de l'agent pourront être évaluées.

\section{Pour en savoir plus}

OCDE (2019), Recommandation du Conseil sur le leadership et les aptitudes de la fonction publique, OECD/LEGAL/0445.

OCDE (2017), "Skills for a High Performing Civil Service", Examens de l'OCDE sur la gouvernance publique, Éditions OCDE, Paris, https://doi.org/10.1787/9789264280724-en.

OECD (2016), “Engaging Public Employees for a High-Performing Civil Service”, Examens de l'OCDE sur la gouvernance publique, Éditions OCDE, Paris. http://doi.org/10.1787/9789264267190-en.

\section{Notes relatives aux graphiques}

Les données relatives à la Colombie, à l'Islande et à la Turquie se réfèrent à l'année 2016. Concernant les données sur Israël, voir http://doi. org/10.1787/888932315602.

6.6. En Autriche, aux Pays-Bas, en Slovénie, en Suisse, en Turquie et au Costa Rica, il existe un régime de gestion des performances identique pour tous les fonctionnaires.

Concernant l'Allemagne, la réponse " oui » pour « Existence d'un régime de gestion des performances pour les cadres supérieurs " et " Système d'évaluation des performances incluant des indicateurs de résultats " s'applique uniquement aux fonctionnaires cadres supérieurs. La réponse "oui " pour "Rémunération liée aux performances » et "Licenciement pour mauvaise performance " ne concerne que les cadres supérieurs employés dans la fonction publique. 
6.6. Caractéristiques du régime de gestion des performances des cadres de haut niveau, 2019

\begin{tabular}{|c|c|c|c|c|c|c|c|c|c|c|}
\hline & \multirow{2}{*}{$\begin{array}{l}\text { Existence d'un régime } \\
\text { de gestion des } \\
\text { performances pour les } \\
\text { cadres de haut niveau }\end{array}$} & \multirow{2}{*}{$\begin{array}{l}\text { Rémunération } \\
\text { sur le résultat }\end{array}$} & \multirow{2}{*}{$\begin{array}{c}\text { Accord de } \\
\text { performance } \\
\text { avec le ministre } \\
\text { (au niveau D1) }\end{array}$} & \multirow{2}{*}{$\begin{array}{l}\text { Accord de performance } \\
\text { avec le directeur } \\
\text { de la fonction publique } \\
\text { (au niveau D1) }\end{array}$} & \multicolumn{4}{|c|}{ Système d'évaluation des performances englobant: } & \multirow[b]{2}{*}{$\begin{array}{l}\text { Promotion en } \\
\text { cas de bonnes } \\
\text { performances }\end{array}$} & \multirow{2}{*}{$\begin{array}{l}\text { Licenciement } \\
\text { en cas de } \\
\text { mauvaises } \\
\text { performances }\end{array}$} \\
\hline & & & & & $\begin{array}{c}\text { Des indicateurs } \\
\text { portant sur les } \\
\text { réalisations }\end{array}$ & $\begin{array}{l}\text { Des indicateurs } \\
\text { portant sur } \\
\text { les produits }\end{array}$ & $\begin{array}{l}\text { Des indicateurs portant } \\
\text { sur l'encadrement }\end{array}$ & $\begin{array}{c}\text { Une } \\
\text { évaluation } \\
\text { à } 360 \text { degrés }\end{array}$ & & \\
\hline Allemagne & $\mathbf{\square}$ & $\mathbf{\square}$ & $\square$ & $\square$ & $\mathbf{\square}$ & $\square$ & $\square$ & $\square$ & $\square$ & $\mathbf{\square}$ \\
\hline Australie & $\overline{\mathbf{a}}$ & $\overline{\mathbf{n}}$ & $\square$ & $\overline{\mathbf{u}}$ & $\bar{\square}$ & $\overline{\mathbf{a}}$ & $\overline{\mathbf{a}}$ & $\bar{\square}$ & $\square$ & $\overline{\mathbf{u}}$ \\
\hline Autriche & $\bar{\square}$ & $\bar{\square}$ & $\square$ & $\bar{\square}$ & $\overline{\mathbf{n}}$ & $\overline{\mathbf{n}}$ & $\bar{\square}$ & $\square$ & $\square$ & $\overline{\mathbf{n}}$ \\
\hline Belgique & $\overline{\mathbf{a}}$ & $\square$ & $\overline{\mathbf{a}}$ & $\square$ & $\overline{\mathbf{\theta}}$ & $\overline{\mathbf{a}}$ & $\overline{\mathbf{a}}$ & $\square$ & $\square$ & $\overline{\mathbf{n}}$ \\
\hline Canada & $\overline{\mathbf{n}}$ & $\overline{\mathbf{n}}$ & $\square$ & $\square$ & $\bar{\square}$ & $\bar{\square}$ & $\bar{\square}$ & $\square$ & $\square$ & $\bar{\square}$ \\
\hline Chili & $\overline{\mathbf{n}}$ & $\overline{\mathbf{n}}$ & $\mathbf{\square}$ & $\square$ & $\bar{\square}$ & $\overline{\mathbf{n}}$ & $\overline{\mathbf{n}}$ & $\square$ & $\square$ & $\overline{\mathbf{n}}$ \\
\hline Corée & $\mathbf{\square}$ & 口 & 口 & $\square$ & 口 & 口 & 口 & 口 & 口 & $\overline{\mathbf{n}}$ \\
\hline Danemark & $\overline{\mathbf{n}}$ & $\overline{\mathbf{n}}$ & $\bar{\square}$ & 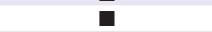 & $\bar{\square}$ & $\bar{\square}$ & $\bar{\square}$ & $\square$ & $\bar{\square}$ & $\bar{\square}$ \\
\hline Espagne & $\overline{\mathbf{a}}$ & $\bar{\square}$ & $\bar{\square}$ & $\square$ & $\square$ & $\square$ & $\square$ & $\square$ & $\square$ & $\overline{\mathbf{a}}$ \\
\hline Estonie & $\overline{\mathbf{a}}$ & $\overline{\mathbf{a}}$ & $\square$ & $\square$ & $\square$ & $\square$ & $\square$ & $\bar{\square}$ & $\square$ & $\square$ \\
\hline Etats-Unis & $\overline{\mathbf{a}}$ & $\overline{\mathbf{a}}$ & $\square$ & $\mathbf{E}$ & $\mathbf{a}$ & 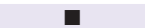 & 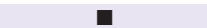 & $\square$ & 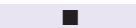 & 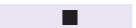 \\
\hline Finlande & 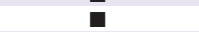 & $\square$ & $\square$ & $\square$ & $\overline{0}$ & $\overline{\mathbf{n}}$ & 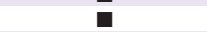 & $\square$ & $\bar{\square}$ & $\overline{0}$ \\
\hline France & $\overline{\mathbf{n}}$ & 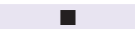 & 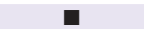 & $\square$ & $\bar{\square}$ & $\bar{\square}$ & $\bar{\square}$ & 口 & $\bar{\square}$ & $\bar{\square}$ \\
\hline Grèce & $\mathbf{\square}$ & $\square$ & च & $\square$ & $\mathbf{a}$ & $\mathbf{\square}$ & $\mathbf{n}$ & $\mathbf{\square}$ & $\mathbf{\square}$ & $\mathbf{n}$ \\
\hline Hongrie & $\square$ & $\square$ & $\bar{\square}$ & $\square$ & $\bar{\square}$ & $\bar{\square}$ & $\bar{\square}$ & $\bar{\square}$ & $\vec{\square}$ & $\square$ \\
\hline Irlande & $\mathbf{\square}$ & $\square$ & $\bar{\square}$ & $\square$ & $\square$ & $\square$ & $\mathbf{\square}$ & $\overline{\mathbf{a}}$ & $\square$ & $\square$ \\
\hline Islande & $\square$ & $\square$ & $\square$ & $\square$ & $\square$ & $\square$ & $\square$ & $\square$ & $\square$ & $\square$ \\
\hline Israël & $\mathbf{a}$ & $\square$ & $\square$ & $\square$ & 口 & $\mathbf{a}$ & $\mathbf{a}$ & 口 & 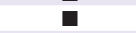 & $\mathbf{a}$ \\
\hline Italie & [ & $\overline{\mathbf{a}}$ & $\overline{\mathbf{a}}$ & $\square$ & $\square$ & $\square$ & $\overline{\mathbf{n}}$ & $\square$ & $\square$ & [ \\
\hline Japon & $\mathbf{\square}$ & $\square$ & $\square$ & $\square$ & $\square$ & $\square$ & $\square$ & $\square$ & $\square$ & $\square$ \\
\hline Lettonie & $\overline{\mathbf{n}}$ & $\bar{\square}$ & 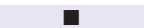 & $\square$ & $\mathbf{a}$ & $\square$ & $\square$ & 口 & $\square$ & $\mathbf{z}$ \\
\hline Lituanie & $\square$ & $\square$ & $\square$ & $\square$ & $\square$ & $\square$ & $\square$ & $\square$ & $\square$ & $\square$ \\
\hline Luxembourg & $\square$ & $\square$ & $\square$ & $\square$ & $\square$ & $\square$ & $\square$ & $\square$ & $\square$ & $\square$ \\
\hline Mexique & $\square$ & $\square$ & $\square$ & $\square$ & $\mathbf{\square}$ & 口 & 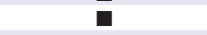 & $\square$ & $\square$ & $\mathbf{\square}$ \\
\hline Norvège & 口 & 口 & 口 & 口 & $\bar{\square}$ & $\bar{\square}$ & $\bar{\square}$ & $\square$ & $\square$ & $\bar{\square}$ \\
\hline Nouvelle Zélande & $\mathbf{a}$ & 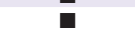 & $\square$ & 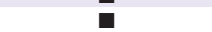 & $\square$ & $\square$ & $\square$ & $\square$ & $\square$ & $\square$ \\
\hline Pays-Bas & $\square$ & $\square$ & $\bar{\square}$ & 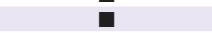 & $\square$ & $\square$ & $\square$ & $\square$ & $\square$ & $\square$ \\
\hline Pologne & $\square$ & $\square$ & $\bar{\square}$ & $\bar{\square}$ & $\square$ & $\square$ & $\square$ & $\square$ & $\square$ & $\square$ \\
\hline Portugal & $\mathbf{\square}$ & $\square$ & $\mathbf{\square}$ & $\square$ & 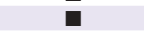 & $\mathbf{\square}$ & $\mathbf{\square}$ & $\square$ & $\square$ & $\mathbf{\square}$ \\
\hline République Slovaque & $\overline{\mathbf{n}}$ & 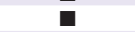 & $\bar{\square}$ & $\square$ & $\bar{\square}$ & $\overline{\mathbf{a}}$ & $\bar{\square}$ & $\square$ & $\square$ & $\overline{\mathbf{n}}$ \\
\hline République Tchèque & $\mathbf{\square}$ & [ & $\square$ & $\square$ & $\overline{\mathbf{u}}$ & $\overline{\mathbf{a}}$ & - & $\square$ & $\square$ & $\bar{\square}$ \\
\hline Royaume-Uni & $\overline{\mathbf{n}}$ & $\overline{\mathbf{n}}$ & $\square$ & 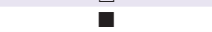 & $\bar{\square}$ & $\overline{\mathbf{a}}$ & 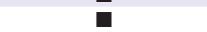 & $\mathbf{\square}$ & $\square$ & 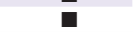 \\
\hline Slovénie & $\square$ & $\overline{\mathbf{a}}$ & $\square$ & $\square$ & $\square$ & $\square$ & $\square$ & $\square$ & $\square$ & $\square$ \\
\hline Suède & 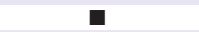 & $\overline{\mathbf{n}}$ & $\mathbf{\square}$ & $\square$ & $\square$ & $\square$ & $\square$ & $\square$ & $\square$ & $\square$ \\
\hline Suisse & $\bar{\square}$ & $\overline{\mathbf{n}}$ & $\overline{\mathbf{n}}$ & $\square$ & $\square$ & $\square$ & $\square$ & $\square$ & $\square$ & $\square$ \\
\hline Turquie & $\square$ & $\square$ & $\mathbf{\square}$ & $\square$ & $\square$ & $\square$ & $\overline{\mathbf{n}}$ & $\square$ & $\mathbf{\square}$ & $\square$ \\
\hline \multicolumn{11}{|l|}{ Total OCDE } \\
\hline Oui - & 25 & 20 & 15 & 7 & 16 & 15 & 16 & 9 & 6 & 19 \\
\hline Non $\square$ & 11 & 16 & 21 & 29 & 20 & 21 & 20 & 27 & 30 & 17 \\
\hline Colombie & a & $\square$ & 口 & $\square$ & $\square$ & $\square$ & $\square$ & $\square$ & $\square$ & $\square$ \\
\hline Costa Rica & $\square$ & $\square$ & $\square$ & $\square$ & $\square$ & $\square$ & $\square$ & $\square$ & $\square$ & $\bar{\square}$ \\
\hline
\end{tabular}

Source : Enquête 2019 de l'OCDE sur la gestion stratégique des ressources humaines (SHRM).

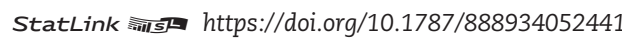

6.7. Existence de relations entre renouvellement de contrat des cadres de haut niveau et des résultats d'une évaluation formelle des compétences, 2019

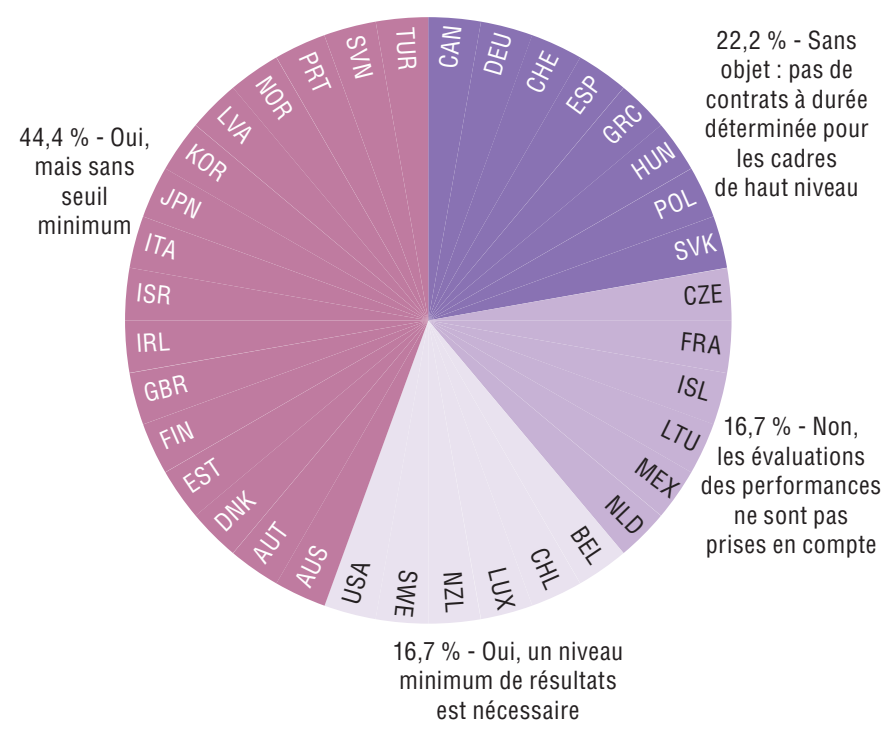

Source: Enquête 2019 de l'OCDE sur la gestion stratégique des ressources humaines (SHRM).

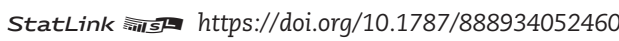

6.8. Existence de politiques pour repérer les cadres à hauts potentiels à un stade précoce de leur carrière, 2019

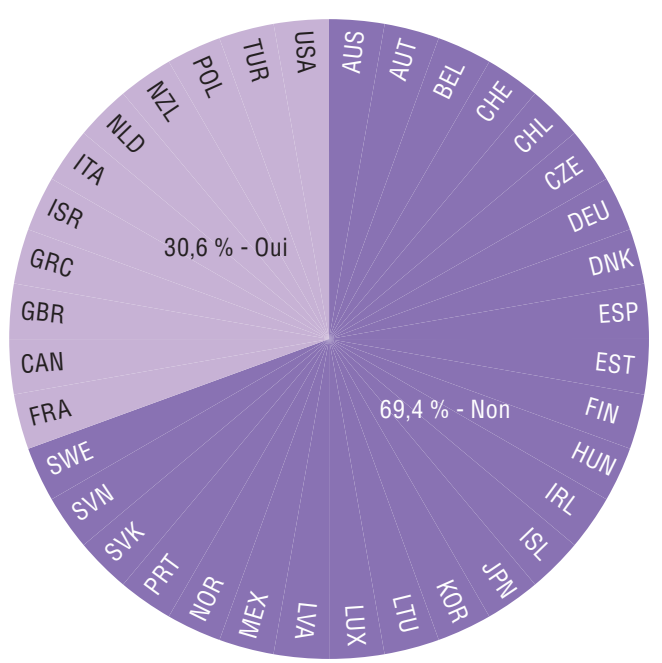

Source: Enquête 2019 de l'OCDE sur la gestion stratégique des ressources humaines (SHRM).

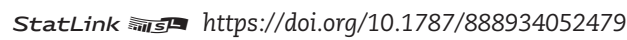




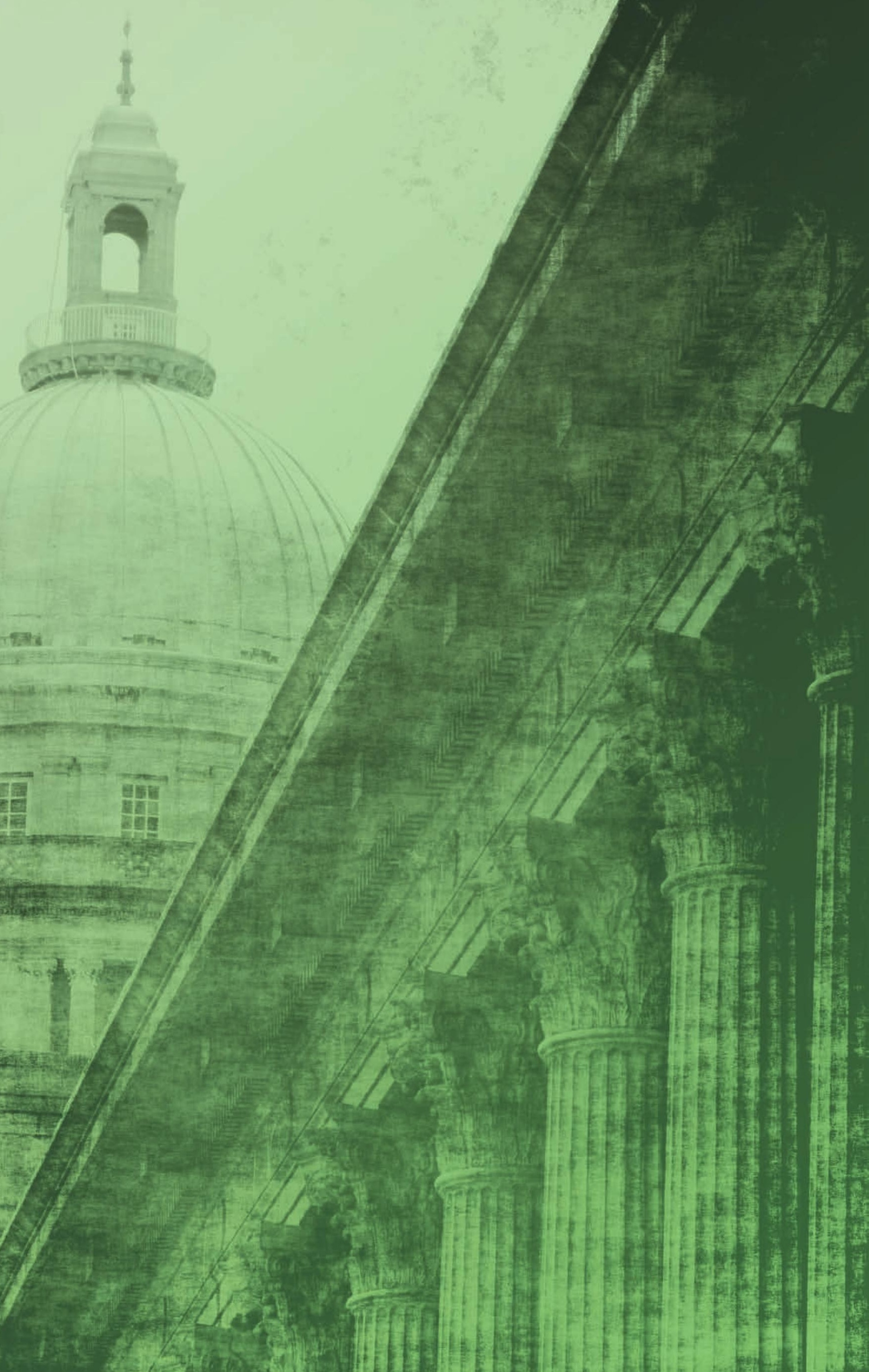




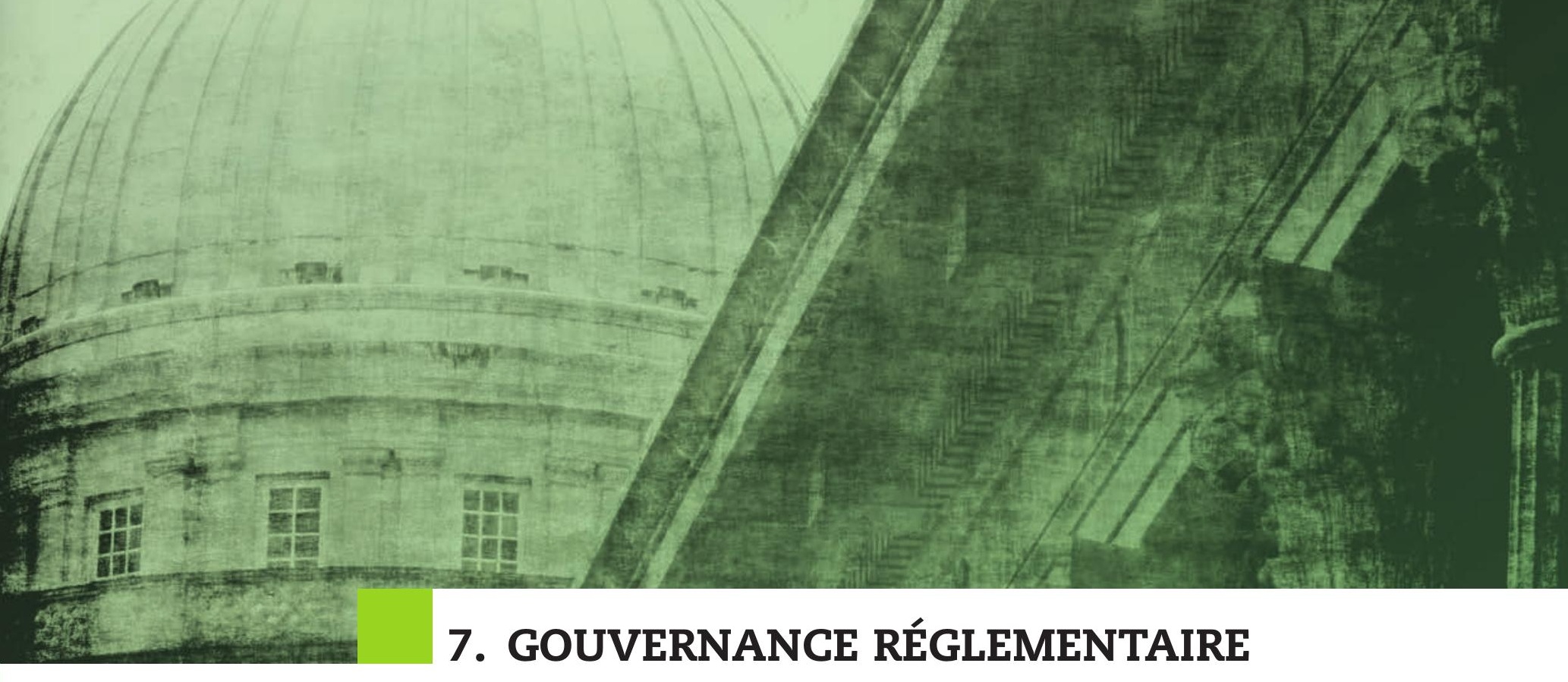

Participation des parties prenantes à l'élaboration de la réglementation

Analyse d'impact de la réglementation

Évaluation a posteriori de la réglementation 
La participation des parties prenantes à l'élaboration de la réglementation consiste à engager les particuliers, les entreprises et la société civile, parmi d'autres, à l'élaboration des textes dans le but d'aboutir à une réglementation axée sur les besoins de la population. Les parties prenantes contribuent au processus en apportant leurs connaissances et leurs éléments d'information concernant les problèmes à régler et les solutions à envisager, ce qui permet aux pouvoirs publics de mieux concevoir la réglementation. La participation des parties prenantes permet également de rendre les textes plus inclusifs et de faciliter l'appropriation des réglementations par la population, ce qui favorise la confiance à l'égard des institutions, la cohésion sociale et le respect des textes.

Bien que les pays de l'OCDE soient globalement attachés à la participation des parties prenantes, une forte marge de progression reste à concrétiser afin d'ouvrir davantage les consultations au grand public et de les rendre plus utiles au sein du processus de l'action publique. Dans tous les territoires étudiés, la participation des parties prenantes est obligatoire pour l'élaboration de certains textes. En général, les exigences formelles et les pratiques de consultation sont moins contraignantes pour les textes réglementaires. Les parties prenantes ne sont pas systématiquement associées en amont pour éclairer les responsables publics sur la nature d'un problème à résoudre et sur les solutions pouvant être envisagées. L'Islande et la Commission européenne ont récemment lancé des réformes visant à élargir le champ d'application de la participation des parties prenantes à différentes étapes du processus d'élaboration de la réglementation.

Les pays ont tout intérêt à progresser sur les terrains de la transparence et du contrôle de la participation des parties prenantes. Par exemple, si de nombreux pays publient en ligne les commentaires formulés dans le cadre des consultations et les transmettent aux décideurs, la plupart n'ont cependant pas pour pratique systématique d'informer à l'avance la population qu'une consultation va être réalisée, ni de fournir un retour d'information sur la façon dont les commentaires ont été pris en compte. De même, sur le plan du contrôle, la majorité des pays ne procède pas à des évaluations régulières des performances de leurs dispositifs d'association des parties prenantes.

Par rapport à 2014, les pays n'ont que légèrement amélioré leurs pratiques en matière de participation des parties prenantes, avec des progrès plus nets pour les textes législatifs que pour les textes réglementaires. Des pays comme la Corée, Israël, l'Italie et les Pays-Bas ont élargi leurs pratiques de consultation et les ont rendues plus accessibles au grand public. En revanche, certains pays semblent être moins performants, voire faire moins bien qu'en 2014 sur le plan de la mise en œuvre de la participation des parties prenantes, d'où un score en baisse.

Les Indicateurs relatifs à la politique et à la gouvernance réglementaires (iREG) constituent une base complète de connaissances sur les améliorations que les pays de l'OCDE ont apportées à leurs pratiques réglementaires à la lumière de la Recommandation du Conseil concernant la politique et la gouvernance réglementaires adoptée en 2012 par l'OCDE. Plus un pays a adopté de pratiques inspirées de la recommandation, plus son score relatif à ces indicateurs est élevé. L'indicateur composite comprend quatre catégories affectées d'une pondération égale : la catégorie " méthodologie » qui évoque les méthodes et outils employés pour la participation des parties prenantes; la catégorie " contrôle et suivi de la qualité " qui traite des dispositifs mis en place pour suivre les pratiques d'association des parties prenantes et pour assurer leur qualité ; la catégorie "adoption systématique " qui porte sur les exigences formelles et sur la fréquence concrète des pratiques d'association des parties prenantes; et la catégorie " transparence » qui suit l'information relative aux principes d'ouverture de l'administration (dits " gouvernement ouvert »). Le score maximal est égal à 1 pour chaque catégorie et le score total pour l'indicateur composite s'échelonne entre 0 et 4. L'indicateur composite iREG relatif à la participation des parties prenantes présenté ci-après est aussi l'un des indicateurs centraux utilisés pour mesurer la dimension " engagement civique » de l'indicateur du viure mieux de l'OCDE.

\section{Methodology and definitions}

Les indicateurs relatifs à la politique et à la gouvernance réglementaires (iREG) s'appuient sur les réponses des délégués auprès du Comité de la politique de la réglementation de l'OCDE et des responsables d'administration centrale aux enquêtes 2014 et 2017 de l'OCDE sur les indicateurs en matière réglementaire. Ces enquêtes ont été menées auprès de 38 pays de l'OCDE ou candidats à l'adhésion et auprès de l'Union européenne. Les données concernent uniquement les projets de textes législatifs et réglementaires à l'initiative du pouvoir exécutif. La plupart des textes législatifs sont issus d'une initiative de l'exécutif dans la majorité des pays de l'OCDE et des pays candidats à l'adhésion, à l'exception de la Corée, du Mexique, de la Colombie et du Costa Rica, pour lesquels l'essentiel des textes sont le résultat d'une initiative parlementaire. Les questions portant sur les textes législatifs sont sans objet pour les États-Unis, étant donné que le pouvoir exécutif américain ne prend jamais l'initiative des textes législatifs. On trouvera un complément d'information sur les indicateurs iREG à l'Annexe E et à l'adresse oe.cd/ireg.

Les textes législatifs sont ceux qui doivent être adoptés par le parlement, tandis que les textes réglementaires peuvent être adoptés par le chef du gouvernement, un ministre ou le Conseil des ministres.

\section{Pour en savoir plus}

OCDE (à paraître), OECD Best Practice Principles for Regulatory Policy: Stakeholder engagement, Éditions OCDE, Paris.

OCDE (2018), Politique de la réglementation : Perspectives de l'OCDE 2018, Éditions OCDE, Paris, https://doi.org/10.1787/ 9789264303072-en.

OCDE (2012), Recommandation du Conseil concernant la politique et la gouvernance réglementaires, Éditions OCDE, Paris, https:// legalinstruments.oecd.org/en/instruments/OECD-LEGAL-0390.

\section{Notes relatives aux graphiques}

Concernant les données sur Israël, voir http://doi.org/10.1787/888932315602 Les données concernant la Lettonie, la Lituanie, la Colombie et le Costa Rica ne sont pas disponibles pour 2014.

7.1. Aucun score national n'est présenté pour les États-Unis, pour lesquels le Congrès est systématiquement à l'origine des textes législatifs. * Dans la majorité des pays de l'OCDE, la plupart des textes législatifs sont élaborés par l'exécutif, sauf pour la Corée, le Mexique, la Colombie et le Costa Rica, où la majorité des textes législatifs sont d'initiative parlementaire. 


\subsection{Participation des parties prenantes à l'élaboration des textes législatifs, 2014 et 2017}

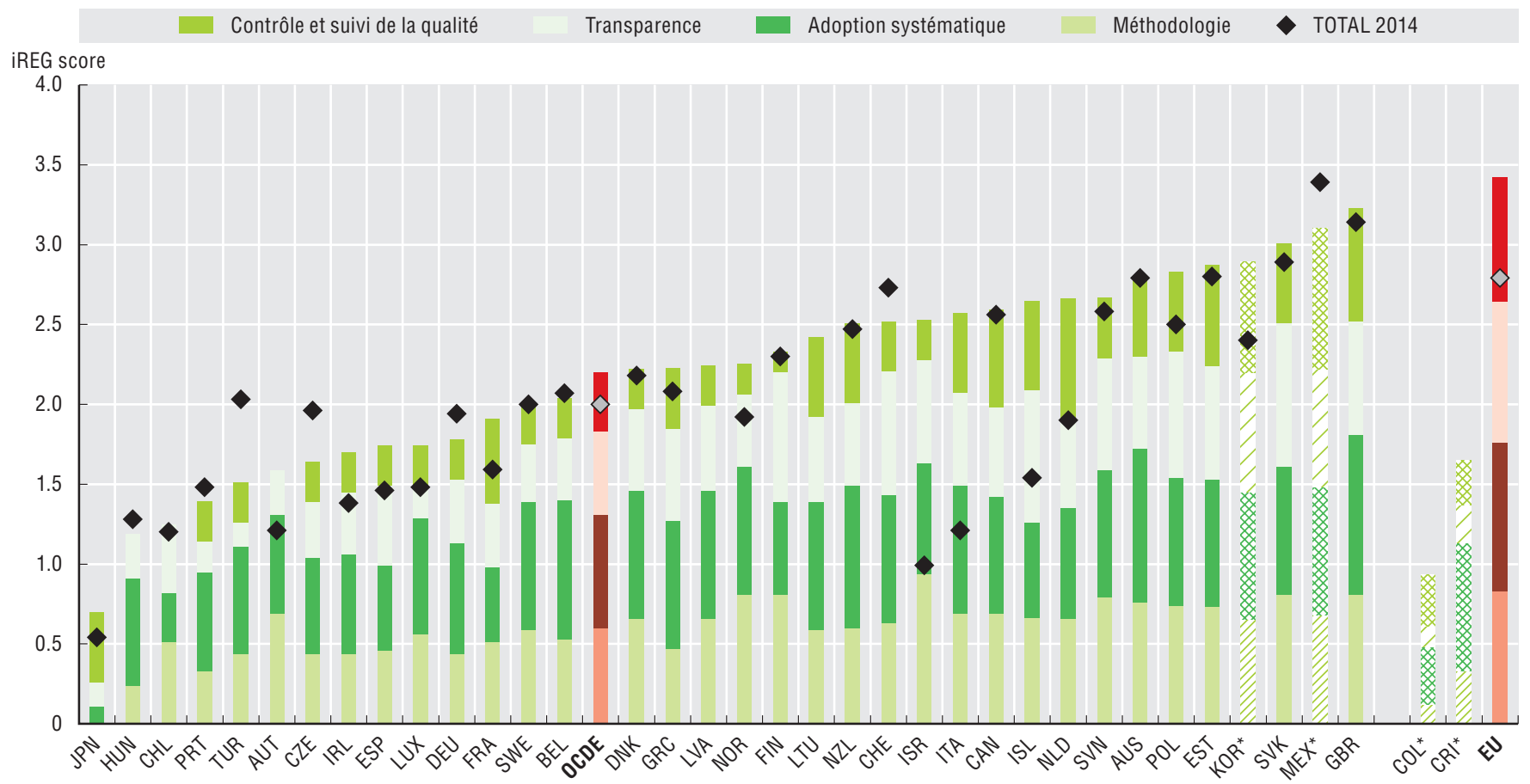

Source : OCDE Indicateurs relatifs à la politique et à la gouvernance réglementaires (iREG) 2015 et 2018, http://oe.cd/ireg.

\subsection{Participation des parties prenantes à l'élaboration des textes réglementaires, 2014 et 2017}

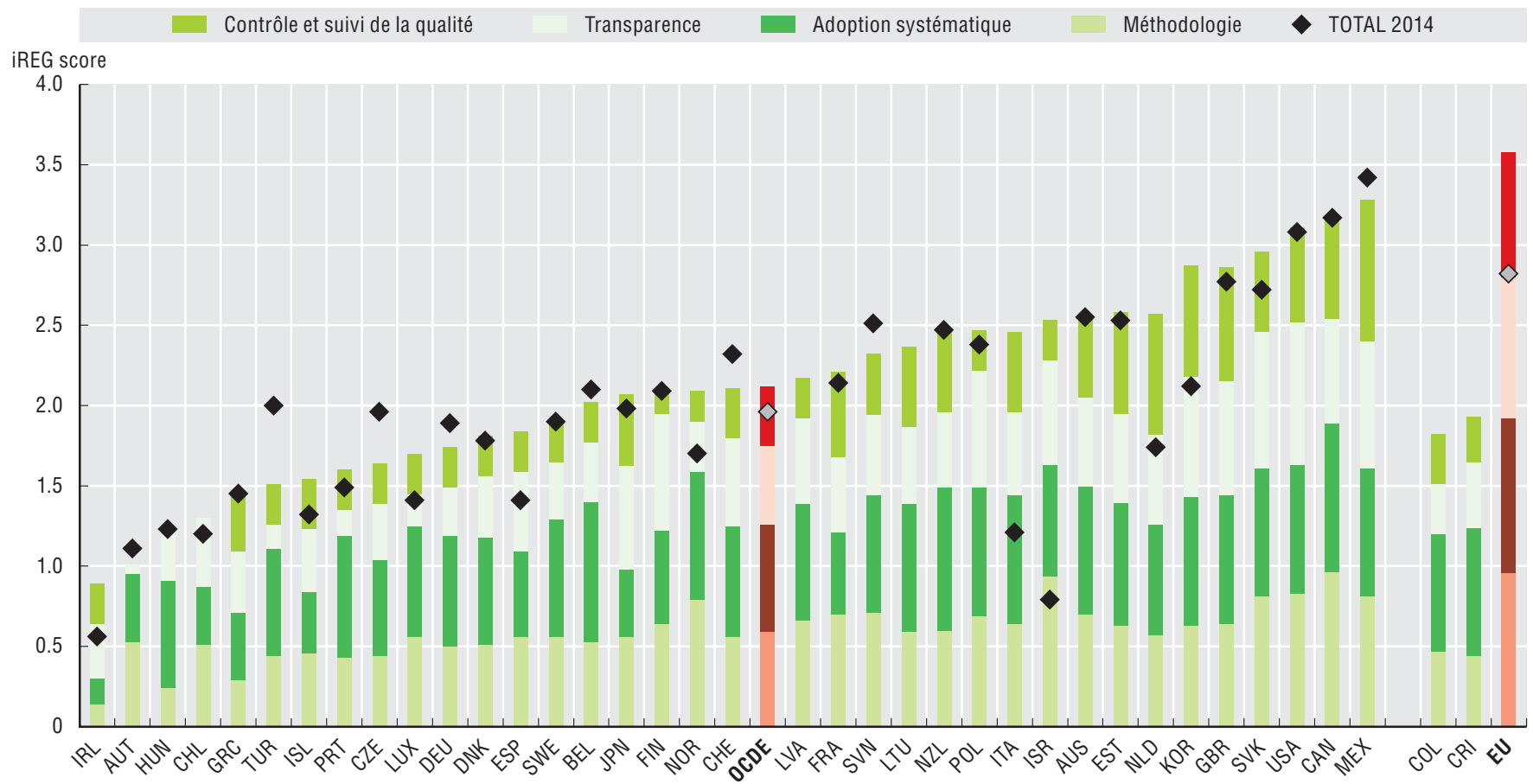

Source : OCDE Indicateurs relatifs à la politique et à la gouvernance réglementaires (iREG) 2015 et 2018, http://oe.cd/ireg. 
L'analyse d'impact de la réglementation (AIR) est un outil crucial qui aide les décideurs à déterminer s'il convient d'adopter un texte et comment, afin d'atteindre leurs objectifs d'action publique. L'AIR permet d'analyser les coûts et les avantages de la réglementation et des autres moyens de réaliser les objectifs poursuivis, afin de trouver la stratégie susceptible de produire l'avantage net le plus grand pour la société. L'AIR dévoile les arbitrages inhérents aux projets de textes et identifie les bénéficiaires probables de la réglementation et ceux qui en supporteront les coûts. L'AIR favorise une action publique fondée sur des données probantes et permet d'éviter les échecs (par exemple dus à une réglementation inutile ou absente). Enfin, l'AIR permet de garder une trace écrite des données probantes qui ont justifié les décisions adoptées et, par conséquent, de mieux placer les pouvoirs publics face à leurs responsabilités.

Globalement, on constate une stagnation, dans l'ensemble des pays de l'OCDE, des progrès accomplis en matière de dispositifs de l'AIR même si certains pays ont réalisé de nettes avancées. En 2017, tous les pays de l'OCDE ou candidats à l'adhésion, de même que la Commission européenne, exigeaient une AIR dans le cadre de l'élaboration d'une sélection de textes. Certains pays ont progressé en matière d'adoption systématique de l'AIR en investissant ces trois dernières années dans l'élargissement du champ d'application des exigences formelles et dans la mise en œuvre de démarches plus proportionnées. À titre d'exemple, le Chili a adopté en 2016 une instruction présidentielle énonçant, pour la toute première fois, une obligation d'AIR pour les ministères dont les portefeuilles touchent aux affaires économiques et le Portugal a renforcé son cadre d'AIR en obligeant les ministères à quantifier l'impact des nouvelles réglementations sur les entreprises et à évaluer les incidences législatives sur les citoyens. D'autres pays, comme l'Espagne, Israël, l'Italie et le Portugal, ont mis en place de nouvelles procédures et lignes directrices d'AIR. Dans un nombre croissant de pays, l'AIR n'existe que dans les projets de textes pour lesquels on prévoit un fort impact. Ainsi, plusieurs pays, tels que par exemple la Corée et le Japon, ont récemment adopté un critère de seuil afin de déterminer si une AIR simplifiée ou détaillée s'impose.

Bien que le domaine du contrôle et du suivi de la qualité reste celui où les pays de l'OCDE ont le moins avancé, certains d'entre eux ont nettement progressé en matière de mise en place des dispositifs de contrôle liés à l'AIR. Ainsi, la Finlande et la Norvège se sont respectivement dotées d'un Conseil finlandais pour l'analyse d'impact de la réglementation et d'un Conseil norvégien pour une meilleure réglementation (Regelrådet), organes indépendants de l'exécutif qui examinent la qualité de certaines AIR et des projets de textes. En 2015, la République slovaque a établi un comité de travail permanent du Conseil législatif qui est rattaché au ministère de l'Économie et est chargé de veiller à la qualité des AIR.

L'indicateur ici présenté fait partie des indicateurs iREG. Il constitue un indicateur crucial de l'OCDE afin de mesurer l'adoption par ses membres de processus d'action publique fondés sur des données probantes. Il se fonde sur les pratiques décrites dans la Recommandation concernant la politique et la gouvernance réglementaires adoptée en 2012 par l'OCDE. Plus un pays a adopté de ces pratiques, plus son score relatif à cet indicateur est élevé. L'indicateur composite comprend quatre catégories affectées d'une pondération égale : la catégorie "méthodologie » qui évoque les différentes analyses réalisées dans le cadre de l'AIR ; la catégorie " contrôle et suivi de la qualité " qui traite des dispositifs mis en place pour suivre les processus d'AIR et assurer leur qualité ; la catégorie " adoption systématique " qui porte sur les exigences formelles et la fréquence concrète de réalisation des AIR ; et la catégorie " transparence " qui suit l'information relative au degré d'ouverture des processus d'AIR. Le score maximal est égal à 1 pour chaque catégorie et le score total pour l'indicateur composite s'échelonne entre 0 et 4.

\section{Methodology and definitions}

Les indicateurs relatifs à la politique et à la gouvernance réglementaires (iREG) s'appuient sur les réponses des délégués auprès du Comité de la politique de la réglementation de l'OCDE et par des responsables d'administration centrale aux enquêtes 2014 et 2017 de l'OCDE sur les indicateurs en matière réglementaire. Ces enquêtes ont été menées auprès de 38 pays de l'OCDE ou candidats à l'adhésion et auprès de l'Union européenne. Les données concernent uniquement les projets de textes législatifs et réglementaires à l'initiative du pouvoir exécutif. La plupart des textes législatifs sont issus d'une initiative de l'exécutif dans la majorité des pays de l'OCDE et des pays candidats à l'adhésion, à l'exception de la Corée, du Mexique, de la Colombie et du Costa Rica, pour lesquels l'essentiel des textes sont d'initiative parlementaire. Les questions portant sur les textes législatifs sont sans objet pour les États-Unis, étant donné que le pouvoir exécutif américain ne prend jamais l'initiative des textes législatifs. On trouvera un complément d'information sur les indicateurs iREG à l'Annexe $\mathrm{E}$ et à l'adresse oe.cd/ireg.

Les textes législatifs sont ceux qui doivent être adoptés par le parlement, tandis que les textes réglementaires peuvent être adoptés par le chef du gouvernement, un ministre ou le Conseil des ministres.

\section{Pour en savoir plus}

OCDE (à paraître), OECD Best Practice Principles for Regulatory Policy: Regulatory Impact Assessment, Éditions OCDE, Paris.

OCDE (2018), Politique de la réglementation: Perspectives de l'OCDE 2018, Éditions OCDE, Paris, https://doi.org/10.1787/ 9789264303072-en.

OCDE (2012), Recommandation du Conseil concernant la politique et la gouvernance réglementaires, Éditions OCDE, Paris, https:// legalinstruments.oecd.org/en/instruments/OECD-LEGAL-0390.

\section{Notes relatives aux graphiques}

Concernant les données sur Israël, voir http://doi.org/10.1787/888932315602 Les données concernant la Lettonie, la Lituanie, la Colombie et le Costa Rica ne sont pas disponibles pour 2014.

7.3. Aucun score national n'est présenté pour les États-Unis, pour lesquels le Congrès est systématiquement à l'origine des textes législatifs. * Dans la majorité des pays de l'OCDE, la plupart des textes législatifs sont élaborés par l'exécutif, sauf pour la Corée, le Mexique, la Colombie et le Costa Rica, où la majorité des textes législatifs sont d'initiative parlementaire. 
7.3. Analyse d'impact de la réglementation pour les textes législatifs en cours d'élaboration, 2014 et 2017

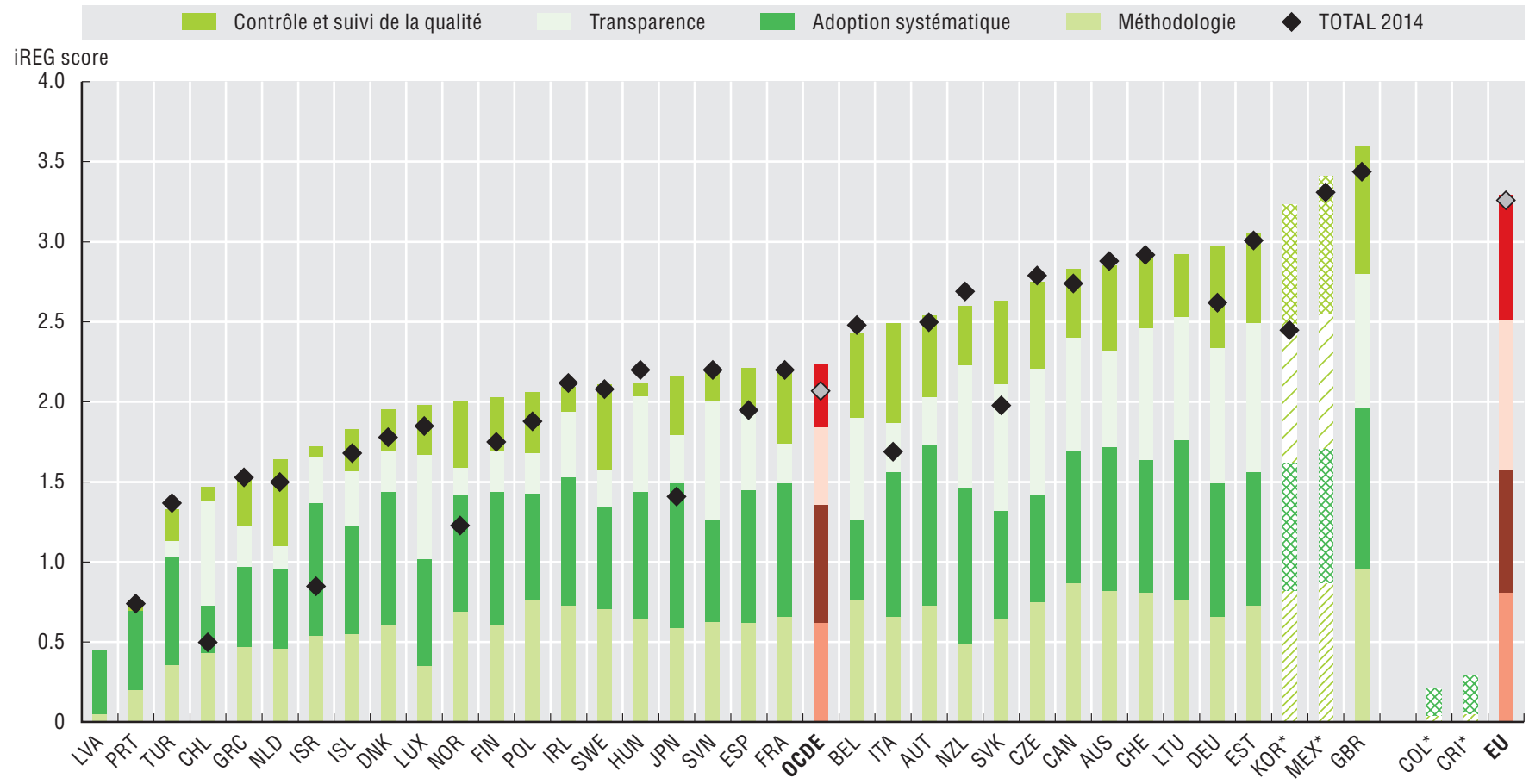

Source : OCDE Indicateurs relatifs à la politique et à la gouvernance réglementaires (iREG) 2015 et 2018, http://oe.cd/ireg.

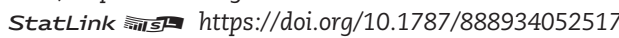

\subsection{Analyse d'impact de la réglementation pour les textes réglementaires en cours d'élaboration, 2014 et 2017}

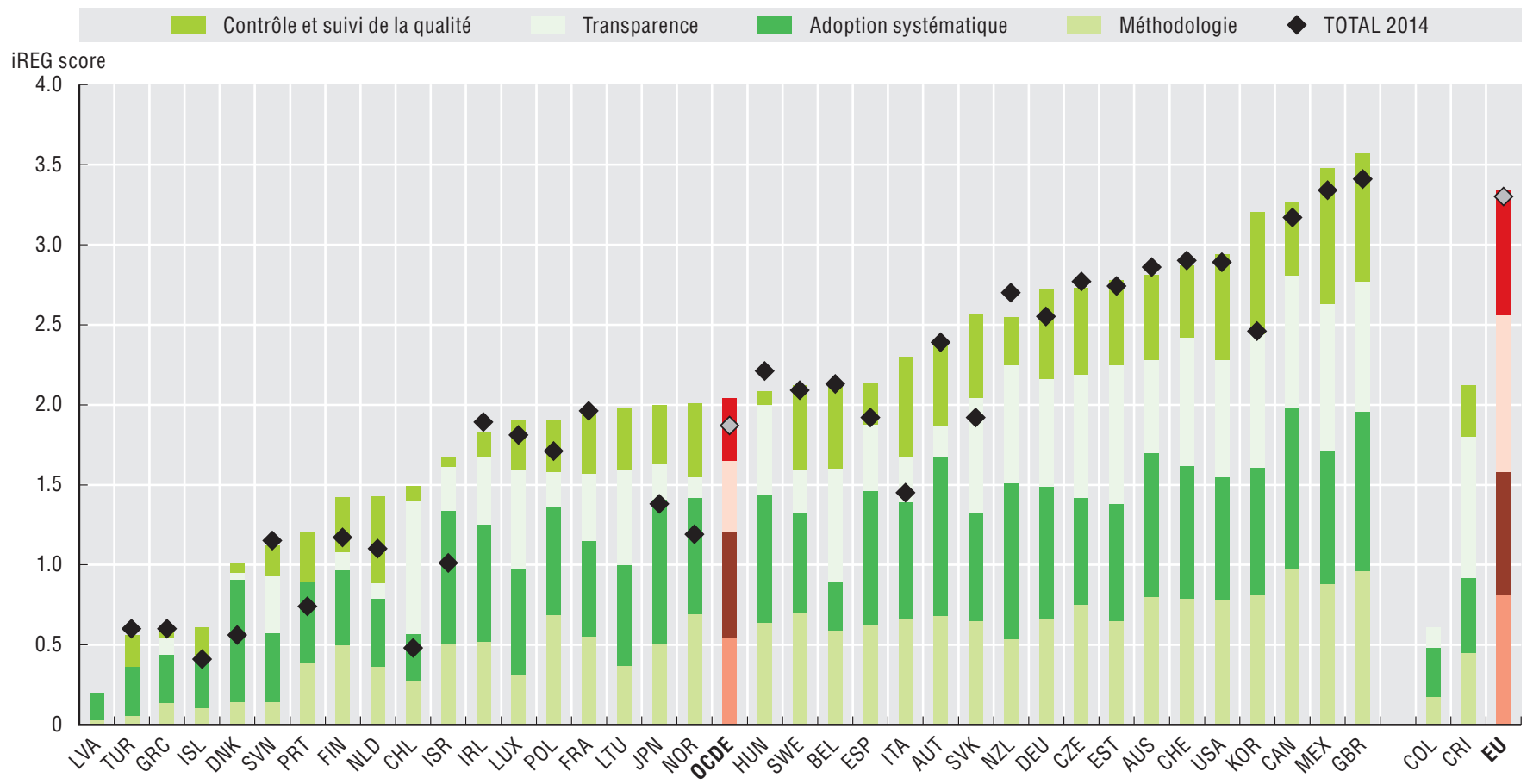

Source : OCDE Indicateurs relatifs à la politique et à la gouvernance réglementaires (iREG) 2015 et 2018, http://oe.cd/ireg. 
Les pays ont intérêt à passer régulièrement en revue leur réglementation existante afin de vérifier que les textes antérieurement adoptés restent adaptés à leur objet. Ce n'est qu'une fois qu'un texte a été mis en œuvre que les autorités peuvent pleinement évaluer ses effets, ses coûts, ses avantages et ses conséquences imprévues. Face à l'évolution rapide de la société et des technologies, même les textes bien conçus peuvent devenir obsolètes et contribuer inutilement à la charge réglementaire, compliquant la vie des particuliers et entravant la marche des entreprises. Les évaluations a posteriori (également appelées " évaluations ex post ") peuvent apporter des renseignements précieux permettant d'améliorer la conception de la réglementation et d'offrir un retour d'information utile pour la planification réglementaire et l'élaboration de nouveaux textes. En outre, elles peuvent renforcer la confiance des parties prenantes à l'égard des pouvoirs publics en améliorant la transparence et la responsabilité en matière d'activité réglementaire.

Les pays de l'OCDE n'investissent encore pas assez dans l'évaluation a posteriori. Par conséquent, le cycle réglementaire demeure incomplet. Les pays désireux d'améliorer leur réglementation axent davantage leurs efforts sur la conception des textes que sur leurs mise en application et évaluation. Ce constat semble valoir aussi bien pour l'évaluation a posteriori des textes législatifs que pour celle des textes réglementaires. En matière d'évaluation a posteriori, l'Australie et le RoyaumeUni sont les pays qui ont le plus investi en termes d'adoption systématique, de méthodologie, de transparence et de contrôle et suivi de la qualité, conformément à la Recommandation concernant la politique et la gouvernance réglementaires. À l'inverse, certains pays de l'OCDE n'ont toujours pas évalué leurs textes afin de déterminer s'ils étaient efficaces et adaptés à leur objet. Tels sont les cas de la Grèce et de la Turquie.

La majorité des pays de l'OCDE n'ont toujours pas adopté de démarche systématique d'évaluation a posteriori, avec des exigences larges et une méthodologie bien établie. En 2017, seuls l'Allemagne, l'Autriche, la Corée, l'Italie et le Royaume-Uni déclaraient exiger de façon systématique que l'on procède à une évaluation a posteriori des textes législatifs et réglementaires et que l'on évalue, dans ce cadre, si les textes examinés avaient atteint l'objectif poursuivi. En matière d'évaluation a posteriori, le contrôle et le suivi de la qualité restent le maillon faible dans la plupart des pays de l'OCDE, bien que quelques progrès aient été constatés chez certains d'entre eux. En revanche, c'est sur le terrain de la transparence que les pratiques des pays de l'OCDE sont les plus avancées : si une évaluation a posteriori est menée, celle-ci est souvent rendue publique.

Ces trois dernières années, les progrès en matière de recours aux évaluations a posteriori par la plupart des pays de l'OCDE sont restés très modestes. Depuis 2014, certains pays ont procédé à des réformes plus larges de leurs pratiques d'évaluation a posteriori. Les principales améliorations ont portées sur le niveau de l'adoption systématique de l'évaluation a posteriori. Par exemple, l'Autriche, l'Espagne et le Japon ont adopté un critère pour déterminer quels textes devaient obligatoirement faire l'objet d'une évaluation a posteriori. Au Japon, par exemple, ce critère détermine en quoi l'évaluation d'impact des réglementations se faisant ex ante exige une évaluation a posteriori. Au Danemark, le Forum des affaires pour mieux légiférer (Business Forum for Better Regulation) a procédé à des analyses approfondies des effets cumulatifs du cadre réglementaire dans différents domaines, dont le transport, le numérique et l'économie circulaire. La Corée et l'Italie ont amélioré le contrôle de leurs systèmes d'évaluation a posteriori en mettant en place un retour d'information régulier sur les performances de leurs systèmes d'évaluation a posteriori.

L'indicateur iREG relatif aux évaluations a posteriori se fonde sur les pratiques décrites dans la Recommandation concernant la politique et la gouvernance réglementaires adoptée en 2012 par l'OCDE. Plus un pays a adopté de ces pratiques, plus son score lié à cet indicateur est élevé. L'indicateur composite comprend quatre catégories affectées d'une pondération égale : la catégorie " méthodologie " qui évoque les différentes analyses réalisées dans le cadre de l'évaluation a posteriori ; la catégorie " contrôle et suivi de la qualité " qui traite des dispositifs mis en place pour suivre la qualité des évaluations a posteriori ; la catégorie " adoption systématique " qui porte sur les exigences formelles et le recours aux différents types d'évaluations a posteriori ; et la catégorie "transparence " qui suit l'information relative au degré d'ouverture des évaluations a posteriori. Le score maximal est égal à 1 pour chaque catégorie et le score total pour l'indicateur composite s'échelonne entre 0 et 4.

\section{Methodology and definitions}

Les indicateurs relatifs à la politique et à la gouvernance réglementaires (iREG) s'appuient sur les réponses des délégués auprès du Comité de la politique de la réglementation de l'OCDE et par des responsables d'administration centrale aux enquêtes 2014 et 2017 de l'OCDE sur les indicateurs en matière réglementaire. Ces enquêtes ont été menées auprès de 38 pays membres de l'OCDE et candidats à l'adhésion et auprès de l'Union européenne. On trouvera un complément d'information sur les indicateurs iREG à l'Annexe E et à l'adresse oe.cd/ireg.

Les textes législatifs sont ceux qui doivent être adoptés par le parlement, tandis que les textes réglementaires peuvent être adoptés par le chef du gouvernement, un ministre ou le Conseil des ministres.

\section{Pour en savoir plus}

OCDE (à paraître), OECD Best Practice Principles for Regulatory Policy: Reviewing the Stock of Regulation, Éditions OCDE, Paris.

OCDE (2018), Politique de la réglementation : perspectives de l'OCDE 2018, Éditions OCDE, Paris, https://doi.org/10.1787/ 9789264303072-en.

OCDE (2012), Recommandation du Conseil concernant la politique et la gouvernance réglementaires, Éditions OCDE, Paris, https:// legalinstruments.oecd.org/en/instruments/OECD-LEGAL-0390.

\section{Notes relatives aux graphiques}

Concernant les données sur Israël, voir http://doi.org/10.1787/ 888932315602. Les données concernant la Lettonie, la Lituanie, la Colombie et le Costa Rica ne sont pas disponibles pour 2014. 
7.5. Évaluation a posteriori des textes législatifs, 2014 et 2017

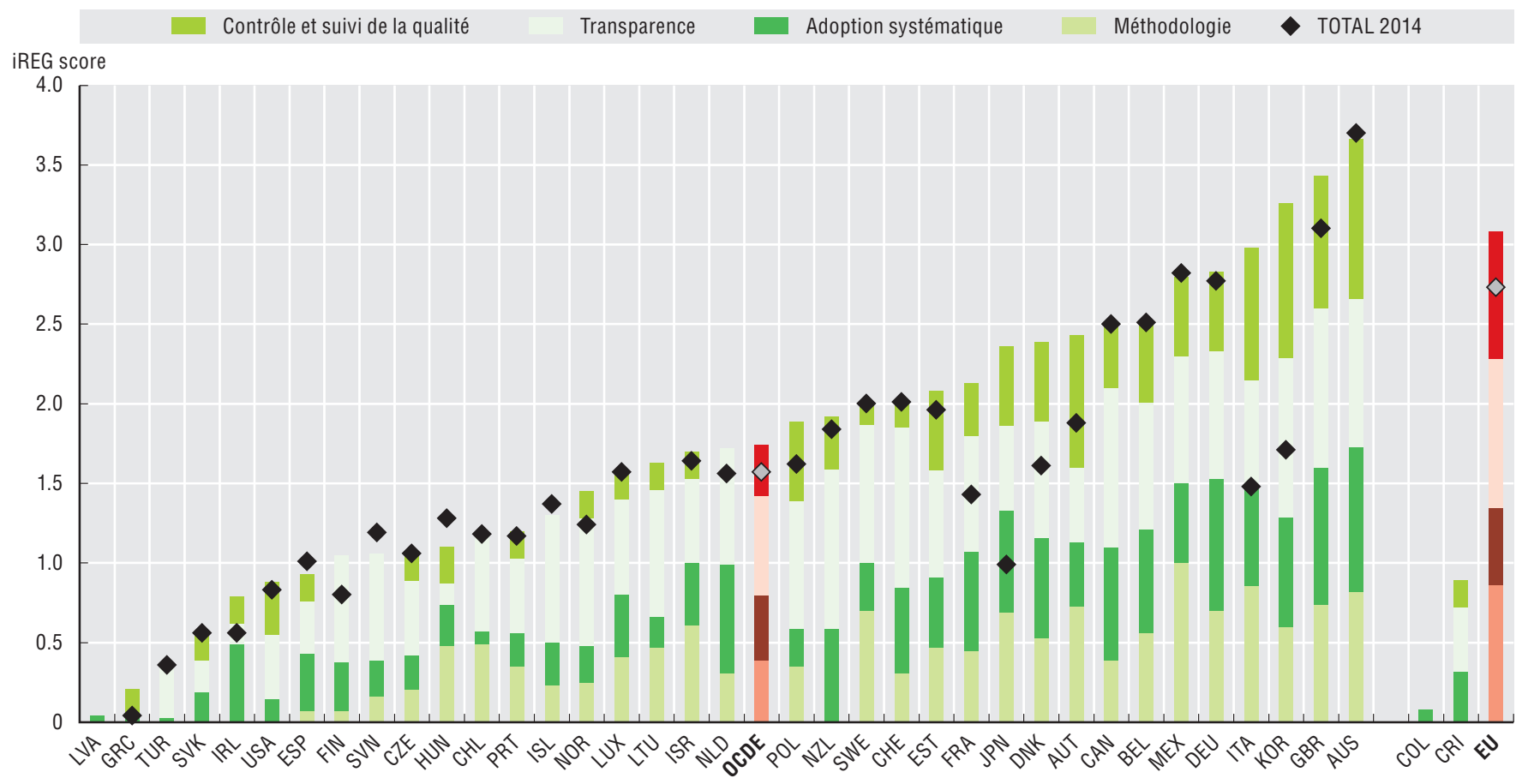

Source : OCDE Indicateurs relatifs à la politique et à la gouvernance réglementaires (iREG) 2015 et 2018, http://oe.cd/ireg.

StatLink 尚ISt https://doi.org/10.1787/888934052555

\section{6. Évaluation a posteriori des textes réglementaires, 2014 et 2017}

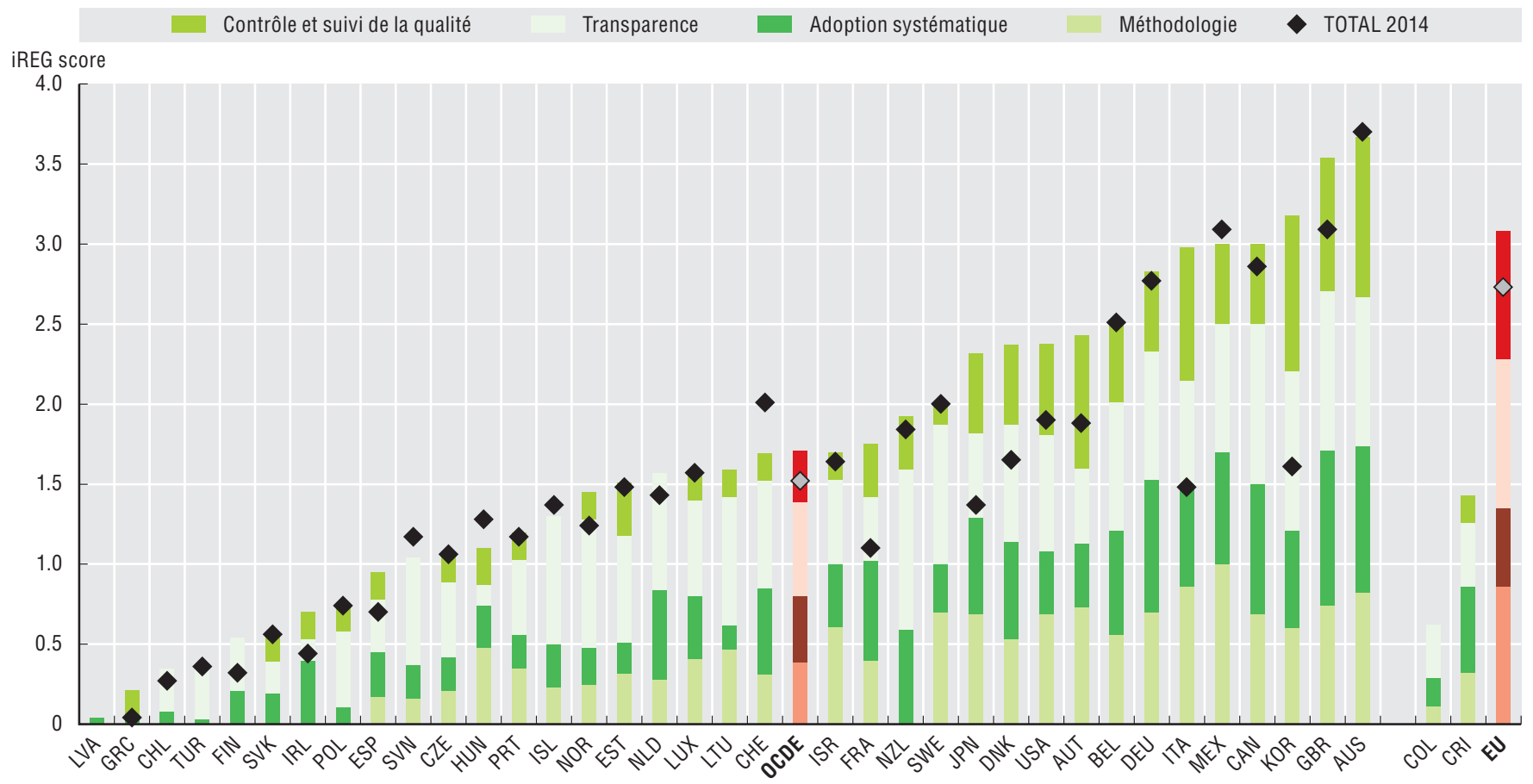

Source : OCDE Indicateurs relatifs à la politique et à la gouvernance réglementaires (iREG) 2015 et 2018, http://oe.cd/ireg. 


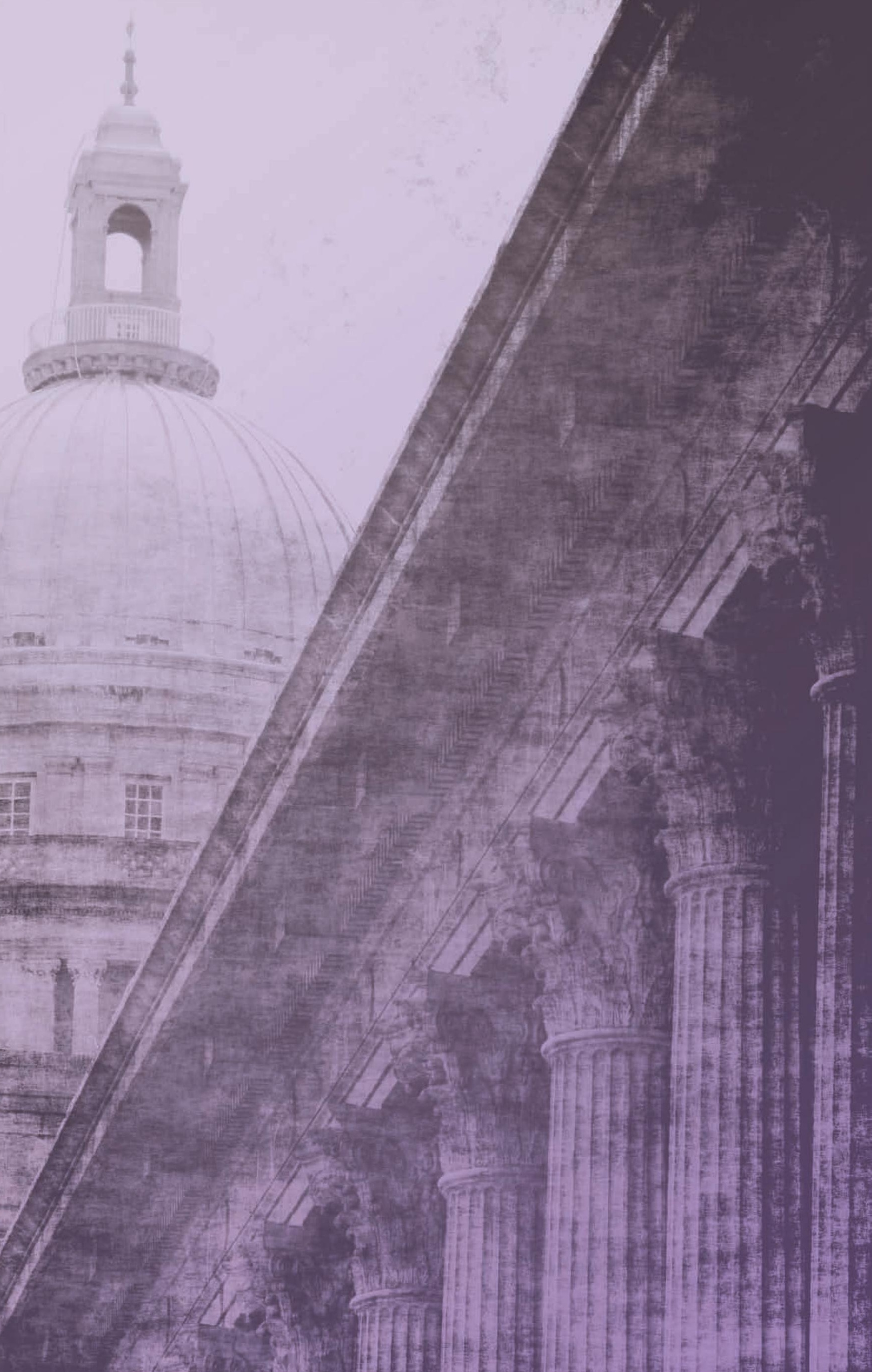


De plus en plus, les administrations publiques s'appuient sur les marchés publics, c'est-à-dire les achats de biens, de services et de travaux par les administrations et entreprises publiques, pour mieux remplir leur mission et poursuivre des objectifs stratégiques plus larges. En plus de se conformer aux principes habituels et aux règles en vigueur, les administrations publiques s'emploient à améliorer l'efficience et l'efficacité de ce volet crucial de leurs activités. D'un point de vue économique, les marchés publics sont considérés comme un levier d'action pour renforcer l'efficience et l'efficacité des dépenses publiques. De surcroît, la réalisation d'objectifs stratégiques plus larges (promotion de l'innovation, durabilité, inclusivité sociale et soutien aux PME, par exemple) constitue une part croissante des stratégies de passation de marchés des administrations publiques.

Du simple fait de leur ampleur, les marchés publics représentent une activité économique majeure. Leur poids économique correspond dans les pays de l'OCDE à environ $12 \%$ du PIB. Il s'échelonne entre 4,9\% au Mexique et 19,5\% aux Pays-Bas et est plus marqué en période de récession économique. De ce fait, dans les trois-quarts des pays de l'OCDE, le poids des dépenses publiques liées aux marchés publics dans le PIB a atteint un pic en 2009, lorsque la plupart d'entre eux ont été frappés par la récession économique. Depuis lors, la part des dépenses liées aux marchés publics dans les pays de l'OCDE a connu une lente diminution mais est resté globalement stable au cours des quatre dernières années, tant en pourcentage du PIB (11,8 \% en 2017) qu'en proportion du total des dépenses des administrations publiques (29,1\% en 2017).

Sous l'effet de contraintes budgétaires, les administrations publiques s'emploient à réformer leurs systèmes de passation des marchés afin d'optimiser ce poste important de dépenses. Très utiles pour les administrations centrales, ces réformes le sont plus encore pour les administrations infranationales qui concentrent dans les pays de l'OCDE $63 \%$ du total des dépenses liées aux marchés publics, étant entendu qu'il existe des différences importantes selon le cadre institutionnel de chaque pays.

Les marchés publics ont des répercussions importantes sur toutes les formes de prestation de services publics, comme l'illustre la ventilation sectorielle des dépenses, qu'il s'agisse de la santé, de la protection de l'environnement, de l'ordre public ou encore des affaires économiques (y compris les infrastructures, les transports, les communications, l'énergie et la R\&D). Les dépenses de santé se taillent la part du lion : elles représentent dans les pays de l'OCDE près du tiers (30\%) des dépenses liées aux marchés publics et même plus de $40 \%$ dans certains pays européens (Allemagne, Belgique, Italie et République slovaque) ainsi qu'au Japon. Plusieurs exceptions sont à noter : aux États-Unis, en Hongrie et en Lettonie les affaires économiques constituent la majeure partie des dépenses liées aux marchés publics. Les affaires économiques (16,3\%), l'éducation (11,7\%), la défense $(10 \%)$ et la protection sociale $(10,2 \%)$ représentent dans les pays de l'OCDE les autres postes les plus importants de dépenses liées aux marchés publics, moyennant des variations importantes d'un pays à l'autre.

\section{Méthodologie et définitions}

Le montant des dépenses des administrations publiques liées aux marchés publics a été estimé à partir de données tirées de la base de données des Statistiques de
l'OCDE sur les comptes nationaux, qui sont établies conformément au Système de comptabilité nationale (SCN). Les dépenses des administrations publiques liées aux marchés publics sont définies comme la somme de la consommation intermédiaire (biens et services achetés par les administrations publiques pour leur propre usage, comme des prestations comptables ou informatiques), de la formation brute de capital fixe (acquisition d'équipements moins ventes d'actifs fixes, par exemple construction de nouvelles routes) et des transferts sociaux en nature opérés par l'intermédiaire de producteurs marchands (achat par les administrations publiques de biens et services produits par des producteurs marchands et fournis aux ménages). Les entreprises publiques ont été exclues de l'estimation des dépenses liées aux marchés publics. Les données relatives aux dépenses des administrations publiques ont été ventilées selon la Classification des fonctions des administrations publiques (COFOG/CFAP) dans l'illustration 8.2 ci-après. On trouvera à l'Annexe $\mathrm{C}$ des précisions sur la nature des dépenses entrant dans chaque catégorie.

\section{Pour en savoir plus}

OCDE (2019), Productivity in Public Procurement, OCDE, Paris, http://www.oecd.org/gov/public-procurement/publications/ productivity-public-procurement.pdf, consulté le 12 février 2019.

OCDE (2015), Recommandation du Conseil sur les marchés publics, OCDE, Paris, https://www.oecd.org/fr/gov/commande-publique/ Recommandation-OCDE-sur-les-marches-publics.pdf.

\section{Notes sur les graphiques}

Concernant les données sur Israël, voir http://doi.org/10.1787/ 888932315602 .

8.1. On ne dispose pas de données pour le Chili. Les données relatives à la Turquie ne sont pas prises en compte dans la moyenne OCDE, dû à certaines séries temporelles manquantes. Aux Pays-Bas, une part importante des dépenses des administrations publiques liées aux marchés publics concerne des transferts sociaux en nature fournis aux ménages par l'intermédiaire de producteurs marchands - ce niveau relativement élevé peut en partie s'expliquer par le système de bourses d'études du pays, ainsi que par son système d'assurance santé obligatoire en vertu duquel l'État subventionne l'achat par les particuliers d'une couverture auprès de prestataires privés.

8.2. On ne dispose pas de données pour l'Australie, le Canada, le Mexique, la Nouvelle-Zélande et la Turquie. Les données relatives au Chili et à l'Islande ne sont pas prises en compte dans le calcul de la moyenne de l'OCDE, dû à certaines séries temporelles manquantes. Les données relatives au Chili comprennent les variations des stocks et acquisitions moins les cessions d'objets de valeur. Les données relatives au Chili et à la Corée portent sur 2016 et non sur 2017.

8.3. (Évolution de la ventilation des dépenses des administrations publiques en matière de marchés publics par fonction, de 2012 à 2017) et 8.4. (Ventilation des marchés publics par niveau d'administration, 2009, 2015 et 2017) sont disponibles en ligne à l'Annexe F. 


\subsection{Poids des marchés publics dans le PIB et dans les dépenses des administrations publiques, 2007, 2009 et 2017}

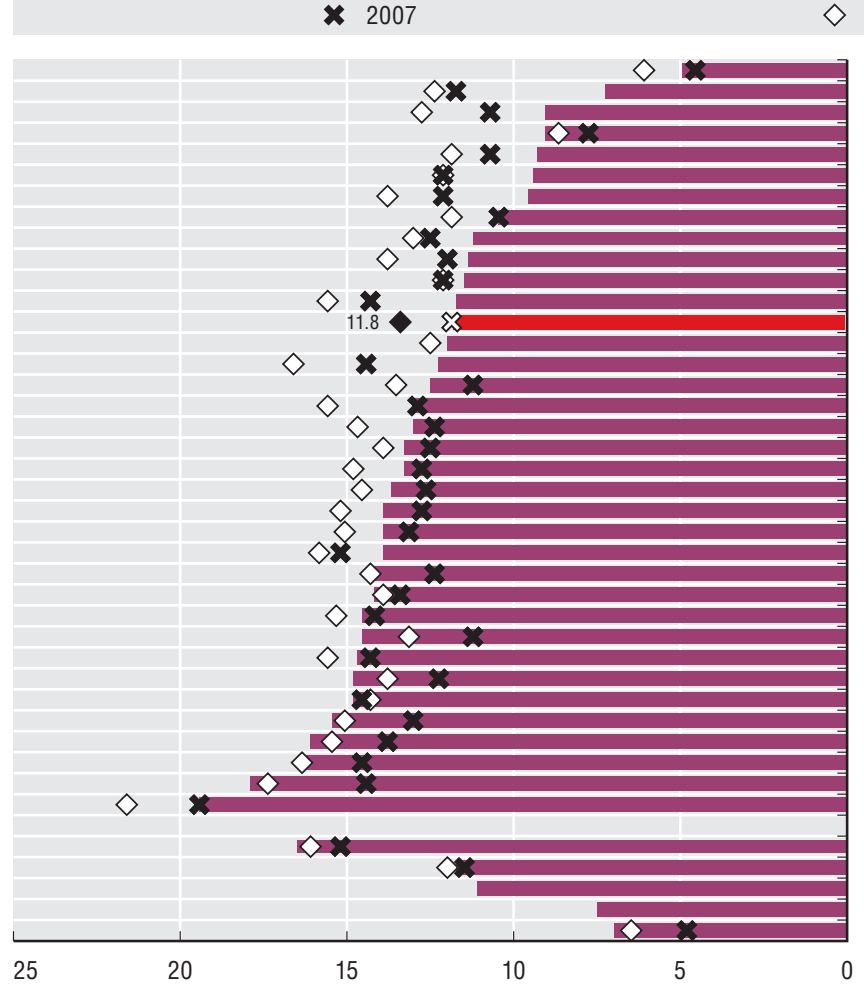

Poids dans le PIB, \%
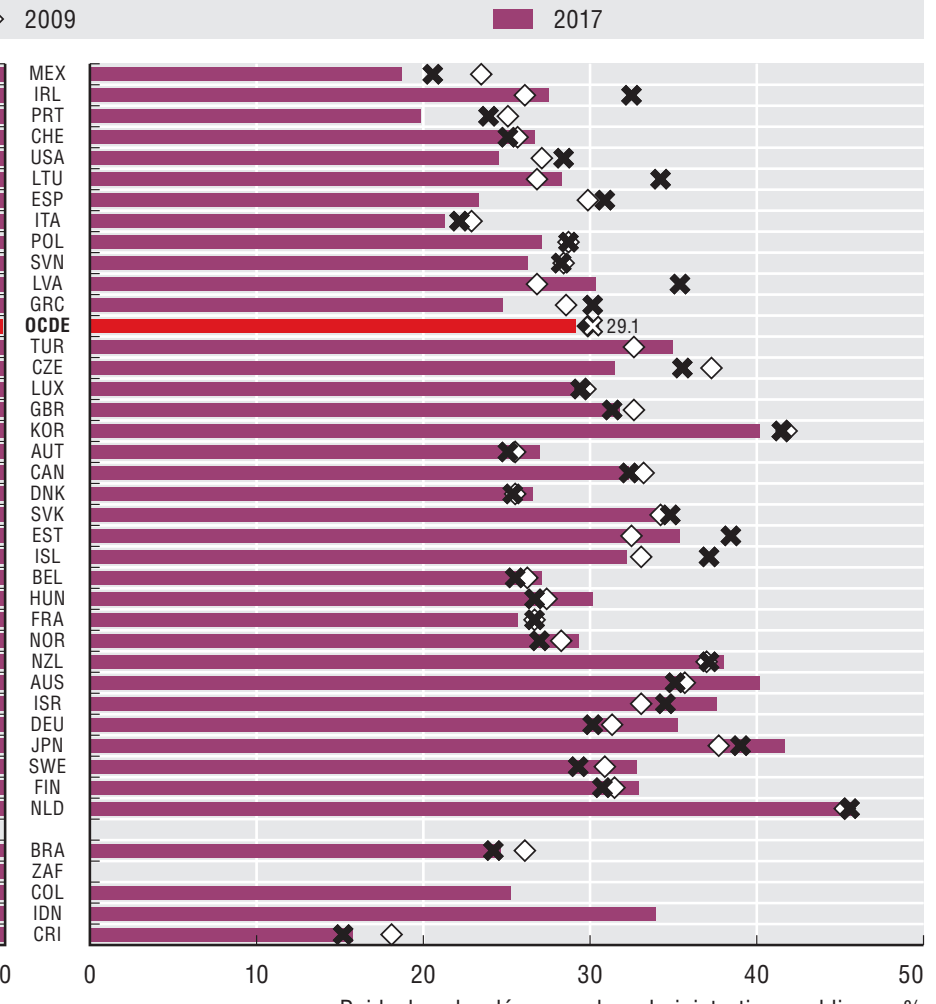

Source: Statistiques de l'OCDE sur les comptes nationaux (base de données). Les données relatives à l'Australie sont tirées des statistiques sur les finances publiques et sur les comptes nationaux fournies par le Bureau australien des statistiques.

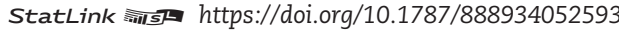

8.2. Ventilation des dépenses des marchés publics par fonction, 2017

\begin{tabular}{|c|c|c|c|c|c|c|c|c|c|c|}
\hline & $\begin{array}{c}\text { Services généraux } \\
\text { des administrations } \\
\text { publiques }\end{array}$ & Défense & $\begin{array}{l}\text { Ordre et sécurité } \\
\text { publics }\end{array}$ & $\begin{array}{c}\text { Affaires } \\
\text { économiques }\end{array}$ & $\begin{array}{c}\text { Protection } \\
\text { de l'environnement }\end{array}$ & $\begin{array}{l}\text { Logement et } \\
\text { équipements } \\
\text { collectifs }\end{array}$ & Santé & $\begin{array}{l}\text { Loisirs, } \\
\text { culture et } \\
\text { culte }\end{array}$ & Éducation & $\begin{array}{c}\text { Protection } \\
\text { sociale }\end{array}$ \\
\hline Allemagne & 10.0 & 4.0 & 3.2 & 9.3 & 2.6 & 1.0 & 40.6 & 2.3 & 6.5 & 20.6 \\
\hline Autriche & 11.8 & 1.5 & 3.1 & 21.9 & 1.3 & 0.7 & 35.8 & 3.9 & 9.2 & 10.7 \\
\hline Belgique & 11.9 & 1.2 & 2.1 & 10.3 & 3.3 & 1.1 & 49.3 & 3.1 & 7.4 & 10.3 \\
\hline Chili & 6.0 & 5.5 & 8.5 & 19.1 & 1.3 & 6.9 & 21.6 & 2.6 & 16.9 & 11.5 \\
\hline Corée & 11.5 & 11.1 & 3.1 & 18.3 & 4.1 & 4.6 & 31.4 & 2.8 & 10.7 & 2.4 \\
\hline Danemark & 14.7 & 5.1 & 2.7 & 11.0 & 1.3 & 0.8 & 31.1 & 5.5 & 11.8 & 16.0 \\
\hline Espagne & 10.6 & 3.7 & 3.3 & 14.0 & 6.9 & 2.8 & 32.8 & 5.8 & 11.5 & 8.5 \\
\hline Estonie & 12.0 & 10.1 & 4.5 & 19.6 & 4.0 & 2.0 & 22.6 & 7.1 & 14.4 & 3.7 \\
\hline Etats-Unis & 9.9 & 20.3 & 6.6 & 22.3 & 0.0 & 2.4 & 14.4 & 1.7 & 19.0 & 3.4 \\
\hline Finlande & 21.8 & 4.6 & 2.2 & 13.3 & 0.6 & 1.1 & 22.8 & 3.8 & 11.6 & 18.3 \\
\hline France & 7.0 & 6.3 & 2.5 & 12.4 & 4.1 & 3.0 & 38.5 & 4.6 & 6.7 & 14.8 \\
\hline Grèce & 16.3 & 7.7 & 1.0 & 29.3 & 3.6 & 1.3 & 31.7 & 2.4 & 5.2 & 1.6 \\
\hline Hongrie & 18.1 & 3.7 & 4.1 & 23.4 & 2.2 & 2.3 & 21.9 & 9.5 & 10.0 & 4.8 \\
\hline Irlande & 5.5 & 0.8 & 4.6 & 15.1 & 3.0 & 4.9 & 34.4 & 4.0 & 9.7 & 17.9 \\
\hline Islande & 8.9 & 0.4 & 3.8 & 19.4 & 2.6 & 2.2 & 25.5 & 9.1 & 20.8 & 7.3 \\
\hline Israël & 6.9 & 25.5 & 3.6 & 2.8 & 2.7 & 1.6 & 31.8 & 4.1 & 10.0 & 11.0 \\
\hline Italie & 11.6 & 3.9 & 3.6 & 9.0 & 7.8 & 3.4 & 44.4 & 4.7 & 5.3 & 6.4 \\
\hline Japon & 6.2 & 3.2 & 1.9 & 14.3 & 5.9 & 2.1 & 44.6 & 1.6 & 6.5 & 13.8 \\
\hline Lettonie & 8.3 & 8.5 & 7.1 & 20.2 & 2.6 & 6.8 & 14.7 & 7.0 & 18.1 & 6.8 \\
\hline Lituanie & 7.7 & 8.3 & 5.0 & 19.2 & 3.0 & 3.4 & 26.0 & 4.8 & 15.3 & 7.4 \\
\hline Luxembourg & 9.8 & 1.0 & 2.3 & 23.6 & 4.3 & 2.8 & 29.7 & 5.7 & 7.6 & 13.2 \\
\hline Norvège & 10.9 & 7.3 & 2.7 & 19.6 & 4.1 & 4.3 & 25.0 & 4.8 & 11.1 & 10.0 \\
\hline Pays-Bas & 6.6 & 2.8 & 3.6 & 11.8 & 5.1 & 1.5 & 35.7 & 3.1 & 8.7 & 21.1 \\
\hline Pologne & 6.5 & 7.0 & 4.4 & 25.2 & 2.3 & 4.2 & 29.4 & 5.4 & 11.6 & 3.9 \\
\hline Portugal & 14.5 & 2.3 & 2.9 & 22.1 & 4.3 & 2.5 & 33.8 & 4.7 & 9.9 & 3.1 \\
\hline République Slovaque & 10.9 & 3.3 & 4.3 & 20.1 & 3.7 & 2.6 & 42.8 & 3.1 & 6.8 & 2.5 \\
\hline République Tchèque & 6.5 & 2.2 & 3.6 & 20.4 & 5.3 & 3.0 & 35.2 & 5.0 & 14.0 & 4.8 \\
\hline Royaume-Uni & 3.4 & 10.2 & 6.3 & 13.2 & 4.4 & 3.2 & 31.6 & 2.9 & 10.9 & 13.8 \\
\hline Slovénie & 11.4 & 2.3 & 3.6 & 20.5 & 2.5 & 3.4 & 33.7 & 5.4 & 13.2 & 4.0 \\
\hline Suède & 19.0 & 4.5 & 2.8 & 13.0 & 1.1 & 3.7 & 21.5 & 2.9 & 15.4 & 16.1 \\
\hline Suisse & 23.1 & 5.9 & 5.8 & 14.7 & 4.2 & 1.4 & 1.6 & 2.9 & 18.7 & 22.0 \\
\hline Total OCDE & 9.2 & 10.0 & 4.3 & 16.3 & 3.0 & 2.4 & 30.1 & 2.8 & 11.7 & 10.2 \\
\hline Costa Rica & 4.4 & 0.0 & 7.4 & 19.5 & 3.5 & 4.3 & 34.1 & 1.8 & 20.4 & 4.7 \\
\hline
\end{tabular}

Sources : Statistiques de l'OCDE sur les comptes nationaux (base de données) ; Statistiques d’Eurostat sur les finances publiques (base de données). 
Lorsqu'il en est fait un usage stratégique, les marchés publics peuvent contribuer à accroître la productivité d'un pays et l'efficience de son secteur public, et à renforcer la confiance envers les institutions. Les pays s'appuient sur les marchés publics pour poursuivre des objectifs stratégiques complémentaires en lien avec le Programme de développement durable à l'horizon 2030. Les marchés publics sont de plus en plus considérés comme un instrument stratégique potentiel et un moyen d'atteindre les objectifs des politiques publiques - par exemple, stimuler l'innovation, développer les marchés publics verts et l'économie circulaire, soutenir l'accès des PME aux marchés publics et promouvoir les comportements éthiques et la conduite responsable des entreprises (CRE).

Tous les pays de l'OCDE ont déclaré avoir adopté des politiques visant des objectifs plus larges tels que les marchés publics verts et 28 pays ont déployé des stratégies ou des politiques en matière de marchés publics écologiques au niveau central. La majorité des pays de l'OCDE se sont dotés de politiques visant à faciliter l'accès des PME aux marchés publics (29) et, dans une moindre mesure, aux marchés publics favorisant l'innovation (26). Ces politiques consistent notamment à éliminer les obstacles à l'utilisation des marchés publics comme moyen d'action stratégique. L'un des principaux obstacles à la réalisation des résultats attendus de la passation de marchés publics en matière d'innovation, d'environnement et de durabilité réside dans le fait que le choix du plus petit prix représente souvent le critère exclusif d'attribution. Dès lors, encourager le recours à des critères d'attribution tenant compte de divers aspects, tels que les coûts de maintenance et d'exploitation tout au long du cycle de vie, contribue à favoriser l'achat de biens et de produits présentant une meilleure performance environnementale tout en favorisant l'innovation. En outre, la complexité des systèmes et processus de passation des marchés publics constitue un obstacle majeur à la participation des PME aux marchés publics, cette complexité les pénalisant tout particulièrement compte tenu de leurs capacités financières, techniques et administratives. Les pays s'emploient à remédier à ce problème en adoptant une variété de mesures, notamment en divisant les marchés en lots, en développant l'usage de la passation électronique des marchés ou en facilitant les soumissions conjointes d'offres associant PME et grandes entreprises.

Entre 2016 et 2018, on a observé une augmentation du nombre de mesures en faveur des marchés publics verts et surtout de la CRE. Plusieurs méthodes sont employées dans les différents pays. Le Canada a par exemple mis en place une certification des fournisseurs dans le cadre de la procédure de soumission aux marchés relevant de l'Initiative d'achat éthique de vêtements (où toute infraction à la certification peut entraîner la rupture du contrat). Aux Pays-Bas, la CRE est désormais un aspect obligatoire de la passation de marchés publics par les entités publiques centrales.

Par ailleurs, un nombre croissant de pays de l'OCDE collecte des données sur l'avancement des objectifs stratégiques des marchés publics et certains prévoient la présentation de rapports à différents niveaux d'administration. C'est notamment le cas en matière de marchés publics verts (73\%) et de soutien aux PME (67\%). En revanche, $30 \%$ seulement des pays mesurent le résultat des processus de passation des marchés publics en lien avec telle ou telle dimension relative à l'innovation ou à la CRE, comme c'est le cas en Corée et en Slovénie. Certains pays comme le Chili, la Corée et le Japon ont adopté des mesures visant à accroître la participation des entreprises dirigées par des femmes aux marchés publics et

\section{Méthodologie et définitions}

Ces données proviennent de l'Enquête 2018 de l'OCDE sur la mise en œuvre des Recommandations 2015 de l'OCDE sur les marchés publics, qui était axée sur les 12 Principes de la recommandation. Un-tiers des pays de l'OCDE ont participé à l'enquête et un pays candidat à l'adhésion (Costa Rica). Les réponses ont été fournies par des délégués nationaux chargés des politiques de passation des marchés publics au sein de l'administration centrale et de hauts responsables de centrales d'achat publiques.

La Commission européenne définit les marchés publics verts ou écologiques comme « un processus de passation de marchés dans le cadre duquel les pouvoirs publics cherchent à obtenir des biens, des services et des travaux dont l'incidence sur l'environnement sur toute leur durée de vie sera moindre que dans le cas de biens, services et travaux à vocation identique mais ayant fait l'objet de procédures de passation de marché différentes ".

La conduite responsable des entreprises fait référence aux moyens mis en œuvre par les entreprises afin de contribuer au progrès économique, environnemental et social visant le développement durable, d'éviter les effet néfastes - liés à leurs propres activités ou à un partenaire commercial au sein de la chaîne de valeur ou d'y remédier.

mesurent également les résultats des processus de passation des marchés publics dans ce domaine.

\section{Pour en savoir plus}

OCDE (2018), SMEs in Public Procurement : Practices and Strategies for Shared Benefits, Examens de l'OCDE sur la gouvernance publique, Éditions OCDE, Paris, https://doi.org/10.1787/ 9789264307476-en.

OCDE (2017), Public Procurement for Innovation: Good Practices and Strategies, OECD Public Governance Reviews, Éditions OCDE, Paris, https://doi.org/10.1787/9789264265820-en.

OCDE (2015), Going Green: Best Practices for Sustainable Procurement, OCDE, Paris, https://www.oecd.org/governance/ethics/Going_ Green_Best_Practices_for_Sustainable_Procurement.pdf.

\section{Notes sur les graphiques}

Les données pour les États-Unis le Luxembourg, la République tchèque, le Royaume-Uni et la Suisse sont manquantes. Concernant les données sur Israël, voir http://doi.org/10.1787/888932315602.

8.5. Cette illustration ne tient pas compte du programme canadien Stratégie d'approvisionnement auprès des entreprises autochtones (SAEA) en tant que politique ou stratégie liée à la conduite responsable des entreprises. Le SAEA a vocation à encourager les entreprises autochtones qualifiées à conclure davantage de marchés avec la puissance publique à l'aide d'un programme prévoyant des marchés obligatoires, des marchés réservés et des activités de développement des capacités des fournisseurs.

8.6. Les données sur la France ne sont pas incluses car les personnes n'ont pas répondu à l'enquête 2016. 
8.5. Élaboration de stratégies/politiques de passation de marchés publics visant à favoriser la réalisation d'objectifs stratégiques complémentaires, 2016 et 2018

\begin{tabular}{|c|c|c|c|c|c|c|c|c|c|c|}
\hline & \multicolumn{2}{|c|}{ Marchés publics verts } & \multicolumn{2}{|c|}{ PME } & \multicolumn{2}{|c|}{ Biens et services innovants } & \multicolumn{2}{|c|}{$\begin{array}{l}\text { Conduite responsable des } \\
\text { entreprises }\end{array}$} & \multicolumn{2}{|c|}{$\begin{array}{l}\text { Entreprises dirigées par des } \\
\text { femmes }\end{array}$} \\
\hline & 2016 & 2018 & 2016 & 2018 & 2016 & 2018 & 2016 & 2018 & 2016 & 2018 \\
\hline Allemagne & • & $\bullet$ & - & - & • & $\bullet$ & 0 & 0 & 0 & 0 \\
\hline Australie & घ & $\bullet$ & • & - & • & $\bullet$ & • & $\bullet$ & 0 & 0 \\
\hline Autriche & $\bullet$ & ๑4 & $\bullet$ & ๑4 & $\bullet$ & $\bullet$ & - & - & 0 & 0 \\
\hline Belgique & $\bullet$ & $\bullet$ & - & $\bullet$ & - & - & - & - & 0 & 0 \\
\hline Canada & - & $\bullet$ & $\bullet$ & - & • & - & 0 & - & 0 & 0 \\
\hline Chili & $\bullet$ & $\bullet$ & $\bullet$ & $\bullet$ & $\circ$ & ○ & $\bullet$ & $\bullet$ & $\bullet$ & $\bullet$ \\
\hline Corée & $\bullet$ & $\bullet$ & $\bullet$ & - & $\bullet$ & - & 0 & $\bullet$ & $\bullet$ & $\bullet$ \\
\hline Danemark & $\bullet$ & $\bullet$ & - & - & - & $\bullet$ & - & - & 0 & 0 \\
\hline Espagne & $\bullet$ & -4 & - & - & - & - & - & - & 0 & 0 \\
\hline Estonie & • & $\bullet$ & - & - & $\bullet$ & $\bullet$ & 0 & 0 & 0 & 0 \\
\hline Finlande & $\bullet$ & •t & • & - & ๑4 & $\bullet$ & ๑4 & $\bullet$ & 0 & 0 \\
\hline France & .. & $\bullet$ & .. & - & .. & - & .. & - & .. & 0 \\
\hline Grèce & - & 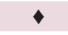 & • & - & 0 & 0 & 0 & 0 & 0 & 0 \\
\hline Hongrie & $\bullet$ & - & - & - & $\bullet$ & $\bullet$ & • & • & - & - \\
\hline Irlande & $\bullet$ & $\bullet$ & - & - & $\bullet$ & - & $\bullet$ & - & - & - \\
\hline Islande & $\bullet$ & • & 0 & 0 & 0 & 0 & 0 & 0 & 0 & 0 \\
\hline Israël & • & • & - & - & 0 & 0 & 0 & 0 & - & $\bullet$ \\
\hline Italie & $\bullet$ & $\bullet$ & 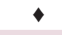 & • & • & - & 0 & 0 & O & 0 \\
\hline Japon & - & $\bullet$ & - & - & 0 & 0 & 0 & 0 & - & - \\
\hline Lettonie & • & • & • & - & 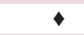 & - & $\bullet$ & $\bullet$ & 0 & 0 \\
\hline Lituanie & - & - & - & - & - & - & - & - & 0 & 0 \\
\hline Mexique & $\bullet$ & $\bullet$ & - & - & $\bullet$ & $\bullet$ & O & 0 & - & $\bullet$ \\
\hline Norvège & • & 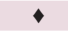 & $\bullet$ & - & ๑4 & - & ๑4 & $\bullet$ & 0 & 0 \\
\hline Nouvelle Zélande & ๑4 & $\bullet$ & $\bullet$ & $\bullet$ & $\bullet$ & Q4 & - & $\bullet$ & 0 & 0 \\
\hline Pays-Bas & - & + & - & + & 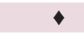 & - & 0 & - & 0 & 0 \\
\hline Pologne & $\bullet$ & $\bullet$ & $\bullet$ & 0 & $\bullet$ & $\bullet$ & $\bullet$ & $\bullet$ & O & 0 \\
\hline Portugal & - & - & - & - & + & - & - & - & 0 & 0 \\
\hline République Slovaque & 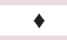 & $\bullet$ & $\bullet$ & $\bullet$ & 0 & $\bullet$ & 0 & 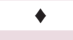 & O & ○ \\
\hline Royaume-Uni & - & .. & - & .. & - & .. & 0 &. & 0 & .. \\
\hline Slovénie & - & - & - & - & - & - & - & - & 0 & 0 \\
\hline Suède & - & - & - & - & - & - & - & - & 0 & 0 \\
\hline Turquie & - & - & - & - & - & - & 0 & 0 & 0 & 0 \\
\hline \multicolumn{11}{|l|}{ Total OCDE } \\
\hline $\begin{array}{l}\text { - Stratégies / politiques } \\
\text { élaborées au niveau central }\end{array}$ & 26 & 28 & 24 & 24 & 20 & 22 & 15 & 18 & 6 & 6 \\
\hline $\begin{array}{l}\text { Stratégies/ politiques/ } \\
\text { internes développées par } \\
\text { certaines entités adjudicatrices }\end{array}$ & 10 & 10 & 8 & 8 & 9 & 8 & 5 & 8 & 1 & 1 \\
\hline - Abrogée & 1 & 0 & 0 & 0 & 0 & 0 & 0 & 0 & 0 & 0 \\
\hline O Jamais élaborée & 0 & 0 & 1 & 2 & 6 & 5 & 14 & 9 & 24 & 24 \\
\hline Costa Rica & - & - & - & - & 0 & - & 0 & - & 0 & 0 \\
\hline
\end{tabular}

Sources : OCDE (2016) « Enquête sur les marchés publics », OCDE (2018) « Enquête sur la mise en oeuvre des Recommandations sur les marchés publics ». StatLink 需s https://doi.org/10.1787/888934052631

8.6. Mesure des résultats des processus de passation des marchés publics au regard des objectifs stratégiques complémentaires, 2016 et 2018

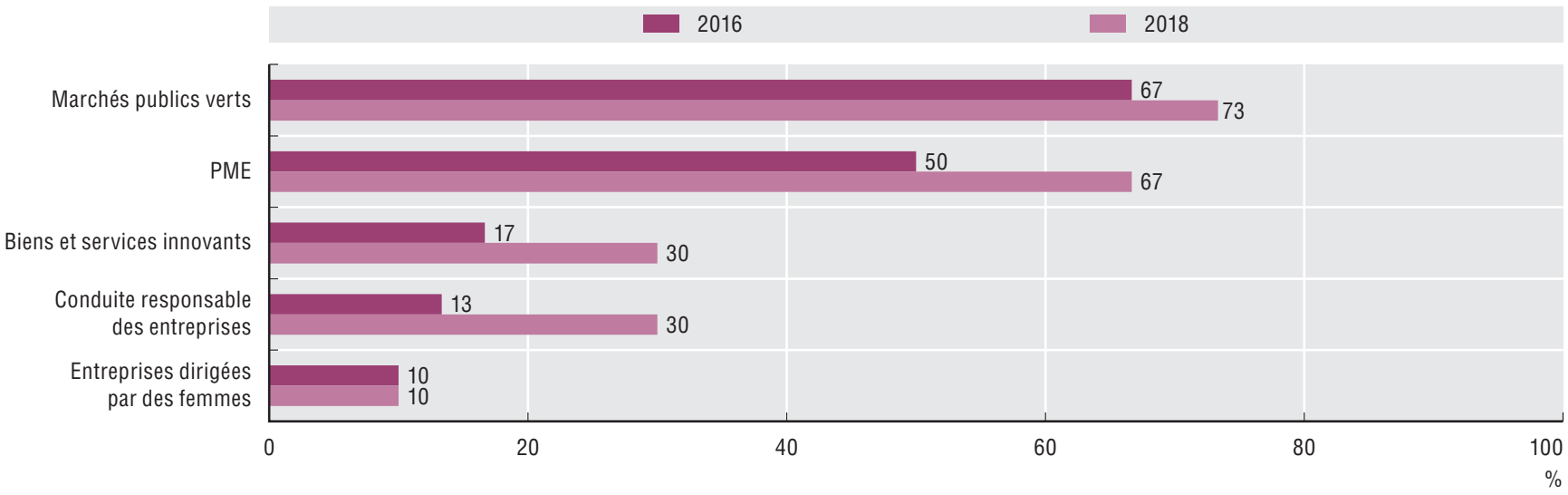

Sources : OCDE (2016) « Enquête sur les marchés publics », OCDE (2018) « Enquête sur la mise en oeuvre des Recommandations sur les marchés publics ».

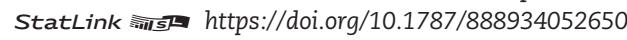


Les systèmes de passation électronique des marchés publics permettent aux administrations publiques non seulement d'améliorer la transparence de la passation des marchés mais aussi de collecter des données cohérentes, actualisées et fiables sur les processus qui s'y rapportent. Ces données peuvent à leur tour alimenter les autres systèmes de technologies de l'information (TI) des administrations publiques via l'échange automatisé de données, et réduire les risques d'erreurs, de fautes et de redondance. En outre, l'intégration à d'autres systèmes numériques publics, comme la facturation électronique, est essentielle pour que les systèmes de passation électronique des marchés soient pleinement fonctionnels à tous les stades du cycle de passation des marchés, y compris pendant les phases d'exécution du marché et de paiement.

La capacité de diffusion des informations augmente avec la prolifération de données et de technologies habilitantes d'où l'augmentation entre 2016 et 2018 de données disponibles relatives aux marchés publics pour le grand public. Cette évolution augmente les possibilités de participation aux processus de passation des marchés publics, les opportunités de recherche bénéfique et de mise en concurrence. Les normes relatives aux données ouvertes, telles que le Standard de données sur la commande publique ouverte (OCDS), contribuent à favoriser l'ouverture de la passation des marchés publics par la publicité, la publication de données et le dialogue avec les différents acteurs, de sorte que les détails du contrat soient traçables et vérifiables, y compris dans le domaine des infrastructures. Toutefois, les administrations publiques doivent trouver le juste équilibre entre l'impératif de responsabilité et de concurrence d'une part et, de l'autre, la protection des secrets commerciaux et le respect de la confidentialité des informations relatives aux prestataires.

Les pays de l'OCDE intègrent de manière croissante leurs systèmes de passation électronique des marchés publics aux autres systèmes informatiques des administrations publiques, tels que les interfaces de budgétisation, les registres du commerciaux fiscaux, les bases de données de sécurité sociale, les systèmes de finances publiques et les progiciels de gestion intégrée (PGI), attestant de la rapidité de la transformation numérique des administrations publiques et de l'intégration des services publics. Si $37 \%$ seulement des pays de l'OCDE ont fait état d'une intégration sous une forme ou sous une autre avec d'autres systèmes informatiques publics dans l'Enquête 2016, ce pourcentage est passé à $72 \%$ dans l'Enquête 2018, les pays ayant de plus en plus tendance à adopter des solutions complètes de passation électronique de marchés publics, couvrant l'ensemble du cycle, depuis la planification et la préparation du marché à l'exécution du contrat et au paiement. En Belgique, au Chili, en France, en Israël, au Portugal et en en Slovénie, le système de passation électronique des marchés publics est relié au progiciel (PGI) de gestion budgétaire et comptable de l'administration centrale. De même, en Autriche, en Estonie, en Grèce, en Lettonie et en République slovaque, le système de passation électronique des marchés publics est rattaché au registre du commerce ; en Estonie et en Lettonie, il est intégré aux registres fiscaux locaux. De nombreux pays ont commencé à connecter les systèmes de passation électronique des marchés aux systèmes de signature et de facturation électronique. Globalement, c'est le système de passation électronique de marchés publics de Corée (KONEPS) qui offre la meilleure connectivité avec des bases de données extérieures, puisqu'il est relié à plus de 200 d'entre elles, dont 65 bases de données d'entités publiques, auxquelles s'ajoutent les interfaces avec des bases de données d'associations professionnelles du secteur privé et de sociétés de notation de crédit ainsi que les systèmes de paiement de banques commerciales. Alors que les systèmes électroniques constituent d'importants vecteurs d'efficience dans le domaine de la passation de marchés publics, force est de constater que seule une minorité de pays de l'OCDE mesurent les gains d'efficience.

\section{Méthodologie et définitions}

Ces données proviennent de l'Enquête 2018 de l'OCDE sur la mise en œuvre des Recommandations 2015 de l'OCDE sur les marchés publics, qui était axée sur les 12 Principes de la recommandation. 31 pays de l'OCDE et un pays candidat à l'adhésion (Costa Rica) ont répondu à l'enquête. Les réponses ont été fournies par des délégués nationaux chargés des politiques de passation des marchés publics au sein de l'administration centrale et de hauts responsables de centrales d'achat publiques. Pour plus de précisions sur l'Enquête 2016 sur les marchés publics, veuillez vous reporter à la section relative aux marchés publics dans l'édition 2017 du Panorama des administrations publiques.

La passation électronique des marchés publics désigne l'intégration de technologies numériques en vue du remplacement ou de la refonte des procédures effectuées sur support papier tout au long du cycle de passation des marchés publics. Le cycle de passation des marchés publics désigne la succession d'activités liées aux marchés publics, depuis l'évaluation des besoins jusqu'au paiement et à la gestion des marchés, en passant par la mise en concurrence et l'attribution du marché, ainsi que toute activité ultérieure de suivi ou d'audit. La disponibilité des documents relatifs aux marchés publics signifie que les documents sont disponibles sans qu'il soit nécessaire d'être enregistré en tant que prestataire.

\section{Pour en savoir plus}

OCDE (2018), Mexico's e-Procurement System : Redesigning CompraNet through Stakeholder Engagement, Examens de l'OCDE sur la gouvernance publique, Éditions OCDE, Paris, https://doi.org/10.1787/9789264287426-en.

OCDE (2016), The Korean Public Procurement Service : Innovating for Effectiveness, Examens de l'OCDE sur la gouvernance publique, Éditions OCDE, Paris, https://doi.org/10.1787/9789264249431-en.

\section{Notes sur les graphiques}

Les données sur les États-Unis, le Luxembourg, la République tchèque et la Suisse ne sont pas disponibles. Concernant les données sur Israël, voir http://doi.org/10.1787/888932315602

8.7. Plusieurs répondants ont souligné la nécessité de protéger les secrets commerciaux et les renseignements commerciaux exclusifs en particulier la teneur du contrat. L'Allemagne a indiqué que les contrats contiennent généralement des informations sensibles que ni les autorités adjudicatrices ni les fournisseurs n'ont le droit de publier. Aux Pays-Bas, le texte des contrats peut être disponible sous une forme adaptée (qui ne mentionne pas, par exemple, la valeur exacte du marché).

8.8. On ne dispose pas de données pour l'Allemagne et l'Irlande. 
8.7. Disponibilité des documents relatifs aux marchés publics, 2016 et 2018

\begin{tabular}{|c|c|c|c|c|c|c|c|c|c|c|}
\hline & \multicolumn{2}{|c|}{ Avis d'appel d'offres } & \multicolumn{2}{|c|}{ Dossier d'appel d'offres } & \multicolumn{2}{|c|}{ Critères d'évaluation } & \multicolumn{2}{|c|}{ Avis d'adjudication } & \multicolumn{2}{|c|}{ Texte du contrat } \\
\hline & 2016 & 2018 & 2016 & 2018 & 2016 & 2018 & 2016 & 2018 & 2016 & 2018 \\
\hline Allemagne & • & $\bullet$ & $\bullet$ & $\bullet$ & $\bullet$ & $\bullet$ & 0 & $\bullet$ & $\bullet$ & 0 \\
\hline Australie & $\bullet$ & $\bullet$ & 0 & O & $\bullet$ & $\bullet$ & $\bullet$ & $\bullet$ & 0 & 0 \\
\hline Autriche & - & $\bullet$ & 0 & $\bullet$ & 0 & - & $\bullet$ & $\bullet$ & 0 & 0 \\
\hline Belgique & $\bullet$ & $\bullet$ & 0 & $\bullet$ & $\bullet$ & $\bullet$ & $\bullet$ & $\bullet$ & O & O \\
\hline Canada & $\bullet$ & $\bullet$ & $\bullet$ & $\bullet$ & $\bullet$ & - & $\bullet$ & $\bullet$ & 0 & 0 \\
\hline Chili & $\bullet$ & $\bullet$ & $\bullet$ & $\bullet$ & $\bullet$ & $\bullet$ & $\bullet$ & - & $\bullet$ & $\bullet$ \\
\hline Corée & - & - & $\bullet$ & $\bullet$ & $\bullet$ & - & $\bullet$ & - & $\bullet$ & $\bullet$ \\
\hline Danemark & • & $\bullet$ & 0 & ○ & • & - & • & - & 0 & 0 \\
\hline Espagne & $\bullet$ & - & $\bullet$ & - & $\bullet$ & - & $\bullet$ & - & 0 & 0 \\
\hline Estonie & • & - & - & - & • & - & - & - & - & - \\
\hline Finlande & - & - & $\bullet$ & - & $\bullet$ & - & 0 & - & - & - \\
\hline France & .. & - & .. & - & .. & - & .. & - & .. & 0 \\
\hline Grèce & - & - & 0 & 0 & - & - & $\bullet$ & - & - & - \\
\hline Hongrie & $\bullet$ & - & 0 & 0 & $\bullet$ & - & $\bullet$ & - & 0 & - \\
\hline Irlande & - & - & 0 & $\bullet$ & - & - & - & - & 0 & 0 \\
\hline Islande & • & - & • & - & • & - & • & - & - & - \\
\hline Israël & • & - & 0 & 0 & - & - & - & - & - & - \\
\hline Italie & • & - & • & $\bullet$ & • & - & $\bullet$ & - & - & - \\
\hline Japon & $\bullet$ & - & $\bullet$ & - & 0 & 0 & $\bullet$ & - & 0 & 0 \\
\hline Lettonie & • & - & - & - & - & - & - & - & - & - \\
\hline Lituanie &.. & - & .. & - & .. & - &.. & - & .. & - \\
\hline Mexique & • & - & $\bullet$ & - & $\bullet$ & - & $\bullet$ & - & 0 & 0 \\
\hline Norvège & - & - & - & $\bullet$ & - & - & - & - & - & - \\
\hline Nouvelle Zélande & • & $\bullet$ & $\bullet$ & $\bullet$ & $\bullet$ & $\bullet$ & $\bullet$ & $\bullet$ & 0 & 0 \\
\hline Pays-Bas & $\bullet$ & $\bullet$ & 0 & 0 & $\bullet$ & - & $\bullet$ & - & 0 & 0 \\
\hline Pologne & - & - & - & $\bullet$ & - & - & $\bullet$ & - & ○ & - \\
\hline Portugal & 0 & $\bullet$ & 0 & $\bullet$ & 0 & - & 0 & 0 & 0 & $\bullet$ \\
\hline République Slovaque & $\bullet$ & $\bullet$ & $\bullet$ & $\bullet$ & $\bullet$ & $\bullet$ & $\bullet$ & $\bullet$ & $\bullet$ & $\bullet$ \\
\hline Royaume-Uni & $\bullet$ & .. & $\bullet$ &.. & $\bullet$ & .. & $\bullet$ &.. & $\bullet$ &.. \\
\hline Slovénie & $\bullet$ & $\bullet$ & 0 & $\bullet$ & $\bullet$ & $\bullet$ & $\bullet$ & $\bullet$ & $\bullet$ & $\bullet$ \\
\hline Suède & $\bullet$ & $\bullet$ & 0 & $\bullet$ & 0 & $\bullet$ & $\bullet$ & $\bullet$ & $\bullet$ & $\bullet$ \\
\hline Turquie & $\bullet$ & $\bullet$ & $\bullet$ & $\bullet$ & $\bullet$ & $\bullet$ & $\bullet$ & $\bullet$ & - & - \\
\hline \multicolumn{11}{|l|}{ Total OCDE } \\
\hline - Oui & 29 & 31 & 18 & 25 & 26 & 30 & 27 & 30 & 17 & 18 \\
\hline Non & 1 & 0 & 12 & 6 & 4 & 1 & 3 & 1 & 13 & 13 \\
\hline Costa Rica & $\bullet$ & $\bullet$ & $\bullet$ & $\bullet$ & $\bullet$ & - & $\bullet$ & - & $\bullet$ & - \\
\hline
\end{tabular}

Sources : OCDE (2016) « Enquête sur les marchés publics », OCDE (2018) «Enquête sur la mise en oeuvre des Recommandations sur les marchés publics ». StatLink 需s https://doi.org/10.1787/888934052669

8.8. Intégration du ou des systèmes de passation électronique des marchés publics avec d'autres systèmes d'administration numérique, 2018

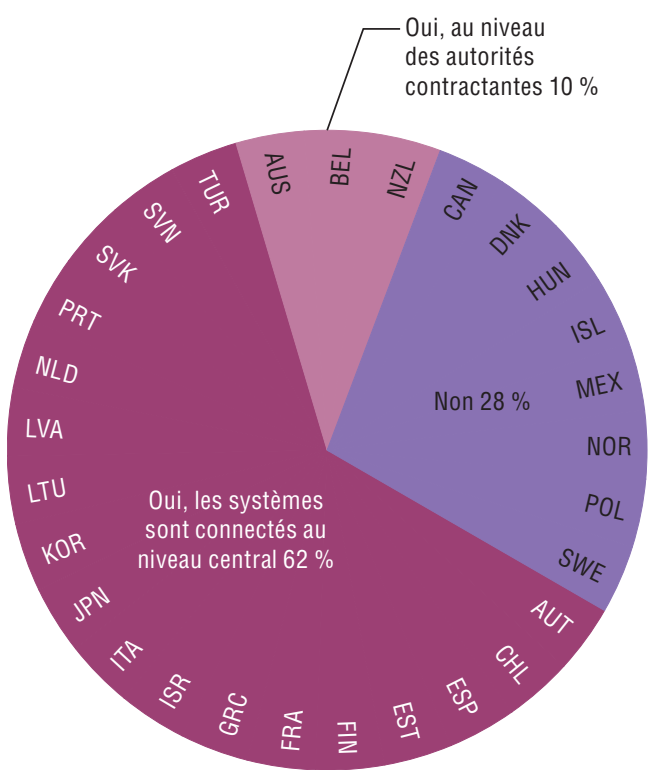

Source: OCDE (2018) « Enquête sur la mise en oeuvre des Recommandations sur les marchés publics".

StatLink त्राजम https://doi.org/10.1787/888934052688
8.9. Mesure des gains d'efficience résultant de l'utilisation de systèmes de passation électronique des marchés publics, 2018

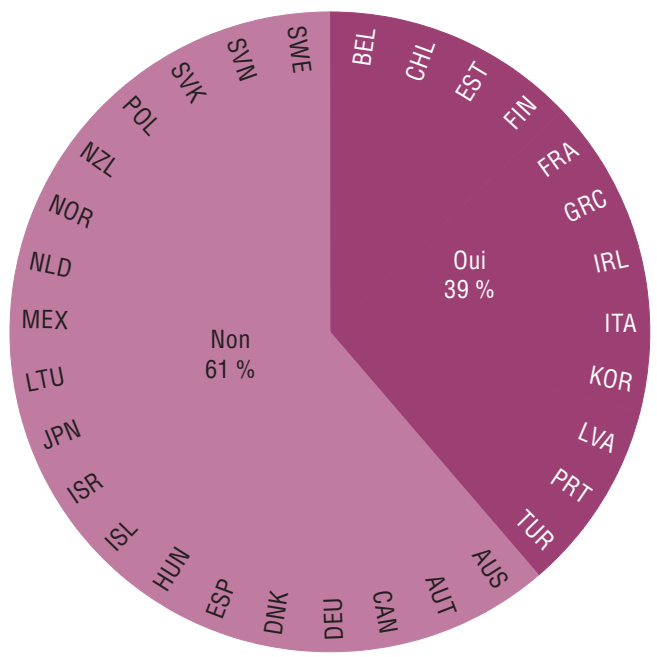

Source: OCDE (2018) «Enquête sur la mise en oeuvre des Recommandations sur les marchés publics ".

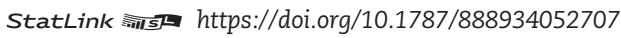


Le principal objectif d'un système de passation des marchés publics est d'assurer une utilisation efficace et efficiente des fonds publics. L'une des principales missions des administrations publiques consiste à veiller à ce que la passation de marchés par les entités publiques soit conforme à ces objectifs. De plus en plus, les pays mesurent l'efficience et le rapport coût-efficacité des marchés publics à l'aide d'indicateurs de performance, à la faveur de la disponibilité croissante des données relatives aux marchés publics. Ces données peuvent contribuer à comparer des autorités adjudicatrices similaires ou à gérer la performance en suivant les évolutions dans le temps et en mesurant les résultats obtenus au regard d'objectifs-cibles prédéfinis. Ces activités structurées de mesure et de gestion de la performance en matière de passation de marchés publics constituent les principaux piliers d'un cadre de gestion de la performance. En outre, lorsque le personnel chargé des marchés publics possède les compétences adéquates, il contribue à la réalisation efficace et efficiente des résultats attendus de la commande publique.

La moitié des pays de l'OCDE indiquent qu'ils analysent les informations et les données relatives à la passation de marchés publics afin de soutenir une action publique stratégique en la matière. Les économies réalisées sur les prix à la suite d'un achat sont l'indicateur de performance le plus couramment mesuré par les autorités adjudicatrices dans les pays de l'OCDE, même si bon nombre d'entre elles mesurent également le coût et le temps consacrés aux processus de passation des marchés publics. Cela étant, $30 \%$ seulement des pays de l'OCDE ont établi des indicateurs clés de performance pour mesurer les résultats des processus de passation de marchés au regard d'objectifs-cibles prédéfinis. De même, seuls $33 \%$ des pays de l'OCDE se sont dotés d'une autorité chargée de gérer le cadre de mesure de la performance, ce qui donne à penser que, dans de nombreux pays de l'OCDE, l'analyse des données et des indicateurs relatifs aux marchés publics relève d'une démarche décentralisée et non systématique.

Les compétences nécessaires aux fonctionnaires chargés des marchés publics varient considérablement entre les pays de l'OCDE, selon les systèmes et niveaux de d'administration et parfois même selon les autorités adjudicatrices. Un système de certification adapté aux compétences pertinentes peut permettre de dispenser des formations régulières et ciblées aux agents publics concernés et améliorer ainsi l'efficacité et la réalisation des objectifs stratégiques de la commande publique. Il doit être étroitement lié aux cadres de compétences qui établissent la liste des compétences essentielles en fonction de l'orientation stratégique générale d'une organisation. C'est pourquoi dans de nombreux pays de l'OCDE, les critères de recrutement dépendent en grande partie des besoins de l'autorité adjudicatrice. Peu nombreux sont les pays de l'OCDE qui se sont dotés de processus de certification ( $21 \%$ ) et de modèles de compétences afin d'établir les critères de recrutement (30\%) des fonctionnaires chargés des marchés publics, les deux dispositifs étant utilisés pour planifier et concevoir la formation des agents publics en question. Au Chili, la centrale d'achats délivre des certificats aux agents publics ayant accompli une formation spécifique. En France, le ministère des Finances a mis au point un modèle complet de compétences assorti des modules de formation correspondants, et supervise l'offre de formation que chaque ministère propose à ses propres agents chargés des marchés publics.

Les catalogues électroniques sont couramment utilisés dans un souci d'efficience pour les achats de faible montant se prêtant à des processus de commande simplifiés ou à une soustraitance directe. Quelques $52 \%$ des pays de l'OCDE déclarent que leurs systèmes de passation électronique des marchés publics comprennent des catalogues électroniques. En Italie Consip - la principale centrale d'achats publique - utilise un catalogue en ligne sophistiqué, le MePA (marché électronique pour l'administration publique), qui a géré 640000 transactions de faible montant en 2017. Autre module d'efficacité répandu : les enchères inversées en ligne qui sont intégrées aux systèmes de passation électronique des marchés publics de $58 \%$ des pays de l'OCDE.

\section{Méthodologie et définitions}

Ces données proviennent de l'Enquête 2018 de l'OCDE sur la mise en œuvre des Recommandations 2015 de l'OCDE sur les marchés publics, qui était axée sur les 12 Principes de la recommandation. 31 pays de l'OCDE et un pays candidat à l'adhésion (Costa Rica) ont participé à l'enquête. Les réponses ont été fournies par des délégués nationaux chargés des politiques de passation des marchés publics au sein de l'administration centrale et de hauts responsables de centrales d'achat publiques.

Les catalogues électroniques présentent les produits et services disponibles à la vente sous format électronique et souvent accompagnés d'illustrations, de la mention du prix et d'une description. L'enchère (électronique) inversée est une méthode qui permet aux opérateurs économiques de soumettre en ligne des prix révisés à la baisse en temps réel. Les économies réalisées sur le prix correspondent généralement à l'écart qui existe entre le prix obtenu au terme du processus d'achat et le prix de référence (par exemple, le prix moyen des offres, le budget maximal alloué, etc.).

\section{Pour en savoir plus}

OCDE (à paraître), Report on the Implementation of the 2015 Recommendation of the Council on Public Procurement, Éditions OCDE, Paris

OCDE (2019), Productivity in Public Procurement: A Case Study of Finland: Measuring the Efficiency and Effectiveness of Public Procurement, http://www.oecd.org/gov/public-procurement/ publications/productivity-public-procurement.pdf.

\section{Notes sur les graphiques}

Les données pour les États-Unis, le Luxembourg, la République tchèque, le Royaume-Uni et la Suisse ne sont pas disponibles. Concernant les données sur Israël, voir http://doi.org/10.1787/888932315602.

8.10. On ne dispose pas de données pour l'Allemagne.

8.11. On ne dispose pas de données pour l'Irlande et l'Italie.. En Norvège, il existe une certification de base des fournisseurs, mais elle est peu utilisée.

8.12. Ce graphique comprend le Japon et l'Italie, où des catalogues en ligne existent dans le système national de passation électronique des marchés publics, mais aussi dans les systèmes propres à certaines autorités adjudicatrices. 
8.10. Cadres de gestion de la performance en matière de passation de marchés publics, 2018

\begin{tabular}{|c|c|c|c|c|}
\hline & $\begin{array}{l}\text { Un système de mesure de la } \\
\text { performance est en place }\end{array}$ & $\begin{array}{l}\text { Un système de mesure de la } \\
\text { performance est axé sur les résultats } \\
\text { des processus de passation des } \\
\text { marchés publics plutôt que sur des } \\
\text { objectifs fixés }\end{array}$ & $\begin{array}{l}\text { L'information est utilisée à l'appui d'une } \\
\text { action publique stratégique en matière } \\
\text { de passation de marchés publics }\end{array}$ & $\begin{array}{l}\text { Une autorité est explicitement } \\
\text { chargée de la gestion des cadres de } \\
\text { performance }\end{array}$ \\
\hline Australie & 0 & 0 & 0 & 0 \\
\hline Autriche & O & 0 & 0 & 0 \\
\hline Belgique & $\bullet$ & - & $\bullet$ & - \\
\hline Canada & $\bullet$ & $\bullet$ & $\bullet$ & $\bullet$ \\
\hline Chili & - & 0 & $\bullet$ & $\bullet$ \\
\hline Corée & - & $\bullet$ & $\bullet$ & 0 \\
\hline Danemark & 0 & 0 & 0 & 0 \\
\hline Espagne & 0 & 0 & 0 & 0 \\
\hline Estonie & 0 & 0 & 0 & 0 \\
\hline Finlande & 0 & 0 & 0 & 0 \\
\hline France & - & $\bullet$ & $\bullet$ & - \\
\hline Grèce & 0 & 0 & $\bullet$ & 0 \\
\hline Hongrie & - & 0 & 0 & 0 \\
\hline Irlande & O & 0 & $\bullet$ & 0 \\
\hline Islande & 0 & 0 & 0 & 0 \\
\hline Israël & 0 & 0 & 0 & 0 \\
\hline Italie & $\bullet$ & - & $\bullet$ & $\bullet$ \\
\hline Japon & O & $\bullet$ & $\bullet$ & 0 \\
\hline Lettonie & - & - & $\bullet$ & $\bullet$ \\
\hline Lituanie & $\bullet$ & 0 & $\bullet$ & $\bullet$ \\
\hline Mexique & 0 & 0 & $\bullet$ & 0 \\
\hline Norvège & 0 & 0 & 0 & 0 \\
\hline Nouvelle Zélande & - & - & $\bullet$ & - \\
\hline Pays-Bas & $\bullet$ & $\bullet$ & $\bullet$ & 0 \\
\hline Pologne & 0 & 0 & $\bullet$ & 0 \\
\hline Portugal & - & 0 & 0 & - \\
\hline République Slovaque & - & $\bullet$ & $\bullet$ & 0 \\
\hline Slovénie & O & 0 & O & O \\
\hline Suède & 0 & 0 & $\bullet$ & $\bullet$ \\
\hline Turquie & $\bullet$ & 0 & $\bullet$ & $\bullet$ \\
\hline \multicolumn{5}{|l|}{ Total OCDE } \\
\hline Oui $\bullet$ & 13 & 9 & 17 & 10 \\
\hline Non $O$ & 17 & 21 & 13 & 20 \\
\hline Costa Rica & $\bullet$ & 0 & 0 & O \\
\hline
\end{tabular}

Source : OCDE (2018) « Enquête sur la mise en oeuvre des Recommandations sur les marchés publics ».

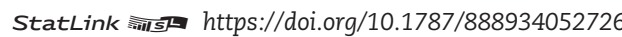

8.11 Processus de certification des fonctionnaires chargés de la passation des marchés publics, 2018

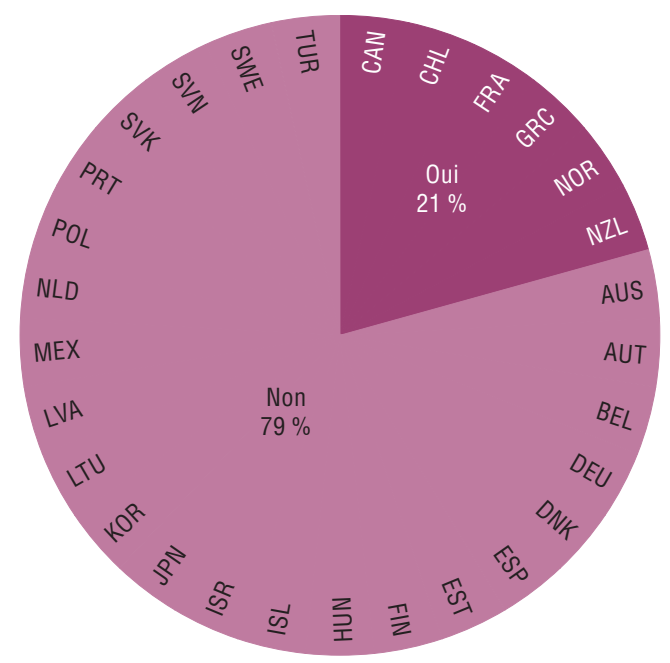

Source : OCDE (2018), Enquête sur la mise en œuure des recommandations sur les marchés publics.

StatLink 게내 https://doi.org/10.1787/888934052745
8.12. Existence de catalogues en ligne en matière de passation des marchés publics, 2018

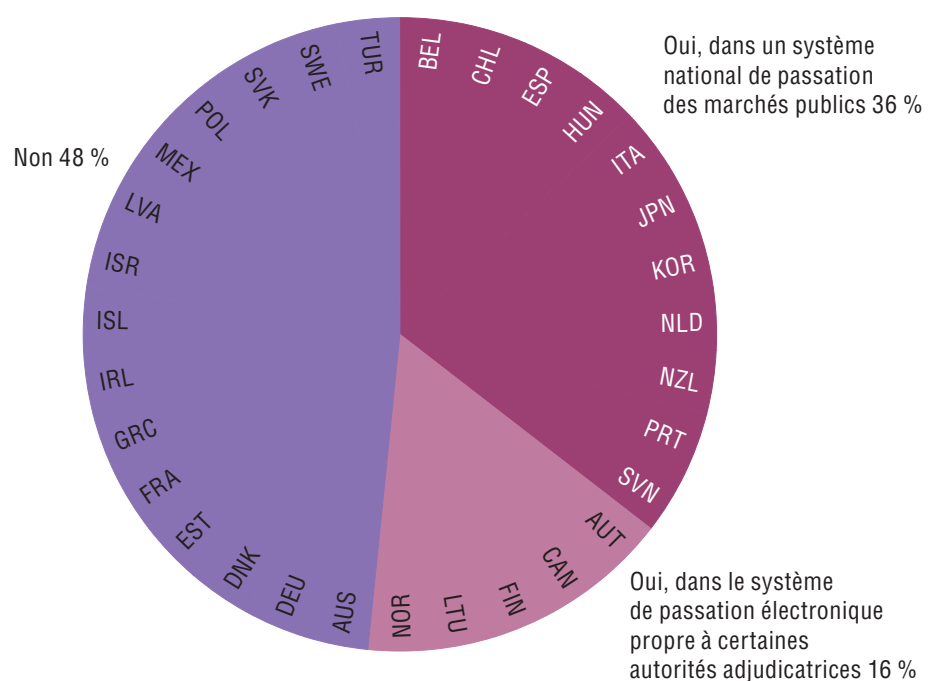

Source : OCDE (2018), Enquête sur la mise en œuvre des recommandations sur les marchés publics.

StatLink 제내 https://doi.org/10.1787/888934052764 
Les marchés publics sont un secteur à haut risque. Le Rapport de l'OCDE sur la corruption transnationale a établi que près des deux-tiers des pots-de-vin versés dans le cadre de transactions internationales concernent des marchés publics. Les pouvoirs publics s'attachent à faire de l'intégrité publique une réponse durable et stratégique face à la corruption. Préserver l'intérêt général est la mission fondamentale des administrations et institutions publiques. Elles doivent s'assurer que les agents publics ne laissent pas leurs liens personnels et leurs intérêts privés compromettre leurs fonctions officielles. Outre les risques de manquement à l'intégrité, bien d'autres risques existent tout au long du cycle de passation des marchés publics, notamment: risque de gaspillage ou d'inefficience à tous les stades du processus de passation de marché (planification, appel d'offres, exécution du contrat, paiement) ; risques liés aux défaillances de l'infrastructure des technologies de l'information (TI), y compris des systèmes de passation électronique des marchés publics et des bases de données ou registres électroniques qui s'y rapportent; risques financiers et risques ou préjudices potentiels pour la réputation et l'image de l'autorité adjudicatrice.

La gestion des risques comprend plusieurs étapes axées sur l'évaluation (de la nature, des causes et des conséquences potentielles des risques) et sur l'atténuation. Seuls $52 \%$ des pays de l'OCDE ont déclaré avoir élaboré une stratégie d'évaluation, de prévention et d'atténuation des risques liés à la passation de marchés publics, mais il arrive également que des politiques de contrôle et d'évaluation du risque à l'échelle de l'ensemble de l'administration publique s'appliquent. Les risques liés à la passation de marchés publics doivent être gérés à un stade précoce, a fortiori lorsque les marchés portent sur des projets complexes et de grande ampleur, comme des infrastructures publiques. Les lois et réglementations régissant les marchés publics s'appliquent au moins en partie aux projets d'infrastructures publiques dans tous les pays de l'OCDE, 61 \% d'entre eux les appliquant à tous les projets d'infrastructures. En complément à l'application des principes et des cadres régissant la passation de marchés publics, des outils sur mesure peuvent aider à identifier et à atténuer les risques souvent liés aux grands projets d'infrastructure tels que les risques d'inefficience, les problèmes de qualité, le dépassement des coûts et la corruption.

Les pays accordent plus d'attention à la mise en place de systèmes et d'instruments destinés à atténuer les risques qui pèsent sur l'intégrité. L'un des principaux risques en la matière dans le contexte de la passation de marchés publics tient aux conflits d'intérêts. Selon les données les plus récentes, dans $90 \%$ des pays de l'OCDE, le cadre réglementaire contient une définition des conflits d'intérêts des responsables des marchés publics. Toutefois, la déclaration de conflits d'intérêts éventuels n'est pas universellement appliquée dans tous les pays de l'OCDE. Les résultats de l'Enquête 2015 sur les recommandations sur les marchés publics montrent que les déclarations indiquant s'il y avait ou non conflit d'intérêts lors d'une procédure de passation de marchés publics étaient réalisées dans $81 \%$ des pays de l'OCDE. Dans leur grande majorité, les pays de l'OCDE s'emploient à assurer l'intégrité dans les marchés publics en radiant les fournisseurs coupables de corruption ou d'autres manquements aux règles d'intégrité. Certains pays comme le Canada se sont dotés de codes de conduite qui récapitulent les règles et politiques applicables en un exposé concis et transparent des attentes de l'administration publique à l'égard de ses agents et de ses fournisseurs. Dans un-tiers environ des pays de l'OCDE, les fournisseurs s'engagent à éviter tout type de corruption lorsqu'ils présentent une offre (" garantie de non-corruption » dans le cadre des documents relatifs aux offres).

\section{Méthodologie et définitions}

Ces données proviennent de l'Enquête 2018 de l'OCDE sur la mise en œuvre des Recommandations 2015 de l'OCDE sur les marchés publics. 31 pays de l'OCDE et un pays candidat à l'adhésion (Costa Rica) ont participé à l'enquête. Les réponses ont été fournies par des délégués nationaux chargés des politiques de passation des marchés publics au sein de l'administration centrale et de hauts responsables de centrales d'achat publiques.

Un conflit d'intérêts désigne un conflit entre la mission publique et les intérêts privés d'un agent public, dans lequel celui-ci possède à titre privé des intérêts qui pourraient influer indûment sur la façon dont il s'acquitte de ses obligations et de ses responsabilités.

Les infrastructures publiques désignent les installations, structures, réseaux, systèmes, usines, biens immobiliers, équipements ou actifs physiques - ainsi que les entreprises qui les utilisent - destinés à fournir des biens publics ou des biens qui répondent à un besoin fondamental dont la responsabilité est confiée à la puissance publique et que le marché ne saurait satisfaire seul.

\section{Pour en savoir plus}

OCDE (2018), Third Progress Report on the Development of the New International Airport of Mexico: Achievements and lessons learned, http://www.oecd.org/centrodemexico/medios/Full\%20 report\%20EN.pdf.

OCDE (2016), Preventing Corruption in Public Procurement, http:// www.oecd.org/gov/ethics/Corruption-Public-ProcurementBrochure.pdf.

OCDE (2015), Recommandation du Conseil sur les marchés publics, https://www.oecd.org/fr/gov/commande-publique/ Recommandation-OCDE-sur-les-marches-publics.pdf.

\section{Notes sur les graphiques}

Les données pour les États-Unis, le Luxembourg, la République tchèque, le Royaume-Uni et la Suisse ne sont pas disponibles. Concernant les données sur Israël, voir http://doi.org/10.1787/888932315602.

8.15. On ne dispose pas de données pour la Finlande et les Pays-Bas. La plupart des répondants ont compris l'expression "risques liés aux marchés publics " au sens de risque de fraude, de corruption et autres manquements aux règles d'intégrité. 
8.13. Mécanismes de prévention et de gestion des conflits d'intérêts chez les fonctionnaires chargés des marchés publics, 2018

\begin{tabular}{|c|c|c|c|c|}
\hline & $\begin{array}{c}\text { Le cadre réglementaire comprend une } \\
\text { définition du conflit d'intérêt pour les } \\
\text { fonctionnaires chargés des marchés } \\
\text { publics }\end{array}$ & $\begin{array}{l}\text { Les fonctionnaires chargés des } \\
\text { marchés publics doivent déclarer leurs } \\
\text { intérêts privés }\end{array}$ & $\begin{array}{l}\text { Les fonctionnaires chargés des } \\
\text { marchés publics doivent déclarer } \\
\text { "l'absence de conflit d'intérêts " ou } \\
\text { notifier l'autorité compétente en cas de } \\
\text { conflit d'intérêts potentiel }\end{array}$ & $\begin{array}{l}\text { Des limitations s'appliquent à certains } \\
\text { fonctionnaires et élus politiques quant à } \\
\text { leur participation aux marchés publics }\end{array}$ \\
\hline Allemagne & 0 & 0 & 0 & O \\
\hline Australie & O & 0 & 0 & O \\
\hline Autriche & O & 0 & 0 & 0 \\
\hline Belgique & O & $\bigcirc$ & $\bigcirc$ & ○ \\
\hline Canada & O & 0 & 0 & ○ \\
\hline Chili & O & $\bigcirc$ & O & ○ \\
\hline \multicolumn{5}{|l|}{ Corée } \\
\hline Danemark & 0 & O & 0 & $\bigcirc$ \\
\hline Espagne & O & 0 & 0 & 0 \\
\hline Estonie & O & $\bigcirc$ & 0 & $\bigcirc$ \\
\hline Finlande & O & 0 & 0 & 0 \\
\hline \multicolumn{5}{|l|}{ France } \\
\hline \multicolumn{5}{|l|}{ Grèce } \\
\hline \multicolumn{5}{|l|}{ Hongrie } \\
\hline \multicolumn{5}{|l|}{ Irlande } \\
\hline Islande & $\bigcirc$ & $\bigcirc$ & $\bigcirc$ & $\bigcirc$ \\
\hline Israël & O & 0 & 0 & 0 \\
\hline \multicolumn{5}{|l|}{ Italie } \\
\hline Japon & 0 & 0 & 0 & 0 \\
\hline \multicolumn{5}{|l|}{ Lettonie } \\
\hline \multicolumn{5}{|l|}{ Lituanie } \\
\hline Mexique & O & 0 & $\bigcirc$ & $\bigcirc$ \\
\hline Norvège & O & 0 & 0 & 0 \\
\hline Nouvelle Zélande & O & 0 & 0 & O \\
\hline \multicolumn{5}{|l|}{ Pays-Bas } \\
\hline \multicolumn{5}{|l|}{ Pologne } \\
\hline \multicolumn{5}{|l|}{ Portugal } \\
\hline \multicolumn{5}{|l|}{ République Slovaque } \\
\hline \multicolumn{5}{|l|}{ Slovénie } \\
\hline Suède & ○ & 0 & ค & $\bigcirc$ \\
\hline Turquie & 0 & 0 & 0 & 0 \\
\hline \multicolumn{5}{|l|}{ Total OCDE } \\
\hline Oui & 28 & 18 & 25 & 18 \\
\hline Non $\bigcirc$ & 3 & 13 & 6 & 13 \\
\hline Costa Rica & 0 & 0 & 0 & 0 \\
\hline
\end{tabular}

Source : OCDE (2018) « Enquête sur la mise en oeuvre des Recommandations sur les marchés publics ».

\subsection{Application aux projets d'infrastructures des lois et réglementations applicables aux marchés publics, 2018}

Oui, partiellement applicables. Des lois et règlements spécifiques (PPP, concessions, etc.) existent pour certains ou pour tous les projets d'infrastructures $39 \%$

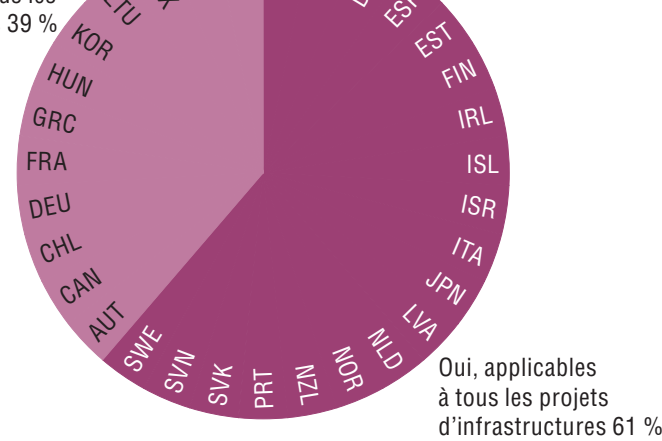

Source : OCDE (2018), Enquête sur la mise en ceuvre des recommandations sur les marchés publics.

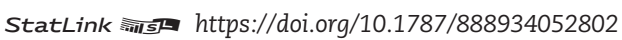

8.15. Existence d'une stratégie d'évaluation, de prévention et d'atténuation des risques liés aux marchés publics, 2018

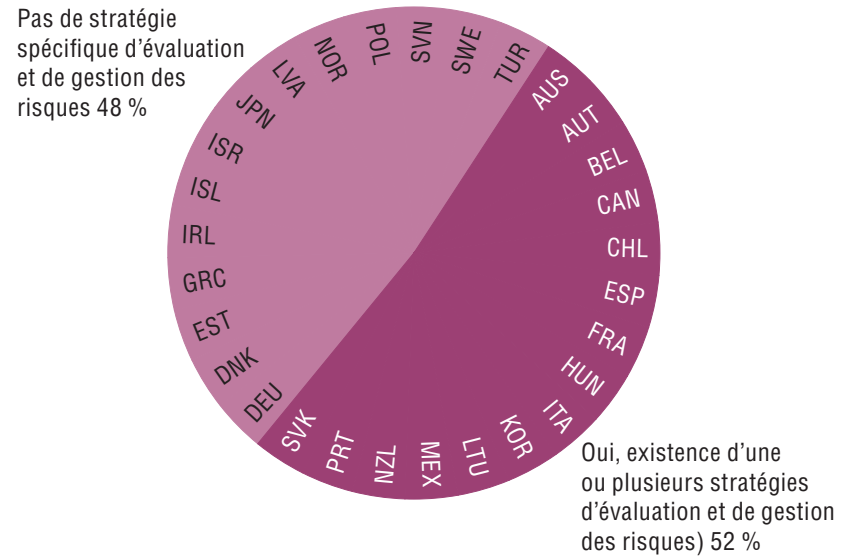

Source : OCDE (2018), Enquête sur la mise en œuvre des recommandations sur les marchés publics.

StatLink त्नाड़ https://doi.org/10.1787/888934052821 


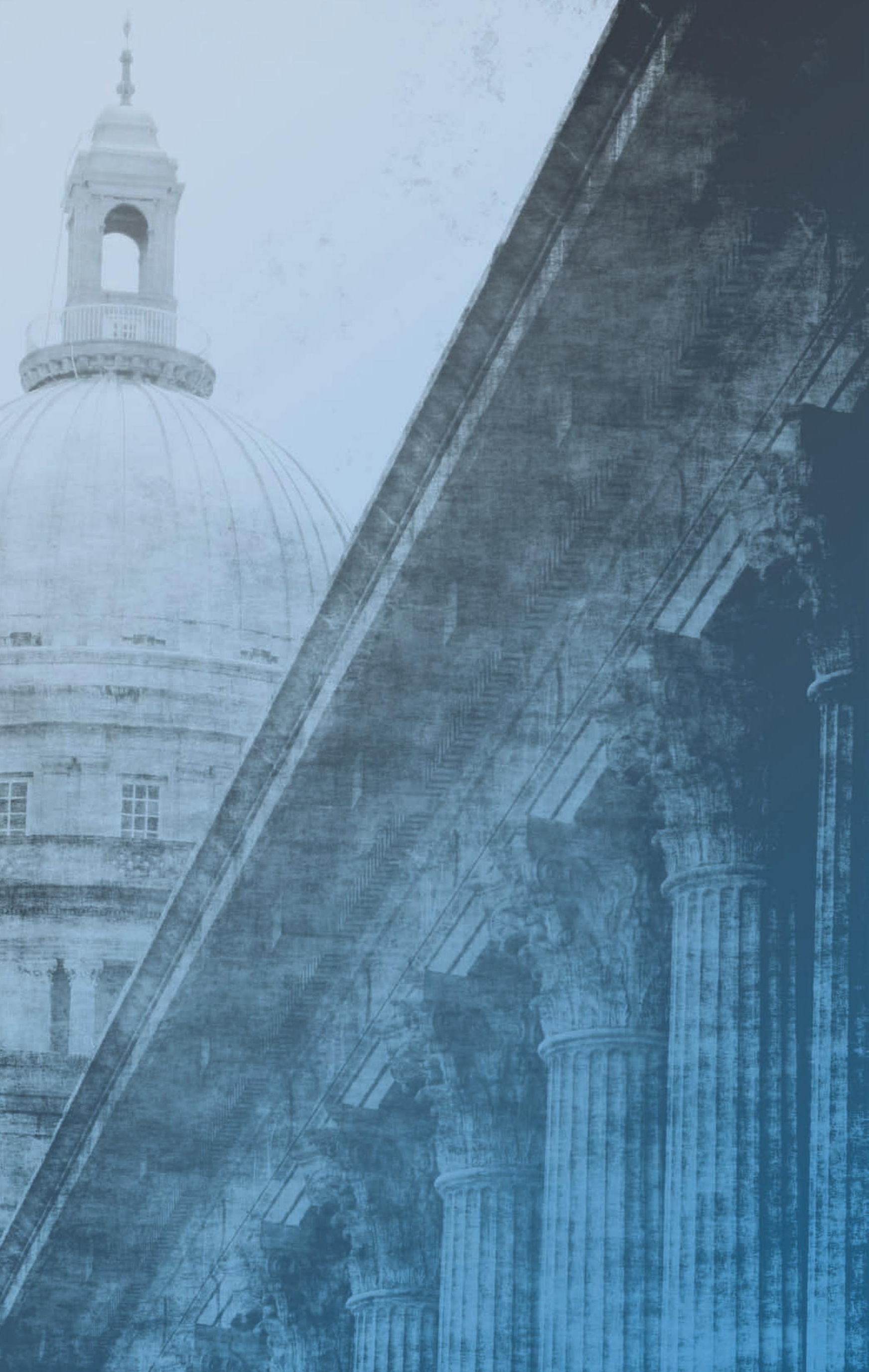




\section{GOUVERNEMENT NUMÉRIQUE} ET DONNÉES PUBLIQUES OUVERTES

L’administration numérique

Données publiques ouvertes : clés de la maturité et de la viabilité

Disponibilité des données : cadres d'action, association des parties prenantes et diffusion des données

Accessibilité des données : des formats ouverts, gratuits et accessibles

Associer les usagers : encourager l'apprentissage et la réutilisation des données ouvertes 
Ces dernières décennies, les pays ont lancé de vastes réformes du secteur public mettant l'accent sur le numérique en vue de gagner en efficience et en efficacité dans les services publics. Dans le cadre de ces réformes, ils ont consacré des ressources considérables à l'adoption de nouvelles pratiques visant à moderniser les services publics et à faire en sorte qu'ils répondent mieux aux besoins des administrés. Ils ont donc mis en place des plateformes en ligne communes à plusieurs entités publiques afin de simplifier les procédures administratives et d'améliorer les interactions avec les administrés.

Bien que des progrès aient déjà été accomplis, un passage complet au numérique augmenterait la cohérence et la coordination des décisions et des activités, au sein de chaque entité publique ainsi qu'entre entités publiques. Il s'agirait de passer de l'administration électronique (systèmes de paiement en ligne de l'impôt, par exemple) à l'administration numérique, notion dans le cadre de laquelle l'utilisation des technologies numériques fait partie intégrante des stratégies de modernisation visant à créer de la valeur publique. L'administration numérique s'appuie sur un écosystème numérique composé d'acteurs publics, d'organisations non gouvernementales, d'entreprises, d'associations de citoyens et d'individus qui favorise la production et l'accessibilité des données, services et contenus au moyen d'interactions avec les administrations (par exemple, les plateformes de données ouvertes communes à plusieurs entités publiques).

La Recommandation du Conseil de l'OCDE sur les stratégies numériques gouvernementales, qui a été adoptée le 15 juillet 2014, offre une base de travail solide pour les pays qui souhaitent mettre en place des dispositifs de gouvernance assurant d'une part un bon leadership et une bonne coordination et favorisant d'autre part l'adoption des décisions à l'échelle du système dans son ensemble.

Selon l'enquête 2019 de l'OCDE sur l'administration numérique, 30 pays de l'OCDE ont confié à une ou plusieurs entités la mission de piloter et de coordonner à l'échelon central ou fédéral les stratégies d'administration numérique. Dans $44 \%$ des pays interrogés, l'entité ou le service chargé de la stratégie numérique appartient au centre de gouvernement ; dans $33 \%$ des pays, c'est le ministère chargé de la coordination qui se charge de cette mission; dans les $23 \%$ de pays restants, c'est un ministère sectoriel qui est responsable. La gestion de ces entités ou services est confiée à un haut fonctionnaire qui porte souvent le titre de directeur de l'information.

L'entité chargée de l'administration numérique peut être dotée d'attributions consultatives (par exemple, coordonner l'élaboration de la stratégie nationale d'administration numérique et suivre sa mise en œuvre) comme d'attributions décisionnelles (par exemple, prioriser les projets basés sur l'usage des technologies de l'information et de la communication (TIC) dans lesquels l'administration investira en priorité et soutenir financièrement leurs développement et mise en œuvre). En moyenne, dans les pays de l'OCDE, ces entités concentrent six des sept attributions consultatives et trois des cinq attributions décisionnelles évoquées dans l'enquête. C'est en Corée, en Hongrie, en Islande, en Israël, au Luxembourg, en République tchèque et en Colombie que ces entités ont les responsabilités les plus larges. À l'inverse, en Belgique, au Mexique et au Costa Rica, elles n'ont qu'un rôle consultatif. Pour sa seule part, le Mexique n'a confié que trois responsabilités à de telles entités.
À l'appui de la mise en œuvre de la stratégie d'administration numérique, trois grands leviers d'action publique ont été identifiés. Ces outils sont importants pour réussir le montage financier et budgétaire et la mise en œuvre des projets numériques : une méthodologie en matière d'études d'opportunité ; un modèle en matière de gestion des projets numériques ; et une stratégie d'achats de technologies numériques à l'échelle de l'administration tout entière. Seuls 11 des 30 pays membres de l'OCDE et un pays candidat à l'adhésion (la Colombie), ont mis en place ces trois leviers d'action dans le cadre de leur stratégie. 21 pays de l'OCDE et 2 pays partenaires (Brésil et Colombie) ont adopté un modèle normalisé de gestion des projets numériques. De plus, 21 pays de l'OCDE ont mis en place des études d'opportunité (par exemple, réalisation d'analyses coût-avantages ou coûtefficacité) ; et 23 ont adopté une stratégie spécifique pour leurs achats de technologies numériques, tandis que 10 indiquent être dotés d'une stratégie d'achats commune à l'ensemble de l'administration.

\section{Méthodologie et définitions}

Les données présentées sous cette rubrique sont issues de l'enquête 2019 de l'OCDE sur l'administration numérique. Cette enquête avait pour objet de suivre la mise en œuvre de la Recommandation sur les stratégies numériques gouvernementales et d'évaluer à cette fin à quel stade les pays en étaient de leur transition entre administration électronique et administration numérique. Le questionnaire d'enquête a été rempli par 30 pays de l'OCDE, 2 pays candidats à l'adhésion (Colombie et Costa Rica) et 1 pays non-membre (Brésil). Les réponses ont été fournies, pour l'essentiel, par de hauts fonctionnaires chargés, au sein de l'administration centrale ou fédérale, du dossier de la transformation numérique du secteur public.

Les leviers d'action publique sont des outils qui peuvent être utilisés par les pouvoirs publics comme moyens d'action dans des secteurs spécifiques afin de produire des changements systémiques.

\section{Pour en savoir plus}

OCDE (à paraître), “Governance of Digital Government”, Digital Government in Peru: Working Closely with Citizens, Éditions OCDE, Paris.

OCDE (à paraître), The Digital Transformation of the Public Sector: Helping Governments Respond to the Needs of Networked Societies, Éditions OCDE, Paris.

OCDE (2014), Recommandation du Conseil sur les stratégies numériques gouvernementales, Éditions OCDE, Paris.

\section{Notes relatives aux graphiques}

Les données concernant l'Australie, les États-Unis, la Pologne, la République slovaque, la Suisse et la Turquie ne sont pas disponibles. Concernant les données sur Israël, voir http://doi. org/10.1787/888932315602 
9.1. Rattachement de l'organe chargé de la stratégie en matière d'administration numérique, 2019

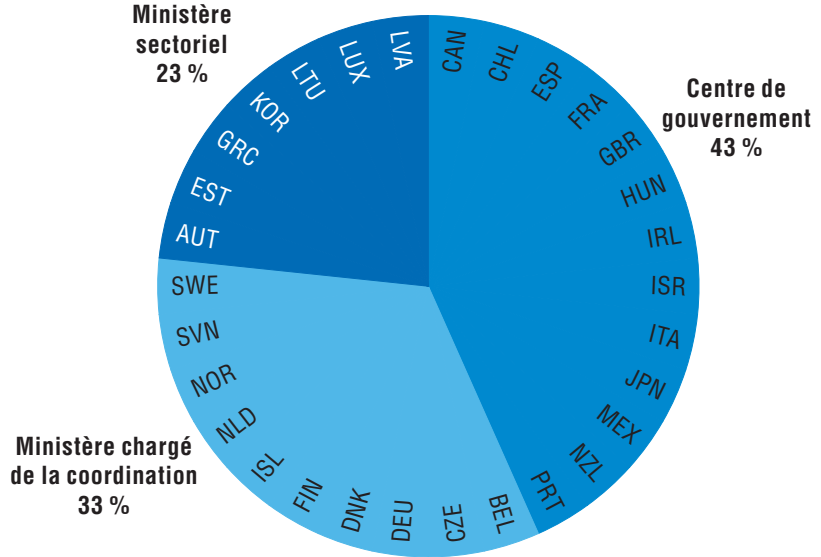

Source : OCDE (2019), Enquête de l'OCDE sur l'administration numérique.

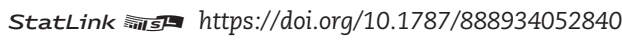

\subsection{Attributions de l'organe chargé de la stratégie d'administration numérique, 2019}

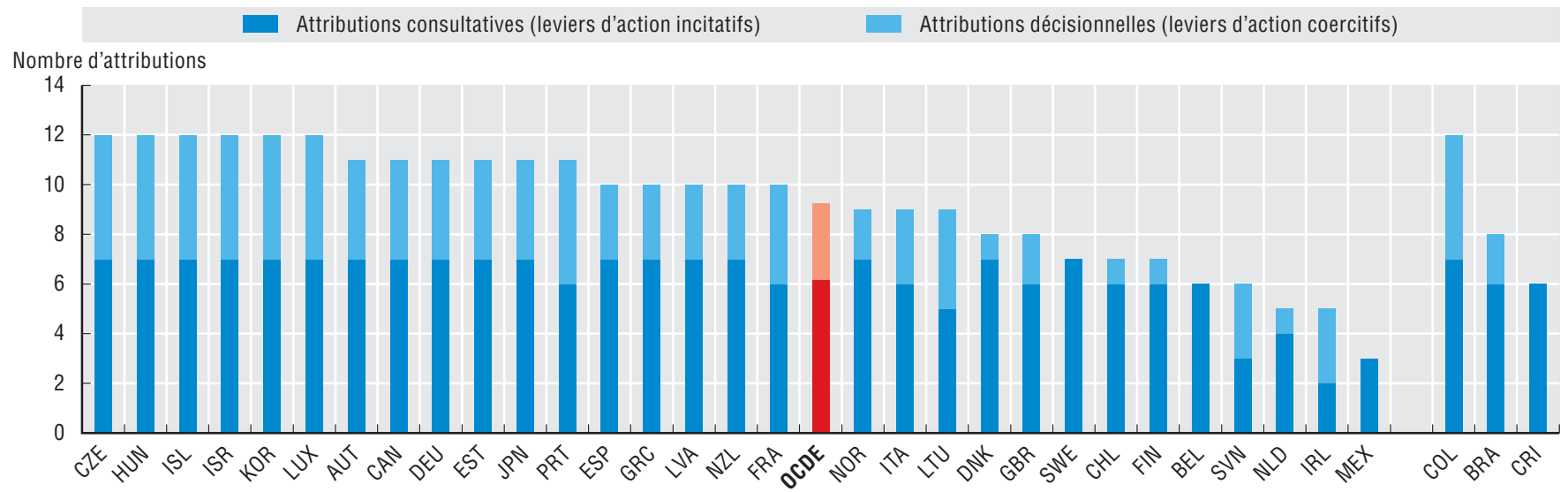

Source : OCDE (2019), Enquête de l'OCDE sur l'administration numérique.

9.3. Recours à des leviers d'action publique normalisés à l'échelon central ou fédéral, 2019

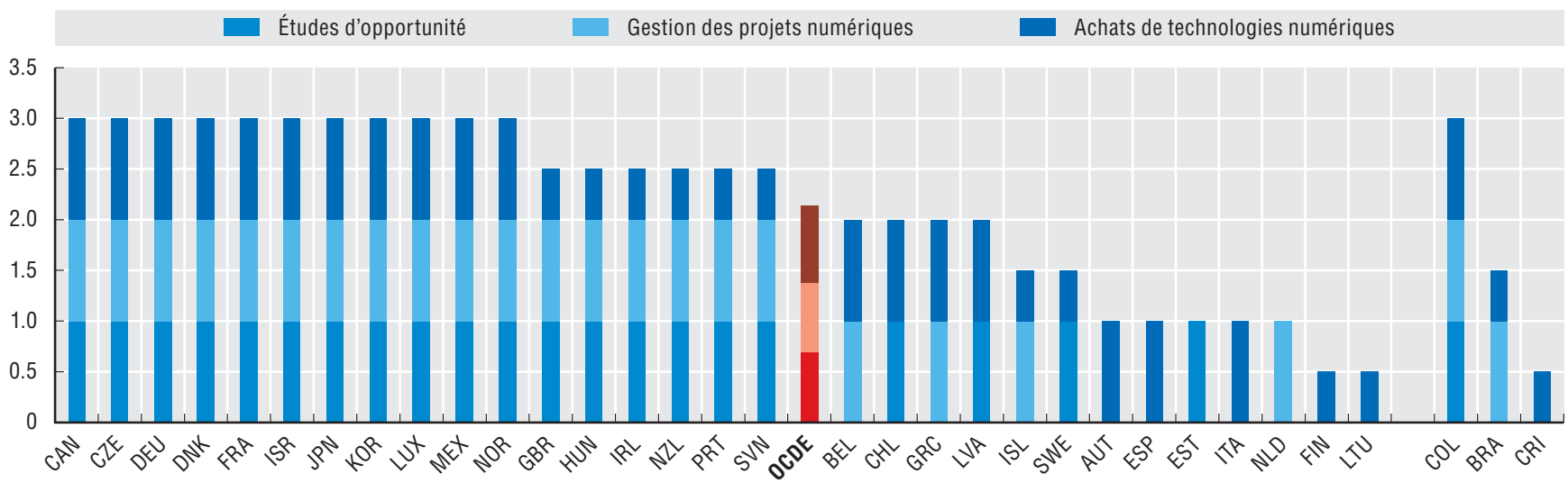

Source : OCDE (2019), Enquête de l'OCDE sur l'administration numérique. 
De nos jours, les données constituent l'une des ressources les plus précieuses pour les sociétés, les économies et les pouvoirs publics. Les politiques d'ouverture des données publiques - on parle aussi de "données publiques ouvertes " ou DPO - se fondent sur des idées et principes qui visent à rendre accessibles à tous les données détenues par les administrations publiques et ce dans des formats ouverts, gratuits et accessibles. Les pratiques des pays de l'OCDE montrent que l'accès aux données publiques peut encourager la participation sociale, créer de nouveaux créneaux pour les entreprises et stimuler l'innovation, de la valeur ajoutée étant ainsi créée à partir de l'information existante.

L'indice OURdata (données publiques ouvertes, utiles et réutilisables) a pour objet d'évaluer et de comparer les politiques d'ouverture des données publiques et leur mise en œuvre. Sa valeur s'échelonne entre 0 et 1, 0 étant le score le plus bas et 1 le meilleur. L'indice se compose de trois indicateurs d'un poids égal : la disponibilité des données, l'accessibilité des données et l'action officielle en faveur de la réutilisation des données. Chacun de ces indicateurs a donc une valeur comprise entre 0 et 0,33. Pour 2019, les valeurs de l'indice OURdata révèlent une maturité croissante générale du processus d'ouverture des données publiques. Sur l'ensemble de la zone OCDE, la valeur moyenne de l'indice est passée de 0,53 en 2017 à 0,60 en 2019, sous l'effet de l'amélioration des trois indicateurs : la disponibilité est passée de 0,18 en 2017 à 0,20 en 2019 ; l'accessibilité, de 0,21 à 0,23 ; et l'action officielle, de 0,15 à 0,17.

Certains pays antérieurement peu performants commencent à rattraper les pays chefs de file que sont la Corée (score global 0,93), la France $(0,90)$ et le Japon $(0,75)$. L'Irlande (avec sa Stratégie nationale d'ouverture des données), la Pologne (avec son Programme d'ouverture des données publiques) et la Slovénie (avec sa Stratégie de développement de l'administration publique) offrent des exemples de stratégies ayant permis d'importants progrès. D'autres pays ont poursuivi leurs progrès depuis 2017, notamment les Pays-Bas et le Canada, obtenant respectivement 0,65 et 0,73 en 2019. En revanche, la Finlande et le RoyaumeUni, qui se situaient en tête du classement en 2017, ont vu leur notation se dégrader sous l'effet de changements des priorités politiques, de réattributions des responsabilités et de baisse du niveau global de soutien politique.

Un renforcement des cadres d'action et une meilleure compréhension de l'importance de l'association des parties prenantes ont conduit à un renforcement de la disponibilité des données dans la plupart des pays de l'OCDE. L'Australie se distingue, puisque la valeur de l'indicateur est passée de 0,14 en 2017 à 0,24 en 2019, essentiellement grâce à un plus grand niveau d'implication des parties prenantes dans la diffusion des données. Si, en général, le nombre de séries de données disponibles n'a que modestement progressé, certains pays ont axé leur action sur une démarche de " publication dans un but précis ", qui consiste à publier les séries de données publiques présentant le plus grand intérêt pour les citoyens et les entreprises (par exemple, registres du commerce, données météorologiques, données sur le budget de l'État). Tel a notamment été le cas de l'Australie, de la République tchèque et de la Slovénie.

L'accessibilité des données est élevée dans toute la zone OCDE. Comme en 2017, le haut du classement est occupé par l'Autriche $(0,32)$, la Colombie $(0,32)$ et la France $(0,31)$. À l'heure actuelle, 30 pays de l'OCDE sur 33 imposent la gratuité des données publiques ; 29 exigent que les données soient disponibles sous licence ouverte; et 31 que les données soient fournies sous une forme lisible sur machine. Les progrès réalisés au niveau des portails de données publiques ouvertes (DPO) ont également contribué au renforcement de l'interaction avec les usagers dans le but de renforcer la qualité et l'exhaustivité des données.

De manière générale, les pouvoirs publics ont aussi intensifié leurs efforts sur le plan de l'action officielle en faveur de la réutilisation des données. Par exemple, sur les 33 pays de l'OCDE, 21 traitent le développement des capacités et des aptitudes au sein de l'administration publique comme une priorité. Les pays sont plus nombreux qu'en 2017 à étudier les incidences possibles de l'ouverture des données publiques, en menant à cette fin des travaux de recherche ou en recueillant des exemples de réutilisations des données. Toutefois, le niveau de soutien à la réutilisation des DPO par les citoyens, entreprises et autres acteurs extérieurs est resté constant.

\section{Méthodologie et définitions}

Les données relatives à l'indice OURdata proviennent de l'enquête 2018 de l'OCDE sur les données publiques ouvertes, à laquelle ont répondu 32 pays de l'OCDE et un pays candidat à l'adhésion (la Colombie). Les réponses ont essentiellement été fournies par de hauts responsables chargés des politiques liées au numérique ou à l'ouverture de l'administration (ou « gouvernement ouvert»). Les données portent uniquement sur les administrations centrales ou fédérales ; elles ne tiennent pas compte des pratiques en place à l'échelon des États fédéraux ou à l'échelon local.

L'indice composite OURdata est fondé sur les principes de la Charte internationale sur les données ouvertes et sur la méthodologie décrite dans les travaux de l'OCDE (Lafortune et Ubaldi 2018). Il se compose de trois indicateurs : la disponibilité des données, l'accessibilité des données et l'action officielle en faveur de la réutilisation des données. Pour chaque indicateur, le score correspond à la moyenne non pondérée de l'ensemble de ces sousindicateurs. L'indice ne mesure pas l'effet de l'ouverture des données publiques sur la situation socio-économique, mais les efforts déployés par les pouvoirs publics pour mettre en place des conditions suffisamment favorables pour permettre et stimuler la réutilisation des données. Pour en savoir plus sur la méthodologie et les données sous-jacentes, voir l'Annexe E.

\section{Pour en savoir plus}

Lafortune, G. et Ubaldi, B. (2018), “OECD 2017 OURdata Index: Methodology and results", Documents de travail de l'OCDE sur la gouvernance publique, $\mathrm{n}^{\circ} 30$, Éditions OCDE, Paris, http://doi. org/10.1787/2807d3c8-en.

OCDE (2019), Rapport sur les données ouvertes publiques : encourager la maturité des politiques de données ouvertes pour un impact durable, Éditions OCDE, Paris, https://doi.org/10.1787/12ea5027-fr.

\section{Notes relatives aux graphiques}

Pour l'année 2017, on ne dispose pas de données concernant la Hongrie, l'Islande et le Luxembourg. Pour l'année 2019, on ne dispose pas de données concernant les États-Unis, la Hongrie, l'Islande et la Turquie. Concernant les données sur Israël, voir http://doi. org/10.1787/888932315602. 
9.4. Indice OURdata (données ouvertes, utiles et réutilisables), 2017 et 2019

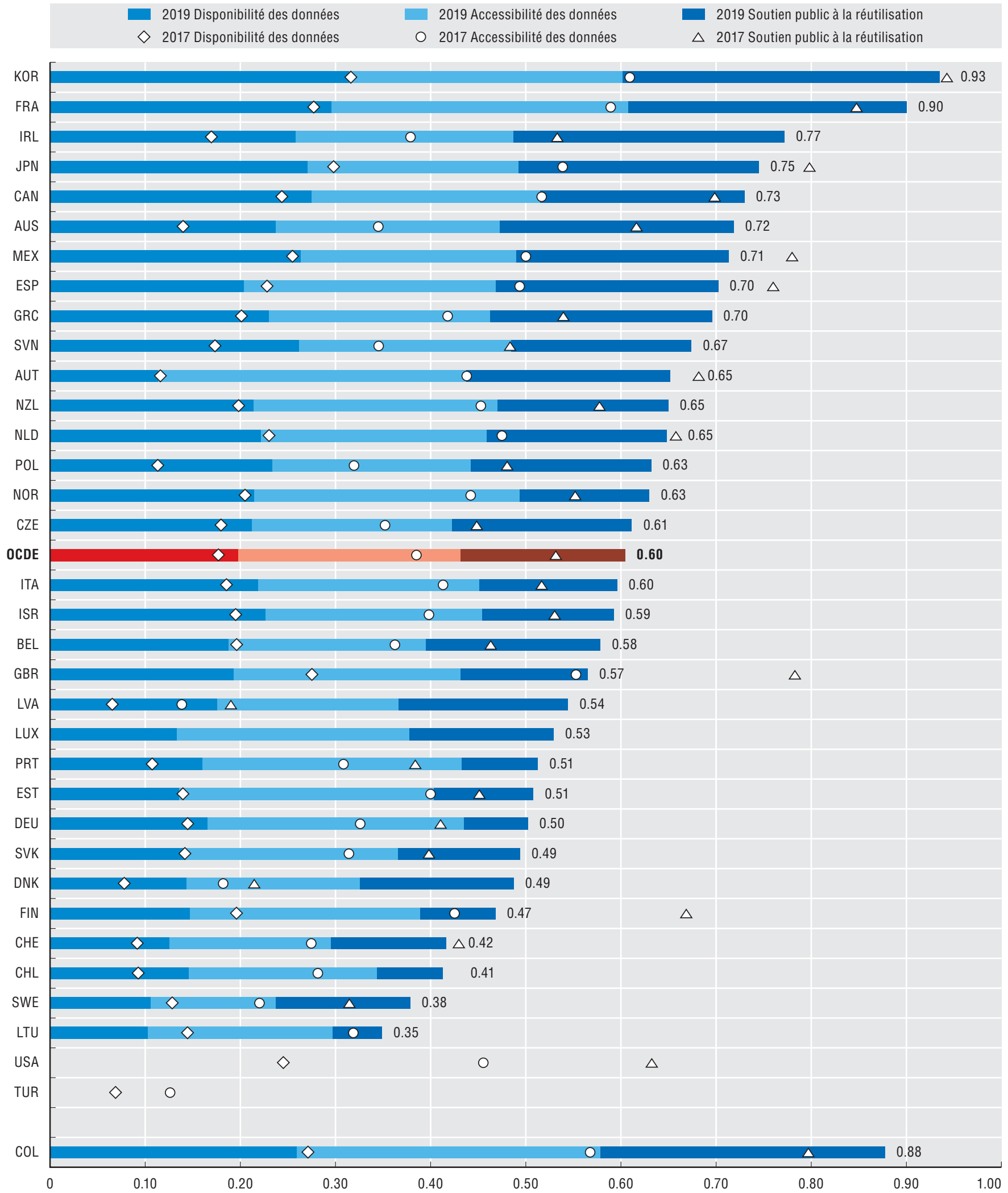

Sources : OCDE (2016 et 2018), Enquête sur les données publiques ouvertes. 


\section{Disponibilité des données : cadres d'action, association des parties} prenantes et diffusion des données

La disponibilité des données publiques est d'une importance capitale pour la mise en œuvre des politiques d'ouverture des données. Une plus grande disponibilité va de pair avec des programmes d'ouverture des données plus robustes et plus viables et des interactions avec les parties prenantes visant la diffusion des données et la publication de séries de données qui présentent un fort intérêt (comme les données sur les infrastructures ou les registres du commerce). Un portail central ou fédéral de données publiques ouvertes permet aux usagers de trouver facilement les données. Il offre à la communauté des usagers et producteurs de données ouvertes un espace pour s'associer avec les pouvoirs publics.

La valeur de l'indicateur relatif à la disponibilité des données s'échelonne entre 0 et 1, 0 étant le score le moins bon et 1 le meilleur. L'indicateur comporte trois sous-indicateurs portant sur le contenu de la politique d'ouverture par défaut ; l'association des parties prenantes à la diffusion des données ; et la mise en œuvre. Chacun de ces sous-indicateurs est doté d'une pondération égale avec un score maximum de 0,33.

Pour l'ensemble de la zone OCDE, la valeur moyenne de disponibilité des données est passée de 0,53 en 2017 à 0,59 en 2019. Cette amélioration résulte de l'intensification des programmes d'ouverture des données et de l'organisation fréquente de consultations des parties prenantes afin d'éclairer la conception des mesures prises en la matière. La plupart des pays de l'OCDE ont progressé sur le plan de la disponibilité de leurs données, y compris ceux dont les scores sont inférieurs à la moyenne, tels que le Chili, dont le score est passé de 0,28 à 0,44; la Suisse, dont le score est passé de 0,28 à 0,38 ; et le Portugal dont le score a augmenté de 0,32 à 0,48.

La plupart des pays de l'OCDE ont progressé sur le plan du contenu de la politique d'ouverture par défaut, puisque leur score moyen est passé de 0,19 en 2017 à 0,22 en 2019. C'est dans les pays ayant récemment adopté des stratégies, programmes ou textes en matière d'ouverture des données à l'échelon central ou fédéral que les scores ont le plus progressé. Ainsi, le score de la Pologne est passé de 0,06 à 0,26 ; celui de l'Irlande, de 0,13 à 0,25 ; et celui de l'Allemagne, de 0,08 à 0,19.

Tous les pays de l'OCDE ont mis en place des exigences explicites pour l'ouverture par défaut des données publiques, à l'exception de l'Autriche et de la Suède. Toutefois, il est important de préciser que l'Autriche fait partie des pays les mieux classés en termes d'accessibilité (voir la page consacrée à l'accessibilité des données). Malgré l'adoption par défaut de principes ouverts, seulement la moitié des pays de l'OCDE ont inclus la mise en œuvre des exigences d'ouverture des données (par exemple, la mise à disposition de données actualisées et lisibles sur machine) dans leurs indicateurs de performance des entités publiques.

Pour la zone OCDE, le score moyen du sous-indicateur relatif à l'association des parties prenantes à la diffusion des données est passé de 0,18 en 2017 à 0,20 en 2019. Certains pays ont progressé. Ainsi, le score de l'Australie est passé de 0,07 à 0,23 et celui du Danemark de 0,03 à 0,17 . En phase avec l'avancée de leurs plans d'ouverture des données, la Slovénie et l'Irlande ont aussi donné la priorité à ce domaine et leurs scores sont respectivement passés de 0,19 à 0,29 et de 0,21 à 0,29 . Les scores élevés du Japon et de la Corée $(0,33)$ résultent probablement du fait qu'ils ont imposé aux entités publiques de consulter régulièrement les usagers des données.

Par rapport à 2017, la quantité de séries de données disponibles a légèrement progressé puisque, sur l'ensemble de la zone OCDE, le score moyen en matière de mise en œuvre est passé de 0,16 en 2017 à 0,18 pour 2019. Les scores de la République tchèque et de la Slovénie sont respectivement passés de 0,08 à 0,21 et de 0,08 à 0,20 , grâce à la publication de séries de données présentant un fort intérêt, tels que des codes postaux et des cartes nationales ou locales. De nombreuses séries de données importantes manquent encore au Danemark $(0,02)$, en Estonie $(0,06)$ et Lituanie $(0,04)$, y compris les données budgétaires ouvertes. Le Canada est le pays de l'OCDE qui publie le plus de séries de données présentant un fort intérêt, ce qui lui vaut d'obtenir un score égal à 0,31 .

\section{Méthodologie et définitions}

La disponibilité des données évalue dans quelle mesure les pouvoirs publics ont adopté et mis en œuvre des exigences formelles visant à favoriser l'ouverture des données publiques à l'échelon central ou fédéral. L'indicateur disponibilité des données correspond aux Principes 1 ("Des données ouvertes par défaut ») et 2 ( " Des données diffusées en temps opportun et exhaustives ") de la Charte internationale sur les données ouvertes. Il se compose des trois sous-indicateurs suivants : le contenu de la politique d'ouverture par défaut; l'association des parties prenantes à la diffusion des données ; et la mise en œuvre. Les trois sousindicateurs sont dotés d'une pondération égale et la valeur de chacun d'entre eux s'échelonne entre 0 et 0,33. La valeur globale de l'indicateur s'échelonne donc entre 0 (score minimum) et 1 (score maximum). Pour obtenir l'indice OURdata global, on recalcule la valeur de l'indicateur relatif à la disponibilité des données de façon à la ramener à un score compris entre 0 et 0,33 . On affecte ensuite à cet indicateur la même pondération qu'aux deux autres indicateurs qui composent l'indice.

Les données relatives à l'indice OURdata et à l'indicateur sur la disponibilité des données proviennent de l'Enquête de l'OCDE sur les données publiques ouvertes. Les réponses ont été fournies, pour l'essentiel, par de hauts responsables chargés des politiques liées au numérique ou à l'ouverture de l'administration (ou " gouvernement ouvert "). Elles reflètent le propre point de vue des pays sur leurs pratiques et procédures actuelles concernant la disponibilité des données. Les données portent uniquement sur les administrations centrales ou fédérales ; elles ne tiennent pas compte des pratiques en place à l'échelon des États fédéraux ou à l'échelon local. L'indice OURdata est un indice composite fondé sur les principes de la Charte internationale sur les données ouvertes ainsi que sur la méthodologie décrite dans les travaux de l'OCDE (Lafortune et Ubaldi 2018).

Pour en savoir plus sur la méthodologie et les données sousjacentes, voir l'Annexe E.

\section{Pour en savoir plus}

Lafortune, G. et Ubaldi, B. (2018), “OECD 2017 OURdata Index: Methodology and results", Documents de travail de l'OCDE sur la gouvernance publique, $\mathrm{n}^{\circ}$ 30, Éditions OCDE, Paris, http://doi. org/10.1787/2807d3c8-en.

OCDE (2016), Open Government Data Review of Mexique : Data Reuse for Public Sector Impact and Innovation, OECD Digital Government Studies, Éditions OCDE, Paris, https://doi. org/10.1787/9789264259270-en.

\section{Notes relatives aux graphiques}

Pour l'année 2017, on ne dispose pas de données concernant la Hongrie, l'Islande et le Luxembourg. Pour l'année 2019, on ne dispose pas de données concernant les États-Unis, la Hongrie, l'Islande et la Turquie.

Concernant les données sur Israël, voir http://dx.doi.org/10.1787/ 888932315602 
Disponibilité des données : cadres d'action, association des parties prenantes et diffusion des données

\subsection{Disponibilité des données, 2017 et 2019}

2019 Contenu de la politique d'ouverture par défaut 2019 Association des parties prenantes à la divulgation des données

$\diamond 2017$ Contenu de la politique d'ouverture par défaut $\quad \bigcirc 2017$ Association des parties prenantes à la divulgation des données

2019 Mise en œuvre

$\triangle 2017$ Mise en œuvre

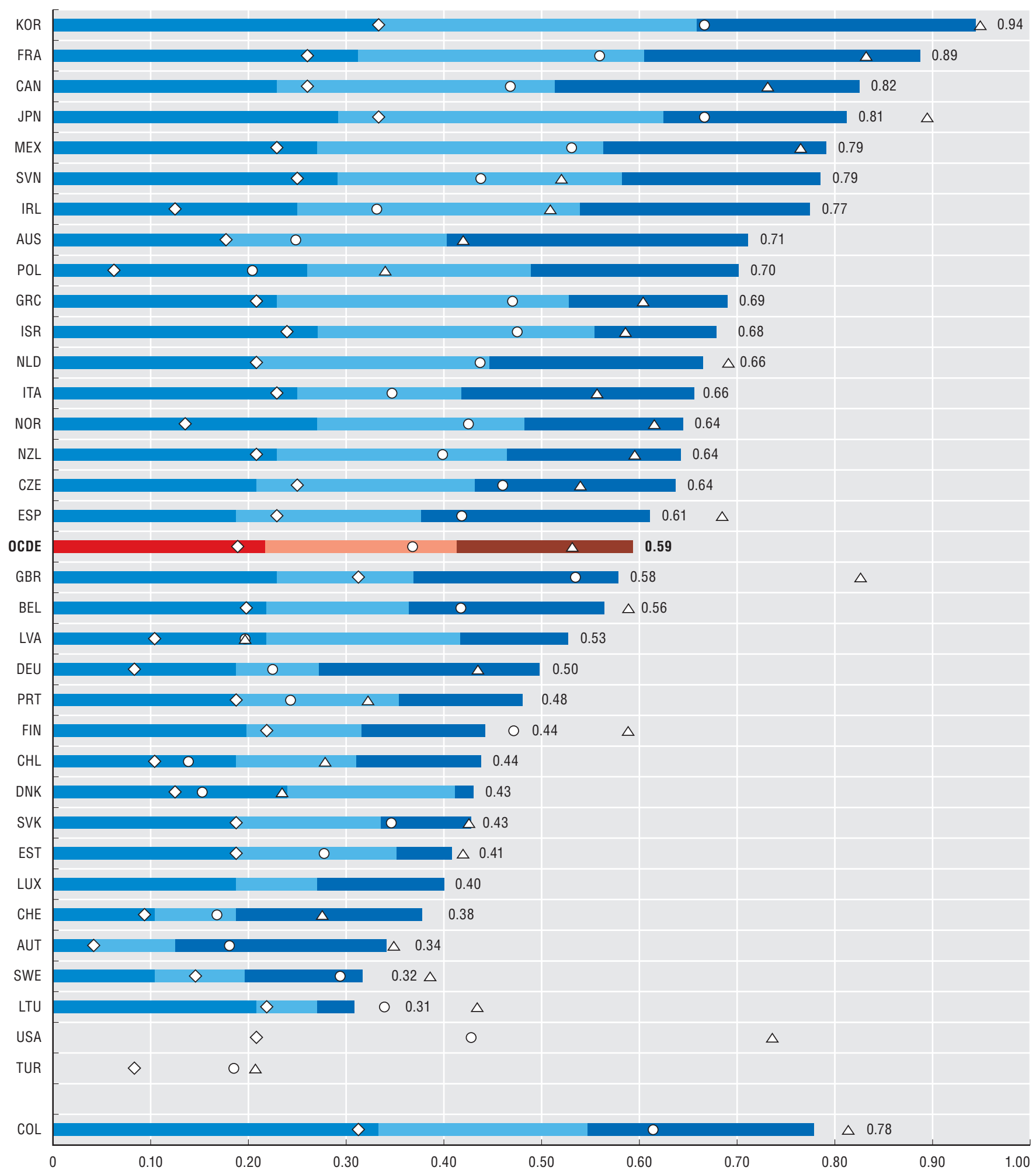

Source : OCDE (2016 et 2018), Enquêtee sur les données publiques ouvertes. 
Afin de permettre aux citoyens, aux entreprises et aux parties prenantes de réutiliser les données publiques, cellesci demandent à être fournies sous des formats et selon des procédures utilisables par quiconque et à toute fin. Pour les rendre accessibles, il convient de diffuser les données gratuitement, sans restriction d'accès et sous une forme lisible sur machine. Dans le but d'améliorer la qualité et l'accessibilité des données, les pouvoirs publics peuvent aussi définir des normes en termes de format et de procédures de publication. De plus, la mise en place de canaux ou autres dispositifs permettant des retours d'information, sur les portails de données publiques ouvertes de l'administration centrale ou fédérale, peut inciter les usagers des données ouvertes à apporter leur contribution.

L'indicateur relatif à l'accessibilité des données publiques s'appuie sur trois sous-indicateurs portant sur les aspects suivants : le contenu de la politique d'accès sans restriction aux données, l'association des parties prenantes au service de la qualité et de l'exhaustivité des données et la mise en œuvre. Chacun de ces sous-indicateurs a une valeur maximale de 0,33. Sur l'ensemble de la zone OCDE, la valeur moyenne de l'indicateur (qui s'échelonne entre 0 et 1) est passée de 0,62 en 2017 à 0,70 en 2019, grâce à des portails de données ouvertes plus sophistiqués qui invitent les usagers à fournir un retour d'information. Dans l'ensemble des pays de l'OCDE, les portails centraux ou fédéraux de DPO sont de plus en plus axés sur l'usager et les plateformes de collaboration, ce qui permet aux utilisateurs d'ajouter des données et des interprétations, par le biais de mécanismes de retour plus avancés Le sous-indicateur relatif à l'association des parties prenantes est par conséquent amélioré.

Des exigences formelles obligeant les entités publiques à fournir les données gratuitement, sans restriction d'accès et sous des formats permettant leur réutilisation, sont courantes dans les pays de l'OCDE. Sur l'ensemble de la zone OCDE, la valeur moyenne du sous-indicateur relatif au contenu de la politique d'accès sans restriction aux données est passée de 0,25 en 2017 à 0,27 en 2019. De plus, sur les 33 pays de l'OCDE, 10 pays obtiennent le plus haut score possible pour cet indicateur $(0,33)$, y compris le Chili, l'Italie et les Pays-Bas, tandis que la Suède reste très en retard, avec un score de 0,06 . Avec l'adoption de sa loi fédérale sur les données ouvertes, l'Allemagne a entrepris l'une des initiatives les plus remarquables de ces dernières années en faveur de l'accessibilité des données, son score étant passé de 0,19 à 0,33.

Beaucoup de pays interagissent sur leurs plateformes de DPO, avec les usagers des données ouvertes et d'autres parties prenantes afin d'améliorer la qualité et l'exhaustivité des données. Sur l'ensemble de la zone OCDE, le score moyen pour le sous-indicateur relatif à l'association des parties prenantes est passé de 0,11 en 2017 à 0,14 en 2019. L'Autriche et la France sont toutes deux dotées de portails de DPO très sophistiqués, ouverts aux contributions des usagers et, par conséquent, ces pays occupent la tête du classement en matière d'accessibilité des données. À l'inverse, les scores totaux du Japon $(0,67)$ et du Mexique $(0,68)$ sur l'accessibilité des données sont inférieurs à la moyenne OCDE, essentiellement en raison de scores plus faibles en matière d'association des parties prenantes (respectivement 0,13 et 0,07 ).

En pratique, la plupart des pays de l'OCDE publient des données accessibles et de grande qualité sur leurs portails centraux ou fédéraux de DPO, y compris la Lettonie qui a lancé son portail en 2017. Sur l'ensemble de la zone OCDE, le score moyen en matière de mise en œuure est passé de 0,27 en 2017 à 0,28 en 2019. Il est intéressant de noter que la Suède fait partie des pays les mieux notés s'agissant de fournir des données accessibles sur son site de DPO (avec un score de 0,30 ), alors même qu'elle a peu mis en place d'exigences formelles applicables aux entités publiques dans le domaine.
Le Danemark $(0,22)$ et la Lituanie $(0,21)$ obtiennent des scores relativement faibles. L'une des explications du score danois tient au fait que les utilisateurs ont besoin de s'enregistrer sur le site afin d'accéder aux données et de les réutiliser.

\section{Méthodologie et définitions}

L'indicateur relatif à l'accessibilité des données permet d'évaluer dans quelle mesure les données publiques sont fournies sous des formats ouverts et réutilisables, avec les métadonnées correspondantes. L'indicateur couvre principalement les Principes 3 ("Des données accessibles et utilisables ") et 4 ("Des données comparables et interopérables") de la Charte internationale sur les données ouvertes. Il se compose de trois sous-indicateurs portant sur les aspects suivants : le contenu de la politique d'accès sans restriction aux données ; l'association des parties prenantes au service de la qualité et de l'exhaustivité des données ; et la mise en œuvre. Les trois sous-indicateurs sont dotés d'une pondération égale et la valeur de chacun d'entre eux s'échelonne entre 0 et 0,33. La valeur globale de l'indicateur s'échelonne donc entre 0 (score minimal) et 1 (score maximal). Pour obtenir l'indice global OURdata, on recalcule la valeur de l'indicateur relatif à l'accessibilité des données de façon à la ramener à un score compris entre 0 et 0,33 . On affecte ensuite à cet indicateur la même pondération qu'aux deux autres indicateurs qui composent l'indice.

Les données relatives à l'indice OURdata et à l'indicateur sur l'accessibilité des données proviennent de l'Enquête de l'OCDE sur les données publiques ouvertes. Les réponses ont essentiellement été fournies par de hauts responsables chargés des politiques liées au numérique ou à l'ouverture de l'administration (ou " gouvernement ouvert "). Elles reflètent le propre point de vue des pays sur leurs pratiques et procédures actuelles concernant la disponibilité des données. Les données portent uniquement sur les administrations centrales ou fédérales; elles ne tiennent pas compte des pratiques en place à l'échelon des États fédéraux ou à l'échelon local. L'indice OURdata est un indice composite fondé sur les principes de la Charte internationale sur les données ouvertes ainsi que sur la méthodologie décrite dans les travaux de l'OCDE (Lafortune et Ubaldi 2018).

Pour en savoir plus sur la méthodologie et les données sous-jacentes, voir l'Annexe E.

\section{Pour en savoir plus}

Lafortune, G. et Ubaldi, B. (2018), “OECD 2017 OURdata Index: Methodology and results", Documents de travail de l'OCDE sur la gouvernance publique, $\mathrm{n}^{\circ} 30$, Éditions OCDE, Paris, http://doi. org/10.1787/2807d3c8-en.

OCDE (2016), Open Government Data in Mexico: The Way Forward, OECD Digital Government Studies, Éditions OCDE, Paris, https://doi.org/10.1787/9789264297944-en.

\section{Notes relatives aux graphiques}

Pour l'année 2017, on ne dispose pas de données concernant la Hongrie, l'Islande et le Luxembourg. Pour l'année 2019, on ne dispose pas de données concernant les États-Unis, la Hongrie, l'Islande et la Turquie.

Concernant les données sur Israël, voir http://dx.doi.org/10.1787/ 888932315602 


\subsection{Accessibilité des données, 2017 et 2019}
2019 Contenu de la politique d'accès sans restriction aux données
2019 Mise en œuvre
O 2017 Association des parties prenantes au service de la qualité et de l'exhaustivité des données
$\diamond 2017$ Contenu de la politique d'accès sans restriction aux données
$\triangle 2017$ Mise en œuvre

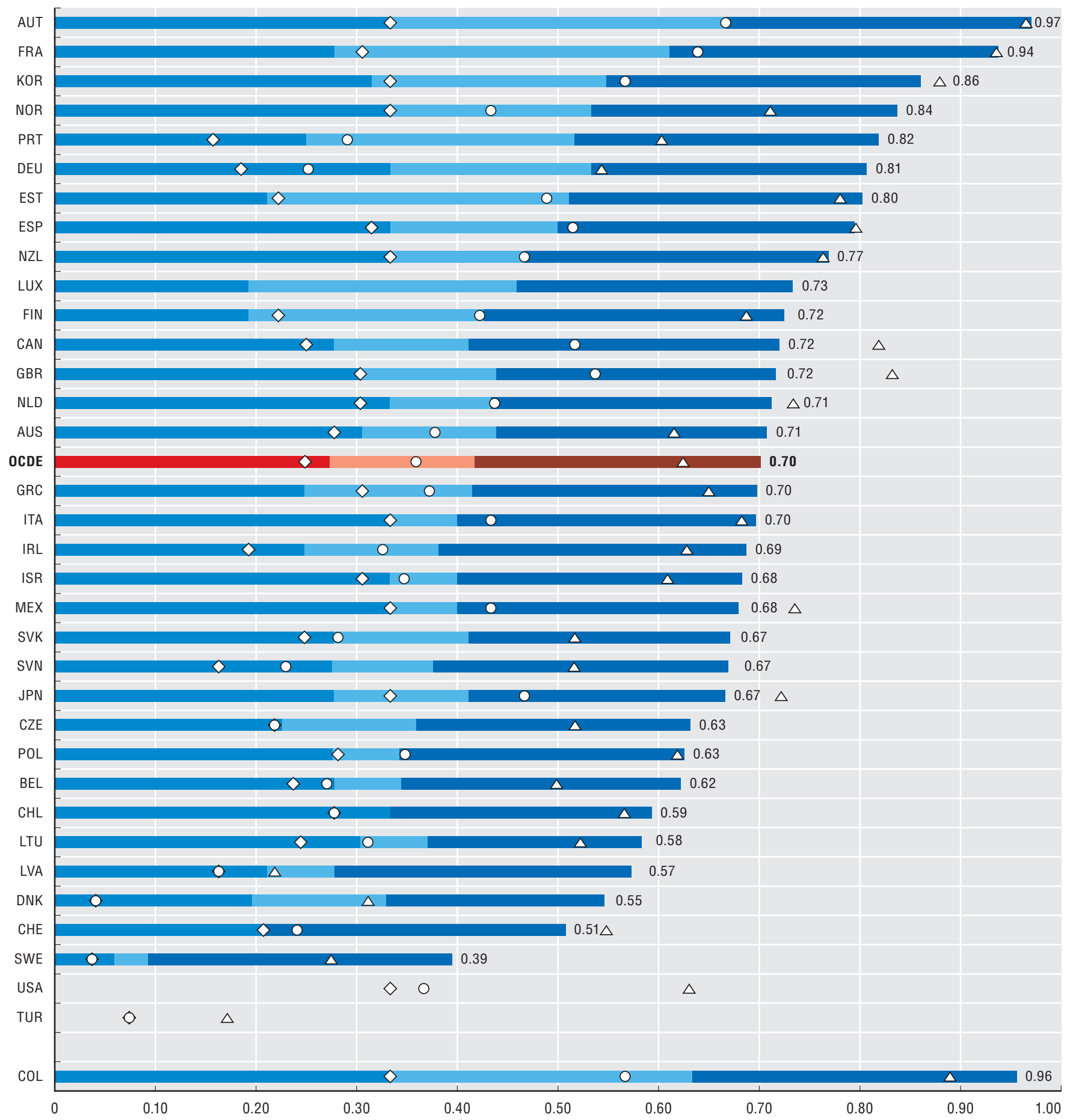

Source : OCDE (2016 et 2018), Enquêtes sur les données publiques ouvertes. 
Afin d'atteindre le plein potentiel de l'ouverture des données publiques, il est essentiel d'encourager, de recueillir et de faire connaître des exemples de réutilisations des données publiques ayant eu des incidences positives sur le bien-être social et économique. En leur qualité de fournisseurs de données, les pouvoirs publics jouent un rôle crucial pour mieux faire connaître les données et encourager leur réutilisation, à l'intérieur comme à l'extérieur du secteur public. À cette fin, ils peuvent renforcer les capacités et les aptitudes présentes au sein du secteur public et organiser des manifestations, concours, programmes et partenariats permettant de stimuler la réutilisation des données par les acteurs extérieurs. Le suivi des incidences sociales et économiques des DPO constitue aussi clé.

L'indicateur relatif à l'action officielle en faveur de la réutilisation des données compte trois sous-indicateurs qui portent sur les initiatives et partenariats visant à promouvoir les données, les programmes de familiarisation avec les données au sein de l'administration et le suivi d'impact. Pour chacun de ces sousindicateurs, le score maximal est égal à 0,33. Sur la zone OCDE, le score moyen pour l'indicateur global est passé de 0,44 en 2017 à 0,52 en 2019 (étant précisé que la valeur du score s'échelonne entre 0 et 1). Les améliorations constatées sur l'ensemble de la zone OCDE sont dues, en grande partie, à l'organisation de programmes et de manifestations sur les DPO à l'intention des agents publics ; à des efforts croissants pour suivre les incidences de l'ouverture des données publiques grâce à des travaux de recherche; et à la mise en ligne d'exemples de réutilisations des données sur les portails centraux de DPO.

La Corée continue d'occuper la tête du classement dans ce domaine et obtient le score le plus élevé pour chacun des trois sous-indicateurs. Même si le soutien à la réutilisation des données progresse de façon générale, certains pays obtiennent de moins bons résultats. En matière de promotion des données, la Norvège et la Finlande voient leurs scores se dégrader entre 2017 et 2019 (ils passent respectivement de 0,24 à 0,08 et de 0,29 à 0,03), car, en 2019 , ces pays ont organisé moins de manifestations associant la société civile et les entreprises qu'en 2017.

Le soutien à la réutilisation des DPO par les acteurs extérieurs est généralement resté stable entre 2017 et 2019, la moyenne OCDE demeurant égale à 0,16 (sur 0,33 ) pour le sousindicateur relatif à la promotion des données. Toutefois, les pays au sein desquels l'ouverture des données publiques est restée une priorité politique importante ces dernières années, parmi lesquels l'Irlande et la Lettonie, ont organisé des activités et des programmes pour favoriser la réutilisation des données par les entreprises et la société civile (par exemple, marathons de programmation, conférences sur l'ouverture des données et manifestations de co-création).

Comme mentionné plus haut, la plupart des pays ont consacré des ressources au renforcement des capacités et des aptitudes en matière de données ouvertes au sein de leurs administrations. Sur l'ensemble de la zone OCDE, le score moyen pour le sousindicateur lié à la familiarisation avec les données au sein de l'administration est passé de 0,17 à 0,21. Par exemple, la NouvelleZélande a réalisé de considérables progrès s'agissant de former ses agents publics aux avantages et à l'utilisation des données ouvertes, ce qui vaut à son score de passer de 0,13 à 0,29.

Dans la droite ligne des progrès accomplis sur le plan des portails de DPO dans les pays de l'OCDE, un plus grand nombre de pays cherchent à recueillir et à diffuser des exemples divers de réutilisations des données afin d'inciter les usagers à faire de même. Par conséquent, le score moyen OCDE du sousindicateur relatif au suivi d'impact est passé de 0,10 en 2017 à 0,14 en 2019. Certains pays ont lancé des travaux de recherche dans le but d'évaluer l'impact socio-économique des DPO sur la situation sociale et économique. Ainsi, le Danemark et la
Lettonie ont étudié les effets économiques de l'ouverture des données géospatiales.

\section{Méthodologie et définitions}

L'indicateur relatif à l'action officielle en faveur de la réutilisation des données permet d'évaluer dans quelle mesure les pouvoirs publics s'emploient à promouvoir la réutilisation des données publiques, à l'intérieur comme à l'extérieur de la sphère publique. Il couvre principalement les Principes 5 ("Amélioration de la gouvernance et de la participation citoyenne ") et 6 (" Développement inclusif et innovation ") de la Charte internationale sur les données ouvertes. Il se compose de trois sous-indicateurs portant sur les aspects suivants : les initiatives et partenariats visant à promouvoir les données ; les programmes de familiarisation avec les données au sein de l'administration; et le suivi d'impact. Chacun de ces sous-indicateurs est doté d'une pondération égale et a donc une valeur comprise entre 0 et 0,33. La valeur de l'indicateur global s'échelonne entre 0 (score minimal) et 1 (score maximal). Pour obtenir l'indice global OURdata, on recalcule la valeur de l'indicateur relatif au soutien public à la réutilisation des données de façon à la ramener à un score compris entre 0 et 0,33 . On affecte ensuite à cet indicateur la même pondération qu'aux deux autres indicateurs qui composent l'indice.

Les données relatives à l'indice OURdata et à l'indicateur sur le soutien public à la réutilisation des données proviennent de l'Enquête de l'OCDE sur les données publiques ouvertes. Les réponses ont essentiellement été fournies par de hauts responsables chargés des politiques liées au numérique ou à l'ouverture de l'administration (ou " gouvernement ouvert »). Elles reflètent le propre point de vue des pays sur leurs pratiques et procédures actuelles concernant les données publiques ouvertes. Les données portent uniquement sur les administrations centrales ou fédérales ; elles ne tiennent pas compte des pratiques en place à l'échelon des États fédéraux ou à l'échelon local. L'indice OURdata est un indice composite fondé sur les principes de la Charte internationale sur les données ouvertes ainsi que sur la méthodologie décrite dans les travaux de l'OCDE (Lafortune et Ubaldi 2018).

Pour en savoir plus sur la méthodologie et les données sous-jacentes, voir l'Annexe E.

\section{Pour en savoir plus}

Lafortune, G. et Ubaldi, B. (2018), “OECD 2017 OURdata Index: Methodology and results", Documents de travail de l'OCDE sur la gouvernance publique, $\mathrm{n}^{\circ} 30$, Éditions OCDE, Paris, http://doi. org/10.1787/2807d3c8-en.

OCDE (2015), Open Government Data Review of Poland: Unlocking the Value of Government Data, OECD Digital Government Studies, Éditions OCDE, Paris, https://doi.org/10.1787/9789264241787-en.

\section{Notes relatives aux graphiques}

Pour l'année 2017, on ne dispose pas de données concernant la Hongrie, l'Islande et le Luxembourg. Pour l'année 2019, on ne dispose pas de données concernant les États-Unis, la Hongrie, l'Islande et la Turquie.

Concernant les données sur Israël, voir http://dx.doi.org/10.1787/ 888932315602 
9.7. Soutien public à la réutilisation des données, 2017 et 2019

$\begin{array}{lll}2019 \text { Initiatives et partenariats visant à promouvoir les données } & 2019 \text { Programmes de familiarisation avec les données au sein de l'administration } \\ \diamond 2017 \text { Data promotion initiatives and patnerships } & \bigcirc \quad 2017 \text { Data literacy programmes in government }\end{array}$

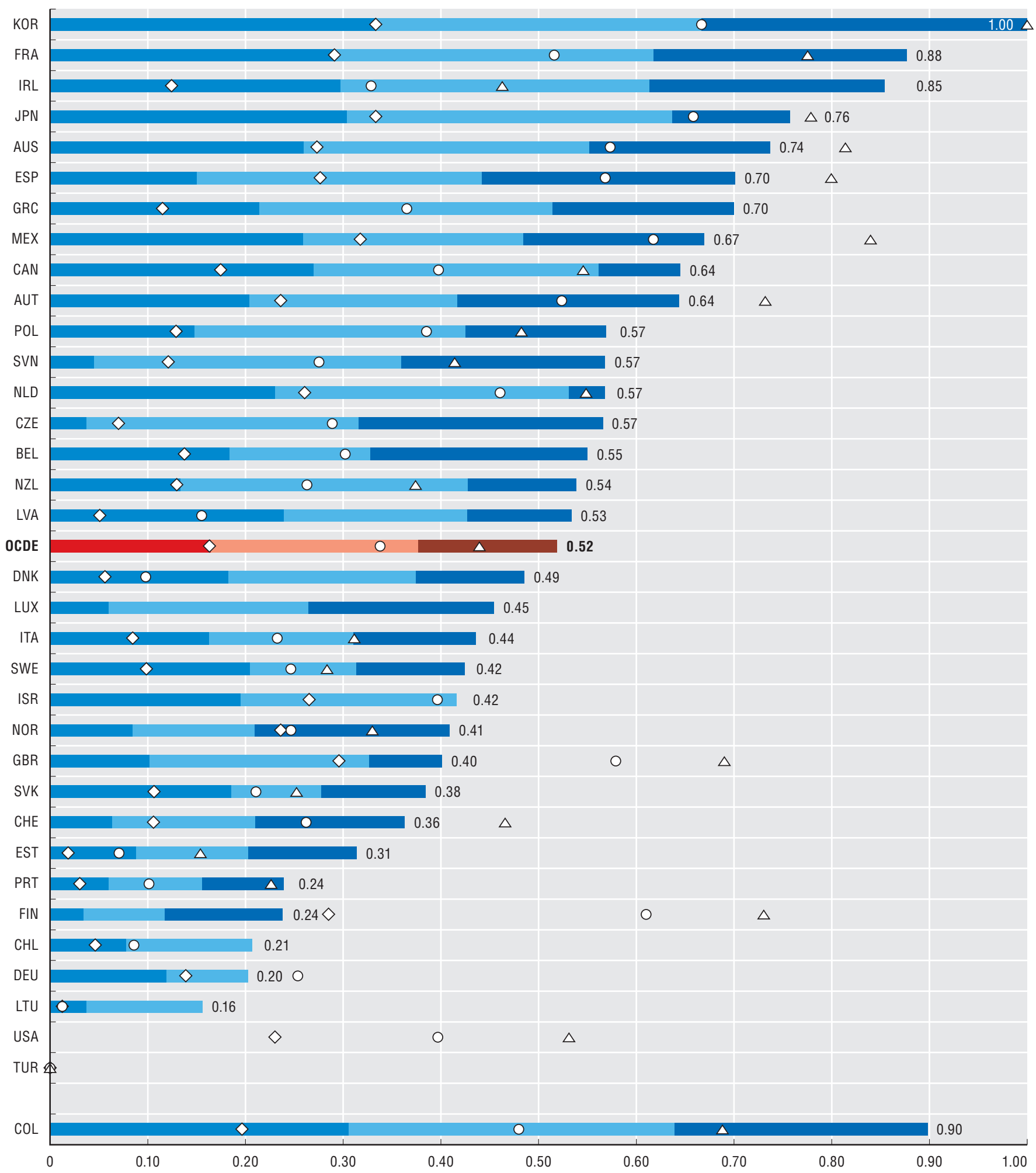

Source : OCDE (2016 et 2018), Enquête sur les données publiques ouvertes. 


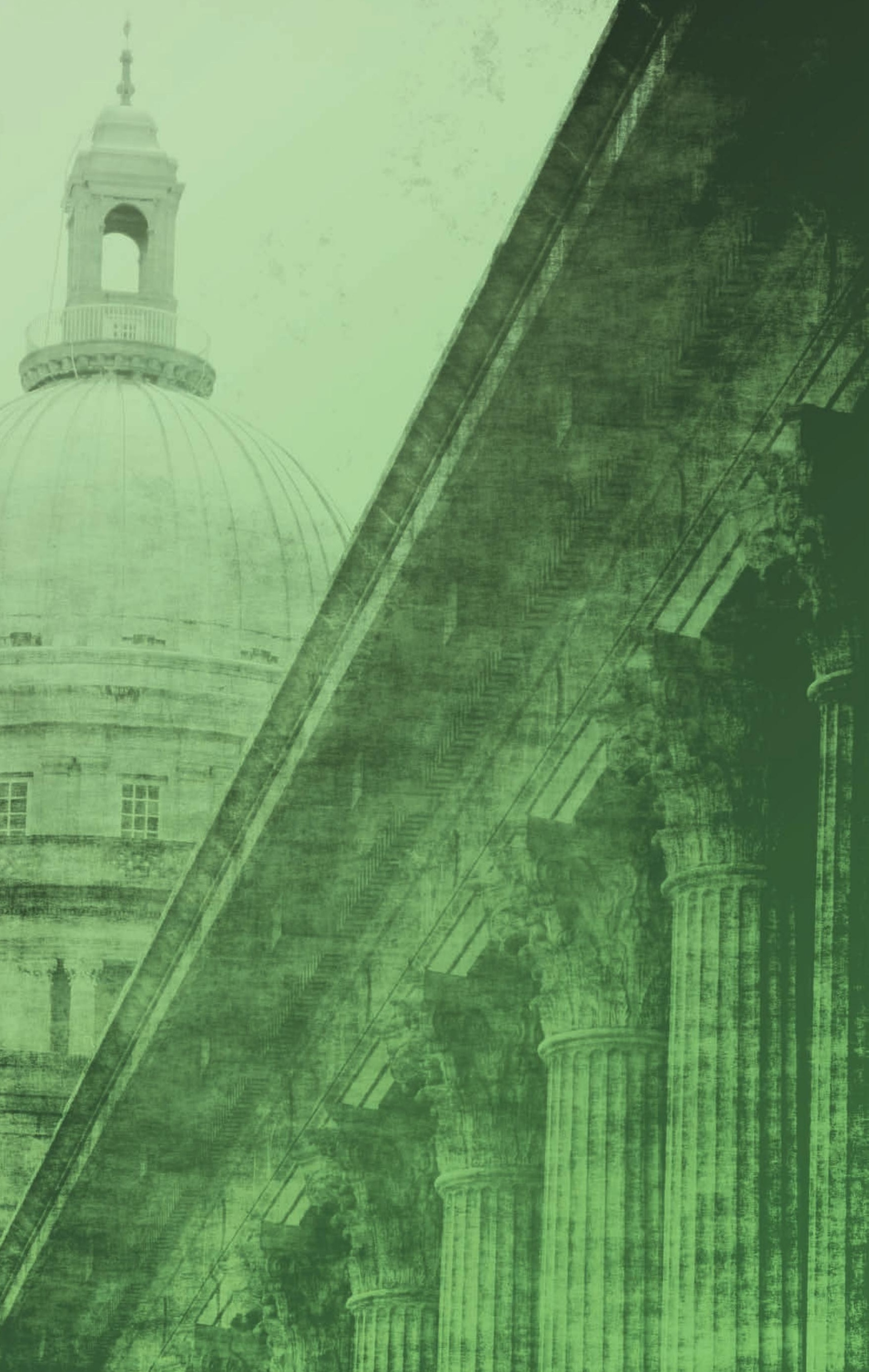




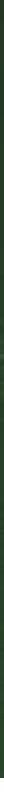

\section{PRINCIPAUX RÉSULTATS} DES POUVOIRS PUBLICS

Confiance à l'égard des pouvoirs publics

Réactivité perçue des administrations publiques

(efficacité politique externe)

Redistribution des revenus et du patrimoine

État de droit

Efficience du secteur public

Rapport coût-efficacité de l'action publique 
On définit la confiance comme la croyance d'une personne selon laquelle une autre personne ou une institution aura le comportement positif auquel elle s'attend (OCDE 2017a). La confiance à l'égard des pouvoirs publics, dite " confiance institutionnelle ", est le fondement de la légitimité des autorités et un facteur-clé pour s'assurer du respect de la réglementation et du système fiscal; elle est essentielle à la mise en œuvre des réformes et à l'aptitude des pouvoirs publics à gouverner sans recourir à la force. Les travaux universitaires s'accordent à dire que la confiance influe sur la relation entre les administrations et les citoyens et qu'elle a des incidences sur les retombées de l'action publique (OCDE 2017b).

La source la plus complète de données internationalement comparables sur la confiance dont on dispose actuellement est l'enquête Gallup World Poll, qui couvre tous les pays membres de l'OCDE et l'ensemble de ses partenaires stratégiques. Les niveaux de confiance connaissent des évolutions très contrastées d'un pays à l'autre, puisqu'ils s'échelonnent entre plus de $70 \%$ au Luxembourg et en Suisse et $20 \%$ ou moins en Grèce et en Lettonie. En moyenne, le taux de confiance s'élevait en 2018 à $45 \%$ pour la zone OCDE, c'est-à-dire à une valeur similaire à celle de 2007 (avant la crise). Néanmoins, dans un groupe de pays - principalement ceux qui ont été durement touchés par la dernière crise financière -, les niveaux de confiance restent nettement inférieurs à ceux d'avant la crise. On constate ainsi un recul durable de la confiance entre 2007 et 2018 en Slovénie (-24 points), en Grèce (-22 points), en Finlande (-20 points) et en Espagne (-19 points). À l'autre extrémité de l'éventail, les niveaux de confiance à l'égard des pouvoirs publics ont progressé sur la même période dans les pays qui ont connu des résultats économiques durablement meilleurs, tels que l'Allemagne (+24 points), la Pologne (+24 points), la Suisse (+22 points) et Israël (+20 points).

$\mathrm{Au}$ sein-même des pays, on constate des différences statistiquement significatives entre les niveaux de confiance des différentes générations. En 2018, les jeunes (15-29 ans) font davantage confiance aux autorités nationales que les citoyens âgés de 50 ans et plus aux Pays-Bas (+15 points), en Finlande ( +8 points), en Lituanie ( +8 points), en Italie ( +8 points) et en Belgique ( 6 points). Ce phénomène peut connaître des explications multiples et propres à chaque pays, mais on peut vraisemblablement considérer que, si les jeunes font davantage confiance aux institutions publiques, c'est parce que la plupart d'entre eux bénéficient ou ont récemment bénéficié du système public d'enseignement. Inversement, dans des pays comme la Hongrie (+26 points), la Turquie (+15 points), la France (+14 points) le Chili (+13 points) et la Pologne (+13 points), les citoyens âgés de 50 ans et plus font davantage confiance aux autorités nationales que les jeunes. Là encore, les facteurs en jeu sont divers, mais ces différences peuvent tenir à ce que les jeunes aient l'impression de ne pas avoir de perspectives d'avenir ou de ne pas pouvoir accéder au même niveau de vie que les générations qui les ont précédés.

Le niveau de confiance à l'égard des pouvoirs publics demeure un élément de mesure crucial des performances des administrations publiques. Toutefois, il s'agit d'une notion pluridimensionnelle, influencée par des facteurs qui ne se limitent pas aux résultats économiques ni aux différences intergénérationnelles : elle dépend aussi de la gouvernance publique, et en son sein l'intégrité du secteur public. Dans les pays de l'OCDE, le niveau de confiance dans les pouvoirs publics présente une forte corrélation négative avec leur degré perçu de corruption. De nouveaux travaux d'analyse et de recueil de données probantes sur la confiance institutionnelle et ses déterminants pourraient aider à définir des politiques sur mesures pour rétablir ou entretenir la confiance, dans la zone OCDE et ailleurs.

\section{Méthodologie et définitions}

Ces données sont tirées de l'enquête Gallup World Poll (GWP) qui se fonde en général sur un échantillon représentatif d'environ 1000 citoyens par pays. Toutefois, pour certains pays, l'échantillon peut être de taille plus réduite ou les citoyens interrogés peuvent ne vivre que dans la capitale ou les plus grandes villes. L'enquête comporte des questions sur la confiance à l'égard des établissements financiers, du système judiciaire, de la police locale, de l'armée et des autorités nationales, ainsi qu'une question sur le degré d'approbation à l'égard des dirigeants politiques. La question relative à la confiance à l'égard des autorités nationales n'opère aucune distinction entre les responsables politiques et les agents publics ; de plus, elle ne précise pas de quelle composante de l'administration nationale il s'agit. On trouvera un complément d'information sur l'enquête Gallup World Poll à l'adresse suivante : http://www.gallup. com/services/170945/world-poll.aspx.

\section{Pour en savoir plus}

OCDE/KDI (2018), Understanding the Drivers of Trust in Government Institutions in Korea, Éditions OCDE, Paris, https://doi.org/ 10.1787/9789264308992-en.

OCDE (2017a), OECD Guidelines on Measuring Trust, Éditions OCDE, Paris, https://doi.org/10.1787/9789264278219-en.

OCDE (2017b), Trust and Public Policy: How Better Governance Can Help Rebuild Public Trust, Examens de l'OCDE sur la gouvernance publique, Éditions OCDE, Paris. https://doi. org/10.1787/9789264268920-en.

\section{Notes relatives aux graphiques}

Concernant les données sur Israël, voir http://doi.org/10.1787/888932315602

10.1. Les données correspondent au pourcentage de réponses positives à la question " Avez-vous confiance dans les autorités nationales ? ". Pour l'Islande, les données portent sur 2017 et non 2018. Pour l'Autriche, la Finlande, l'Irlande, la Norvège, le Portugal, la République slovaque, la Slovénie et la Suisse, les données portent sur 2006 et non 2007. Pour l'Islande et le Luxembourg, elles portent sur 2008 et non 2007.

10.2. On ne dispose pas de données concernant la tranche d'âge des 15-29 ans pour l'Australie, le Japon, l'Islande et la Slovénie. Pour le groupe des 15-29 ans, concernant la Finlande et la Nouvelle-Zélande, les données portent sur 2017 et non 2018

10.3. Les données correspondent au pourcentage de réponses positives à : " La corruption est-elle répandue au sein des pouvoirs publics de ce pays ? ». Pour l'Islande, les données portent sur 2017 et non 2018. 
10.1. Confiance à l'égard des autorités nationales en 2018 et évolution par rapport à 2007

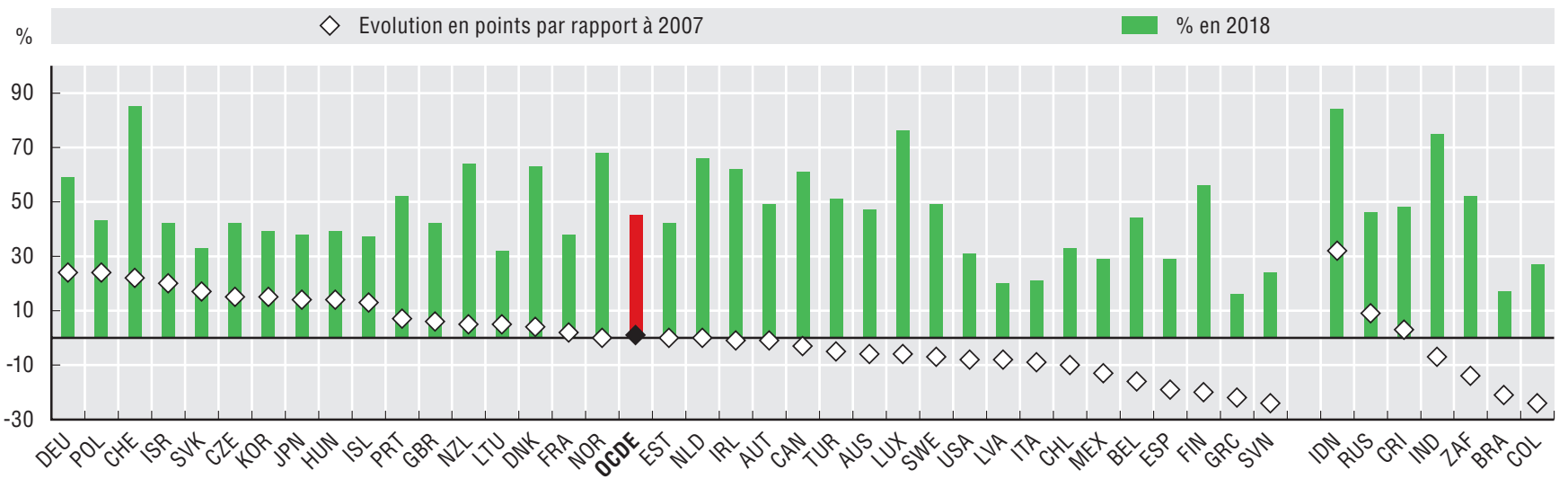

Source : Gallup World Poll, 2018.

StatLink नints https://doi.org/10.1787/888934052954

10.2. Confiance à l'égard des autorités nationales selon la tranche d'âge, 2018

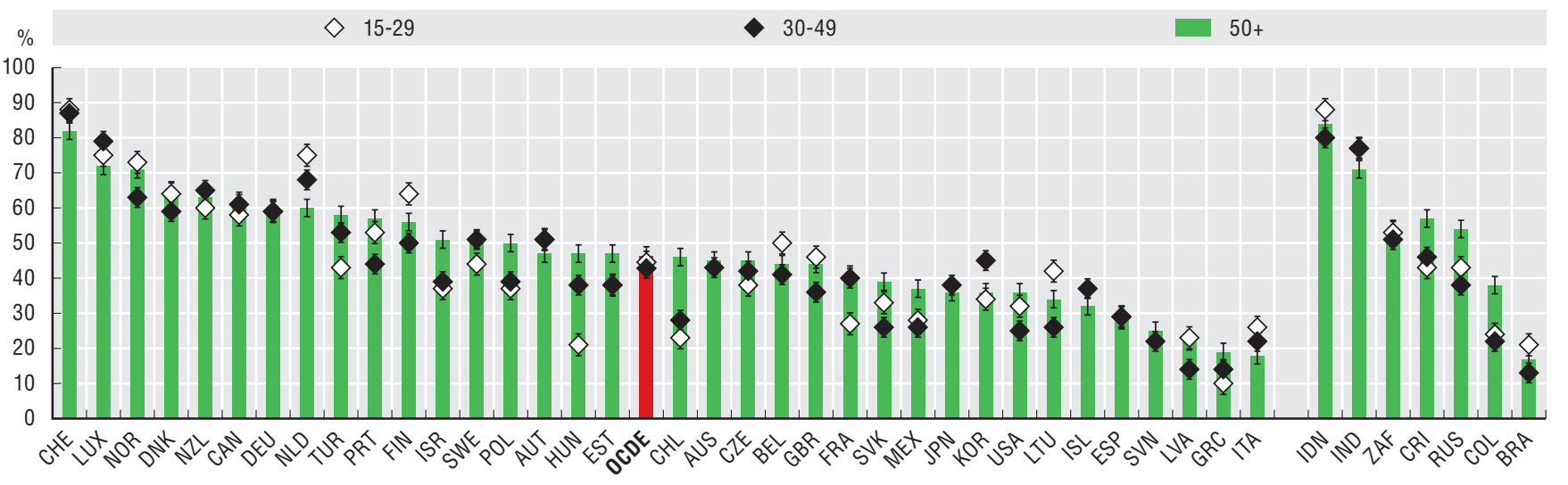

Source : Gallup World Poll, 2018

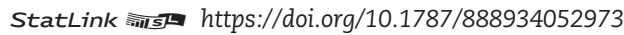

10.3. Corrélation entre la confiance à l'égard des autorités nationales et la corruption des pouvoirs publics dans les pays de l'OCDE, 2018

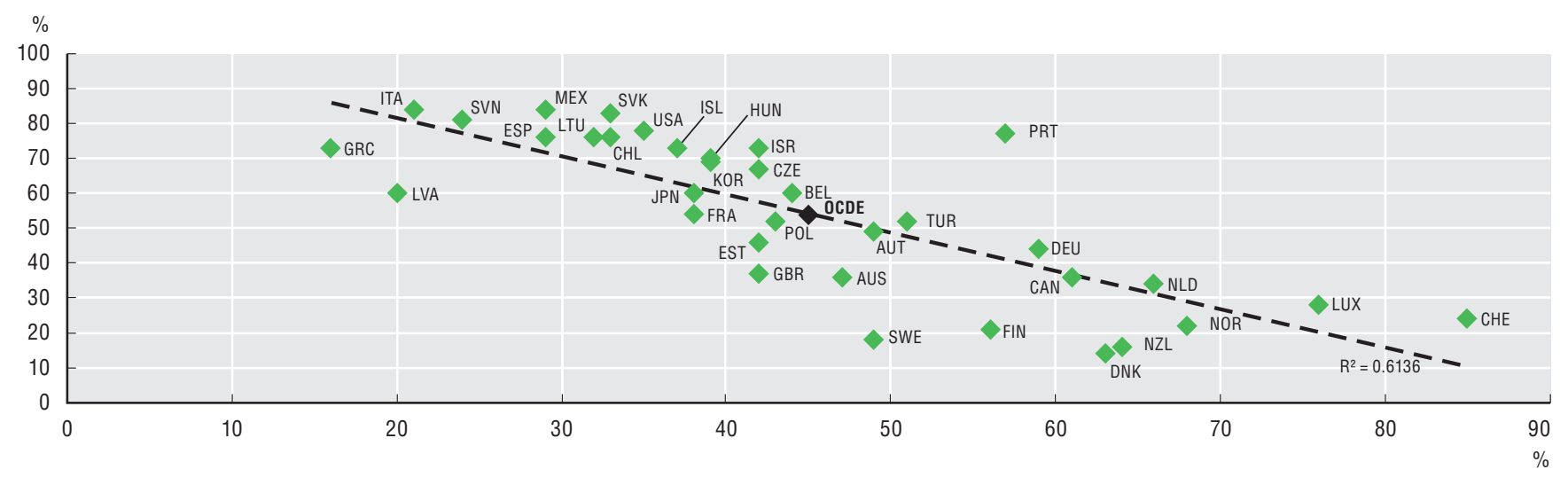

Source : Gallup World Poll, 2018. 
L'efficacité politique externe fait référence à la conviction que l'on a son mot à dire sur l'action des pouvoirs publics. Ceci est important, car les citoyens souhaitent que leurs avis et leurs besoins soient pris en compte dans les décisions des administrations publiques. Ainsi, le sentiment d'efficacité politique externe peut être renforcé ou amoindri en fonction du vécu des administrés face à certaines administrations ou de leur impression que les institutions ne sont pas à l'écoute de leurs besoins (par exemple, en raison de processus d'action publique et de décisions gouvernementales en rupture avec les préférences de l'opinion publique). Le sentiment d'efficacité politique externe est de la plus haute importance pour les systèmes démocratiques, puisqu'il est lié à la conviction que le changement politique et social est possible avec, pour les citoyens, la possibilité de jouer un rôle dans l'avènement de ce changement. Cette conviction est aussi associée à l'idée selon laquelle accomplir ses devoirs civiques vaut la peine (OCDE 2017).

Une longue tradition existe s'agissant de mesurer le sentiment d'efficacité politique externe dans le cadre des enquêtes relatives à la participation politique. Ainsi, aux États-Unis, l'enquête American National Electoral Study rassemble ces données tous les deux ans depuis 1952. Plus récemment, des enquêtes sociales générales (par exemple l'Enquête sociale européenne - ESS) et des enquêtes sur les compétences des adultes (par exemple l'enquête du Programme pour l'évaluation internationale des compétences des adultes PIAAC) ont ajouté ce sujet à leur panoplie d'éléments de mesure. Selon les dernières données disponibles, qui portaient sur 23 pays (essentiellement européens) de l'OCDE, seulement $37 \%$ des citoyens en moyenne, considéraient avoir une influence sur l'action des pouvoirs publics en 2016. On constate néanmoins de fortes disparités entre les pays, puisque ce pourcentage s'échelonne entre environ $74 \%$ en Suisse, pays doté d'une longue tradition de démocratie directe et environ 10 \% seulement en Italie. De 2014 à 2016, le pourcentage de la population estimant pouvoir influer sur l'action des autorités a surtout progressé en Islande $(+24,5$ points) et en Allemagne (+14,4 points) ; à l'inverse, il a connu ses baisses les plus marquées en Pologne (-12,4 points) et en Suède $(-9,9$ points).

D'après les travaux universitaires publiés sur ce thème, le sentiment d'efficacité politique externe est corrélé à la participation citoyenne, à la satisfaction à l'égard de la démocratie, et à la confiance à l'égard des institutions publiques telles que le parlement. Les citoyens qui ont le sentiment de pouvoir influer sur les autorités sont aussi ceux qui présentent les plus forts niveaux de satisfaction à l'égard de la démocratie et de confiance dans le parlement (OCDE à paraître). La Suisse, les Pays-Bas et les pays nordiques tels que la Finlande, la Norvège, et la Suède enregistrent les niveaux les plus élevés de sentiment d'efficacité politique externe, de satisfaction à l'égard de la démocratie et de confiance dans le parlement. À l'inverse, dans les pays de l'Europe du Sud et de l'Est tels que l'Italie, le Portugal, la Pologne, la Lituanie et la Slovénie, les citoyens font moins confiance à leurs élus et sont généralement moins satisfaits de la démocratie.

\section{Méthodologie et définitions}

L'ESS est une enquête scientifique transnationale menée dans toute l'Europe depuis sa création en 2001. Elle est réalisée tous les deux ans, sous forme d'entretiens en face à face avec de nouveaux échantillons transversaux récemment sélectionnés. Chaque pays doit rassembler un échantillon d'au moins 1500 personnes. Pour les petits pays (moins de 2 millions d'habitants), ce nombre est abaissé à 800 . L'enquête vise à mesurer les attitudes, les opinions et les comportements des citoyens d'horizons divers qui vivent dans les pays européens.

\section{Pour en savoir plus}

Borgonovi, F. et Pokropek, A. (2017), "Mind the gap: The mediating role of literacy and education in explaining disparities in external political efficacy in 22 countries", Intelligence, vol. 62, mai 2017, pp. 125137, http://doi.org/ 10.1016/j.intell.2017.03.006

González, S. (à paraître), "Statistical accuracy of responsiveness measures in public governance", Documents de travail de l'OCDE sur la gouvernance publique, Éditions OCDE, Paris.

OCDE (2017), Comment va la vie ? 2017 : mesurer le bien-être, Éditions OCDE, Paris, https://doi.org/10.1787/how_life-2017-fr.

\section{Notes relatives aux graphiques}

Concernant les données sur Israël, voir http://doi.org/10.1787/888932315602.

10.4. Les données de 2016 font référence au pourcentage de personnes ayant répondu " un peu ", " beaucoup " ou "énormément " à "À quelle hauteur estimez-vous que le système politique [du pays concerné] permet à des personnes comme vous d'avoir leur mot à dire sur l'action des autorités ? ". S'agissant des scores pour 2014, ils correspondent aux pourcentages de réponses d'une valeur égale ou supérieure à 5 , sur une échelle qui allait de 0 à 10 (pas du tout ou tout à fait). Les données présentées ici sont des calculs de l'OCDE fondés sur les septième et huitième éditions de l'ESS, dans le cadre desquelles on disposait de données pour 21 pays européens. On dispose de données sur l'Italie dans la huitième édition de l'enquête, mais pas dans la septième. On dispose de données sur le Danemark dans la septième édition de l'enquête, mais pas dans la huitième. On ne dispose de données sur Israël que dans la huitième édition de l'enquête.

10.5. Les données se réfèrent au pourcentage de personnes ayant répondu 5 ou plus sur une échelle de 0 (pas de confiance du tout) à 10 (confiance totale) à : " Dans l'ensemble, dans quelle mesure êtes-vous satisfait du fonctionnement de la démocratie dans votre pays?"

10.6. Les données se réfèrent au pourcentage de personnes ayant répondu 5 ou plus sur une échelle de 0 (pas de confiance du tout) à 10 (confiance totale) à : «Quelle confiance accordez-vous à chacune des institutions, au parlement de votre pays ? ». 
10.4. Avoir son mot à dire sur ce que fait le gouvernement, 2016 et 2016

En \% de la population

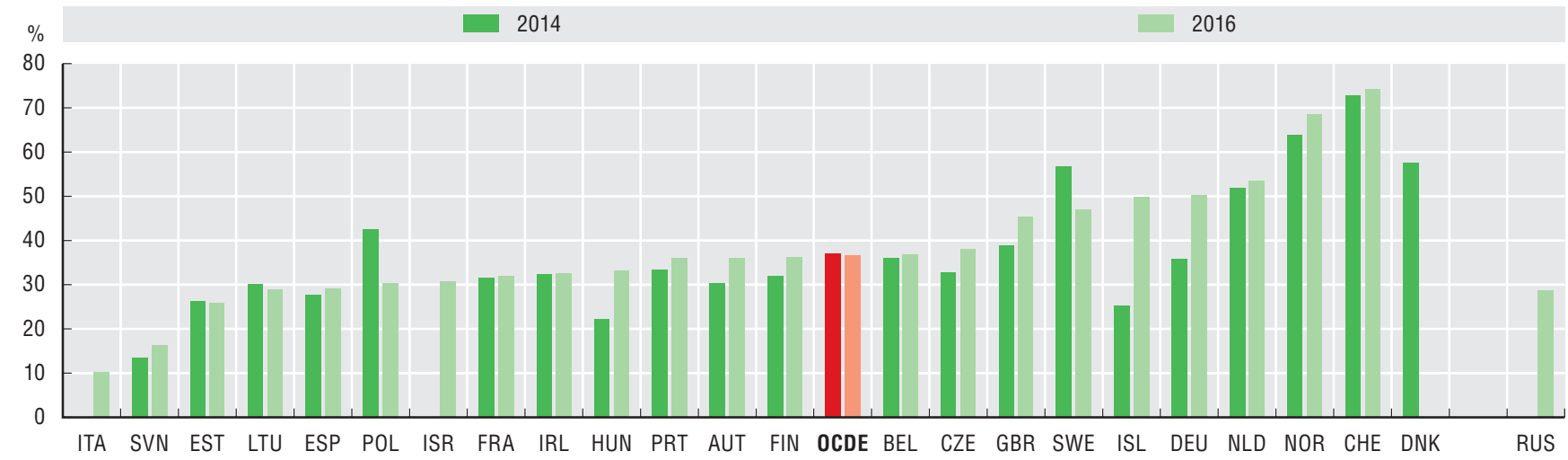

Source : Calculs de l'OCDE à partir des éditions 7 et 8 de l'Enquête sociale européenne.

10.5. Sentiment d'efficacité politique et satisfaction à l'égard de la démocratie, 2016

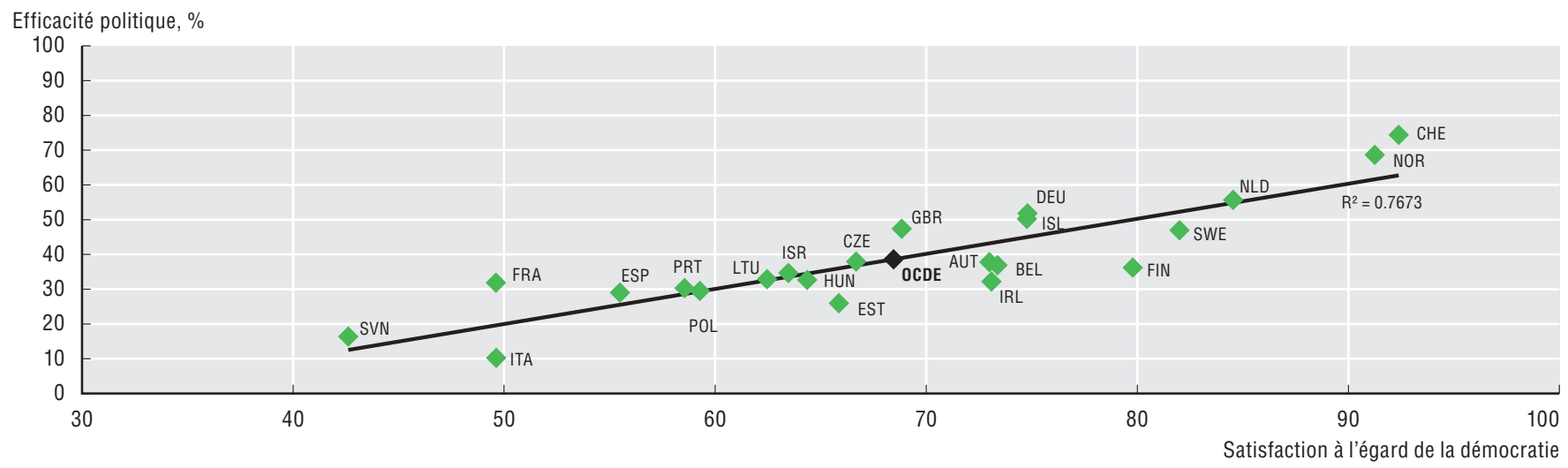

Source : Enquête sociale européenne, 2016.

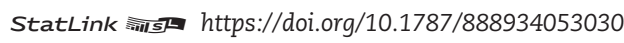

10.6. Sentiment d'efficacité politique et confiance à l'égard du parlement, 2016

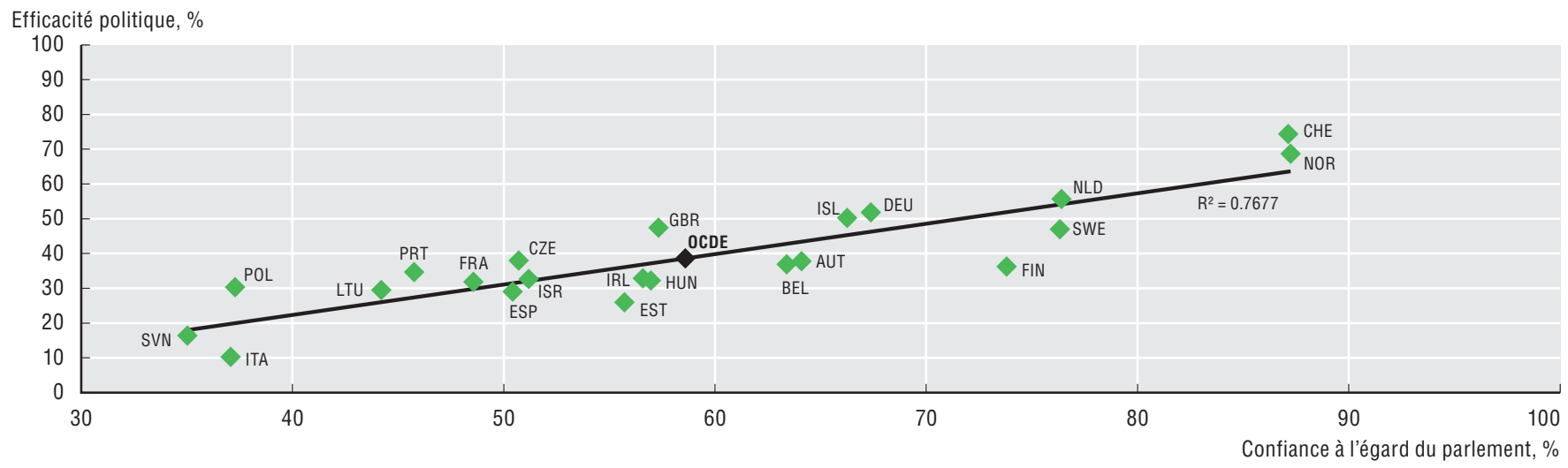

Source : Enquête sociale européenne, 2016. 
L'un des effets les plus répandus de la crise économique de 2007-2008 a été la montée en puissance des inégalités de revenu, qui ont atteint des niveaux historiquement élevés dans de nombreux pays de l'OCDE. Le creusement des inégalités de revenu peut influer sur les conditions matérielles, la formation de capital humain et l'accès aux possibilités économiques de ceux qui occupent les tranches inférieures de l'échelle de distribution des revenus. L'accentuation des inégalités peut aussi conduire à une perte de confiance dans les institutions et dans leur aptitude à mettre en œuvre des politiques qui profitent au plus grand nombre plutôt qu'à une élite. La capacité des pouvoirs publics à rendre le jeu plus équitable, en assurant l'égalité des chances et un niveau de vie minimum, est d'une importance cruciale pour éviter les troubles sociaux et favoriser le bien-être.

Les pouvoirs publics peuvent jouer un rôle plus ou moins important de renforcement ou de réduction des inégalités de revenus, par l'impôt (exonérations d'impôt, par exemple) et par les transferts (allocations ou subventions, par exemple). Le coefficient de Gini est l'élément classique de mesure des inégalités ; il représente la répartition des revenus au sein de la population d'un pays donné. Sa valeur s'échelonne entre 0 (cas de figure où tous les ménages percevraient les mêmes revenus) et 1 (cas de figure où un seul ménage percevrait l'intégralité des revenus). En 2016, le niveau moyen des inégalités de revenus avant impôts s'établissait à 0,47 dans les pays de l'OCDE; il passait à 0,32 après l'intervention des pouvoirs publics. C'est dans les pays européens dotés de systèmes unifiés de protection sociale tels que la Finlande (24,6 points), l'Irlande (23,4 points) et la Belgique (23,3 points) que le niveau de redistribution est le plus élevé.

Le patrimoine des ménages est réparti de façon plus inégale que le revenu des ménages, en raison d'une concentration très forte au sommet de l'échelle de répartition du patrimoine. Sur l'ensemble des pays de l'OCDE, les $10 \%$ de ménages les plus riches détiennent $52 \%$ du patrimoine net total alors que les $10 \%$ d'individus au plus fort revenu ne concentrent que $24 \%$ du revenu total. Les inégalités de patrimoine, telles que mesurées par la part du patrimoine net détenue par les ménages situés dans la tranche supérieure des $10 \%$, sont particulièrement fortes aux États-Unis (79\%) et aux Pays-Bas (68\%) ; c'est au Japon (41\%) et en République slovaque (34\%) qu'elles sont les plus faibles.

Tenir compte de la distribution du patrimoine, au-delà de la seule distribution des revenus, permet d'obtenir une vision plus complète des ressources économiques qui permettent de faire face à tout changement de revenu. Au sein des pays de l'OCDE, on trouve beaucoup de citoyens qui, tout en n'étant pas considérés comme pauvres en termes de revenu, sont économiquement vulnérables en cas de perte soudaine de revenu (liée au chômage, à une rupture familiale ou à l'apparition d'un handicap par exemple). Si ces citoyens cessent brusquement de percevoir un revenu, ils n'ont pas suffisamment d'actifs financiers liquides pour se maintenir audessus du seuil de pauvreté pendant au moins trois mois. C'est en Lettonie $(62,2 \%)$ et en Grèce $(55,4 \%)$ que la vulnérabilité économique est la plus marquée. À l'inverse, c'est au Japon $(13,6 \%)$ et en Corée $(4,3 \%)$ qu'elle est la plus limitée (Balestra et Tonkin 2018). La forte concentration du patrimoine et les niveaux élevés de vulnérabilité économique illustrent le rôle crucial des pouvoirs publics en matière de réduction des inégalités de revenus.

\section{Méthodologie et définitions}

Les données sont tirées des bases de données de l'OCDE sur la distribution des revenus et la distribution des richesses (patrimoine). On mesure la redistribution des revenus en comparant, pour la population d'âge actif, le coefficient de Gini pour le revenu marchand des ménages (c'est-à-dire le revenu total tiré de sources marchandes avant les transferts monétaires publics et les impôts sur les ménages) et le coefficient de Gini pour le revenu marchand disponible des ménages (c'est-à-dire après les transferts publics directs et les impôts directs). Le patrimoine net englobe aussi bien les actifs immobiliers, tels que les résidences secondaires et l'immobilier d'investissement, que les actifs financiers nets des éléments de passif (par exemple dispositifs d'épargne retraite, épargnes personnelles et héritages). On ne tient pas compte du patrimoine-retraite constitué des droits au titre du régime de sécurité sociale et des plans de retraite professionnels à prestations définies. Un individu est pauvre en termes de patrimoine s'il appartient à un ménage dont le patrimoine financier liquide n'est pas suffisant pour se maintenir au-dessus du niveau de pauvreté durant au moins trois mois. Les individus pauvres en termes de patrimoine mais qui ne sont pas pauvres en termes de revenu sont ici décrits comme économiquement vulnérables. La vulnérabilité économique ne tient pas compte des transferts sociaux (par exemple allocations chômage) que les citoyens peuvent recevoir face à certains imprévus. On considère qu'un individu est pauvre en termes de revenu quand le revenu du ménage auquel il appartient est inférieur à un certain seuil, en l'occurrence, inférieur à 50 \% du revenu médian national avant impôt (seuil de pauvreté en termes de revenu).

\section{Pour en savoir plus}

Balestra, C. et Tonkin, R. (2018), "Inequalities in household wealth across OECD countries: Evidence from the OECD Wealth Distribution Database", OECD Statistics Working Papers, $\mathrm{n}^{\circ}$ 2018/01, Éditions OCDE, Paris, https://doi.org/10.1787/ 7e1bf673-en.

\section{Notes relatives aux graphiques}

Concernant les données sur Israël, voir $h$ ttp://doi.org/10.1787/888932315602.

10.7. Les données sur le Canada, la Corée, le Chili, les États-Unis, la Finlande, Israël, la Norvège, le Royaume-Uni et la Suède, concernent 2017 ; 2014 et non 2016 pour la Nouvelle-Zélande. Les données sur l'Islande, le Japon, la Suisse et la Turquie concernent 2015 et non 2017. Le revenu marchand est le revenu après impôt et avant transferts pour le Mexique et la Turquie ; les données ne sont donc pas strictement comparables.

10.8. Les données portent sur 2016 pour les États-Unis, la Finlande, la Lettonie, les Pays-Bas et le Royaume-Uni ; sur 2014 pour l'Australie, la Hongrie et la Nouvelle-Zélande ; et sur 2012 pour le Japon. Dans certains cas, des problèmes d'ordre méthodologique peuvent influer sur les positions relatives des pays.

10.9. Les données portent sur 2016 le Canada ; sur 2014 pour l'Allemagne, l'Australie, l'Autriche, la Belgique, le Chili, la France, la Grèce, la Hongrie, l'Italie, le Japon, la Lettonie, le Luxembourg, la Norvège, la NouvelleZélande, la Pologne, la République slovaque et la Slovénie ; sur 2013 pour l'Estonie, les États-Unis, la Finlande, l'Irlande et le Portugal ; et sur 2012 pour l'Espagne. Dans certains pays, les données sur les revenus tirées de l'enquête sur la richesse sont en retard sur les données relatives aux actifs, ce qui peut affecter la comparaison entre pays. 
10.7. Inégalités de revenus entre les ménages avant et après impôts et transferts publics, 2016

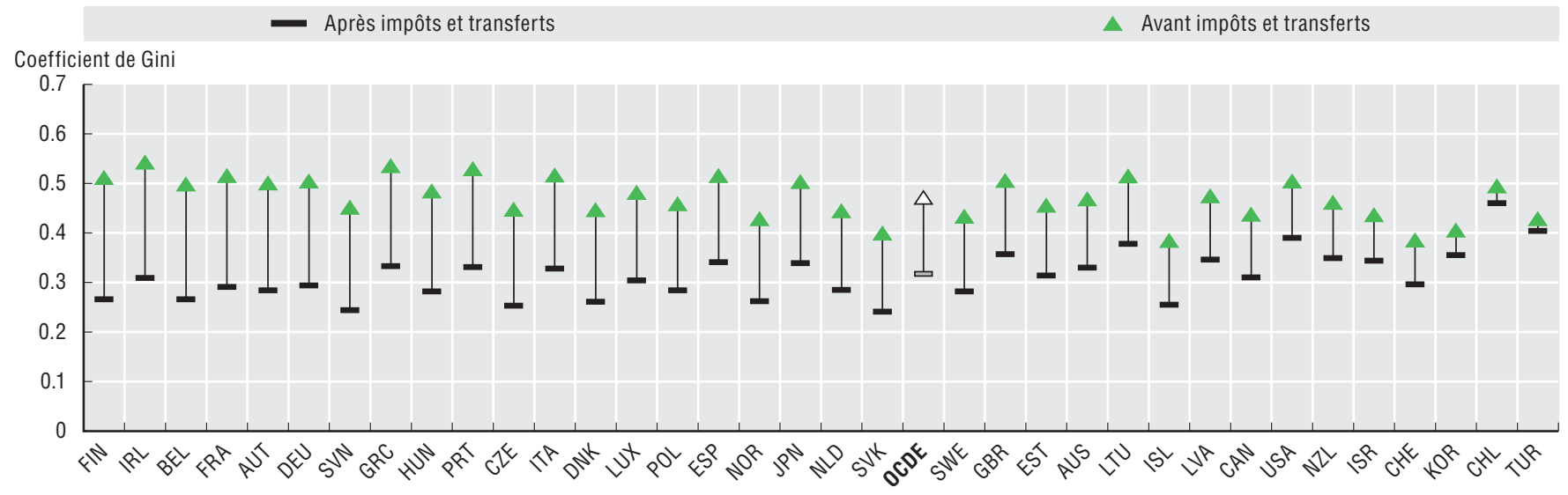

Source: Base de données de l'OCDE sur la distribution des revenus.

StatLink त्ञाI https://doi.org/10.1787/888934053068

10.8. Parts du revenu et du patrimoine des ménages détenues par la tranche supérieure de $10 \%$ des ménages, 2015 ou année la plus proche

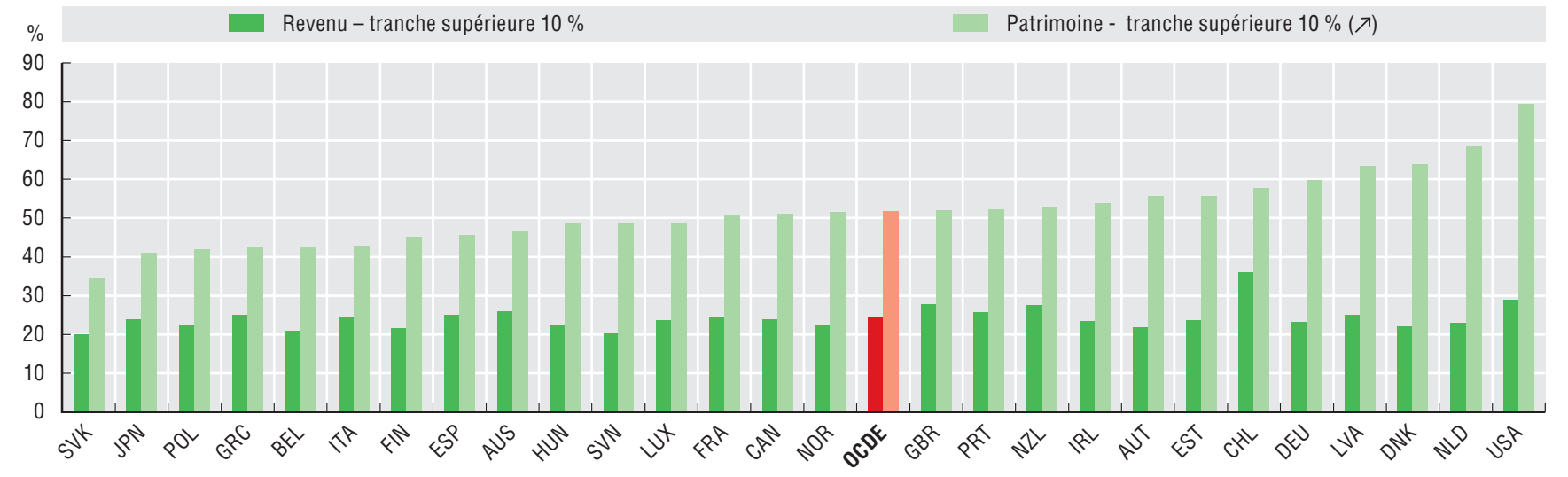

Source : Balestra et Tonkin (2018), à partir des bases de données de l'OCDE sur la distribution des richesses et sur la distribution des revenus.

StatLink नiाlst https://doi.org/10.1787/888934053087

\subsection{Pourcentage d'individus en situation de pauvreté en termes de revenu ou de patrimoine,} 2015 ou année la plus proche

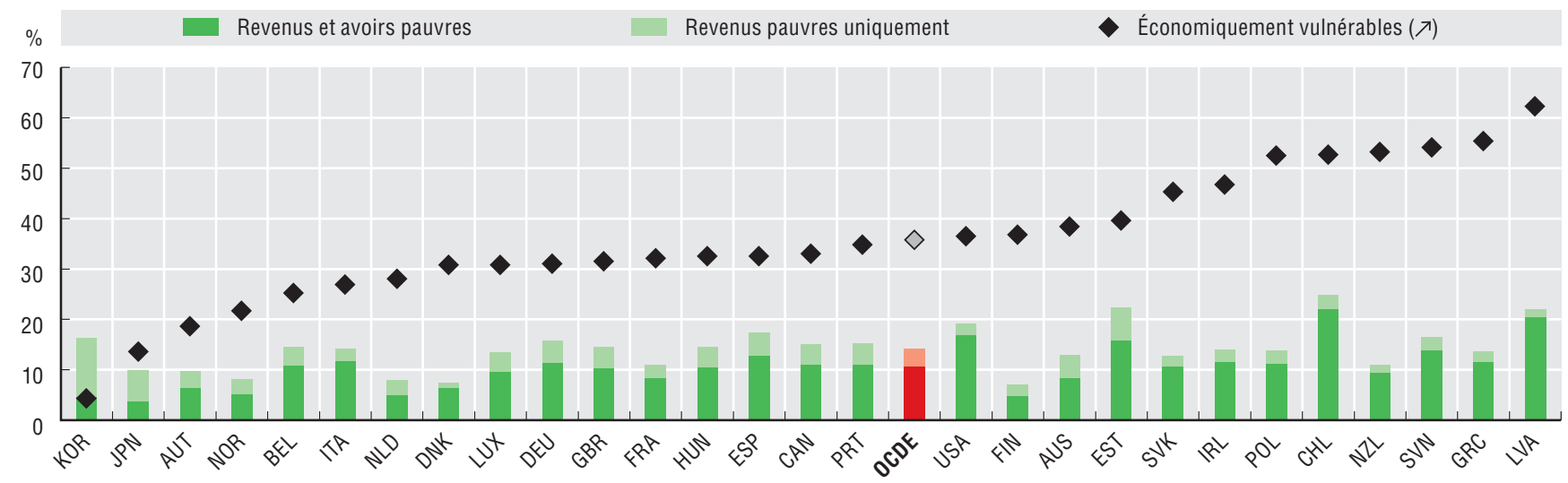

Source : Balestra et Tonkin (2018), à partir de la base de données de l'OCDE sur la distribution des richesses. 
Le principe de l'égalité de tous devant la loi constitue le fondement des systèmes démocratiques. L'état de droit est la notion selon laquelle les mêmes règles, procédures et principes s'appliquent à tous les individus et à toutes les organisations, y compris l'administration elle-même. En pratique, ce concept est inscrit dans des lois, codes et procédures qui garantissent un traitement équitable par les institutions et un accès égal à la justice. Sa mise en application exige ainsi de la prévisibilité, de la fiabilité et de la responsabilité au niveau du système juridique. En soi, l'état de droit est un concept pluridimensionnel qui recouvre des aspects aussi divers que les droits fondamentaux, l'ordre et la sécurité, l'absence de corruption et l'ouverture de l'administration (ou " gouvernement ouvert "). La plupart de ces aspects sont aussi considérés comme des éléments cruciaux d'une bonne gouvernance, car ils sont indispensables au maintien de l'ordre public, au développement économique et à la bonne marche de la fourniture des biens et services publics.

Le World Justice Project (WJP) propose l'une des approches les plus systématiques en termes de conception et de mesure de l'état de droit au niveau mondial. Selon cette méthodologie, le respect de l'état de droit s'appuie sur quatre principes universels : 1) L'administration comme les acteurs privés doivent répondre de leurs actes devant la loi ; 2) Les textes applicables sont clairs, publiés, stables et justes ; ils sont appliqués de manière uniforme et ils protègent les droits fondamentaux, y compris le droit à la sécurité des personnes, des contrats et des biens ainsi que certains droits humains essentiels ; 3) Les procédures de promulgation, d'administration et de mise en application des textes sont accessibles, justes et efficientes ; 4) La justice est rendue en temps utile par des représentants et des intervenants neutres compétents, irréprochables sur le plan éthique et indépendants qui sont accessibles, dotés de ressources appropriées et représentatifs de la composition des populations au service desquelles ils sont placés (WJP 2019). Le WJP mesure l'état de droit à partir de huit facteurs qui visent à évaluer différents aspects de ce concept. On présente ici les résultats pour deux de ces facteurs : les limites aux pouvoirs de l'administration et les droits fondamentaux. Les scores s'échelonnent entre 0 et 1, 1 étant le plus haut score possible et 0 le plus bas score possible.

Le facteur relatif aux limites aux pouvoirs de l'administration évalue dans quelle mesure ceux qui gouvernent sont limités dans leur action par la loi. Il englobe les moyens, aussi bien constitutionnels qu'institutionnels, qui limitent les pouvoirs de l'administration et de ses représentants et agents et qui les obligent à répondre de leurs actes devant la loi. Ce facteur englobe également les dispositifs de contrôle extérieurs à l'administration, tels que la liberté et l'indépendance de la presse (WJP 2019). Pour la zone OCDE, le score moyen est de 0,74 . Plusieurs pays nordiques tels que le Danemark $(0,95)$, la Norvège $(0,94)$, la Finlande $(0,92)$ et la Suède $(0,87)$ obtiennent des scores particulièrement élevés pour ce facteur. À l'autre extrémité de l'éventail, le Mexique $(0,47)$ et la Turquie $(0,29)$ obtiennent des scores inférieurs à 0,5 . Parmi les pays candidats à l'adhésion à l'OCDE et les grandes économies non membres, seul le Costa Rica $(0,78)$ obtient un score supérieur à la moyenne de la zone OCDE. En effet, il dispose de dispositifs robustes permettant de superviser en toute indépendance l'action de l'État, y compris la chambre constitutionnelle de sa Cour suprême (OCDE 2015).

Le facteur relatif à la protection des droits fondamentaux recouvre l'efficacité de la mise en application du droit, la régularité de la procédure ainsi que le respect d'une gamme de droits fondamentaux de l'être humain et du travail qui découlent du droit international. Comme pour le facteur précédent, les pays de l'OCDE obtiennent un score moyen relativement élevé $(0,76)$. Toutefois, des disparités existent entre les pays et le score s'échelonne entre 0,9 ou plus au Danemark et en Finlande et moins de 0,6 en Hongrie, au Mexique et en Turquie. En général, il existe une forte corrélation positive entre les deux facteurs (c'est-à-dire limites aux pouvoirs de l'administration et droits fondamentaux). Cette corrélation montre que les pays qui ont mis en place des contre-pouvoirs face à l'administration garantissent aussi les droits fondamentaux.

\section{Méthodologie et définitions}

Le World Justice Project collecte des données au moyen d'un ensemble de questionnaires basés sur le cadre conceptuel de l'indice de l'état de droit. Les questions sont posées à des échantillons représentatifs du grand public et à des experts du droit interagissant régulièrement avec leurs institutions nationales. Pour le grand public, un échantillon probabiliste de 1000 habitants est sélectionné dans chacun des 126 pays. Pour les experts du droit, 30 experts locaux par pays en moyenne sont interrogés. Ce sont systématiquement de grands instituts de sondage locaux qui réalisent l'enquête. On dispose de données pour 28 pays de l'OCDE et pour 2 candidats à l'adhésion à l'OCDE (la Colombie et le Costa Rica) ainsi que pour 6 partenaires stratégiques de l'Organisation. Toutes les variables utilisées pour chacun des facteurs sont codées et normalisées de façon à obtenir un score compris entre 0 et 1, 1 étant le plus haut score possible et 0 le plus bas score possible. Pour plus d'informations sur les variables utilisées pour construire l'indice, voir https://worldjusticeproject.org/our-work/research-and-data/ wjp-rule-law-index-2019/methodology.

\section{Pour en savoir plus}

OCDE (2015), Costa Rica: Good Governance, from Process to Results, Examens de l'OCDE sur la gouvernance publique, Éditions OCDE, Paris, https://doi.org/10.1787/9789264246997-en.

World Justice Project (2019), Rule of Law Index 2019. World Justice Project, Washington, DC, https://worldjusticeproject.org/sites/ default/files/documents/WJP-ROLI-2019-Single\%20Page\%20 View-Reduced_0.pdf.

\section{Notes relatives aux graphiques}

On ne dispose pas de données pour l'Irlande, l'Islande, Israël, la Lettonie, la Lituanie, le Luxembourg, la République slovaque et la Suisse. 
10.10. Limites aux pouvoirs de l'administration, 2019

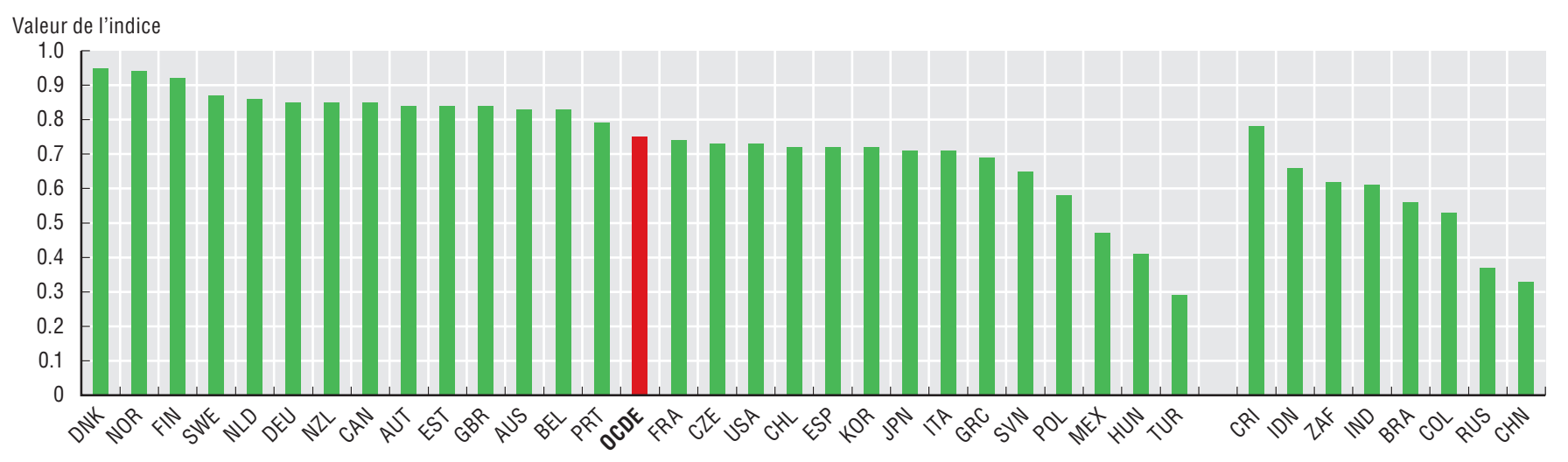

Source : World Justice Project, Rule of Law Index 2019.

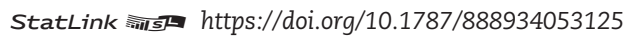

10.11. Droits fondamentaux, 2019

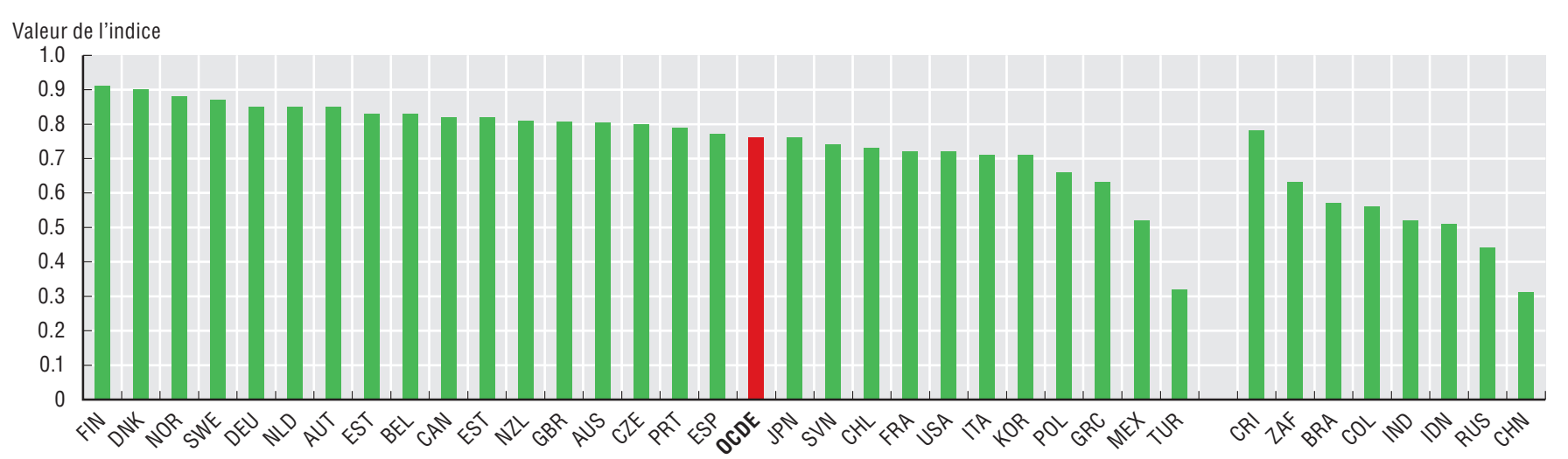

Source : World Justice Project, Rule of Law Index 2019

StatLink न्ताst https://doi.org/10.1787/888934053144

10.12. Limites aux pouvoirs de l'administration et droits fondamentaux, 2019

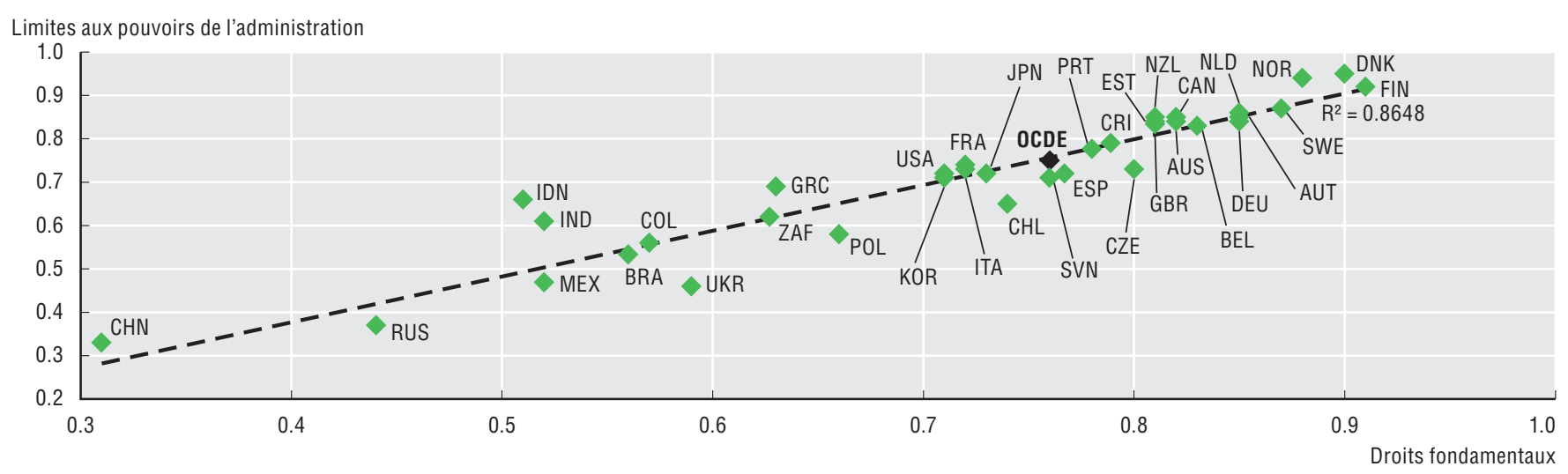

Source : World Justice Project, Rule of Law Index 2019 
L'efficience est le rapport entre un ou plusieurs intrants (ou facteurs de production) et un ou plusieurs extrants (ou produits). Dans un souci d'utilisation plus raisonnée de l'argent du contribuable, et parce que l'efficience est devenue un critère de hiérarchisation entre les nombreuses priorités qui existent en matière de dépenses, les pouvoirs publics et les entités publiques sont soumis à une pression constante en matière de réalisation de gains d'efficience. Par exemple, on attend des administrations fiscales qu'elles collectent dans les meilleurs délais l'ensemble des impôts dus par les citoyens et les entreprises. Dans le secteur de la santé, plusieurs éléments permettent de mesurer l'efficience des soins, y compris un indicateur-clé relatif à la durée moyenne de séjour à l'hôpital (DMS). Toutes choses égales par ailleurs, y compris et surtout les résultats sur le plan de la santé, un séjour plus court réduit les ressources nécessaires et le coût par sortie, ce qui permet de traiter un plus grand nombre de patients avec des ressources données. Toutefois, des séjours plus courts exigent généralement une intensité de services plus élevée et coûtent cher par journée d'hospitalisation. Des séjours trop courts peuvent aussi compromettre les résultats obtenus sur le plan de la santé ou s'avérer préjudiciables au confort du patient ou à son rétablissement.

En 2017, la durée moyenne de séjour à l'hôpital, toutes causes confondues, s'établissait à 7,5 jours dans la zone OCDE. C'était en Turquie que la durée d'hospitalisation était la plus courte (4,1 jours) et en Corée qu'elle était la plus longue (18,5 jours). En moyenne, la DMS a baissé d'environ un jour ces dix dernières années. Toutefois, certains pays ont connu des réductions plus importantes, comme les Pays-Bas (où elle a diminué de 5,8 jours entre 2006 et 2017, la Finlande (4,6 jours) et la NouvelleZélande (3,6 jours). Les pays ont appliqué différentes stratégies pour réduire la DMS tout en préservant ou en améliorant la qualité des soins. Ils ont notamment diminué le nombre de lits d'hôpitaux, développé des programmes de sortie précoce permettant aux patients de recevoir la suite de leurs soins à domicile et favorisé des procédures chirurgicales moins invasives (OCDE 2019). Néanmoins, la durée d'hospitalisation s'est allongée dans certains pays tels que la Corée ( $+3,6$ jours) où faute d'établissements spécialisés dans l'accueil des personnes âgées, celles-ci sont traitées à l'hôpital.

Les impôts versés par les citoyens et les entreprises sont la principale source de financement de la prestation des services publics. Par conséquent, l'activité de recouvrement de l'impôt est très importante pour les administrations publiques. Le processus de recouvrement de l'impôt comporte plusieurs étapes : l'enregistrement, le calcul, la vérification, la collecte et le contentieux. L'étape du calcul englobe toutes les activités liées au traitement des déclarations d'impôt (établissement des avis d'imposition, remboursement, mises en demeure, relevés, etc.) ; elle reflète donc les activités de l'administration fiscale et ses performances. Quand un particulier ou une entreprise dépose une déclaration d'impôt, une dette d'impôt se crée et devient exigible. L'administration fiscale a tout intérêt à faciliter le processus de recouvrement grâce aux outils électroniques, aux déclarations pré-remplies ainsi qu'à tout autre moyen permettant de réduire les délais de recouvrement tout en contribuant à la réduction des coûts.

D'après les dernières données disponibles, sur l'ensemble de la zone OCDE, le pourcentage de respect des délais de déclaration pour l'impôt sur le revenu des personnes physiques (IRPP) s'établit à $87,9 \%$. Ce pourcentage s'échelonne entre $100 \%$ au Danemark, en Espagne et en Norvège et moins de $25 \%$ au Mexique. En dépit de ce pourcentage relativement faible, l'administration fiscale mexicaine a travaillé pour rattraper son retard sur ses homologues en mettant notamment en œuvre une modernisation récente de l'outil technologique permettant de remplir les déclarations d'IRPP. Cette modernisation permet de pré-remplir les déclarations, de calculer automatiquement le montant de l'impôt dû et de raccourcir les délais de remboursement (OCDE 2019). S'agissant des déclarations remplies par les entreprises pour le calcul de l'impôt sur les sociétés (IS), le taux de respect des délais de déclaration s'établit à $81,8 \%$ en moyenne, ce qui est inférieur au pourcentage atteint pour l'IRPP. Ce pourcentage moindre peut s'expliquer par la complexité plus grande du système applicable aux sociétés, et par des retards dans la clôture des comptes annuels des entreprises. L'Espagne est également très bien classée sur le plan du taux de respect des délais de déclaration pour l'IS (100\%). Cela peut s'expliquer, en partie, par le fait que les entreprises ont l'obligation de recourir exclusivement à des outils électroniques pour obtenir un numéro d'identification fiscale, s'inscrire au registre des contribuables et rendre des comptes à l'administration.

\section{Méthodologie et définitions}

La durée moyenne de séjour (DMS) fait référence au nombre moyen de jours que les patients passent à l'hôpital. Elle est obtenue en divisant le nombre total de jours passés par l'ensemble des patients hospitalisés au cours d'une année par le nombre de sorties (toutes causes confondues). Les admissions avec sortie le jourmême ne sont pas prises en compte.

On définit l'IRPP comme l'impôt prélevé sur le revenu net (revenu brut déduction faite des abattements autorisés) et les gains en capital des personnes physiques. On définit l'IS comme l'impôt prélevé sur le bénéfice net (revenu brut déduction faite des abattements autorisés) et les gains en capital des entreprises. On calcule le pourcentage de déclarations respectant les délais en divisant le nombre de déclarations fournies dans les délais par le nombre total de déclarations qui auraient dû être fournies.

\section{Pour en savoir plus}

OCDE (à paraître), Panorama de la santé 2019 : indicateurs de l'OCDE, Éditions OCDE, Paris.

OCDE (2019), Tax Administration 2019: Comparative Information on OECD and other Advanced and Emerging Economies, Éditions OCDE, Paris, https://doi.org/10.1787/74d162b6-en.

\section{Notes relatives aux graphiques}

10.13. Les données présentées pour l'Autriche, le Danemark, les ÉtatsUnis, la France et la Nouvelle-Zélande portent sur 2016; les données présentées pour la Grèce portent sur 2013 et non 2017 ; les données présentées pour la Corée portent sur 2005 et non 2006. On ne dispose pas de données pour le Japon car elles se réfèrent exclusivement à la durée du séjour en soins curatifs (aigus).

10.14. Les données présentées pour la Hongrie portent sur 2016. 
10.13. Durée moyenne du séjour hospitalier, toutes causes confondues, 2006 et 2017

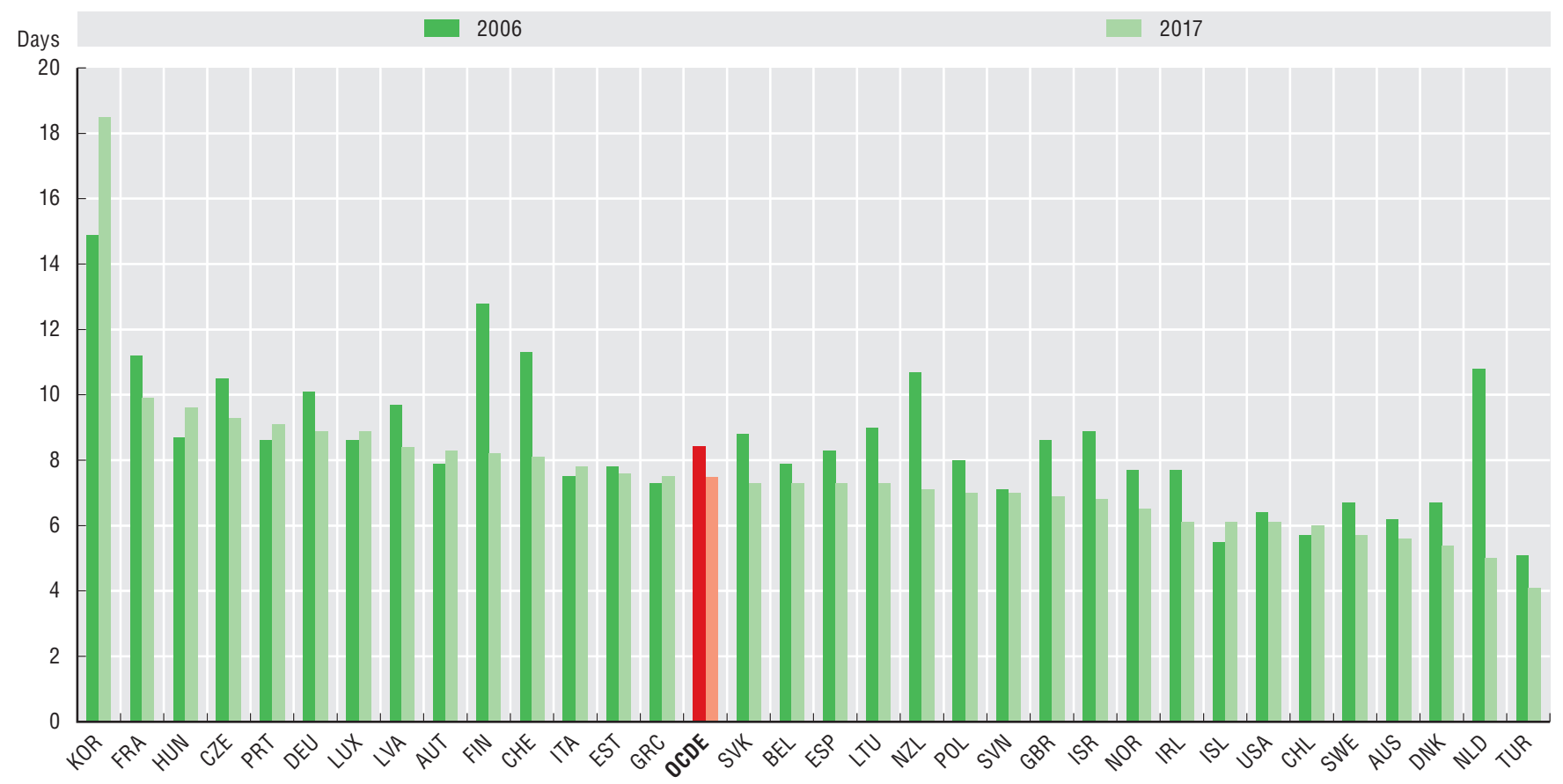

Source : Statistiques de l'OCDE sur la santé (base de données).

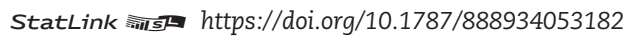

10.14. Taux de respect des délais de déclaration concernant l'impôt sur le revenu des personnes physiques et l'impôt sur les sociétés, 2017

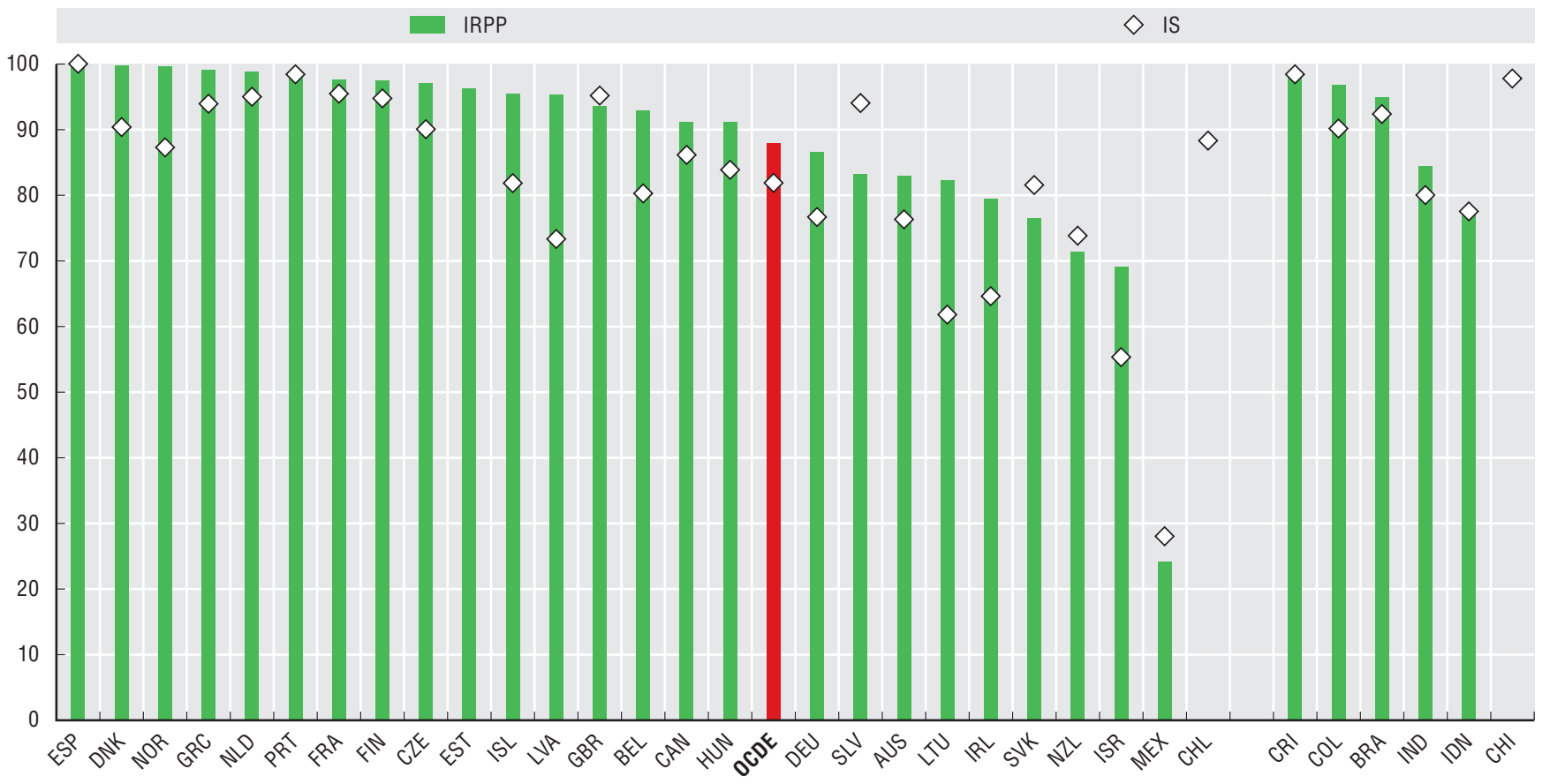

Source : OCDE (2019), base de données sur l'administration de l'impôt. 
Les administrations publiques ont pour mission de veiller à ce que les services publics soient assurés, soit directement, soit en encadrant l'action de prestataires privés. Les travaux universitaires utilisent la notion de rapport coût-efficacité pour mettre en balance les intrants (humains et financiers) et les réalisations sectorielles. Ainsi, les réalisations obtenues sur le plan de la santé et de l'éducation (gains de santé, d'apprentissage, d'avantages financiers nets et d'amélioration de la satisfaction à l'égard des services) résultent de différents facteurs extérieurs dont les politiques publiques menées et des services publics assurés.

\section{Soins de santé}

Le rapport entre les dépenses totales de santé (ou les seules dépenses publiques de santé, qui représentent environ $75 \%$ des dépenses totales de santé) et l'espérance de vie à la naissance constitue un critère général d'appréciation de l'efficacité des dépenses de santé, même si de nombreux autres facteurs influent sur l'espérance de vie des individus. Des dépenses de santé plus élevées sont associées à une plus longue durée de vie, même si c'est avec des rendements décroissants. Au-delà d'un certain seuil, les écarts internationaux sur le plan de l'espérance de vie s'expliquent par des choix politiques et par des facteurs externes tels que l'environnement et le mode de vie de l'individu.

Au Japon, en Italie, en Espagne, en Israël et en Corée, l'espérance de vie est relativement élevée au regard du niveau des dépenses de santé. À l'inverse, aux Etats-Unis, et dans une moindre mesure, en Lettonie, au Mexique et en Hongrie, l'espérance de vie est inférieure aux prévisions de dépenses de santé. Aux États-Unis, ce phénomène s'explique notamment par les facteurs suivants : le très fort morcellement du système de santé américain, au sein duquel les ressources consacrées à la santé publique et aux soins primaires sont relativement faibles; des comportements porteurs d'incidences en termes de santé, y compris des taux d'obésité et le nombre de décès provoqués par des accidents de la route; et des niveaux plus élevés de pauvreté et d'inégalités de revenus que dans la plupart des autres pays de l'OCDE. Entre 2015 et 2017, la crise des opioïdes et le ralentissement de la baisse de la mortalité cardiovasculaire, a contribué à une baisse de l'espérance de vie américaine entre 2015 et 2017 (Raleigh 2019). En Hongrie, en Lettonie et en Lituanie, le niveau relativement faible de l'espérance de vie s'explique principalement par une plus forte exposition à des facteurs de risque (tabagisme, consommation excessive d'alcool et régime alimentaire malsain), surtout chez les hommes.

\section{Éducation}

La création de capital humain et le renforcement des compétences représentent deux objectifs cruciaux pour le secteur de l'éducation, en raison de leurs effets bénéfiques sur les perspectives d'emploi et les revenus tout au long de la vie. Il existe plusieurs moyens possibles de mesurer le rapport coût-efficacité du secteur de l'éducation. L'une des possibilités consiste à examiner quel est, pour l'administration, le rendement financier net (coûts moins avantages financiers) tout au long de la vie professionnelle des bénéficiaires de l'éducation. En moyenne de la zone OCDE, le rendement financier net public lié à l'obtention d'un diplôme de l'enseignement supérieur s'établit à environ 148200 USD pour un homme et à 77300 USD pour une femme. Pour un homme, c'est au Luxembourg que ce rendement financier public est le plus élevé (373 300 USD) ; pour une femme, c'est aux Pays-Bas (223 600 USD). Les écarts de rendement financier net public selon le sexe des individus s'expliquent par le fait que les femmes perçoivent des rémunérations moins élevées et connaissent un taux d'emploi plus faible. Cet état de fait peut aussi découler, entre autres, de différences en termes de secteur d'activité, d'évolution de carrière et de type de métier et de contrat, d'une conciliation difficile entre vie professionnelle et vie de famille et d'un écart de rémunération entre hommes et femmes, pour des emplois similaires.

\section{Méthodologie et définitions}

L'espérance de vie à la naissance mesure le nombre moyen d'années pendant lequel un nouveau-né peut espérer vivre en l'absence d'évolution des taux de mortalité. Les dépenses courantes de santé totales correspondent à la consommation finale de produits et services de santé, à l'exclusion des investissements dans les infrastructures de santé. Ce chiffre englobe les dépenses des acteurs tant publics que privés consacrées aux biens et services médicaux, aux programmes de prévention et à l'administration de la santé. Les valeurs attribuées aux coûts et aux avantages de l'éducation se fondent sur l'écart de situation entre les hommes ou femmes qui ont obtenu un diplôme de l'enseignement supérieur et ceux ou celles qui ont obtenu un diplôme du second cycle de l'enseignement secondaire. Dans le cadre de cette analyse, les coûts englobent à la fois les coûts directement engagés par les administrations publiques en matière d'éducation et leur manque à gagner en termes d'impôts sur le revenu ; et les avantages correspondent aux impôts sur le revenu et aux cotisations sociales encaissés en plus. Pour les administrations publiques, les coûts directs représentent la plus grande part des coûts publics totaux de l'enseignement supérieur, même si l'on ne tient pas compte dans cet indicateur des prêts étudiants. On définit les diplômés de l'enseignement supérieur comme les individus qui ont atteint au moins l'un des niveaux suivants au sein de la Classification internationale type de l'éducation (CITE) : enseignement supérieur de cycle court (CITE 5), niveau licence ou équivalent (CITE 6), niveau master ou équivalent (CITE 7) ou niveau doctorat ou équivalent (CITE 8).

\section{Pour en savoir plus}

OCDE (2019), Regards sur l'éducation 2019 : les indicateurs de l'OCDE, Éditions OCDE, Paris, https://doi.org/10.1787/6bcf6dc9-fr.

OCDE (2017), Panorama de la santé 2017 : les indicateurs de l'OCDE, Éditions OCDE, Paris, https://doi.org/10.1787/health_glance2017-fr.

Raleigh, V. (2019), "Trends in life expectancy in EU and other OECD countries: Why are improvements slowing?", Documents de travail de l'OCDE sur la santé, $\mathrm{n}^{\circ} 108$, Éditions OCDE, Paris, https://doi.org/10.1787/223159ab-en.

\section{Notes relatives aux graphiques}

Concernant les données sur Israël, voir http://doi.org/10.1787/ 888932315602

10.15. Toutes les données relatives aux dépenses courantes de santé par habitant portent sur 2017

10.16. Les résultats se fondent sur les revenus d'activité nets des adultes diplômés de l'enseignement supérieur (comparés aux revenus d'activité nets des adultes diplômés de l'enseignement secondaire). Les données relatives au Chili, à la France, à l'Italie et à la République tchèque portent sur 2015 et celles sur les aux Pays-Bas portent sur 2014. 
10.15. Espérance de vie à la naissance et dépenses de santé totales par habitant, 2017 ou année la plus proche

Espérance de vie à la naissance (en années)

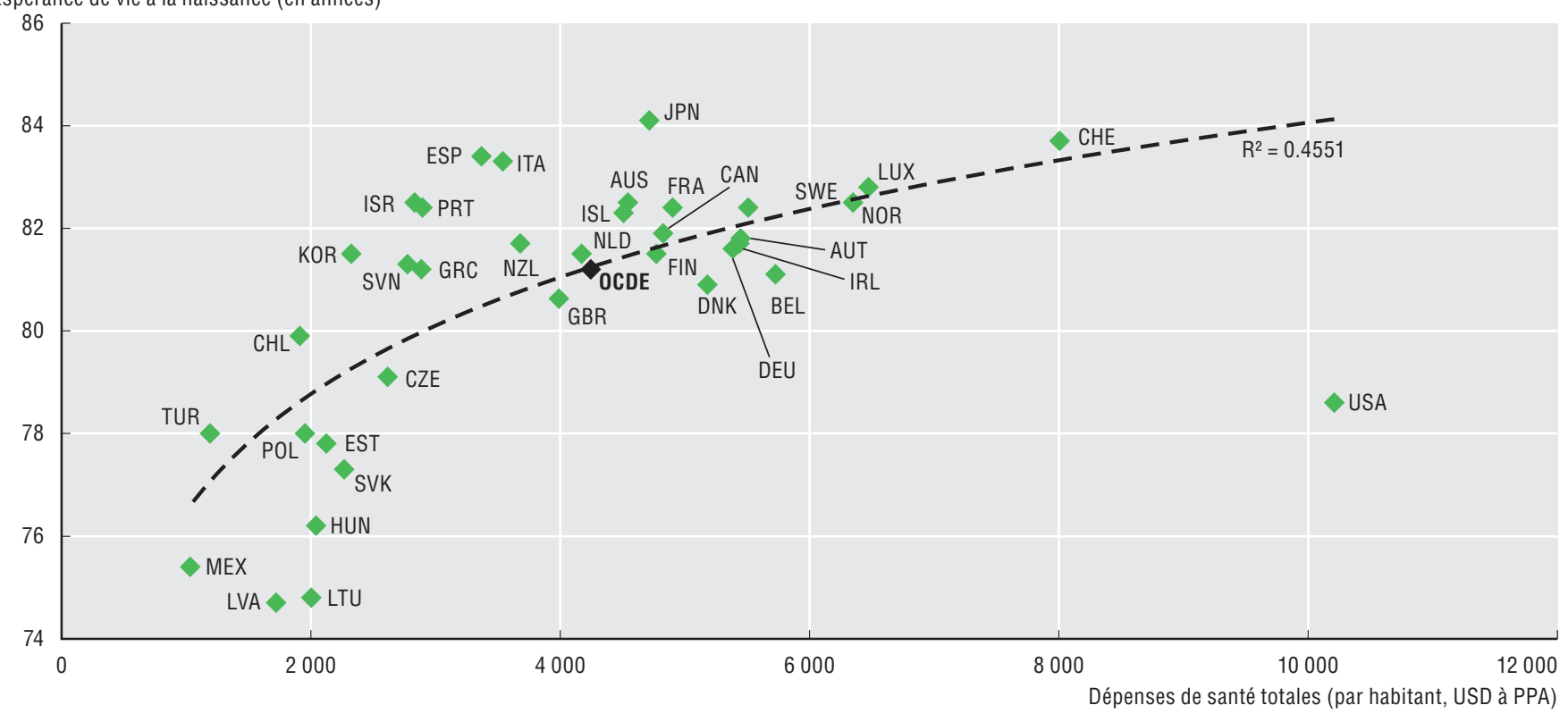

Source : Statistiques de l'OCDE sur la santé (base de données).

StatLink नillst https://doi.org/10.1787/888934053220

10.16. Coûts et avantages publics de l'obtention d'un diplôme d'enseignement supérieur par un homme ou une femme, 2016

Par rapport au rendement de l'obtention d'un diplôme d'enseignement secondaire, en équivalents USD à PPA pour le PIB. Les coûts et avantages futurs sont actualisés à un taux de $2 \%$.

Avantages totaux des hommes Coûts totaux des hommes

Rendement financier net

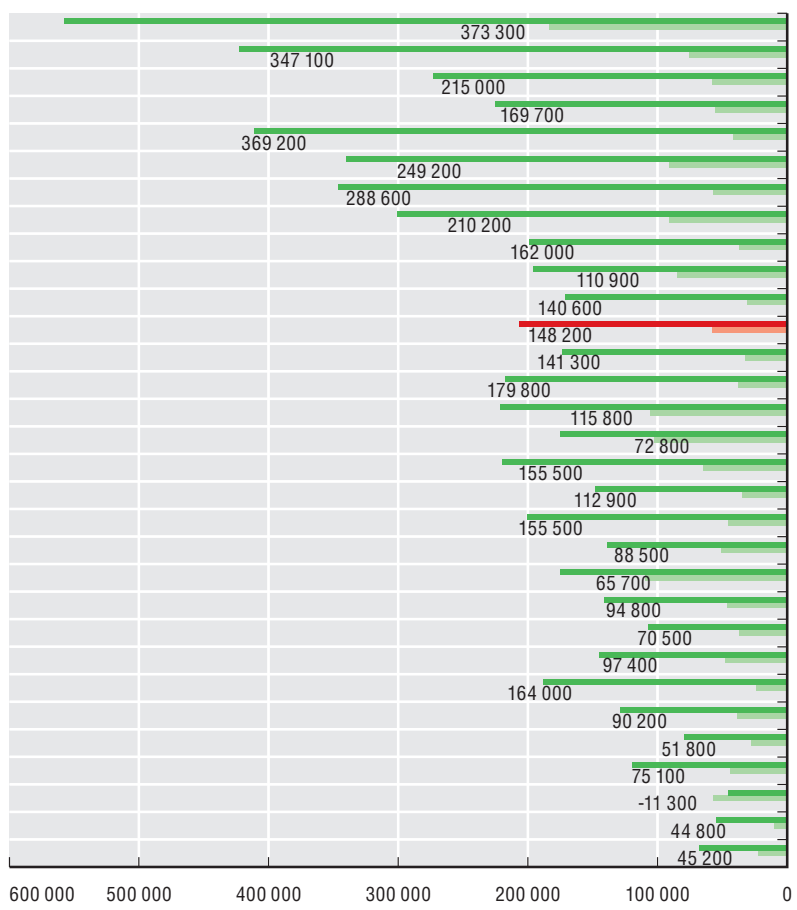

Avantages totaux des femmes

Coûts totaux des femmes

Rendement financier net

Source: Base de données Regards sur l'éducation.

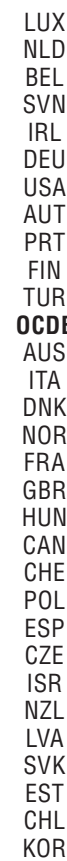

NLD

BEL

SVN

DEU

USA

PRT

FIN

TUR

AUS

ITA
DNK

NOR

GUR

CAN

CHE

ESP

CZE

NZL

LVA

EST

CHL

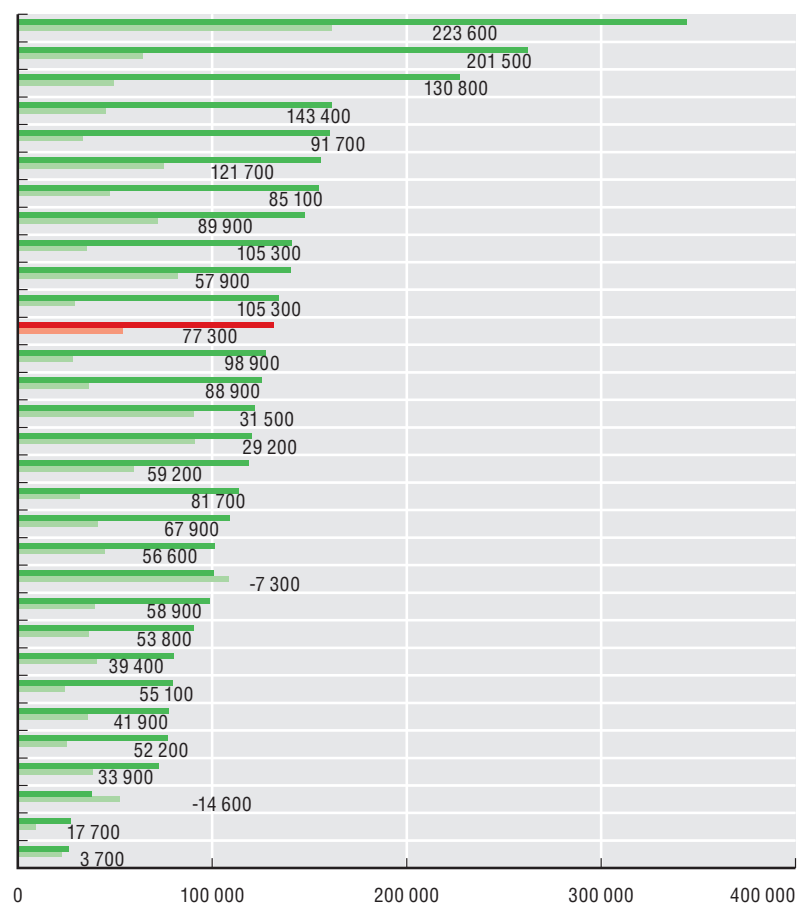




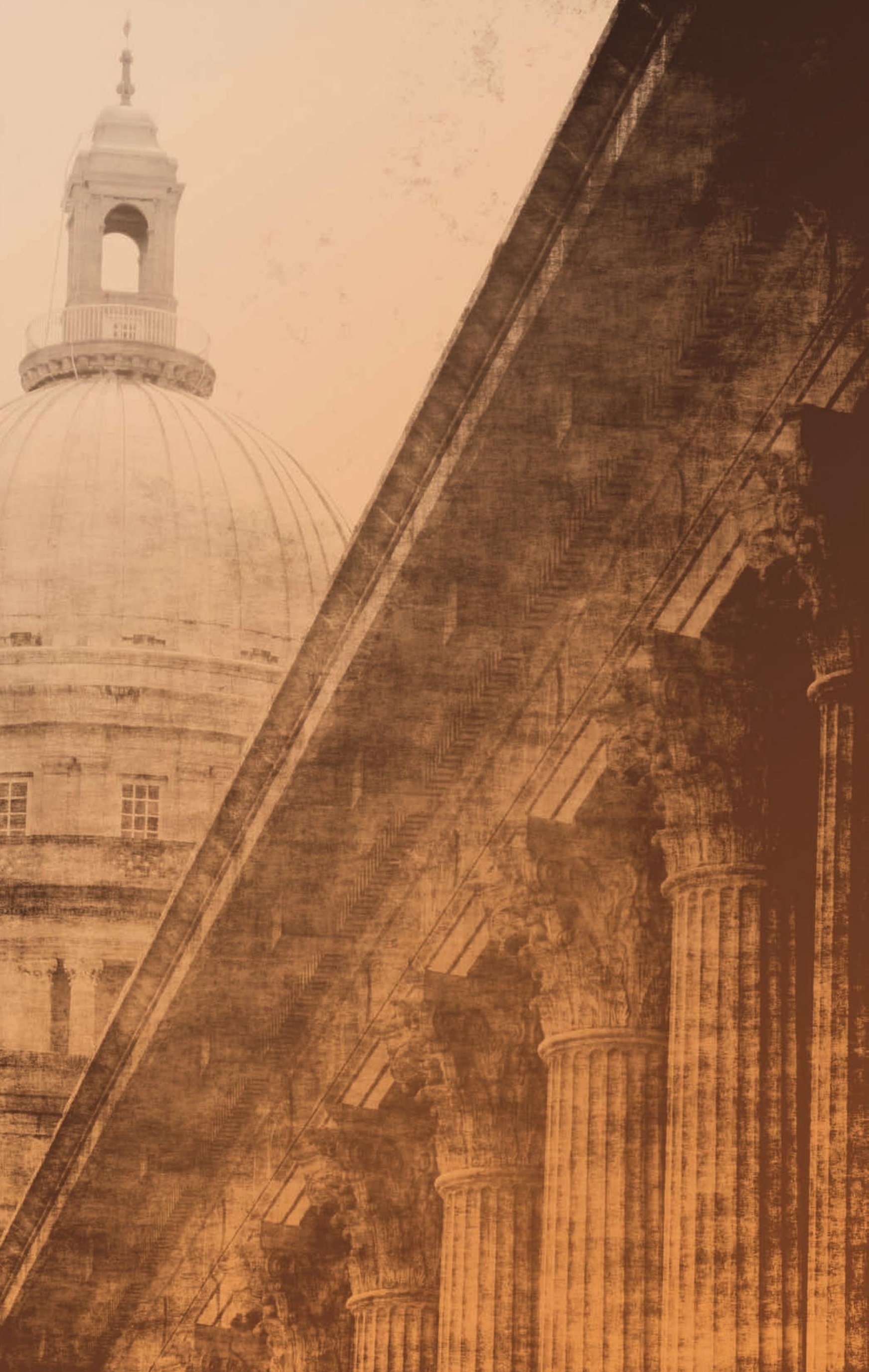




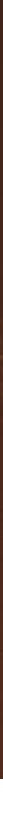

\section{SERVIR LES CITOYENS}

Tableaux de bord des services rendus aux citoyens

Satisfaction des citoyens à l'égard des institutions et des services publics

Accessibilité financière et géographique des soins de santé Accessibilité financière à l'éducation

Accès aux services juridiques et judiciaires

Réactivité des systèmes de santé face aux besoins des patients Réactivité des systèmes éducatifs face aux besoins des élèves Rapidité des services de justice civile Qualité des soins de santé Résultats scolaires et équité dans l'éducation Efficacité et équité des systèmes judiciaires 
Ce chapitre décrit les performances des pays de l'OCDE sur le plan de l'accessibilité, de la réactivité et de la qualité des services publics et se fonde pour cela sur le cadre d'analyse " Au service des citoyens » de l'OCDE. Les tableaux de bord proposent une synthèse des performances du système national (accès, réactivité et qualité) par le biais d'une sélection d'indicateurs sectoriels portant sur l'éducation, la santé et la justice. Les indicateurs retenus illustrent la manière dont la performance des services publics peut être comparée, même lorsqu'ils sont organisés de manière distincte et abordent différents aspects de la vie sociale et individuelle. Bien que les classements par pays soient fournis, ceux-ci sont calculés pour comparer des indicateurs qui diffèrent en termes d'unités de mesure et de phénomènes sous-jacents. Par conséquent, les tableaux de bord ne donnent pas une image unifiée des pays qui offrent les meilleurs services globaux et ne sont pas prévus à cet effet.

Les tableaux de bord des services rendus aux citoyens ont été introduits dans l'édition 2017 du Panorama des administrations publiques et les indicateurs ont été sélectionnés par des experts de l'OCDE sur chaque sujet. Les critères étaient les suivants : 1) adéquation (l'indicateur représente le concept à mesurer), 2) pertinence politique, 3) disponibilité et couverture des données, et 4) interprétabilité des données (c'est-à-dire qu'il n'existe aucune corrélation entre la mesure d'une valeur plus élevée ou plus basse et une performance meilleure ou plus mauvaise). Les indicateurs sélectionnés ont pour but de donner un aperçu des aspects pertinents pour chaque secteur. C'est pour cette raison que le choix des mesures diffère d'un secteur à l'autre (par exemple, la scolarisation pour l'éducation et la couverture des soins de santé pour la santé sont des mesures d'accès). Dans cette édition, les mesures choisies pour les tableaux de bord sont les mêmes que dans l'édition 2017 du Panorama des administrations publiques. Seules celles qui ont été actualisées sont présentées dans ce chapitre.

Cadre OCDE « Au service des citoyens"

\begin{tabular}{|c|c|c|}
\hline Accessibilité & Réactivité & Qualité \\
\hline Accessibilité financière & Courtoisie et traitement & Efficacité de la prestation des services et des réalisations \\
\hline $\begin{array}{l}\text { - couverture des soins de santé } \\
\text { - besoins de santé non satisfaits } \\
\text { - Poids du reste à charge dans la consommation } \\
\text { des ménages } \\
\text { - dépenses privées consacrées à l'éducation } \\
\text { (de l'enseignement primaire au supérieur) } \\
\text { - taux de scolarisation des enfants de } 4 \text { ans } \\
\text { - taux d'accès à l'enseignement supérieur } \\
\text { - La justice civile est accessible et abordable } \\
\text { - Les mécanismes alternatifs de règlement des } \\
\text { litiges sont accessibles, impartiaux et efficaces }\end{array}$ & $\begin{array}{l}\text { - Un médecin régulier les a impliqués autant qu'ils } \\
\text { le voulaient dans les décisions de traitement ou de } \\
\text { soins }\end{array}$ & $\begin{array}{l}\text { - taux de mortalité - infarctus aigu du myocarde (crise cardiaque } \\
\text { - taux de mortalité - accident vasculaire cérébral (AVC) } \\
\text { - taux de mortalité - cancer du sein chez les femmes } \\
\text { - mortalité sous trente jours après une hospitalisation pour } \\
\text { - un IAM } \\
\text { - admission à l'hôpital pour asthme et maladie pulmonaire } \\
\text { obstructive chronique (MPOC) chez les adultes } \\
\text { - admission à l'hôpital pour insuffisance cardiaque congestive } \\
\text { (ICC) chez l'adulte } \\
\text { - scores PIRLS } \\
\text { - application effective de la justice civile }\end{array}$ \\
\hline $\begin{array}{l}\text { Proximité géographique } \\
\text { - densité de médecins par région TL2 }\end{array}$ & $\begin{array}{l}\text { Correspondance entre les services et les besoins } \\
\text { spécifiques } \\
\text { - pourcentage de jeunes sortant de l'enseignement } \\
\text { primaire et supérieur âgés de } 18 \text { à } 24 \text { ans qui ne } \\
\text { travaillent pas } \\
\text { - écoles manquant drenseignants qualifiés pour } \\
\text { enseigner aux élèves ayant des besoins spécifiques } \\
\text { dans le premier cycle de l'enseignement secondaire } \\
\text { - pourcentage de décisions prises au niveau de l'école } \\
\text { dans le premier cycle de l'enseignement secondaire }\end{array}$ & $\begin{array}{l}\text { Cohérence de la prestation des services et des réalisations } \\
\text { - score PIRLS pour les étudiants qui fréquentent une école } \\
\text { où plus de } 75 \% \text { des étudiants entrent avec quelques } \\
\text { compétences en lecture et en écriture } \\
\text { - score PIRLS selon le sentiment d'appartenance à l'école } \\
\text { et pourcentage d'élèves manifestant un fort sentiment } \\
\text { d'appartenance }\end{array}$ \\
\hline Accès à l'information & Rapidité & Sécurité/sûreté \\
\hline $\begin{array}{l}\text { - pourcentage de personnes ayant bénéficié de } \\
\text { conseils juridiques et ayant pris des mesures } \\
\text { pour résoudre leurs litiges au cours des } 2 \\
\text { dernières années } \\
\text { - trois raisons principales de ne pas tenter } \\
\text { d'obtenir l'assistance d'un avocat pour résoudre } \\
\text { un litige } \\
\text { - raisons pour ne pas agir en cas de un litige } \\
\text { - les mécanismes alternatifs de résolution des } \\
\text { conflits sont accessibles, impartiaux et efficaces }\end{array}$ & $\begin{array}{l}\text { - attente deux mois ou plus pour un rendez-vous chez } \\
\text { - un spécialiste } \\
\text { - intervention chirurgicale pour fracture de la hanche } \\
\text { - temrès l'admission à l'hôpital } \\
\text { commerciales, administratives et autres (non contesté) } \\
\text { - délai de règlement des affaires civiles et commerciales } \\
\text { contentieuses (première instance) } \\
\text { - temps de décision pour les affaires administratives } \\
\text { (cour suprême) }\end{array}$ & $\begin{array}{l}\text { - les personnes n'utilisent pas la violence pour régler leurs } \\
\text { griefs personnels }\end{array}$ \\
\hline
\end{tabular}

Note : Les indicateurs en italique sont inclus dans les tableaux de bord.

\section{Interprétation des tableaux de bord}

Chaque tableau de bord se concentre sur une dimension du cadre et compare tous les services (éducation, santé et justice). Pour chaque indicateur, les pays sont classés en trois quantiles en fonction de leur performance : 1) tiers supérieur (vert) ; 2) tiers intermédiaire (orange) ; 3) tiers inférieur (rouge).

On indique, à titre de complément d'information, le rang de chaque pays par rapport aux autres pays pour lesquels on dispose de données, de manière à fournir des informations supplémentaires sur les performances (le pays avec la meilleure performance est classé numéro 1). Si plusieurs pays affichent la même valeur pour un indicateur, ils se voient attribuer le même rang.

Quand on dispose d'indications sur l'évolution du score, des flèches indiquent si le bilan du pays s'est amélioré ( $\uparrow$ ), s'est dégradé $(\downarrow)$ ou est resté stable $(\rightarrow)$. La dernière ligne du tableau indique l'année de base et l'année de référence, à des fins de comparaison. 


\section{Accessibilité des services}

La plupart des pays de l'OCDE ont mis en place une couverture des soins de santé universelle, dans le cadre de régimes d'assurance privés ou publics. Par rapport à 2013, le taux de couverture est resté stable dans les pays de tête du classement. En Grèce, sous l'effet de la crise, 30 \% de la population environ a perdu en 2013 son accès aux soins. Pour remédier à cette situation, les autorités ont adopté en 2016 une législation destinée à assurer le financement du régime et à rétablir la couverture universelle. En Hongrie, une série de réformes, comprenant la dissolution du ministère de la Santé, s'est traduite par un recul du taux de couverture (passé d'une couverture universelle en 2011 à 94 \% en 2017). Celui-ci a en revanche augmenté aux États-Unis et au Mexique et grâce, respectivement, au Patient Protection and Affordable Care Act (PPACA) en 2010 et à l'introduction et à l'expansion du Seguro Popular en 2004. Leur taux de couverture des soins de santé se situait aux environs de 90 \% en 2017.

L'éventail des services couverts par les régimes d'assurance maladie et le poids des frais de santé supportés in fine par le patient (le “ reste à charge ») varient selon les pays de l'OCDE. Néanmoins, le poids du reste à charge dans la consommation des ménages n'indique pas, en soi, si les citoyens ont accès aux soins. Il est, par exemple, élevé en Autriche, en Espagne et en Suisse, même si ces pays ont le plus faible pourcentage de besoins non satisfaits. À l'inverse, le poids du reste à charge est relativement faible en Slovénie et au Royaume-Uni, mais les listes d'attente y sont plus longues car le nombre de médecins en exercice y est inférieur à celui d'autres pays.

Dans tous les pays de l'OCDE, les systèmes éducatifs assurent l'accès universel à la scolarité pour les enfants en âge de scolarisation obligatoire, âge qui varie toutefois selon les pays. L'accès aux établissements d'éducation de la petite enfance et d'enseignement supérieur dépend en partie des ressources publiques mises à disposition pour leur financement. La Belgique est l'un des pays où les sources privées contribuent pour la plus faible part au financement de l'enseignement, du cycle primaire aux études supérieures et compte parmi ceux qui affichent les taux d'inscription les plus élevés dans l'enseignement préélémentaire et supérieur. Au contraire, les États-Unis sont lourdement tributaires des financements privés (à la fois avant et après transferts intersectoriels), ce qui se traduit par des taux d'inscription relativement bas dans ces deux cycles (préélémentaire et supérieur).

La part élevée du financement privé dans certains pays est due aux bourses et transferts aux individus ou aux établissements privés. Le Royaume-Uni, par exemple, affiche un taux de scolarisation de $100 \%$ dans l'enseignement préélémentaire car tous les enfants ont droit, dès l'âge de quatre ans, à quinze heures d'accueil gratuit dans un établissement public ou privé. En 2016 , le Chili a adopté une loi permettant l'accès gratuit des étudiants économiquement défavorisés à l'enseignement supérieur, alors qu'ils se voyaient auparavant accorder des bourses et des prêts. La majorité des étudiants devraient bénéficier de cette réforme en 2020. En 2017, de tous les pays membres de l'OCDE, le Chili était celui qui affichait le taux d'inscription d'élèves nationaux le plus élevé. Afin d'accéder à la justice, les citoyens doivent à la fois être informés de leurs droits et des mécanismes existant pour régler leurs litiges et être en mesure d'assumer les coûts liés aux procédures. Selon le World Justice Project (WJP), c'est aux Pays-Bas, en Allemagne et en Suède que les obstacles à l'accès à la justice civile (en termes de sensibilisation, d'équité de traitement et de financement) sont les moins nombreux. Le Mexique, les États-Unis et le Royaume-Uni sont, à l'inverse, les pays où les citoyens se heurtent aux plus grandes difficultés à cet égard. Les mécanismes alternatifs de règlement des litiges (MARL) représentent un moyen pour régler les différends sans recourir aux tribunaux. L'enquête menée par le WJP auprès d'experts porte sur l'intégrité des arbitres, les coûts et les délais des MARL et la mise à exécution des accords amiables conclus à la suite de contentieux commerciaux. Les pays proposant les MARL les plus accessibles sont la Corée, le Japon et la Norvège. Les MARL les moins accessibles se situent en Hongrie, au Mexique, et en Suède.

\section{Qualité des services}

La prestation de services publics vise à améliorer la qualité de vie et le bien-être des citoyens dans différents domaines. Les systèmes de santé sont par exemple chargés de protéger les citoyens des risques sanitaires, tandis que le système judiciaire remplit pour sa part une fonction importante en garantissant l'état de droit et le respect des droits humains, ce qui donne à la population un sentiment de sécurité.

Les indicateurs sélectionnés pour les tableaux de bord Servir les Citoyens sont axés sur la qualité des politiques de santé dans leur ensemble y compris la prévention. En ce sens, ils diffèrent de ceux utilisés dans Panorama de la Santé, où la qualité des soins fournis aux patients est adressée (par exemple, hospitalisations évitables, traumatismes obstétricaux). Cette distinction est pertinente car les indicateurs affichés dans les tableaux de bord Servir les Citoyens reflètent également les attitudes de la population en matière de soins personnels (par exemple suivre le calendrier des visites médicales recommandés). Dans cette publication, la qualité des services de soins de santé fournis aux patients est adressée dans les deux pages sous ce nom.

Les pays de l'OCDE sont parvenus à réduire la mortalité associée aux maladies contagieuses. Les principales causes de décès sont les crises cardiaques, les accidents vasculaires cérébraux (AVC) et les cancers, qui sont souvent liés au mode de vie et aux habitudes des individus, tels que le tabagisme ou certaines pratiques alimentaires. Les actions de prévention, les interventions précoces et le traitement de ces maladies figurent au premier rang des stratégies mises en œuvre par les systèmes de santé pour diminuer la mortalité qui leur est associée. Ces dernières années, la plupart des pays ont réussi à réduire le nombre de décès associés à ces maladies.

L'Espagne compte parmi les pays qui affichent la plus faible mortalité liée aux crises cardiaques, aux AVC et aux cancers du sein. Les autres pays de l'OCDE pour lesquels on dispose de données obtiennent des résultats supérieurs aux autres pour une ou deux de ces maladies, et inférieurs pour le reste. Le Mexique et la Turquie, par exemple, font partie des pays qui enregistrent la plus faible mortalité liée au cancer du sein, et l'une des mortalités les plus élevées associées aux crises cardiaques. On constate le contraire en Belgique et aux Pays-Bas.

S'agissant du système judiciaire, le WJP recueille des données sur la mise en application du droit dans le monde entier au travers d'enquêtes menées auprès d'experts et auprès du grand public. Les questions portent sur la probabilité que les individus se fassent eux-mêmes justice ; que les autorités influencent un juge dans le cadre d'une action contre l'État ; et que les décisions de justice soient exécutées. La Suède et la Norvège sont les pays qui obtiennent les meilleurs résultats sur ces différents plans ; la Grèce, le Mexique et la Turquie sont, à l'inverse, ceux où la mise en application du droit laisse le plus à désirer. Certains pays obtiennent de meilleurs résultats dans un domaine plutôt que d'autres. En Hongrie, par exemple, les citoyens ont moins tendance à se faire justice eux-mêmes que dans d'autres pays de l'OCDE, mais l'influence des pouvoirs publics sur les procès y est plus forte, et les décisions de justice y sont moins mises à exécution. 
Tableau de bord 1. Accessibilité des services publics

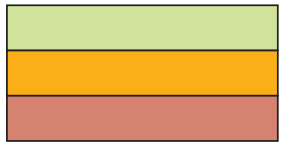

Tiers supérieur

Tiers intermédiaire

Tiers inférieur

Notes : Les pays sont présentés par ordre alphabétique. Le numéro figurant dans les cases indique le positionnement de chaque pays par rapport à l'ensemble des pays pour lesquels on dispose de données.

\begin{tabular}{|c|c|c|c|c|c|c|c|c|c|c|c|c|c|c|c|}
\hline \multirow{4}{*}{\begin{tabular}{|l} 
Indicateur \\
Allemagne \\
\end{tabular}} & \multicolumn{6}{|c|}{ Santé } & \multicolumn{5}{|c|}{ Éducation } & \multicolumn{4}{|c|}{ Justice } \\
\hline & \multicolumn{6}{|c|}{ Accessibilité financière } & \multicolumn{5}{|c|}{ Accès à l'enseignement } & \multicolumn{4}{|c|}{$\begin{array}{c}\text { Accès au système judiciaire et à } \\
\text { l'information juridique }\end{array}$} \\
\hline & \multicolumn{2}{|c|}{$\begin{array}{l}\text { Couverture des } \\
\text { soins de santé }\end{array}$} & \multicolumn{2}{|c|}{$\begin{array}{l}\text { Besoins de soins de } \\
\text { santé non satisfaits } \\
\text { (écart entre bas } \\
\text { et hauts revenus) }\end{array}$} & \multicolumn{2}{|c|}{$\begin{array}{c}\text { Poids du reste à } \\
\text { charge dans la } \\
\text { consommation des } \\
\text { ménages }\end{array}$} & \multicolumn{2}{|c|}{\begin{tabular}{|c|} 
Dépenses privées \\
consacrées à \\
l'éducation (de \\
l'enseignement \\
primaire au supérieur)
\end{tabular}} & \multicolumn{2}{|c|}{$\begin{array}{l}\text { Taux de scolarisation } \\
\text { des enfants de } 4 \text { ans }\end{array}$} & \multirow{2}{*}{$\begin{array}{c}\begin{array}{c}\text { Taux d'accès à } \\
\text { l'enseignement } \\
\text { supérieur }\end{array} \\
20\end{array}$} & \multicolumn{2}{|c|}{$\begin{array}{l}\text { La justice civile } \\
\text { est accessible } \\
\text { et abordable }\end{array}$} & \multicolumn{2}{|c|}{$\begin{array}{l}\text { Les mécanismes alternatifs } \\
\text { de règlement des litiges sont } \\
\text { accessibles, impartiaux } \\
\text { et efficaces }\end{array}$} \\
\hline & 1 & $\uparrow$ & 3 & $\uparrow$ & 11 & $\rightarrow$ & 18 & $\rightarrow$ & 13 & $\rightarrow$ & & 2 & $\uparrow$ & 5 & $\downarrow$ \\
\hline Australie & 1 & $\rightarrow$ & s.o. & & 16 & $\uparrow$ & 33 & $\downarrow$ & 24 & $\uparrow$ & s.o. & 20 & $\uparrow$ & 9 & $\downarrow$ \\
\hline Autriche & 2 & $\rightarrow$ & 1 & $\rightarrow$ & 30 & $\downarrow$ & 6 & $\rightarrow$ & 18 & $\uparrow$ & 13 & 10 & $\uparrow$ & 20 & $\uparrow$ \\
\hline Belgique & 4 & $\downarrow$ & 9 & $\uparrow$ & 25 & $\rightarrow$ & 7 & $\downarrow$ & 2 & $\rightarrow$ & 9 & 6 & $\uparrow$ & 15 & $\uparrow$ \\
\hline Canada & 1 & $\rightarrow$ & s.o. & & 13 & $\downarrow$ & 26 & $\rightarrow$ & s.o. & & s.o. & 23 & $\uparrow$ & 16 & $\downarrow$ \\
\hline Chili & 7 & $\downarrow$ & s.o. & & 31 & $\rightarrow$ & 34 & $\uparrow$ & 26 & & 2 & 13 & $\uparrow$ & 21 & $\uparrow$ \\
\hline Corée & 1 & $\rightarrow$ & s.o. & & 34 & $\downarrow$ & 30 & $\uparrow$ & 7 & $\uparrow$ & s.o. & 19 & $\uparrow$ & 3 & $\downarrow$ \\
\hline Danemark & 1 & $\rightarrow$ & 7 & $\uparrow$ & 17 & $\rightarrow$ & s.o. & & 3 & $\rightarrow$ & 4 & 4 & $\uparrow$ & 4 & $\rightarrow$ \\
\hline Espagne & 2 & $\rightarrow$ & 2 & $\uparrow$ & 26 & $\rightarrow$ & 24 & $\downarrow$ & 5 & $\rightarrow$ & 6 & 5 & $\uparrow$ & 14 & $\uparrow$ \\
\hline Estonie & 6 & $\uparrow$ & 20 & $\downarrow$ & 19 & $\downarrow$ & 9 & $\downarrow$ & 20 & & s.o. & 7 & $\uparrow$ & 8 & $\uparrow$ \\
\hline États-Unis & 10 & $\uparrow$ & s.o. & & 14 & $\uparrow$ & 32 & $\rightarrow$ & 31 & $\rightarrow$ & 25 & 27 & $\downarrow$ & 18 & $\downarrow$ \\
\hline Finlande & 1 & $\rightarrow$ & 17 & $\downarrow$ & 24 & $\downarrow$ & 1 & $\rightarrow$ & 28 & $\uparrow$ & 21 & 17 & $\uparrow$ & 19 & $\rightarrow$ \\
\hline France & 2 & $\rightarrow$ & 6 & $\uparrow$ & 3 & $\uparrow$ & 16 & $\rightarrow$ & 1 & $\rightarrow$ & s.o. & 15 & $\uparrow$ & 7 & $\uparrow$ \\
\hline Grèce & 1 & $\uparrow$ & 19 & $\uparrow$ & 32 & $\rightarrow$ & 8 & 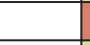 & 30 & $\uparrow$ & 26 & 16 & $\uparrow$ & 23 & $\downarrow$ \\
\hline Hongrie & 7 & $\downarrow$ & 5 & $\uparrow$ & 28 & $\uparrow$ & 21 & $\uparrow$ & 11 & $\uparrow$ & 27 & 24 & $\uparrow$ & 27 & $\downarrow$ \\
\hline Irlande & 1 & $\rightarrow$ & 13 & $\uparrow$ & 12 & $\uparrow$ & 10 & $\downarrow$ & 1 & $\uparrow$ & S.o. & s.o. & & s.o. & \\
\hline Islande & 1 & $\rightarrow$ & 14 & $\uparrow$ & 15 & $\uparrow$ & 5 & $\uparrow$ & 6 & $\uparrow$ & 16 & s.o. & & s.o. & \\
\hline Israël & 1 & $\rightarrow$ & s.o. & & 18 & $\downarrow$ & 23 & $\uparrow$ & 4 & & 15 & s.o. & & s.o. & \\
\hline Italie & 1 & $\rightarrow$ & 9 & $\uparrow$ & 21 & $\downarrow$ & 15 & $\rightarrow$ & 15 & $\downarrow$ & 24 & 22 & $\rightarrow$ & 25 & $\rightarrow$ \\
\hline Japon & 1 & $\rightarrow$ & s.o. & & 10 & $\downarrow$ & 29 & $\rightarrow$ & 12 & $\uparrow$ & 5 & 11 & $\uparrow$ & 2 & $\uparrow$ \\
\hline Lettonie & 1 & & 18 & $\uparrow$ & 33 & $\downarrow$ & 11 & $\uparrow$ & 17 & & s.o. & s.o. & & s.o. & \\
\hline Lituanie & 8 & & 8 & $\uparrow$ & 20 & $\rightarrow$ & 14 & $\rightarrow$ & 25 & & 8 & s.o. & & s.o. & \\
\hline Luxembourg & S.o. & & 3 & $\uparrow$ & 2 & $\rightarrow$ & 3 & $\rightarrow$ & 14 & $\downarrow$ & 28 & S.o. & & s.o. & \\
\hline Mexique & 11 & $\downarrow$ & s.o. & & 23 & $\uparrow$ & 25 & $\downarrow$ & 21 & $\uparrow$ & 23 & 28 & $\downarrow$ & 28 & $\downarrow$ \\
\hline Norvège & 1 & $\rightarrow$ & 7 & $\downarrow$ & 22 & $\downarrow$ & 2 & $\downarrow$ & 8 & $\rightarrow$ & 12 & 9 & $\downarrow$ & 1 & $\uparrow$ \\
\hline Nouvelle-Zélande & 1 & $\rightarrow$ & s.o. & & 4 & $\downarrow$ & 28 & $\rightarrow$ & 10 & $\uparrow$ & 1 & 8 & $\uparrow$ & 12 & $\rightarrow$ \\
\hline Pays-Bas & 2 & $\uparrow$ & 2 & $\uparrow$ & 8 & $\uparrow$ & 22 & $\rightarrow$ & 9 & $\downarrow$ & 14 & 1 & $\uparrow$ & 6 & $\rightarrow$ \\
\hline Pologne & 9 & $\uparrow$ & 16 & $\uparrow$ & 9 & $\rightarrow$ & 13 & $\rightarrow$ & 27 & $\uparrow$ & 7 & 21 & $\uparrow$ & 13 & $\uparrow$ \\
\hline Portugal & 1 & $\rightarrow$ & 10 & $\uparrow$ & 29 & $\rightarrow$ & 20 & $\uparrow$ & 19 & $\uparrow$ & 19 & 14 & $\uparrow$ & 10 & $\uparrow$ \\
\hline Rép. slovaque & 1 & $\rightarrow$ & 3 & $\uparrow$ & 5 & $\downarrow$ & 17 & $\rightarrow$ & 23 & $\uparrow$ & 17 & 18 & $\downarrow$ & 11 & $\uparrow$ \\
\hline Rép. tchèque & 5 & $\rightarrow$ & 11 & $\downarrow$ & 6 & $\uparrow$ & 19 & $\rightarrow$ & 29 & $\uparrow$ & 22 & s.o. & & s.o. & \\
\hline Royaume-Uni & 1 & $\rightarrow$ & 15 & $\downarrow$ & 7 & $\rightarrow$ & 31 & $\downarrow$ & 1 & $\uparrow$ & 10 & 26 & $\downarrow$ & 17 & $\downarrow$ \\
\hline Slovénie & 1 & $\rightarrow$ & 15 & $\downarrow$ & 1 & $\rightarrow$ & 12 & $\rightarrow$ & 22 & & 11 & 12 & $\uparrow$ & 22 & $\downarrow$ \\
\hline Suède & 1 & $\rightarrow$ & 8 & $\uparrow$ & 27 & $\rightarrow$ & 4 & $\rightarrow$ & 16 & $\rightarrow$ & 18 & 3 & $\uparrow$ & 26 & $\downarrow$ \\
\hline Suisse & 1 & $\rightarrow$ & 4 & $\uparrow$ & 35 & $\downarrow$ & s.o. & & 32 & $\uparrow$ & 3 & s.o. & & s.o. & \\
\hline Turquie & 3 & $\uparrow$ & 12 & $\uparrow$ & s.o. & & 27 & $\downarrow$ & 33 & $\uparrow$ & s.o. & 25 & $\uparrow$ & 24 & $\downarrow$ \\
\hline Année & 2017 & 2013 & 2018 & 2014 & 2017 & 2013 & 2016 & 2012 & 2017 & 2012 & 2017 & 2019 & 2015 & 2019 & 2015 \\
\hline
\end{tabular}

Notes : Les pays sont présentés par ordre alphabétique. Le numéro figurant dans les cases indique le positionnement de chaque pays par rapport à l'ensemble des pays pour lesquels on dispose de données. S'agissant de la couverture des soins de santé, le groupement a été effectué comme suit - tiers supérieur : couverture comprise entre $95 \%$ et $100 \%$; tiers intermédiaire : couverture comprise entre $90 \%$ et $95 \%$; et tiers inférieur : couverture inférieure à $90 \%$. Les données se rapportent à l'année 2015, sauf celles concernant l'Espagne et le Japon, qui sont celles de 2014. Les données sur les revenues consacres á des dépenses médicaux dans les dépenses finales des ménages pour le Chili datent de 2014. En Australie, en Nouvelle-Zélande, au Royaume-Uni et Aux États-Unis, la part relativement élevée des dépenses privées consacrées à l'éducation est associée à une proportion importante des étudiants bénéficiant de prêts et de bourses. Les données sur les dépenses privées consacrées à l'éducation en Grèce datent de 2015. Concernant les données sur Israël, voir : http://doi.org/10.1787/888932315602. Pour les autres indicateurs, les détails relatifs aux données seront fournis dans les rubriques correspondantes.

Sources : OCDE (2019, Staistiques sur la santé (base de données) ; Eurostat (2019) ; OCDE (2019), Regards sur l'éducation ; World Justice Project (2019), Indice de l'état de droit (RuLi). 


\section{Tableau de bord 2. Qualité des services publics}

Tiers supérieur

Tiers intermédiaire

Tiers inférieur

Notes : Les pays sont présentés par ordre alphabétique. Le numéro figurant dans les cases le positionnement de chaque pays par rapport à l'ensemble des pays pour lesquels on dispose de données.

\begin{tabular}{|c|c|c|c|c|c|c|c|c|c|c|c|c|}
\hline \multirow{3}{*}{$\begin{array}{l}\text { Indicateur } \\
\text { Allemagne }\end{array}$} & \multicolumn{6}{|c|}{ Santé } & \multicolumn{6}{|c|}{ Justice } \\
\hline & \multicolumn{2}{|c|}{$\begin{array}{l}\text { Taux de mortalité } \\
\text { - infarctus aigu }\end{array}$} & \multicolumn{2}{|c|}{$\begin{array}{l}\text { Taux de mortalité - } \\
\text { accident vasculaire } \\
\text { cérébral (AVC) }\end{array}$} & \multicolumn{2}{|c|}{$\begin{array}{l}\text { Taux de mortalité - } \\
\text { cancer du sein } \\
\text { chez les femmes }\end{array}$} & \multicolumn{2}{|c|}{$\begin{array}{l}\text { Bonne mise en } \\
\text { application de } \\
\text { la justice civile }\end{array}$} & \multicolumn{2}{|c|}{$\begin{array}{l}\text { Absence d'influence } \\
\text { indue des pouvoirs } \\
\text { publics sur la } \\
\text { justice civile }\end{array}$} & \multicolumn{2}{|c|}{$\begin{array}{l}\text { Non-recours à } \\
\text { la violence en } \\
\text { cas de griefs }\end{array}$} \\
\hline & 23 & $\uparrow$ & 13 & $\uparrow$ & 28 & $\downarrow$ & 5 & $\uparrow$ & 3 & $\uparrow$ & 12 & $\uparrow$ \\
\hline Australie & 14 & $\uparrow$ & 10 & $\uparrow$ & 9 & $\downarrow$ & 10 & $\downarrow$ & 4 & $\downarrow$ & 13 & $\downarrow$ \\
\hline Autriche & 24 & $\uparrow$ & 6 & $\uparrow$ & 18 & $\uparrow$ & 8 & $\downarrow$ & 9 & $\rightarrow$ & 9 & $\uparrow$ \\
\hline Belgique & 9 & $\uparrow$ & 12 & $\uparrow$ & 24 & $\downarrow$ & 9 & $\uparrow$ & 11 & $\uparrow$ & 17 & $\downarrow$ \\
\hline Canada & 17 & $\uparrow$ & 3 & $\uparrow$ & 12 & $\downarrow$ & 13 & $\rightarrow$ & 7 & $\uparrow$ & 7 & $\uparrow$ \\
\hline Chili & 31 & $\uparrow$ & 30 & $\downarrow$ & 8 & $\uparrow$ & 19 & $\uparrow$ & 21 & $\downarrow$ & 27 & $\downarrow$ \\
\hline Corée & 6 & $\uparrow$ & 20 & $\uparrow$ & 1 & $\uparrow$ & 7 & $\downarrow$ & 18 & $\downarrow$ & 20 & $\downarrow$ \\
\hline Danemark & 5 & $\uparrow$ & 18 & $\uparrow$ & 27 & $\downarrow$ & 4 & $\uparrow$ & 2 & $\rightarrow$ & 2 & $\rightarrow$ \\
\hline Espagne & 6 & $\uparrow$ & 20 & $\uparrow$ & 5 & $\downarrow$ & 23 & $\uparrow$ & 22 & $\rightarrow$ & 21 & $\uparrow$ \\
\hline Estonie & 8 & $\uparrow$ & 19 & $\uparrow$ & 11 & $\downarrow$ & 16 & $\uparrow$ & 10 & $\rightarrow$ & 21 & $\uparrow$ \\
\hline États-Unis & 16 & $\uparrow$ & 11 & $\uparrow$ & 9 & $\downarrow$ & 17 & $\uparrow$ & 19 & $\downarrow$ & 16 & $\rightarrow$ \\
\hline Finlande & 25 & $\uparrow$ & 22 & $\uparrow$ & 9 & $\downarrow$ & 6 & $\rightarrow$ & 8 & $\uparrow$ & 5 & $\rightarrow$ \\
\hline France & 2 & $\uparrow$ & 2 & $\uparrow$ & 21 & $\downarrow$ & 11 & $\uparrow$ & 15 & $\uparrow$ & 22 & $\uparrow$ \\
\hline Grèce & 22 & $\uparrow$ & 29 & $\uparrow$ & 19 & $\downarrow$ & 25 & $\downarrow$ & 23 & $\downarrow$ & 26 & $\downarrow$ \\
\hline Hongrie & 28 & $\uparrow$ & 31 & $\uparrow$ & 30 & $\downarrow$ & 26 & $\downarrow$ & 27 & $\downarrow$ & 8 & $\uparrow$ \\
\hline Irlande & 29 & $\uparrow$ & 17 & $\uparrow$ & 29 & $\downarrow$ & s.o. & & s.o. & & s.o. & \\
\hline Islande & 18 & $\uparrow$ & 7 & $\uparrow$ & 28 & $\uparrow$ & s.o. & & S.0. & & s.o. & \\
\hline Israël & 3 & $\uparrow$ & 4 & $\uparrow$ & 23 & $\downarrow$ & s.o. & & s.o. & & s.o. & \\
\hline Italie & 8 & $\uparrow$ & 24 & $\uparrow$ & 17 & $\downarrow$ & 28 & $\downarrow$ & 20 & $\downarrow$ & 25 & $\uparrow$ \\
\hline Japon & 1 & $\uparrow$ & 13 & $\uparrow$ & 3 & $\uparrow$ & 12 & $\uparrow$ & 16 & $\uparrow$ & 3 & $\downarrow$ \\
\hline Lettonie & 27 & $\uparrow$ & 35 & $\uparrow$ & 26 & $\downarrow$ & s.o. & & S.0. & & s.o. & \\
\hline Lituanie & 11 & $\uparrow$ & 34 & $\uparrow$ & 13 & $\downarrow$ & s.o. & & S.0. & & s.o. & \\
\hline Luxembourg & 10 & $\uparrow$ & 5 & $\uparrow$ & 22 & $\downarrow$ & s.o. & & S.0. & & s.o. & \\
\hline Mexique & 33 & $\downarrow$ & 21 & $\uparrow$ & 4 & $\uparrow$ & 27 & $\rightarrow$ & 26 & $\downarrow$ & 28 & $\downarrow$ \\
\hline Norvège & 20 & $\uparrow$ & 9 & $\uparrow$ & 6 & $\downarrow$ & 2 & $\uparrow$ & 1 & $\downarrow$ & 4 & $\uparrow$ \\
\hline Nouvelle-Zélande & 30 & $\uparrow$ & 23 & $\uparrow$ & 16 & $\downarrow$ & 15 & $\downarrow$ & 12 & $\uparrow$ & 11 & $\uparrow$ \\
\hline Pays-Bas & 7 & $\uparrow$ & 16 & $\uparrow$ & 26 & $\downarrow$ & 3 & $\rightarrow$ & 6 & $\downarrow$ & 18 & $\rightarrow$ \\
\hline Pologne & 12 & $\uparrow$ & 25 & $\uparrow$ & 20 & $\uparrow$ & 20 & $\uparrow$ & 25 & $\downarrow$ & 19 & $\uparrow$ \\
\hline Portugal & 13 & $\uparrow$ & 28 & $\uparrow$ & 10 & $\downarrow$ & 24 & $\uparrow$ & 14 & $\uparrow$ & 24 & $\uparrow$ \\
\hline Rép. slovaque & 26 & $\downarrow$ & 33 & $\downarrow$ & 25 & $\uparrow$ & s.o. & & S.0. & & s.o. & \\
\hline Rép. tchèque & 19 & $\uparrow$ & 26 & $\uparrow$ & 14 & $\downarrow$ & 18 & $\downarrow$ & 17 & $\uparrow$ & 6 & $\rightarrow$ \\
\hline Royaume-Uni & 15 & $\uparrow$ & 15 & $\uparrow$ & 22 & $\downarrow$ & 14 & $\rightarrow$ & 13 & $\rightarrow$ & 15 & $\uparrow$ \\
\hline Slovénie & 27 & $\downarrow$ & 27 & $\uparrow$ & 28 & $\downarrow$ & 21 & $\uparrow$ & 24 & $\downarrow$ & 14 & $\uparrow$ \\
\hline Suède & 21 & $\uparrow$ & 14 & $\uparrow$ & 7 & $\downarrow$ & 1 & $\uparrow$ & 5 & $\rightarrow$ & 1 & $\uparrow$ \\
\hline Suisse & 4 & $\uparrow$ & 1 & $\uparrow$ & 15 & $\downarrow$ & s.o. & & s.o. & & s.o. & \\
\hline Turquie & 32 & & 32 & & 2 & & 22 & $\uparrow$ & 28 & $\downarrow$ & 23 & $\uparrow$ \\
\hline Année & 2017 & 2006 & 2017 & 2006 & 2017 & 2006 & 2019 & 2015 & 2019 & 2015 & 2019 & 2015 \\
\hline
\end{tabular}

Notes : Les pays sont présentés par ordre alphabétique. Le numéro figurant dans les cases le positionnement de chaque pays par rapport à l'ensemble des pays pour lesquels on dispose de données. Les données sur l'infarctus aigu du myocarde, les maladies cérébrovasculaires et la mortalité par cancer du sein en Australie, en Belgique, au Chili, en Estonie, en Finlande, en Allemagne, en Grèce, en Israël, au Japon, en Corée, au Luxembourg, en Norvège, en Pologne, au Portugal, en Espagne, aux États-Unis, au Royaume-Uni, en Suède, en Suisse, et en Turquie et se rapportent à 2016 au lieu de 2017. Les données pour le Canada, le Danemark, la France, l'Irlande, l'Italie, la Lettonie et la Slovénie ne sont pas disponibles. Les données pour l'Australie datent de 2004 au lieu de 2005. Les données pour le Portugal portent sur 2007 au lieu de 2005. Pour les données concernant Israël, voir http://doi. org/10.1787/888932315602. Des détails sur les données d'autres indicateurs sont fournis dans les sections correspondantes.

Sources : OCDE (2019), Staistiques sur la santé (base de données) ; World Justice Project (2019), Indice de l'état de droit (RuLi). 
Les hôpitaux et les établissements d'enseignement sont des institutions publiques dans le cadre desquelles la population est en contact direct avec les services publics. Bien qu'une proportion plus réduite de la population soit amenée à traiter avec les tribunaux, ceux-ci remplissent une fonction essentielle en garantissant les droits fondamentaux et en veillant à ce qu'un système de contre-pouvoirs soit mis en place. Le degré de satisfaction des citoyens à l'égard des services publics est considéré comme un bilan essentiel de l'activité gouvernementale et fait donc l'objet d'un suivi au sein de la plupart des pays de l'OCDE désirant améliorer leurs services publics. À ce jour, il n'existe cependant pas de méthode internationale normalisée pour mener des enquêtes à ce sujet.

L'enquête Gallup World Poll recueille régulièrement des données relatives au degré de satisfaction des citoyens en ce qui concerne l'éventail de services publics, notamment dans les domaines de la santé, de l'éducation et de la justice. Bien que de nombreux facteurs puissent influencer les réponses aux enquêtes d'opinion - contact récent avec des fonctionnaires, lassitude des personnes interrogées et styles de réponse, par exemple -, cet ensemble de données permet de comparer les perceptions des citoyens dans le temps et entre les différents pays de l'OCDE.

Le degré de satisfaction à l'égard des services de santé et d'éducation ainsi que le niveau de confiance envers le secteur judiciaire ont, dans l'ensemble, légèrement augmenté dans les pays de l'OCDE au cours de la décennie écoulée. En 2018, $70 \%$ des citoyens se déclaraient satisfaits s'agissant de l'accès aux services de santé existant dans leur région, proportion qui est restée stable depuis 2007. Par ailleurs, environ $66 \%$ des citoyens se disaient satisfaits du système éducatif et des établissements d'enseignement, taux qui marque une hausse de 3 points de pourcentage par rapport à 2007. Enfin, $56 \%$ avaient confiance dans le système judiciaire et les tribunaux, soit une hausse de 4 points.

Les niveaux de satisfaction les plus élevés à l'égard du système de santé sont observés aux Pays-Bas (90\%), en Belgique (89\%), en Norvège (89\%), au Danemark (88\%) et en Suisse $(88 \%)$; les plus faibles sont constatés au Chili (40\%), en Lettonie (40\%) et en Grèce (42\%). Alors que les pays qui connaissent le plus haut taux de satisfaction assurent une couverture publique quasi-universelle des soins de santé, le taux de couverture du Chili compte parmi les plus bas de l'OCDE. Toujours dans le domaine de la santé, le degré de satisfaction des citoyens a progressé en Estonie (+21 points) et en Lituanie (+19 points). Un important déclin de la satisfaction des citoyens est observé au Japon (-13 points), en France (-10), en Grèce (-10) et au Luxembourg (-10).

Les plus hauts taux de satisfaction à l'égard des établissements d'enseignement et du système éducatif sont observés en Norvège (87\%), en Suisse (85\%), au Danemark (84 \%) et en Finlande (84\%) ; les plus faibles sont constatés en Turquie (35\%), en Lituanie (43\%) et en Hongrie (48\%). La satisfaction a le plus progressé aux Pays-Bas (+16 points), en Suisse (+12 points) et en Israël (+12 points); la plus forte baisse est, à l'inverse, enregistrée en Turquie (-17 points).

Une majorité de citoyens dit avoir confiance dans le système judiciaire en Norvège (89\%), au Danemark (87\%) et en Suisse (82 \%). À l'inverse, le Chili (24\%) et la Lettonie (28\%) enregistrent les plus faibles taux de confiance à l'égard des institutions judiciaires. Il se peut que le degré de confiance à l'égard du système judiciaire soit influencé par le ressenti à l'égard d'autres institutions publiques chargées de faire respecter la loi, comme le parquet ou la police, avec lesquels les citoyens sont peut-être plus souvent en contact (voir la double page 166, "Confiance à l'égard des institutions »).

\section{Méthodologie et définitions}

Ces données sont tirées de l'enquête Gallup World Poll, qui est généralement basée sur un échantillon représentatif de 1000 citoyens dans chaque pays. On trouvera un complément d'information sur cette enquête à l'adresse suivante : www.gallup.com/home.aspx.

Les styles de réponse sont des tendances comportementales des personnes interrogées qui ne sont liées ni au contenu des questionnaires, ni à leur présentation, et qui faussent les résultats ; par exemple, certaines personnes choisissent constamment les extrêmes de l'échelle de réponse ou le point intermédiaire. On observe une lassitude des répondants lorsque la motivation et l'attention des participants au sondage diminuent sous l'effet d'un nombre excessif de questions posées, phénomène qui produit des données de faible qualité.

Les données relatives au degré de satisfaction à l'égard des soins de santé ou de l'éducation correspondent au pourcentage de réponses "Satisfait " à la question : " Dans la ville ou la zone où vous habitez, êtes-vous satisfait ou insatisfait de l'accès à des soins de santé ou au système éducatif ou aux établissements scolaires ? ".

Pour ce qui est de la justice, les données correspondent au pourcentage de réponses positives à la question : "Dans ce pays, avez-vous confiance dans chacune des entités suivantes ? (...) Et dans le système judiciaire et les tribunaux? ". Les données sont l'expression de la proportion des personnes interrogées qui ont répondu « oui ».

\section{Pour en savoir plus}

Murtin, F., et al. (2018), “Trust and its determinants: Evidence from the Trustlab experiment", OECD Statistics Working Papers, $n^{\circ}$ 2018/02, Éditions OCDE, Paris.

OCDE/KDI (2018), Understanding the Drivers of Trust in Government Institutions in Korea, Éditions OCDE, Paris, https://doi. org/10.1787/9789264308992-en.

\section{Notes relatives aux graphiques}

Les pays sont classés par ordre décroissant en fonction de leur moyenne nationale. Les données sont celles de 2006 pour les pays suivants : Autriche, Finlande, Irlande, Norvège, Portugal, République slovaque, Slovénie et Suisse ; pour le Luxembourg, il s'agit des données de 2008. Concernant les données sur Israël, voir http://doi. org/10.1787/888932315602.

11.3. Les données relatives à la Corée ne sont pas présentées en raison de problèmes de fiabilité. L'OCDE s'emploiera à améliorer la qualité des données relatives au système judiciaire et aux tribunaux. Les données relatives aux tribunaux en Afrique du Sud sont celles de 2006 et non 2007

11.4. (Satisfaction des citoyens à l'égard de la police locale, 2018) est disponible en ligne à l'Annexe F. 
11.1. Satisfaction des citoyens à l'égard du système de santé, 2007 et 2018

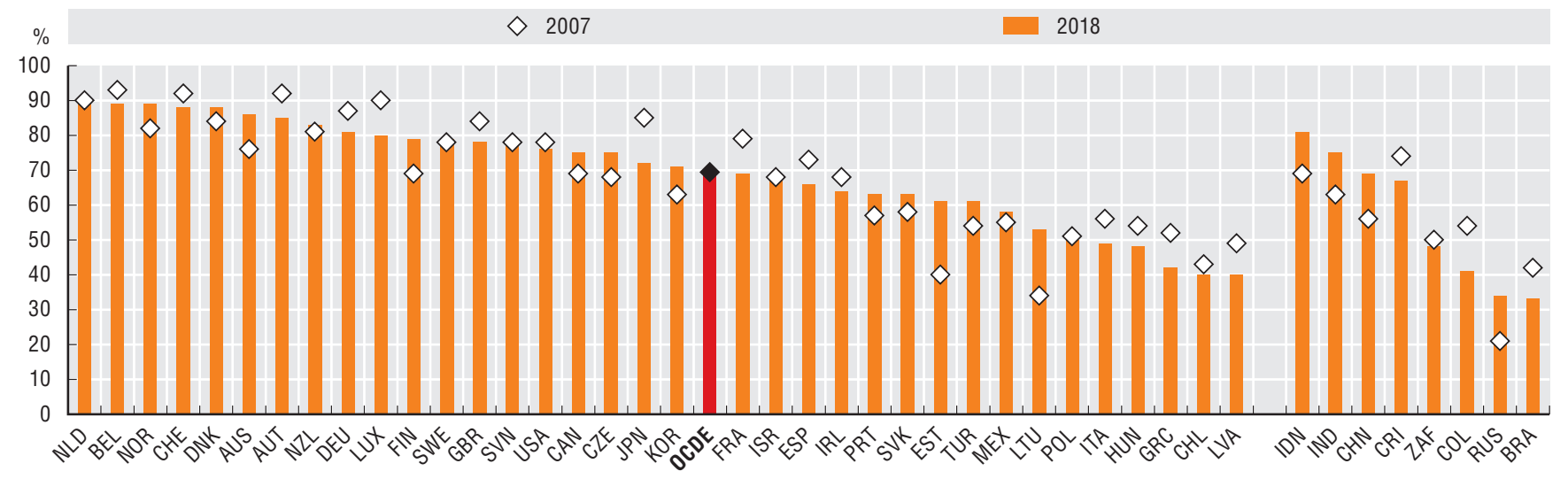

Source : Gallup World Poll 2018 (base de données)

StatLink नints https://doi.org/10.1787/888934053258

11.2. Satisfaction des citoyens à l'égard du système éducatif et des établissements d'enseignement, 2007 et 2018

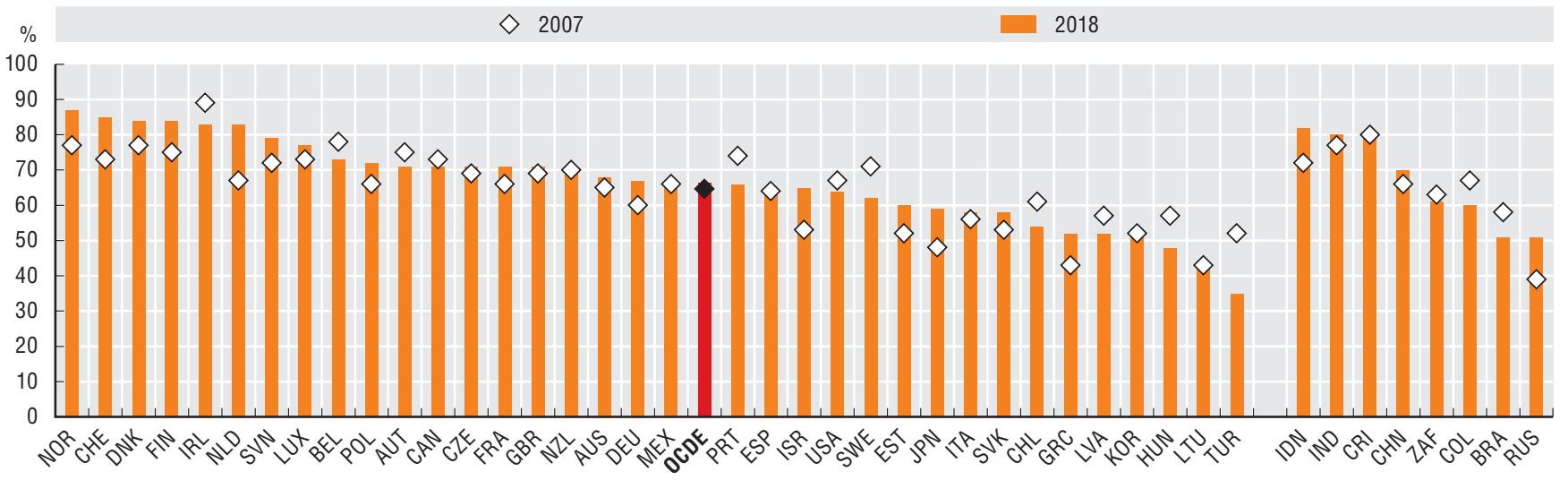

Source : Gallup World Poll 2018 (base de données)

StatLink न्ताIs] https://doi.org/10.1787/888934053277

11.3. Confiance des citoyens à l'égard du système judiciaire et des tribunaux, 2007 et 2018

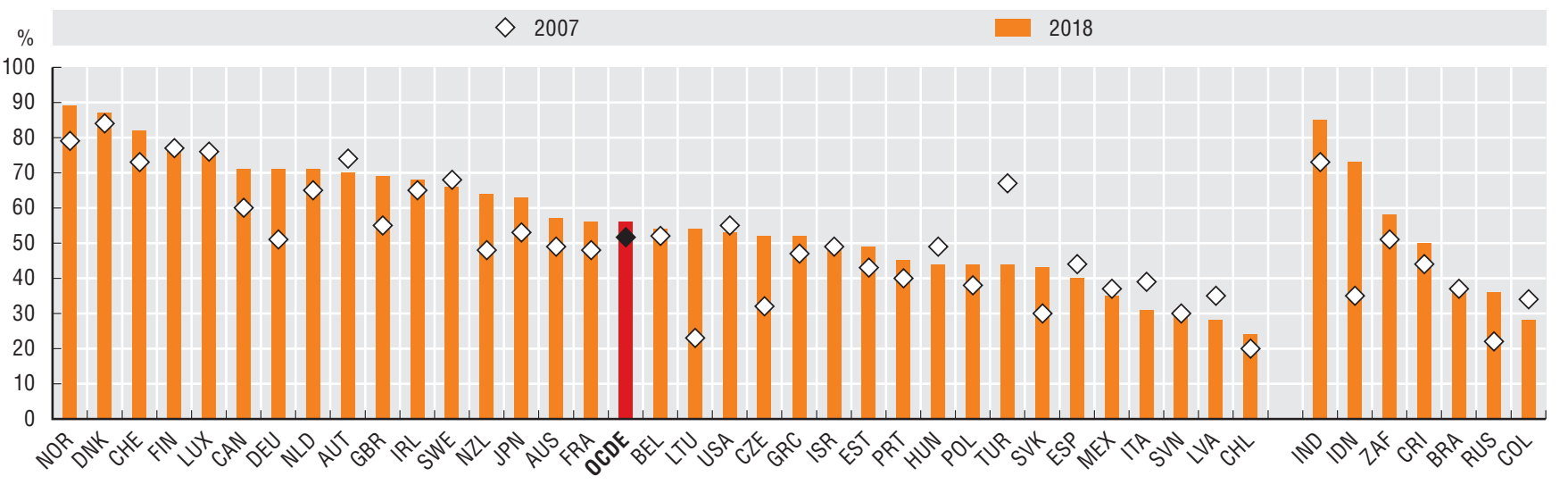

Source : Gallup World Poll 2018 (base de données) 
La plupart des pays de l'OCDE ont instauré une couverture universelle (ou quasi-universelle) pour un ensemble fondamental de services de santé qui comprend habituellement les consultations médicales et les soins hospitaliers. Cette couverture a généralement été mise en place au travers de systèmes nationaux de santé ou de régimes sociaux d'assurance maladie, quoiqu'un petit nombre de pays (Pays-Bas et Suisse) aient fait appel à des régimes privés et obligatoires d'assurance maladie. Malgré tout, des problèmes d'accessibilité physique ou financière font parfois obstacle à l'utilisation des services de santé. Ces problèmes sont essentiellement liés aux dépenses restant à la charge des patients (ou "reste à charge "), à l'éloignement des services de santé ou aux délais pour obtenir un rendez-vous.

Les besoins de soins de santé non satisfaits sont un indicateur pertinent de ces problèmes d'accessibilité. En Europe, dans le cadre de l'enquête EU-SILC (Statistics on Income and Living Conditions), on demande aux personnes interrogées s'il leur est arrivé, au cours des 12 mois précédents, de renoncer à consulter un médecin, pour différentes raisons. En 2018, 2,7 \% de la population des pays européens de l'OCDE ont fait état de besoins de soins non satisfaits en raison des coûts, de l'éloignement ou des délais. Ce pourcentage atteint 4,6\% pour les citoyens à faibles revenus. En moyenne, $16,4 \%$ des personnes interrogées en Estonie et 8,8 \% des personnes interrogées en Grèce indiquent ne pas avoir pu satisfaire leurs besoins de soins. C'est en Grèce, en Lettonie, en Turquie et en Belgique que l'inégalité d'accès aux soins est la plus prononcée.

En 2017, dans les pays de l'OCDE, le ménage moyen a directement consacré près de $3 \%$ de ses revenus à des services médicaux (proportion qui est restée stable depuis 2013). Le reste à charge le plus élevé est observé en Suisse et en Corée, pour lesquels $6 \%$ des revenus des ménages sont consacrés à des dépenses d'ordre médical. En France, au Luxembourg et en Slovénie, les dépenses que les ménages dédient à la santé représentent moins d'un tiers de celles des ménages suisses.

Les politiques publiques peuvent atténuer la charge financière pesant à cet égard sur les ménages. En République slovaque, le poids du reste à charge a diminué par rapport à 2013, suite à l'instauration de mesures visant à limiter ces paiements pour les personnes atteintes de maladies chroniques et pour les catégories vulnérables, ainsi qu'au durcissement des règles concernant les frais complémentaires facturés par les prestataires privés (OCDE et Observatoire européen 2017a). En Lettonie, entre 2009 et 2011, le montant du reste à charge a été réduit pour les ménages à faible revenu dans le cadre du Programme de réforme de la sécurité sociale et du secteur social, qui les a exemptés de toute participation aux frais de santé et a subventionné les produits pharmaceutiques. Après 2011, le programme a recentré son champ d'application sur certains groupes de patients, ce qui a eu pour effet d'augmenter les paiements directs et de rétablir le reste à charge à son niveau de 2009 (OCDE et Observatoire européen 2017b).

La pénurie de médecins dans certaines régions peut entraîner un allongement des délais d'attente ou contraindre les patients à effectuer des déplacements importants pour accéder aux services de santé (OCDE 2019). Le nombre global de médecins et leur répartition géographique varient considérablement selon les pays de l'OCDE. En 2017, on comptait deux médecins en exercice pour 1000 habitants en Corée, Pologne et Turquie, alors que ce chiffre était nettement supérieur dans des pays comme l'Autriche et la Grèce. Dans de nombreux pays, comme les États-Unis, la Grèce, la République tchèque et la République slovaque, on observe une forte concentration des médecins autour de la capitale nationale.

\section{Méthodologie et définitions}

Ces données relatives aux besoins de soins non satisfaits sont tirées de l'enquête EU-SILC, dans le cadre de laquelle on demande aux personnes interrogées si, au cours des 12 mois précédant l'enteretien il leur est arrivé de ne pas bénéficier d'un examen médical alors qu'elles en ressentaient le besoin. Les données présentent uniquement le nombre de répondants qui n'ont pu obtenir cet examen en raison de l'éloignement, des délais d'attente ou des coûts. Les personnes à faibles revenus correspondent au quintile le plus pauvre de la population, les personnes à revenus élevés au quintile le plus riche.

Le reste à charge des patients correspond aux frais que ces derniers financent directement à partir de leurs revenus lorsque des services ou traitements médicaux ne font pas partie des prestations prises en charge dans le cadre de régimes d'assurance publics et privés ou lorsqu'ils le sont partiellement (participation aux frais). Il recouvre également, dans certains pays, des estimations des paiements non officiels fournis aux prestataires de soins. Le nombre de médecins comprend les généralistes et les spécialistes qui ont exercé durant l'année, dans des établissements publics ou privés. Les données concernant la Grèce et le Portugal couvrent également ceux qui n'exercent pas (d'où une forte surestimation). La densité de médecins correspond au ratio du nombre de médecins par rapport à la population dans une région donnée. Le niveau territorial 2 correspond aux macrorégions (provinces par exemple).

\section{Ouvrages de référence}

OCDE (2019, à paraître), Panorama de la santé 2019 : indicateurs de l'OCDE, Éditions OCDE, Paris.

OCDE/Observatoire européen des systèmes et des politiques de santé (2017a), Slovak Republic: Country Health Profile 2017, State of Health in the EU, Éditions OCDE, Paris/Observatoire européen des systèmes et des politiques de santé, Bruxelles.

OCDE/Observatoire européen des systèmes et des politiques de santé (2017b), Latvia: Country Health Profile 2017, State of Health in the EU, Éditions OCDE, Paris/Observatoire européen des systèmes et des politiques de santé, Bruxelles.

\section{Notes relatives aux graphiques}

11.5. Les données concernant l'Allemagne, la France, l'Irlande, la Lituanie, le Luxembourg, la Norvège, le Royaume-Uni et la Suisse portent sur l'année 2017 ; celles de l'Islande portent sur l'année 2016 et non 2018.

11.6. Les données relatives à l'Australie, au Brésil, au Costa Rica et à la Fédération de Russie sont celles de 2016 et non 2017. Celles concernant le Chili et la Turquie ne sont pas présentées. Concernant les données sur Israël, voir http://doi.org/10.1787/888932315602.

11.7. Les données concernant l'Estonie, les États-Unis, la Finlande, la Grèce, l'Italie, le Japon, la Lituanie, la Nouvelle-Zélande, le RoyaumeUni, la Slovénie, la Suède et la Turquie portent sur l'année 2010 et non 2015. Les données sur la Chine et la Fédération de Russie portent sur l'année 2013. Le niveau territorial 2 comprend les macro-régions (par exemple, les provinces).

Concernant les données sur Israël, voir http://doi.org/10.1787/888932315602. 
11.5. Besoins de soins non satisfaits (pour examens médicaux) selon le niveau de revenu, 2017

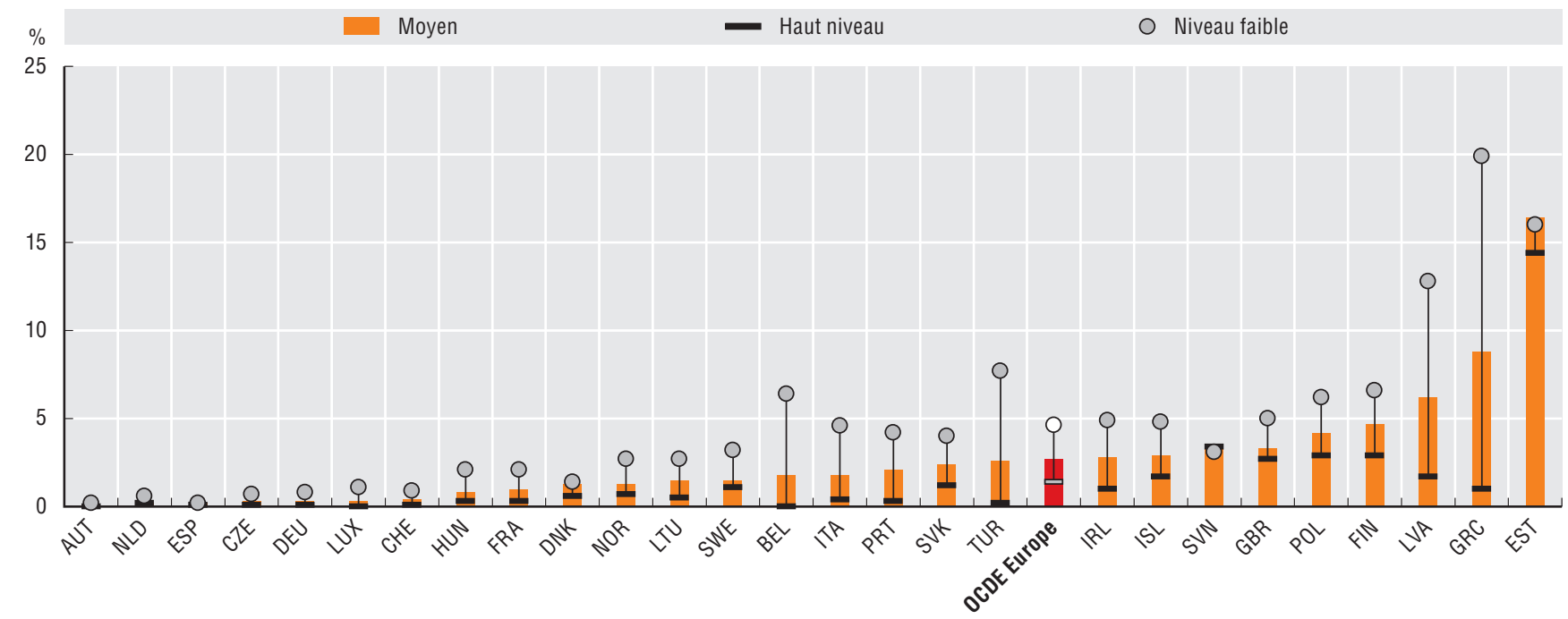

Source : European Union Statistics on Income and Living Conditions (EU-SILC) (base de données), 2019.

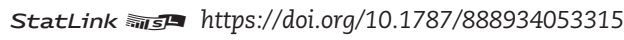

11.6 Poids du reste à charge des patients dans les dépenses finales des ménages, 2013 et 2017

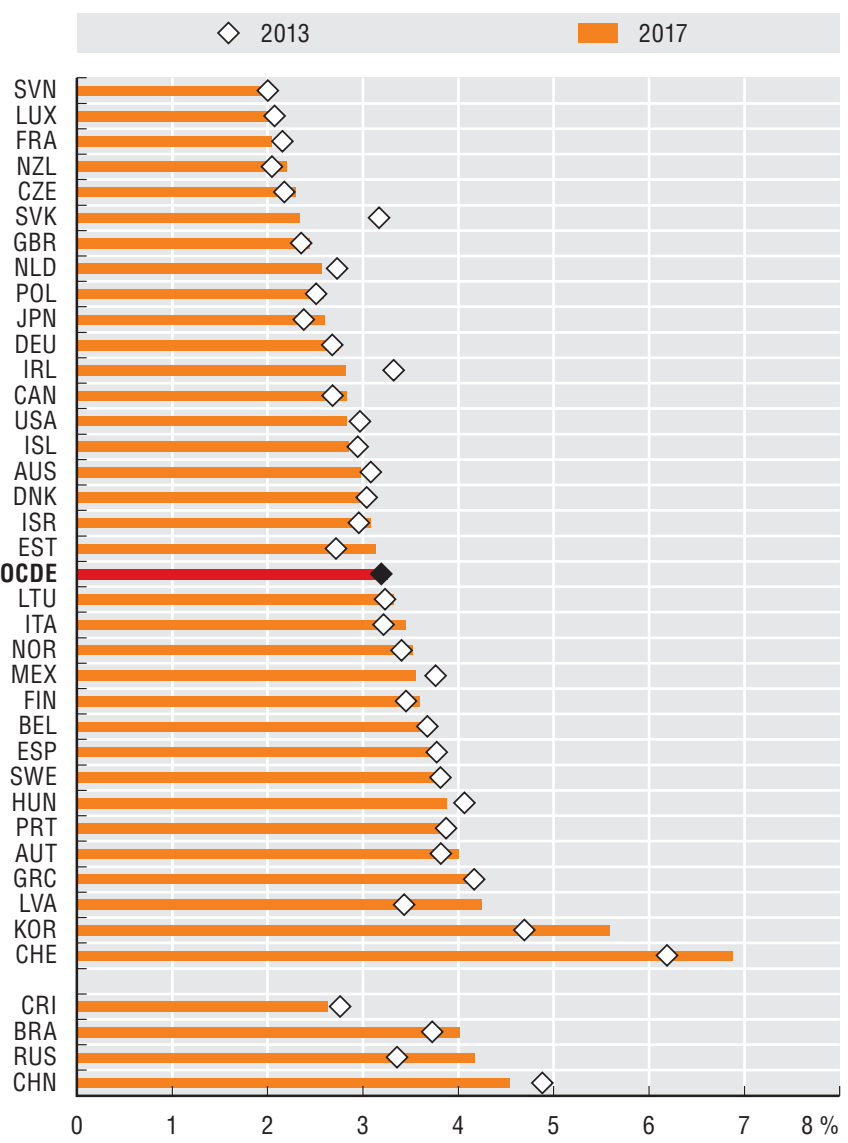

Source: OCDE (2019), Statistiques sur la santé (base de données), OCDE (2019), Base de données des comptes nationaux de l'OCDE, 2019

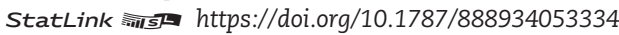

11.7 Densité de médecins dans les régions TL2, 2015 ou année la plus proche

Nombre de médecins en exercice pour 1000 habitants

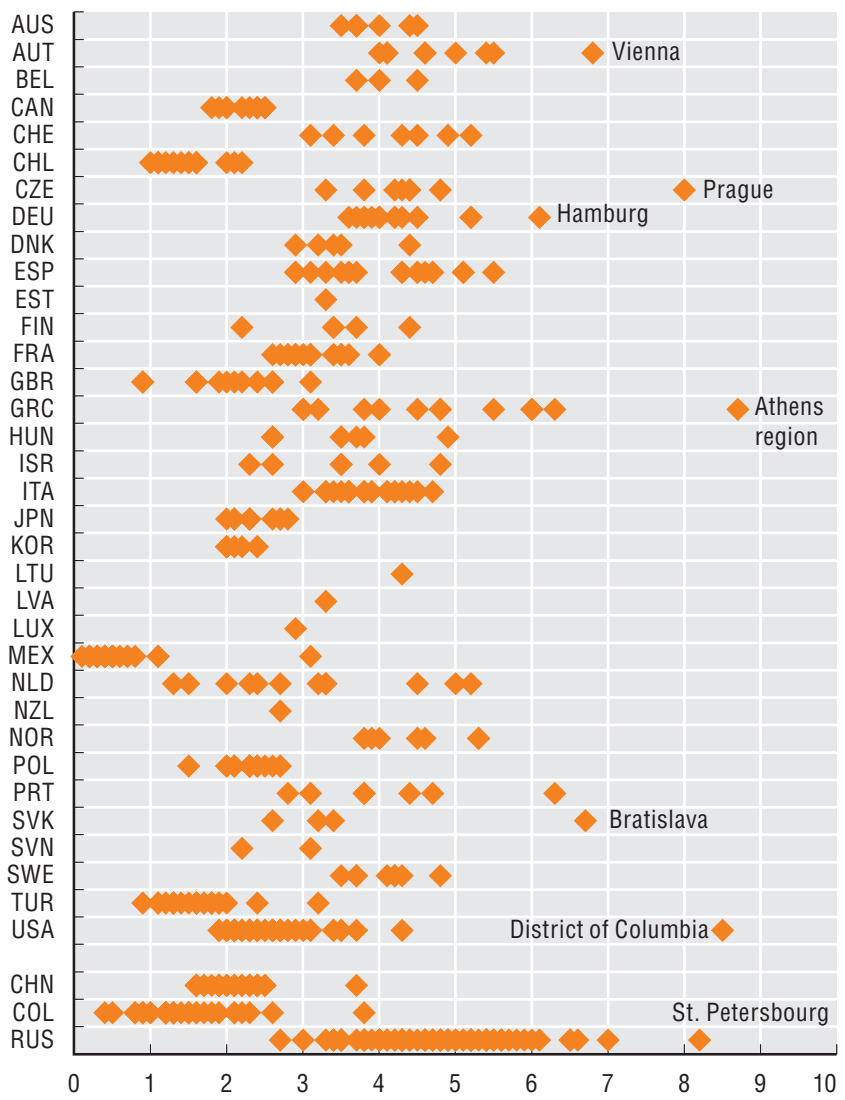

Source: OCDE (2019), Statistiques sur la santé (base de données)

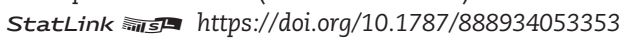


Les systèmes éducatifs des pays de l'OCDE assurent un accès universel à l'enseignement primaire et secondaire, la scolarisation étant obligatoire de 6 à 16 ans dans la majorité d'entre eux (OCDE 2018). Dans la plupart des pays, le système d'enseignement est constitué à la fois d'établissements publics et privés, ce qui permet aux parents de faire leur choix en fonction de leurs moyens financiers et de leurs préférences. L'accès à l'enseignement supérieur dépend, entre autres, du diplôme obtenu à la fin du cycle secondaire (surtout dans les pays où il existe un système d'orientation dans le cadre duquel les élèves sont répartis en différents groupes selon leurs résultats scolaires), des avantages nets que semble présenter le prolongement des études, des places disponibles et des possibilités de financement (programmes de prêts, bourses).

Le taux de scolarisation des enfants de 3 et 4 ans a augmenté ces dernières années dans toute la zone OCDE, malgré des disparités entre pays. En moyenne, en 2017, $89 \%$ des enfants de quatre ans et $79 \%$ des enfants de trois ans ont fréquenté des établissements d'éducation de la petite enfance (ou primaire). Si la scolarisation est désormais généralisée dès l'âge de trois ans dans plusieurs pays de l'OCDE, elle atteint un taux proche de 100 \% en France. Depuis 2012, la Grèce et la Pologne ont enregistré une augmentation de 16 et 18 points de pourcentage, respectivement, des taux de scolarisation des enfants de quatre ans. Pour la même période, bien que seul un peu plus d'un tiers des enfants de quatre ans fréquentent l'école en Turquie, le taux de scolarisation y a toutefois progressé de 17 points.

En moyenne, les financements privés (c'est-à-dire l'ensemble des ressources non publiques) représentent moins d'un tiers du budget de l'éducation. S'agissant des cycles d'enseignement primaire, secondaire et post-secondaire (non supérieur), moins de $10 \%$ des financements des pays de l'OCDE provenaient en 2015 de sources privées. Ce pourcentage atteignait environ $18 \%$ pour l'enseignement préprimaire et plus du triple pour l'enseignement supérieur (31\%).

Le financement des systèmes d'enseignement varie considérablement entre les pays de l'OCDE, ce qui peut être expliqué par l'existence de politiques éducatives différentes. En Finlande, Norvège et Suède, l'enseignement primaire, secondaire et post-secondaire est exclusivement financé par des fonds publics, tandis qu'en Turquie $25 \%$ des coûts sont supportés par des ressources privées. La Belgique, l'Irlande et le Luxembourg financent l'enseignement préprimaire exclusivement à partir de fonds publics, alors qu'au Japon, plus de la moitié des dépenses proviennent de fonds privés.

Certains pays favorisent l'accès à l'enseignement supérieur, en investissant des fonds publics à ce niveau. En Autriche, en Finlande, en Islande, au Luxembourg et en Norvège, la part des fonds privés consacrés à l'enseignement supérieur comptait en 2016 pour $8 \%$ au maximum, alors qu'elle atteignait au moins 64 \% des dépenses totales au Chili, aux États-Unis, au Japon et au Royaume-Uni. Les dépenses publiques consacrées à l'enseignement supérieur représentent plus de 1,8 \% du PIB en Autriche, en Finlande, en Norvège et en Suède (contre une moyenne de 1,2 \% dans la zone OCDE), ce qui permet aux universités de ces pays de dispenser un enseignement gratuit, ou d'appliquer des frais de scolarité très faibles.

Les étudiants désireux d'obtenir un diplôme d'enseignement supérieur s'inscrivent généralement dans un établissement d'enseignement supérieur dès la fin du cycle secondaire. Dans l'ensemble, près de la moitié des citoyens des pays de l'OCDE se sont inscrits à un cursus d'enseignement supérieur avant l'âge de 25 ans. C'est le cas de $70 \%$ de la population au Chili. Il arrive que certains décident de poursuivre leurs études ultérieurement afin d'actualiser leurs compétences ou d'en acquérir de nouvelles. Au Danemark et en Suisse, si près de $70 \%$ des habitants (étudiants internationaux exclus) se sont inscrits dans l'enseignement supérieur au cours de leur vie, 50 \% environ l'ont fait avant l'âge de 25 ans. Le taux de première inscription dans l'enseignement supérieur est en moyenne de $65 \%$ dans la zone OCDE, mais seulement de $58 \%$ lorsque l'on exclut les étudiants internationaux. En Nouvelle-Zélande, il est de $89 \%$, mais tombe à $61 \%$ si l'on ne tient pas compte de ces derniers.

\section{Méthodologie et définitions}

Pour tous les graphiques, les données sont tirées de la collecte de données statistiques sur l'éducation réalisée par l'Unesco, l'OCDE et Eurostat (UOE). Les dépenses privées englobent toutes les dépenses directes consacrées aux établissements d'enseignement, qu'elles soient partiellement compensées par des subventions publiques ou non. La classification des niveaux d'éducation suit la Classification internationale type de l'éducation (CITE) de 2011. L'éducation de la petite enfance (CITE 0) recouvre deux sortes de programmes: les programmes de développement éducatif de la petite enfance (CITE 01) et les programmes d'enseignement préprimaire (CITE 02). Les programmes d'éducation de la petite enfance répondent à une démarche éducative et s'adressent aux enfants n'ayant pas encore l'âge de suivre un enseignement primaire. Une inscription complète signifie qu'au moins $90 \%$ de la population est inscrite à un certain âge. On obtient le taux net d'accès à l'enseignement pour un âge donné en divisant le nombre de nouveaux inscrits de cet âge par la population totale du même âge. On calcule la somme des taux nets d'accès en additionnant les taux obtenus pour chaque âge.

\section{Pour en savoir plus}

OCDE (2019), Regards sur l'éducation 2019 : les indicateurs de l'OCDE, Éditions OCDE, Paris, https://doi.org/10.1787/6bcf6dc9-fr.

OCDE (2017), Starting Strong 2017: Key OECD Indicators on Early Childhood Education and Care, Éditions OCDE, Paris.

\section{Notes relatives aux graphiques}

Concernantles données sur Israël, voir http://doi.org/10.1787/888932315602.

11.8. Les données relatives au Chili et à la Colombie se rapportent à l'année 2017. On ne dispose pas de données pour le Danemark, la Grèce et la Suisse. Les chiffres correspondent aux pourcentages après transferts en provenance du secteur public.

11.9. Les données sont présentées par ordre décroissant, selon le taux de scolarisation des enfants de 4 ans. Les données concernant l'Afrique du Sud se rapportent à l'année 2016.

11.10. On ne dispose pas de données pour l'Australie, le Canada, la Corée, l'Estonie, la France, l'Irlande, la Lettonie et la Turquie. 
11.8. Part des dépenses privées consacrées à l'éducation après transferts, 2016

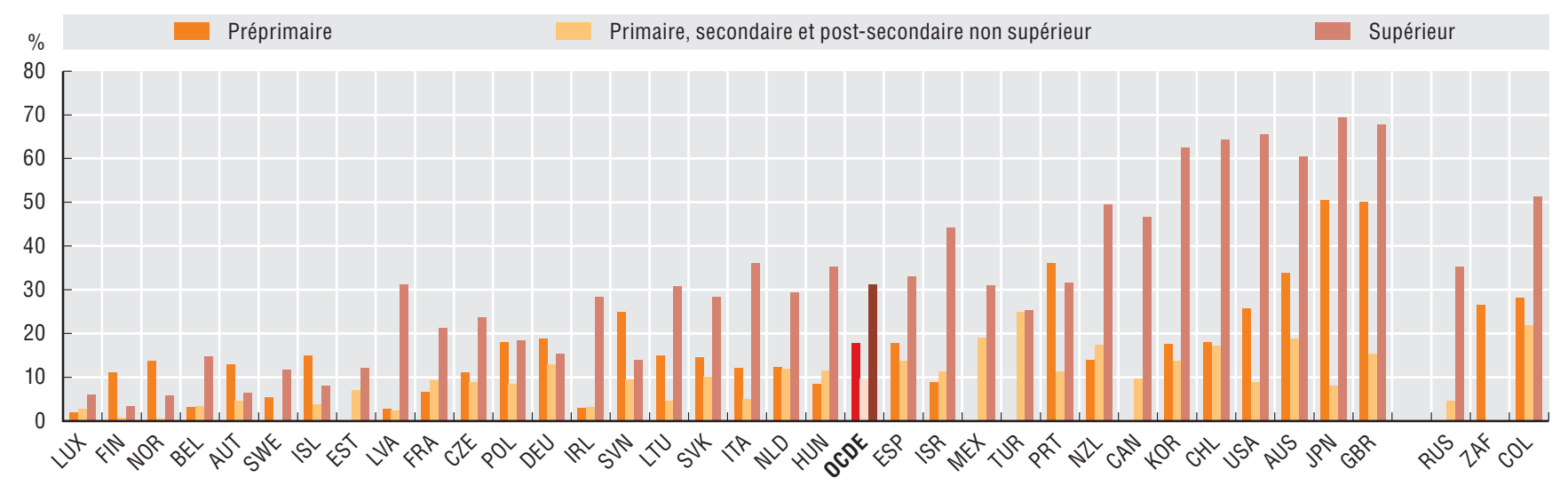

Source : Regards sur l'éducation, 2019.

StatLink नints https://doi.org/10.1787/888934053372

11.9. Taux de scolarisation des enfants de trois et quatre ans dans une structure d'éducation de la petite enfance et dans l'enseignement préprimaire, 2017

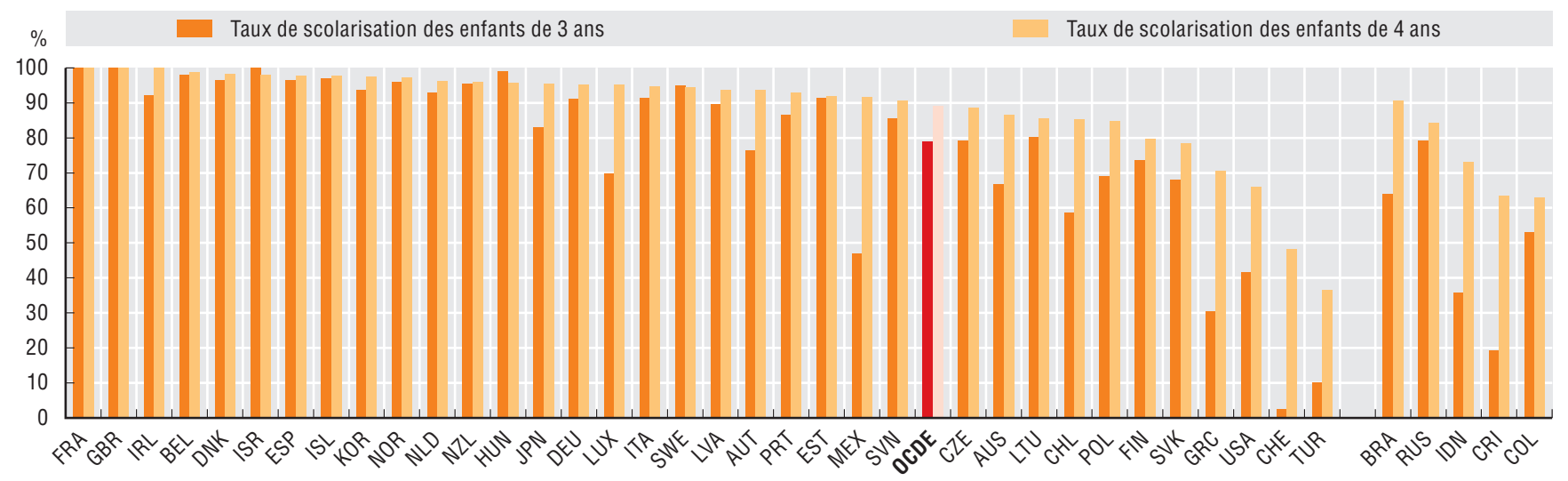

Source : Regards sur l'éducation, 2019.

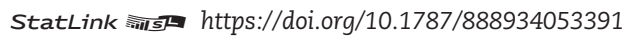

11.10. Taux de première inscription dans l'enseignement supérieur, 2017

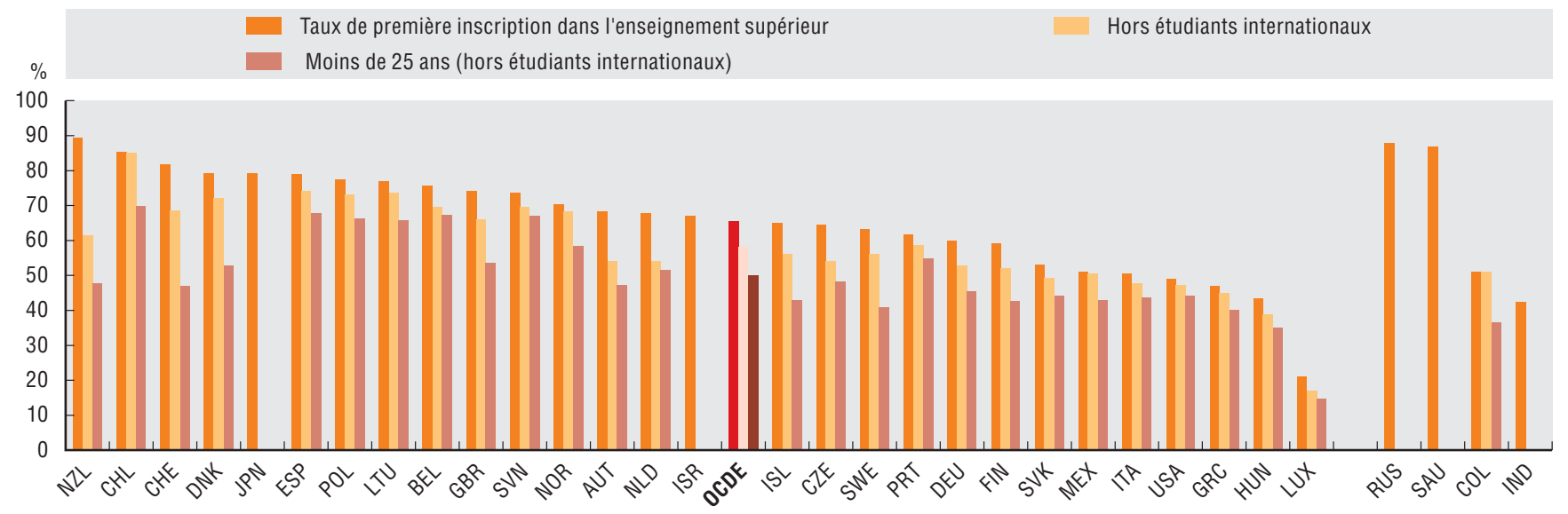

Source : Regards sur l'éducation, 2019. 
L'accès à la justice désigne la possibilité offerte aux particuliers et aux entreprises de réclamer et d'obtenir le juste règlement de leurs problèmes d'ordre juridique par l'intermédiaire d'un large éventail de services juridiques et judiciaires. Ces services juridiques englobent des services d'information, de conseil et de représentation devant des mécanismes de règlement des litiges officiels (comme les tribunaux) et alternatifs, et des dispositifs de mise en application (OCDE 2019). Une place plus importante est désormais accordée à l'autonomisation, qui permet aux individus de participer efficacement au système judiciaire et renforce leur aptitude à comprendre le droit et à en faire usage par eux-mêmes (OCDE 2019). L'état de droit exige une justice impartiale et non discriminatoire. Sans égalité d'accès aux services juridiques et judiciaires, une forte proportion de la population serait exclue et vulnérable.

Les enquêtes sur les besoins juridiques sont utiles, en ce qu'elles permettent aux responsables publics de comprendre le vécu des citoyens en quête de justice, leur parcours et les obstacles auxquels ils se heurtent pour régler un différend. Dans le cadre de ces enquêtes, on demande notamment aux personnes interrogées si elles ont été confrontés à des problèmes juridiques tout au long de la période de référence, si elles ont fait appel à une assistance juridique (si oui, de quel ordre) et si elles ont tenté de régler le litige, et de quelle manière. Certains pays ont intégré des modules portant sur les besoins juridiques dans leurs enquêtes auprès des ménages, mais ces informations ne sont pas régulièrement recueillies (OCDE/Open Society Foundations 2019). Depuis 2016, le World Justice Project collecte des données sur l'accès à la justice dans le cadre du sondage General Population Poll mené partout dans le monde, ce qui permet de procéder à des comparaisons entre pays.

En 2018, 59 \% en moyenne des personnes interrogées dans la zone OCDE ont signalé avoir connu un litige au cours des 24 mois précédents. Parmi ces individus, $32 \%$ ont eu recours à une assistance juridique, essentiellement auprès de parents ou d'amis - qu'ils aient été des professionnels du droit ou non. Il existe de fortes disparités entre les pays de l'OCDE à cet égard : si, au Pays-Bas, près de la moitié des personnes connaissant un litige ont sollicité des conseils juridiques, seules $16 \%$ d'entre elles l'ont fait en Turquie.

$14 \%$ seulement des personnes connaissant un litige ont décidé de recourir à un mécanisme officiel, comme les tribunaux, la police, les services publics, les autorités religieuses ou communautaires. En Espagne, $22 \%$ des personnes interrogées y ont fait appel, ce qui a été le cas de moins de $8 \%$ d'entre elles en Finlande, en Hongrie, en Norvège et au Royaume-Uni. La principale raison invoquée par les personnes en litige qui s'étaient abstenues de solliciter une assistance juridique est qu'elles ne jugeaient pas difficile de le régler elles-mêmes (54\% en moyenne). Ce pourcentage va de $74 \%$ des répondants au Chili à $39 \%$ en Belgique. Dans l'OCDE, près de $30 \%$ des personnes interrogées en moyenne ont signalé s'être heurtées à des obstacles - manque d'information sur la possibilité de bénéficier de conseils ou sur les services à contacter à cette fin, éloignement et crainte de solliciter une aide juridique ou des frais que celle-ci entraînerait. Quelques 40 \% des personnes interrogées en Belgique et $18 \%$ au Chili ont signalé avoir été confrontées à l'une de ces difficultés.

Sur l'ensemble de la zone OCDE, $28 \%$ des personnes interrogées n'ont pas fait appel à un tiers pour régler leur problème d'ordre juridique parce qu'elles estimaient pouvoir le résoudre seules. Aux États-Unis et au Royaume-Uni, 37 \% d'entre elles en ont témoigné, contre $19 \%$ au Japon. En moyenne, $21 \%$ des personnes interrogées dans la zone OCDE ont déclaré ne pas juger leur litige assez important ou l'estimer assez facile à régler. Ce fut le cas de $35 \%$ des répondants en Pologne.

\section{Méthodologie et définitions}

Ces données proviennent de l'enquête General Population Poll conduite par le World Justice Project en 2017 et 2018. Elles se fondent sur un échantillon de 1000 personnes interrogées dans les trois plus grandes villes de chaque pays. Une technique d'échantillonnage par quotas a été utilisée et les entretiens ont été menés en ligne et en face à face.

Les litiges couvrent tous les différends qu'un particulier peut avoir, entre autres, avec des prestataires de services, l'administration publique, son employeur, ses voisins ou ses parents. Il peut s'agir de fautes professionnelles, d'abus de pouvoir de la part des services de police, de licenciements abusifs, de différends fonciers ou de divorces. Dans le cadre de l'enquête, on a demandé aux personnes qui ont déclaré s'être heurtées à des problèmes de cet ordre si elles avaient sollicité les conseils d'une personne ou d'un organisme, ou l'intervention d'un tribunal ou d'un autre médiateur. On a ensuite demandé à celles qui avaient répondu par la négative d'en expliquer les raisons. Celles-ci ont notamment invoqué les motifs suivants : elles ne jugeaient pas le problème important ; elles estimaient les conseils inutiles ; elles redoutaient les frais qu'il faudrait engager ; elles ne savaient pas à qui s'adresser. Les personnes n'ayant pas contacté de médiateur ne l'avaient pas fait, entre autres, parce qu'elles pensaient que le problème pouvait être résolu sans faire appel à un iers, que la procédure serait longue et bureaucratique, ou parce qu'elles en craignaient les conséquences.

\section{Pour en savoir plus}

OCDE (2019), Equal Access to Justice for Inclusive Growth: Putting People at the Centre, Éditions OCDE, Paris, https://doi. org/10.1787/597f5b7f-en.

OCDE/Open Society Foundations (2019), Legal Needs Surveys and Access to Justice, Éditions OCDE, Paris, https://doi.org/10.1787/ g2g9a36c-en.

\section{Notes relatives aux graphiques}

Les données pour l'Autriche, le Canada, le Chili, le Danemark, l'Estonie, la Finlande, la Grèce, la Hongrie, l'Italie, le Mexique, la NouvelleZélande, la Norvège, le Portugal, la République tchèque, la Slovénie, le Brésil et l'Indonésie sont celles de 2017 et non de 2018. Les données pour l'Islande, l'Irlande, Israël, la Lettonie, la Lituanie, le Luxembourg, la République slovaque et la ne sont pas disponibles.

11.13. L'enquête de 2017 ne comportait pas de questions sur les raisons pour lesquelles les répondants n'avaient pas pris de mesures en vue de régler leurs litiges.

Les barres d'erreur affichent une erreur standard. 
11.11. Pourcentage d'individus ayant bénéficié d'une assistance juridique et ayant pris des mesures en vue de régler un litige au cours des deux dernières années, 2018

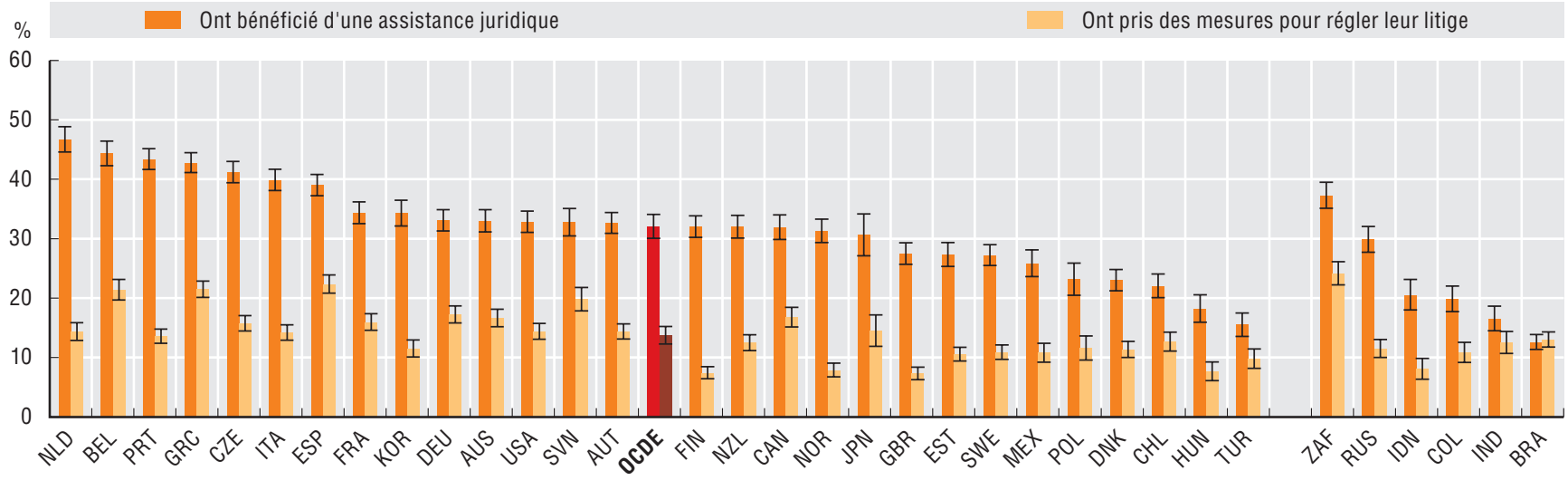

Source : World Justice Project, General Population Poll 2018.

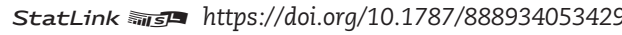

11.12. Trois principaux motifs invoqués par les individus n'ayant pas cherché à bénéficier d'une assistance juridique pour régler un litige, 2018

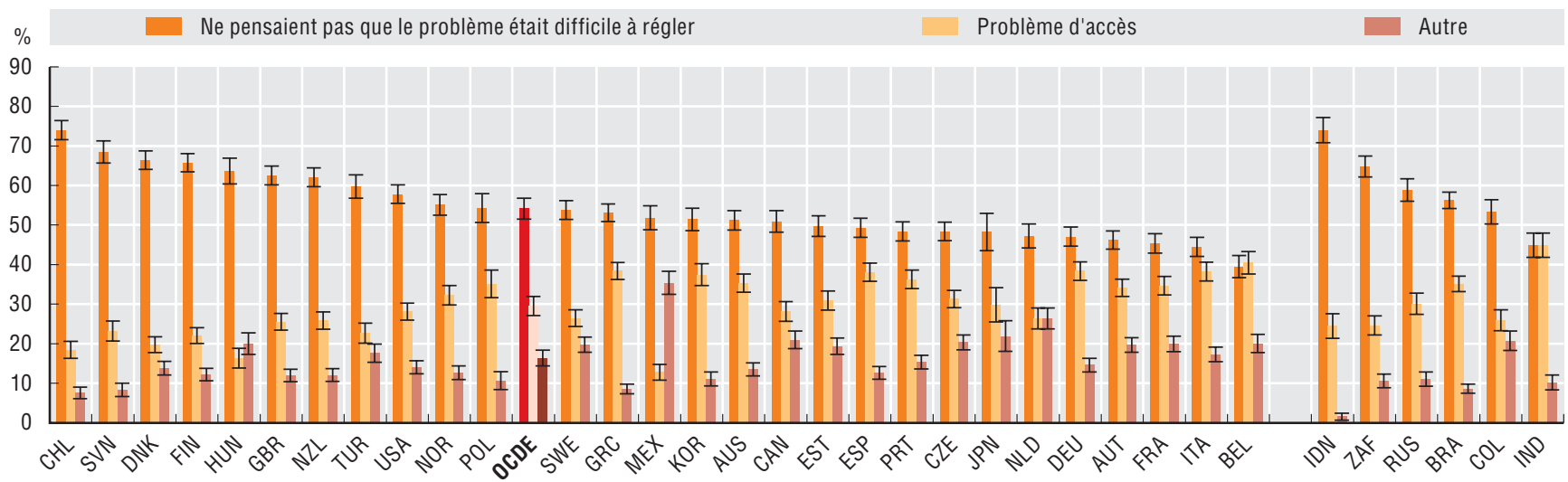

Source : World Justice Project, General Population Poll 2018.

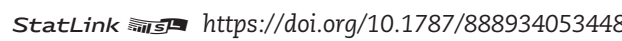

11.13. Motifs invoqués par les individus n’ayant pas pris de mesure pour régler un litige, 2018

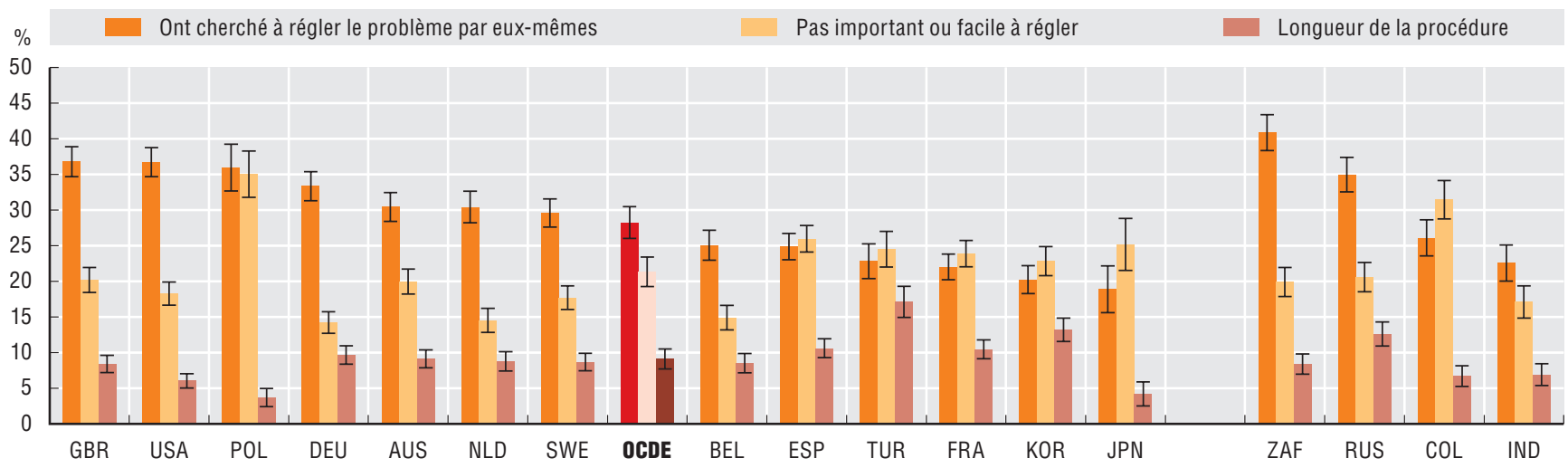

Source : World Justice Project, General Population Poll 2018. 
Les systèmes de santé, qui assurent l'accès aux services médicaux, ont par ailleurs pour objectif d'améliorer l'expérience des patients en interaction avec des prestataires de soins, d'autant que cette expérience fait désormais partie de leur grille d'évaluation des performances. Les systèmes de santé s'efforcent de donner une place plus centrale aux patients, ce qui suppose de les faire participer aux décisions concernant leur prise en charge et d'encourager la responsabilité et la transparence de la part des prestataires.

Étant donné l'importance que revêtent l'évaluation, le suivi et l'utilisation des retours des patients pour améliorer le fonctionnement des systèmes de santé, de nombreux pays recueillent des éléments de mesures de l'expérience déclarée par les patients (PREM) auprès d'échantillons de patients ayant reçu des soins hospitaliers ou ambulatoires. L'OCDE a lancé en 2018 l'initiative PaRIS (Patient-Reported Indicators Surveys Enquête sur les indicateurs fondés sur les retours des patients). L'enquête International Health Policy Survey du Commonwealth Fund collecte en outre des données sur l'expérience des patients dans 11 pays de l'OCDE. Celle de 2017 a porté sur les personnes âgées de 65 ans et plus.

La communication entre les médecins et les patients est l'un des aspects pris en considération dans les PREM. Pour faire des patients des coproducteurs de leur propre santé, il est indispensable de leur fournir les informations pertinentes et de leur permettre d'influer sur leur traitement, surtout en ce qui concerne les soins auto-administrés. Dans l'enquête de 2017 du Commonwealth Fund, sur l'ensemble de la zone OCDE, en moyenne $59 \%$ des patients âgés indiquaient que leur médecin traitant les faisait participer aux décisions concernant leur prise en charge et leur traitement. Quelques $73 \%$ des patients aux États-Unis et $72 \%$ en Nouvelle-Zélande ont indiqué être consultés par leur médecin, alors que seuls $26 \%$ d'entre eux le sont en France.

De longs délais d'attente peuvent conduire à une aggravation des symptômes des patients (Siciliani, Borowitz et Moran 2013) et ont une incidence défavorable sur leur expérience. Selon l'enquête de 2017 du Commonwealth Fund, 15 \% des seniors en moyenne ont dû attendre deux mois ou plus avant d'obtenir un rendez-vous avec un spécialiste sur recommandation d'un généraliste. En Suisse et aux Pays-Bas, $5 \%$ des personnes interrogées seulement ont déclaré avoir dû attendre aussi longtemps, proportion qui atteint $22 \%$ en Norvège et $28 \%$ au Canada.

Une intervention chirurgicale est généralement nécessaire pour réparer ou remplacer l'articulation de la hanche après une fracture. Il est généralement admis qu'une intervention chirurgicale rapide optimise les résultats pour le patient et limite les risques de complications (OCDE à paraître). En moyenne, $81 \%$ des personnes qui avaient besoin d'une arthroplastie de la hanche ont été opérées dans un délai de deux jours en 2017 (chiffre en progression de trois points de pourcentage par rapport à 2012). C'était le cas de plus de $96 \%$ des patients au Danemark, en Norvège et aux Pays-Bas, qui affichent les taux les plus hauts, mais de moins de la moitié des patients en Lettonie et au Portugal. C'est en Israël que la plus forte amélioration est observée, ce taux étant passé de $68 \%$ en 2012 à $89 \%$ en 2017.

\section{Méthodologie et définitions}

L'intervention chirurgicale après admission à l'hôpital pour une fracture de la hanche est un indicateur qui correspond au pourcentage de patients âgés de 65 ans et plus hospitalisés au cours d'une année donnée pour fracture du col du fémur qui ont été opérés dans les deux jours suivant leur admission.

Les données des graphiques 11.14 et 11.15 proviennent de l'enquête International Health Policy Survey of Older Adults (portant sur les personnes âgées de 65 ans et plus) de 2017 du Commonwealth Fund, qui couvre 11 pays de l'OCDE. Les entretiens ont été réalisés par téléphone entre mars et juin (à l'exception de la Suisse où ils ont été réalisés en ligne). Les échantillons allaient de 500 à 7000 individus. Les données ont été pondérées pour en assurer la représentativité. Pour en savoir plus, consulter le site www.commonwealthfund.org.

\section{Pour en savoir plus}

Fujisawa, R. et Klazinga, N. (2017), "Measuring patient experiences (PREMS) : Progress made by the OCDE and its member countries between 2006 and 2016", Documents de travail de l'OCDE sur la santé, $n^{\circ} 102$, Éditions OCDE, Paris, https://doi.org/10.1787/893a07d2-en.

OCDE (2019, à paraître), Panorama de la santé 2019 : indicateurs de l'OCDE, Éditions OCDE, Paris.

Siciliani, L., Borowitz, M. et Moran, V. (dir.) (2013), Waiting Time Policies in the Health Sector: What Works?, Études de l'OCDE sur les politiques de santé, Éditions OCDE, Paris.

\section{Notes relatives aux graphiques}

11.14. La question «Dans quelle mesure votre médecin traitant ou le personnel médical que vous consultez vous font-ils participer autant que vous le souhaitez aux décisions concernant votre prise en charge ou votre traitement? " a été posée aux personnes interrogées qui ont indiqué avoir un ou plusieurs médecins traitants.

11.15. La question "Après que l'on vous ait recommandé de consulter un spécialiste, ou que vous ayez décidé de le faire, combien de jours, semaines ou mois avez-vous dû attendre avant d'obtenir un rendezvous ? " n'a été posée qu'aux personnes interrogées qui avaient signalé avoir consulté ou avoir eu besoin de consulter un spécialiste au cours des deux années précédentes.

11.16. Les données pour l'Allemagne, les Pays-Bas, le Portugal et la République tchèque sont celles de 2011 et non de 2012. Les données pour les Pays-Bas et l'Islande sont celles de 2016, de 2015 pour le Costa Rica, l'Italie, la Finlande, et la Suisse et de 2014 pour la Belgique et la Norvège, au lieu de 2017. Pour les données concernant Israël, voir http://doi.org/10.1787/888932315602. 
11.14. Pourcentage de patients estimant que leur médecin traitant les fait participer autant qu'ils le souhaitent aux décisions concernant leur prise en charge ou leur traitement, 2017

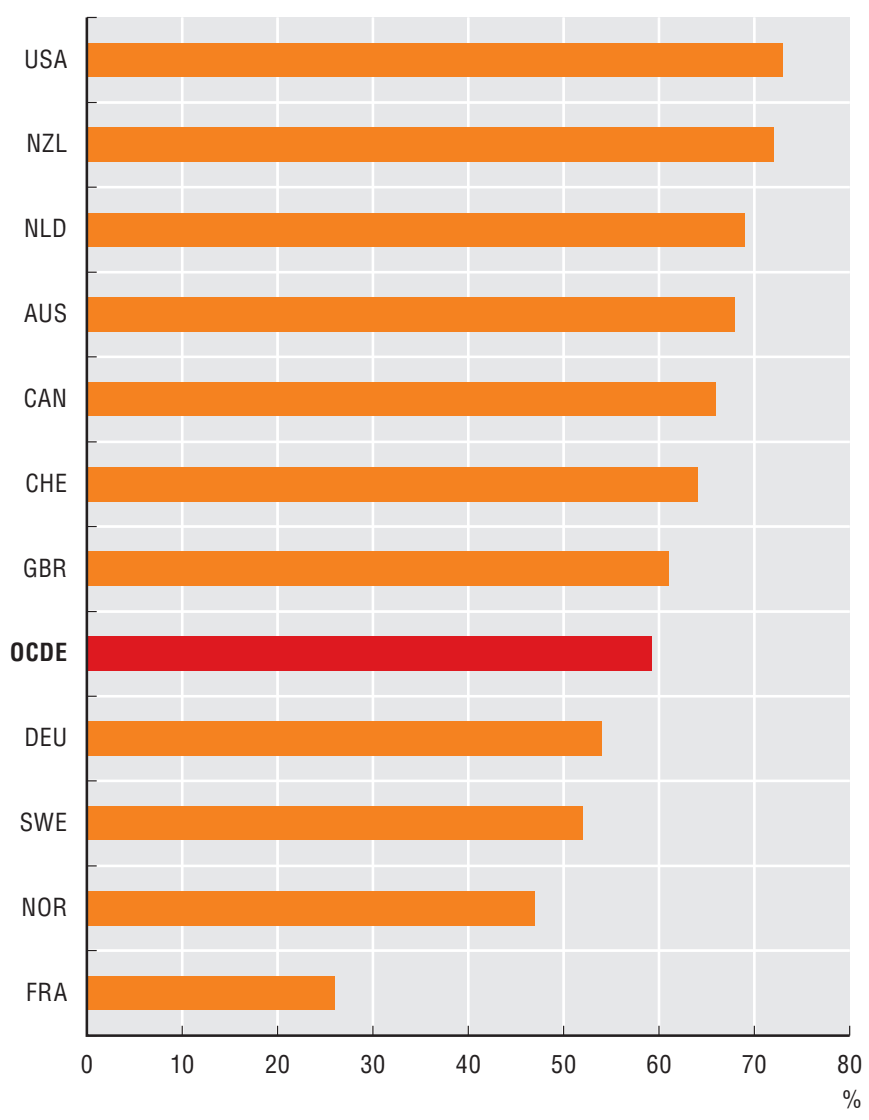

Source : Enquête International Health Policy Survey of Older Adults, 2017, Commonwealth Fund

StatLink +intsh https://doi.org/10.1787/888934053486
11.15. Pourcentage de patients ayant dû attendre deux mois ou plus pour obtenir un rendez-vous chez un spécialiste, 2017

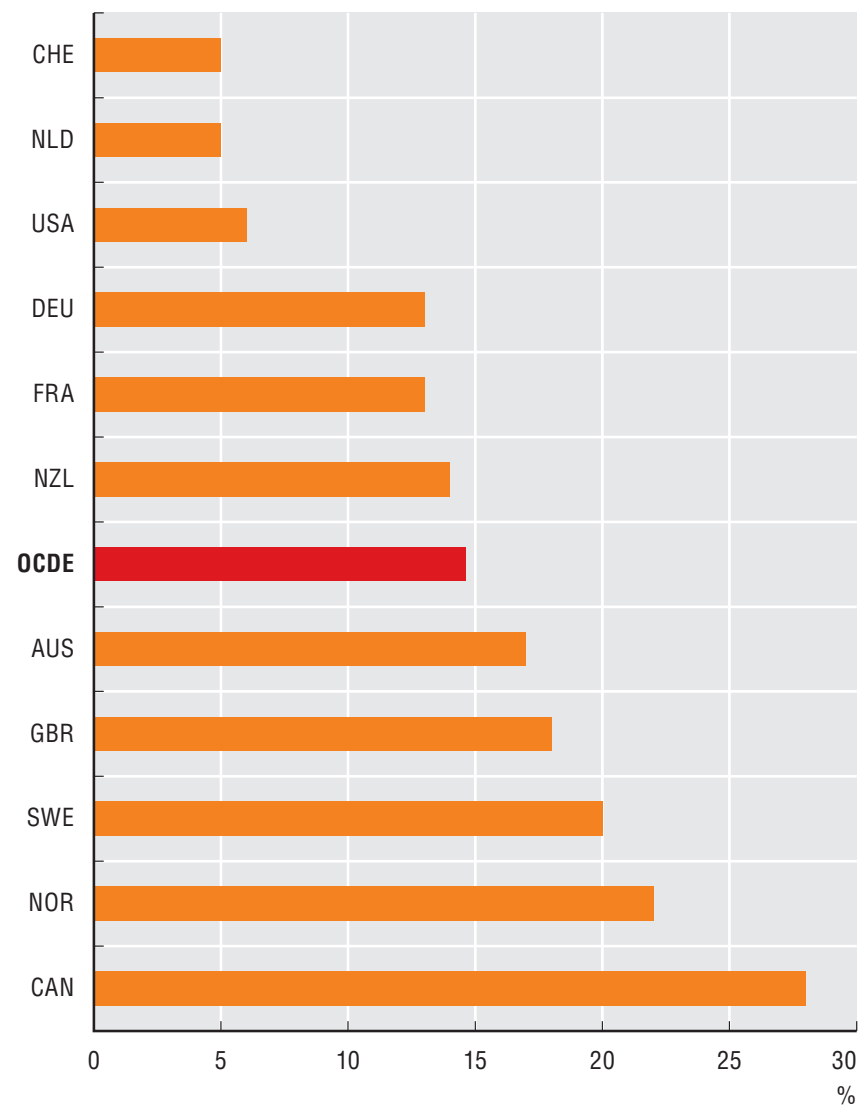

Source : Enquête International Health Policy Survey of Older Adults, 2017, Commonwealth Fund

StatLink त्राज https://doi.org/10.1787/888934053505

11.16 Opération chirurgicale après hospitalisation pour une fracture de la hanche, 2012 et 2017 (ou année la plus proche)

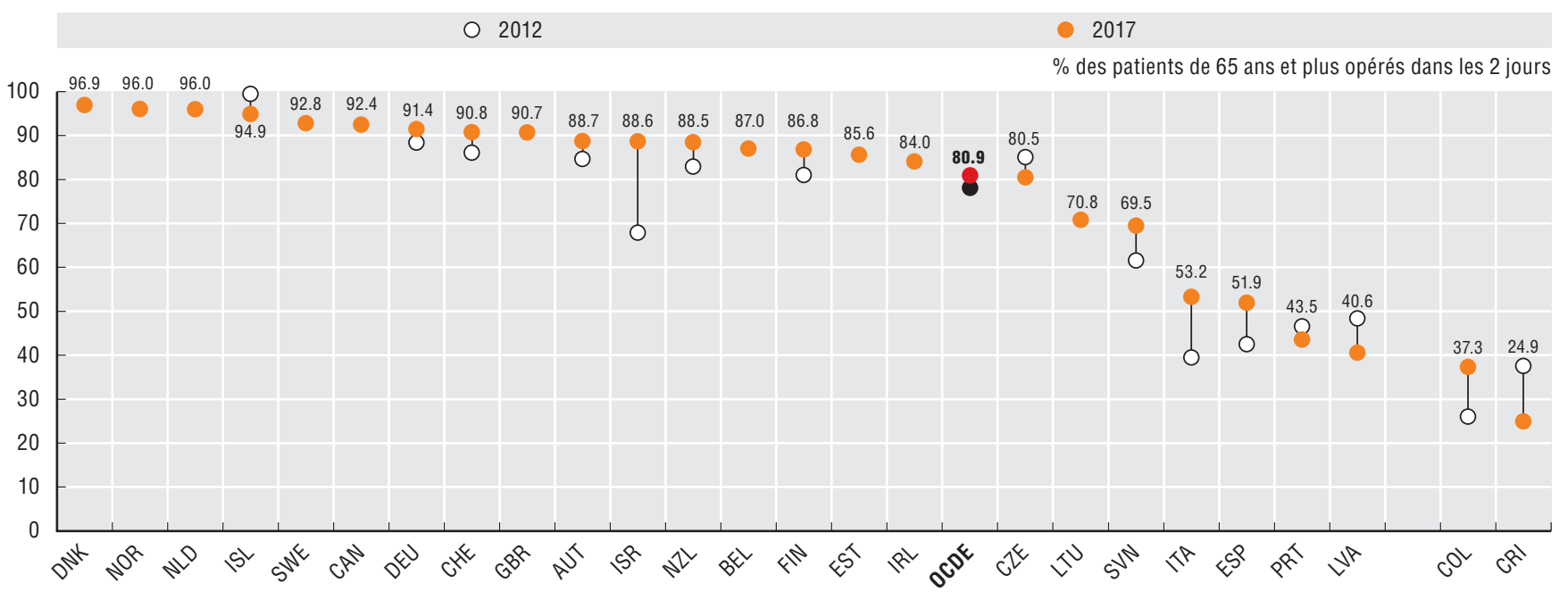

Source : Panorama de santé, 2019. 
Le marché du travail évolue rapidement : quelques emplois peu qualifiés sont progressivement remplacés par des processus automatisés. Les nouveaux emplois exigent une connaissance des technologies de l'information et de la communication des compétences analytiques et de la créativité. Le système éducatif aide à préparer la jeunesse aux emplois de demain. Dans ce contexte, la réactivité consiste à assurer l'égalité des chances en matière d'éducation, face à des élèves issus de milieux divers, aux niveaux de revenus différents et aux conditions de vie distinctes.

Les jeunes sans emploi et sortis du système éducatif (ni étudiant, ni employé, ni stagiaire - NEET) sont l'un des principaux problèmes auxquels se trouvent confrontés les pays de la zone OCDE, tout particulièrement à la suite de la crise de 20072008. Dans les pays européens de l'OCDE, 5,2 \% des jeunes âgés de 18 à 24 ans relevaient de cette catégorie en 2018 (chiffre qui marque une amélioration de 1,7 point par rapport à 2012). Au total, 10,7 \% des jeunes âgés de 18 à 24 ans avaient quitté l'école avant ou dès la fin du premier cycle du secondaire (c'est-à-dire des. jeunes prématurément déscolarisés) en 2018, soit une baisse de 2,1 points par rapport à 2012. La plupart des pays ont réussi à diminuer le nombre de jeunes dans cette situation. La Grèce, l'Irlande et le Portugal l'ont réduit de moitié.

En 2010, l'Allemagne a lancé l'initiative des chaînes de formation pour aider les jeunes à passer de l'école à la formation professionnelle. L'initiative comprend une analyse potentielle pour aider les jeunes à découvrir leurs propres forces et talents. Par exemple, les journées d'ateliers axées sur la pratique leur offrent une vue d'ensemble pratique dans environ 18 domaines d'activité et leur permettent d'identifier des emplois correspondant à leurs sources d'intérêt.

Les établissements d'enseignement ont besoin d'enseignants du premier cycle du secondaire qualifiés pour faire face aux besoins des étudiants et pour les encourager à poursuivre leurs études. Dans la zone OCDE, $31 \%$ des chefs d'établissement font état d'un manque d'enseignants dotés des compétences nécessaires pour enseigner à des élèves présentant des besoins particuliers (c'est-à-dire ceux qui sont désavantagés mentalement, physiquement ou émotionnellement). De plus, $18 \%$ ont déclaré manquer de personnel qualifié pour enseigner dans des contextes multiculturels, et $16 \%$ n'ont pas assez d'enseignants dotés des compétences nécessaires pour prendre en charge des élèves défavorisés sur le plan socioéconomique.

La gouvernance des établissements scolaires est aussi un facteur qui influe sur la réactivité du système éducatif. Dans la zone OCDE, $38 \%$ des décisions en moyenne sont prises au niveau de l'établissement d'enseignement ou après consultation avec celui-ci ; 23 \% sont arrêtées par l'établissement selon un cadre défini par les autorités supérieures; et $10 \%$ sont prises en toute autonomie.

Le degré d'autonomie accordé aux établissements varie selon les pays de l'OCDE, certains leur concédant un faible pouvoir de décision et d'autres encourageant une gouvernance décentralisée. Aux Pays-Bas, les établissements sont autorisés à prendre $92 \%$ des décisions, onze fois plus qu'en Grèce et en Turquie. En Angleterre, ils prennent $48 \%$ des décisions en toute autonomie, alors que $33 \%$ des pays de l'OCDE environ ne leur accordent aucun pouvoir de décision.

\section{Méthodologie et définitions}

Les données utilisées pour le graphique 11.17 sont issues des cycles 2018 et 2012 de l'Enquête sur les forces de travail de l'Union européenne (EFT UE), une grande enquête par sondage auprès des ménages (plus de 1.7 million d'entretiens) menée chaque trimestre auprès de personnes âgées de 15 ans et plus. Selon la définition d'Eurostat, les jeunes ayant quitté prématurément le système d'éducation et de formation sont les personnes âgées entre 18 et 24 ans dont le niveau d'étude ne dépasse pas le premier cycle du secondaire (niveaux 0-3C de la CITE 2011) et qui n'ont suivi aucun enseignement ni aucune formation au cours des quatre semaines précédant l'entretien. L'indicateur est exprimé sous forme de pourcentage des personnes âgées de 18 à 24 ans qui satisfont à ces critères sur l'ensemble de la population de cette tranche d'âge. Une personne âgée de 18 à 24 ans qui ne suit pas d'études et n'a pas d'emploi au moment considéré est classée dans la catégorie des NEET (ni étudiant, ni employé, ni stagiaire).

Les données relatives au graphique 11.18 proviennent de l'édition 2018 de l'Enquête internationale de l'OCDE sur l'enseignement et l'apprentissage (TALIS). L'enquête se compose de deux questionnaires, dont l'un est adressé aux chefs d'établissement et l'autre aux enseignants. Deux cents établissements au moins ont été sélectionnés pour chaque pays. Le graphique rend compte des résultats de l'enquête envoyée aux chefs d'établissement du premier cycle de l'enseignement secondaire (CITE 2), dans laquelle il leur était demandé : "Dans quelle mesure les problèmes suivants limitent-ils actuellement la capacité de votre établissement à offrir un enseignement de qualité ? ".

Les données du graphique 11.19 proviennent de l'enquête sur la prise de décision menée en 2017 par l'OCDE auprès d'un panel d'experts du premier cycle de l'enseignement secondaire dans chaque pays. L'enquête portait sur les niveaux de décision au sein des établissements de ce cycle et sur leur degré d'autonomie, par type d'autorité. Les domaines couverts étaient l'organisation de l'enseignement (par exemple temps d'enseignement), la gestion du personnel (par exemple recrutement), la planification et les structures (par exemple création d'écoles) et l'affectation et l'utilisation des ressources. Les résultats sont indiqués en pourcentage de l'ensemble des décisions.

\section{Pour en savoir plus}

Carcillo, S. et al. (2015), "NEET Youth in the Aftermath of the Crisis : Challenges and Policies", Documents de travail de l'OCDE sur les questions sociales, l'emploi et les migrations, $n^{\circ} 164$, Éditions OCDE, Paris, https://doi.org/10.1787/5js6363503f6-en.

\section{Notes relatives aux graphiques}

Concernantles données sur Israël, voir http://doi.org/10.1787/888932315602.

11.17. Les données ne sont disponibles que pour les pays européens de l'OCDE.

11.18. : Les pays sont classés par ordre croissant en fonction de la pénurie d'enseignants qualifiés pour enseigner aux élèves présentant des besoins particuliers. Les données pour le Canada ne concernent que l'Alberta; les données pour le Royaume-Uni se rapportent uniquement à l'Angleterre. Les données pour l'Allemagne, la Grèce, l'Islande, l'Irlande, le Luxembourg, la Pologne et la Suisse ne sont pas disponibles.

11.19. Pour la Belgique, la présentation des données est divisée entre Belgique francophone et Belgique néerlandophone; pour le Royaume-Uni, elle est divisée entre Angleterre (Ang.) et Écosse. 
11.17. Pourcentage de jeunes âgés de 18 à 24 ans ayant quitté prématurément le système d'éducation et de formation et actuellement sans emploi, 2012 et 2018

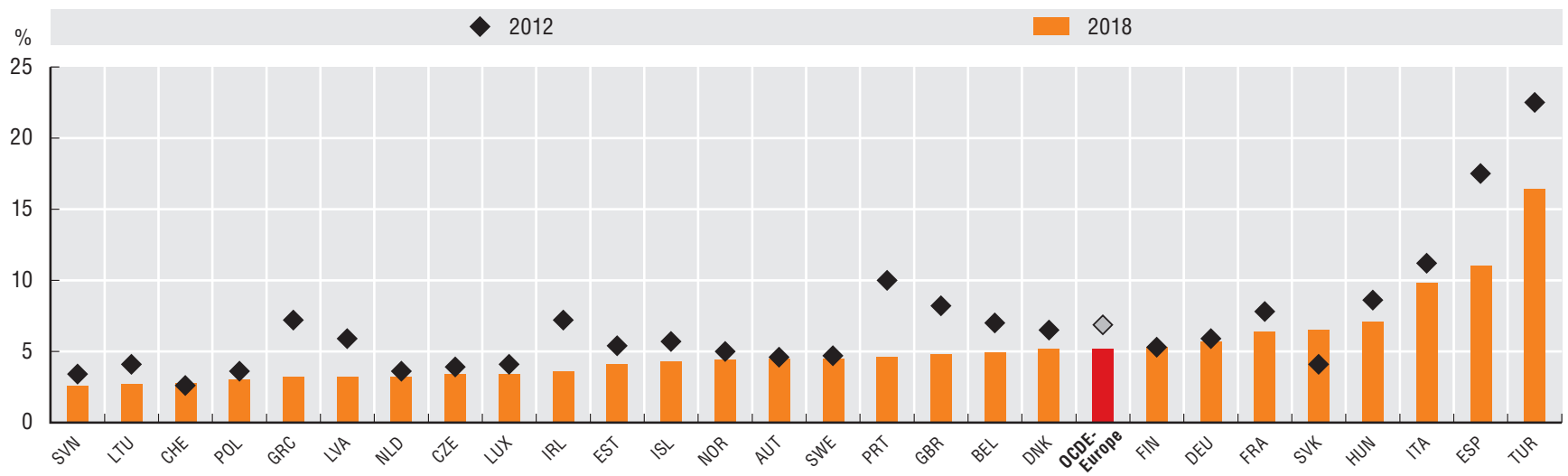

Source : Enquête sur les forces de travail de l'Union européenne, 2018 et 2012

StatLink 茼 $h$ https://doi.org/10.1787/888934053543

\subsection{8. Établissements manquant d'enseignants qualifiés pour prendre en charge des élèves présentant des besoins} particuliers dans le premier cycle de l'enseignement secondaire, 2018

Pourcentage de chefs d'établissement indiquant que le manque d'enseignants qualifiés limite " dans une certaine mesure » ou « beaucoup » la capacité de leur établissement à offrir un enseignement de qualité

Difficultés socioéconomiques

Contexte multiculturel

Besoins particuliers

Percentage of principals reporting that shortages of qualified teachers hinder the school's capacity to provide quality instruction "quite a bit"”

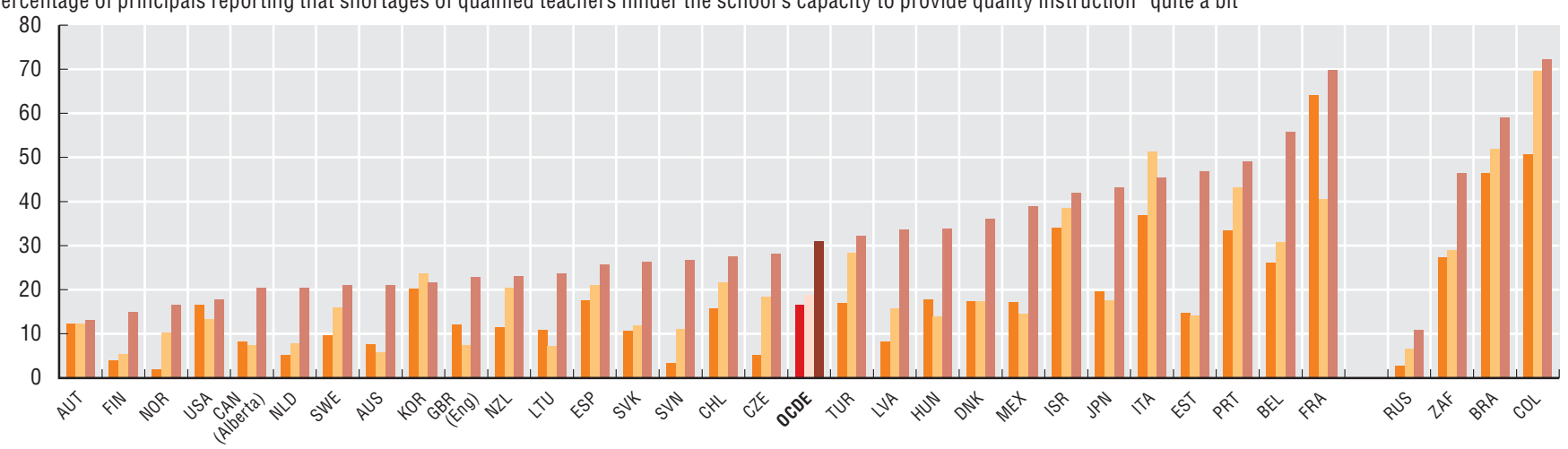

Source : OCDE (2018), Enquête internationale sur l'enseignement et l'apprentissage

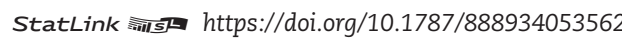

\subsection{Pourcentage de décisions prises à l'échelon des établissements dans le premier cycle} de l'enseignement secondaire public, 2017

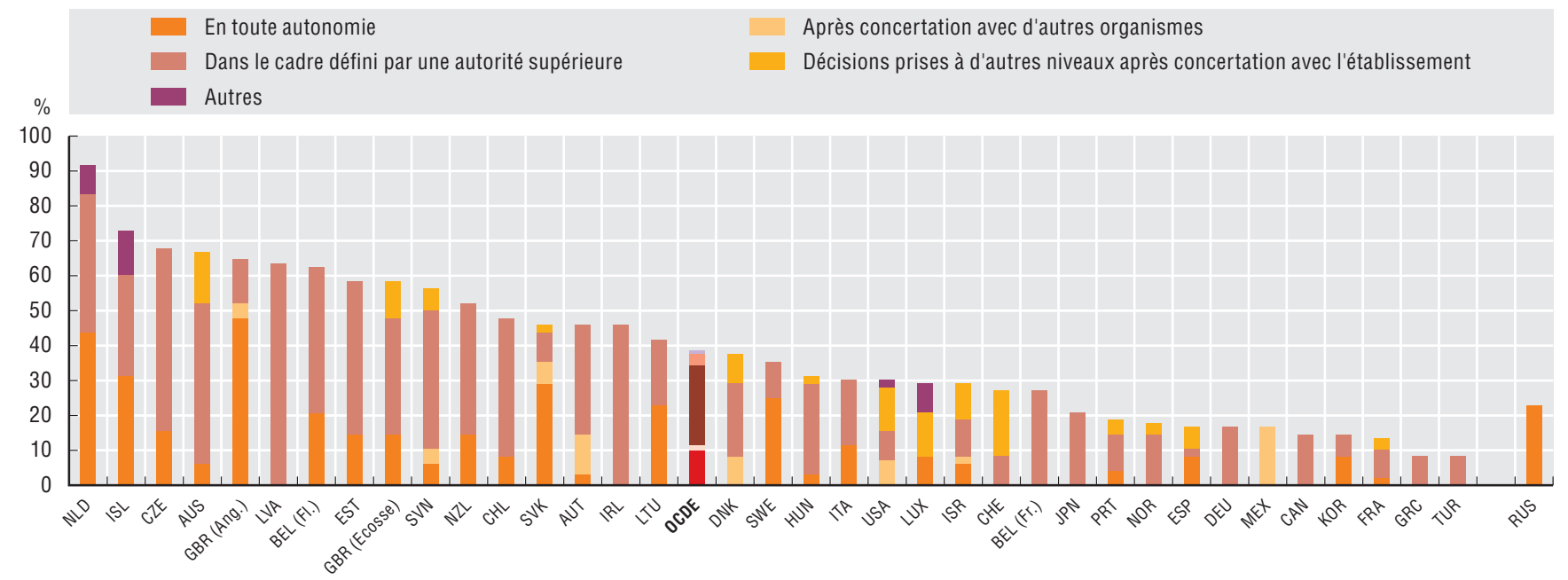

Source: Regards sur l'éducation, 2018. 
L'état de droit implique d'appliquer les mêmes règles impartiales aux particuliers, institutions et entités. Son absence peut compromettre l'aptitude des individus à faire valoir leurs droits et à régler leurs problèmes juridiques. Or, ces derniers sont très courants - ils surgissent dans la vie quotidienne et relèvent le plus souvent du droit civil. Les retards en la matière pénalisent les citoyens de diverses manières, avec des répercussions sur leur budget, leur productivité, leur santé, leur emploi et leur entourage, et ils risquent de les dissuader de s'appuyer sur le droit pour régler leurs litiges futurs. Un système judiciaire réactif garantit que le " bon " éventail de services soit fourni aux " bons " usagers, dans les " bons » domaines du droit, au « bon » endroit et au « bon " moment (OCDE 2019).

Les facteurs qui font obstacle à la résolution rapide des litiges sont principalement le manque de juges et d'autres professionnels compétents, le manque de salles d'audience, le recours limité aux mécanismes alternatifs de règlement des litiges ainsi que d'autres problèmes liés à l'insuffisance des fonds disponibles. Outre les contraintes matérielles, le manque de souplesse des systèmes judiciaires (qui n'autorisent pas la réaffectation des dossiers), l'inefficacité des règles de procédure (qui, par exemple, ne permettent pas de poursuivre les témoins qui refusent de témoigner), une affectation inefficace des ressources et la faiblesse du recours aux TIC entravent la gestion des dossiers.

La Commission européenne pour l'efficacité de la justice (CEPEJ) recueille des données sur la durée estimative des affaires civiles, commerciales, administratives et autres (qui ne tombent pas sous le coup de la justice pénale), c'est-à-dire leur délai de traitement (disposition time, DT). L'indicateur DT permet d'estimer le nombre maximum de jours nécessaires à la résolution d'une affaire au sein d'un territoire donné, compte tenu du nombre d'affaires résolues par an et des affaires pendantes.

S'agissant des affaires non pénales non contentieuses (procédures non contestées), les durées de traitement les plus courtes ont été observées en 2016 au Danemark (21 jours), en Estonie (40) et en Lituanie (41). C'est, à l'inverse, en France, en Italie et en Israël qu'elles sont les plus longues (plus de 300 jours). Le délai nécessaire pour arrêter une décision a augmenté en République tchèque $(+39$ jours par rapport à 2012), en France (+37) et en Pologne (+35). Par rapport à 2012, le DT a diminué de 161 jours en Suisse et de 120 jours en République slovaque.

En ce qui concerne les affaires civiles et commerciales contentieuses, c'est en Lituanie (88 jours), au Luxembourg (91) et en Suisse (107) que les DT étaient les plus courts en 2016. À l'inverse, les délais de décision les plus longs sont observés en Grèce (plus de 18 mois pour résoudre une affaire), en Italie (près de 18 mois) et en Turquie (plus d'un an). Les plus fortes améliorations sont intervenues en République slovaque (307 jours de moins qu'en 2012), au Portugal (80 jours) et en Italie (-76). Les délais pour résoudre les cas se sont, à l'inverse, allongés en Turquie ( 245 jours de plus) et en Grèce (141 jours de plus).

S'agissant du contentieux administratif, les pays faisant preuve de la plus grande rapidité en 2016 ont été la Lituanie (72 jours), Israël (101 jours), l'Estonie (108 jours), la Suède (108 jours) et la Hongrie (109 jours). Les plus lents (2,5 ans et plus) ont été la Grèce, l'Italie et le Portugal. La situation s'est améliorée en République slovaque (- 530 jours par rapport à 2012). La Grèce a aussi ramené ses délais de 1520 jours en 2012 à 1086 en 2016. En Slovénie, au contraire, le DT a plus que doublé, passant de 130 jours en 2012 à 282 en 2016.

\section{Méthodologie et définitions}

Ces données sont tirées de la base de données de la Commission européenne pour l'efficacité de la justice (CEPEJ), qui rend compte de l'évaluation des systèmes judiciaires pour 2016 et les années antérieures. La durée des procédures correspond au délai estimatif nécessaire pour qu'une affaire soit tranchée, c'est-à-dire au délai nécessaire à un tribunal pour arriver à une décision en première instance. On obtient cette durée en divisant le nombre d'affaires pendantes d'une année donnée par le nombre d'affaires résolues au cours de la même période, puis en le multipliant par 365. Cet indicateur ne fournit pas d'information sur le délai moyen de traitement d'une affaire, mais il permet d'estimer la durée d'une affaire au sein d'un territoire spécifique.

Le contentieux civil et commercial correspond aux litiges opposant des parties - un divorce contentieux, par exemple. En revanche, les affaires non contentieuses portent sur des procédures - injonctions de paiement, par exemple - non contestées. Les affaires commerciales relèvent de la compétence de juridictions commerciales spécialisées dans certains pays et des juridictions civiles dans d'autres. Le contentieux d'ordre administratif oppose les citoyens aux autorités locales, régionales ou nationales. Les affaires administratives relèvent de la compétence de juridictions administratives spécialisées dans certains pays et des juridictions civiles dans d'autres.

L'administration de la justice et la répartition des responsabilités entre les tribunaux varient selon les pays ; il convient donc d'interpréter les comparaisons internationales avec prudence. En effet, les types de tribunaux et d'affaires pris en compte peuvent différer, de même que les méthodes de collecte et de catégorisation des données.

\section{Pour en savoir plus}

CEPEJ (2018), « Systèmes judiciaires européens - Efficacité et qualité de la justice ", Commission européenne pour l'efficacité de la justice (CEPEJ), Conseil de l'Europe, Strasbourg, https:// rm.coe.int/rapport-avec-couv-18-09-2018-fr/16808def9d.

OCDE (2019), Equal Access to Justice for Inclusive Growth: Putting People at the Centre, Éditions OCDE, Paris, https://doi. org/10.1787/597f5b7f-en.

RECJ (2011), Timeliness report 2010-2011, Réseau européen des Conseils de la Justice, Bruxelles.

\section{Notes relatives aux graphiques}

Les pays sont rangés par ordre croissant des délais nécessaires, en nombre de jours, lors de la dernière année disponible.

Pays non européens de l'OCDE : données non disponibles. Concernant les données sur Israël, voir http://doi.org/10.1787/888932315602.

11.22. Les données relatives au Royaume-Uni couvrent uniquement l'Angleterre et le pays de Galles. 
11.20. Délai de résolution des affaires civiles, commerciales, administratives et autres, 2012,2014 et 2016

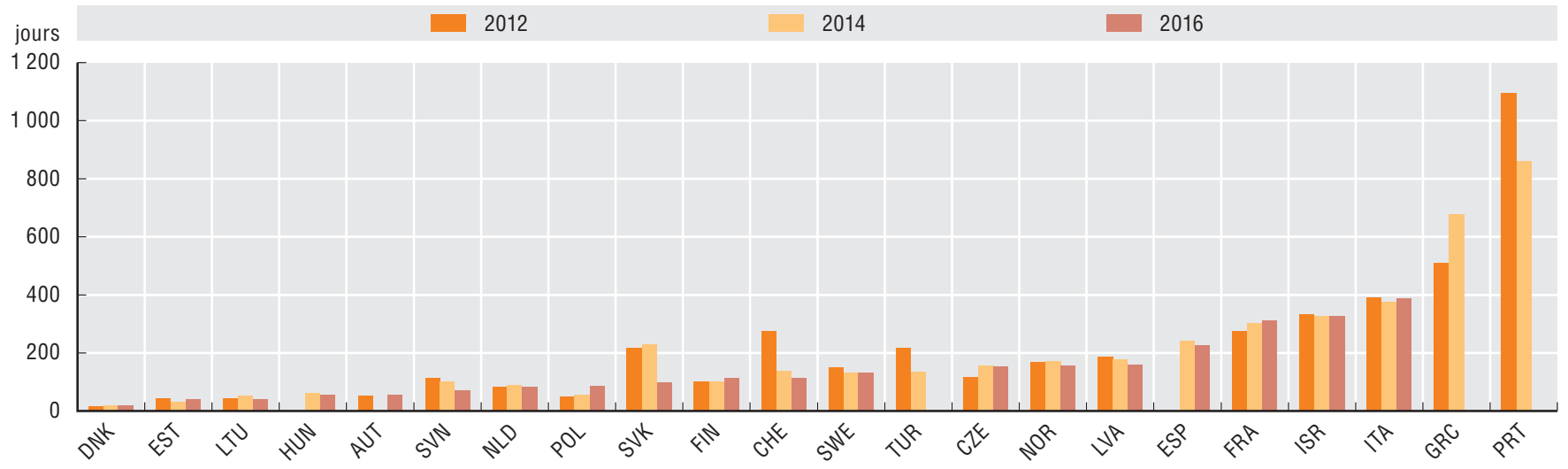

Source : Base de données des systèmes judiciaires européens, 2019

11.21. Délai de résolution des affaires contentieuses civiles et commerciales, 2012, 2014 et 2016

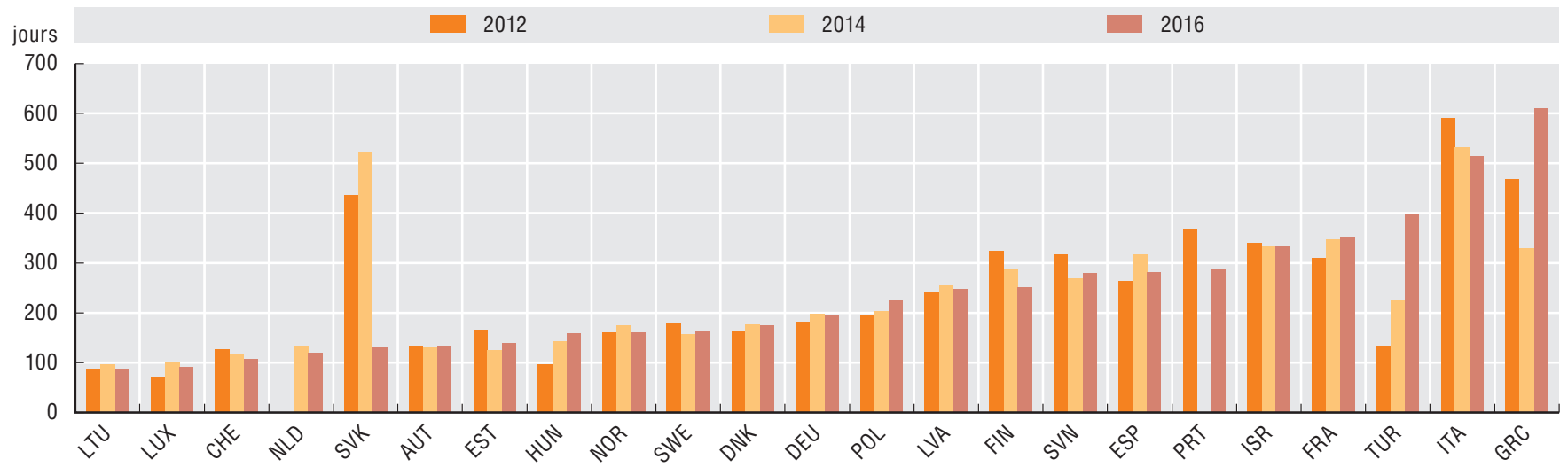

Source : Base de données des systèmes judiciaires européens, 2019

11.22. Délai de résolution des affaires administratives, 2012, 2014 et 2016

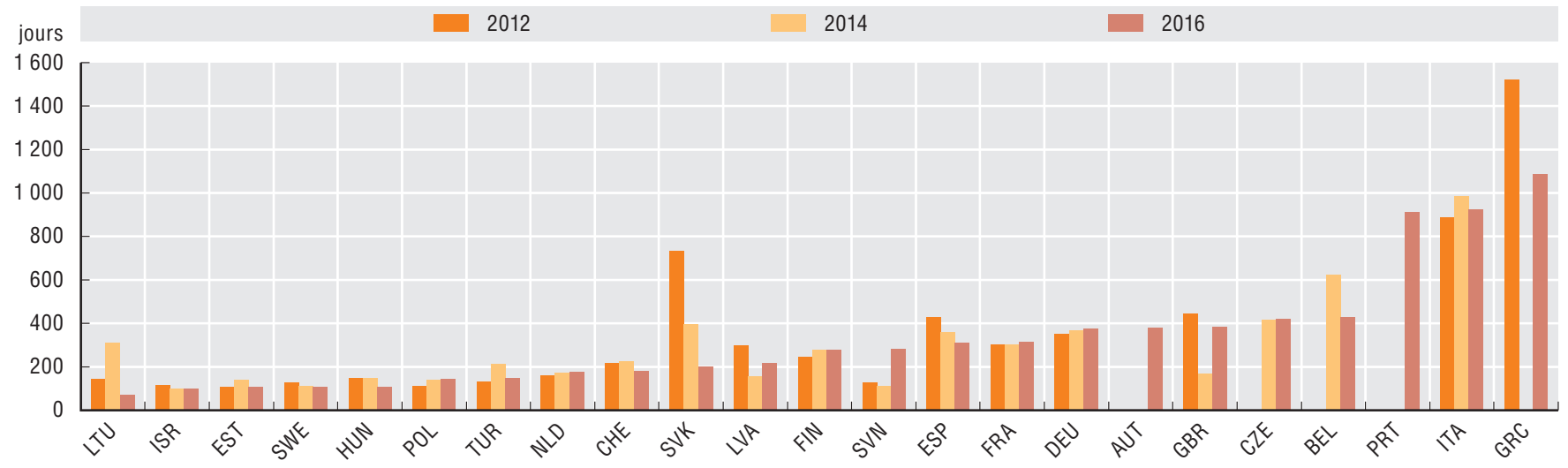

Source : Base de données des systèmes judiciaires européens, 2019 
Il appartient au système de santé d'éviter les problèmes de santé (prévention) et de traiter les problèmes aigus ou chroniques lorsqu'ils surgissent (traitement). La qualité des soins de santé renvoie à l'aptitude du système à obtenir des réalisations souhaitables (des vies plus longues et en meilleure santé, par exemple) et à éviter tout effet négatif résultant des processus de soins eux-mêmes (Kelley et Hurst 2006).

Le taux de mortalité dans les 30 jours suivant une hospitalisation pour infarctus aigu du myocarde (IAM ou crise cardiaque) est un indicateur reconnu, qui permet d'évaluer les réalisations liées aux soins hospitaliers. Sur l'ensemble des pays de l'OCDE, pour les IAM, le nombre de décès pour 100 hospitalisations de personnes âgées de 45 ans et plus a reculé de 2,6 entre 2007 et 2017 ; une amélioration est observable dans la plupart des pays, notamment le Chili et la République slovaque $(-5,7$ décès) et les Pays-Bas $(-5,4)$. Seul le Mexique fait état d'une hausse modique de ce chiffre. l'Australie, le Danemark, l'Islande, la Norvège, les Pays-Bas, et la Suède affichent les taux de mortalité les plus faibles (tous inférieurs à $4 \%$ ).

Les soins primaires sont généralement le premier contact des patients avec le système de santé ; ils ont pour objet de prévenir, de diagnostiquer de façon précoce et de prendre en charge les affections courantes. L'asthme, la bronchopneumopathie chronique obstructive (BPCO) et l'insuffisance cardiaque congestive (ICC) sont des affections de longue durée répandues dans les pays de l'OCDE. Il existe pour ces trois pathologies des traitements éprouvés qui peuvent, pour la plupart, être dispensés au niveau des soins primaires. Un système de soins primaires qui fournit des services accessibles et de qualité peut réduire les épisodes aigus de dégradation de l'état de santé des personnes qui en sont atteintes et réduire le nombre d'hospitalisations inutiles (OCDE 2019).

Dans les pays de l'OCDE, le taux d'hospitalisation pour asthme et BPCO était de 183 pour 100000 habitants en 2017. Le Chili, l'Italie, le Japon, le Mexique et le Portugal affichaient des taux inférieurs à la moitié de la moyenne de l'OCDE ; ceux de la Hongrie et de la Turquie, à l'inverse, étaient presque deux fois plus élevés. La Lettonie est le pays qui enregistrait le taux le plus élevé pour l'asthme (93 hospitalisations pour 100000 habitants) ; la Hongrie, celui qui affichait le taux le plus haut pour la BPCO (354 pour 100000 habitants).

Sur l'ensemble des pays de l'OCDE, le taux d'hospitalisation pour ICC était de 233 pour 100000 habitants en 2017. Le taux le plus bas était observé au Mexique (57), suivi de la Corée (88) et du Chili (96). C'est la Pologne qui affichait le taux le plus élevé d'hospitalisations évitables pour cette pathologie (510 pour 100000 habitants), devant la Lituanie (502) et la République slovaque (479).

\section{Méthodologie et définitions}

Le taux de létalité des IAM correspond au pourcentage de patients âgés de 45 ans et plus qui décèdent dans les 30 jours qui suivent leur hospitalisation. Les taux présentés au graphique 11.23 font référence aux décès intervenus dans l'hôpital où le patient a été initialement admis. Les taux sont standardisés par rapport à l'âge et au sexe.

Les indicateurs présentés correspondent au nombre d'admissions hospitalières avec un diagnostic principal d'asthme, de BPCO ou d'ICC de personnes âgées de 15 ans et plus pour 100000 habitants. Les taux ont été standardisés par rapport à l'âge et au sexe en fonction de la population 2010 de la zone OCDE âgée de 15 ans et plus. Les hospitalisations faisant suite à un transfert depuis un autre hôpital et pendant lesquelles le patient décède ne sont pas prises en compte dans les calculs, car ces situations sont jugées difficilement évitables.

\section{Pour en savoir plus}

Kelley, E. et Hurst, J. (2006), "Health Care Quality Indicators Project : Conceptual Framework Paper", Documents de travail de l'OCDE sur la santé, n² 23, Éditions OCDE, Paris, https://doi. org/10.1787/440134737301.

OCDE (2019, à paraître), Panorama de la santé 2019 : les indicateurs de l'OCDE, Éditions OCDE, Paris.

\section{Notes relatives aux graphiques}

Concernantles données sur Israël, voir http://doi.org/10.1787/888932315602.

11.23. Les données concernant l'Autriche, la Belgique, le Chili, la Corée, le Japon, la Lettonie et le Royaume-Uni portent sur l'année 2008 ; les données sur la Finlande, la France, le Mexique, la Slovénie et la Colombie sur l'année 2009 et non 2007. Les données sur la France, l'Italie, le Luxembourg et la Nouvelle-Zélande concernent 2015, pour l'Australie, l'Islande et les Pays-Bas 2016 au lieu de 2017. Les données indiquées pour le Royaume-Uni portent uniquement sur l'Angleterre et sont présentées au niveau de groupements d'hôpitaux (les trusts).

11.24 et 11.25. Les données pour l'Australie, les États-Unis, l'Islande, les Pays-Bas et la Pologne portent sur 2016 ; les données sur la France, le Luxembourg, la Suisse et la Turquie (seulement le graphique 11.24) concernent 2015 ; les données sur la Belgique (seulement le graphique 11.24) et la Nouvelle-Zélande concernent 2014 ; les données sur la Hongrie portent sur 2012 et le Japon 2011 et non 2017 ; les données sur la Grèce et la Lettonie sont non disponibles ; Luxembourg et Japon, données non disponibles pour le graphique 11.25. 
11.23. Taux de mortalité dans les 30 jours suivant une hospitalisation pour IAM, 2007 et 2017

$$
\text { ○ } 2007
$$

Taux standardisé par rapport à l'âge et au sexe pour 100 admissions de personnes âgées de 45 ans et plus

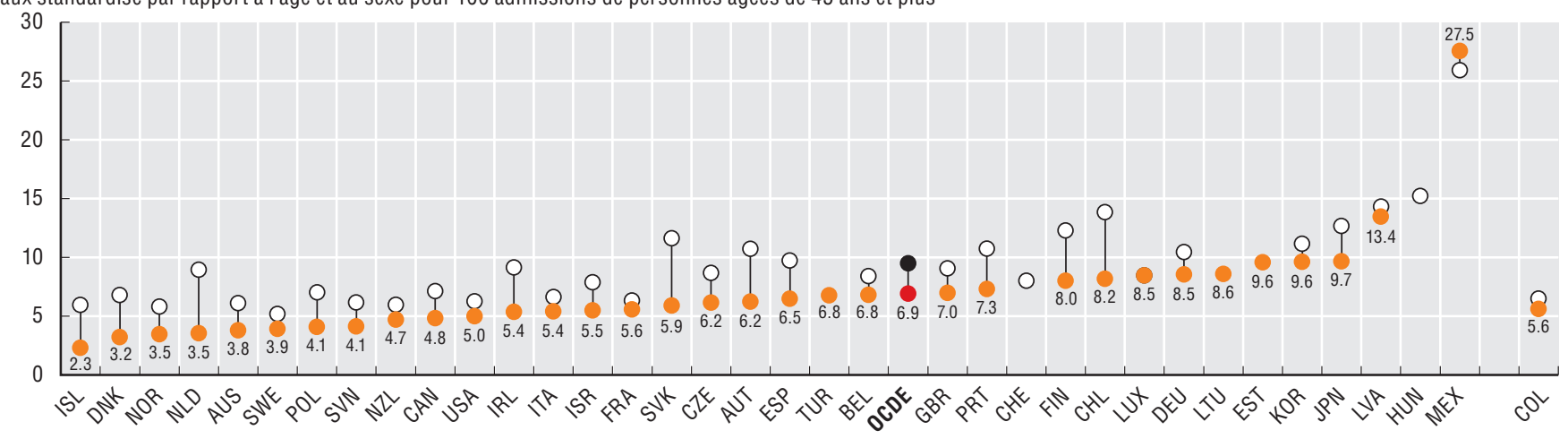

Source : OCDE (2019), Statistiques de l'OCDE sur la santé (base de données)

11.24. Hospitalisations pour asthme et BPCO parmi la population adulte, 2017 (ou année la plus proche)

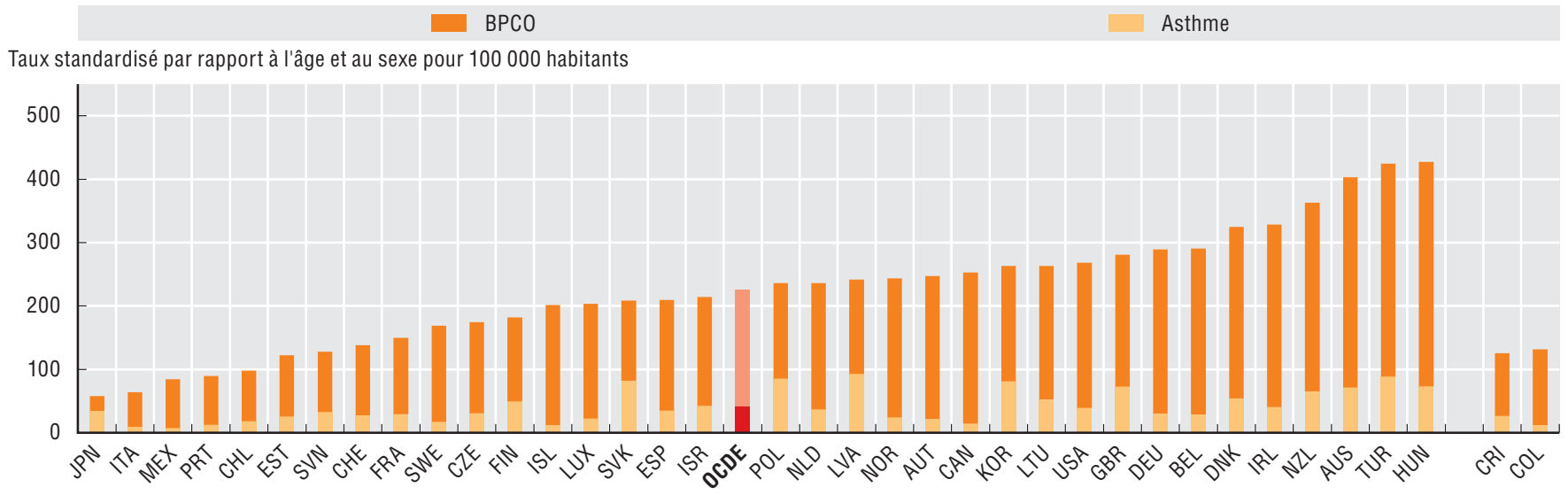

Source : OCDE (2019), Statistiques de l'OCDE sur la santé (base de données)

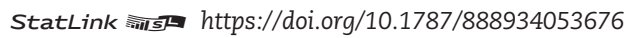

11.25. Hospitalisations pour insuffisance cardiaque congestive (ICC) parmi la population adulte, 2017 (ou année la plus proche)

Taux standardisés en fonction de l'âge et du sexe pour 100000 habitants

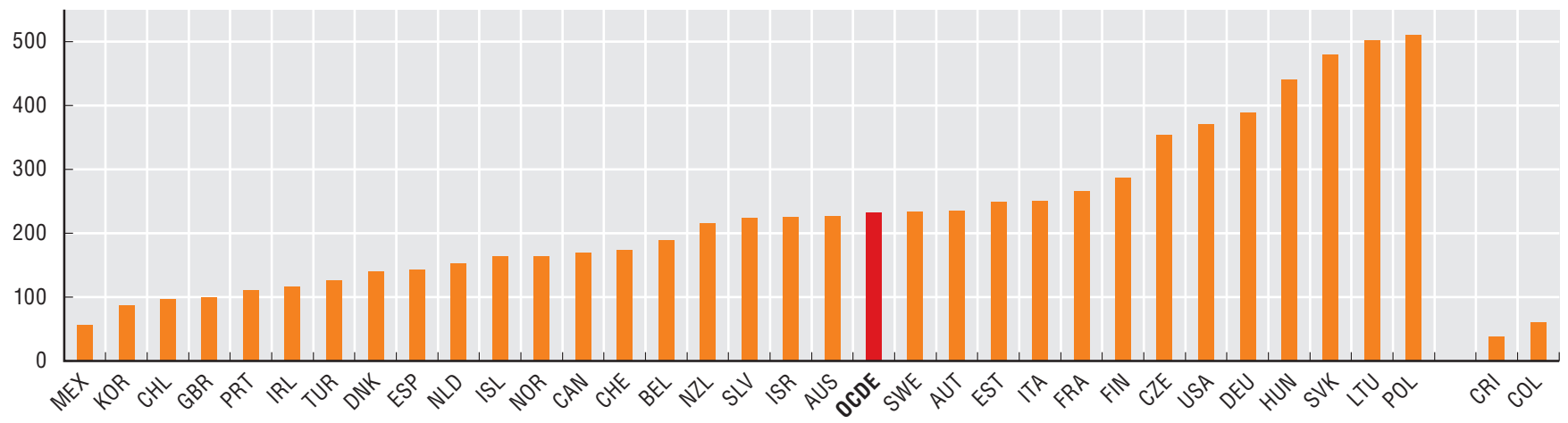

Source : OCDE (2019), Statistiques de l'OCDE sur la santé (base de données) 
Le système éducatif a pour mission de doter les individus de connaissances, compétences et outils dont ils auront besoin pour évoluer tout au long de leur vie. On peut mesurer la qualité de l'enseignement à l'aune de l'efficacité avec laquelle ils fournissent aux élèves les compétences nécessaires pour réussir dans la société dans laquelle ils vivent. Les investissements éducatifs qui ciblent les plus jeunes présentent plus d'intérêt que ceux qui interviennent à un stade plus tardif, et un climat scolaire favorable est indispensable pour offrir de meilleures chances aux enfants socio-économiquement défavorisés (OCDE 2012).

Il existe deux grandes évaluations internationales en matière d'éducation : le Programme international de l'OCDE pour le suivi des acquis des élèves (PISA), les études Tendances en matière d'études internationales de mathématiques et de sciences (TIMSS) et l'Étude de progrès en alphabétisation en lecture internationale (PIRLS) de l'Association internationale pour l'évaluation du rendement scolaire (IEA). L'enquête PISA porte sur les élèves de 15 ans ; l'étude TIMSS, sur les élèves effectuant leur quatrième et leur huitième année de scolarité ; l'étude PIRLS, sur les élèves de 4 e année (âgés de 13 à 14 ans). Ces évaluations offrent des comparaisons entre pays et permettent d'identifier les différences entre les catégories d'élèves et d'établissements.

Dans l'ensemble, le classement issu de l'étude PIRLS menée en 2016 dans les pays de l'OCDE participants est resté stable par rapport à 2011 : la Finlande et l'Irlande occupent le haut du classement et la France et la Wallonie (Belgique) enregistrent les scores les plus bas des pays de l'OCDE participants. Les plus fortes améliorations sont observées en Lituanie (+22 points sur l'échelle de résultats), en Australie (+17), en Hongrie (+15), en Irlande $(+15)$ et en Espagne (+15), et les plus fortes baisses au Portugal (-13) et en Israël (-11). Les résultats de cette enquête sont fortement corrélés à ceux de l'enquête PISA menée en 2015 dans les pays participants, ce qui indique que la performance moyenne des systèmes éducatifs reste stable au fils des années de scolarisation, et que ces évaluations sont fiables.

Les enfants qui ont accès à une éducation préscolaire sont plus susceptibles d'améliorer leurs compétences tout au long de la vie et de connaître une situation plus favorable. Les écarts ne se résorbent généralement pas au cours de la scolarité. L'étude PIRLS met en évidence les établissements où plus de $75 \%$ des élèves entrent dans l'enseignement primaire munis de compétences de base en lecture - la lecture de quelques mots, par exemple. Dans les pays de l'OCDE, $21 \%$ des élèves fréquentent des établissements de ce type - cette proportion étant de $96 \%$ en Irlande, et de $94 \%$ en Irlande du Nord. C'est au Chili que l'écart de résultats est le plus prononcé entre les élèves qui fréquentent des établissements où la majorité des enfants entament leur scolarité pourvus de compétences de base et la moyenne. La situation inverse s'observe en Israël, en Italie et en Pologne ; dans ces pays, toutefois, moins de $15 \%$ des élèves fréquentent ce type d'établissement où la majorité entrent dans le primaire avec des compétences de base en lecture.

L'intégration des élèves à l'école peut expliquer les variations de performance. Le questionnaire de l'étude PIRLS évalue leur sentiment d'appartenance en leur demandant, entre autres, s'ils se plaisent à l'école et si les enseignants les traitent de manière équitable. Si $82 \%$ des élèves portugais ont déclaré un fort sentiment d'appartenance, ce n'était le cas que pour $42 \%$ des élèves en République tchèque. Dans la plupart des pays de
l'OCDE, ceux qui expriment un fort sentiment d'appartenance obtiennent de meilleurs résultats que ceux qui expriment un faible sentiment d'appartenance. Les écarts les plus importants sont constatés en Irlande du Nord et en Finlande. Israël est le seul pays où les élèves qui déclarent un faible sentiment d'appartenance obtiennent de meilleurs résultats que ceux qui se disent très intégrés.

\section{Méthodologie et définitions}

Ces données sont tirées de l'étude PIRLS de 2016. Celleci a été réalisée tous les cinq ans par l'IEA, qui produit également l'étude TIMSS. En ce qui concerne la couverture, 50 pays et 11 régions de référence y ont participé en 2016, soit 346852 élèves au total. L'évaluation a porté sur quatre processus de compréhension de l'écrit : retrouver des informations explicitement énoncées, en tirer des déductions simples, interpréter et intégrer des idées et des informations et évaluer et analyser le contenu et les éléments textuels. L'échelle des acquis de l'étude PIRLS a été établie en 2001, sur la base des résultats de l'ensemble des pays participants. Elle est comprise entre 300 et 700 points, le point central, qui correspond à la moyenne des résultats globaux en 2001, étant de 500.

Le questionnaire portant sur le contexte scolaire demandait aux chefs d'établissement d'indiquer le pourcentage d'élèves qui entraient en $1^{\text {re }}$ année capables de reconnaître la plupart des lettres de l'alphabet, de lire quelques mots, de lire des phrases, de lire une histoire, d'écrire les lettres de l'alphabet et d'écrire quelques mots. Le graphique 11.27 montre seulement les résultats des établissements dont $75 \%$ des élèves au moins possédaient au minimum trois de ces compétences.

Le questionnaire relatif au contexte de vie des élèves demandait aux enfants déclarer leur accord avec les énoncés suivants : " J'aime bien être à l'école "; " Je me sens en sécurité à l'école »; "Je me sens à ma place dans cette école ", "Les enseignants de cette école sont justes avec moi ", "Je suis fier/fière d'être dans cette école ". Un sentiment élevé d'appartenance signifie que l'élève se déclare " tout à fait d'accord " avec trois de ces énoncés et « plutôt d'accord » avec les deux autres.

\section{Pour en savoir plus}

OCDE (2013), Équité et qualité dans l'éducation : comment soutenir les élèves et les établissements défavorisés, Éditions OCDE, Paris, https://doi.org/10.1787/9789264028050-fr.

\section{Notes relatives aux graphiques}

La présentation de la Belgique est scindée en deux : Belgique/Wallonie (Fr) et Belgique/Flandre (Fl). Les données fournies pour le RoyaumeUni correspondent à l'Angleterre et à l'Irlande du Nord.

Corée, Estonie, Grèce, Islande, Japon, Luxembourg, Mexique, Suisse et Turquie : données non disponibles. Concernant les données sur Israël, voir http://doi.org/10.1787/888932315602. 
11.26. Progrès en alphabétisation en lecture internationale (PIRLS), 2011 et 2016

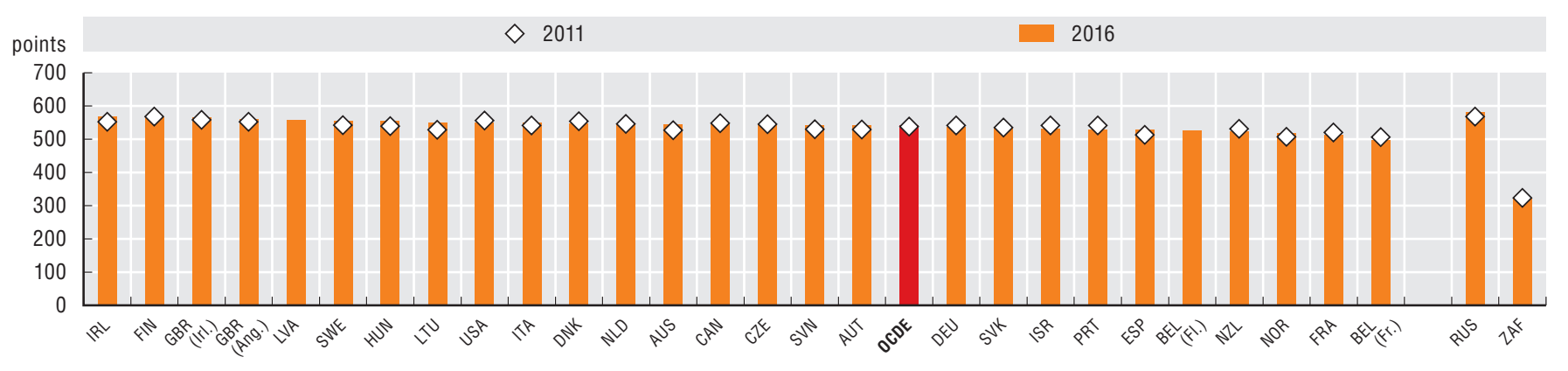

Source: Étude PIRLS (Progress in International Reading Literacy Study), 2016

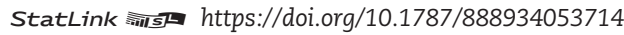

11.27. Résultats de l'étude PIRLS concernant les élèves qui fréquentent un établissement où plus de $75 \%$ d'entre eux sont entrés pourvus de quelques compétences en lecture et en écriture, 2016

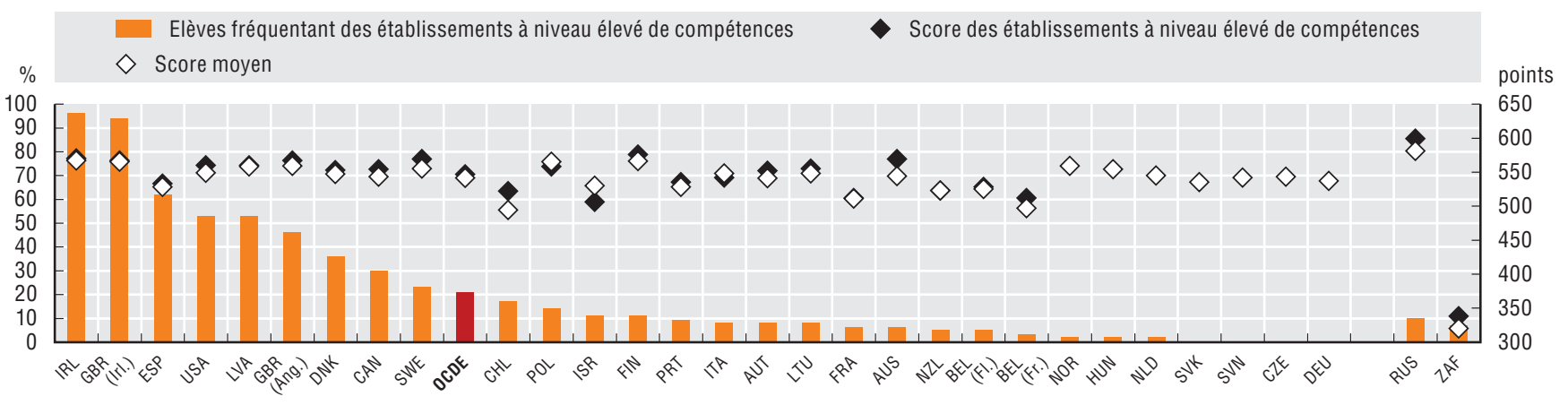

Source: Étude PIRLS (Progress in International Reading Literacy Study), 2016

StatLink ails https://doi.org/10.1787/888934053733

11.28. Score de l'étude PIRLS selon le sentiment d'appartenance et pourcentage d'élèves déclarant un fort sentiment d'appartenance, 2016

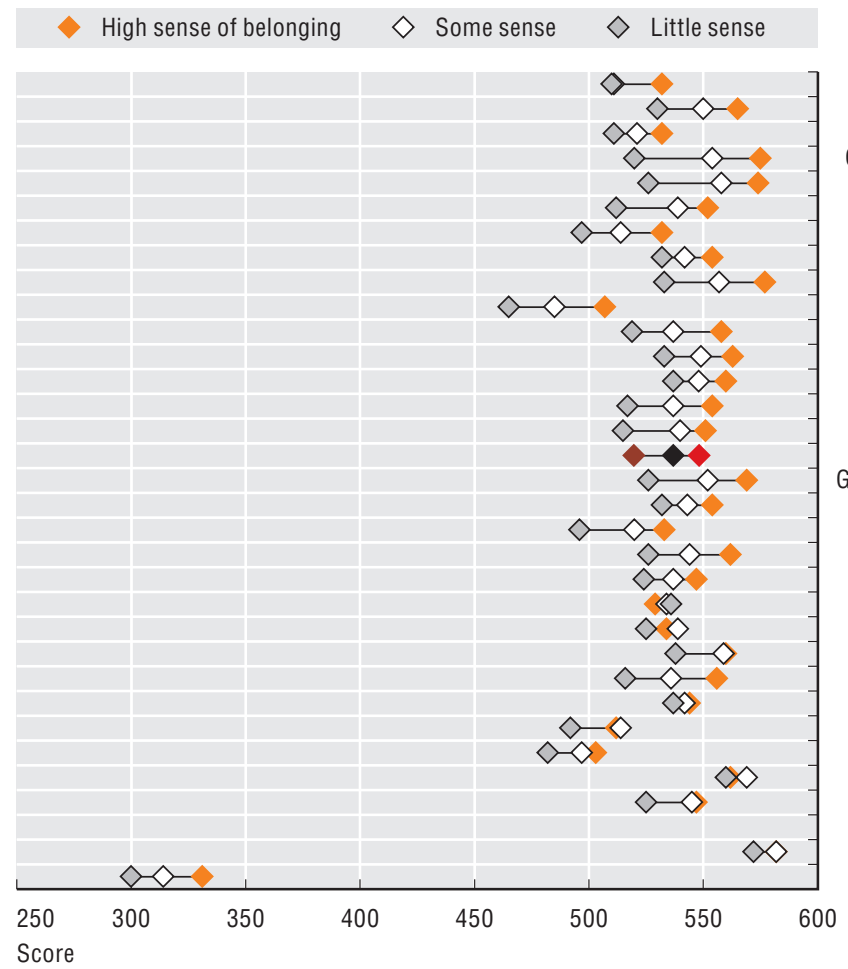

Source: Étude PIRLS (Progress in International Reading Literacy Study), 2016

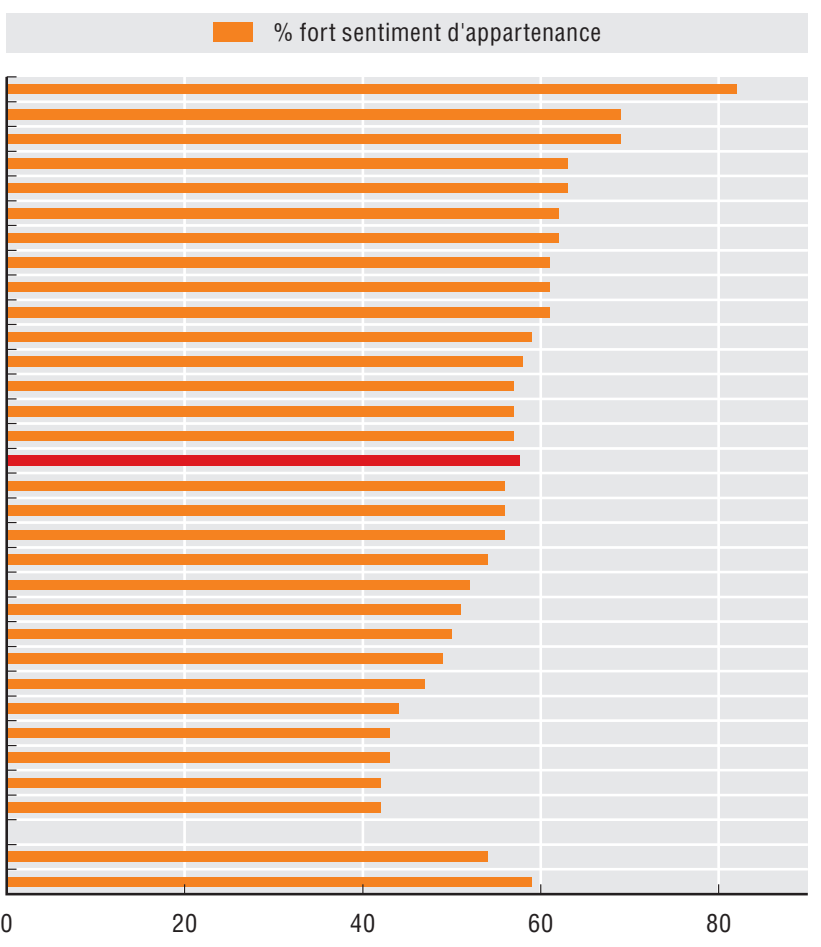

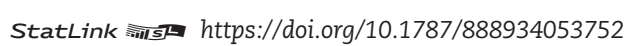


Les systèmes judiciaires ont pour mission d'assurer la protection des droits des citoyens et de garantir la satisfaction de leurs besoins juridiques. Pour évaluer leur efficacité et leur équité, il faut tenir compte de l'éventail complet des services à assurer, depuis l'accessibilité de l'information juridique et de l'aide juridique jusqu'aux mécanismes formels (tribunaux) et alternatifs de règlement des litiges et aux dispositifs d'exécution (OCDE 2019).

Les tribunaux demeurent une composante essentielle des systèmes judiciaires ; ils servent pour les particuliers et les entreprises, à résoudre leurs litiges et à protéger et à faire respecter leurs droits. Or, le bon fonctionnement des tribunaux et la juste application du droit exigent des juges indépendants de toute pression extérieure. Une enquête menée en 2017 auprès des juges européens a montré que des pressions indues pourraient être exercées par leurs supérieurs hiérarchiques au sein des tribunaux et par les parties et leurs avocats. Un tiers des répondants disaient en outre douter que les conseils de la justice disposent des mécanismes appropriés pour défendre leur indépendance (RECJ 2017).

L'absence d'influence indue de l'exécutif sur les affaires traitées par la justice administrative et la bonne mise en application de la justice civile, telles que mesurées par l'indice de l'état de droit (RuLi) du World Justice Project (WJP), sont fortement corrélées. En moyenne, les scores des pays de l'OCDE pour ces deux indicateurs ont légèrement fléchi entre 2015 et 2019. C'est la Belgique qui a le plus progressé sur ces deux plans $(+0,09$ points pour l'absence d'influence indue et $+0,14$ pour la bonne mise en application du droit).

Parmi les différentes formes de justice assurées par le système judiciaire, la justice pénale est l'une des plus délicates, dans la mesure où elle influe sur la liberté des individus. Dans les affaires pénales, deux droits humains fondamentaux se télescopent: la sécurité personnelle et la présomption d'innocence. Les victimes ont le droit d'exiger qu'une enquête soit faite et que l'auteur des faits soit poursuivi, pour se prémunir, et prémunir la société, de toute menace ultérieure ; et l'accusé a droit à une procédure équitable dans le cadre de laquelle toutes les garanties seront respectées. À titre d'exemple, toute détention en amont du procès doit être évitée et, quand elle intervient, doit être la plus courte possible, pour éviter toute atteinte aux libertés individuelles. Les décisions de justice doivent être rapides et équitables, afin de garantir le respect de ces deux droits.

La rapidité et l'efficacité de la justice pénale sont fortement corrélées à une moindre tendance des justiciables à se faire justice eux-mêmes (" autojustice "), selon les indicateurs du WJP. Globalement, les résultats des pays de l'OCDE sont restés stables sur ces deux points. La plus forte amélioration est observée en Turquie $(+0,20$ pour le non-recours à l'autojustice, et $+0,05$ pour la qualité de la justice), juste avant l'Allemagne $(+0,05,+0,07)$. Les plus fortes baisses sont, à l'inverse, constatées en Corée $(-0,17,-0,02)$ et en Grèce $(-0,09,-0,05)$. En Slovénie, il semble que les citoyens aient moins recours à la violence pour régler des griefs personnels, même si le système de justice pénale paraît moins efficace qu'en $2015(+0,18,-0,12)$.

Le sentiment selon lequel la délinquance est maîtrisée s'est, en moyenne, renforcé dans les pays de l'OCDE par rapport à 2015. Le WJP prend en compte plusieurs facteurs, et examine notamment si les habitants disent se sentir en sécurité lorsqu'ils marchent seuls la nuit dans la rue, ou s'ils ont déclaré avoir été victimes d'un cambriolage et/ou d'un vol. La majorité des pays affichent des résultats supérieurs à la moyenne et ont enregistré des améliorations, surtout la Norvège $(+0,05)$, la Hongrie $(+0,05)$ et la Slovénie $(+0,04)$.

\section{Méthodologie et définitions}

Les conseils de la justice sont définis dans la Résolution de Budapest (2008) du Réseau européen des conseils de la justice (RECJ) comme des institutions indépendantes ou autonomes des pouvoirs législatif et exécutif de l'État et les responsables de l'administration de la justice.

Pour les trois graphiques, les données proviennent de l'indice de l'état de droit (RuLi) mis au point par le World Justice Project. Cet indice repose sur les réponses à une enquête générale d'opinion réalisée auprès de 1000 répondants (échantillon représentatif) des trois plus grandes villes de chaque pays, ainsi qu'à une enquête menée auprès d'experts du droit civil (praticiens et universitaires). Une note comprise entre 0 et 1 est attribuée à chaque composante de l'indice ; plus la note est élevée, plus le résultat est satisfaisant. Un complément d'information peut être consulté à l'adresse suivante : worldjusticeproject.org/ruleoflaw-index.

On évalue l'absence d'influence indue en évaluant à quel point il est probable qu'un administré gagne un procès contre l'État ; que l'État respecte cette décision ; et qu'il cherche à influencer le tribunal. Pour évaluer la bonne mise en application de la justice civile, on interroge sur la mise à exécution des décisions de justice et sur leur rapidité.

On évalue l'efficacité et la rapidité du système judiciaire pénal en interrogeant sur le délai nécessaire pour amener un suspect devant les tribunaux et sur la durée de la détention en amont du procès, et en demandant dans quelle mesure les auteurs d'infractions violentes sont interpellés et traduits devant les tribunaux. Le recours à la violence consiste, par exemple, à intimider ou à attaquer l'auteur d'une infraction.

Pour évaluer la maîtrise de la délinquance, on interroge notamment les citoyens sur leur sentiment de sécurité lorsqu'ils se déplacent à pied la nuit et on leur demande, entre autres, s'ils ont été victimes d'une infraction au cours de l'année/des trois années précédentes (selon la question).

\section{Pour en savoir plus}

Réseau européen des conseils de la justice (2017), Enquête 20162017 sur l'indépendance des juges, RECJ, Bruxelles.

OCDE (2019), Equal Access to Justice for Inclusive Growth: Putting People at the Centre, Éditions OCDE, Paris, https://doi. org/10.1787/597f5b7f-en.

\section{Notes relatives aux graphiques}

Islande, Israël, Lettonie, Lituanie, Luxembourg, République slovaque et Suisse : données non disponibles.

11.32. (Application effective de la justice civile et absence d'influence indue du gouvernement, 2015 et 2019) et 11.33 (Efficacité/opportunité du système de jugement des tribunaux de justice pénale et l'utilisation de la violence pour remédier à des griefs personnels, 2015 et 2019) sont disponibles en ligne à l'Annexe F. 
11.29. Bonne mise en application de la justice civile et absence d'influence indue de l'exécutif, 2019

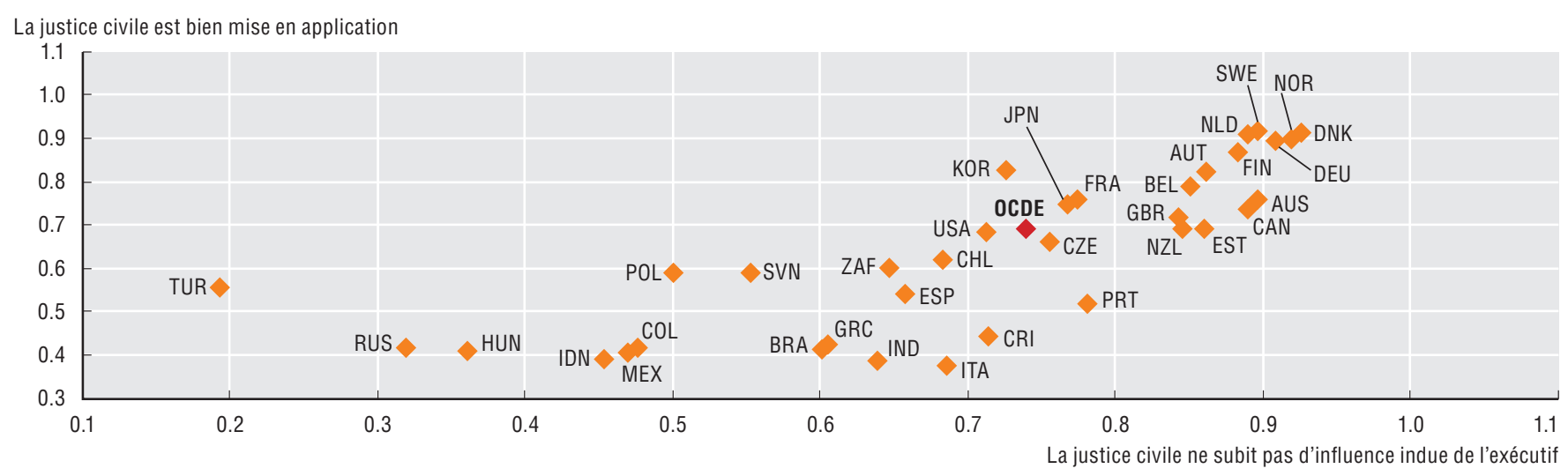

Source : World Justice Project (2019), Rule of Law Index 2019.

StatLink 角ISt https://doi.org/10.1787/888934053771

11.30. Efficacité/rapidité de décision des tribunaux de justice pénale et degré de recours à la violence pour régler des griefs personnels, 2019

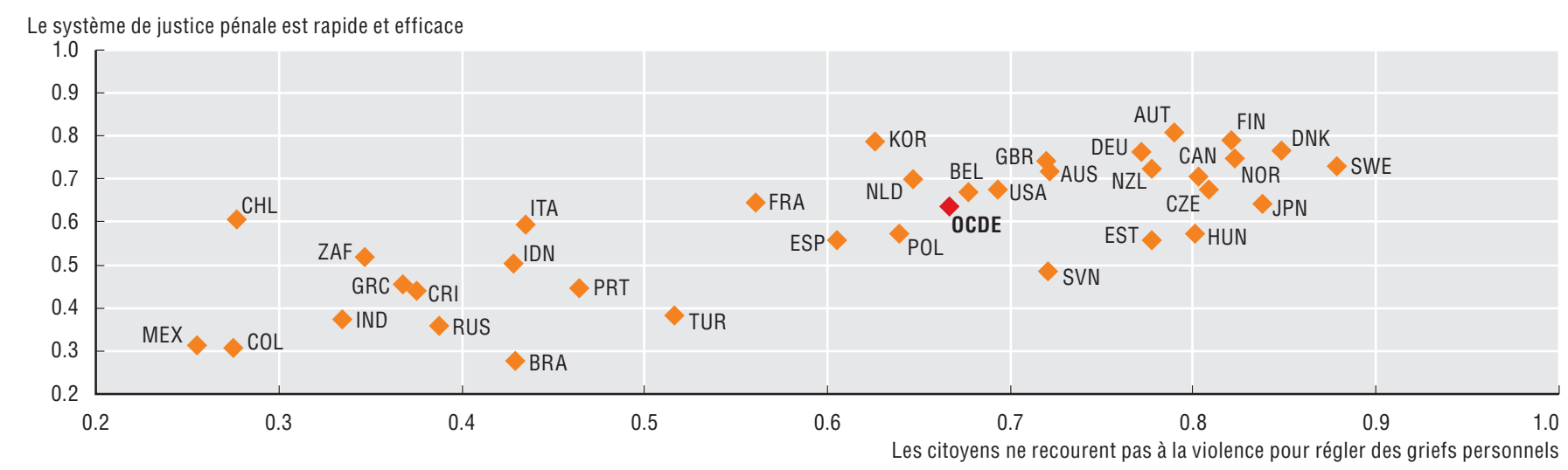

Source : World Justice Project (2019), Rule of Law Index 2019.

StatLink ants https://doi.org/10.1787/888934053790

11.31. Maîtrise de la délinquance, 2015 et 2019

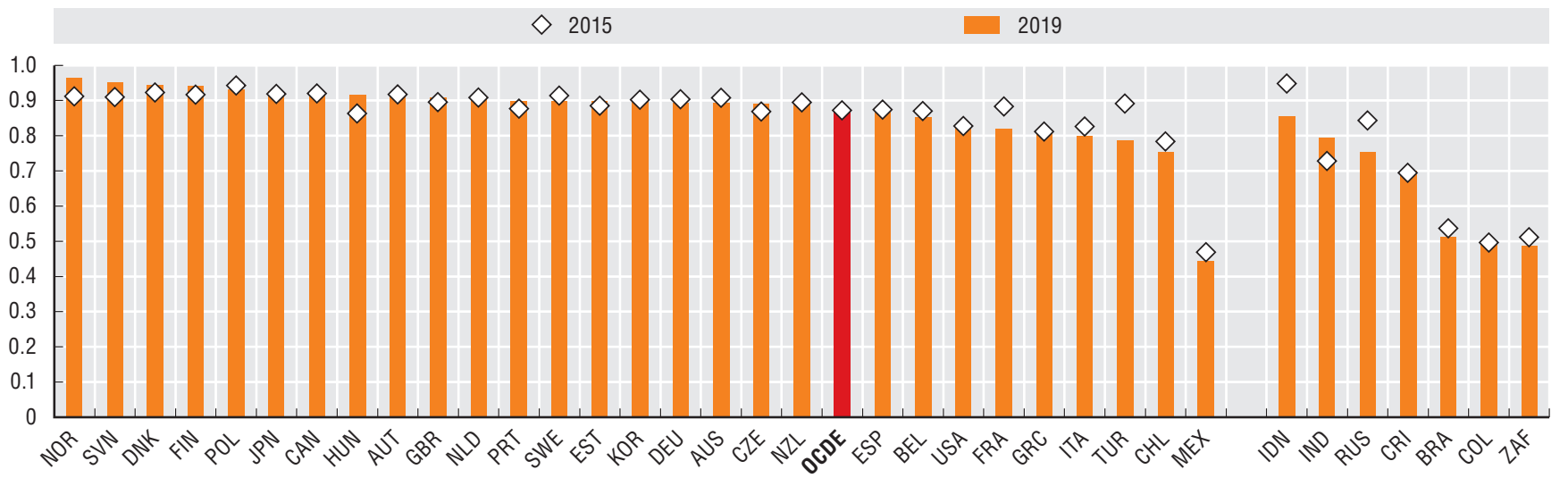

Source : World Justice Project (2019), Rule of Law Index 2019. 



\section{Systèmes et sources d'information sur les comptes publics au sein des statistiques sur les comptes nationaux}

Tableau A.1.1. Systèmes et sources d'information financière par pays

\begin{tabular}{|c|c|c|}
\hline Pays & Comptes publics non financiers & Comptes publics financiers \\
\hline \multicolumn{3}{|c|}{ Pays membres de l'OCDE } \\
\hline Allemagne & $\begin{array}{l}\text { ESA2010 ; comptes nationaux annuels de l'OCDE, comptes des } \\
\text { administrations publiques }\end{array}$ & $\begin{array}{l}\text { ESA2010 ; statistiques d'Eurostat sur les finances publiques, } \\
\text { comptes financiers annuels des administrations publiques, } \\
\text { consolidés* }^{*}\end{array}$ \\
\hline Australie & $\begin{array}{l}\text { SNA2008; comptes nationaux annuels de l'OCDE, comptes des } \\
\text { administrations publiques }\end{array}$ & $\begin{array}{l}\text { SNA2008; comptes nationaux annuels de l'OCDE, comptes de } \\
\text { patrimoine financier, consolidés }\end{array}$ \\
\hline Autriche & $\begin{array}{l}\text { ESA2010 ; comptes nationaux annuels de l'OCDE, comptes des } \\
\text { administrations publiques }\end{array}$ & $\begin{array}{l}\text { ESA2010 ; statistiques d'Eurostat sur les finances publiques, } \\
\text { comptes financiers annuels des administrations publiques, } \\
\text { consolidés* }\end{array}$ \\
\hline Belgique & $\begin{array}{l}\text { ESA2010; comptes nationaux annuels de l'OCDE, comptes des } \\
\text { administrations publiques }\end{array}$ & $\begin{array}{l}\text { ESA2010 ; comptes nationaux annuels de l'OCDE, comptes de } \\
\text { patrimoine financier, consolidés }\end{array}$ \\
\hline Canada & $\begin{array}{l}\text { SNA2008; comptes nationaux annuels de l'OCDE, comptes des } \\
\text { administrations publiques }\end{array}$ & $\begin{array}{l}\text { SNA2008; comptes nationaux annuels de l'OCDE, comptes de } \\
\text { patrimoine financier, consolidés }\end{array}$ \\
\hline Chili & $\begin{array}{l}\text { SNA2008; comptes nationaux annuels de l'OCDE, comptes des } \\
\text { administrations publiques }\end{array}$ & $\begin{array}{l}\text { SNA2008 ; comptes nationaux annuels de l'OCDE, comptes de } \\
\text { patrimoine financier, non consolidés }\end{array}$ \\
\hline Corée & $\begin{array}{l}\text { SNA2008; comptes nationaux annuels de l'OCDE, comptes des } \\
\text { administrations publiques }\end{array}$ & $\begin{array}{l}\text { SNA2008 ; comptes nationaux annuels de l'OCDE, comptes de } \\
\text { patrimoine financier, consolidés }\end{array}$ \\
\hline Danemark & $\begin{array}{l}\text { ESA2010 ; comptes nationaux annuels de l'OCDE, comptes des } \\
\text { administrations publiques }\end{array}$ & $\begin{array}{l}\text { ESA2010 ; statistiques d'Eurostat sur les finances publiques, } \\
\text { comptes financiers annuels des administrations publiques, } \\
\text { consolidés* }^{*}\end{array}$ \\
\hline Espagne & $\begin{array}{l}\text { ESA2010 ; comptes nationaux annuels de l'OCDE, comptes des } \\
\text { administrations publiques }\end{array}$ & $\begin{array}{l}\text { ESA2010 ; comptes nationaux annuels de l'OCDE, comptes de } \\
\text { patrimoine financier, consolidés }\end{array}$ \\
\hline Estonie & $\begin{array}{l}\text { ESA2010 ; comptes nationaux annuels de l'OCDE, comptes des } \\
\text { administrations publiques }\end{array}$ & $\begin{array}{l}\text { ESA2010 ; statistiques d'Eurostat sur les finances publiques, } \\
\text { comptes financiers annuels des administrations publiques, } \\
\text { consolidés* }^{*}\end{array}$ \\
\hline États-Unis & $\begin{array}{l}\text { SNA2008; comptes nationaux annuels de l'OCDE, comptes des } \\
\text { administrations publiques }\end{array}$ & $\begin{array}{l}\text { SNA2008 ; comptes nationaux annuels de l'OCDE, comptes de } \\
\text { patrimoine financier, consolidés }\end{array}$ \\
\hline Finlande & $\begin{array}{l}\text { ESA2010; comptes nationaux annuels de l'OCDE, comptes des } \\
\text { administrations publiques }\end{array}$ & $\begin{array}{l}\text { ESA2010 ; statistiques d’Eurostat sur les finances publiques, } \\
\text { comptes financiers annuels des administrations publiques, } \\
\text { consolidés* }^{*}\end{array}$ \\
\hline France & $\begin{array}{l}\text { ESA2010 ; comptes nationaux annuels de l'OCDE, comptes des } \\
\text { administrations publiques }\end{array}$ & $\begin{array}{l}\text { ESA2010 ; statistiques d’Eurostat sur les finances publiques, } \\
\text { comptes financiers annuels des administrations publiques, } \\
\text { consolidés* }^{*}\end{array}$ \\
\hline Grèce & $\begin{array}{l}\text { ESA2010 ; comptes nationaux annuels de l'OCDE, comptes des } \\
\text { administrations publiques }\end{array}$ & $\begin{array}{l}\text { ESA2010 ; comptes nationaux annuels de l'OCDE, comptes de } \\
\text { patrimoine financier, consolidés }\end{array}$ \\
\hline Hongrie & $\begin{array}{l}\text { ESA2010; comptes nationaux annuels de l'OCDE, comptes des } \\
\text { administrations publiques }\end{array}$ & $\begin{array}{l}\text { ESA2010 ; comptes nationaux annuels de l'OCDE, comptes de } \\
\text { patrimoine financier, consolidés }\end{array}$ \\
\hline
\end{tabular}




\section{Tableau A.1.1. Systèmes et sources d'information financière par pays (suite)}

\begin{tabular}{|c|c|}
\hline Pays & Comptes publics non financiers \\
\hline Islande & $\begin{array}{l}\text { SNA2008; comptes nationaux annuels de l'OCDE, comptes des } \\
\text { administrations publiques }\end{array}$ \\
\hline Irlande & $\begin{array}{l}\text { ESA2010; comptes nationaux annuels de l'OCDE, comptes des } \\
\text { administrations publiques }\end{array}$ \\
\hline Israël & $\begin{array}{l}\text { SNA2008; comptes nationaux annuels de l'OCDE, comptes des } \\
\text { administrations publiques }\end{array}$ \\
\hline Italie & $\begin{array}{l}\text { ESA2010; comptes nationaux annuels de l'OCDE, comptes des } \\
\text { administrations publiques }\end{array}$ \\
\hline Japon & $\begin{array}{l}\text { SNA2008; comptes nationaux annuels de l'OCDE, comptes des } \\
\text { administrations publiques }\end{array}$ \\
\hline Lettonie & $\begin{array}{l}\text { ESA2010 ; comptes nationaux annuels de l'OCDE, comptes des } \\
\text { administrations publiques }\end{array}$ \\
\hline Lituanie & $\begin{array}{l}\text { ESA2010 ; comptes nationaux annuels de l'OCDE, comptes des } \\
\text { administrations publiques }\end{array}$ \\
\hline Luxembourg & $\begin{array}{l}\text { ESA2010 ; comptes nationaux annuels de l'OCDE, comptes des } \\
\text { administrations publiques }\end{array}$ \\
\hline Mexique & $\begin{array}{l}\text { SNA2008; comptes nationaux annuels de l'OCDE, comptes des } \\
\text { administrations publiques }\end{array}$ \\
\hline Nouvelle-Zélande & $\begin{array}{l}\text { SNA2008; comptes nationaux annuels de l'OCDE, comptes des } \\
\text { administrations publiques }\end{array}$ \\
\hline Norvège & $\begin{array}{l}\text { SNA2008; comptes nationaux annuels de l'OCDE, comptes des } \\
\text { administrations publiques }\end{array}$ \\
\hline Pays-Bas & $\begin{array}{l}\text { ESA2010 ; comptes nationaux annuels de l'OCDE, comptes des } \\
\text { administrations publiques }\end{array}$ \\
\hline Pologne & $\begin{array}{l}\text { ESA2010 ; comptes nationaux annuels de l'OCDE, comptes des } \\
\text { administrations publiques }\end{array}$ \\
\hline Portugal & $\begin{array}{l}\text { ESA2010 ; comptes nationaux annuels de l'OCDE, comptes des } \\
\text { administrations publiques }\end{array}$ \\
\hline
\end{tabular}

République tchèque ESA2010; comptes nationaux annuels de l'OCDE, comptes des administrations publiques Comptes publics financiers

SNA2008; comptes nationaux annuels de l'OCDE, comptes de patrimoine financier, consolidés

ESA2010 ; comptes nationaux annuels de l'OCDE, comptes de patrimoine financier, consolidés

SNA2008; comptes nationaux annuels de l'OCDE, comptes de patrimoine financier, consolidés

ESA2010 ; statistiques d'Eurostat sur les finances publiques, comptes financiers annuels des administrations publiques, consolidés*

SNA2008; comptes nationaux annuels de l'OCDE, comptes de patrimoine financier, consolidés

ESA2010 ; statistiques d'Eurostat sur les finances publiques, comptes financiers annuels des administrations publiques, consolidés*

ESA2010 ; comptes nationaux annuels de l'OCDE, comptes de patrimoine financier, consolidés

ESA2010 ; statistiques d'Eurostat sur les finances publiques, comptes financiers annuels des administrations publiques, consolidés*

SNA2008; comptes nationaux annuels de l'OCDE, comptes de patrimoine financier, non consolidés

SNA2008; comptes nationaux annuels de l'OCDE, comptes de patrimoine financier, non consolidés

SNA2008; comptes nationaux annuels de l'OCDE, comptes de patrimoine financier, consolidés

ESA2010 ; statistiques d'Eurostat sur les finances publiques, comptes financiers annuels des administrations publiques, consolidés*

ESA2010 ; comptes nationaux annuels de l'OCDE, comptes de patrimoine financier, consolidés

ESA2010 ; statistiques d'Eurostat sur les finances publiques, comptes financiers annuels des administrations publiques, consolidés*

ESA2010 ; statistiques d'Eurostat sur les finances publiques, comptes financiers annuels des administrations publiques, consolidés*

République slovaque ESA2010; comptes nationaux annuels de l'OCDE, comptes des administrations publiques

ESA2010 ; statistiques d'Eurostat sur les finances publiques, comptes financiers annuels des administrations publiques, consolidés*

Royaume-Uni ESA2010; comptes nationaux annuels de l'OCDE, comptes des administrations publiques

Slovénie

ESA2010 ; comptes nationaux annuels de l'OCDE, comptes des administrations publiques

ESA2010 ; comptes nationaux annuels de l'OCDE, comptes de patrimoine financier, consolidés

ESA2010 ; statistiques d'Eurostat sur les finances publiques, comptes financiers annuels des administrations publiques, consolidés*

Suède ESA2010; comptes nationaux annuels de l'OCDE, comptes des administrations publiques

ESA2010 ; statistiques d'Eurostat sur les finances publiques, comptes financiers annuels des administrations publiques, consolidés*

Suisse

SNA2008; comptes nationaux annuels de l'OCDE, comptes des administrations publiques

Turquie SNA2008; comptes nationaux annuels de l'OCDE, comptes des administrations publiques

SNA2008; comptes nationaux annuels de l'OCDE, comptes de patrimoine financier, consolidés

SNA2008; comptes nationaux annuels de l'OCDE, comptes de patrimoine financier, consolidés

Pays candidats à l'adhésion à l'OCDE

Colombie

SNA1993 ; comptes nationaux annuels de l'OCDE, comptes des administrations publiques

Costa Rica

SNA2008 ; comptes nationaux annuels de l'OCDE, comptes des administrations publiques

Russie SNA1993 ; comptes nationaux annuels de l'OCDE, comptes des administrations publiques

SNA2008; comptes nationaux annuels de l'OCDE, comptes de patrimoine financier, consolidés

SNA1993 (GFSM2001/86)

SNA2008; comptes nationaux annuels de l'OCDE, comptes de patrimoine financier, non consolidés

${ }^{*}$ Pour les comptes publics financiers de ces pays, on a pris pour référence les statistiques d'Eurostat sur les finances publiques, qui reflètent les dernières données actualisées validées (qui sont transmises à Eurostat deux fois par an). Pour les autres pays, les dernières données actualisées validées sont versées à la base de données des Statistiques de l'OCDE sur les comptes nationaux; on a donc pris cette base de données pour référence. 
ANNEXE B

\section{Méthodologie utilisée pour les agrégats relatifs aux recettes}

Le tableau ci-dessous fournit des données détaillées sur la façon dont les agrégats relatifs aux impôts, aux cotisations sociales nettes, aux ventes, aux aides et autres recettes présentés au Chapitre 2, « Finances publiques et économie ", ont été construits à partir des données issues des statistiques de l'OCDE relatives aux comptes nationaux.

\section{Tableau B.1. Agrégats relatifs aux recettes}

\begin{tabular}{|c|c|c|}
\hline $\begin{array}{l}\text { Intitulé dans le Panorama des } \\
\text { administrations publiques }\end{array}$ & Intitulé dans le Système de comptabilité nationale & $\begin{array}{l}\text { Code dans la base de données de l'OCDE } \\
\text { relative aux comptes nationaux (Principaux } \\
\text { agrégats des administrations publiques) }\end{array}$ \\
\hline \multicolumn{3}{|l|}{ Impôts } \\
\hline Impôts indirects & Impôts sur la production et les importations, à recevoir & GD2R \\
\hline Impôts directs & $\begin{array}{l}\text { Impôts courants sur le revenu et le patrimoine, à } \\
\text { recevoir }\end{array}$ & GD5R \\
\hline Impôts en capital & Impôts en capital & GD91R \\
\hline Cotisations sociales nettes & Cotisations sociales nettes & GD61R \\
\hline \multirow[t]{2}{*}{ Ventes } & $\begin{array}{l}\text { Production marchande et production pour usage final } \\
\text { propre }\end{array}$ & GP11_P12R \\
\hline & Paiements au titre de la production non marchande & GP131R \\
\hline \multicolumn{3}{|l|}{ Aides et autres recettes } \\
\hline \multirow[t]{2}{*}{ Aides courantes et aides à l'équipement } & Autres transferts courants, à recevoir & GD7R \\
\hline & $\begin{array}{l}\text { Autres transferts en capital et aides à l'investissement, } \\
\text { à recevoir }\end{array}$ & GD92R_D99R \\
\hline Subventions & Autres subventions sur la production, à recevoir & GD39R \\
\hline Revenus de la propriété & Revenus de la propriété, à recevoir & GD4R \\
\hline Total des recettes & Total des recettes & GTR \\
\hline
\end{tabular}




\section{Classification des fonctions des administrations publiques (CFAP/COFOG)}

Conçue par l'OCDE, la Classification des fonctions des administrations publiques (CFAP ou COFOG) classe les données sur les dépenses des administrations publiques provenant du Système de comptabilité nationale selon l'objet auquel les fonds sont consacrés. Comme le montre le tableau C.4, la CFAP de niveau 1 répartit les données sur les dépenses selon 10 groupes ou sous-secteurs « fonctionnels » de dépenses (comme les affaires économiques, l'enseignement ou la protection sociale) et la CFAP de niveau 2 subdivise chaque groupe du niveau 1 en neuf sous-groupes au maximum. On dispose de données correspondant au niveau 1 de la CFAP pour 32 des 36 pays membres de l'OCDE (selon la disponibilité de données chronologiques) mais, s'agissant du niveau 2, on ne dispose actuellement de données que pour 26 pays de l'OCDE membres de l'Union européenne, plus Israël et le Japon*.

\section{Tableau C.1. Niveaux 1 et 2 de la CFAP (COFOG)}

\begin{tabular}{ll}
\hline Niveau 1 & Niveau 2 \\
\hline Services généraux des & Fonctionnement des organes exécutifs et législatifs, affaires financières et fiscales, affaires étrangères \\
administrations publiques & Aide économique extérieure \\
& Services généraux \\
& Recherche fondamentale \\
& R-D concernant des services généraux des administrations publiques \\
& - Services généraux des administrations publiques n.c.a. \\
& Opérations concernant la dette publique \\
& Transferts de caractère général entre administrations publiques \\
\hline
\end{tabular}

\footnotetext{
* On ne dispose pas de données quant aux dépenses correspondant au niveau 1 de la CFAP pour le Canada, le Chili et le Mexique. Jusqu'à une époque récente, les données correspondant au niveau 2 de la CFAP étaient disponibles dans certains offices statistiques nationaux, mais n'étaient pas recueillies par les organisations internationales. De plus, les données correspondant à ce niveau n'étaient pas toujours pleinement comparables d'un pays à l'autre, car le guide relatif au SCN des Nations Unies et le Manuel de statistiques de finances publiques du Fonds monétaire international ne donnaient que peu d'indications pratiques sur l'application des concepts de la CFAP. Toutefois, en 2005, Eurostat a mis en place un groupe de travail chargé de rédiger un manuel sur l'application de la CFAP aux données relatives aux dépenses figurant dans les comptes nationaux et de débattre du recueil de données correspondant au niveau 2 de la CFAP pour les pays européens. On ne dispose pas de données correspondant au niveau 2 de la CFAP pour la Turquie, ni pour les membres de l'OCDE non européens, à l'exception d'Israël et du Japon. Par ailleurs, dans certains pays membres de l'UE, ces données sont disponibles seulement pour certaines divisions de la CFAP. Des efforts sont en cours afin de parvenir à un accord avec ces pays sur la communication de ces données à l'OCDE.
} 
Tableau C.1. Niveaux 1 et 2 de la CFAP (COFOG) (suite)

\begin{tabular}{|c|c|}
\hline Niveau 1 & Niveau 2 \\
\hline Défense & $\begin{array}{l}\text { - Défense militaire } \\
\text { Défense civile } \\
\text { - Aide militaire à des pays étrangers } \\
\text { - R-D concernant la défense } \\
\text { - Défense n.c.a. }\end{array}$ \\
\hline Ordre et sécurité publics & $\begin{array}{l}\text { - Services de police } \\
\text { - Services de protection civile } \\
\text { - Tribunaux } \\
\text { - Administration pénitentiaire } \\
\text { - } \text {-D concernant l'ordre et la sécurité publics } \\
\text { Ordre et sécurité publics n.c.a. }\end{array}$ \\
\hline Affaires économiques & $\begin{array}{l}\text { - Tutelle de l'économie générale, des échanges et de l'emploi } \\
\text { - Agriculture, sylviculture, pêche et chasse } \\
\text { - Combustibles et énergie } \\
\text { Industries extractives et manufacturières, construction } \\
\text { - Transports } \\
\text { - Communications } \\
\text { - Rutres branches d'activité } \\
\text { - R concernant les affaires économiques } \\
\text { - Affaires économiques n.c.a. }\end{array}$ \\
\hline Protection de l'environnement & $\begin{array}{l}\text { - Gestion des déchets } \\
\text { - Gestion des eaux usées } \\
\text { - Lutte contre la pollution } \\
\text { - Préservation de la diversité biologique et protection de la nature } \\
\text { - R-D dans le domaine de la protection de l'environnement } \\
\text { - Protection de l'environnement n.c.a. }\end{array}$ \\
\hline $\begin{array}{l}\text { Logements et équipements } \\
\text { collectifs }\end{array}$ & $\begin{array}{l}\text { - Logements } \\
\text { Équipements collectifs } \\
\text { - Alimentation en eau } \\
\text { Éclairage public } \\
\text { - L-D dans le domaine du logement et des équipements collectifs } \\
\text { - Logements et équipements collectifs n.c.a. }\end{array}$ \\
\hline Santé & $\begin{array}{l}\text { - Produits, appareils et matériels médicaux } \\
\text { - Services ambulatoires } \\
\text { - Services hospitaliers } \\
\text { - Services de santé publique } \\
\text { - R-D dans le domaine de la santé } \\
\text { - Santé n.c.a. }\end{array}$ \\
\hline Loisirs, culture et culte & $\begin{array}{l}\text { - Services récréatifs et sportifs } \\
\text { - Services culturels } \\
\text { - Services de radiodiffusion, de télévision et d'édition } \\
\text { - R-D dans le domaine des loisirs, de la culture et du culte } \\
\text { - Loisirs, culture et culte n.c.a. }\end{array}$ \\
\hline Enseignement & $\begin{array}{l}\text { - Enseignement préélémentaire et primaire } \\
\text { - Enseignement secondaire } \\
\text { - Enseignement postsecondaire non supérieur } \\
\text { - Enseignement supérieur } \\
\text { - Services annext non défini par niveau à l'enseignement } \\
\text { - R-D dans le domaine de l'enseignement } \\
\text { - Enseignement n.c.a. }\end{array}$ \\
\hline Protection sociale & $\begin{array}{l}\text { - Maladie et invalidité } \\
\text { - Vieillesse } \\
\text { Survivants } \\
\text { - Famille et enfants } \\
\text { Chômage } \\
\text { - Exgement } \\
\text { - R-D dans le domaine de la protection sociale } \\
\text { - Protection sociale n.c.a. }\end{array}$ \\
\hline
\end{tabular}

n.c.a. : éléments non classés ailleurs 


\title{
Classification et définition des professions
}

\begin{abstract}
La classification qui suit est tirée de l'enquête 2016 de l'OCDE sur la gestion stratégique des ressources humaines (SHRM), qui a fait l'objet d'une actualisation en 2019 ; cette enquête se fondait sur les mêmes définitions qu'une enquête réalisée en 2016 sur la composition des effectifs de l'administration centrale/fédérale. Cette classification distingue quatre grands niveaux hiérarchiques au sein de l'administration.

La classification et la définition des professions sont une adaptation de la Classification internationale type des professions (CITP 08) établie par l'Organisation internationale du Travail (OIT). Les définitions complètes peuvent être consultées à partir du lien suivant : https://www.ilo.org/public/french/bureau/stat/isco/index.htm.

Si une adaptation a été nécessaire, c'est parce que tous les pays ne suivent pas le modèle de la CITP pour classer les professions au sein de leur fonction publique ; en effet, les professions exercées à l'échelon national peuvent varier en fonction des spécificités du cadre juridique et administratif applicable.
\end{abstract}

\section{Tableau D.1. Classification et définition des professions}

\begin{tabular}{|c|}
\hline Les cadres de haut niveau \\
\hline $\begin{array}{l}\text { Les cadres de niveau D1 (qui relèvent de la catégorie } 1112 \text { de la CITP-08) sont des fonctionnaires de très haut rang situés juste en dessous du ministre ou du } \\
\text { secrétaire d'État. Ils peuvent faire partie de la haute fonction publique et/ou être nommés par le gouvernement ou le chef du gouvernement. Ils conseillent le } \\
\text { gouvernement en matière d'action publique, supervisent l'interprétation et la mise en œuvre des politiques publiques et, dans certains pays, ont des pouvoirs } \\
\text { exécutifs. Les cadres D1 peuvent être autorisés à assister à certaines réunions du Cabinet ou du Conseil des ministres, mais ils n'appartiennent pas à ces instances. } \\
\text { Ils assurent une direction et une gestion globales auprès du ministre/secrétaire d'État ou pour un domaine administratif particulier. Dans les pays ayant un système } \\
\text { d'agences autonomes, des pouvoirs décentralisés, des organisations dans lesquelles le nombre de niveaux hiérarchiques est réduit et des cadres jouissant d'une } \\
\text { certaine autonomie, le niveau D1 correspond au rang des directeurs généraux. }\end{array}$ \\
\hline $\begin{array}{l}\text { Les cadres de niveau D2 (qui relèvent des catégories } 11 \text { et } 12 \text { de la CITP-08) sont situés immédiatement en dessous des cadres D1. Ils formulent et révisent les } \\
\text { politiques et planifient, dirigent, coordonnent et évaluent les activités générales du ministère ou d'une direction/d'une unité, avec l'aide d'autres cadres. Ils peuvent } \\
\text { faire partie de la haute fonction publique. Ils donnent des orientations en matière de coordination et de gestion du programme de travail et des directives aux équipes } \\
\text { de spécialistes de différents domaines. Ils fixent les objectifs, stratégies et programmes pour l'unité administrative/le département qu'ils supervisent. }\end{array}$ \\
\hline Les cadres moyens (qui encadrent au moins 3 personnes) \\
\hline $\begin{array}{l}\text { Les cadres de niveau D3 (qui relèvent de la catégorie } 12 \text { de la CITP-08) sont situés immédiatement en dessous des cadres D2. IIs planifient, dirigent et coordonnent } \\
\text { le fonctionnement général d'une direction/d'une unité administrative au sein du ministère, avec l'aide d'autres cadres, en suivant généralement les lignes directrices } \\
\text { établies par un conseil d'administration ou un organe de direction. Ils dirigent et encadrent des équipes de spécialistes dans leur domaine spécifique. Ces } \\
\text { responsables élaborent et encadrent le programme de travail d'unités, de divisions ou de domaines d'action et gèrent le personnel correspondant. Ils établissent et } \\
\text { gèrent les budgets, contrôlent les dépenses et veillent à l'efficience dans l'utilisation des ressources. Ils suivent et évaluent les performances des différentes équipes } \\
\text { de spécialistes. }\end{array}$ \\
\hline $\begin{array}{l}\text { Les cadres de niveau D4 (qui relèvent de la catégorie } 121 \text { de la CITP-08) sont situés immédiatement en dessous des cadres D3. Ils formulent et prodiguent des } \\
\text { conseils d'action et assurent une planification stratégique et financière. Ils établissent et dirigent des procédures opérationnelles et administratives et adressent } \\
\text { des avis aux cadres supérieurs. Ils supervisent la sélection, la formation et les performances du personnel, établissent les budgets et supervisent les opérations } \\
\text { financières, contrôlent les dépenses et veillent à l'efficience dans l'utilisation des ressources. Ils dirigent des équipes spécifiques de spécialistes au sein d'une unité. }\end{array}$ \\
\hline $\begin{array}{l}\text { Les cadres de niveau D5 (catégorie facultative) (qui relèvent des catégories } 1211,1212 \text { et } 1213 \text { de la CITP-08) sont situés immédiatement en dessous des cadres D4. } \\
\text { II peut s'agir de spécialistes senior dont la responsabilité première consiste à piloter l'exécution du programme de travail et à superviser le travail d'autres spécialistes } \\
\text { et de spécialistes junior. }\end{array}$ \\
\hline $\begin{array}{l}\text { Les cadres de niveau D6 (catégorie facultative) (qui relèvent des catégories 1211, } 1212 \text { et } 1213 \text { de la CITP-08) peuvent être des spécialistes dont la responsabilité } \\
\text { première consiste à piloter l'exécution du programme de travail et à superviser le travail d'autres spécialistes ou de spécialistes junior. }\end{array}$ \\
\hline
\end{tabular}




\section{Table D.1. Classification and definition of occupations (suite)}

\begin{tabular}{|c|}
\hline Les spécialistes \\
\hline $\begin{array}{l}\text { Les économistes/analystes des politiques senior (qui relèvent des catégories } 242 \text { et } 2422 \text { de la CITP-08) n'exercent pas de fonction de direction (ils encadrent trois } \\
\text { personnes au maximum) et occupent un rang supérieur à celui des analystes junior et du personnel administratif/de secrétariat. Ils sont généralement tenus d'avoir un } \\
\text { diplôme universitaire. Ils assurent certaines fonctions de pilotage pour un domaine de travail ou divers projets et conçoivent et analysent des politiques pour guider } \\
\text { l'élaboration, la mise en œuvre et la modification d'actions et de programmes officiels. Ces spécialistes examinent les politiques et textes existants afin de détecter les } \\
\text { anomalies et les dispositions dépassées. Ils analysent et formulent des options d'action et ils établissent des documents d'information et des recommandations en } \\
\text { vue de faire évoluer les politiques existantes. Par ailleurs, ils évaluent l'impact, les conséquences financières et la faisabilité politique et administrative des politiques } \\
\text { publiques. Les agents de cette catégorie ont la possibilité de progresser dans leur carrière jusqu'au rang de cadres. Leurs domaines de compétence peuvent aller du } \\
\text { droit, de l'économie, de la politique, de l'administration publique ou des relations internationales à l'ingénierie, à l'environnement, à la pédagogie, à l'économie de la } \\
\text { santé, etc. Les économistes/analystes des politiques senior ont au moins cinq années d'expérience professionnelle. }\end{array}$ \\
\hline $\begin{array}{l}\text { Les économistes/analystes des politiques junior (qui relèvent des catégories } 242 \text { et } 2422 \text { de la CITP-08) occupent un rang supérieur à celui du personnel } \\
\text { administratif/de secrétariat. Ils sont généralement tenus d'avoir un diplôme universitaire. Ils n'exercent aucune fonction de direction. Ils conçoivent et analysent } \\
\text { des politiques pour guider l'élaboration, la mise en œuvre et la modification d'actions et de programmes officiels. Ces spécialistes examinent les politiques et textes } \\
\text { existants afin de détecter les anomalies et les dispositions dépassées. Ils analysent et formulent des options d'action et ils établissent des documents d'information } \\
\text { et des recommandations en vue de faire évoluer les politiques existantes. Par ailleurs, ils évaluent l'impact, les conséquences financières et la faisabilité politique et } \\
\text { administrative des politiques publiques. Leurs domaines de compétence peuvent aller du droit, de l'économie, de la politique, de l'administration publique ou des } \\
\text { relations internationales à l'ingénierie, à l'environnement, à la pédagogie, à l'économie de la santé, etc. Les économistes/analystes des politiques junior ont moins de } \\
\text { cinq années d'expérience professionnelle. }\end{array}$ \\
\hline Personnel de secrétariat \\
\hline $\begin{array}{l}\text { Les secrétaires (employés de bureau, fonctions générales) (qui relèvent des catégories } 411 \text { et } 4110 \text { de la CITP-08) ne sont généralement pas tenus d'avoir un diplôme } \\
\text { universitaire, même s'ils sont nombreux à être diplômés de l'enseignement supérieur. Ils assurent un large éventail de tâches administratives en rapport avec des } \\
\text { opérations de trésorerie, l'organisation de déplacements, des demandes d'information et l'organisation de rendez-vous. Ils enregistrent, préparent, trient, classent } \\
\text { et saisissent des informations ; trient, ouvrent et envoient le courrier ; préparent des rapports et des courriers ; enregistrent la remise de matériel au personnel ; } \\
\text { répondent aux demandes de renseignement par téléphone ou en ligne ou les transmettent à qui de droit ; vérifient des chiffres ; établissent des factures et enregistrent } \\
\text { les détails des opérations financières réalisées ; saisissent des informations sur ordinateur et relisent et corrigent des épreuves. Certains aident à la préparation des } \\
\text { budgets, au suivi des dépenses et à la rédaction de contrats et de bons de commande. Ceux qui occupent les rangs hiérarchiques les plus élevés, et qui supervisent } \\
\text { les travaux de membres du personnel administratif, sont exclus de cette catégorie. }\end{array}$ \\
\hline
\end{tabular}




\section{Méthodologie employée pour les indices composites sur la budgétisation sensible au genre et sur les politique et gouvernance réglementaires et pour l'indice Ourdata (des données ouvertes, utiles et réutilisables)}

Les indices composites étroitement définis présentés dans le Panorama des administrations publiques constituent le meilleur moyen de synthétiser des informations d'ordre qualitatif éparses. "Il est beaucoup plus facile d'interpréter des indices composites que d'essayer de dégager une tendance commune à partir de nombreux indicateurs distincts " (OCDE/EU/JRC 2008). Toutefois, leur conception et leur utilisation peuvent prêter à controverse. En effet, ces indices sont facilement et fréquemment interprétés de façon erronée par les utilisateurs : faute de transparence suffisante sur la façon dont ils sont créés, il est difficile de déterminer ce qu'ils mesurent réellement.

L'OCDE a pris plusieurs dispositions pour éviter ou prendre en compte les problèmes couramment posés par ces indices. Les indices composites présentés dans la présente publication suivent les étapes définies dans le Handbook on Constructing Composite Indicators (Nardo et al. 2008) et nécessaires à la bonne construction d'indices composites ou synthétiques.

Chaque indice composite repose sur un cadre théorique représentant un concept reconnu dans le domaine traité. Les variables composant les indices sont sélectionnées en fonction de leur pertinence à l'égard de ce concept. Chaque indice est élaboré en étroite coopération avec le groupe OCDE d'experts compétent, et on demande notamment aux experts leur avis sur le choix des variables de l'indice et sur les modalités de pondération.

De plus, on procède à plusieurs analyses statistiques afin d'assurer la validité et la fiabilité des indicateurs composites.

- Les questions d'enquête utilisées pour élaborer les indices sont les mêmes pour tous les pays, et les indices sont donc comparables.

- Différentes méthodes visant à imputer les valeurs manquantes ont été examinées.

- Tous les sous-indicateurs et variables ont été normalisés pour assurer la comparabilité.

- Pour la construction des composites, tous les sous-indices ont été agrégés suivant une méthode linéaire, selon la méthodologie acceptée.

- Une analyse factorielle en composantes principales est réalisée pour confirmer les hypothèses relatives aux concepts sous-jacents en train d'être mesurés.

- Les variables redondantes sont exclues, afin d'éviter tout double comptage et toute surpondération. 
- On calcule aussi l'alpha de Cronbach afin de mesurer les corrélations entre items.

- Enfin, une analyse de sensibilité basée sur une simulation Monte-Carlo est menée afin d'établir la solidité des indices selon différentes options de pondération.

Des annexes détaillées sur chacun des indices composites présentés dans cette édition du Panorama des administrations publiques, y compris les variables et les pondérations utilisées pour construire chaque indicateur, peuvent être consultées en ligne, à l'adresse http://www. oecd.org/gov/govataglance.htm.

\section{Références}

Nardo et al. (2005), Tools for composite indicators building, Rapport EUR 21682 EN, Centre commun de recherche, Ispra.

OCDE/EU/JRC (2008), Handbook on Constructing Composite Indicators and User Guide, Éditions OCDE, Paris https://doi.org/10.1787/9789264043466-en. 


\section{ANNEXE F}

\section{Graphiques supplémentaires disponibles en ligne}

\section{Chapitre 2. Finances publiques et économie}

- 2.5. Transferts nets en capital, en pourcentage du PIB [https://doi.org/10.1787/888934053828]

- 2.12. Taux annuel moyen de progression en termes réels de la dette publique par habitant, 2007-17 et 2017-18 [https://doi.org/10.1787/888934053847]

- 2.24. Composition des recettes de l'administration centrale, 2017 et 2018 [https://doi. org/10.1787/888934053866]

- 2.25. Composition des recettes des administrations d'États fédérés, 2017 et 2018 [https://doi. org/10.1787/888934053885]

- 2.26. Composition des recettes des administrations locales, 2017 et 2018 [https://doi. org/10.1787/888934053904]

- 2.32. Ventilation des dépenses des administrations publiques par fonction, 2017 [https://doi. org/10.1787/888934053923]

- 2.33. Évolution de la ventilation des dépenses des administrations publiques, par fonction, de 2007 à 2017 [https://doi.org/10.1787/888934053942]

- 2.34.Ventilation des dépenses publiques, par fonction de la catégorie « Services généraux des administrations publiques ", 2017 [https://doi.org/10.1787/888934053961]

- 2.35. Ventilation des dépenses publiques, par fonction de la catégorie "Ordre et sécurité publics ", 2017 [https://doi.org/10.1787/888934053980]

- 2.36. Ventilation des dépenses publiques, par fonction de la catégorie "Affaires économiques ", 2017 [https://doi.org/10.1787/888934053999]

- 2.37. Ventilation des dépenses publiques, par fonction de la catégorie « Enseignement », 2017 [https://doi.org/10.1787/888934054018]

- 2.38 Ventilation des dépenses de l'administration centrale par fonction, 2017 [https://doi. org/10.1787/888934054037]

- 2.39 Ventilation des dépenses des administrations d'États fédérés par fonction, 2017 [https://doi.org/10.1787/888934054056]

- 2.40 Ventilation des dépenses des administrations locales par fonction, 2017 [https://doi. org/10.1787/888934054075]

- 2.42. Ventilation des dépenses de l'administration centrale par opération économique, 2017 et 2018 [https://doi.org/10.1787/888934054094]

- 2.45. Évolution de la répartition des recettes publiques entre les niveaux d'administration, de 2007 à 2017 [https://doi.org/10.1787/888934054113] 
- 2.46. Évolution de la ventilation des dépenses des administrations publiques entre les niveaux d'administration, de 2007 à 2017 [https://doi.org/10.1787/888934054132]

- 2.50. Investissement public en pourcentage de l'investissement total, 2007 et 2017 [https:// doi.org/10.1787/888934054151]

- 2.51. Composition de l'investissement des administrations publiques par fonction, 2017 [https://doi.org/10.1787/888934054170]

- 2.55. Composition des dépenses d'externalisation des administrations publiques, 2017 et 2018 [https://doi.org/10.1787/888934054189]

- 2.58. Ventilation des dépenses publiques par fonction de la catégorie " Protection sociale ", 2017 [https://doi.org/10.1787/888934054208]

- 2.59. Ventilation des dépenses publiques, par fonction de la catégorie « Santé », 2017 [https:// doi.org/10.1787/888934054227]

- 2.60. Évolution de la ventilation des dépenses publiques par fonction de la catégorie "Protection sociale », de 2009 à 2017 [https://doi.org/10.1787/888934054246]

- 2.61. Évolution de la ventilation des dépenses publiques par fonction de la catégorie "Santé ", de 2009 à 2017 [https://doi.org/10.1787/888934054265]

\section{Chapitre 4. Institutions}

- 4.3 Rôle du centre de gouvernement et d'autres entités dans le préexamen des questions soumises au conseil des ministres, 2016 [https://doi.org/10.1787/888934054284]

- 4.4 Mécanismes de coordination des politiques, 2016 [https://doi.org/10.1787/888934054303]

- 4.7 Étapes du cycle de l'action publique auxquelles le centre de gouvernement consulte les parties prenantes, 2016 [https://doi.org/10.1787/888934054322]

- 4.10 Les cadres juridiques dans lesquels s'inscrit l'évaluation générale des politiques publiques, 2018 [https://doi.org/10.1787/888934054341]

\section{Chapitre 5. Pratiques et procédures budgétaires}

- 5.15. Prise en compte des conséquences environnementales et climatiques dans les projets d'infrastructure publique, 2018 [https://doi.org/10.1787/888934054360]

\section{Chapitre 8. Marchés publics}

- 8.3. Évolution de la ventilation des dépenses des administrations publiques en matière de marchés publics par fonction, de 2012 à 2017 [https://doi.org/10.1787/888934054379]

- 8.4. Ventilation des marchés publics par niveau d'administration 2009, 2015 et 2017 [https:// doi.org/10.1787/888934054398]

\section{Chapitre 11. Servir les citoyens}

- 11.4. Satisfaction des citoyens à l'égard de la police locale, 2018 [https://doi.org/10.1787/ 888934054417]

- 11.32. Bonne mise en application de la justice civile et absence d'influence indue de l'exécutif, 2015 et 2019 [https://doi.org/10.1787/888934054436]

- 11.33. Efficacité/rapidité de décision des tribunaux de justice pénale et degré de recours à la violence pour régler des griefs personnels, 2015 et 2019 [https://doi.org/10.1787/888934054455] 
ANNEXE G

\section{Membres du groupe de direction}

\begin{tabular}{|c|c|c|c|}
\hline Pays & Nom & Titre/position & Ministère \\
\hline Autriche & M. Michael Kallinger & $\begin{array}{l}\text { Chef d'unité pour le } \\
\text { développement administratif }\end{array}$ & Chancellerie fédérale, Service public et innovation administrative \\
\hline Belgique & M. Jacques Druart & $\begin{array}{l}\text { Chef de la coordination } \\
\text { internationale }\end{array}$ & Service public federal, stratégie et appui \\
\hline Canada & Mme. Erica van Wyngaarden & $\begin{array}{l}\text { Analyste, Politique stratégique, } \\
\text { priorités et planification }\end{array}$ & Secrétariat du conseil du Trésor \\
\hline Chili & M. Raimundo Monge & $\begin{array}{l}\text { Chef de division de la coordination } \\
\text { interministérielle }\end{array}$ & Ministère de la présidence \\
\hline Corée & M. Kiyeon Hwang & Conseiller & Délégation permanente de la Corée auprès de l'OCDE \\
\hline Finlande & Mme. Katju Holkeri & $\begin{array}{l}\text { Conseiller financier, département } \\
\text { de la gouvernance publique }\end{array}$ & Ministère des finances \\
\hline France & M.Daniel Yves Taupenas & $\begin{array}{l}\text { Conseiller en gouvernance } \\
\text { publique, affaires sociales et } \\
\text { développement régional }\end{array}$ & Répresentation permenente de la France auprès de l'OCDE \\
\hline Hongrie & Mme. Zsuzsanna Gregor & Première secrétaire & Délegation permanente de la Hongrie auprès de l'OCDE \\
\hline Italie & M. Stefano Pizzicanela & $\begin{array}{l}\text { Directeur des relations } \\
\text { internationales }\end{array}$ & Départament de l'administration publique \\
\hline Japon & M. Maki Takashi & Premier secrétaire & Délégation permanente du Japon auprès de l'OCDE \\
\hline Norvège & M. John Noseid & Conseiller principal & Agence pour la gestion publique et l'administration numérique \\
\hline Pays-Bas & M. Frans Van Dongen & Gestionnaire de programme & Ministère de l'Interieur et des Relations extérieures \\
\hline Royaume-Uni & M. Matt Kerlogue & $\begin{array}{l}\text { Chef de l'analyse stratégique } \\
\text { et internationale, Groupe de la } \\
\text { fonction publique }\end{array}$ & Secrétariat du Cabinet \\
\hline Suède & M. Arvid Perbo & Analyste & Agence suédoise de la gestion publique \\
\hline
\end{tabular}




\section{Glossaire}

\section{Termes ou expression}

Administrations publiques

\section{Autorité budgétaire centrale $(\mathrm{ABC})$}

Biens et services collectifs

\section{Utilisation dans le Panorama des administrations publiques}

Le secteur des administrations publiques se compose des groupes suivants d'unités institutionnelles résidentes : a) toutes les unités appartenant à l'administration centrale, aux administrations d'États fédérés et aux administrations locales ; b) toutes les institutions sans but lucratif non marchandes contrôlées par des administrations publiques ; c) les régimes de sécurité sociale, qu'il s'agisse d'unités institutionnelles distinctes ou de parties d'une administration centrale, d’État fédéré ou locale.

Le secteur ne comprend pas les sociétés publiques, même quand la totalité du capital de ces sociétés est détenue par des administrations. il ne comprend pas non plus les quasi-sociétés qui sont possédées et contrôlées par des administrations publiques. En revanche, les entreprises non constituées en sociétés qui appartiennent à des administrations publiques et qui ne sont pas des quasi-sociétés restent partie intégrante de ces administrations et elles doivent donc être incluses dans le secteur des administrations publiques (Système de comptabilité nationale 2008).

L'Autorité budgétaire centrale $(A B C)$ est une entité publique (ou plusieurs entités coordonnées) située au niveau de l'administration centrale, nationale ou fédérale et chargée de tenir et de gérer le budget national ou fédéral. Dans de nombreux pays, l'ABC est rattachée au ministère des Finances. Ses responsabilités précises peuvent varier selon les pays mais, de façon générale, l'ABC est chargée de formuler les propositions budgétaires, de mener les négociations budgétaires, d'affecter ou de réaffecter les ressources, de veiller au respect des textes budgétaires et de procéder à des évaluations des performances ou à des examens de l'efficience. l'Autorité régule l'exécution du budget mais n'assume pas nécessairement de fonction de gestion de la trésorerie l'amenant à procéder à des paiements à partir de fonds publics. Enfin, elle joue un rôle très important en suivant et en contrôlant la discipline budgétaire d'ensemble (nationale) et en assurant le contrôle des dépenses effectuées à partir du budget.

Biens et services qui bénéficient à la communauté dans son ensemble. Les dépenses publiques consacrées à la défense et au maintien de l'ordre relèvent des biens et services collectifs. 
Biens et services individuels

Budget

Budget du citoyen

Cadre général d'emploi de la fonction publique

Centre de gouvernement (COG)

Confiance

Efficacité
Biens et services bénéficiant avant tout aux individus. Tel est, par exemple, le cas des programmes d'éducation, de santé ou d'assurance sociale.

État exhaustif des plans financiers de l'État présentant les dépenses, les recettes, le déficit ou l'excédent et la dette. Le budget constitue le principal document de politique économique du gouvernement, illustre comment le gouvernement prévoit de mettre les ressources publiques au service de ses objectifs et, dans une certaine mesure, traduit ses priorités.

Un «budget du citoyen » est défini ici comme une synthèse en langage simple des principaux volets du budget annuel présenté au parlement. Ce document devrait se suffire à lui-même et expliquer la teneur du projet de budget annuel et les effets escomptés. Même si ce guide comporte des liens vers ou des références à des documents plus détaillés, le lecteur devrait pouvoir le comprendre sans être obligé de se référer à ces autres documents ou d'en connaître la teneur.

Il énonce généralement les conditions d'emploi de la plupart des agents publics et, en tout état de cause, de la plupart des agents statutaires. Selon cette définition, les agents occasionnels ne relèvent pas du Cadre général d'emploi de la fonction publique. Il convient de noter que, dans un certain nombre de pays, tous les agents, y compris ceux qui sont recrutés pour une courte durée, relèvent du Cadre général d'emploi de la fonction publique, à quelques exceptions près (il y a peu, voire pas d'agents occasionnels dans ce genre de cas).

L'expression " centre de gouvernement " désigne la structure administrative qui est au service de l'exécutif (c'est-à-dire du Président ou du Premier ministre ainsi que du Cabinet dans son ensemble). Le centre de gouvernement connaît des appellations très diverses selon les pays: secrétariat général, secrétariat du Cabinet, chancellerie, bureau ou ministère de la présidence, bureau du Conseil des ministres, etc. Dans de nombreux pays, le centre de gouvernement est composé de plusieurs unités aux fonctions diverses. Le rôle du centre de gouvernement est étroitement lié à celui du pouvoir exécutif lui-même, c'est-à-dire orienter les ressources de l'État (financières, législatives, réglementaires et même militaires) afin d'accomplir une mission reflétant une vision politique et correspondant à un mandat des citoyens.

On considère de manière générale qu'avoir confiance, c'est avoir une opinion positive de l'action d'un individu ou d'une organisation. La confiance conduit à considérer que l'on peut compter sur l'autre pour agir conformément à nos attentes dans des circonstances précises. Bien que la confiance puisse être fondée sur l'expérience personnelle, il s'agit, dans la plupart des cas, d'un phénomène éminemment subjectif.

Degré de réalisation des objectifs définis pour une activité donnée (OECD Glossary of Statistical Terms, en anglais uniquement). 
Efficience

Emploi total

Ensemble de données

Équivalent temps plein (ETP)

État fédéral

États unitaires

Fonctionnaire

Genre

Gouvernance

Indicateur

Indice composite
Obtention d'un produit maximum à partir d'un niveau déterminé de ressources affecté à l'exercice d'une activité (OECD Glossary of Statistical Terms, en anglais uniquement).

L'emploi total couvre toutes les personnes exerçant une activité productive qui entre dans la nomenclature des activités productives des comptes nationaux. Cela inclut les individus qui, pendant une période de temps pouvant être spécifique et brève, étaient dans les catégories suivantes : employés/salariés et travailleurs indépendants.

Ensemble d'indicateurs ou de variables concernant un sujet unique (la qualité de la réglementation, par exemple).

Nombre d'emplois en équivalent temps plein, défini comme le nombre total d'heures travaillées, divisé par la moyenne annuelle des heures travaillées dans des emplois à temps plein (OECD Glossary of Statistical Terms, en anglais uniquement).

Pays ayant délimité, dans sa constitution, une division de l'autorité politique entre une administration centrale et plusieurs administrations régionales ou d'États fédérés autonomes.

Pays n'ayant pas délimité, dans leur constitution, de division de l'autorité politique entre une administration centrale et plusieurs administrations régionales ou d'États fédérés autonomes. Cependant, les États unitaires peuvent comporter des divisions administratives incluant des niveaux d'administration locaux, provinciaux ou régionaux.

Employé de l'État disposant d'un contrat permanent ou à long terme et qui resterait employé de l'État en cas d'alternance politique. Par ailleurs, les fonctionnaires sont des employés couverts par un cadre juridique public spécifique ou d'autres dispositions spécifiques.

Comportements et attentes résultant d'une construction et d'un apprentissage d'ordre social associés aux personnes de sexe féminin et de sexe masculin. Toutes les cultures interprètent et élaborent les différences biologiques entre femmes et hommes au sein d'un ensemble d'attentes sociales quant aux comportements et aux activités considérés comme acceptables et quant aux droits, aux ressources et au pouvoir attribués aux femmes et aux hommes. Tout comme la race, l'ethnicité et la classe, le genre est une catégorie sociale qui détermine en grande partie les possibilités offertes à l'individu. il influe sur sa participation à la vie sociale et économique.

L'exercice de l'autorité politique, économique et administrative.

« (...) mesure quantitative ou qualitative dérivée d'une série de faits observés pouvant indiquer des positions relatives (par exemple, d'un pays) dans un domaine donné. Évalué à des intervalles réguliers, un indicateur peut indiquer le sens d'une évolution à travers différentes unités et au cours du temps » (Nardo et al. 2005).

Indicateur formé par la compilation d'indicateurs individuels en un indice unique, sur la base d'un modèle sous-jacent (Nardo et al. 2005). 
Informations sur la performance

Institution

budgétaire

indépendante (IBI)

Intrants

Main-d'oeuvre

Portail centralisé

de données

publiques

ouvertes

Processus de

secteur public

Productivité
Les informations sur la performance peuvent provenir aussi bien d'entités publiques que d'entités non publiques et être d'ordre tant qualitatif que quantitatif. Les informations sur la performance consistent en des éléments de mesure/des indicateurs/des données d'ordre général sur les intrants, les processus, les produits et les réalisations des politiques/programmes/organismes publics et elles peuvent être utilisées, in fine, pour évaluer leur efficacité, leur rapport coût-efficacité et leur efficience. Les informations sur la performance peuvent être tirées : de statistiques ; d'états financiers ou de rapports d'activité d'organismes publics ; de rapports sur la performance publiés par des organismes publics ; d'évaluations de politiques, de programmes ou d'organismes ; ou d'examens des dépenses, par exemple.

Organe indépendant financé sur fonds publics et placé sous l'autorité du pouvoir exécutif ou du pouvoir législatif qui contrôle et analyse de manière non partisane les politiques et résultats budgétaires et, dans certains cas, formule des conseils en la matière. Les IBI jouent un rôle prospectif en matière de diagnostic ex ante (à la différence des institutions de contrôle des finances publiques, qui jouent un rôle tout aussi indispensable de contrôle ex post).

Unités de travail, de capital, de biens et de services utilisées dans la production de biens et de services. " En prenant l'exemple des services de santé, les intrants sont définis comme le temps de l'équipe médicale et non médicale, les médicaments, l'électricité et les autres intrants achetés et l'utilisation de capital relative à l'équipement et aux locaux utilisés. » (Lequiller 2005).

La main-d'oeuvre ou population active du moment comprend toutes les personnes qui remplissent les conditions requises pour être incluses parmi les personnes pourvues d'un emploi ou les chômeurs durant une brève période de référence spécifiée (OECD Glossary of Statistical Terms, en anglais uniquement).

Le portail centralisé de données publiques ouvertes des administrations centrales ou fédérales ("guichet unique") constitue un point d'accès unique aux données des administrations publiques. Les données peuvent être consultées, soit directement sur le portail, soit indirectement, l'utilisateur étant redirigé vers l'adresse où se situent les données (le site web d'un ministère par ex.).

Structures, procédures et dispositifs de gestion largement appliqués au sein du secteur public.

La productivité est généralement définie comme le ratio entre une mesure en volume de produit et une mesure en volume d'utilisation d'intrants (OECD Glossary of Statistical Terms, en anglais uniquement). Les économistes distinguent la productivité totale, c'est-à-dire les produits totaux divisés par le ou les intrants (pondérés), de la productivité marginale, c'est-à-dire l'évolution des produits divisée par l'évolution du ou des intrants (pondérés) (Coelli et al. 1999). 
Produit

(" output")

Produit intérieur brut (PIB)

Réalisation

(" outcome")

Règle budgétaire

Secteur public

Services publics
Aux fins de l'évaluation des performances des administrations publiques, les produits sont définis comme les biens ou services fournis par celles-ci (par exemple, les heures d'enseignement assurées, les allocations sociales calculées et versées) (OECD Glossary of Statistical Terms, en anglais uniquement).

Instrument classique de mesure de la valeur des biens et services produits par un pays au cours d'une période donnée. Plus précisément, le PIB peut être défini comme la somme des valeurs ajoutées brutes de toutes les unités institutionnelles résidentes qui exercent des activités de production (augmentée de la partie des impôts moins les subventions sur les produits qui n'est pas déjà incluse dans l'évaluation de la production); comme la somme des emplois finals de biens et de services (c'est-à-dire de tous les emplois à l'exception de la consommation intermédiaire), mesurés aux prix d'acquisition, diminuée de la valeur des importations de biens et de services; ou comme la somme des revenus primaires distribués par les unités productrices résidentes (OECD Glossary of Statistical Terms, en anglais uniquement).

Fait référence à ce qui est obtenu, en dernière analyse, grâce à une activité. Les réalisations reflètent les résultats, souhaités ou non, de l'action des administrations, mais des facteurs extérieurs à l'action des administrations entrent également en jeu (OECD Glossary of Statistical Terms, en anglais uniquement).

Aux fins du présent ouvrage, l'OCDE emploie une définition similaire à celle de la Commission européenne. Une règle budgétaire numérique consiste en une contrainte pesant à titre permanent sur les agrégats des finances publiques (par exemple, les règles portant uniquement sur l'exercice en cours sont exclues).

Le secteur public comprend les administrations publiques et les sociétés publiques. Les quasi-sociétés qui sont contrôlées par des administrations publiques sont regroupées avec les sociétés dans le secteur des sociétés non financières ou dans le secteur des sociétés financières et font donc partie des sociétés publiques (Système de comptabilité nationale 2008).

Services réalisés au bénéfice de la population ou des institutions publiques. Les administrations assurent des services publics aux administrés, soit directement (dans l'enceinte du secteur public), soit en finançant la prestation des services en question par le secteur privé. La notion de service public est associée à un consensus social selon lequel certains services doivent être accessibles à tous les citoyens, indépendamment de leurs ressources financières. Même quand des services publics ne sont ni assurés, ni financés par des acteurs publics, ils sont généralement assujettis, pour des raisons d'ordre social et politique, à une réglementation plus poussée que la plupart des secteurs économiques. 
Système de comptabilité nationale

Système européen des comptes

Transferts monétaires

Variable
Le Système de comptabilité nationale (SCN) se compose d'une série cohérente de comptes macro-économiques, de comptes de patrimoine et de tableaux articulés et coordonnés qui s'appuient sur un ensemble de concepts, définitions, nomenclatures et règles de comptabilisation approuvés au plan international. En 2009, la Commission de statistique des Nations unies a adopté un ensemble révisé de normes internationales pour la compilation des comptes nationaux : le Système de comptabilité nationale 2008, qui se substitue à la version 1993 du SCN. Pour en savoir plus sur la révision récente du cadre du SCN et sa mise en œuvre par les pays, se référer à l'Annexe A.

Cadre comptable compatible au plan international utilisé par les membres de l'Union européenne pour décrire de façon systématique et détaillée une économie totale (c'est-à-dire une région, un pays ou un groupe de pays), ses composantes et ses relations avec d'autres économies totales (OECD Glossary of Statistical Terms, en anglais uniquement). Ce cadre comptable est pleinement compatible avec le Système de comptabilité nationale (SCN).

Prestations versées par les administrations aux individus qui y ont droit et n'ayant pas à être consacrées à un bien ou à un service spécifique. Les transferts monétaires englobent, par exemple, les pensions, les allocations chômage et l'aide au développement.

Caractéristique d'une unité observée pouvant revêtir diverses valeurs et à laquelle peut être assignée une mesure numérique ou une catégorie relevant d'une classification (par exemple, revenu, âge, poids, etc., et " profession ", " secteur ", " maladie », etc.) (OECD Glossary of Statistical Terms, en anglais uniquement). 



\section{Panorama des administrations publiques 2019}

Le Panorama des administrations publiques fournit des données fiables et comparables à l'échelle internationale sur les activités des administrations publiques et leurs résultats au sein des pays membres de l'OCDE. Dans la messure du possible, elle présente aussi des données pour l'Afrique du Sud, le Brésil, la Chine, la Colombie, le Costa Rica, la Fédération de Russie, l'Inde et l'Indonésie. Cette revue est la seule source de données disponibles dans certains domaines de la gouvernance publique. Elle couvre les intrants, les processus et les indicateurs sur les produits et réalisations obtenues ainsi que des informations contextuelles sur chaque pays.

L'édition 2019 inclut des indicateurs portant sur les intrants qui sont axés sur les finances publiques et l'emploi public ; les processus incluent des données sur les institutions, les pratiques et procédures budgétaires, la gestion des ressources humaines, la gouvernance de la réglementation, les marchés publics, et l'ouverture et la transition numérique de l'administration. Les réalisations couvrent les principaux résultats des pouvoirs publics (ex. la confiance, la réduction des inégalités) et les indicateurs portant sur l'accessibilité, la réactivité et la qualité des services publics de l'éducation, de la santé et de la justice, ainsi que sur le degré de satisfaction des citoyens à leur égard. Les indicateurs de gouvernance sont particulièrement utiles pour surveiller et évaluer le progrès des administrations publiques dans les réformes du secteur public.

Veuillez consulter cet ouvrage en ligne : $h$ ttps://doi.org/10.1787/8be847c0-fr.

Cet ouvrage est publié sur OECD iLibrary, la bibliothèque en ligne de l'OCDE, qui regroupe tous les livres, périodiques et bases de données statistiques de l'Organisation.

Rendez-vous sur le site www.oecd-ilibrary.org pour plus d'informations. 